University of Louisville ThinkIR: The University of Louisville's Institutional Repository

Electronic Theses and Dissertations

$8-2019$

\title{
Opioid and stimulant use among a sample of corrections-involved drug users : seeking an understanding of high-risk drug decisions within a system of constraint.
}

Kirsten Elin Smith

University of Louisville

Follow this and additional works at: https://ir.library.louisville.edu/etd

Part of the Other Social and Behavioral Sciences Commons

\section{Recommended Citation}

Smith, Kirsten Elin, "Opioid and stimulant use among a sample of corrections-involved drug users : seeking an understanding of highrisk drug decisions within a system of constraint." (2019). Electronic Theses and Dissertations. Paper 3259.

https://doi.org/10.18297/etd/3259

This Doctoral Dissertation is brought to you for free and open access by ThinkIR: The University of Louisville's Institutional Repository. It has been accepted for inclusion in Electronic Theses and Dissertations by an authorized administrator of ThinkIR: The University of Louisville's Institutional Repository. This title appears here courtesy of the author, who has retained all other copyrights. For more information, please contact thinkir@louisville.edu. 
OPIOID AND STIMULANT USE AMONG A SAMPLE OF CORRECTIONS-INVOLVED DRUG

USERS: SEEKING AN UNDERSTANDING OF HIGH-RISK DRUG DECISIONS WITHIN A SYSTEM

OF CONSTRAINT

By

Kirsten Elin Smith

B.S.W. University of Kentucky, 2014

M.S.W. University of Kentucky, 2015

\begin{abstract}
A Dissertation
Submitted to the Faculty of the

Raymond A. Kent School of Social Work of the University

of Louisville in Partial Fulfillment of the Requirements for

the Degree of

Doctor of Philosophy in Social Work
\end{abstract}

Social Work

University of Louisville

Louisville, Kentucky

August 2019 
Copyright 2019 by Kirsten E. Smith

All rights reserved 



\section{OPIOID AND STIMULANT USE AMONG A SAMPLE OF CORRECTIONS-INVOLVED DRUG}

USERS: SEEKING AN UNDERSTANDING OF HIGH-RISK DRUG DECISIONS WITHIN A SYSTEM

\section{OF CONSTRAINT}

\section{By}

Kirsten Elin Smith

B.S.W. University of Kentucky, 2014

M.S.W. University of Kentucky, 2015

A Dissertation Approved on

July 16, 2019

By the following Dissertation Committee:

\begin{tabular}{c}
\hline Adrian Archuleta, Ph.D., chair \\
\hline Martin T. Hall, Ph.D. \\
\hline George E. Higgins, Ph.D. \\
\hline Thomas Lawson, Ph.D. \\
\hline Michele Staton, Ph.D.
\end{tabular}




\section{DEDICATION}

To the many people addicted to drugs who are unquestioningly blamed for the harms that they suffer and for the harms they cause others to suffer; for those punished not once, but twice, for that which they could not help.

My family, who kept me alive through the worst of times, and in spite of myself.

My husband, Jesse, a man of infinite patience and love.

My grandfather, Randolph Onus Boring, who left the only copy of his dissertation on a bus, thus inspiring me to do otherwise. 


\section{ACKNOWLEDGMENTS}

This dissertation was completed only as a result of the support and direction from many important people. Foremost, I would like to recognize the incredible guidance, insight, and energy given to me by my committee chair, Dr. Adrian Archuleta. Prior to helping curate this dissertation process, Dr. Archuleta, as an instructor, nurtured my potential as an academic. As his student, I began developing several important skills that were essential in helping complete this project. Though always earnest, forthright, and unfailing in his rapid-fire insight and wit, Dr. Archuleta was exceedingly generous with his time throughout this process. His strategic blend of encouragement and pessimism was given to me in the quality and quantity that I needed at critical points and, without which, I would have been lost. Since I have known him, Dr. Archuleta has always taken the time to nurture my strengths and help me learn how to gird my more unreasonable instincts. He continually gave of himself so that my best tendencies could be developed, and

my worst tendencies could be refocused into what was useful and needed for a given task. For this, I thank him.

I would next like to thank Dr. Michele Staton, from the University of Kentucky, who has, for many years now, given me the mentorship, time, encouragement, and expertise that I needed. The opportunities that Dr. Staton provided me with changed my path in profound ways. Prior to meeting Dr. Staton, my life could have easily been one defined by several unfortunate foregone conclusions; that of another unhappy statistic. Subsequent to meeting and learning from Dr. Staton, my life was transformed into one of unquestionable redemption. An academic of purpose, poise, intelligence, and diligence, Dr. Staton is an exemplar, and a scholar whom I hope to continue to learn from as my career evolves. For all of the many doors that she opened that would have likely otherwise remined shut, I am forever grateful.

I would also like to acknowledge and express my deep gratitude to the other committee members for all of their help, time, and encouragement, both prior to and during the completion of this dissertation: 
Dr. Martin T. Hall, Dr. George E. Higgins, and Dr. Thomas Lawson. Their insight and expertise during this process was instrumental; each gave me exactly what I needed in order to complete this program and this dissertation successfully. More importantly, and more enduringly, each provided me with a knowledge, insight, and opportunity for approaching challenging lessons, and learning from them, so that I could become a more capable, responsible, and thoughtful academic. These scholars provided me with a type of wisdom that transcends this dissertation, and which will undoubtedly change the tenor of my future work for the better. For this, I am truly thankful.

Dr. Riaan van Zyl, while not on this committee, had a profound influence on me when I was his student and is someone I would like to acknowledge. Dr. van Zyl imparted not only expert knowledge and skill, but also modeled a disposition and intellect that is worth emulating. My intellectual curiosity was emboldened by his instruction. Importantly, too, my belief that intellectual curiosity must be coupled with intellectual rigor and intellectual honesty, and then brought to bear on all aspects of life, found a confederate in Dr. van Zyl and reminded me, during moments when I most needed reminding, that I was not alone in this belief.

I also want to acknowledge and thank Dr. Seana Golder for her time and mentorship, and the many other professors and staff in the Kent School of Social Work who made the last few years of my academic life not only possible, but also productive and enjoyable. To them all, I owe a great deal. Thank you, as well, to the University of Kentucky's Center on Drug and Alcohol Research (CDAR) and the Kentucky Department of Corrections for allowing me to examine data collected as part of the Criminal Justice Kentucky Treatment Outcome Study (CJKTOS). In particular, I would like to thank the CJKTOS project manager, Erin Winston and CJKTOS research associate, Martha Tilson, for their assistance in the acquisition of the data and other materials used to complete this project, as well as for their support and much-appreciated good humor.

Lastly, and with great affection, I would like to acknowledge cherished mentorship from two intellectual mutants. First, Robert Walker, M.S.W., the brilliant, exact, and obstinate stoic. Robert Walker changed the way I dared to think from the first conversation forward. Though never a formal student during his professorship at the University of Kentucky, Robert Walker, over many years, taught me a new language, a language of the sort that does not interest most enough to learn. He helped me--joyfully, 
painstakingly--practice speaking it each time we met, all the while suffering my many interruptions and fits. Any success I achieve is a direct result of his investment in me. Second, Dr. David Epstein, the brilliant, optimistic, and generally agreeable eccentric, and chief of the Real-world Assessment, Prediction, and Treatment Unit at the National Institute on Drug Abuse's Intramural Research Program. Dr. Epstein provided me with encouragement, expertise, and valuable insight throughout this process; always reminding me that there was a light at the end of this tunnel. He is a senior scientist whom I admire more than words can express and a mentor who I look forward to many years of creative and productive work with. 


\title{
ABSTRACT \\ OPIOID AND STIMULANT USE AMONG A SAMPLE OF CORRECTIONS-INVOLVED DRUG USERS: SEEKING AN UNDERSTANDING OF HIGH-RISK DRUG DECISIONS WITHIN A SYSTEM OF CONSTRAINT
}

\author{
Kirsten Elin Smith
}

July 16, 2019

In the United States, high-risk drug use remains a significant social problem. Opioids and stimulants are two drug classes that have contributed to substantial recent increases in drug-related arrests, overdose, and mortality. Kentucky has been particularly devastated by high rates of opioid and stimulant use. Opioid and stimulant effects, while highly rewarding, can result in adverse consequences. Still, some people choose to use these drugs, and choose to continue using even after experiencing adverse consequences, such as incarceration. The aim of this study was to explore high-risk drug use among a sample of corrections-involved adults in Kentucky and to identify endogenous and exogenous factors with the potential to have influenced drug-related decision-making prior and subsequent to incarceration. Attention was paid to understanding concomitant opioid and stimulant use and heroin use. Survey data collected as part of an ongoing corrections-based substance use treatment program outcomes study were examined. The final sample $(\mathrm{N}=1,563)$ included adults released into Kentucky counties between 20122017. Non-parametric statistical tests and multinomial logistic regression were used to identify factors associated with opioid, stimulant, and concomitant use; binary logistic regression was used to identify factors associated with heroin use. Results indicate that opioid and stimulant use was endemic in this sample, though rates of use subsequent to incarceration were lower than pre-incarceration rates. 
During the 30-day period prior to incarceration, $29.0 \%$ of participants reported concomitant use, $28.5 \%$ reported opioid use, and $18.0 \%$ reported stimulant use. During the one-year post-release period, $11.9 \%$ of participants reported concomitant use, $12.5 \%$ reported opioid use, and $8.3 \%$ reported stimulant use. During this post-release period, $10.7 \%$ reported heroin use. Concomitant and heroin use positively correlated with many factors with the potential to adversely influence cognition and constrain choice. Similar relationships between many of these factors and outcomes involving other drug or no drug use were not observed.

Behavioral economics, a molar view of choice and behavior, was used to conceptualize how factors in the lives of participants had the potential to influence and constrain decision-making in respect to high-risk drugs. Findings are discussed in light of how they may inform future research, policy, and practice. 
TABLE OF CONTENTS

PAGE

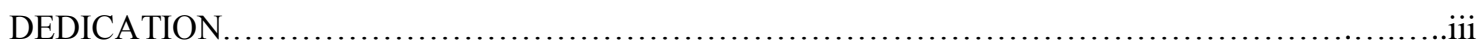

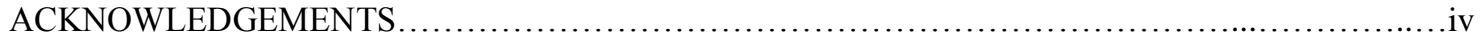

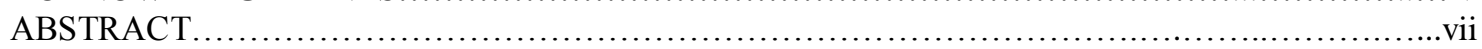

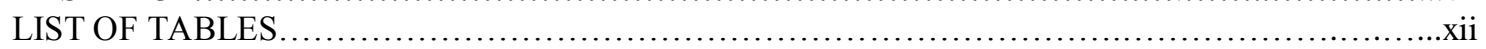

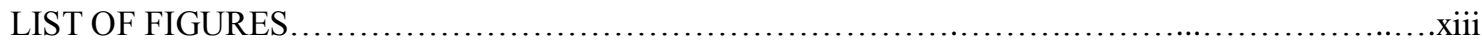

\section{CHAPTER}

I. INTRODUCTION TO SOCIAL PROBLEM AND CONCEPTUAL

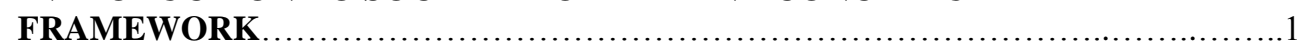

Purpose of Exploratory Study, and the Kentucky Context..................................

Selection and Description of Conceptual Framework..................................... 12

The High-Risk Nature of Opioid and Stimulant Use.......................................20

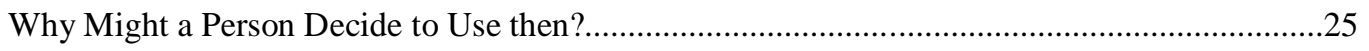

The Road to Hell is Paved with Good Intentions.................................................29

II. SOME INFLUENCES ON DRUG-RELATED DECISION-MAKING AND

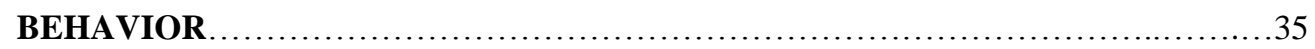

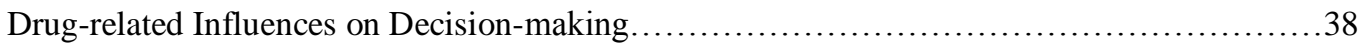

Constraints on Decision-making Capacity Among Opioid and Stimulant Users:

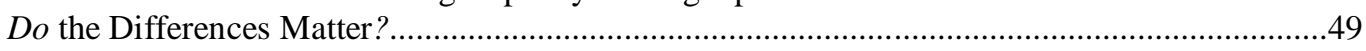

Demographic Characteristics with Potential to Influence Decision-making....................53

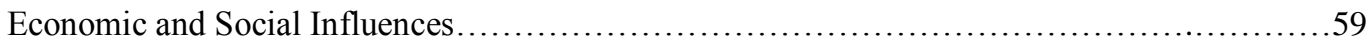

Stress, Psychiatric Symptoms, Pain and Misregulation...................................67

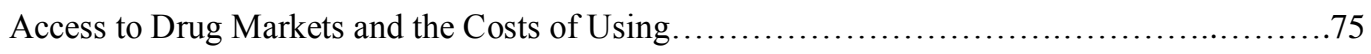

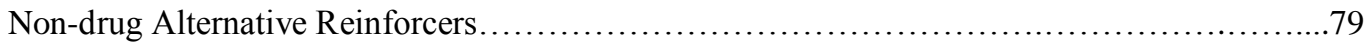


Restricted Opportunity for Accessing Non-Drug Alternatives............................ 84

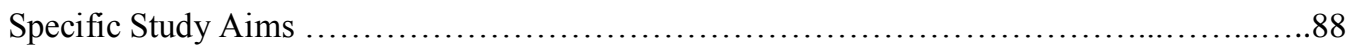

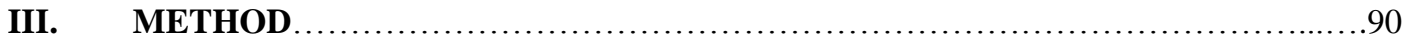

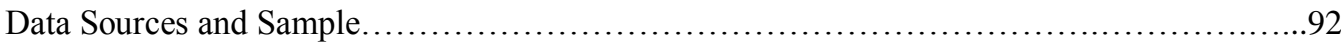

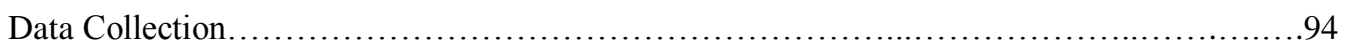

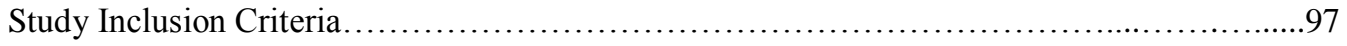

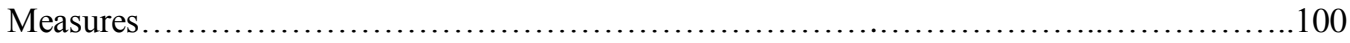

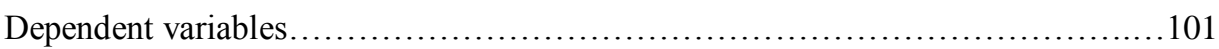

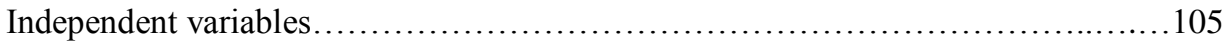

Control variables..............................................................

Descriptive variables........................................................ 120

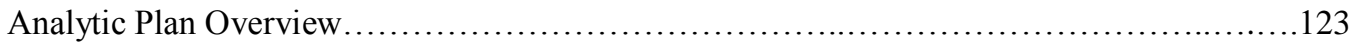

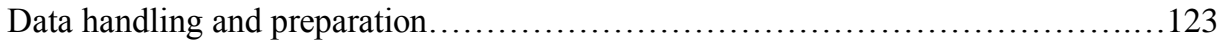

Analytic plan development...................................................124

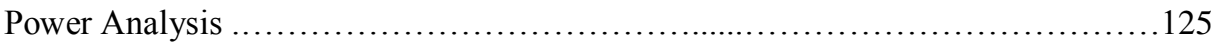

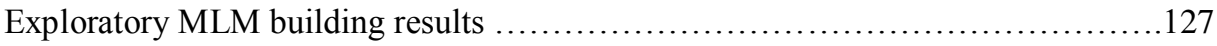

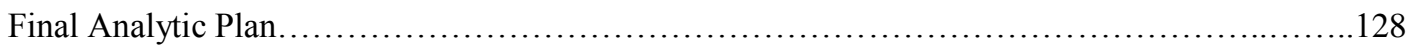

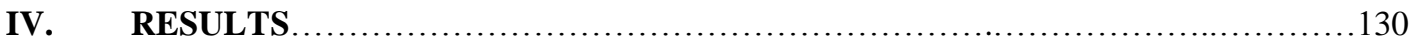

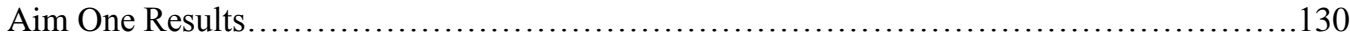

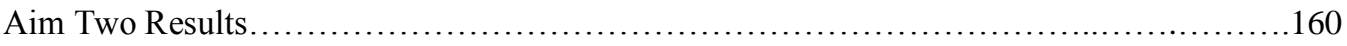

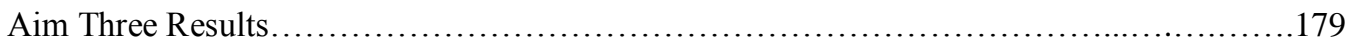

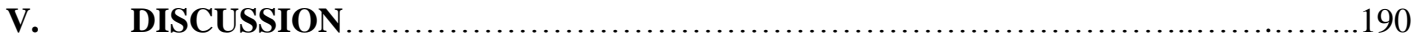

Some Potential Differences in Drug-related Decision-making Prior and Subsequent to

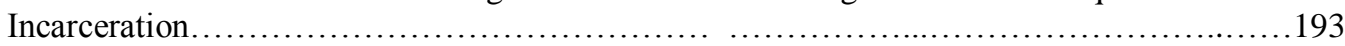

Drug-related Influences on Decision-making.........................................200

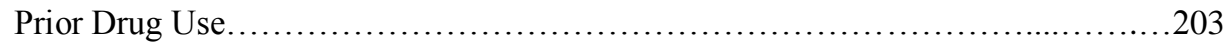

Versatile Drug Use, Preference, and Drug Use Severity............................208

Drug Substitutes, Compliments, and Risk Amidst Changing Markets.......................211

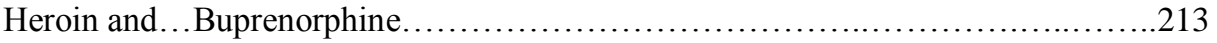


Additional Endogenous Factors with the Potential to Influence Decision-making.....

Non-drug Alternative Reinforcers.

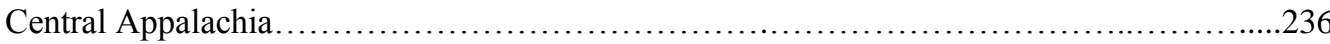

Ambiguous Risk.....................................................240

Broader Implications and Future Directions............................................. 242

The Road Back to Hell is Still Paved with Good Intentions.......................242

Additional Considerations for Future Work...............................245

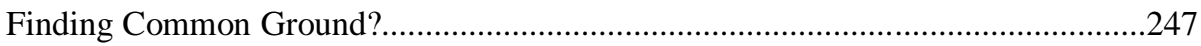

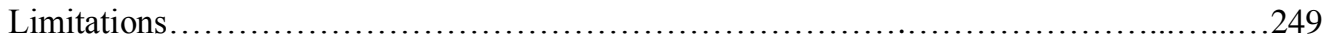

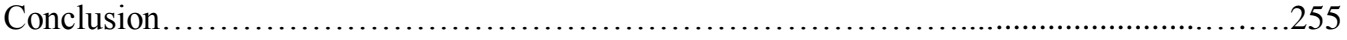

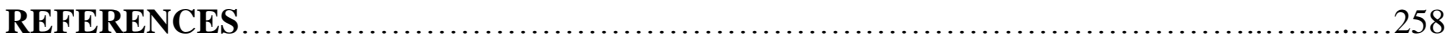

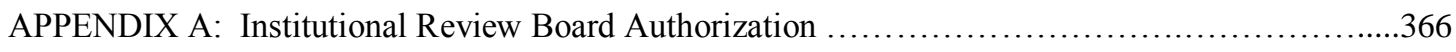

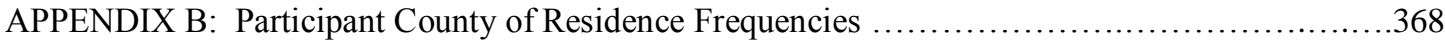

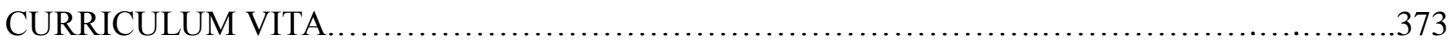




\section{LIST OF TABLES}

TABLE

PAGE

1. Sample Characteristics and Drug Use Group Means, Proportions, and

Between-group Differences Prior to Incarceration.

2. Sample Characteristics and Drug Use Group Means, Proportions, and Between-group Differences Subsequent to Incarceration

3. Results of Multinomial Logistic Regression Examining Opioid and Stimulant

Use During the 30-day Pre-incarceration Period

4. Results of Multinomial Logistic Regression Examining Opioid and Stimulant

Use During the One-year Post-release Period

4.1. Results of Multinomial Logistic Regression Examining Past 30-day

Pre-incarceration Drug Use and Past-year Post-release Drug Use

5. Group Means, Proportions, and Between-group Differences Among Heroin and Non-heroin users for One-year Post-release Period

6. Results from Binary Logistic Regression Examining Heroin Use for the

One-year Post-release Period

6.1. Results of Binary Logistic Regression Examining Past-year Other Drug use and Past-year Heroin Use During One-year Post-release Period 


\section{LIST OF FIGURES}

FIGURE

PAGE

1. Concept model of endogenous and exogenous factors with potential to influence drug-related decision-making

2. CJKTOS sample size for FYs 2013-2017, removal of cases, and final sample size

3. Participant counties of residence prior to incarceration with $\leq 10$ observations per county.

3.1. Participant counties of residence subsequent to incarceration with $\leq 10$ observations per county.

4. Participant counties of residence prior to incarceration with $>10$ observations

per county.

4.1. Participant counties of residence subsequent to incarceration with $>10$ observations per county.

5. Opioid and stimulant use during the 30-day pre-incarceration period by Appalachia and Non-Appalachia Residence.

6. Opioid and stimulant use during the one-year pre-incarceration period by Appalachia and Non-Appalachia Residence.

7. Opioid and stimulant use during the 30-day pre-incarceration period by urban influence designation.

8. Opioid and stimulant use during the one-year post-release period by Appalachia and Non-Appalachia residence.

9. Opioid and stimulant use during the one-year post-release period by urban influence designation.

10. Percent change in odds of past 30-day concomitant use prior to incarceration.................164

11. Percent change in odds of past 30-day opioid use prior to in incarceration. .165 
12. Percent change in odds of past 30-day stimulant use prior to incarceration.

13. Percent change in odds of concomitant use during one-year post-release period.

14. Percent change in odds of opioid use during one-year post-release period

15. Percent change in odds of stimulant use during one-year post-release period.

16. Percent change in odds of concomitant use during one-year post-release period by pre-incarceration drug use.

17. Percent change in odds of opioid use during one-year post-release period by pre-incarceration drug use.

18. Percent change in odds of stimulant use during one-year post-release period by pre-incarceration drug use.

19. Percent change in odds for heroin use during the one-year post-release period.

20. Percent change in odds for heroin use during the one-year post-release period by co-used drug. 


\section{CHAPTER I \\ INTRODUCTION TO SOCIAL PROBLEM \\ AND CONCEPTUAL FRAMEWORK}

\section{High-risk Drug Use}

Risky, or risk-taking, behavior is defined by Turner et al. (2004) as a volitional behavior with a potentially negative outcome in which the danger is recognized. Risk has been further conceptualized as outcome invariance (Real \& Caraco, 1986). For instance, if two choices have equal value, the one with greater variance may be considered to have higher risk (Daly \& Wilson, 2001). Use of addictive drugs is one type of volitional high-risk behavior (Bickel \& Marsch, 2001). During a drug-related decision, use of high-risk drugs is one of many possible behavioral outcomes. Other potential outcomes include the substituted use of a relatively less risky but still desired drug, or no drug use, with the person otherwise engaged in behaviors unrelated to drugs (Vuchinich \& Heather, 2003). The use of addictive high-risk drugs constitutes a significant economic, social, legal, and medical problem in the United States (Degenhardt et al., 2014; Schuchat, Houry, \& Guy, 2017; Volkow \& Collins, 2017). Although use of any drug entails some risk for the person using it, certain drugs are associated with comparatively greater risk. Opioids (e.g., heroin, non-medical prescription opioids) and psychostimulants (e.g., amphetamines, cocaine) are two drug classes that have several unique risks associated with their use, making them potentially more dangerous in both the short- and long-term, relative to other licit drugs (e.g., caffeine, nicotine, kratom, alcohol), prescription drugs (e.g., benzodiazepines), and illicit drugs (e.g., cannabis, psilocybin, LSD) (Bachi, Sierra, Volkow, Goldstein, \& Alia-Klein, 2017; Chen, Storr, \& Anthony, 2009; Paulozzi, 2012; Tomassoni et al., 2017). For instance, analyzing 1991-2011 National Health Interview Survey data, Walker et al. (2017) found that among a nationally representative sample, lifetime use of heroin and cocaine was significantly associated with excess mortality risk. More broadly, opioids and stimulants account for a disproportionate 
number of drug overdose fatalities in the US. (Jalal et al., 2018). Examining data for drug-related overdose deaths between 1979-2016, Jalal et al. (2018) found exponential growth for all drug-related overdose fatalities, with peak mortality rates for drug classes distinguished by time, geographic region, and population characteristics (e.g., sex, race). Heroin, semisynthetic prescription opioids (e.g., oxycodone), and synthetic opioids (e.g., fentanyl) contributed to a significant portion of accidental overdose deaths, as did cocaine and amphetamine drugs. Between 2016 and 2017, opioid overdose fatalities increased approximately 30\% (Vivolo-Kantor et al., 2010). Presently in the US, 130 people a day die from an opioidrelated overdose, with opioid misuse comprising a nearly $\$ 80$ billion a year cost (Centers for Disease Control and Prevention [CDC]). Since, 2012, cocaine-related overdose fatalities have also resulted in significant losses, particularly among non-Hispanic Blacks (Shiels, Freedman, Thomas, \& de Gonzalez 2018).

Considering this nearly 40 -year exponential growth, two relevant conclusions might be made. First, the current "opioid epidemic" may be considered one of many interconnected drug epidemics, albeit a larger and possibly more insidious one, due to the continued need for analgesic drugs in the treatment of chronic pain in the US (Bowser, Fullilove, \& Word, 2017; Dowell, Haegerich, \& Chou, 2016). Second, both stimulants and opioids have been leading causes of drug-related overdose fatalities historically.

Evidence suggests that in addition to stimulant-related overdose fatalities, stimulants may soon increase as a contributor of non-overdose drug-related fatalities, as many people who developed problematic cocaine and amphetamine use in the 1970s-1990s are growing older and are therefore more likely to start experiencing delayed consequences of use (Levandowski et al., 2016; Shiels et al., 2018; Yarnell, 2015). Taking a broader view of the problem of high-risk drug use in the US, rather than considering separable high-risk drug epidemics, it becomes clear that both opioid and stimulant use persist as leading contributors to poor outcomes for users and that both remain complicated social, economic, medical, and legal challenges to understand and address (Plunk, Grucza, \& Peglow, 2018).

\section{Opioid and Stimulant Use in the United States}

\section{Opioid Use}

Opioids are defined here as any natural (i.e., derived the poppy [Papaveraceae] family; e.g., codeine, morphine, heroin), semi-synthetic (i.e., substances produced by chemical synthesis; e.g., 
OxyContin, Suboxone), or fully-synthetic (e.g., fentanyl, methadone) substance that acts as a partial or full agonist at one of the primary human opioid receptors ( $\mu$-opioid receptor, MOR; $\delta$-opioid receptor, DOR; and $\mathrm{\kappa}$-opioid receptor, KOR) and results in physiological and psychoactive effects (Feng et al., 2012; Pergolizzi, LeQuang, Berger, \& Raffa, 2017; Taber, Neubert, \& Rheingold, 2002). In humans, opioids produce both analgesic and euphoric effects (Webster, Johnson, Stauffer, Setnik, \& Ciric, 2011). These effects are rewarding and memorable, and therefore behaviorally reinforcing, whereby people may be motivated to use again, even when it requires considerable effort or expense (Everitt \& Robbins, 2005). Adverse consequences associated with opioid use and misuse have been studied and speculated about for some time; though the full scope of possible short- and long-term consequences is still incompletely understood (Baldo, 2016; Cheng \& Lee, 2016; Kreek, 1996; Laorden, Fuertes, GonzálezCuello, \& Milanés, 2000). Given the scale of opioid use and misuse in the US, adverse consequences cannot help but have an outsized effect if left insufficiently understood and addressed (Epstein, Heilig, \& Shaham, 2018) In 2017, an estimated 6.0 million people in the US aged 12 and older reported past-month illicit prescription opioid use and nearly half a million of this same age group reported past-month heroin use (Substance Abuse Mental Health Services Administration [SAMHSA], 2017). Over the past two decades, rates of opioid prescribing and prescription opioid diversion increased, contributing to increases in opioid-related mortalities (Brady et al. 2015; Dart et al. 2015; Kolodny et al. 2015; Oquendo \& Volkow, 2018). In 2012 the annual prescribing rate for opioids peaked at 81.3 per 100 persons and remains elevated at a rate of 58.5 per 100 persons as of 2017 (CDC 2019).

Wide-ranging efforts have been made over the past decade to address prescription opioid misuse. These have included the dismantling of predatory "pill mills", increased access to treatment for opioiddependent offenders, harsher criminal sanctions for opioid dealers, revised CDC opioid prescribing guidelines, mandated prescriber participation in drug monitoring systems, and scientific advances in abusedeterrent opioid pill formulations (CDC, 2018; Freeman, Goodin, Troske, \& Talbert, 2015; Lin et al. 2018; Makary et al. 2017; McCarthy 2017; Powell and Pacula, 2017; Worley, 2012). Despite these efforts, heroin use in the US has increased significantly since 2010 (Compton et al. 2016; Kanouse \& Compton, 2015). Both prescription opioid and heroin use persist as significant public health threats. Between 1999 and 2016 the age-adjusted rate of drug overdose deaths involving natural and semisynthetic opioids (e.g., oxycodone) 
increased from $1.0 \%$ to $4.4 \%$ (Hedegaard, Warner, \& Miniño, 2017). Heroin and fentanyl-adulterated heroin overdose mortalities have also increased, with over 15,000 Americans dying from heroin-related overdose in 2017 alone (CDC, 2017b). Use of diverted buprenorphine (i.e., Suboxone) has also become a concerning new phenomenon within the broader opioid crisis (Molfenter et al., 2019).

Among high-risk drugs of abuse, opioids are more likely than other drugs to result in overdose and overdose-related mortalities, and also to be perceived by survivors as comparatively worse overdose experiences (Bohnert et al., 2018; Dowell, Noonan, \& Houry, 2017). As the opioid-related death toll has increased in the US, no socioeconomic or demographic group has been spared and no region has been left unaffected (Alexander, Barbieri, \& Kiang, 2017; Plunk et al., 2018; Shiels et al., 2018). Areas in the southeastern US, including regions within and adjacent to Central Appalachia, have been particularly impacted by prescription opioid and heroin use over the past two decades (Jonas et al. 2012; Moody, Satterwhite, \& Bickel, 2017; Slavova et al. 2017; Staton et al. 2018).

One result of the dramatic uptick in opioid-related mortalities over a short period of time is that increased attention is being paid to the problem and more public resources are being devoted to addressing it (Collins, Koroshetz, \& Volkow, 2018; Madras, 2017). Yet, even with increased attention and resources aimed at understanding and mitigating the current opioid epidemic, some of the most fundamental and enduring questions about the factors with the potential to influence a person's decision to use opioids despite associated risks, and to persist in use despite adverse consequences, remain insufficiently answered.

\section{Psychostimulant Use}

Psychostimulants are defined here as any natural (i.e., derived from coca [Erythroxylaceae] family; e.g., cocaine), natural but processed (e.g., hydrochloride salt cocaine, bicarbonate of sodium solution, a.k.a. "crack" cocaine), or synthetic (e.g., 3,4-methylenedioxymethamphetamine, pharmaceutical methamphetamine) substance that acts primarily on human dopamine and serotonin receptors and results in physiological and psychoactive effects. (Johanson \& Fischman, 1989; Kalivas, 2007; Koob, 1992). Stimulants produce a range of effects, including increased alertness, euphoria, locomotor activity, endurance, and arousal (Rush \& Baker, 2001; Rush, Stoops, \& Sevak, 2010; Uhl, Halll, \& Sora, 2002).

As of 2017 in the US, an estimated 2.2 people aged 12 or older reported past-month powder and/or crack cocaine use, while 1.8 million reported past-month prescription stimulant use, and 774,000 reported 
past-month methamphetamine use (SAMHSA, 2017). Nationally, there has been an upward trend in the use of stimulant drugs. For instance, the number of people aged 12 or older who reported past-month cocaine use increased from 1.6 million in 2012 to 2.2 million in 2017, an increase of over half a million people (SAMHSA, 2012). There have been slight increases in prescription stimulant and methamphetamine use as well. For example, between 2012 and 2017, the number of people aged 12 or older reporting past-month prescription stimulant use increased from 1.2 million to 1.8 million, whereas past-month amphetamine use among people 12 or older increased between 2012 and 2017, from approximately 440,000 to 774,000, respectively (SAMHSA, 2012, 2017).

Cocaine's addictive potential has been well-documented for decades; however, increases in use in the 1970s and 1980s resulted in greater attention among researchers, medical professionals, and law enforcement (Associated Press, 1982; Fagan \& Chin, 1989; Gawin, Allen, \& Humblestone, 1989; Marriott, 1989). This was partially a result of a sharp increase in cocaine-related emergencies and mortalities. Rates of cocaine-related deaths increased four-fold between 1976-1981 (CDC, 1982). In 1985, the smokable derivative of cocaine, "crack", emerged as a serious drug of concern across many communities, particularly metro areas and within communities with a disproportionate number of people of color (Fryer, Levitt, \& Murphy, 2013). Between the late 1980s and early 1990s, the total number of cocaine users in the US increased, in part due to new adopters of crack cocaine (Cornish \& O'Brien, 1996; O'Malley, Bachman, \& Johnston, 1988). This increase contributed to a rippling of adverse social, legal and health consequences (including drug-related violence) for users, their families, and their communities (Blumstein, 1995; Fagan, 1994; MacGregor et al., 1987).

Availability and use of diverted prescription stimulants, such as methamphetamine, methylphenidate (i.e., Ritalin), or dextroamphetamine (i.e., Adderall), increased in the 1990s and 2000s as these drugs became more widely used in the pharmacotherapy of attention deficit disorders (Wilens et al., 2008). In the 1990s, clandestine labs, spanning drug cartels to home kitchens, began producing "street meth" (e.g., "crystal meth", “ice”, or "stove top meth") (Cunningham, Liu, \& Callaghan, 2009; Maxwell \& Rutkowski, 2008; Zernike, 2006). Thereafter, the availability and use of methamphetamine increased, becoming a fixture of many communities across the US, though use has often been more prevalent in smaller, mid-sized, and rural communities (Dombrowski, Crawford, Khan, \& Tyler, 2016; Habecker, 
Welch-Lazoritz, \& Dombrowski, 2018; Lambert, Gale, \& Hartley, 2008; Roussell, Holmes, \& AndersonSprecher, 2013).

\section{Concomitant Drug Use}

Another form of high-risk drug use includes concomitant use of more than one drug (Zoorob, 2018). Polydrug use is a well-documented phenomenon among people with SUD, versus the general population, who may be more likely to use drugs recreationally, in moderation, and individually (verses concomitantly); however, less is known about controlled users, as they are seldom institutionalized and studied (Zinberg, 1984; Zinberg, Harding, \& Winkeller, 1977; Waldorf, Reinarman, \& Murphy, 1992). Oftentimes, people with SUD also present comorbidly with at least one other SUD (Anthony, Warner, \& Kessler, 1994; Kandel, Chen, Warner, Kessler, \& Grant, 1997). Polydrug use can include co-ingestion of more than one drug at the same time. For example, drinking alcohol while smoking cannabis, or injecting opioids and stimulants simultaneously (e.g., "speedballing", "goofballing”) (Dolan, Black, Malow, \& Penk, 1991; Latkin, Edwards, Davey-Rothwell, Yang, \& Tobin, 2018). It can also include contemporaneous use of multiple drugs over a particular time period (Kandel, Yamaguchi, \& Chen, 1992).

As opioid and stimulant use constitute high-risk forms of drug use singularly, co-use of these drugs is especially concerning. Specifically, opioids used in combination with other drugs, such as benzodiazepines and alcohol, is more likely to result in depressed respiration and possibly death (Calcaterra, Glanz, \& Binswanger, 2013; Hobelmann \& Clark, 2016; Jones, Mogali, \& Comer, 2012). Similarly, cocaine used in combination with alcohol is known to significantly increase the likelihood of experiencing adverse health effects, including cardiac events and hyperthermia (McCance-Katz et al., 1993). When used together, opioids and stimulants demonstrate more potent effects than either drug used alone, in part by synergistically increasing euphoria and decreasing unpleasant subjective side effects produced by each drug, a result that can increase overall abuse potential and encourage continued dosing (Jasinski \& Preston, 1986; Guzman \& Ettenberg, 2004; Leri, Bruneau, \& Stewart, 2003). The co-use of opioids and stimulants, popular among many polydrug users, is associated with increased likelihood of fatal and non-fatal overdose (Coffin et al., 2003; Kaye \& Darke, 2004; Warner, Trinidad, Bastian, Miniño, \& Hedegaard, 2016). 
While there may be some important within-group homogeneity across substance users, there may also be some considerable heterogeneity, including among polydrug users generally and polydrug users who use opioids and stimulants specifically (Bickel, Crabbe, \& Sher, 2010; Shaw, Shah, Jolly, \& Wylie, 2008, Terracciano, Löckenhoff, Crum, Bienvenu, \& Costa, 2008; Tetrault et al., 2008). This cautions against drawing overly broad generalizations about between- or within-group differences based on type of drug used. It is likely that people who use multiple drugs over a given time period or during a single drugtaking episode, despite any overall tendency toward versatile drug use, are influenced by a variety of factors. It is also likely that polydrug users may indicate greater preference for certain drugs, and continue to choose to use them, even as they also choose to use other, less preferred drugs (Smith \& Lawson, 2017). It is important to keep in mind that concomitant drug use, and particularly concomitant use of opioids and stimulants, entails extreme risk. Such risk has increased in recent years as fentanyl and fentanyl analogues have proliferated in the illicit drug supply, and are now found as additives and adulterants in opioid and stimulant drugs alike (Dai, Abate, Smith, Kraner, \& Mock, 2019; Gladden, 2016; Nolan, Shamasunder, Colon-Berezin, Kunins, \& Paone, 2019; O’Donnell et al., 2017).

\section{Purpose of Exploratory Study, and the Kentucky Context}

Because of the risks associated with opioids, stimulants, and concomitant use of opioids and stimulants, it is important to work toward developing a better understanding of what factors have the potential to influence decisions to use these high-risk drugs. This includes considering not only what factors may have influenced initial drug use, but also what factors may influence continued decisions to use high-risk drugs among adults with established histories of drug use and other high-risk behaviors.

Although specific study aims will be introduced in the following chapter, the primary purpose of this study is to explore high-risk drug use among a sample of corrections-involved adults in Kentucky and to identify and discuss endogenous and exogenous factors with the potential to have influenced drugrelated decision-making prior and subsequent to incarceration. This will be achieved by identifying the prevalence of opioid and stimulant use and correlates of use. Of interest is identifying what individual (endogenous) and contextual (exogenous) factors were present in the lives of participants that had the potential to influence decisions to use high-risk drugs during both time periods. 
Kentucky is an ideal state for such exploration, as it has experienced high opioid prescribing and diversion rates and continues to experience historically high rates of opioid-related morbidities and mortalities, in both rural and urban areas (CDC 2017b; Faryar et al. 2017; Hall et al. 2013; Kentucky Office of Drug Control Policy 2016; Luu et al. 2018). In 2016, the number of opioid-related overdose fatalities in Kentucky increased to 33.5 per 100,000, ranking the state fifth highest in the nation (CDC 2017a). Opioid users in Kentucky are situated in an interesting ecological context, where rates of opioid prescribing are comparatively greater to other states, even as heroin use continues to increase (Slavova et al., 2017). In 2016, approximately 176,000 adults in Kentucky reported prescription opioid misuse and 17,000 reported heroin use (SAMHSA, 2017).

Reflective of the broader US, barriers to the equitable provision of comprehensive, scientificallyinformed drug treatment remain in Kentucky (Epstein et al., 2018; Moody et al., 2017; Patrick et al., 2019). The availability of, and access to, medication-assisted treatment (MAT) is highly variable across the state. Although some Kentucky counties have few buprenorphine prescribers and experience challenges to implementing and delivering MAT, others have experienced significant increases in buprenorphine prescribing (Kentucky Cabinet for Health and Family Services, 2018a; Substance Abuse Mental Health Services Administration, 2018). Depending on where a person lives in the state, they may have more or less difficulty accessing MAT and, relatedly, comparatively greater or lesser access to purchasing buprenorphine illicitly. Diverted buprenorphine use remains a growing but underexplored phenomenon in the region and is important to consider, as it constitutes either a form of opioid misuse or medically unsupervised use for self-treatment, both of which are potentially dangerous (Carroll, Rich, \& Green, 2018; Cicero, Ellis, \& Chilcoat, 2018; Daniulaityte, Carlson, Brigham, Cameron, \& Sheth, 2015; Mitchell, Gryczynski, \& Schwartz, 2018; Smith et al., 2019).

In addition to opioids, stimulant use remains a problem in many Southeastern regions, including Kentucky (Courtwright, 1983; Stoops et al., 2007; Stoops, Staton-Tindall, Mateyoke-Scrivner, \& Leukefeld, 2005; Stover, Winstanley, Zhang, \& Feinberg, 2018). As of 2016, approximately 59,000 adults in Kentucky reported cocaine use (SAMHSA, 2017), and methamphetamine use persists across urban and rural regions alike (Croft, Foppe, Huffines, \& Subedi, 2018; Fernandez, Gohmann, \& Pinkston, 2018). 
As is the case in other states, many people in Kentucky who use opioids and stimulants become involved with the criminal justice system (Golder et al., 2014; Hall et al., 2018; Staton, Leukefeld, \& Webster, 2003; Stoops, Staton-Tindall, Mateyoke-Scrivner, \& Leukefeld, 2005). Kentucky’s high rates of opioid and stimulant use have contributed to the state's increased incarceration and community supervision rates, relative to other states. Currently, Kentucky incarcerates 948 people per 100,000, making it eighth in the world for per capita incarceration rates (Prison Policy Initiative, 2018). As of 2017, approximately 63,800 Kentucky adults were on community supervision (Bureau of Justice Statistics, 2018). These rates partially reflect the personal blame sometimes assigned to opioid and stimulant users and the moral and punitive approach historically taken to understanding and addressing drug use (Corrigan, 2000; Crisp, Gelder, Rix, Meltzer, \& Rowlands, 2000; Corrigan \& Wassel, 2008; Matheson et al., 2014). For medically untreated opioid users released from controlled environments, the risks of resumed use can be deadly. Considering findings from a meta-analysis conducted by Merrall et al. (2010), Epstein and colleagues (2018 )concluded that "for addicts who are incarcerated, failure to provide agonist treatment can convert a short jail term to a death sentence."

Because high-risk drug use is a complex behavioral outcome, and not attributable to any one factor, it can be challenging to exculpate or explain to the general public. It may be more challenging to explain a person's continued use despite harsh drug-related consequences, such as incarceration. It is therefore important when exploring high-risk drug use among a sample of corrections-involved adults in Kentucky that endogenous and exogenous factors with the potential to influence a person's decision to use opioids or stimulants despite known risks are examined. However, even if it is presumed that risks associated with opioids and stimulants are completely unknown to people prior to any decision to use them (however unlikely that is), the question of what factors might influence a decision to use even in the absence of perceived risk remains an interesting and important one, as the consequences associated with use are severe, ranging from incarceration to death.

In order to help identify and understand some of the factors with the potential to influence decision-making about high-risk drugs among a sample of corrections-involved drug users in Kentucky, a behavioral economic approach will be used. Though behavioral economics is described in subsequent sections, it is briefly introduced here as an approach that characterizes the allocation of behavior within "a 
system of constraint", with particular emphasis on conditions that influence choice and the consumption of goods, including drugs (Bickel \& Marsch, 2000; Bickel, Green, \& Vuchinich, 1995, p. 258).

\section{Self-reported Opioid and Stimulant Use as Decisional Outcomes}

For this exploratory study, the self-reported use of high-risk drugs, such as opioids and stimulants, will be conceptualized as decisional outcomes, a behavior reflective of prior choice. Given the nature of choice and behavior, it is not possible to identify and measure all of the factors that influence decisionmaking in real-time in the real world, or to retrospectively construct accurate models of how a person came to make decisions. This may be even more difficult for illicit commodities, such as drugs. However, it is possible to discern unitary or aggregated decisions and their behaviors (i.e., decisional outcomes), along with some of the factors that potentially influenced them. This can be accomplished in several ways using available data sources, including self-report data, institutional records (e.g., hospital emergency department admissions), and publicly available statistics (e.g., Drug Enforcement Administration data on drug price, purity, and availability estimates). Decisions pertaining to opioids and stimulants can be recognized post hoc, due to the dichotomized behavioral outcome itself. In other words, use of a drug establishes that a decision about that drug was made. Opioid or stimulant use reported for a given time period indicates that a decision was made to use these drugs at some point during this time period. In the case of repeated or continued use of a given drug over a given time period, multiple decisions would have been made. A decisional outcome for a given time period that does not involve opioid or stimulant use, but rather other drug use or no use, suggests several possibilities. The first possibility is that opioids and stimulants were directly encountered by the person and that the person chose not to use them. The second possibility is that opioids and stimulants were not directly encountered by the person, but that the person still had the potential to contemplate their use (by virtue of knowing that these drugs do exist and are procurable), and that the person decided not to procure and use them.

Examples of the latter possibility abound in the real-world, even if they do not in lab conditions, where decision tasks often have mutually exclusive real or hypothetical choices presented to subjects (Cohen \& Blum, 2002; Ratcliff \& McKoon, 2008). For instance, chocolate cake and apple pie are two deserts that the vast majority of adults residing in the US have some awareness of. Even if an adult has never decided to eat chocolate cake or apple pie, they understand that both are available types of food. 
People routinely make decisions based on information such as availability. If no stores in a person's town sold cakes or pies, then this would change how decisions about cakes and pies, as choices, are made. Some people may decide that it is simply too much effort to drive to another town to purchase these items not sold in close proximity to them. Other, more curious, people might decide that it is worth a trip. If these cakes and pies were illegal to purchase, then the nature of the decision would change still further. It would involve not only more effort, but greater risk. But in all cases, the choices of chocolate cake or apple are available to decision-makers. Similarly, knowing that opioids and stimulants are potentially procurable is a type of information that drug users and would-be users have.

Decisions to use opioids and stimulants does not suggest that decisions to use other licit, or comparatively less risky illicit drugs could not also have been made during a given time period. Use of drugs with well-known risks and use of drugs known to have comparatively less risk are not mutually exclusive. In examining self-reported drug use prior and subsequent to incarceration, use of drugs other than opioids and stimulants might also be identified. Additionally, concomitant use of opioids and stimulants may also be identified. Accordingly, part of this study includes exploring the problem of highrisk drug use by identifying more than one possible drug use outcome. These outcomes include opioid use, stimulant use, concomitant opioid and stimulant use, other drug use that excludes opioids and stimulants, and no drug use. The latter two outcomes can be considered as comparatively less risky, as will be discussed further below.

In practice, each decision to use drugs brings with it an increased probability of experiencing acute adverse events (e.g., injury, overdose, arrest, drug deal "gone bad", blackout, driving under the influence, etc.), delayed economic and health consequences (e.g., more money spent on more drugs, damage to vital organs, dependence), and myriad opportunity costs. Therefore, it is important not only to identify if highrisk drugs were used, and to identify some of the endogenous and exogenous factors with the potential to influence decisions to use, but also to assess participants' overall drug use. As will be subsequently discussed, any solitary instance of use indicates a decision to use, but it may be that many decisions were made to use the same drugs during a given time period. The difference between deciding to use heroin once and deciding to use it 100 times is important (Herrnstein \& Prelec, 1991; Heyman, 2003). The degree of drug exposure, taken to be the frequency and regularity with which drugs were used, may itself come to 
reflect not just the behavioral referent of a decision, or may decisions, but also an additional influence on subsequent drug-related decision-making. In other words, regarded episodically, a drug-related decision and its behavioral outcome of "use" may be treated as a dependent variable, but when there are numerous decisions and many instances of use (particularly for the same class of drugs) over a given time period, a question is then raised about how the history of drug use comes to influence contemporaneous or future drug-related decisions.

\section{Selection and Description of Conceptual Framework}

There are roughly two broad approaches used to guide the study and treatment of drug use and to situate the phenomenon within a larger conceptual paradigm. The first can be approximately summarized as the "brain disease model of addiction" (BDMA), whereby neurobiological changes that develop from continued drug-taking are believed to contribute to a person's development of and persistence in problematic use despite adverse consequences, due to the intrinsically rewarding effects of drugs (Volkow \& Koob, 2015). The second can be approximately summarized as the "disorder of choice" approach, whereby a person may choose to use drugs, and to continue to choose to use drugs despite adverse consequences, due to endogenous and exogenous factors that influence and constrain decision-making capacity and choice (Bickel et al., 2014; Heyman, 2009, 2013b; Hyman, 2005, 2007). In this approach, the intrinsically rewarding effects of drugs are but one among many factors of with the potential to influence use. Despite ongoing debates and important differences in what both approaches emphasize, the two approaches have much conceptual overlap and are often in empirical, if not philosophical, agreement about some important aspects of drug use and addiction (Berridge, 2017; Bickel et al., 2014; Fenton \& Wiers, 2017; Hyman 2007; Leshner, 2001; Lewis, 2017; Shaffer et al., 2004; Szalavitz, 2017). Though both provide useful methods for understanding addiction, the "disorder of choice" approach, under which behavioral economics is subsumed, may provide additional explanatory power. This is because behavioral economics seeks to explain both drug use and addiction, whereas the BDMA focuses on pathology without equal focus on non-pathological use, and because the behavioral economic approach emphasizes the importance of considering how dynamic conditions can influence drug-related decision-making and drug use at one time point and across time (Bickel, 2014; Higgins, Silverman, \& Heil, 2008; Heyman, 2009; Müller \& Schumann, 2011). By utilizing a behavioral economic approach, the neurobiological changes that 
result from continued drug use (that some refer to as "disease") are not overlooked, as this approach understands the same phenomenon as a reinforcer pathology that can be influenced by many additional factors of a person's life (Bickel et al., 2014, 2016; Heyman, 2009; MacKillop, 2016).

\section{What Exactly is Behavioral Economics, and Why is it Relevant?}

Broadly, behavioral economics conceives that people's available choices, cognition, decisionmaking, and behaviors are influenced and constrained by a variety of factors and across time, and that many of these influences may be beyond a person's complete awareness or control (Chaloupka, Emery, \& Liang, 2003; McClure, Laibson, Loewenstein, \& Cohen, 2004; Newell \& Shanks, 2014; Tomer, 2007). Fundamentally, and in relation to decisions involving drugs, behavioral economics is concerned with how a person's history, environment, cognitive capacities, psychology, preferences, and available choices shape drug-related decisions (and drug use) in light of rewards and costs associated with all available choices (Camerer \& Loewenstein, 2004; Vuchinich \& Heather, 2003). In other words, high-risk drug use and addiction can be, among other things (e.g., habituated responding), described as one type of goal-directed behavior resulting from a goal-directed decision-making process (Brown \& Madsen, 2018; de Wit, 2018). As Vuchinich \& Heather (2003) explain, behavioral economics is:

A system of specific concepts that applies general principles of relativism and molarity to understanding the use of psychoactive substances. In general, the value of substance use, and the extent to which it is preferred, can be viewed as a function of the benefit/cost ratio of substance consumption in relation to the benefit/cost ratios of other available activities (p. 5).

This molar view of choice simply means that activities, such as drug use, can involve local behaviors as well as behaviors extended in time, and that all behavior constitutes choice (Baum, 2004). The latter premise rests on the idea that in all situations, even the most constrained or restricted ones, more than one behavioral option is available (Baum, 1974). "In the molar view, behavior is a choice and choice a behavior. Whether local or extended, a relative response rate constitutes an allocation of behavior among alternatives and is a primary feature of behavior" (Baum, 2004, p. 355). Activities are considered here to be comprised of other activities and, in kind, to potentially comprise other activities. Activities have three properties: First, each part of an activity stands in relation, as a part, to its whole; second, parts cohere to function as a whole (toward some end); third, activities are subject to scaling (i.e., procuring heroin, fixing 
heroin into a syringe, injecting the heroin; then, done with enough frequency, transitioning from someone who has injected heroin to someone addicted to heroin). Because activities are extended in time, measuring or otherwise making sense of a behavior involves considering what other behaviors precede and follow it and what else stands in relation to it. Again, a person who uses cocaine once differs from a person who uses cocaine every hour or every day. A person who studies for school once a week differs from someone who studies for school each day. The frequency of behavioral allocation provides information about activities which are comprised of many choices/behaviors. Importantly, decisions to use drugs are not made in a vacuum. In addition to many other potentially influential factors, constraints on drug availability, access to non-drug alternatives (e.g., satisfying relationships), and perceived or real likelihood of cost (e.g., parole revocation, overdose), all stand to influence drug-related decisions, at one time point and over time (Bickel et al., 2014; Caprioli et al., 2009; Greenwald \& Hursh, 2006; Klapproth, 2012).

In his overview of behavioral economics, Tomer (2007) states that:

The word, strand, is the right one for thinking about [behavioral economics]. A strand is a part that is bound together (as in a rope) to form a whole. Behavioral economics consists of quite a few strands as well as individual practitioners whose work does not fit neatly into any one of these strands. Because there is enough commonality in these strands, they do form a whole (p. 469). More recently, Bickel et al. (2014) referred to behavioral economics as "a discipline that hybridizes economics and psychology... that can be considered as the application of economic concepts and approaches to the molar study of individuals' choices and decisions" (p. 643). Although behavioral economics began its departure from classical and neoclassical economics many decades ago, the pace of departure has accelerated in the last decade as behavioral economic researchers increasingly incorporate insights and methods from non-economic disciplines (e.g., psychology, neuroscience) (Becker, 2013; Thaler \& Ganser, 2015; Tomer, 2007). Behavioral economics, and its extension neuroeconomics, continue to reconsider and refine fundamental assumptions of economics, in order to establish a more realistic understanding of decision-making and behavior, including drug use (Heshmat, 2015; Kahneman, 2003; Melrose \& Monterosso, 2015; Thaler \& Ganser, 2015). With increased knowledge about cognitive systems underlying information processing and decision-making (e.g., dual-process theories) and improved appreciation for how context can greatly influence decision-making, behavioral economics has 
reconceptualized certain assumptions about how people make decisions involving drugs and other commodities (Evans \& Stanovich, 2013; Kahneman, 2011). The application of behavioral economics to drug use and addiction has proliferated over the past decade and is increasingly used to help explain people's persistence in drug use despite risk and cost (Vuchinich \& Heather, 2003).

\section{Specific Rationale for Utilizing a Behavioral Economic Approach}

Accordingly, a behavioral economic approach is helpful for organizing an exploration of high-risk drug use among corrections-involved adults, both in terms of how it may help explain some findings and in terms of its potential to contextualize high-risk drug use, something often left undone when discussing and researching drug use. A more thorough contextualization of decision-making in relation to high-risk drug use may accomplish several things. For one, it may call into question the degree to which a person can be regarded as culpable or blameworthy for their use. Further, it may do this without necessarily calling into question a person's agency, responsibility, and inherent dignity, which has important philosophical, policy, and clinical implications, several of which will be discussed in Chapter 5 (Heyman, 1996; Pickard, 2017).

A behavioral economic approach was chosen to help conceptualize the phenomenon of high-risk drug use, formulate exploratory study aims, and interpret findings for roughly three reasons. First, this approach can help to scrutinize and explain different types of high-risk drug use. For instance, particular opioid or stimulant drugs may differ in their relative availability and value to corrections-involved people in Kentucky and may be more or less accessible at different times and regions within the state. This approach can help explain why decision-making about particular drugs might differ between people and potentially change over time.

Second, this approach permits conceptualization of opioid and stimulant use as "decisional outcomes". These can be understood as the behavioral referents of drug-related decisions (the other discernable behavioral referents of the decision being "no use", or the use of non-opioid and non-stimulant

drugs). This means that a dichotomized outcome (e.g., "use" versus "no use") for particular drug can be examined and discussed in terms of what factors may have influenced the decision to use during a given time period, rather than merely influenced the probability that use would be observed without also acknowledging the role of a decision-making agent. This proximate step prior to use, the decision, is ultimately what all antecedents are influencing. By placing increased attention on decision-making and by 
emphasizing its importance as the penultimate act of interest, interventions may be better developed to help people improve their decision-making capacities in both local and global ways (e.g., improving cognitive functioning, affect regulation, mindfulness, interoceptive awareness, inhibition, etc.) and policies can be developed to help "nudge" people into making more adaptive decisions (Aharonovich et al., 2006; Ahn et al., 2014; Chetty, 2015; Dunn et al., 2010; Naqvi \& Bechara, 2010). By highlighting the capacity for some aspects of drug users' decision-making and behavior to change under the right conditions (rather than suggesting that drug users have an incurable, chronic brain disease), self-efficacy may be better nurtured.

Third, this approach understands that opioid and stimulant use can reflect trade-offs between short-term rewards of use and long-term benefits that develop from not using. In this study, at least two long-term rewards can be discerned (i.e., not incurring morbidities or mortalities related to these drugs; not becoming incarcerated for using these drugs) (Brinkley-Rubinstein et al., 2017; Dublin \& Von Korff, 2018; Merrall et al., 2010). People in the US are disincentivized from using opioids and stimulants, yet, many people in this sample made a decision to use these drugs despite disincentives. Behavioral economics can help make sense of a person's pursuit of reward in situations involving uncertainty and risk due to the fact that this approach considers what endogenous and exogenous factors may have contributed to how drug and non-options were framed, valued, and assessed (Bickel \& Marsch, 2001; Heshmat, 2015; Kahneman, 20013, 2011). That the context within which people make drug-related decisions is emphasized in this approach is also helpful, given that opioid and stimulant use cannot be divorced from the complex lives of people and the uniquely complex set of circumstances related to choice that corrections-involved people often find themselves in.

To date, a behavioral economic approach for understanding decision-making and behavior has not been used as a conceptual lens by social science researchers concerned with examining opioid, stimulant, and other drug use among corrections-involved people using secondary data sources. Many controlled human and non-human animal studies have helped to advance behavioral economic and neuroeconomic understandings of drug use. Such lab-based experiments are essential for helping describe and elucidate discrete drug-related choice and behavior. They are nonetheless limited, oftentimes unable to sufficiently model the factors that shape choice, influence decision-making, and reinforce behavior among people in their everyday lives (e.g., threat of incarceration, social status, isolation and loneliness, desire to regain 
child custody, fear of contracting HIV, poverty, chronic back pain, etc.) (Heilig, Epstein, Nader, \& Shaham, 2016; Venirro, et al., 2018). The vast majority of people who use opioids and stimulants make a decision to do so within the complicated and changing conditions of their life, not within a controlled study or under hypothetical (or otherwise artificial) conditions.

While it is crucial to continue studying drug-related decision-making and drug use using preclinical animal models, even if they cannot fully account for real-world decision-making conditions, it is also crucial that in parallel to such lab-based work researchers outside of the basic sciences and economic disciplines increasingly seek to understand high-risk drug use by conceptualizing the phenomenon in a manner that takes into fuller account the inherent complexity of drug use. Part of this complexity requires more direct acknowledgement and study of the fact that the drug use is only ever a byproduct of the drug decision, and that both occur within broader conditions.

\section{Necessary, but not Sufficient}

Therefore, instead of asking what factors are associated with observing an outcome of opioid use during a given time, it may be helpful for social science researchers to begin asking what factors have the potential to influence a person's decision to use opioids during a given time, as this helps to situate the problem with greater coherence and provides a way to achieve greater clarity by acknowledging the fact that the problem of high-risk drug use isn't necessarily the primary problem. Rather, it is the person's decision to use high-risk drugs and the conditions that give rise to the decision. It is coherent in the sense that unless drugs are forcibly given to someone, a person does indeed choose them. The drug use behavior does not exist without the prior decision. The decision does not exist without the conditions. If high-risk drug use is a problem, it is only secondary to decisions to use high-risk drugs. That drug use can assume the character of compulsion, but also may not ever have assume this character for many, may confuse how it should be conceptualized in relation to choice. However, if the decision to use drugs is also recognized as more or less voluntary (verses compelled), then it becomes possible to talk about constraint across levels (i.e., a person was compelled to decide to purchase and inject heroin despite a desire to not use heroin, or despite a desire to not make such a decision). The possibility is suggested here that the behavior of "use" cannot be performed without prior decision, however constrained and compelled the decision was. Put differently, there may be no "freedom of will" at any level, not just at the level of 
observable behavior of use. Here, desires, intentions, choice, and behavior would all be constrained, but decision would still precede action.

This idea, that decision precedes action, is intuitive, yet, it is not always considered and is less frequently identified as an object of investigation, particularly among social scientists. This is the case for a variety of important problems. For example, the problem of hepatitis $\mathrm{C}$ infection that results from injecting drugs and sharing drug equipment is secondary to the injection itself. The observed outcome of disease is clear and measurable, and the sufficient conditions that actualize it (i.e., injecting drugs, sharing injecting equipment) are not typically confused with it. It is understood that one precedes the other, and that reducing new IDU-related hepatitis $\mathrm{C}$ infection rates means reducing IDU or syringe sharing. Since decisions are made prior to injecting drugs and sharing syringes, one legitimate focus might be on the factors that influence these high-risk decisions. In other words, to consider what factors are present so as to influence the decision to inject with a used syringe.

To date, myriad "risk factors" have been identified as positively correlated with drug use and misuse, often considered as contributors to observed drug use outcomes (Dube et al., 2003; Kreek, Nielsen, Butelman, \& LaForge, 2005; Nation \& Heflinger, 2006). Although the presence of these risk factors may be sufficient contributors to the development of drug use and misuse, none of them are necessary

(Newcomb, Maddahian, \& Bentler, 1986). It is the decision to use drugs that is necessary for use to occur. Additional necessary, but not sufficient, conditions include the capacity to act and the actual existence of drugs as commodities. ${ }^{1}$ In other words, even if it may require considerable effort to procure a high-risk drug, they do (and must) exist as procurable commodities in order to be used. Many other factors with the potential to influence drug-related decision-making are also only ever sufficient, but not necessary. Indeed, there is a vast array of possible influences on decision-making. The same factors may not reliably influence decision-making across people uniformly, or across the same person over time. Still, it may be that some conditions do influence high-risk decision-making involving opioids and stimulants among more people more of the time. Of course, the presence or absence of these factors could still be conceptualized in terms

\footnotetext{
${ }^{1}$ By "capacitary to act", it is meant that not merely agency, in the most general sense, is implied, but that also, more specifically, the requisite capability for particular forms of high-risk drug use are accounted for. For example, swallowing a prescription opioid requires less procedural knowledge than preparing the same pill for intravenous injection. A person must, at a minimum, be capable of drug self-administration.
} 
of "risk" or "protection", though they need not be. In some cases, in which the factors influencing decisionmaking are more proximate (i.e., milliseconds) it may be better not to think in terms of "risk", but rather in terms of "constraint". Irrespective of how endogenous and exogenous factors with the potential to influence drug-related decision-making are termed, they are worth considering. And, it is possible to do so using existing research on cognition, decision-making, and drug use.

Shifting the focus of the discussion toward factors with the potential to influence drug-related decision-making--instead of remaining focused on factors with the potential to influence its behavioral referent ("use") unmoored to the decisions themselves-- gets closer to the actual phenomenon of interest. As discussed in Chapter 5, such investigatory refocus may have the potential to help inform interventions that might attenuate high-risk drug use at its more proximate and potentially extricable roots.

\section{Coarse-grained Approach}

Exploring opioid and stimulant use by identifying endogenous and exogenous factors with the potential to influence drug-related decision-making can be accomplished to the extent that available data sources are able to discern and indicate, however indirectly, the presence or absence of such factors. It is important to emphasize that attempting to identify endogenous and exogenous factors with the potential to influence drug-related decision-making constitutes a "coarse-grained" approach, whereby conceptual expanse is gained at the expense of measurement detail and specificity. In the case of a secondary data analyses of survey data, such measurement detail is already largely absent. Coarse-grained models are sometimes used in science to simplify otherwise inscrutably complex systems (Marrink, Risselada, Yefimov, Tieleman, \& De Vries, 2007; Noid et al., 2008). People can be conceptualized as stochastic, selforganized complex systems, even if they cannot yet be measured or modeled as such (Bak, 2013; Bullmore \& Sporns, 2009; Wagenmakers, van der Maas, \& Farrell, 2012; Rubinov, Sporns, Thivierge, \& Breakspear, 2011). For this reason, the general study of dynamic human decision-making and behavior will, for the foreseeable future, remain coarse-grained and even somewhat philosophical, irrespective of the degree to which measurement error is present or absent. It is also for this reason that it may be reasonable to assume a coarse-grained approach when exploring high-risk drug use among a sample of corrections-involved adults.

For the exploratory study proposed here, many lines of rapidly-developing research can be brought to bear in helping understand and think about the endogenous and exogenous factors in people's everyday 
lives with the potential to influence high-risk drug-related decisions. Using behavioral economics as a conceptual approach can help orient the study and its findings to this body of literature. Because data examined in this exploratory study were collected as part of an ongoing outcomes evaluation, the objective was not to pursue empirical validation of specific behavioral economic concepts. Rather, this study sought to apply ideas central to behavioral economics and the molar view of choice to the available data, and to use existing literature to help think about possible ways that endogenous and exogenous factors may have influenced decision-making about opioids and stimulants among participants across time periods. Many of these factors will be discussed in Chapter 2. As discussed below, the risk associated with these drugs is significant. This significant risk means that a decision to use opioids and stimulants is nothing less than puzzling, unless it is situated within the broader context of a people's lives.

\section{The High-Risk Nature of Opioid and Stimulant Use}

\section{Other Drug Use}

Before describing some of the specific risks associated with opioids and stimulants, it is worth briefly considering some of the risks associated with other commonly used drugs. Alcohol use, for instance, constitutes one of the greatest financial and social burdens on society. It results in more morbidities and mortalities in the U.S. than illicit drugs, due to the sheer volume of alcohol consumers (CDC, 2019; Jinjuvadia \& Liangpunsakul, 2015). Excluding accidents, most alcohol-related morbidities and mortalities develop gradually (CDC, 2019). Alcohol poisoning can result in death; however, this accounted for only $1.9 \%$ of annual alcohol related fatalities in the U.S. between 2006-2010 (CDC, 2019).

Many potential social, psychological, educational, and occupational consequences can result from alcohol use; but these too may manifest gradually, suggesting many potential points for prevention or attenuation (Lee, Geisner, Patrick, \& Neighbors, 2010 Patton et al., 2013; Schulenberg \& Maggs, 2002). Some of the delayed adverse consequences of alcohol use can be attributed to the fact that alcohol is licit and socially normed, meaning that the criminalization, stigmatization, and social exclusion of alcohol users and people with alcohol use disorder (AUD) occurs to a lesser degree compared to people who use opioids or stimulants, or who have respective use disorders for these drugs (Janulis, Ferrari, \& Fowler, 2013; MacArthur, Jacob, Pound, Hickman, \& Campbell, 2017; Pachankis et al., 2017). The availability and price of alcohol is also such that many people who use or misuse it may not need to resort to serious criminal 
offending or extreme measures to obtain the drug, even if there are fluctuations in alcohol prices that may nevertheless influence consumption (Felson \& Staff, 2017; Mericle, Karriker-Jaffe, Gupta, Sheridan, \& Polcin, 2016; Ornstein \& Levy, 1983; Shortt, Rhynas, \& Holloway, 2017). For instance, someone wanting to consume alcohol need not meet a drug dealer. Since the repeal of the $18^{\text {th }}$ amendment, which prohibited non-medical sale of alcohol, people in the US over the age 21 may drink without direct threat of legal consequences (Okrent, 2010).

Lastly, with the exception of homemade spirits (e.g., "moonshine"), alcohol products sold in the US are held to strict regulatory guidelines and are subject to government monitoring to ensure that the package contents are concordant with labeling, advertising, etc. (Alcohol and Tobacco Tax and Trade Bureau, 2019; Holstege, Ferguson, Wolf, Baer, \& Poklis, 2004). This means that consumers have access to information which may help them assess at least some risk. While information about opioids and stimulants is informally available to users within illicit drug markets and online, it may be unreliable and, given the nature of illicit drug markets, subject to change rapidly (Duxbury, 2018; Orsolini, Papanti, Corkery, \& Schifano, 2017 Treloar \& Abelson, 2005; Rönkä \& Katainen, 2017). Further, information may only be available about specific illicit drug products via specific mediums (e.g., word-of-mouth, online, etc.) or about specific products sold by specific distributors (e.g., heroin advertised as "fentanyl-free" on the Deep Web and verified by customer reviews) (Becker, 2017; Quintana Mathé, 2017). In other words, illicit drug information is not reliable or durable, nor is it equally accessible to all would-be consumers. Taken together, the choice to use alcohol is one that entails serious risk, but it is one that, when compared to opioids and stimulants, has less relative risk.

Other illicit drugs, such as psychedelics, benzodiazepines, barbiturates, and cannabis, are also used by people in the general population recreationally, experimentally, or for the self-treatment of health symptoms, in addition to being used by people with SUDs (Andersson, Persson, \& Kjellgren, 2017; Jeffers et al., 2015; Ogborne, Smart, Weber, \& Birchmore-Timney, 2000). Though these drugs may pose health and legal risks for users, they tend to have, overall, fewer social and medical consequences compared to opioids and stimulants, including being comparatively less stigmatized (Brown, 2015; Hall, 2015; Meurk, Carter, Partridge, Lucke, \& Hall, 2014; Pachankis et al., 2017; Patorno, Glynn, Levin, Lee, \& Huybrechts, 2017; U.S. Department of Justice, 2015). Novel psychoactive substances (NPSs), such as synthetic 
cathinones or cannabinoids, are used far less frequently than opioids and traditional psychostimulants, but are increasingly associated with adverse side effects and remain high-risk drugs of concern (Cohen \& Weinstein, 2018). However, recent evidence suggests that, compared to traditional drugs, their availability and use is gradually declining and that they are not preferred over traditional drugs (Blackman \& Bradley, 2017; Smith \& Staton, 2018; Smith \& Stoops, 2019). Thus, while NPS use is high-risk, prevalence rates currently remain far lower than other drugs (Mathews, Jeffries, Hsieh, Jones, \& Buckne, 2019; Palamar, Rutherford, \& Keyes, 2019). This, coupled with their relative lack of appeal to many users, means that NPSs may be considered as concerning primarily among a narrow subpopulation of drug users, compared to opioids and stimulants, which are more popular and which have over a century of documented associated risks (Chaldecott, 1907; Scheltema, 1910; Wood, 1904).

\section{Indeterminate (but Potentially Perceived) Risks of Opioids and Stimulants}

The problem of high-risk drug use in the US has recently received increased local and national attention, as rates of drug-related mortalities have come to surpass mortalities attributed to firearms, car accidents, and HIV/AIDS at their respective peaks (Blackford, 2018; Katz, 2017). Among the general public and drug-using populations, opioid and stimulant use is known to have potential for health and legal consequences (Draus \& Carlson, 2009; McGovern \& McGovern, 2011; Norden et al., 2009; Small, Rhodes, Wood, \& Kerr, 2007). In order to procure these drugs without prescription or by chance, one must first become aware that they are available for purchase through black markets and informal peer networks (Jonas et al., 2012) ${ }^{2}$. This means that potential legal consequences, even if underestimated or only vaguely perceived, are still known. As for potential health consequences, public health messaging around opioid and stimulant use has increased significantly in the past decade (Hernandez, Meyers-Ohki, Farkas, \& Rotrosen, 2018; Parker, Strunk, \& Fiellin, 2018).

Although all drugs have been generically profiled in public awareness and prevention campaigns for decades (e.g., DARE program), cocaine/crack cocaine and methamphetamines have been presented to US citizens via graphic and disturbing images by the Drug Enforcement Administration and the Partnership

\footnotetext{
2 "By chance" alludes to the fact that people may inadvertently come across prescription drugs and illicit drugs without meaning to. For instance, discovering them in a parent's medicine cabinet or among a sibling's possessions, etc.
} 
for Drug-Free America (Buchannan \& Wallack, 1998; Linnemann \& Wall, 2013; Marsh, Copes, \& Linnemann, 2017; West \& O’Neal, 2004). News media and politicians have also drawn attention to the devastation that cocaine/crack cocaine and methamphetamine use can contribute to, though with a noticeably different tenor than that used in recent profiles of opioid use, and white opioid users in particular (Netherland \& Hansen, 2016). For crack cocaine specifically, US media helped facilitate the creation of caricaturized social constructs that developed around both real and tragic realities, as well as exaggerated myths and misinformation (Brownstein, 1996; Hartman \& Golub, 1999; Reinarman \& Levine, 2017). Images and catch-phrases advertising the risk of crack cocaine use became commonplace, with many depreciatory and cautionary phrases persisting in the cultural lexicon (e.g., "crack babies", "crack whores", “crack mothers") (Lyons \& Rittner, 1998; Meyers, 2004). In addition to crack cocaine, methamphetamine use has been profiled in stark, graphic, and derogatory terms (Marsh et al., 2017; Peterson, Gubrium, Fiddian-

Green, 2018). The media and politicians have portrayed methamphetamine use as a public menace rather than public health crisis, with methamphetamine use frequently associated with "white trash" (Hartigan, 2013; Jenkins, 1994; Weidner, 2009). Film and TV have also portrayed some of the risks associated with opioids and stimulants, even as they have simultaneously glamorized their use (Allen \& Alberici, 2018; Cape, 2003; Garriott, 2016).

It should be noted that while both opioid and stimulant users have been blamed, caricaturized, maligned, and punished (as well as pejoratively labeled as "junkies", "crack-heads", "tweakers", etc.), the public health messaging that predominates the current opioid epidemic was not as pronounced during the crack cocaine epidemics of the 1980s and 1990s. Despite that crack use was deemed a "plague" during this era, public safety, in the form of deterrence and retributive justice, was given greater priority than public health (Furst, Johnson, Dunlap, \& Curtis, 1999; Lloyd, 2013; Mauer, 2004; Reinarman \& Levine, 1997). Jail and prison terms, including mandatory minimum sentences, were given to crack cocaine users and sellers more frequently than treatment referrals (Graham, 2010 Kautt \& Spohn, 2002). Beginning in the early 2000s, the same became true for many methamphetamine users who began receiving jail and prison terms, including mandatory minimum sentences, for methamphetamine-related offenses (Graham, 2010). Currently, people who sell opioids, irrespective of any personal use, are receiving harsh federal 
punishments, including life without the possibility of parole; again, based on mandatory minimum statutes (Duncan, 2013; Mack, 2016; Offices of the United States Attorneys, 2017). However, in addition to punitive measures, public health measures are also being promoted in earnest, with the hopes of mitigating the opioid crisis (Collins et al., 2018).

Beyond legal consequences advertising the risk of opioid and stimulant use, additional types of knowledge about the risks of use are available. These include information provided by drug-using peers and first-hand observations, as many people who come to use opioids or stimulants have also witnessed use of these drugs in their own families or communities (Alexander, Piazza, Mekos, \& Valente, 2001; Barnard \& McKeganey, 2004; Mohler-Kuo, Lee, \& Wechsler, 2003). More directly, people can form experiential knowledge after initial or continued use. Should users experience adverse effects, they will have observed some potential consequences of use. In 2017, a majority of people in the US aged 12 or older $(71.3 \%$ 94.5\%) believed that trying cocaine or heroin even occasionally was associated with "great risk" (SAMHSA, 2017). Thus, it may be concluded that, irrespective of the validity or quality of available information about opioids and stimulants, most US citizens associate opioids and stimulants with some measure of danger. For people who have encountered institutions or treatment facilities, the risks associated with these drugs may be known with even greater specificity. Although most people may not be able to articulate the specific risks associated with opioid or stimulant use, it is still reasonable to suggest that there is some indeterminate degree of understanding that opioid and stimulant use is not "risk-free", in that there is considerable uncertainty associated with use.

Some potential risks of opioid and stimulant use that may be discerned to varying degrees by people in the US include: 1) The potential to develop dependence or addiction, due to the high abuse liability of opioids and stimulants (Epstein, Preston, \& Jasinski, 2006; Higgins, 1997; Koob \& Volkow, 2010); 2) The potential to develop problems in occupational, social, and daily functioning as a result of misuse or dependence (Cunha, Bechara, de Andrade, \& Nicastri, 2011; Henry, Minassian, \& Perry, 2010; Hoffmann \& Larison, 1999; Leshner, 1999); 3) This can be extended to potential discord in relationships, in that it is likely that not all of the people in one's life will be supportive of opioid and stimulant use, even if some are. In other words, smoking meth may upset a spouse or injecting heroin may result in rebuke from one's mother. Some social censure might be expected (Amato \& Rogers, 1997; Orford, Copello, 
Velleman, \& Templeton, 2010); 4) Here, social consequences of opioid and stimulant use may include stigmatization, social exclusion, rejection, and discrimination (Brown, 2015; Kennedy-Hendricks et al, 2017; Pachankis et al., 2017). Stigmatization is public, persistent over generations, and consequential; it is why many users do not publicly disclose their drug-using identity voluntarily (Chalmers, Lancaster, \& Hughes, 2016; Pachankis, 2007;

Quinn \& Chaudoir, 2009; Ross et al., 2007); 5) Potential short- and long-term financial problems associated with opioid and stimulant use. This can include consequences from money spent on drugs, as well as from potentially poorer lifetime employment and earning trajectories. (Mark, Woody, Juday, \& Kleber, 2001; Simon et al., 2001); 6) The potential to incur injury or health conditions related to use (e.g., infectious diseases or abscesses), including the potential for drug overdose and death (Binswanger, Kral, Bluthenthal, Rybold, \& Edlin, 2000; Cornish \& O'Brien, 1996; O'Donnell, Gladden, \& Seth, 2017; Zibbell et al., 2018); 7) Lastly, the purchase, possession, and use of opioids and stimulants is illegal, meaning that a person is aware of the increased likelihood of coming into contact with law enforcement and becoming involved in the criminal justice system (Mumola \& Karberg, 2006; Webster, Dickson, Mannan, \& Staton, 2018). Moreover, opioid and stimulant use is expensive and often necessitates extralegal income-generating endeavors, including criminal ones (Biernacki, 1979; Schwartz et al., 2008).

In Kentucky, between 2015-2016, approximately $76.5 \%$ of adults reported perceiving some risk from using cocaine at least once a month (SAMHSA, 2017). For this same period, approximately $89.1 \%$ of adults in Kentucky perceived some risk from "trying heroin once or twice" (SAMHSA, 2017). This means that within this region a majority of adults associated risk with at least one opioid and/or stimulant drug. Given the wide-ranging scope and severity of the aforementioned risks associated with opioids and stimulants, and the fact that most people are aware of these risks, it is unclear what factors could sufficiently influence a person to willingly use, or to continue to use, opioid or stimulant drugs.

\section{Why Might a Person Decide to Use, then?}

As noted earlier, it is likely that a variety of endogenous and exogenous factors increase the likelihood that a person decides to use opioids or stimulants, whereby short-term reward is pursued despite possible future costs. It is important to remember, though, that the nature of risk is that it exposes one to, but does not guarantee, unfavorable outcomes (Daly \& Wilson, 2001). One commonality among many of 
the risks described above is that their actualization, wherein the risk of harm becomes experienced harm, can be delayed. This is important for several reasons. First, because unlike a person touching their hand to a hot stove, the association between opioids or stimulants and harm (i.e., punishment learning) is often indirect (Skinner, 1938, 1953). Opioids and stimulants, however, have directly reinforcing effects (Bickel et al., 2014; van Ree, Gerrits, \& Vanderschuren, 1999; Woolverton \& Johnson, 1992). In other words, the reward of using them is not only salient, but directly associated, making learning rapid and the encoding of memories about these pleasurable drug experiences instantiated in key brain areas for "quick retrieval" in the future (Baler \& Volkow, 2006; Stewart, de Wit, \& Eikelboom, 1984). Conversely, larger future rewards (or punishments) are abstract and more difficult to envisioned clearly (e.g., episodic future thinking, episodic counterfactual thinking) or with the same salience as proximate rewards or punishments, particularly those for which there are episodic memories (i.e., associated conditioning), which serve to influence decision-making and behavior (O'Donnell, Daniel, \& Epstein, 2017; Schacter, Benoit, De Brigard, \& Szpunar, 2015).

Second, many risks associated with high-risk drug use and many benefits associated with not using high-risk drugs are realized in the future, meaning that consequences or contingencies are not perceived in the same way as immediate "sure things" (Platt \& Huettel, 2008). This decision-making under uncertainty means that a person may be less able to accurately assess and value information about high-risk choices and may experience less motivation to make decisions in the present that help achieve or avoid particular future outcomes, which are only ever contingent (Kahneman \& Tversky, 2013).

A decision to use opioids or stimulants constitutes an intertemporal choice, whereby people make a decision at one time point with awareness that this decision influences the availability of other possibilities in the future (Berns, Laibson, \& Loewenstein, 2007; Kable \& Glimcher, 2007). Utility, which can be thought of as the value (e.g., enjoyment, usefulness, pleasure satisfaction, etc.) associated with a given choice, may not always be maximized in the long-term by people (Herrnstein \& Prelec, 1992; Machina, 1989). Oftentimes, future rewards and punishments associated with choices cannot be fully anticipated or accurately assessed, even if some risk/benefit is discernable (e.g., anticipated, remembered, inferred) (Broome, 1991; Loewenstein \& Elster, 1992; Zauberman, Ratner, \& Kim, 2008). Intertemporal choice poses problems for people in instances where choosing the option with the greatest local utility (i.e., 
immediate value/reward) decreases overall utility (i.e., value/reward over time) (Herrnstein \& Prelec, 1992). This mismatched utility is not always the case for addictive drugs, but often can be. By choosing to use drugs repeatedly, drugs can become overvalued, and non-drugs undervalued. This can be considered as reinforcer pathology (Bickel et al., 2001). Thus, the risk, but not certainty, of future punishments and rewards associated with opioids and stimulants, coupled with their immediate or local appeal, for which the outcome expectancies of reward are often clear, makes high-risk decisions about drugs as challenging as they are consequential. Importantly, intertemporal choice, particularly between immediately rewarding (but risky) local outcomes and future contingencies, demands significant cognitive effort and flexibility to navigate (e.g., self-control, reframing, inhibition, working memory, metacognition, etc.) (Baumeister \& Vohs, 2007; Heatherton \& Wagner, 2011; Nichols \& Wilson, 2015; Westbook \& Braver, 2015; Yeung \& Summerfield, 2012). As will be discussed, many factors can influence and impair needed cognitive capacities.

The dynamic between immediate versus future, certain versus uncertain, poses a challenge for people, in that they must evaluate choices that cannot help but be weighted differently based on past history (and how past experiences are remembered during decision-making), present states and context, and beliefs about themselves, their abilities, and their future (i.e., prospection, self-projection, self-efficacy) (Buckner \& Carroll, 2007; Kahneman, 2003; Kahneman \& Tversky, 2013 Krueger \& Dickson, 1994). Because the utility of opioids or stimulants is also relative and changing, it is important to consider not only what factors influence decision-making generally, but also what factors might reinforce use or no use of particular opioid and stimulant drugs. This could include considering what additional drugs might be available to people as substitutes (i.e., comparable replacements) or compliments (e.g., i.e., commonly coused drugs), as well as what rewarding non-drug alternatives (e.g., relationships, opportunity, jobs) are accessible (Bickel, Madden, \& Petry, 1998; Etten, Higgins, Budney, \& Badger, 1998; Morral, Iguchi, \& Belding, 1999; Quick, Pyszczynski, Colston, \& Shahan, 2011; Rogers et al., 2008; Waldorf et al., 1992; Yates, Bardo, \& Beckmann, 2019). Given the multitude of influences on decision-making and behavior, it is likely that many factors constrain people's choices and ability to choose in ways that maximize wellbeing over time. 
Even holding conditions constant, the starting point for making a decision between a smaller, immediate reward and a potentially larger, later reward is an uphill battle. This is because people and other animals evolved in states of uncertainty and scarcity to develop greater bias toward immediate consumption of rewarding things (e.g., food) over rewards situated in the future and rewards associated with greater trade-offs, uncertainty, or effort (Bickel, 2014; Kagel, Green, \& Caraco, 1986; Rosati, 2017). It is also an uphill battle because some types of cognition are automatic, unconscious, and heavily influenced by factors that may not be readily perceived by the person (e.g., physiology, affect, surroundings) (Bubier \& Drabick, 2008; Evans, 2008; Kahneman \& Egan, 2011). This type of cognition can be fast and useful, but comes at a cost; it can make information processing (e.g., assessment, valuation, prediction, etc.) and decisions prone to inaccuracies, bias, and error (Kahneman \& Egan, 2011; Lee, 2013). Cognitive processes that are slower, more deliberative, and concerned with intertemporal or abstract reasoning rely more heavily on higherlevel cognition (e.g., attention, working memory and executive function) (Ash \& Wiley, 2006; Evans \& Stanovich, 2013). This type of cognition is both more effortful, but also necessary for the coordination of goal-directed behavior that is not habituated (Kahneman, 2011; Koechlin \& Hyafil, 2007; Engle \& Kane, 2004; McCabe, Roediger, McDaniel, Balota, \& Hambrick, 2010). In addition to being more laborious, this type of cognition is also influenced by a wide-range of endogenous and exogenous factors that can contribute to decision-making error. These two cognitive systems are believed to work in concert, but the latter system is crucial for deliberation and for inhibition.

Since decision-making relies on a person's cognitive capacities (e.g., cognitive flexibility, inhibitory control, prospection), their cognitive repertoire, and the factors with the potential to influence it during decision-making, are of interest (Stanovich \& West, 2000). As will be discussed in subsequent chapters, many traits and states can bias people toward greater impulsiveness or poorer self-regulation, making an already difficult job of weighing benefit/risk during a decision even more difficult (Baumeister, 2014; Frederick, 2005; Vohs \& Baumeister, 2016). For instance, people with fewer resources who live amidst or routinely experience highly stressful circumstances may be ill equipped to engage in attentional control, impulse inhibition, and deliberative decision-making, and may be more likely to prefer immediately rewarding choices, rather than choices with delayed reward (Mani, Mullainathan, Shafir, \& Zhao, 2013; Shah, Mullainathan, \& Shafir, 2012; Shah, Shafir, \& Mullainathan, 2015; Spears, 2011). 
Ultimately, some people may have or experience more factors that bias them toward a decision to use drugs than others. Yet, even considering only populations that may be statistically more likely to touch a hot stove in the first place, and to repeat touching it despite adverse consequences, there remains the question of what factors influence people within this group to use opioids or stimulants specifically. After all, highly rewarding effects can be produced from other drugs. It may be that there are important endogenous and exogenous factors that are implicated as influences on decisions to use opioids or stimulants, compared decisions to use other drugs or to not use drugs at all.

\section{The Road to Hell is Paved with Good Intentions}

Monterosso \& Lou (2010) and others (Rachlin, 1995) articulate that, "goal-directed behavior can be conceived on a continuum from the molecular (e.g., trying to hit a nail with a hammer) to the molar (e.g., trying to build a house)". For instance, a person may want and plan to inject heroin at a particular moment (molecular) but prefers and plans not to become addicted to heroin and not to incur costs associated with heroin addiction (molar).

Ultimately, a person's drug use involves choices and behaviors with the potential to reach across a lifetime. A person may have an immediate goal (to use heroin), that conflicts with other long-term goals (to stay out of prison indefinitely). Melrose et al., (2015) suggest that high-risk drug use and addiction is "a function of the individual's overall set of motivations", meaning that a person has multiple motivations for behavior and that these motivations can be at odds in one moment (ambivalence), or between selves over time. For instance, a person in the present may be conflicted about using or not using (this is called a "synchronic model of conflict"), or a person may value smoking crack cocaine more in the present, even if their future self would value it less, and instead value health and family more (this is called a "diachronic model of conflict") (Melrose et al., 2015). In other words, for people who use high-risk drugs, there can be inconsistencies between what a person values or conceives of as being in their best interest (at least some of the time) and their decisions and behaviors, which are discordant with such valuation and conception.

The melioration, or "primrose path", behavioral economic model of drug use (Herrnstein \& Prelec, 1992 ) is in keeping with this understanding of inconsistent or conflicting preferences, and helps explain how a person who is motivated to avoid drug-related problems, or gain benefits that develop from not using, can still act in a manner (i.e., using high-risk drugs) so as to increase the likelihood that such 
problems will materialize. In this model, people choose the option with the highest local reinforcement; that is, the highest local or immediate utility. Here, the choice selected among available options is the one perceived as the most immediately desirable or satisfying. Particularly for people who are drug dependent, drugs often have the highest local reinforcement value compared to other choices.

As is often the case with decisions, people make them repeatedly. On a daily basis, people decide what to eat for dinner, how to use their leisure time, what route to take to work, etc. Decisions such as these can be repeated for many years. In the case of choosing to use a high-risk drug or choosing not to, and to instead pursue some non-drug alternative (e.g., jogging, work, watch TV, spend time with friends), if using the drug has the highest perceived local utility, then that is what will be chosen--again and again. Over some finite time (a month, a year, a lifetime), the overall or average utility associated with the choice to use a drug inevitably decreases, due to the nearly unavoidable negative long-term consequences of drug use and/or due to physiological changes that render the drug less rewarding (e.g., tolerance) or even punishing (e.g., hyperalgesia) (Li, Depoortere, \& Emmett-Oglesby, White, 2004). It is important to note three points. First, the local utility of a drug will always be higher than other choices, so long as the drug is most preferred in the immediate (which is often the case for regular drug users). Second, the overall utility of using the drug decreases with repeated decisions over time. Third, in the case of addictive drugs, preference and enjoyment can become decoupled, meaning that a person can come to prefer what they no longer enjoy (Robinson \& Berridge, 1993). Along this "primrose path", a person is always one who is myopic or "meliorating", always choosing the option with the highest local utility (immediate reward), even though repeatedly making this choice decreases the overall utility as a result. In order to maximize overall utility, a person would need to repeatedly, over time, choose the option with the lower local utility (doing something other than use, that isn't worse than using). This model illustrates that immediate and long-term motivators may conflict and may require people to choose an option with less local utility in order to maximize overall utility. As Heyman (2003) notes:

Because one cigarette does not cause cancer and one shot of heroin does not condemn a user to a 'junkie life style', a person can quite correctly reason that since it's 'just for one last time,' the drug is the best choice. However, a series of 'one-last times' turns into a relapse (p. 99). 
It is easy to see, then, how people who use drugs may end up experiencing severe, even ruinous consequences from use even as they did not truly intend to achieve such ends. On this path, some people may be better able to reverse their course once the overall utility of using a particular drug has decreased. A person would then need to repeatedly choose a non-drug option, even if it had a lower local utility relative to the drug. In order to do this, a person would need to, among other things reduce present-bias and increase inhibition. Though all people may have time-inconsistent preferences, people with greater present bias may demonstrate greater inconsistency, wherein the value of the immediate reward is weighted more heavily than a future reward (O'Donoghue \& Rabin, 1999; Takeuchi, 2011). In order to develop more timeconsistent preferences, a person would need to cognitively reframe the relative local utilities. Doing so may promote decisions that maximize overall utility. As will be discussed, many factors can influence a person's cognition, their drug and non-drug choices, and their decision-making. It is not so simple for some people to "reframe" choices. Certain endogenous and exogenous factors can make it far more difficult for a person to become less present-biased and more future-oriented. These factors can also make it more difficult for a person to choose the option with less local, but overall greater, utility.

An example to help illustrate is to consider someone who prefers eating sugary foods over other, non-sugary foods. In this model, the sugary foods would always have the greatest local reinforcement for the person, and therefore the highest local utility. However, over time, the person may grow lethargic, gain weight, develop diabetes, or experience some other decrease in their overall well-being related to the consumption of sugary foods. The person may then come to understand that the overall utility of sugary foods has decreased, even as the local utility of sugary foods remains constant (i.e., no less appealing in the immediate). At whatever point the overall utility of sugary foods clearly decreases, motivation to make different choices can develop and may potentially be nurtured, even if the relative appeal of sugary foods remains unchanged. Yet, motivation to change course after a decrease in overall utility is realized is still bound within the context of the person's history and everyday circumstances. Consider in this example how decision-making might be influenced and constrained by several factors. These factors could include living in a home or community with a high density of sugary foods, or where other potentially rewarding alternatives to sugary foods are lacking. They could also include not having the capacity to adequately plan alternative strategies for acquiring non-sugary foods, due to lack of resources and constraints on cognition 
brought about by stress or scarcity. Many additional factors might also be identified that make selfregulation more or less difficult. Several such endogenous and exogenous factors with the potential to influence decision-making are discussed in Chapter 2.

This "primrose path" model is a simplified way to understand several general points, which will be helpful to remember as decisional outcomes involving opioids and stimulants among correctionsinvolved adults are explored. First, is that the options and outcomes in the immediate are distinct, whereas options and outcomes in the future are indistinct and contingent. For example, the proximate rewarding effects of methamphetamines are almost certain, but all else is speculation that requires cognitive effort to envision and become behaviorally motivated by. Second, the local utility of a drug is only appreciable in the greater context of a person's life. Decision-making about specific drugs during a particular time period is not dissociable from prior and contemporaneous circumstances, such as other available choices, or from a person's prospects for or beliefs about the future. Third, not all people are myopic or meliorating, or at least not in respect opioids or stimulants. Some people would never find themselves on such a path. Fourth, there is reason to believe that people who do end up on this path in relation to opioids or stimulants may be less able to reverse their course due to some endogenous and exogenous factors that impair decisionmaking capacities more generally. Some of these factors may have predated drug use, while other may have developed as a result of use. Finally, this "primrose path" is not one that a person traverses due to immorality, willful defiance, or self-sabotage. Rather, it can be seen as a series of intertemporal decision errors pertaining to drugs that arise, in part, from time-inconsistent preferences (Kim \& Lee, 2011). After all, most people do not desire or intend to become addicts. Rachlin (2003) reflects on such conflicting intertemporal preferences and some of the possible unintended consequences thusly:

The alcoholic does not choose to be an alcoholic. He prefers not to be one. His preference ordering is: 1) Not being an alcoholic, 2) Drinking tonight, 3) Not drinking tonight. He just chooses to drink tonight, and tonight, and tonight—-and ends up as an alcoholic without ever having chosen to be one (147).

The lifestyle and future fate of an opioid or stimulant user may not be what is preferred and then chosen from a menu of options, but may nevertheless be what is received through solitary decisions over time. There is value in emphasizing the possibility that people who end up with drug-related consequences 
did not choose this less rewarding path. Such emphasis has moral implications for how society addresses high-risk drug users, including how policies and interventions are developed and delivered.

\section{Exploratory Study Relevance}

Insufficient consideration of the many factors that can influence decision-making may lead to inaccurate conclusions about decision-making agents. Among correction-involved adults with a history of drug use, some will make decisions to use opioids and stimulants. Some will continue to use these drugs, despite the serious risks associated with use and despite experiencing significant consequences. Failing to consider the breadth of conditions with the potential to influence decisions to use high-risk drugs may contribute to the perpetuation of inaccurate attributions of corrections-involved people's overall motives or intentions, whereby they may be viewed as more culpable, blameworthy, and morally responsible for their drug use and drug-related offenses (Corrigan, 2000, 2005; Lacey \& Pickard, 2012; Meurk et al., 2014).

Inaccurate intentions or attributions assigned to corrections-involved opioid and stimulant users can result in less pragmatic, humane, and effective interpersonal and systemic responses. For instance, evidence suggests that sanctions, rather than treatment, are more likely to be administered for drug use that is perceived as a willful act of "abuse" (Kelly \& Westerhoff, 2010). That drug users and correctionsinvolved people are often stigmatized and socially devalued means that they are potentially more likely to be blamed for using, while the mitigating endogenous and exogenous factors that influence decisions to use are less likely to be fully considered (Barry, McGinty, Pescosolido, \& Goldman, 2014; Crawford, 2012; Earnshaw, 2013; van Boekel, 2015). Such blame and stigmatization can contribute to increased anxiety, shame, social withdrawal, and maladaptive forms of coping--including continued opioid or stimulant use (Ahern, Stuber, \& Galea, 2007; Birtel, Wood, \& Kempa, 2017; Luoma, O'Hair, Kohlenberg, Hayes, \& Fletcher 2010; Ritsher, Otilingam, \& Grajales, 2003; Schomerus et al., 2011).

Ultimately, there are many limitations to a coarse-grained exploration of high-risk drug use among corrections-involved people using the methods proposed for this study. These are important and are discussed in Chapter 5. Challenges and limitations notwithstanding, examination of self-report data has the potential to be used as a means for fostering thoughtful discussions about decision-making and for forming critical questions about the appropriateness of attributing blameworthiness to corrections-involved drug users, who are often punished and stigmatized for their use and criminal offending (Maruna \& King, 2009; 
McCorkle, 1993; Phillips \& Shaw, 2013; Pickard, 2011, 2017). Historically, the morality, decency, and worth of people who use drugs, or who become corrections-involved, has been doubted, and their actions treated as willful defiance and public threat; little consideration was given to their welfare (Foucault, 2012). For centuries, the collective instinct when responding to drug use, criminal offending, and other "deviant" behavior has been to punish for its own sake, or to punish in order to absolve or cure (De Tocqueville, 2003; Foucault, 2003; 2012). Given this long-standing collective instinct, it is important to draw attention the fact that there are many factors with the potential to influence and constrain a person such that they would choose to use high-risk drugs and choose to continue to use at nearly all costs. By articulating the potential range and omnipresence of such influence and constraint in this preliminary study, it may be possible in future work to also consider how responsibility and culpability for high-risk drug use, and attendant criminal offending, may be more readily dissociated (Pickard, 2017). 


\section{CHAPTER II}

\section{SOME INFLUENCES ON DRUG-RELATED DECISION-MAKING AND BEHAVIOR}

In the previous chapter, the risks associated with opioids and stimulants were described along with how decisions involving drugs constitute intertemporal choice. Intertemporal choice involving opioids and

stimulants inevitably differs across people and time periods, given that the circumstances of people's lives are wide-ranging and dynamic. This variance and potential for change means that, during drug-related decision-making, people will have many drug and non-drug options to evaluate, each choice differing in its probability of risk and reward in the immediate and the long-term. In short, decision-making about highrisk drugs, such as opioids or stimulants, for which the range of outcomes is never completely certain, requires that many cognitive and affective capacities be brought to bear.

Recall that self-reported use of a drug for a given time period indicates that a decision about using this drug was made during this time period. In this chapter some factors with the potential to influence a person's ability to make decisions that involve risk and trade-offs between current and future rewards will be explored, along with how the options and opportunities available to people can constrain the overall set of drug and non-drug choices. In thinking about some of the factors that might be present prior or contemporaneous to high-risk decision-making so as to influence the likelihood that opioids or stimulants would be used, several factors specific to the person, their history, and the greater context within they live might be identified. Several such factors will be explored in this chapter in at least one of two ways. The first way includes identifying factors that might increase the likelihood of risky decision-making generally. These are factors that might diminish cognitive control, limit a person's set of choices, and otherwise influence intertemporal choice such that a person makes decisions that do not maximize well-being over time. Such poorer decision-making could include choosing to use, or to continue to use, high-risk drugs. It 
could also include choosing any option with high local but lower overall utility. These are factors that can influence cognitive capacities and decision-making more generally. The second way includes identifying factors that might increase the likelihood that opioids or stimulants specifically might be chosen, as opposed to choosing drugs with less relative risk, or choosing not to use drugs.

\section{Always Today, Never Tomorrow}

As noted earlier, because the future is uncertain, and therefore also less salient then the present, people may discount the utility of future rewards, typically referred to as delay or temporal discounting, where the perceived utility of a choice is reduced as time increases (Ainslie, 1992; Bickel et al., 2007; Rachlin, 2006). Temporal discounting can include discounting future rewards and punishments alike. While some economists understand a person's discount rate to be time-consistent and exponential, behavioral economists consider instead that people's discounting, and drug users' discounting in particular, can be hyperbolic (or quasi-hyperbolic) and time-inconsistent (O'Donoghue \& Rabin, 1999, 2002; Rachlin, 2006). When discounting is hyperbolic, people weight the "time until reward" is received as cost, and will choose a smaller, immediate reward over a larger delayed reward (e.g., $\$ 20$ today versus $\$ 40$ in three days) (Ainslie, 1992). As the delay in the second option increases, there is a decrease in preference for this delayed outcome, even as remote outcomes still do retain some small value. A person might also choose an option in the present for which there is larger later cost (e.g., $\$ 20$ today that must be repaid two-fold the following week). In other words, people can be inconsistent in their preferences as a function of time. This can be detected, in part, by preference reversal if the choice is between two outcomes in the future; in this case, the person will select the larger amount (e.g., \$20 in one year or \$40 in one year and three days). Typically, though, the future self prefers the larger, later amount, while the present self prefers the smaller, sooner amount (O'Donoghue \& Rabin, 1999). This dynamic inconstancy indicates that the value assigned to choices varies in proportion to how far away the person is from receiving the outcome associated with a given choice (Ainslie \& Haslam, 1992; Green, Myerson, Lichtman, Rosen, \& Fry, 1996; Loewenstein \& Elster, 1992). The rewarding effects of drugs, of course, can be swift. Although many costs associated with drug use can be realized immediately (e.g., overdose, adverse side effects), others are realized only in the future (e.g., parole violation, liver failure, divorce), including ones that develop as a result of repeated solitary decisions over time (e.g., dependence, addiction). 
The "primrose path" model described in the previous chapter, in which people shift decisions and behaviors to choices with the highest local utility, and without the ability to always adequately assess the utility of their distributed choices, can also be applied to decisions pertaining to other phenomena that are less destructive than addictive drugs. Distributed choice may still be suboptimal for these other decisions; but it may not be life-ruining and lethal. As Herrnstein \& Prelec (1991) note, "In its most egregious form, the slippery slope of distributed choice leads to addiction, which is to say, a devastating level of overindulgence in some commodity or activity" (p.149).

People who have steeper discount rates can be considered as more present-biased or "temporally myopic", and therefore more likely to be meliorating consumers, in which they choose the option with the highest unitary return, thus propelling them further down this unintended trajectory (Bickel \& Marsch, 2001). Present-bias and steeper discounting are more common among current and remitted drug users, including opioid and stimulant users (Heil, Johnson, Higgins, \& Bickel, 2006; Kirby \& Petry, 2004; Kirby, Petry, \& Bickel, 1999; Petry, 2001; Petry, Bickel, \& Arnett, 1998; Reynolds, 2006). Petry et al. (2002) observed not only poorer decision-making performance among a sample of heroin users, evidenced by higher rates of choosing options with high immediate, but low overall reward, but also found that heroin users were less future oriented, including less capable of predicting events in the distant future and coherently organizing future events. It is important to note that while some people may evidence greater present-bias and steeper delay discounting rates for certain intertemporal decisions, they may not do similarly for others. Relatedly, a person may exercise greater self-regulation in one domain than in another. Thus, although a person may have some global propensity toward present-bias and delay discounting, and less ability to self-regulate or inhibit impulses, there can also be differences that depend on the choices themselves, other cognitive capacities, and the context in which learning and decision-making occurs (Mishel, 1973; Mischel, Ebbesen, \& Raskoff-Zeiss, 1972; Mishel et al., 2010).

However, evidence suggests that some people are simply more prone toward impulsivity and present-bias, such that irrespective of the nature of reward, they will more often tend to choose the option with highest immediate or local utility, as future rewards and costs are weighted differently than immediate or near-term outcomes (Madden \& Bickel, 2010; Petry, 2001). Increasing evidence suggests not only a strong positive correlation between impulsivity, delay discounting, and drug use (de Wit, 2009; Madden \& 
Bickel, 2010; Verdejo-García, Lawrence, \& Clark, 2008), but also a likely causal relationship (in some cases mediated by poorer executive function, including working memory), evidenced by steep discounting observed prior to drug use initiation (Anokhin, Golosheykin, Grant, \& Heath, 2011; Audrain-McGovern et al., 2009; Khurana et al., 2013; MacKillop et al, 2001). Although some groups may be more prone to temporal discounting, certain endogenous and exogenous factors can also contribute to this tendency.

\section{Drug-related Influences on Decision-making}

When exploring factors with the potential to influence opioid and stimulant use among a sample of corrections-involved adults with a history of drug use — who by virtue of belonging to this group may already be estimated to have a propensity toward greater present bias and delay discounting-- it is important to consider additional factors that may have been present prior to or contemporaneous with time periods when decisions about opioids and stimulants were made. Prior drug use, including prior use of opioids or stimulants, is one such factor.

\section{Early Drug Use Initiation}

Early drug use initiation is defined here as psychoactive drug intoxication at or prior to age 14. Age 14 is consistent in the literature as a critical developmental juncture, in that there is less frontocortical density and less neural integration or "coupling" of brain areas prior and during this time (Gogtay et al., 2004; Shaw et al., 2008). Early drug use initiation has been associated with a greater likelihood of developing drug-related problems and more severe use in later adolescence and adulthood, including dependence, SUD, and IDU (Anthony \& Petronis, 1995; Chen et al., 2009; McCabe, West, Morales, Cranford, \& Boyd, 2007; Trenz et al., 2012). It is therefore necessary to consider some of the possible implications of early drug use initiation on cognition and drug-related decision-making during adulthood.

Initial drug use can be considered as a form of experimentation and exploration. As such, it signals some proclivity toward curiosity. While curiosity is a universal human characteristic, it is particularly pronounced in youth (Piaget, 2005). Beyond age-typical curiosity, early drug use suggests the presence of pre-morbid traits known to correlate with steeper discounting and risk-taking, such as impulsivity, sensation-seeking, and novelty-seeking (Cloninger, 1987; Cloninger et al., 1988; Ebstein \& Belmaker, 2002; Ersche et al., 2010; Lejuez et al., 2010; MacPherson, Magidson, Reynolds, Kahler, \& Lejuez, 2010; Wingo et al., 2016). In addition to being associated with early drug use initiation, these 
characteristics have been implicated, singularly or in a synergistic manner, in the development and perpetuation of problematic drug use and addiction to a variety of drugs, including opioids and stimulants (Belin, Mar, Dalley, Robbins, \& Everitt, 2008; Dawe \& Loxton, 2004; MacPherson et al., 2010; Madden et al., 1999; Vuchinich \& Simpson, 1998).

Early drug exposure also indicates that neurophysiological and epigenetic changes transpired during critical phases of rapid neurocognitive development (Albertson, Schmidt, Kapatos, \& Bannon, 2006; Andersen \& Navalta, 2004; Cadet, 2016; de Wit, 2009; Guerri \& Pascual, 2010; Stanwood \& Levitt, 2004). This is important, in that during adolescence a person's brain evidences greater plasticity and is not yet fully developed, meaning that some neural, genetic, cognitive, and behavioral changes may have enduring impacts on later functioning if not addressed and ameliorated; particularly when other factors (e.g., stress, deviant peer influence, parental SUD) or morbidities are present (Chambers, Taylor, \& Potenza, 2003; Champagne, 2010; Fagiolini, Jensen, \& Champagne, 2009; Post, 2016; Mistry, Bawor, Desai, Marsh, \& Samaan, 2014; Tarter, Kirisci, Habeych, Reynolds, \& Vanyukov, 2004; Vaughn, Beaver, DeLisi, Perron, \& Schelbe, 2009; Witt, 2010). Incorporated within this latter point is the fact that opioids and stimulants are highly rewarding, meaning that they might lead to extreme "wanting" or craving among those that use them (i.e., sensitization of incentive salience; Robinson \& Berridge, 2000), which includes dramatically altering learning and behavior (stimulus-response habituation; Robbins \& Everitt, 1999), in either case, biasing a person toward continued use. Consequently, a decision not to use drugs one was previously exposed to becomes more difficult make.

The degree or frequency of drug use subsequent to initiation is important. Consider how a person who uses cocaine once at age 14 may differ from someone who used cocaine intermittently at age 14 and again in young adulthood, and how this person would differ still further from a person who used cocaine at age 14 and regularly throughout adolescence and later adulthood. Moreover, someone who used cocaine prior to age 14 might be expected to experience an overall poorer SUD trajectory in later adolescence and adulthood, in part due to a longer exposure window (Anthony \& Petronis, 1995; Grant \& Dawson, 1998; Gruber, DiClemente, Anderson, \& Lodico, 1996; Tapert \& Brown, 1999).

The commonality here is that early age of drug use initiation indicates a greater likelihood of observing other traits associated with increased risk-taking and less adaptive decision-making in adulthood. 
Regardless of whether early age of initiation indicates the pre-morbid presence of traits (e.g., cognitive, personality, genetic) associated with poorer executive control, propensity toward use, and greater likelihood for developing SUD, or that early use casually contributes to the development of these things, the implications of early age of initiation (e.g., greater impulsivity, poorer drug-related decision-making in adulthood) are similar. This is particularly true in regard to impulsivity and lack of self-regulatory capacity (King \& Chassin, 2007; Tarter et al., 2003), as evidence suggests that impulsivity can be considered marker for SUD development and other addictive behaviors (Petry, 2001; Verdejo-García, Lawrence, \& Clark, 2008).

Impulsivity involves several components. These include action with less forethought; immediate gain at risk of larger, future loss; and "disposition toward rapid, unplanned reactions to internal or external stimuli without regard to the negative consequences of reactions" (Ainslie, 1974; Dickman, 1993; Moeller et al., 2001). DeYoung \& Rueter (2016) consolidate the definition into two necessary parts: 1) The impulse, urge, motivation, or desire to act, and 2) A lack of inhibition, restraint, or control of the impulse (e.g., failure of self-regulation). Impulsivity is central to consider when discussing high-risk decision-making, in that people with higher impulsivity/poorer self-regulation are typically more likely to demonstrate other executive dysfunction (e.g., poorer working memory), have steeper discount rates, and exhibit greater risk propensity/neurodisinhibition (Hinson, Jameson, Whitney, 2003; Nichols \& Wilson, 2015; Semple, Zians, Grant, \& Patterson, 2005; Tarter et al., 2003). These have obvious implications for decision-making and risk-taking in relation to opioids and stimulants. Further, there is support for the idea that people who evidence greater impulsivity may perceive time differently than people with greater capacity for selfregulation/impulse control, wherein the former group overestimate the duration of time intervals and commensurately devalue delayed rewards at steeper rates (Berlin \& Rolls, 2004; Rubia, Halari, Christakou, \&Taylor, 2009; Wittmann \& Paulus, 2007, 2009). In other words, for these people, the future may feel like an eternity away and potential future states may resonate less intensely. Impulsivity, which involves premature decision and behavior, thus makes sense as a correlate of delay discounting, particularly in relation to drug use, where potent effects are gained in real-time (Paasche, Weibel, Wittmann, \& Lalanne, 2018). 
Because early age of drug use initiation often positively correlates with other high-risk behaviors (e.g., smoking, unprotected sex, driving under the influence) and with disorders which positively correlate with SUD and diminished self-regulatory control (e.g., attention deficit hyperactivity disorder [ADHD]), people who began using earlier in life may be considered potentially more impulsive and less capable of self-regulation during decision-making in adulthood than people who began using later in life (Barkley, Edwards, Laneri, Fletcher, \& Metevia, 2001; Eslami-Shahrbabaki, Fekrat, \& Mazhari, 2015 Stueve \& O’donnell, 2005; McGue et al., 2001; Vaughn et al., 2009).

Considering early drug use initiation as a marker or proxy for impulsivity and poorer selfregulation is not without limitations. Wittmann \& Paulus (2007) offer a reminder that "impulsivity is determined by a complex set of processes and consists of multiple components,... [but that] nevertheless, impulsivity can be conceptualized as a pattern of behavior for which the potential of negative consequences has limited influence on the planning of actions". Among a sample of corrections-involved people, early drug use initiation can be considered as one of many indicators of such a behavioral pattern.

\section{Drug Preferences, Drug Use, and Severity of Use}

A person's drug preferences, drug-related decisions, and drug behaviors can change over time. For instance, a person might use heroin or cocaine heavily for several years and then later moderate or discontinue use (Lewis, 2015; Robins, Helzer, \& Davis, 1975; Waldorf et al., 1992). However, when exploring factors with the potential to influence decision-making about opioids and stimulants during a particular time period, it is important to identify people's prior and contemporaneous use of these drugs, the severity of their use, and their drug preferences.

\section{Reinforcer Pathology and Drug Preference}

People who exhibit greater present bias, impulsivity, delay discounting, and poorer self-regulation may be more likely to initiate drug use and persist in use. Despite these tendencies, it is important to consider that consumption of certain commodities may not ever lead to problems. In other words, the utility associated with unitary choices and their distribution (aggregation) may not appreciably differ. It is important to keep in mind that in addition to endogenous and exogenous factors that influence decisions to use opioids and stimulants, the drugs themselves comprise a unique form of consumption. Bickel et al. (2011) describe how the consumption of highly rewarding commodities, such as drugs, can result in 
reinforcer pathology, defined as a "1) the persistently high valuation of a reinforcer, broadly defined to include tangible commodities and experiences; and/or 2) the excessive preference for the immediate acquisition or consumption of a commodity despite long-term negative outcomes" (p.407). In simplest terms, a reinforcer can be understood as the presence or absence of a stimulus that results in associative learning, and subsequent increases or decreases in particular behavioral responses (Sutton \& Barto, 2018; Thorndike, 1998).

People who misuse drugs, and persist in use despite costs, come to overvalue drugs in the extreme and demonstrate preference for immediate receipt of drugs. Thus, while certain factors, such as impulsivity and poorer executive control, may influence a person's overall cognitive repertoire and decision-making capacities, the rewarding characteristics of the drugs themselves must be considered in relation to these other factors (Bickel et al., 2007, 2011; Bickel, Mueller, Jarmolowicz, 2011; Jentsch \& Taylor, 1999; Monterosso et al., 2007; Stafford, LeSage, \& Glowa., 1998; Xu et al., 2009). For some people, opioids and stimulants may produce rewarding effects that are greater relative to other drugs, therefore intensely motivating behavior toward the use of these, but not necessarily all other drugs with abuse liability (Bickel, Marsch, \& Carroll, 2000; Kreek, Bart, Lilly, Laforge, \& Nielsen, 2005; Kreek et al., 2012).

As highlighted in Chapter 1, many drug users can best be considered as polydrug users. For instance, someone who used prescription opioids frequently during the past-year may have also used heroin during the same period with even greater frequency, but may have used buprenorphine with less frequency. They may also have used MDMA, inhalants, alcohol, and a variety of other drugs (Daniulaityte, Falck, Wang, \& Carlson, 2009; Leri et al., 2003). Despite this polydrug use, it is likely that this person has a preferred drug of choice (O’Connor \& Berry, 1990). As will be discussed, exposure to or accessibility of a particular drug, while important, is not the only potential influence on drug-related decision-making. Preference is also important to consider, as drug choices stand in relation to one another and vary in their utility and reinforcing effects.

Although it might be measured in many ways, drug preference can be understood as the drug associated with the greatest behavioral response, which, for people, might be characterized as the drug used with greatest frequency or the drug that people spend more resources to acquire when other drug choices are also available (Katz, 1990). However, because many factors can constrain people's choices during a 
given time period, and therefore decrease use (e.g., not due to lack of preference but to decreased supply), it is also important to determine what drug a person would rate as their preferred drug of choice. Although stated preferences can diverge from choice and behavior, there is often strong correlation (Smith \& Staton, 2018). Consider how a person who prefers chocolate ice cream might always choose chocolate ice cream when it is available, but might eat vanilla or strawberry ice cream if chocolate is unavailable. This same person, however, would not eat pistachio ice cream, even if it was the only flavor of ice cream available. Indeed, because preference is in many ways conceptually similar, if not interchangeable, with utility (Bentham, 1996), a person's preferred drug necessarily implies that it has a higher local utility compared to other drugs. Although such a preference doesn't suggest that this drug is preferred to non-drug choices, it does suggest that this preference will influence drug-related decision-making. If a person prefers heroin, then this preference influences their decision to use heroin. For instance, it would be less likely that, given a choice between heroin and a substitute for heroin, which could include a drug with similar pharmacology (e.g., prescription opioids, buprenorphine) or a drug with dissimilar pharmacology that still produced rewarding effects, a person would not choose heroin.

\section{Prior Drug Use and Frequency of Use}

When exploring factors with the potential to influence decision-making about opioids and stimulants for a particular time period, prior and contemporaneous use of opioids and stimulants may serve as possible influences. For example, a person may have used prescription opioids every month for the pastyear or every day for the past month. Or, they could have used them seldomly, perhaps one month out of the past year and three days out of the past month. Clearly, there are differences between the two. Prior or contemporaneous use of prescription opioids influences current and future decisions about prescription opioids. At a minimum, any use of this drug indicates a willingness to use prescription opioids.

Frequent and regular use indicates the possibility that prescription opioid use may have, to some degree, become habituated over time (Nelson \& Killcross. 2006; Robbins \& Everitt, 1999). By habituation, it is meant that certain behaviors, in this case the acquisition and use of prescription opioids, have been established as a result of stimulus-response learning, thus making future similar behaviors easier to perform and dissimilar future behaviors (in relation to prescription opioids) more difficult to preform (Gardner, 2015). To help illustrate, consider how many common behaviors become habituated: Eating the same meal 
for breakfast; putting contact lenses in after brushing teeth, beginning with the right eye; watching Netflix, eating chocolate, and drinking pinot noir every night after putting the children to bed; taking the same route home from work; using Apple operating software instead of Microsoft; ordering pizza every Friday night from the same restaurant (Aarts, Verplanken, \& Van Knippenberg, 1998; Graybiel, 2008; Ouellette \& Wood, 1998; Polites \& Karahanna, 2012). Habits are efficient and certainly not always pathological (Desrochers, Jin, Goodman, \& Graybiel, 2010). In the absence of habits, significant cognitive energy would be wasted processing previously encountered information, deliberating, forming goals, making decisions, and re-learning behaviors; indeed, maintaining habits requires less cognitive effort than inhibiting or "overriding" them, with habits strengthening over time (Gardner, 2015; Graybiel, 2008; Wood \& Rünger, 2016). When prescription opioid use becomes habituated, however, decision-making about this drug can be considered as significantly biased toward continued use and no longer necessarily associated with goaldirected behavior; it may even be considered compulsive (de Wit, 2018). In relation to prescription opioids, cognition and behavior would be more implicit, automated, and reflexive, and less explicit and deliberative (Bechara, Noel, \& Crone, 2006). Habits may still be consonant with goals or intentions, but they may also interfere with goal-directed behavior (Wood \& Neal, 2007; see also Chapter 5 for further discussion).

Additionally, more frequent and regular use of a drug (i.e., greater exposure) is associated with decreased capacity to regulate attention, affect, and make deliberative decisions about that drug (Bechara, 2005; Naqvi \& Bechara, 2010). This does not suggest that habituated behaviors, including drug taking, occur involuntarily or without an agent (e.g., a person with capacity to act), or without agency, defined as the exercise or manifestation of the capacity to act (Bandura 2006; Stanford Encyclopedia of Philosophy, 2015; Wood \& Rünger, 2016). Rather, it implies that the cognitive effort or self-regulation needed to "do otherwise" is much greater, and much more difficult to come by, given that habituation is shaped and constrained from inputs at multiple levels (Zimmerman, 2013).

Thus, someone who has used prescription opioids frequently over the past-year or month, and who currently prefers prescription opioids to other choices, would require more cognitive energy and time when making a decision to not use prescription opioids following this time period; particularly if the general circumstances or context of their past use had not also changed (Lally, Van Jaarsveld, Potts, \& Wardle, 2010; Wood \& Neal, 2007). Ultimately, this recent prior exposure to prescription opioids would serve as an 
influence on decision-making, such that the person would be more likely to choose to use prescription opioids rather than a novel or infrequently used drug. This doesn't mean that the person would always choose the same drug or self-administer drugs in the same manner, as habits and patterns of use can change and reform over a person's life (Des Jarlais et al., 2007; Neaigus et al., 2001). Certainly, though, the presence of other factors will enable or frustrate both goal-directed and habituated behavior. For instance, in order for a person to make other decisions and act on them, there would have to be alternative choices to opioids with presumably greater perceived local utility (e.g., other drugs, other activities), or the overall utility of prescription opioids would have to be recognized by the person as lowered as a result of continued use (e.g., tolerance). In order to discern, reflect, and act upon this information, however, the person would need higher-level cognitive capacities (e.g., attentional control, prospection, counterfactual and abstract reasoning, self-regulation, etc.), both to identify the discrepancy between the local and overall utility of prescription opioids, but also to help enable decisions and enact behaviors discordant with habituated action. In other words, greater capacity to inhibit or "override" the habituated behavior and choose the option with greater overall utility.

\section{Severity of Drug Use}

Drug exposure, or frequency of use, is not synonymous with drug use severity. Indeed, some people may use drugs frequently or with regularity over days, months, or years, but in a moderated or otherwise controlled manner (Parker, Williams, \& Aldridge, 2002; Waldorf et al., 1992; Zinberg, Jacobson, \& Harding, 1975; Zinberg, 1984). Severity of use can be defined in several ways. DSM-V diagnostic criteria for SUD, indicators such as craving, persistence in use despite a desire or attempts to quit, and withdrawal are used to help diagnose both the presence and severity of the disorder (APA, 2013). The severity of a person's drug use is relevant in that greater severity may indicate transition from goal-directed to habituated behavior in relation to drugs, as described above (Nelson \& Killcross, 2006 Zapata, Minney, $\&$ Shippenberg, 2010). More frequent use and greater severity of use correlate with considerable neurobiological changes known to impair areas of the brain associated with capacities crucial for decisionmaking (e.g., risk assessment, valuation, emotional regulation, self-regulation, attention, memory information-gathering) (Blanchard, Hayden, \& Bromberg-Martin, 2015; Koob \& Volkow, 2010; Strait, Sleezer, \& Hayden, 2015). Therefore, the qualities of drug use severity (e.g., craving, unsuccessful attempts 
to quit) occurring prior to or contemporaneous with drug-related decisions indicates the presence of significant influences on decision-making capacity, whereby a person becomes more biased toward use even if a multitude of other possible choices are available and even if the subjective feeling of choice or phenomenological sense of ability to choose freely is present (Lau, Hiemisch, \& Baumeister, 2015). Here, a person with greater using severity might be considered to have fewer degrees of freedom for volitional action compared to a person with less severity (Dennett, 2015; O’Connor, 2009).

Ultimately, greater drug use severity would likely influence a person to continue to make unitary (local) decisions over global ones that, in the aggregate, decrease overall utility, whereas someone with less severity might have greater purchase on the cognitive capacities needed to reframe choices, inhibit habituated behaviors, and choose another option with less local, but greater overall, utility (Heyman \& Dunn, 2002).

\section{Craving and Utility}

One specific quality of drug use severity worth highlighting is craving (i.e., drug wanting), in part because it often correlates with use (Robinson \& Berridge, 1993; Tsui, Anderson, Strong, \& Stein, Sayette, 2016). Throughout this discussion, utility has been used primarily to mean "decision utility", the choice with the highest preference assigned to it; however, "experienced utility" (which is closely related to decision utility, but less frequently considered; see Kahneman, Wakker, \& Sarin, 1997), may also be relevant, in that it reflects the choice that brings about the greatest satisfaction experientially. In other words, the utility for a decision based on one's preference is separable from one's actual experience of that choice. What is preferred or desired is not always synonymous with what is enjoyable or satisfying. These differences in utility are consonant with the well-known idea that "wanting" and "liking" can become cognitively decoupled among drug users (Berridge, Robinson, \& Aldridge, 2009). "Remembered utility" refers to the satisfaction one gets after having made a choice and experienced it (Kahneman et al., 1997).

Generally, after having made choices and experienced their outcomes, a person forms memories that enable them to make predictions about similar choices when making decisions in the future.

Berridge \& O’Doherty (2014) consider that, typically, "wanting” or "desire” would be informed by memories, would coherently map on to experiences, and that, "for such cognitive desires, decision utility=predicted utility, and predicted utility=remembered utility" (p.342) . However, incentive salience, 
which is a cognitive process of wanting or craving that develops with repeated drug use (informed by both current physiology/neurobiological states and learned cue-induced stimulus response), is a reliable and potent behavioral motivator (Robinson \& Berridge, 1993). For drug users, "situations exist where cuetriggered decision utility>remembered utility from the past, and similarly decision utility>predicted utility for future reward value (Berridge \& Aldrridge, 2008). In other words, it is possible to 'want' (and choose) what is not expected to be liked, nor remembered to be liked, as well as what is not actually liked when obtained" (Berridge \& O’Doherty, 2014, p. 324).

Again, craving is understood as a state of incentive salience. It is cognitive and visceral, it influences the perception of time, the relative value assigned to a drug, and potentiates behavioral responses (Bechara, Dolan, \& Hindes, 2002; Grant et al., 2000; Loewenstein, 2000; Sayette, 2005; Volkow et al, 1991; Wang et al., 2012). It has been proposed by Redish et al., (2015) as reflecting a possible interaction between systems of valuation characteristic of deliberation and Pavlovian conditioning. For people with extensive or pathological drug use, craving is one state that can influence decision-making by essentially cognitively decoupling a unitary choice from both past and future (e.g., cue-triggered decision utility>remembered utility from the past; decision utility>predicted utility for future reward value). Of course, for some, there may still be a desire for drugs in which incentive salience is not a component (e.g., decision utility=predicted utility, and predicted utility=remembered utility).

For polydrug users, the person's preferred drug and drugs most frequently used in the past would serve, along with severity of use (e.g., craving, attempts to quit), as influences on decisions about drugs. Again, preference may become separable from experience, but greater prior use of a drug and craving for that drug are likely to be associated. For example, incentive salience for a person who used alcohol every day, but used cocaine only every five months, would likely correspond to different alcohol versus cocaine wanting, assuming no prior cocaine dependence at an earlier time. In this way, a person would have greater or fewer degrees of freedom when making a decision about particular drugs based, in part, on their prior exposure to these drugs. A person who exhibited this same alcohol and cocaine use behavior over a year would more likely come to "want" cocaine in the traditional sense, but "want" alcohol in the pathological, behavior-inducing sense. 
Taken together, it is reasonable to believe that drug-related decision-making is likely influenced by incentive salience and other drug-related factors described above if, during a given time period, a person reports experiencing greater severity of use (e.g., craving, failed attempts to quit using following a period of regular drug use, withdrawal, etc.) and also reports ongoing drug use during this period.

\section{Sorites Paradox}

Recall that on the "primrose path", where decisions can be considered both unitarily as well as in the aggregate (i.e., many choices distributed over a time period), it is possible for a person to reverse course by choosing options with lower local, but higher overall utility through cognitive reframing and other efforts (e.g., seeking treatment, forming new non-drug habits, etc.) after the costs associated with continued use become apparent (Herrnstein \& Prelec, 1992; Heyman, 2003, 2013). However, such cognitive reframing and behavioral change might be more or less difficult depending on how many unitary choices have been made to use thus far. As Heyman (2003) articulates:

[A]lcoholism and abstinence (or controlled drinking) are states that reflect the cumulative effects of many smaller decisions ... [where] no one decision is decisive. This could be considered, among other ways, in terms of severity of use and exposure (Heyman \& Dunn, 2002) (p. 103).

In other words, greater drug exposure and severity of use develop. "Frequent" use doesn't begin as "frequent" and "severe" doesn't begin as "severe". These states or properties are acquired and therefore have the capacity to intensify or dissolve. Although earlier discussion considered how these states can influence decision-making, it failed to reconcile when these states change into something else. Similar irreconcilability exists for the idea of solitary versus aggregated decisions and local versus overall utility: There is the injection of heroin, and then there is the heroin addiction. It is therefore important to note that when exploring factors with the potential to influence opioid and stimulant use in real-world conditions, it is not possible to know the juncture at which a person--as necessarily both a state and a verb, as both the decision-maker and the embodied aggregate of his decisions--becomes precisely defined and influenced by prior drug use or using severity, or where the person is relative to past and future decisions. Instead, the parameters can only be drawn so as to define the field, or time period, of interest (which can still be arbitrary) during which solitary decisions are being made and in which their distributed effects are bounded (e.g., a day, a year, etc.), and to then identify what else stands in relation to those decisions. This doesn't 
suggest that with access to perfect information, or the acquisition of enough data points over time, decisions could not be plotted. In theory, they could. In practice, they seldom are outside of experimental conditions (and even within) due to obvious data collection limitations. Even here, choice is constrained to levels that are inconsistent with choice in the real-world.

Therefore, important conceptual and measurement limitations remain, in that any cut-off point between "many unitary decisions to use heroin" and their distributed effect of "becoming a heroin addict" is still only ever obliquely, and rather inadequately, discernable. This is due, in part, to insufficient information, diagnostic and measurement limitations, lingering disagreement about what addiction is best defined as, and the fact that observable symptoms or indicators of pathologies such as "heroin addiction" may also be attributable to other pathologies. Some "heroin addiction" indicators may be attributable to

other sources, including to unitary decisions having nothing to do with heroin. Put differently, the observed effects of distributed choice to use heroin (i.e., heroin addiction or OUD) present with features that are similar to other pathologies with distinct etiologies and neural correlates (Brooks, Lochner, Shoptaw, \& Stein, 2017; Cuthbert, 2015; Kwako, Momenan, Litten, Koob, \& Goldman). This is noted not to disparage any particular diagnostic method or detract from behavioral economic accounts of drug use and addiction, which have tremendous parsimony and empirical support, but rather to note, as others have, some of the unavoidable tension between theory and data (Bickel, Jarmolowicz, Mueller, \& Gatchalian, 2011). Because this study is exploratory, it is important to emphasize the fact that much of what is worth discussing about the social problem of high-risk drug use approach cannot yet be sufficiently modeled or explicated in terms of real-world conditions. Limitations will be considered further in Chapter 5.

\section{Possible Constraints on Decision-making Capacity Among Opioid and Stimulant Users: Do the Differences Matter?}

Prior drug exposure, drug use severity, and preference may also be influential in ways that vary according to drug type. Unsurprisingly, pharmacological differences between drugs have the potential to influence a drug user's neurocognitive capacities differentially. As decisions and behaviors only occur as a result of brains, the underlying neurobiology of drug use is recognized as relevant among researchers studying choice and behavior (Bickel et al., 2007). A person who frequently uses and prefers alcohol will experience different neurobiological changes than a person who frequently uses and prefers opioids or 
stimulants. For instance, a person who frequently uses opioids or stimulants, but infrequently uses alcohol, would not develop Wernicke-Korsakoff syndrome (Dodge, Jacobson, \& Jacobson, 2016).

Even within opioid and stimulant drug classes, between-drug differences might be observed. For example, methamphetamine-dependent people have been found to have impaired risk-related processing due to alterations in brain areas necessary for higher-level cognitive functioning (e.g., working memory, self-regulation, attention, planning), interoception, and time perception compared to non-users and other drug users (including opioid users), and to evidence greater impulsivity and uncertainty salience (Ersch et al., 2005; Gonzalez, Bechara, \& Martin, 2007; Gowin et al., 2013; Leland, Arce, Feinstein, \& Paulus, 2006; Leland \& Paulus, 2005; Pollatos, Laubroc, \& Wittmann, 2014; Semple et al., 2005). One meta-analysis found that people with active and remitted methamphetamine use disorder exhibited moderate cognitive impairment across multiple domains, with the largest deficits observed for impulsivity/reward processing and social cognition (Potvin et al., 2018). Further, some evidence indicates that people with greater impulsivity and sensation-seeking report stronger rewarding effects from amphetamines (e.g., greater liking, wanting, and euphoria) (Kelly et al., 2006). Among MDMA users, attentional control, long-term memory, and sensory gating (i.e., ability to filter out non-relevant distractions and sensory information) were also found to be impaired, with deficits persisting over time (Lundqvist, 2005).

Compared to healthy controls and other drug users (e.g. cannabis, opioids), cocaine dependent people have performed worse on decision-tasks (e.g., making less advantageous choices, commission errors) that require information acquisition, working memory, prediction, and attention (Cunha, Bechara, de Andrade, \& Nicastri, 2011; Kjome et al., 2010; Mejía-Cruz, Green, Myerson, Morales-Chainé, \& Nieto, 2016). When compared to non-users and cannabis users, cocaine users performed worse on decision tasks, even after a short period of abstinence (Verdejo-Garcia et al., 2007). Among a clinical sample, crack cocaine users scored higher on measures of impulsivity and risk-taking compared to heroin users (Bornovalova, et al., 2005). However, some evidence also suggests that cocaine users' performance can improve over time when valued contingencies (e.g., money) are introduced (Vadhan, Hart, Haney, van Gorp, \& Foltin, 2009).

More generally, stimulant users (cocaine, amphetamines) have demonstrated less adaptive and dynamic cognitive functioning (i.e., no increased shift toward frontocortical processing), and poorer 
decision-making and behavioral performance during tasks that require learning, prediction, and cognitive flexibility, resulting in less adaptive error responding (Paulus, 2002; Paulus, Lovero, Wittman, \& Leland, 2008; Simon et al., 2001). Evidence remains mixed, though, for the degree to which cognitive functioning, impulsivity, working memory, and decision-making remains impaired or improves over time after stimulant use is reduced or discontinued, and whether cognitive, self-regulatory, and decision-making deficits are a contributor to or consequence of stimulant use (Frazer, Richards, \& Keith, 2018; Hulka et al., 2015; Kalechstein, Newton, \& Green, 2003; McCann et al., 2008; Potvin, Stavro, Rizkallah, \& Pelletier, 2014; Potvin et al., 2018; Salo et al., 2009; Stewart et al., 2013; Wang et al., 2013).

Chronic opioid use can alter and impair neurophysiology and neuroanatomy in areas associated with emotion, self-regulation, risk-taking, and goal-directed behavior (Baldacchino, Balfour, Passetti, Humphris, \& Matthews, 2012; Pandria, Kovatsi, Vivas, \& Bamidis, 2018). Among a sample of opioid users, poor performance on decision tasks measured at treatment admission predicted post-treatment relapse (Passetti, Clark, Mehta, Joyce, \& King, 2008). In another study, patients receiving treatment for OUD made riskier decisions compared to controls and exhibited decisional deficits associated with impaired executive function (Brand, Roth-Bauer, Driessen, \& Markowitsch, 2008). Additionally, current and former opioid users, including people maintained on agonist therapies, have evidenced steeper discount rates and less adaptive decision-making (Biernacki et al., 2018; Wollman et al., 2017), including discounting delayed heroin at higher rates than money (Madden, Petry, Badger, \& Bickel, 1997). Heroin users have also shown to persist in poorer strategic task performance where learning and error correction would be expected (Orrnstein et al. 2000). One study examining impulsivity and strategic decision-making, found that active heroin users performed worse compared to healthy controls, people who take prescription opioids licitly (and do not have OUD), and abstinent heroin users receiving MMT (Baldacchino, Balfour, \& Matthews, 2015). In another study, current and abstinent (1 year) opioid users and stimulant users performed worse than controls in terms of risk on decision tasks, but did not significantly differ by druguse group, meaning that both groups may be considered to have increased likelihood for some cognitive impairment, even after use is discontinued (Ersche et al., 2005).

Taken collectively, though, findings provide support for the idea that both former and current opioid users have impairments in decision-making, working memory, attention, and inhibition/self- 
regulation, but with arguably greater variance across samples than across samples of stimulant users ( $\mathrm{Li}$ et al., 2013; Yan, et al., 2014; Zheng et al., 2013). Specifically, evidence points to potentially worse cognitive effects for amphetamine (including "street meth") users, compared to cocaine and opioid users, which may be partially attributable to greater exposure to synthetic compounds that produce greater neurotoxic effects (McConnell et al., 2015; Nordahl, Salo, \& Leamon, 2003; Potvin et al., 2018). Badiani et al. (2011) considered the possibility that despite the fact that cognitive impairments (e.g., memory, cognitive flexibility, decision-making) are observed in both opioid and stimulant users, and share common neurobiological substrates (Bechara, 2005; Fernandez-Serrano, Pérez-García, M., Schmidt Río-Valle, \& Verdejo-Garcia, 2010; Fu et al., 2008; McNamara, Dalley, Robbins, Everitt, \& Belin, 2010; Muriach et al., 2010; Orrnstein et al. 2000; Tramullas, Martínez-Cué, \& Hurlé, 2008), there are nevertheless noteworthy differences in the degree of neuroanatomical difference and neurocognitive impairment between opioid and stimulant users, particularly for impulsivity and cognitive flexibility. The authors note that the preponderance of (albeit limited) evidence suggests that the latter group may exhibit significant and potentially longer-lasting deficits (Ersche et al., 2005; Ersche, Clark, London et al., 2005; London, Robbins, \& Sahakian, 2006; Ersche, Roiser, Robbins, \& Sahakian, 2008). Further, discontinuation of stimulants is associated with increased deficits in inhibition, whereas impulsivity did not increase with opioid discontinuation (Liu, Heitz, \& Bradberry, 2009; Liu et al., 2011; McNamara et al., 2010). It may therefore be reasonable to believe that frequent stimulant use, more so than frequent opioid use, would adversely influence a person's decision making and ability to self-regulate, even after periods of abstinence.

When examining opioid and stimulant use during particular time periods, prior and contemporaneous exposure to these drugs might be considered then, not only in terms of potential influences described in previous sections (e.g., preference, habituation, craving), but also in terms of how use of these high-risk drugs may beget further high-risk decision-making in general.

A limitation to some of the research described above is that opioid and stimulant users may also co-use other drugs. However, when concomitant use of opioids and/or stimulants was examined, people using opioids and/or stimulants still performed worse than alcohol and other drug users, including other polydrug users who did not use opioids or stimulants (Nixon, Paul, \& Phillips, 1998). Additionally, some 
findings indicate that dependence on multiple drugs is not associated with increased delay discounting (Businelle, McVay, Kendzor, \& Copeland, 2010) and that cognitive effects from other drugs may not necessarily be additive to opioids or stimulants. For instance, one study examining cognitive abilities found alcohol users performed better than stimulant-only users and alcohol/stimulant co-users, but that stimulantonly users performed worse than both the alcohol-only group and the alcohol/stimulant co-use group (Lawton-Craddock, Nixon, \& Tivis, 2003). However, as findings cannot definitively clarify if cognitive deficits associated with opioid and stimulant use pre-dated drug use initiation, resulted from, or were exacerbated by prolonged exposure to these drugs, opioid and stimulant use may be considered as a generic and approximated potential indicator of cognitive deficits. Irrespective of whether these deficits pre-dated or developed from use, evidence suggests a strong association between opioid and stimulant use and executive dysfunction, such that prior exposure to these drugs indicates that important decision-making deficits may be present.

\section{Demographic Characteristics with Potential to Influence Decision-making}

\section{Age and Sex}

There are two demographic characteristics, younger age and male sex, that are commonly associated with risk-taking behavior generally and greater likelihood to use illicit drugs specifically (APA, 2013; Blazer \& Wu, 2009; Gullone, Moore, Moss, \& Boyd, 2000; Miller, Naimi, Brewer, \& Jones, 2007).

Younger age is positively correlated with greater impulsivity, sensation-seeking, novelty-seeking, and risk-taking (Reimers, Maylor, Stewart, \& Chater, 2009; Spear, 2000; Steinberg et al., 2004, 2008). Evidence suggests that while some younger people may underestimate risk, others may perceive it and their own vulnerability to unfavorable outcomes, yet, may still often pursue risky action (Benthin, Slovic, \& Severson, 1993; Cohn, Macfarlane, Yanez, \& Imai, 1995; Gardner \& Steinberg, 2005). Evidence also suggests that reward salience is greater in youth, and that younger people may be more reward-sensitive and punishment-insensitive compared to older adults (Doremus-Fitzwater, Varlinskaya, \& Spear, 2010; Spear, 2000). Further, younger people have less crystallized intelligence that is needed, along with fluid intelligence, to assess risk and make decisions (Li, Baldassi, Johnson, \& Weber, 2013). Younger people have also simply made fewer decisions, including decisions involving uncertainty and risk, than older adults, indicating a general inexperience. Additionally, younger people also tend to be more present-biased 
and to have steeper delay discounting rates on the same task compared to older individuals (Green, Myerson, \& Ostaszewski, 1999; Meier \& Sprenger, 2010; Steinberg et al., 2009). This means that younger age may be an indicator of poorer decision-making capacity, greater risk-taking, and present-bias.

Many of these age-related differences are partially attributable to the related fact that, unlike adults over the age of 26 , people in the first two and a half decades of their life do not yet have a fully matured prefrontal cortex, the region of the brain instrumental in higher-level cognition (Gogtay et al., 2004; Spear, 2007). Younger people are generally more likely to experiment with drugs, including opioids and stimulants, and to persist in use despite adverse effects (Gfroerer \& Brodsky, 1992). This is one reason why rates of dependence are far lower among people aged 50 and older in the US (Blazer \& Wu, 2009).

However, as "baby boomer" cohorts age, rates of SUD among older persons is expected to increase due to the larger number of young people who initiated use in the 1960s (Han, Gfroerer, Colliver, \& Penne, 2009; Wu \& Blazer, 2011). There remains, too, the phenomenon of late-adopters to illicit drug use and the development of opioid and stimulant use disorders later in life (Arndt, Clayton, \& Schultz, 2011; Lofwall, Schuster, \& Strain, 2008). Meaning that there are exceptions to general trends.

Male sex, both before and after puberty, is frequently and strongly correlated with greater impulsivity/self-regulatory failure, steeper discount rates, and risk-taking behavior, including drug use (Byrnes, Miller, \& Schafer, 1999; Cottle, Lee, \& Heilburn, 2001; Hosseini-Kamkar \& Morton, 2014; Myerson, Green, van den Berk-Clark, \& Grucza, 2015; Gershon \& Gershon, 2002). Taken together, evidence suggests that younger age and male sex may be factors with the potential to influence decisionmaking about high-risk drugs like opioids and stimulants.

\section{Some Additional Indirect Indicators of Cognitive Functioning}

As noted above, how a person makes intertemporal decisions depends in part on a person's ability to engage deliberative and higher-level cognition. In addition to age- and sex-related influences on decision-making capacities and neurocognitive deficits that developed subsequent to drug exposure, a person may have additional attributes that influence cognition, risk-taking, self-control, and decisionmaking (Verdejo-García et al., 2008).

Deficits in neurocognitive functioning and executive control could have developed as a result of a number of factors (e.g., early childhood exposure to pre- or perinatal environmental neurotoxins, trauma, 
injury, abuse etc.) (Coles et al., 1997; Kim et al., 2013; Meehan \& Mannix, 2010; Perry, Pollard, Blakley, Baker, \& Vigilante, 1995). For instance, lead and other neurotoxin exposure is a large but overlooked problem in the US, despite the fact that lead exposure is associated with a cascade of neurocognitive and affective problems that include disinhibition, intellectual disabilities, behavioral problems, attentional deficits, and poor self-regulation (Cecil et al., 2008; Goodlad, Marcus, \& Fulton, 2013). Such exposure is believed to have a causal contribution to conduct disorders, drug use, and to violent and non-violent crime (Boutwell et al. 2016; Farrington, 2018). This example highlights a few possibilities. First, there can be a

wide-range of cognitive function and dysfunction among people generally and among corrections-involved drug users in particular. Second, not all cognitive dysfunction is necessarily attributable to drug use. Third, such dysfunction is still nevertheless relevant to consider as a potential influence on risk-taking and decision-making using available indicators. Oftentimes, the causal contributors to neurological deficits remain unknown and only indirect indicators of impairment are observed and assessed.

\section{Learning Disability and Poor Educational Performance}

Irrespective of what ultimately contributed to the development of cognitive deficits, many young people who experience deficits in cognition, impulse control, attention, and memory experience greater challenges in school, including conduct problems, poor performance on standardized tests, and unfavorable teacher evaluations (Mattison, Hooper, \& Carlson, 2006). Increasingly, per state and federal mandates, children who consistently underperform against normative standards are assessed for learning disabilities (LDs) (Bowen \& Rude, 2006). LDs, defined as "a neurobiological disorder of cognitive and/or language processing caused by atypical neurocognitive functioning", have been associated with less adaptive cognitive functioning and poorer academic performance compared to controls (Mayes, Calhoun, \& Crowell, 2000; Rubinsten, 2009; Silver et al., 2008). There is also a high rate of co-occurrence for LDs, ADD/ADHD, and externalizing disorders (DuPaul \& Volpe, 2009; Semrud-Clikeman et al., 1992). Further, some research has found that young adult and adult participants who self-report having a LD and/or ADHD perform worse on neurocognitive tests and evidence greater stress and psychiatric symptoms (Elbin et al., 2013; Wilson, Deri-Armstrong, Furrie, \& Walcot, 2009; Zuckerman, Lee, Odom, Solomon, \& Sills, 2013). Children with LDs or ADD/ADHD are sometimes enrolled into special education (SE) curriculum (Turnbull, 1995). SE services may be provided to students for a number of reasons, ranging from 
intellectual and developmental disabilities, including LDs, to poor academic performance (Freeman \& Alkin, 2000; Turnbull, 1995).

Having been diagnosed with a LD or having been enrolled in SE may indicate the presence of some deficits in executive functioning, including inhibitory control, as there are high rates of co-occurrence for LDs and ADD/ADHD (Barkley et al., 2001; Biederman et al., 2004). LDs, ADD/ADHD, and SE enrollment are vastly overrepresented among both corrections-involved people and drug users (Allison et al., 1999; APA, 2013; Blanton \& Dagenais, 2007; Bryan, Freer, \& Furlong, 2007; Cottle et al., 2001; Kavanagh, Rowe, Hersch, Barnett, \& Reznik, 2010). It is important to note that this association between LD diagnosis, SE enrollment, and high-risk behavior in adulthood may be partially attributable to neurocognitive deficits, but may also be due to a variety of other psychosocial and economic factors (Coles et al., 1997; Raine et al., 2005; Spencer et al., 2005). Indeed, while LD and SE enrollment may provide an indirect indicator of mild intellectual impairments or deficits in cognitive functioning (e.g., working memory, self-regulation) which can impede decision-making, they may also indicate other things, such as race-based structural and systemic inequities, teacher bias, and childhood poverty (Altarac \& Saroha, 2007; Artiles \& Trent, 1994; Skiba et al., 2008). There is the further possibility that the effects of being diagnosed with a LD and the effects of enrollment into SE curricula may contribute to, rather than necessarily reflect, cognitive challenges or developmental delays. For instance, being diagnosed with a stigmatized condition or state, such as LD diagnosis or SE enrollment, can increase stress, negative affect, which can hinder learning (Bender, Rosenkrans, \& Crane, 1999; Kistner, Haskett, White, \& Robbins, 1987; Mishna, 2003; Shifrer, 2013; Tabassam \& Grainger, 2002). Students with intellectual disabilities often fare worse in SE than in integrated classrooms (Freeman \& Alkin, 2000). In this way, irrespective of cognitive difficulties prior to LD diagnosis or SE enrollment, the diagnosis and enrollment may themselves contribute to important developmental delays that can have a lasting impact on cognition and decision-making.

\section{Difficulties with Comprehension, Concentration, and Memory}

In addition to using LD diagnosis or SE enrollment as an indirect indicator of cognitive deficits with the potential to influence decision-making capacities, there are some specific markers of poorer cognitive functioning that might be discerned through the self-report. For instance, self-reported difficulties in concentration, comprehension, and memory during the same time periods that drugs were used may 
indicate that a person was experiencing important constraints on their cognitive abilities (Bechara \& Martin, 2004; Dreer, DeVivo, Novack, Krzywanski, \& Marson, 2008). This may be particularly true if the person does not attribute these difficulties to acute drug intoxication.

During intertemporal choice, it is necessary to weigh benefits against risks across a range of options and probabilities, and to consider one's preferences over time. Impairments in executive control, including deficits in comprehension, concentration, and memory can all adversely influence a person's capacity to do this. For example, concentration, which is the attentional process that involves focusing on a particular task while also gating non-relevant inputs, is implicated in working memory capacity, defined as the coordination of multiple short-term memory systems' negotiation of attentional control to maintain information in a dynamic, readily retrievable fashion (Baddeley, 1992; Engle, 2002). Working memory is crucial for decision-making, particularly decision-making that involves risk and uncertainty (Paulus et al., 2001). Memory impairments self-reported by a person may implicate impairments in multiple other cognitive areas, as some of the same networks within the brain concerned with memory are also implicated in related functions, such as prospection, autobiographical memories, and counterfactual simulation of events, all which can influence a person's tendency toward present-bias and delay discounting, and their ability to make intertemporal choices (Abraham \& Bubic, 2015; Schacter, Benoit, De Brigard, \& Szpunar, 2015; Spreng, Mar, \& Kim, 2009).

\section{Incidence of Head Injury}

In addition to these indicators, a history of traumatic brain injury (TBI) or mild traumatic brain injury (mTBI) may be an indicator of poorer cognitive function in areas necessary for impulse control, emotional regulation, and decision-making (Bechara \& Van Der Linden, 2005; Bogod, Mateer, \& Macdonald, 2003; Dockree et al., 2006; Fellows, 2006; Kim, 2002). While people with mTBI evidence affective and cognitive dysregulation contemporaneous to injury, symptoms often resolve within 3-6 months (Belanger, Vanderploeg, Curtiss, \& Warden 2007; Mayer, Mannell, Ling, Gasparovic, \& Yeo, 2011). However, people with a history of multiple head injuries may exhibit progressive neurocognitive degeneration (McKee et al., 2009). Longer-lasting deficits in cognition and behavior related to head injury may be expressed in terms of poorer self-regulation, concentration, and decision-making and may, in some cases, co-occur with anxiety and depressive symptoms (Arciniegas, Topkoff, \& Silver, 2000; Greve et al., 
2001; Newcombe et al., 2011; Walker, Hiller, Staton, \& Leukefeld, 2003). Self-regulation is increasingly understood as a causal mechanism in successful intertemporal choice (e.g., not selecting the smaller, sooner reward over the larger, future reward), and is associated with frontocortical areas that are especially sensitive to head trauma (Figner et al., 2010). Head injuries are also overrepresented among correctionsinvolved populations and drug users (Miller et al., 1992; Hestad, Updife, Seines, \& Royall, 1995; Walker, Staton, \& Leukefeld, 2003). Among younger corrections-involved people, higher self-reported rates of repeated TBI/mTBI positively correlated with higher rates of repeat offending (Williams, Cordan, Mewse, Tonks, \& Burgess, 2010). When assessing the effects of drug use on decision-making, self-reported history of head injury has been controlled for as a possible confounding variable (Ersche et al., 2005).

It is possible that people may self-report a history of multiple head injuries in addition to LD diagnosis and/or SE enrollment. In assessing TBI/mTBI, LDs and ADD/ADHD are increasingly recommended as factors to consider as potential confounders in the assessment process (Elbin et al., 2013; McCroy et al., 2009). Similarly, in assessing neurocognitive impairment, LD and ADD/ADHD in young people, self-reported mTBI/TBI may be controlled for among other factors (e.g., abuse), underscoring its potential impact on cognitive functioning, including memory and concentration (Cicerone, 1996; Raine et al., 2005; Walker et al., 2003). It may be that a greater number of these indicators suggests that there are multiple factors present with the potential to influence a person's ability to make decisions involving opioids and stimulants. It also suggests that people with more of these indicators may be less enabled to deliberate and engage in the types of higher-level cognition needed to minimize risk and make decisions that maximize well-being over time, rather than choosing more immediate rewards.

\section{Vague and Distal, but Potentially Relevant}

Sophisticated, but still coarse-grained, conceptualizations of how multiple distal and proximate factors can influence decision-making and behavior are increasingly articulated, even as perfect information needed for fine-grained, more precise modeling of human behavior remains inaccessible. However, many potential coarse-grained inferences may be made using information about a person's history. Such history can provide indirect clues as to what factors may have influenced cognition, decisionmaking, and behavior during particular time periods. Although there are limitations to exploring selfreported accounts of a person's life and to examining indirect indicators with the potential to influence 
decision-making (such as head injury, LD diagnosis, SE enrollment), there is also some merit. The Adverse Childhood Events Questionnaire (Felitti et al., 1998) is one example of how the dichotomized presence or absence of a set of conditions during childhood can be used to make directional hypotheses about, and to help understand, behavioral outcomes in adulthood (Baglivio et al., 2015; Dube et al., 2003; Julian et al., 2018). Just as the self-reported presence of sexual abuse during childhood increases the odds of observing drug use problems in adulthood, so too might self-reported incidence of head injury or SE enrollment be used to consider the greater likelihood of observing impaired or high-risk decision-making in adulthood. The centrality of such distal, and sometimes imprecise, factors is increasingly made explicit during presentence investigations and death penalty mitigation defenses (Haney, 2007). Abuse, family interactions, mTBI, lead exposure, $\mathrm{LD}$, and poor educational performance in early life are all considered as potentially mitigating factors in a person's later commission of crime (Freedman, 2007; Haney, 1994, 2007). Given this precedent, head injury, LD diagnosis, and SE enrollment may be justifiably explored as potential influences on decision-making about high-risk drugs, though understood to be limited in what they have capacity to approximate and indicate.

\section{Economic and Social Influences}

\section{Scarcity and Economic Hardship}

Some researchers have proposed that scarcity is a state that changes the way people think and behave, including how they prioritize and solve problems, perceive choices, and make decisions (Mani, Mullainathan, Shafir, \& Zhao, 2013; Shah, Mullainathan, \& Shafir, 2012; Shah, Shafir, \& Mullainathan, 2015; Spears, 2011). For people who experience scarcity, problems may appear larger, more urgent, and may often require greater attention, emotion, and energy to address--all of which can influence attentional control, perception of time, and the ability to make intertemporal decisions that maximize long-term wellbeing (Jabs and Devine, 2006; Kahneman, 1973; Karlan, McConnell, Mullainathan, \& Zinman, 2016; Shah et al., 2012; Zhu \& Ratner, 2015). In other words, there is a quality of present-bias among people who are resource-scarce. Problems encountered by people who experience scarcity also require greater cognitive effort to address, in that trade-offs among limited resources necessitates careful deliberation that is not required to the same degree for people with abundant resources (Shah et al., 2012). Deprivation and scarcity can also increase cognitive load (e.g., "effort" involved in information processing), which can 
further constrain a person's capacity to engage in deliberative thinking during decision-making--a process that necessarily involves conceptualizing rewards/costs, weighing uncertainty, and considering preferences between a person's current and future self (Hinson et al., 2003; Kurth-Nelson, Bickel, \& Redish, 2012; Ward \& Mann, 2000; Vinoo, Ly, \& Soman, 2016). Increased cognitive load has been associated with subsequently reduced cognitive capacity, or "bandwidth", for self-regulation and with the ability to exercise "willpower", both of which can hinder a person's capacity to make decisions that will maximize overall utility, instead of choosing options with the highest local utility (Baumesiter, 2002a; Hofmann, Schmeichel, \& Baddeley, 2012; Mani et al., 2013; Mullainathan \& Shafir 2013; Schilbach, Schofield, \& Mullainathan, 2016).

For a person who may already be attempting to self-regulate their drug use, by moderating or abstaining, ego depletion might also occur, in which sustained efforts at self-control can become partially and temporarily depleted (Baumeister, 2014). This temporary depletion in self-control can contribute to an increased desire for drugs and a failure to self-regulate when willpower is expended (Baumeister, 2014). For instance, a drug user who achieved abstinence in a controlled environment and subsequently reenters the community hoping to maintain abstinence, would need to exert considerable planning and selfregulation. For some, it may be that continued efforts to not use drugs exacerbate the very ability needed to not use. When a current or former drug user is living in scarcity, they may experience increased ego depletion and cognitive load daily and across a variety of domains (e.g., not buying the sunglasses they want because they are too expensive and not shoplifting them due to the potential consequences; inhibiting desire to approach the dealer on the block near their house; deciding between buying groceries and paying the gas bill; deciding which household member will get to use the car today and who will take public transportation; saying "no" to old using friends who want to come over). For someone who is resource scarce, all of these seemingly small deliberations and inhibitions can exert a greater cognitive toll, making already difficult decision-making more taxing.

Experiencing poverty and acute economic hardship both include aspects of scarcity. Lower earnings and transient periods of economic distress or hardship are associated with greater choice polarization, stress response, impaired cognitive functioning, and poorer self-regulation (Bernheim, Ray, \& Yeltekin, 2015; Fry, Langley, \& Shelton, 2017; Hackmam \& Farah, 2009; Hunt, 2010; Loibl, 2017; Mani 
et al., 2013; Nobel, McCandliss, \& Farah, 2007; Zhu \& Ratner, 2015). Across studies, low-income and poverty are associated with increased stress, negative affect, anxiety, depressive symptoms, and diminished cognitive capacity and control during decision-making (Green, Myerson, Lichtman, Rosen, \& Fry, 1996; Santiago, Wadsworth, \& Stump, 2011; Haushofer \& Fehr, 2014; Spears, 2001; Weich \& Lewis; 1998). Evidence suggests that it is the state of scarcity that results in suboptimal decision-making and cognition, not necessarily the converse (Mullainathan \& Shafir, 2013; Shah et al., 2012; Sheehy-Skeffington, 2018). One result is that choices with higher local utility, but lower overall utility, are more likely to be made, thus potentially reinforcing some sources of scarcity and poverty (e.g., borrowing from a payday lender, purchasing lottery tickets, putting off a doctor's visit, delaying minor repairs that become major repairs over time, spending on smaller essential and non-essential goods in the immediate instead of saving) (Bair, 2005; Clotfelter, Cook, Edell, \& Moore, 1999; Mullainathan \& Shafir, 2009). Among heroin users, decisions have been shown to become more risk-prone as a function of deprivation (Bickel, Giordano, \& Badger, 2004). Although all people tend to temporally discount, the consequences of this can be disproportionately greater among people with fewer resources (Bertrand, Mullainathan, \& Shafir, 2004). Greater temporal discount rates have also been observed in conditions where people experience acute financial hardship or loss (Haushofer, Schunk, \& Fehr, 2013).

Mani et al. (2013) conducted two complementary experiments that help elucidate how low-income and financial hardship could potentially influence cognition and inhibitory control. In the first study, the authors found that lower-income participants (e.g., \$20,000 annual income) and higher-income participants (e.g., \$70,000 annual income) performed similarly on measures of fluid intelligence and cognitive control (inhibition) when randomized to an "easy condition", in which they were primed by making hypothetical financial decisions involving smaller, less significant sums of money. However, when randomized to a "hard condition", in which participants were primed by making hypothetical financial decisions involving larger, more consequential sums of money, low-income participants performed worse than high-income participants. Specifically, an interaction was observed for low-income + hard condition, whereby lowerincome participants making challenging economic decisions performed poorer than all other groups. This study, which controlled for test/math anxiety among other things, was repeated using incentives for correct responses and similar findings were observed, with effect sizes for the low-income + hard condition robust 
across experiments (Cohen's $d 0.88-0.94)$. In other words, when making high-risk/high-consequence decisions, those with the least performed the worst.

In the second experiment, Mani et al. (2013) found that farmers in India experienced greater financial hardship pre-harvest, compared to post-harvest, and that, after controlling for stress, farmer, and month fixed effects, there were significant differences between periods of economic hardship (pre-harvest) and economic stability/prosperity (post-harvest) on measures of fluid intelligence and cognitive control. In a study by Carvalho et al. (2016), a similar "liquidity constraint" on decision-making was found, whereby greater present-bias was observed for low-income households tested before payday, rather than after. Unsurprisingly, spending was also higher after payday. This finding is consonant with the notion that changes in liquidity constraints can account for changes in self-control. It also does not exclude the possibility that scarcity itself influences self-control and decision-making, highlighting the need for more research in this area.

Still, taken collectively, there is support for the idea that being low-income is associated with poorer cognitive functioning under decision-making conditions, and that transient periods of economic hardship and distress are associated with declines in cognitive functioning. Having more unmet needs may conceptually correspond to a decision-making pattern referred to as "satisficing", in which a person tends to seek out options with the potential to meet immediate needs (e.g., an available choice with high local utility), rather than maximizing utility by seeking out optimal long-term outcomes (Mishra \& Lalumière, 2010; Schwartz et al., 2002; Simon, 1956; Todd \& Gigerenzer, 2000). For low-income people, or people experiencing financial difficulties, this satisficing tendency may be rational and helpful in the short-term, in that it minimizes labor expenditures and, prioritizes "good enough" options over "best solutions". This makes existential sense, of course. Interestingly, satisficing, compared to maximizing, has been found to correlate with greater perceived happiness and satisfaction (Schwartz et al., 2002). A related finding suggests that while there is a ceiling on the "money equals happiness" correlation $(\$ 75,000$ annually), perceived life satisfaction increases exponentially with income (Khaneman \& Deaton, 2010). The implication is that people earning less $(<\$ 75,000)$ may be both less happy and less satisfied with their life and, as discussed earlier, may experience scarcity mindsets and satisficing tendencies. 
One decision-making aspect related to income that is of additional interest here is that for opioid and stimulant users, increased spending power or relief from transitory economic distress may actually increase purchase and use of drugs (Bretteville-Jensen \& Sutton, 1996; Petry, 2000; Greenwald \& Steinmiller, 2014; Roddy \& Grenwald, 2009; Roddy, Steinmiller, \& Greenwald, 2011; Shaner et al., 1995). This is due to the fact that demand for opioids and stimulants, among regular users of these drugs, can be income elastic, meaning that purchases will increase at rates higher than rates of income increase, with people demonstrating greater income elastic demand for drugs they prefer, are dependent on, or believe to be of higher quality (Goudie, Sumnall, Field, Clayton, \& Cole, 2007; Petry \& Bickel, 1998).

Many drug users and corrections-involved people experience forms of scarcity, including lowincome and economic hardship (Garland, Wodahl, \& Mayfield, 2011; Mulia, Schmidt, Bond, Jacobs, \& Korcha). Further, because using a drug can serve an immediate need, and may assume a form of satisficing, but because the distributed choice of repeated drug use can be suboptimal or devastating, a scarcity mindset or satisficing tendency may be problematic. It is therefore important to consider how factors in a person's life, such as income and economic distress, might be understood in terms of scarcity, but also how they may function to influence drug-related decision making in other ways, such as providing greater or lesser drug purchasing power.

\section{Community-wide Scarcity and Harsh Environments}

Scarcity of flexible resources and socioeconomic disparities may also be observed at the level of neighborhood, community, county, or region. For instance, some areas enjoy greater economic prosperity than others, whereby there are more households with higher earnings and fewer households living in poverty (Brown \& Hirschl, 1995; Thorne, Tickamyer, \& Thorne, 2004). Regional poverty and regional economic inequality are both associated with regional crime, violence, social mistrust, poorer self-rated health, and higher rates of non-natural and premature morbidities and mortalities, including higher infant mortality rates (Blakely, Lochner, \& Kawachi, 2002; Graif, Gladfelter, \& Matthews, 2014; James \& Cossman, 2006; Hsieh \& Pugh, 1993; Patterson, 1991; Santiago et al., 2011; Sparks, McLaughlin, \& Stokes, 2009; Wilkinson \& Pickett, 2009; Yen \& Kaplan, 1999). Such environments are defined as "harsh" by Frankenhuis et al. (2016), who suggest that people living within such harsh places cannot develop a 
sense of certainty about their lives or futures, and that such uncertainty changes their cognition.

Frankenhuis et al. (2016) note that:

In such conditions, present-orientation may be adaptive. This orientation can psychologically manifest in: first, vigilance used to detect threats and opportunities, second, impulsive reactions (little deliberation) in order to respond quickly; and third, steep future discounting to motivate the capture of immediate benefits, as future rewards are less likely to be cashed in" (pg.77). ${ }^{3}$

In other words, growing up or living in high-stress, high-mortality, low-resource, and uncertain conditions may contribute to a person being more present-biased, in which they will discount the future at higher rates, and potentially take more risks when it comes to securing immediate rewards (e.g., sex, food, drugs) or avoiding perceived harms (Griskevicius et al., 2013; Griskevicius, Tybur, Delton, \& Robertson, 2011).

Evidence has shown that both lower-income areas (e.g., neighborhoods, counties, regions) and areas with greater economic inequality have high associated rates of morbidities and mortalities, meaning that such counties can be considered as "harsh", using criteria proposed by Frankenhuis and colleagues (2016) (Cheng \& Kindig, 2012; Franzini, Ribble, \& Spears, 2001; Huynh, Parker, Harper, Pamuk, \& Schoendorf, 2005; McLaughlin \& Stokes, 2002; Muramatsu, 2003). The implication is that people living within those areas would be expected to be more present-biased and risk-taking.

\section{Subjective Socioeconomic Comparison and Stress}

Scarcity, however, is not limited to economic circumstances. People may experience scarcity across flexible resources, which are resources that are dynamic, valuable, and applicable across situations (Phelan, Link, \& Tehranifar, 2010). In addition to money, these resources might include time, knowledge, education, and status, closely associated with power, defined here as "asymmetric control over valued outcomes and resources" (Joshi \& Fast, 2014; Phelan et al., 2010). People who have fewer of these resources and, more importantly, who perceive themselves as having fewer of these resources in relation to others in society, often experience greater subjective stress, poorer physical and psychological health, and

\footnotetext{
${ }^{3}$ Vis-à-vis evolution; in terms of biological fitness, not in terms of more adaptive health or well-being. Indeed, health and well-being, in the immediate and long-term, may actually decrease in order to better ensure transmission of genes under such conditions.
} 
greater impulsiveness/decreased self-regulation (Adler, Epel, Castellazzo, \& Ickovics, 2000; Kessler \& Cleary, 1980; Marmot, 2004; Singh-Manoux, Marmot, \& Adler, 2005).

Lower subjective social status, experiencing social isolation as a result of lower status, and encountering others that one feels subordinate to, particularly when the socially dominant other is encountered in a domain that they control (i.e., social defeat; e.g., the boss at work, a wealthy customer's home, probation/parole officer), may all result in increased stress response, defined as the body's physiological reaction to a real or perceived stressor that has the potential to influence health, cognition, and behavior (Adler, Epel, Castellazzo, \& Ickovics, 2000; Abbott et al, 2003; Cole, Logan, \& Walker, 2011; Covington \& Miczek, 2005; De Kloet, Joëls, \& Holsboer, 2005; Koob, 2009; McEwen \& Gianaros, 2010; Sapolsky, Alberts, and Altmann, 1997; Selye, 1975, 1998). Conversely, people who have, or perceive themselves to have, more relative power, resources, and status have been shown to have better health and to exhibit heightened executive functioning, abstract reasoning, and increased goal-directed focus (Overbeck \& Park, 2006; Smith, Dijksterhuis, \& Wigboldus, 2008; Smith \& Trope, 2006; Wilkinson \& Marmot, 2003).

Among non-human animals, higher social rank and social dominance have been associated with less alcohol, cocaine, and opioid consumption, whereas lower and/or marginalized social status is associated with increased drug-taking (Heilig, Epstein, Nader, Shaham, 2016; Kuhar, 2002; McKenzieQuirk \& Miczek, 2008; Nader et al., 2012; Wolffgramm \& Heyne, 1995). Pre-clinical models examining hierarchical status, social defeat, and drug use have found that cocaine serves as a behavioral reinforcer in subordinate, but not higher-ranking primates (Covington \& Miczek, 2005; Morgan et al., 2002; Nader et al., 2006), that rats increase alcohol, cocaine, and cocaine + heroin self-administration following social defeat or exposure to social stress (Caldwell \& Riccio, 2010; Cruz, Quadros, Hogenelst, Planeta, \& Miczek, 2011), and that morphine- and cocaine-induced place preference is reinstated following exposure to various stressors, including social stressors (Do Couto et al., 2006; Do Couto, Aguilar, Lluch, Rodríguez-Arias, \& Miñarro, 2009). Among non-human primates, holding a lower rank in the social hierarchy is also associated with higher stress (at resting levels, as measured by glucocorticoids), whereas higher rank, particularly in stable social hierarchies, is associated with the converse, and with greater cortical integration and coupling 
(Gesquiere et al., 2011; Noonan et al., 2014; Sapolsky, 2017; Sallet et al., 2011)4. Increasingly, addiction researchers are emphasizing the importance of modeling social stressors, including low social status, in terms of how they may influence drug-taking (Heilig et al., 2016).

Relative to decision-making, the importance of real or perceived higher social status is summarized in part by Joshi \& Fast (2014) who state that, "Power activates a high-level construal orientation (Magee, Milliken, \& Lurie, 2010; Smith, Dijksterhuis, \& Wigboldus, 2008; Smith \& Trope, 2006). High-level construal expands temporal horizons (Troupe \& Liberman, 2010), which results in the perception of the distant future as being closer and imminent and induces a sense of connection with the future self (Kantan, 2011)". One implication is that people who have a lower perceived social rank are more likely to be present-biased, and therefore potentially more likely to temporally discount future rewards for smaller, immediate ones. Another implication is that people with real and perceived lower rank are, in general and without acute provocation, more physiologically aroused and "stressed" than higher ranking, more powerful people. This, as noted before, impairs cognitive ability. Corrections-involved drug users may subjectively perceive themselves as having an equal status to others in society. However, this should not be assumed, due to the fact that drug users and corrections-involved people are highly stigmatized and socially devalued in the US (Barry et al., 2014).

In sum, experiencing forms of resource scarcity, such as economic distress or having a lower subjective social rank, along with living in a "harsh" region that fosters a sense of uncertainty about the future, all have the potential to influence cognition and high-risk decision-making. This is because all of these factors are associated with increases in stress response and temporal discounting, and with decreases in capacities needed for future-orientation, self-regulation, and other higher-level cognition (Bartels \& Rips, 2010; Ersner-Hershfield, Garton, Ballard, Samanez-Larkin, \& Knutson, 2009; Frederick et al., 2002).

\footnotetext{
${ }^{4}$ Cortical integration or coupling is relevant to note due to the fact that it indicates what might be considered a "healthy brain". That is, a brain better equipped for cognitive flexibility and higher-level cognition, including working memory, attention, emotional regulation, abstract thinking, impulse control, valuation, etc.
} 


\section{Stress, Psychiatric Symptoms, Pain, and Misregulation}

\section{Stress-related Health Effects}

Although stress response can be immediately adaptive, in terms of increasing a person's capacity to respond to threats and facilitating memory consolidation of specific threats, it disrupts homeostatic functioning and increases allostatic load, which has significant deleterious short- and long-term effects including impaired memory and cognition, fatigue, pain, lowered immunological function, affective dysregulation, elevated blood pressure, and premature mortality (Arnsten, 2000; Boyce, 2004; Chattarji, Tomar, Suvrathan, Ghosh, \& Rahman, 2015; De Kloet et al., 2005; Goymann \& Wingfield, 2004; Juster, McEwen, \& Lupien, 2010; Lupien, Maheu, Fiocco, \& Schramek, 2007; Lupien, McEwen, Gunnar, \& Heim, 2009; McEwen, 1998, 2009; Sapolsky, 2004, 2005; Sapolsky, Alberts, \& Altmann, 1997). In a variety of human and non-human studies, chronic stress, acute stress exposure, self-reported stress, and greater stress reactivity, have been associated with craving, addiction, and relapse to drugs, including opioids and stimulants (Back et al., 2010; Garland, Franken, \& Howard, 2012; Kennedy et al., 2015; Leri et al., 2004; Preston et al., 2017; 2018; Sinha , 2001,2008; Sinha \& Jastreboff, 2013; Sorge, Rajabi, \& Stewart, 2005). Taken together, these findings suggest that people who experience stress (if only ever physiologically and not psychologically) may be influenced to use drugs, use them at higher rates, and resume use (e.g., lapse, relapse) during periods of greater stress.

Chronic exposure to stress is also associated with insensitivities to changes in outcomes and with behaviors that are more habituated and rigid (and therefore less goal-directed) (Dias-Ferreira et al., 2009; Liston et al., 2006; Schwabe \& Wolf, 2009, 2011; Soares et al., 2012). This is problematic, in that decisions involve contingencies. Imagining and deliberating various contingencies requires higher-level cognitive functioning and flexibility, including the capacity to utilize available information, generate abstractions, and initiate behavioral change in response to outcome change. For example, if someone was informed that instead of their next positive drug screen resulting in a one-week jail sanction it resulted in a 4-year prison term, a person would need to be able to attend to, understand, and envision these possibilities. They would also need to have the requisite cognitive flexibility for modifying decision-making patterns and behavior in light of this information. When decisions involve uncertainty, which is often the case during intertemporal 
choice involving drugs, stress and stress-related health effects can increase, potentially further hindering the decision-making process (Morgado, Sousa, \& Cerqueira, 2015; Peters, McEwen, \& Friston, 2017). If a person's life is characterized by uncertainty, as many corrections-involved drug users' lives are, then stress may generally be higher.

Interestingly, for some drug users, and for some people with subclinical psychopathy, underactivation rather than overactivation of visceral states (e.g., such as increased heart rate, stomach upset, perspiration) or "somatic markers" can adversely impair decision-making, in that the "cues" needed to help anticipate reward and punishment are inadequate or absent (Bechara \& Damasio, 2002; van Honk, Hermans, Putman, Montagne, \& Schutter, 2002). Thus, there is no reason to believe that there is complete homogeneity across drug users who are resource-scarce or who have been exposed to stressors in terms of how they are cognitively or behaviorally influenced by these states. Identifying the presence of healthrelated stress symptoms, that include somatic as well as psychological indicators, during a given time period may be one way to discern if cognitive states are being adversely influenced by stress response.

\section{Psychiatric Symptoms}

In addition to the slower, deliberative, and higher-level cognition necessary for decision-making discussed earlier, faster, automatic cognition also facilitates adaptive decision-making (Evans, 2008).

Emotions, or feeling states, can help a person intuit, reason, and make decisions, in part through viscera or somatic signals that steer them away from danger and risk (Bechara \& Damasio, 2002; Damasio, 1994, 2012). The somatic marker hypothesis posits that while decision-making is a process that often requires conscious, deliberative cognition, it is also a process facilitated by unconscious physiological states and their emotional referents (Bechara \& Damasio, 2005).

As with stress, feeling states--spanning affect, mood, and somatization---including those characteristic of anxiety and depressive disorders--can impair neurocognitive function, including working memory, attention, interoceptive accuracy, time perception, and risk assessment, thus influencing decisionmaking processes (Darke, 1988; Dias-Ferreira et al., 2009; Furman et al., 2013; Gohier et al., 2009; Harvey et al., 2004; Marazziti, Consoli, Picchetti, Carlini, \& Faravelli, 2010; Nebes et al., 2000; Schulz \& Vögele, 2015; Schwabe \& Wolf, 2013; Scott et al., 2015; Shackman et al., 2006; Wittmann \& Paulus, 2008). For instance, heightened emotional arousal corresponds to affective, rather than deliberative, decision-making, 
which is associated with faster, simplified decision-making, risk-taking, and delay discounting (Figner, Mackinlay, Wilkening, \& Weber, 2009; Figner \& Weber, 2011). This makes some sense, in that affective, automatic/associative cognition is less effortful and demanding than deliberative reasoning and behavioral inhibition (Sanfey \& Chang, 2008). But it is important to note that emotional states are not, as a rule, unhelpful during decision-making, given that the ability to make decisions relies heavily on emotion (Bechara \& Damasio, 2005). Rather, it is important to understand that emotional states and psychological disorders have the capacity to alter cognition and decision-making processes.

For instance, time perception can be influenced by affective states. During depressive episodes, people can experience time passing more slowly, whereas people in manic or anxious states may perceive time passing more quickly; all of which can influence attention, present-bias, and delay discounting, thereby increasing the probability of suboptimal decision-making (Bschor et al., 2004; Murphey et al., 2001; Zauberman, Kim, Malkoc, \& Bettman, 2009). Although investigations have examined how emotions common to psychiatric disorders, such as anxiety and depression, influence decision-making, the evidence is mixed (Paulus \& Yu, 2012; Seymour \& Dolan, 2008). For instance, some data suggest that anxiety-prone individuals are more risk averse and demonstrate biased attention to possible threats (Dugas, Gagnon, Ladouceur, \& Freeston, 1998; MacLeod \& Mathews, 1988; Maner et al., 2007). Although Rounds et al. (2007) found impulsive choice increased following increased exposure to social anxiety, this finding was not replicated (Jenks \& Lawyer, 2015).

People with depression have been shown to be less biased toward and responsive to reward, to be less accurate in evaluating outcome probabilities, and to experience more conflict during decision-making (Cella, Dymond, \& Cooper, 2010; Elliott et al., 1996; Han et al., 2014; Harlé, Allen, \& Sanfey, 2010; Lempert \& Pizzagalli, 2010; Murphy et al., 2001; Pizzagalli, Bogdan, Ratner, \& Jahn, 2007; Rubinsztein, Michael, Underwood, Tempest, \& Sahakian, 2006; Smoski, et al., 2008; van Randenborgh, de Jong-Meyer, \& Hüffmeier, 2010). People with depressive symptoms are believed to have enhanced somatic markers for negative, but not positive stimuli, making punishment learning potentially more rapid and biasing them toward risk aversive decisions (Smoski, et al., 2008). However, during experimentally induced emotional states, Raghunathan \& Pham (1999) found that people in the "sad" condition were biased toward highrisk/high-reward decisions, whereas people in the "anxious" condition were biased toward low-risk/low 
reward options. Further, among a sample of people with major depressive disorder (MDD), participants with MDD discounted rewards at steeper rates and were insensitive to increases in reward changes (Pulcu et al., 2013), which is in keeping with the finding of higher discount rates among depressed participants with and without suicidal ideation and previous suicide attempts observed by Dombrovski et al. (2011). Feelings of hopelessness and detachment from one's future have been proposed as possibly contributing to temporal discounting (Pulcu et al., 2013). In other words, for a person without resonant connection to their future self, it may be easier to discount their future self's preferences.

Paulus \& Yu (2012) conclude that, overall, findings suggest both trait-like (i.e., related to psychiatric disorder) and state-like (i.e., internal state and mood) decision-making dysfunction in relation to anxiety and depression. This means that both transient affective or mood states, as well as more enduring or reoccurring psychiatric disorders (which can, of course, still include these states), have the potential to influence decision-making. Anxiety and depressive symptoms are highly prevalent among both opioid and stimulant users (Grant et al., 2004; Merikangas et al., 1998). Given that drug use can impair brain areas associated with emotional processing and self-regulation, and given that abstinence can induce anxiety and depressive symptoms, it is possible that both active and abstinent drug users may experience affective and mood states that influence decision-making (Fernández-Serrano, Lozano, Pérez-García, \& Verdejo-García, 2010; Verdejo-García, Rivas-Pérez, Vilar-López, \& Pérez-García, 2007). However, because findings are preliminary and inconclusive, more work is needed to understand the associations between anxiety and depressive disorder symptoms and high-risk decision-making and behavior, including the use of opioids or stimulants. Nevertheless, symptoms related to anxiety and depression might still be explored as possible influences on decisions to use high-risk drugs, as the presence of negatively valanced states may influence decision-making in other ways, as will be discussed subsequently.

\section{Chronic Pain}

Chronic pain is defined here as a condition in which "signals triggered by aversive stimuli and/or damaged tissue persist over time, beyond their normal duration" (Yoris et al., 2018). Typically, pain that persists for three or more months may be considered as chronic (Cleeland \& Ryan, 1994). Chronic pain conditions, which often have high prevalence rates among people who are older, have fewer resources, SUD history, and corrections-involvement, can also potentially influence decision-making (Dunn, Brooner, 
\& Clark, 2014; Dunn, Finan, Tompkins, Fingerhood, \& Strain, 2015; Johannes, Le., Zhou, Johnston, \& Dworkin, 2010; Reingle-Gonzalez, Walters, Lerch, \& Taxman, 2015; Rosenblum et al., 2003; Sapolsky, 2004, Kristenson et al., 2004; Mallik-Kane \& Visher, 2008; Smith et al., 2019). People who experience chronic pain have been shown to have impaired cognitive function and impaired decision-making, as indicated by poorer performance on decision-making simulations compared to healthy controls (Apkarian et al., 2004; Elvemo, Nilsen, Landrø, Borchgrevink, \& Håberg, 2017; Landrø et al., 2013; Walteros et al., 2011).

Ordinarily, a multitude of physiological reactions occur prior to and during decision-making, both automatically and unconsciously, as well as consciously in the form of emotions or feeling states which, as noted earlier, can help guide learning, decision-making, and behavior (Bechara \& Damasio, 2005; Berridge, 2007; Van den Bergh, Zacharioudakis, \& Petersen, 2018). Recall that, as explained by dual process models of cognition, decision-making relies on more than one type of cognition (i.e., a variety of automatic, more visceral signals, and deliberative, higher-level cognitive functions), and that choice involving uncertainty is more cognitively demanding than choice with certainty and choice associated with habituation (Damasio, 1994; Evans, 2008). During complex tasks, such as intertemporal choice, cognitive systems may work together, rather than in explicit “competition” (Monterosso \& Luo, 2010). Recall, too, that physiology and "feeling states" guide decision-making so as to improve adaptation and performance amidst uncertainty, ambiguity, and change (Bechara \& Damasio, 2005; Damasio, 1994). Consider, for instance, the usefulness of disgust as an emotional state in the avoidance of spoiled food or refuse (Curtis, De Barra, \& Aunger, 2001; Nesse, 1994). Consider, too, the usefulness of fear. When faced with perceived threat, pupils dilate to improve sight and to signal arousal, heart rate increases to pump needed blood to the body's muscles for movement, and glucocorticoid production increases, readying the body for swift action and the consolidation of memory around threat (Clinchy, Sheriff, \& Zanette, 2013; Leuchs, Schneider, Czisch, \& Spoormaker, 2017; Mobbs et al., 2009; Neese, 1994; Perry et al., 1995; Schaefer, Larson, Davidson, \& Coan, 2014; Sapolsky, 2003; Schulkin, Morgan, \& Rosen, 2005). This state may indeed become interpreted as fear and associated with anxiety; however, the phenomenology of such states could also be appraised as excitement and associated with pleasure (Garfinkel \& Critchley, 2016). Thus, a 
person's ability to accurately sense, detect, identify and appraise somatic states is important to decisionmaking and behavior (e.g., one may "avoid" due to fear and "approach" due to excitement).

Skin conductance response (SCRs), or electrodermal response, is one type of physiological reaction or "somatic marker" that occurs quickly and in response to arousing endogenous or exogenous stimuli (Dawson, Schell, \& Courtney, 2011; Lykken \& Venables, 1971). SCRs can help guide and optimize decision-making via anticipatory signaling, as evidenced by the graded relationship between SCR and other somatic marker signaling prior to choice, wherein people with weak or no signaling are more likely to make the riskiest choices (Crone, Somsen, Beek, \& Van Der Molen, 2004). Evidence indicates that people with chronic pain are impaired in anticipatory SCR generation before making unfavorable outcomes (Elvemo et al., 2014). People who experience chronic pain have also been found to have less cortical volume in areas related to decision-making, emotion, and reward compared to healthy controls (Elvemo et al., 2014; Smallwood et al., 2013). Apkarian et al. (2004) propose that "chronic pain should be considered a 'cognitive state' ....[that] may be competing with other cognitive abilities", including emotional regulation, risk-assessment, inhibition, and decision-making, due to the preferential recruitment of the prefrontal cortex in chronic pain (Apkarian et al., 2001a-c; Apkarian et al., 2002). Still, such findings should be interpreted with caution, due not only to their correlational nature, but also due to the fact that differences in some neural areas associated with chronic pain vary by sex and potentially other factors as well (Gupta et al., 2016).

More generally, though, a variety of chronic pain conditions are associated with reductions in interoceptive sensibility, the ability to consciously detect and "make sense of" physiological changes, and with interoceptive accuracy, the ability to reliably detect interoceptive signals, with an inverse correlation observed between pain symptom severity and interoceptive accuracy (Borg et al., Di Lernia et al., 2016; Duschek, Montoror, \& Reyes del Paso, 2015; Farb \& Logie, 2018; Mehling et al., Pallatos et al., 2011). Findings cautiously interpreted from preliminary work suggest the possibility that people who experience chronic pain may also encounter disruptions in cognition, emotional regulation, and decision-making as a result of disruptions in interoceptive sensibility and accuracy (Dunn et al., 2010; Füstös, Gramann, Herbert, \& Pollatos, 2012). 
Of additional interest here is that some burgeoning research suggests that stimulant dependent people may have increased or hyperinteroceptive accuracy (de la Fuente et al., 2019). It may be, too, that other drug use is associated with hyperinteroceptive accuracy, or with hypointeroceptive accuracy. Ultimately, interoceptive signaling that helps inform decision-making may become disrupted from drug use (Bechara \& Damasio, 2002). However, some findings suggest that while opioid users evidence poorer decision-making, it may not be due to lower SCRs. For instance, Biernacki et al., (2018) found no differences in SCR between opioid users and non-users. Contrary to other findings, opioid users who evidenced emotional reactivity on par with healthy controls, and who were found to have higher anticipatory SCR than controls, still made significantly risker decisions (Biernacki et al., 2018). This suggests that even when somatic markers are present, drug users may have deficits in interoceptive awareness (i.e., the metacognitive awareness of accurately detecting somatic states) needed to clarify feeling states, consider their significance, and use them to guide choice (Craig, 2002). In other words, the signal is there, but not being adaptively "picked up" or interpreted.

Considering how chronic pain may influence decision-making, as a result of altered somatic marker signaling or impaired interoception is just one of several ways that chronic pain might influence decision-making in relation to high-risk drugs, particularly opioids. Unsurprisingly, chronic pain is associated with increased odds of illicit opioid and other drug use, including use among correctionsinvolved people (Alford et all, 2016; Cole \& Logan, 2010; Hall et al., 2016; Prater, Zylstra, \& Miller, 2002; Vowles et al., 2015). In addition to chronic pain potentially influencing risk-taking and decision-making, via impaired somatic markers as described above, chronic pain may influence the perceived utility of opioids due to their analgesic properties and may increase the likelihood that a person might choose to use of these drugs despite associated risks (Volkow \& McLellan, 2016). In other words, being in a state of pain can motivate behaviors believed to mitigate pain or increase pleasure (Leknes \& Tracey, 2008).

People with pain conditions who also have a history of SUD and/or who are receiving MAT are often undertreated for their pain, meaning that illicit use of opioids may become an appealing option (Baldacchino, Gilchrist, Fleming, \& Bannister, 2010; Dunn et al., 2014; Hines, Theodorou, Williamson, Fong, \& Curry, 2008; Jamison, Kauffman, \& Katz, 2000; Karasz et al., 2004; Rosenblum et al., 2003). Motivation to use opioids to alleviate pain does not suggest that people who choose to take opioids to 
address pain, licitly or illicitly, would not also be influenced by other factors, such as negative affective states or craving (Butler, Fernandez, Benoit, Budman, \& Jamison, 2008; Garland, Brown, \& Howard, 2016; Havens et al., 2009; Martel, Dolman, Edwards, Jamison, \& Wasan, 2014; Martel, Jamison, Wasan, \& Edwards, 2014; Tsui et al., 2016). Though some evidence suggests that pain severity does not necessarily correlate with opioid craving (Martel et al., 2016). Put differently, the local utility, or relative value, of opioids could increase due in part to a person's chronic pain, but chronic pain may not be the only factor influencing how opioids are valued.

\section{Misregulation}

As noted earlier, neurobiological changes from drug use can make people potentially less able to engage in the kind of self-regulation and higher-level cognition (e.g., prospection, deliberation, inhibition, cognitive reappraisal, mindfulness) needed to mitigate or "reframe" negatively valanced states (Baldacchino et al., 2012; Bechara, 2003; Buhle et al., 2014; Bush, Luu, \& Posner, 2000; Hoffman \& Vohs, 2016; Schoenbaum, Roesch, \& Stalnaker, 2006). However, not all decisions to use high-risk drugs can be considered as a failure to cognitively reframe or self-regulate, or as a temporary deficit in willpower (e.g., ego depletion). People may choose to use drugs in what they may perceive to be pragmatic ways. These could include using as means to improve mood, cope with stress, anxiety, or depression, reduce pain, mitigate drug-related harms, lose weight, or to increase endurance and performance (Alford et al., 2016; Assanangkornchai, Muekthong, Sam-Angsri, \& Pattanasattayawong, 2007; Baumeister et al., 1994; Conklin \& Perkins, 2005; Daniulaityte, Carlson, \& Kenne, 2006; Greely et al., 2008; Levine et al., 2010; Kopetz, Woerner, \& Briskin, 2018; O’Connor \& Berry, 1990; Smith \& Lawson, 2017; Tice, Bratslavsky, \& Baumeister, 2001). Such choices, when they have a high local but potentially low overall utility, can be conceptualized as misregulation, a self-regulation failure that includes attempted control or regulation, but in a way that fails to achieve the desired longer-term outcome (Baumeister et al., 1994; Baumeister \& Heatherton, 1996). For instance, a person may intend to moderate or discontinue their drug use, but may still use, "lapsing" or "relapsing", not due to a self-regulatory failure, but rather due to choosing to use drugs to cope with negatively valanced states. As noted throughout this discussion, states and contexts can influence a person's cognition and decision-making capacities. They can also influence the relative value of opioids and stimulants, with value attributable to potentially multiple factors (e.g., taking opioids to 
simultaneously achieve a feeling of euphoria, to cope with stress, and to blunt lower back pain so as to be able to function at work and remain employed) (Redish, Schulheiss, \& Carter, 2015). Misregulation is in harmony with the concept of satisficing and is also consonant with the idea that drug use may have a high local utility and may be rational for someone experiencing stressful or painful states, even as overall utility still decreases (Kopetz et al., 2018; Rachlin, 1997). In exploring high-risk drug use, it is important to understand if people making decisions to use drugs are doing so in part to reduce unpleasant states. Identifying such influence on decision-making has some practical implications, insofar as tendencies toward misregulation may be more amenable to intervention compared to other influences.

\section{Access to Drug Markets and the Costs of Using}

As discussed earlier, the area that a person lives in can influence a person's perception of risk and their decision-making capacities. Importantly, it can also determine what drugs are available or more readily accessible, along with some of the costs associated with acquiring them. Costs can be measured in terms of money, time, effort, and opportunity cost (e.g., money spent on heroin cannot also be money spent on rent, time spent obtaining heroin is not time spent with children) (Bickel et al., 2014; Caulkins \& Reuter, 2004). Indeed, regions can sometimes differ considerably in terms of drug availability (e.g., drug type, number of different suppliers, time required to acquire drug), all of which can influence demand and consumption (Roddy \& Greenwald, 2009; Taylor et al., 2011). This can include differences in accessibility of alcohol (e.g. "dry" versus "wet" counties), illicit drugs, and drugs that can be obtained via prescription (Cicero, Surratt, Inciardi, \& Munoz, 2007; Cox, Motheral, Henderson, \& Mage, 2003; Furst, Herrmann, Leung, Galea, \& Hunt, 2004; Rigg \& Monnat, 2015; Webster, Pimentel, \& Clark, 2008).

Differences in drug availability and cost may be attributable to features of a geographic location that impact drug distribution networks and the burden of drug acquisition (e.g., proximity to metro areas, highway systems, "pill mills", pain clinics, MAT providers, etc.), but may also be attributable to variability in the demographic and health characteristics of a region's population (Coleman, 2012; Furst, 2004; Paterline, 2013; Inciardi, Surratt, Cicero, \& Beard, 2009; Inciardi, Surratt, Kurtz, \& Burke, 2006). For example, areas densely populated with older, disabled, or chronically ill people may provide more opportunities for diversion of prescription drugs into illicit markets (Green et al., 2013; Inciardi et al., 2009; Rigg, Kurtz, \& Surratt, 2012). Appalachian and rural areas in Kentucky have had historically high rates of 
disability, cancer, and chronic health conditions, as well as rates of opioid prescribing and diversion that rank among the highest in the nation (Inciardi, Surratt, Lugo, \& Cicero, 2007; Luu et al., 2018; Social Security Administration, 2016; Wilson, Ryerson, Singh, \& King, 2016). Since greater exposure to drugs is positively correlated with increased likelihood for misuse, the ambient level of a given drug in accessible markets, including licit prescription drugs, is important to consider as a possible influence on drug-related decisions (Cicero et al., 2007; Daniulaityte et al., 2006). Indeed, prescription drugs provide considerable opportunity for diversion and use for a variety of drug classes, including stimulants (McCabe, Teter, \& Boyd, 2006). In one study Daniulaityte et al. (2009) found that prescription tranquilizer and prescription stimulant use were the strongest predictors of prescription opioid use. Steep increases in drug prescribing in the 1990s and 2000s contributed to subsequent increases in prescription drug diversion and misuse (Inciardi \& Cicero, 2009).

For categorically illicit drugs, if a person lives in a place where there is a decreased availability of, say, cocaine, but an abundance of methamphetamine, then not only would the price of cocaine be expected to be higher and the price of methamphetamine lower, but additional costs associated with the acquisition of cocaine, in terms of labor and opportunity costs, would also be expected to increase (Kleiman, 1992). Drug preferences and the rewarding effects of drugs are important to consider here. Numerous pre-clinical studies have documented that the rewarding effect of a drug is positively correlated with learned behavioral responses, indicating increased willingness to work to obtain the drug (Everitt \& Robbins, 2005). This means that people will be likely to spend more money and work harder for drugs associated with greater reward (and less punishment), or which are preferred relative to other drug choices (Herrstein, 1961, 1970; Katz, 1990).

It is also consonant with the idea of own-price elasticity of demand, which is the variation in demand to a commodity in response to variation in price (Bickel et al., 1992) $)^{5}$. Demand may be elastic, wherein increases in price significantly decrease consumption (e.g., a 35\% price increase for OxyContin pills results in a 70\% reduction in OxyContin pill purchases), or demand may be inelastic, wherein increases in price may only result in marginal decreases (e.g., a 35\% price increase of OxyContin pills results in a 9\% reduction in purchases). Heroin and cocaine are two specific drugs with price inelastic

\footnotetext{
${ }^{5}$ Understood to be holding other factors constant.
} 
demand, though demand and consumption is still responsive to fluctuations in price (e.g., at some cost they would be unattainable) (Bretteville-Jensen \& Sutton, 1996; Bretteville-Jensen \& Biørn, 2003; Gallet, 2014; Olmstead, Alessi, Kline, Pacula, \& Petry, 2015; Roddy \& Greenwald, 2009; Saffer \& Chaloupka, 1999). If cocaine was a person's preferred drug, then demand for cocaine for this person would be expected to be more inelastic. The person would be more likely to expend greater effort and resources to obtain cocaine should costs increase, with only very extreme or prohibitive costs modifying consumption. For some people with strong drug preferences, decisions to use their preferred drug may persist over time, irrespective of broader drug trends and fluctuations in price or availability (Brecht, Huang, Evans, \& Hser, 2008; Falck, Wang, \& Carlson, 2007). In addition to paying higher prices in local drug markets to acquire a preferred drug, a person might expend more effort (e.g., driving greater distances to purchase it), or might try accessing the drug on bourgeoning avenues such as the Internet and Deep Web (Gilbert \& Dasgupta, 2017; Kassab \& Rosen, 2019; Quintana Mathé et al., 2017).

However, a person who prefers cocaine might also choose to substitute it with other drugs. These could include drugs with stimulant properties (e.g., amphetamines, cathinones), but could also include drugs with dissimilar pharmacology, but for which the associated costs are less than that of cocaine. In other words, changes in costs associated with one drug affect the consumption of others (i.e., cross-price elasticity of demand) (Bickel et al., 1995). For instance, following increased restriction of methamphetamine precursors in the US, methamphetamine cost temporarily rose, purity declined, and rates of methamphetamine-related service utilization and arrests decreased, while rates of cocaine use increased in some, but not all local drug markets (Borders et al., 2008; Cunningham, \& Liu, 2003, 2005, 2008; Dobkin, \& Nicosia, 2009; McKetin, Sutherland, Bright, \& Norberg, 2011.

For people who prefer or frequently use heroin and cocaine concomitantly, (e.g., "speedballing"), it is conceivable that as demand for cocaine decreases due to increased price that heroin demand would also decrease (Ball \& Ross, 1991; Leri, Bruneau, \& Stewart, 2003). For this group, heroin and cocaine could be considered as compliments rather than substitutes, meaning that they are co-used with regularity and can be considered concurrent reinforcers (Bickel, DeGrandpre, \& Higgins, 1995). For instance, alcohol and cocaine and nicotine and caffeine have been considered as compliments, demonstrating concurrently reinforcing effects and negative cross-price elasticity of demand (Bickel, Hughes, De Grandpre, Higgins, \& 
Rizzuto, 1992; Carrol, Rousaville, \& Bryant, 1993; McCance-Katz et al., 1993). Again, it may be that cocaine is simply substituted for another stimulant, such as methamphetamine (e.g., "goofballing"), or that demand for heroin is independent, with changes in cocaine prices not resulting in commiserate changes in heroin demand and consumption. Research has demonstrated, though, that polydrug users are willing to substitute drugs across drug classes, even as drugs such as heroin and cocaine have demonstrated inelastic demand among regular users (Olmstead et al., 2015; Petry, 2001; Sumnall, Tyler, Wagstaff, \& Cole, 2004).

Over the last decade, drug substitution has occurred en masse. Many prescription opioid users began purchasing heroin as prescription opioid supply decreased, prices increased, and abuse-deterrent pill formulations proliferated (Evans et al., 2018; Compton et al., 2016; Mallatt, 2017; Mars et al., 2014; Peavy et al., 2012; Rutkow et al., 2015). One result has been increased heroin demand, greater availability, lower prices, and increased consumption (Cicero, Ellis, Surratt, \& Kurtz, 2014; Kanouse \& Compton, 2015; Kilmer et al., 2014). Additionally, the supply of fentanyl and fentanyl analogues have proliferated, due in part to fentanyl's potency and low distribution burden (Armenian, Vo, Barr-Walker, \& Lynch, 2018; Ciccarone, 2017; Misailidi et al., 2018). However, the use of prescription opioids remains higher than heroin in some areas, particularly rural areas, even as rates of heroin use in suburban and rural areas continue to steadily increase (Borders \& Wen, 2018; Cicero, Ellis, Surratt, \& Kurtz, 2014; Day, Conroy, Lowe, Page, \& Dolan, 2006; Havens et al., 2009; Wunsch, Nakamoto, Behonick, \& Massello, 2009). The use of stimulant drugs differs across metro and non-metro areas and geographic regions, with cocaine often more available in metro areas, but methamphetamine increasingly available across localities (Brownstein, Mulcahy, Fernandes-Huessy, Taylor, \& Woods, 2012; Gfroerer, Larson, \& Colliver, 2007; Stover, Winstanley, Zhang, \& Feinberg, 2018). It is important to emphasize, though, that cocaine has, to varying degrees, remained accessible in rural areas (Booth, Leukefeld, Falck Wang, \& Carlson, 2006; Borders, Booth., Stewart, Cheney, \& Curran, 2015).

It is also worth noting, that not all people who have used prescription opioids or who have access to heroin would choose to substitute the latter for the former, as heroin may be perceived to have higher relative risk (Daniulaityte, Falck, \& Carlson, 2012; Inciardi et al., 2009; Inciardi \& Cicero). Additionally, some drug users, including polydrug users, may have developed drug preferences and patterns of use over time that make them less likely to substitute their preferred drug for other drugs with similar psychoactive 
effects and more likely to persist in the use of their primary drug. Evidence suggests that long-time cocaine and crack cocaine users may be less likely to use other stimulant drugs, and that co-use of cocaine and other stimulants is low among groups with greater cocaine use severity or longer use history (Booth et al., 2006; Borders et al., 2008; Falck et al. 2007; Wu et al., 2009). Further, drug users for whom cocaine is their primary drug, have persisted in cocaine use over time (>10 years), whereas primary users of methamphetamine and heroin showed gradual decline in respective use for the same duration of time (Brecht et al., 2008).

Lastly, it is important to consider that preference and decisions to use can be shaped by perceived drug quality and cost, with people reversing preferences for drugs based on increases or decreases in price and effort needed to obtain the drug (Caulkins, 1995; Goudie et al., 2007; Jacobs \& Bickel, 1999). Preference for drugs may develop around a variety of factors beyond subjective effects. Conversely, some people may simply be less discriminant in their drug choice and therefore more willing to substitute preferred drugs with less preferred, but more accessible and affordable, drugs despite differences in subjective effects, quality, and known risk (e.g., substituting with fentanyl, synthetic cathinones, etc.) (Mars, Rosenblum, \& Ciccarone, 2018; Smith \& Staton, 2018).

In sum, drug accessibility and cost comprise part of the larger "system of constraint" within which people make decisions and allocate resources, as options in accessible drug markets provide choices with differing values, risks, and costs--all of which can motivate behavior differentially (Bickel, Mueller, MacKillop, \& Yi, 2016). Although the region that a person lives in puts them in proximity to different markets, and therefore different drug choices, this proximity is just one factor that shapes drug-related decision-making and drug use. It is therefore also important to also consider the non-drug choices available to people.

\section{Non-drug Alternative Reinforcers}

As noted throughout this discussion, numerous aspects of a person's history and everyday life have the potential to influence cognition and choice, and the potential to reinforce behavior. Recall that opioids and stimulants are potent reinforcers that can result in reinforcer pathology. This is due to the rewarding drug effects that can contribute to repeated use and/or habituated use that persists despite adverse consequences, including criminal justice involvement (Bickel, et al., 2011; Everitt \& Robbins, 2005). 
Aspects of a person's life apart from drugs may also be considered in terms of how they reinforce behavior. The importance of considering the role of non-drug alternative reinforcers using behavioral economics can be summarized by Bickel et al. (2014) thusly:

[The] behavioral economics perspective gives equal weight to the larger environmental context within which addictive behavior takes place and, more specifically, the alternative reinforcers to which a person has access....A behavioral economics perspective is a molar account of addiction, focusing on aggregates of behavior, not instances, and both the endogenous conditions of the organism and exogenous characteristics of their environment. Put simply, a person's internal motivational state and the alternative reinforcers available in the environmental context are theorized to jointly determine the decision to use or not to use a drug. From this perspective, the yin and yang of addiction are high levels of endogenous factors (high [drug] demand and impulsive discounting) and low levels of exogenous factors (alternative reinforcers). Furthermore, a recursive etiological process is proposed to be operative for alternative reinforcers over the course of the development of a substance use disorder. For example, an individual may begin drinking recreationally and as part of a diverse repertoire of positively and negatively reinforcing behaviors. But, as use escalates and negative consequences mount (e.g., dissolution of a relationship, loss of a job, legal difficulties), the availability of these alternative reinforcers diminish, which commensurately increases the reinforcing effects of alcohol (Rachlin, 1997). This recursive etiological feedforward loop illustrates the classic vicious cycle of addiction (e.g., the primrose path model of addiction formulated by Hernstein \& Prelec, 1992) (p.654).

Many preclinical models have demonstrated that non-drug alternative reinforcers (e.g., saccharin, food, water, access to conspecifics/social interaction) are chosen over drugs such as cocaine or morphine, depending on the dose and effort associated with choice (Ahmed, 2010; Tarou \& Bashaw, 2007; Cantin et al., 2010; Lenoir, Sere, Cantin, Ahmed, 2007; Venniro, Zhang, Shaham, \& Caprioli, 2017; Zernig, Kummer, \& Prast, 2013). Among humans, non-drug reinforcers could include a wide-range of things. Nondrug alternatives might include exercise, money, work, school, close relationships with family or friends, hobbies, educational participation, or group membership (e.g., church, civic organizations, or mutual aid/12-Step groups) (Comer et al., 1998; Donlin, Knealing, \& Silverman, 2008; Etten, Higgins, Budney, \& 
Badger, 1998; Greenwald \& Steinmiller, 2009; Higgins, Bickel, \& Hughes, 1994; Morral, Iguchi, \& Belding, 1999; Petry, Tedford, J., \& Martin, 2001; Quick, Pyszczynski, Colston, \& Shahan, 2011; Rogers et al., 2008; Waldorf et al., 1992; Zlebnik, \& Carroll, 2015). Although the possible number of non-drug alternative reinforcers that people could encounter is almost limitless, and could conceivably include abstractions (e.g., religious belief, life meaning, values), one type of non-drug alternative that may serve as a particularly effective behavioral reinforcer is social interactions and close connection with others (Laudet \& White, 2008; Ostafin \& Feyel, 2019). Although social interaction and close relationships are often associated with enhanced health and well-being, choice of relationships over drugs is not a given when the two are placed in direct competition. This is reflected indirectly by high divorce rates and low rates of sustained family reunification among active or recently remitted drug users (Brook \& McDonald, 2009; Brook, McDonald, Gregoire, Press, \& Hindman, 2010; Collins, Ellickson, \& Klein, 2007; Lloyd \& Akin, 2014).

Still, prosocial interactions and relationships may constitute one of the most potent non-drug alternatives a person could gain access to, given that social interaction, play, and bonding are intrinsically rewarding within and across sexes and species, and have demonstrated potential to attenuate drug use ${ }^{6}$ (Fritz et al., 2011; Heilig et al., 2016; Kohtz, Lin, Smith, \& Aston-Jones, 2018; Peirce, Frone, Russell, Cooper, \& Mudar, 2000; Smith, 2012; Strickland \& Smith, 2014; Vanderschuren, Achterberg, \& Trezza, 2016; Zernig, Kummer, \& Prast, 2013; Venniro et al., 2018).

Ultimately, non-drug alternatives help define the range of choices available to people, but also have the potential to be rewarding in the short- and long-term (e.g., relationships, school, hobbies) (Herrnstein \& Prelec, 1991). There is the possibility, then, that certain non-drug alternatives can have both a high local and overall utility (Herrnstein \& Prelec, 1991). This is important in terms of their potential to influence decisions at one time point and across time, as well as in terms of providing satisfying, but durable, alternatives to drug use. Additionally, non-drug alternatives may reinforce behavior in terms of opportunity cost, wherein the opportunity cost for a given choice is defined as equal to the best alternative

\footnotetext{
${ }^{6}$ It is worth mentioning that, for people, studies have consistently found money to also be a potent behavioral reinforcer as well (see Higgins et al., 2008). Money, somewhat depressingly, was also ranked by Americans as one of the things that "gives their life meaning", with money outranking family and other activities for some respondents (Pew Research Center, 2018).
} 
not selected (Bickel et al., 1993, 2014). When drugs are in direct competition with a set of potentially rewarding alternatives, the opportunity cost of drug use increases and may motivate decisions not to use drugs; for instance, if food and a drug were in direct competition, the opportunity cost of choosing the drug would be not eating and, over time, eventual starvation (Caprioli, Zeric, Thorndike, \& Venniro, 2015).

For corrections-involved people, community supervision and drug court (which includes drug monitoring and sanctions) have the capability to serve as additional behavioral reinforcers (Marlowe \& Wong, 2008; Petry et al., 2006). Here, some abstinence-promoting behaviors may be reinforced by threat of unfavorable consequences. For someone who uses heroin while on probation, the opportunity costs associated with use and subsequent punishment may be extreme, such as incarceration.

In real-world circumstances, it is not always clear when a decision to use drugs is in direct competition with alternatives. For instance, a person could choose to use hydrocodone while simultaneously engaging in other rewarding activities (Vanderschuren, Spruijt, Hol, Niesink, \& Van Ree, 1995). In other words, taking hydrocodone and pursuing non-drug choices are not necessarily mutually exclusive. Often, instead of direct competition between drugs and non-drug alternatives, there are contingencies that a person must navigate when making decisions. For example, a man's romantic partner threatens to divorce him if he continues to smoke crack cocaine each weekend. Because smoking crack and being married are not necessarily mutually exclusive, it is possible for him to do both, until the point where they do become mutually exclusive (e.g., the partner leaves, with reunification explicitly contingent on abstinence from crack cocaine). Additionally, the credibility and likelihood of the partner's threat coming true will influence its reinforcing effects, with less credible threats (e.g., divorce) or rewards (e.g., staying married) being discounted in terms of probability (Rachlin et al., 1991).

In this scenario the man loves his partner and does not want her to leave. He discontinues his crack cocaine use. Here, the presence of the man's partner in his life is rewarding and her stipulations, that in order for her to stay he must stop smoking crack, are sufficiently reinforcing. This rewarding non-drug alternative that has been put in direct competition with drugs has resulted in a decision to not use crack. Considering this same scenario between drugs and a relationship with another person, but where the relationship is not characterized as close or where the quality of it has deteriorated, suggests that the nondrug alternative may not have a similarly reinforcing effect (Homish, Leonard, Kozlowski, \& Cornelius, 
2009). To put this in perspective, consider the effort a person might undertake for their child, but not for an acquaintance or co-worker. Consider, too, the difference in magnitude of reward received interacting with a partner, child, or close friend compared to that received from interactions with strangers or acquaintances.

Take another example of a man who smokes crack cocaine on the weekends. In this scenario, however, the man is on probation and subject to drug monitoring. The man knows the date he will see his probation officer next month, but does not know for certain if he will be drug tested on this next visit. He also knows that his probation officer could make an unannounced visit to randomly drug test him. He has been told that if he has another positive drug test, his probation will be officially "violated" and a judge will determine his sentence. He could receive a minimum of one year and a possible maximum of six years in prison. Here, there are clear potential losses, but the probability of those consequences occurring is unclear. Further, the certain risk involved in meeting with the probation officer is one month away. The reinforcing effect of the probation officer and drug testing is real, but it is in the future, and the results of the interaction uncertain. This non-drug alternative reinforcer is thus influenced by the steepness of the man's temporal and probability discounting which, as discussed earlier, may be influenced by many other factors. For this man on probation, there is a certainty that he will be drug tested in the future and that a negative drug screen will not result in arrest. There is no certainty of when the drug screening will occur or what the exact consequences of a positive drug screen would be. Here, the magnitude of the reinforcer may vary as a function of time (e.g., the closer to the next appointment with his officer the less likely he is to smoke crack cocaine).

Both examples illustrate how the uncertainty associated with a behavioral reinforcer for which the effects are delayed makes decision-making more complicated, and also implies that people may be biased toward choosing drugs due to the fact the drug effects are nearly immediate and approximately certain. ${ }^{7}$ Importantly, the risk of choosing drugs in both examples is clear, due to the uncertainty and severity of potential outcomes. In both examples, there are potential rewards (e.g., intact relationship, intimacy, companionship, not having probation violated, remaining free) and losses (e.g., divorce, incarceration) that

\footnotetext{
${ }^{7}$ By "approximately certain" it is meant that there is an outcome expectancy for drug reward or effect in a generic sense; it is, of course, inevitable that the potency of drug effects vary across doses due to changes in drug quality, as well as physiological states and contexts (e.g., possibility of have been sold ineffectual drugs, hormonal fluctuations, etc. ) (Kepler, Kest, Kiefel, Cooper, \& Bodnar, 1989).
} 
must be considered, in addition to the reinforcing effects of crack cocaine. In other words, smoking crack cocaine each weekend does not occur in a vacuum. In both examples, the opportunity cost of choosing not to smoke crack cocaine would simply be not getting to smoke crack cocaine ${ }^{8}$. For some, such an opportunity cost may be perceived as too great due to the overvaluation of the drug in the immediate, the undervaluation of non-drug alternatives.

\section{Restricted Opportunity for Accessing Non-Drug Alternatives}

Ultimately, it is the presence or absence, and the potential for variation, of these non-drug alternative reinforcers that defines the choices available to a person and serves to influence what decisions are made. One way to think about the role of alternative reinforcers is to consider that the more that a person has, or the more they have the opportunity to access, the greater the potential opportunity costs. Another way to think about them is to consider that in the absence of rewarding alternatives, drugs may retain a high local and conceivably, under extreme conditions, even a higher overall utility; in such extreme circumstances the overall utility, in the traditional sense, becomes irrelevant or nonsensical. Without, or with far fewer, non-drug choices the rewarding effects from drugs may be greater, potentially contributing to reinforcer pathology (Ahmed, Lenoir, \& Guillem, 2013; Bickel et al., 2011). A person who does not have friends or family would not be able to choose these relationships over drugs. If a person does not have a romantic partner to enjoy the company of, then there is no reward to be had and no partner-loss to incur in its absence. It simply does not exist as a non-drug alternative and thus cannot motivate decision-making and behavior in relation to drugs.

This idea may be extended to more broadly consider how the absence of abundant opportunity for accessing non-drug alternatives might influence decision-making. As noted in the first chapter, in order to influence decision-making, people need only know that certain options exist and that there is opportunity and ability to access them in order to motivate behavior (recall the chocolate cakes and apple pies). Thus, while a person may not currently have non-drug alternatives such as close friends, employment, group affiliation, or educational participation, they still have potential to acquire these things. However, for

\footnotetext{
${ }^{8}$ It is conceivable that there are also some other hidden opportunity costs under particular circumstances and for particular drugs. If a person is using opioids so as not to go into withdrawal or not to be crippled by pain so that they can go to work and keep their job, then there would be a string of related opportunity costs. In this example of irregular crack cocaine use, this would be less likely.
} 
people with less proximity to opportunities for such acquisition, non-drug alternatives may remain limited, constraining choice proportionately. In line with some of the findings discussed earlier related to the detrimental effects of social isolation, studies have demonstrated that, compared to impoverished environments, enriched environments with greater access to rewarding non-drug alternatives (e.g., social interaction, food, activities) can attenuate drug-taking (Bardo, Neisewander, \& Kelly, 2013; Imperio et al., 2018; Marlatt, Baer, Donovan, \& Kivlahan, 1988; Nader \& Banks, 2014; Panebianco, Gallupe, Carrington, \& Colozzi, 2016; Strickland \& Smith, 2015; Solinas, Chauvet, Thiriet, El Rawas, \& Jaber, 2008; Venniro et al., 2018). Yates et al. (2017) found that in enriched environments, demand elasticity for cocaine increased. Similarly, Hofford et al. (2017) found that value and reinforcer strength for remifentanil was lower among rats housed in enriched environments.

People who live in resource scarce, economically distressed, or otherwise "harsh" regions (e.g., higher poverty, greater prevalence of violence, disability, mortality, fewer treatment providers, etc.), or in areas with less social cohesion and fewer opportunities for prosocial connection, may be considered to live in impoverished (verses enriched) areas, in that they have comparatively less accessibility to non-drug alternatives (e.g., relationships, education, employment, money, school, group affiliation, entertainment) (Dew, Elifson, \& Dozier, 2007; Erickson, VanLooy, von Schrader, \& Bruyère, 2018; Monnat, 2018; Zoorob \& Salemi, 2017). Highly urban or highly rural areas, including those within central Appalachia, may be considered "harsh" environments, wherein some opportunities for accessing rewarding non-drug alternatives are restricted, despite the fact that close social, cultural, and spiritual connections can be found in both locales (Case \& Deaton, 2017; Diddle \& Denham, 2010; Donnermeyer, Jobes, \& Barclay, 2006; Eller, 2008; Lee, Maume, \& Ousey, 2003; Meit, Heffernan, Tanenbaum, \& Hoffmann, 2017; Oser, Harp, O'Connell, Martin, \& Leukefeld, 2012; Snell-Rood \& Carpenter-Song, 2018; Tonkiss, 2005; Williams, Palar, \& Derose, 2011). It is perhaps unsurprising that rates of SUD, and rates of opioid and stimulant use in particular, are high in these areas (Moody, Satterwhite, \& Bickel, 2017). Sadly, prolific drug use within a region may come to further qualify it as "harsh", insofar as drug use can initiate and perpetuate a cascade of loss and breakdown in social cohesion (Mack, Jones, \& Ballesteros, 2017; Monnat, 2018; Orsi, YumaGuerrero, Sergi, Pena, \& Shillington, 2018; Oser et al., 2011; Zoorob \& Salemi, 2017). This means that 
drug use can potentially be understood as both cause and consequence of harsh environments. Bleakness begetting bleakness.

As noted earlier, living in "harsh" conditions can foster a sense of uncertainty about one's future, influencing cognition and decision-making. In the last two decades, many rural and urban regions have experienced rapid changes due to globalization, deindustrialization, shifting demographics, and technological innovation, meaning that some areas that may have already engendered feelings of uncertainty about the future, had an increased potential to do so (Case \& Deaton, 2017; McLean, 2016). In the presence of such existential uncertainty, and in the absence of opportunity for accessing rewarding nondrug alternatives, opioids and stimulants, despite their deadly risk, may still hold value. This is not to say that suburban communities necessarily guarantee access to opportunity or ensure protection from conditions which might create a scarcity mentality (Allard, 2017). Therefore, when identifying how the region a person lives in might shape their decision-making, it is important to avoid overgeneralizations and to consider possible gradations in "harshness" between regions.

The counter to all this is that people may have few non-drug alternatives, have only very weakly reinforcing non-drug alternatives, or may live in areas with fewer opportunities for accessing rewarding non-drug alternatives, but may still choose to not ever use opioids or stimulants, to moderate their use, or to discontinue use without prospects changing; and that people with abundant access to non-drug reinforcers may still choose to use opioids and stimulants (Bozarth, Murray, \& Wise, 1989); Davis, 2014; Grant, 2007; Premac, 2017; Miller, 2004). Indeed, there are ultimately many factors with the potential to influence decisions to use and decisions not to use. The importance of considering alternative reinforcers, however, is articulated in research showing that the presence or absence of certain conditions reliably influences behavior (Dayan \& Balleine, 2002). It is also articulated by the possibility that the presence of rewarding non-drug alternatives, like a job, relationships, group participation,--and the sense of belonging, responsibility, and meaning that can come with these things (Laudet \& White, 2008; Petry et al., 2001; Pew Research Center, 2018)-- has the capacity to make people more vested in their lives, helping to produce what Waldorf et al. (1992) refer to as "a stake in the conventional life". This concept is important, because implicit in it is a connection between a person's current and future self. For people who have a stake in the conventional life, who are truly vested in their everyday roles and in their future, the breadth of opportunity 
costs from using high-risk drugs has the potential to be nothing less than heartbreaking. For people who continue to use high-risk drugs without access to the types of rewarding alternatives that might foster a stake in the conventional life, the unrealized human potential is no less heartbreaking.

\section{Study Aims Overview}

Having discussed how a behavioral economic approach considers that both endogenous and exogenous factors influence drug-related decision-making and behavior, and having detailed several factors with the potential to exert such influence in relation to opioids and stimulants, it is important to reiterate that these factors can vary across people, and that people's lives are always in a state of flux. The same factors that may have contributed to a decision to use opioids or stimulants at one time may not be present at a later time. For corrections-involved people with a history of drug use, decision-making about opioids and stimulants may change subsequent to losses incurred as a result of their use, such as incarceration.

Given the high-risk nature of opioid and stimulant use, and the many constraints on decisionmaking and behavior, it is important to identify, explore, and describe what factors with the potential to influence drug-related decision-making and behavior are associated with opioid and stimulant use among corrections-involved adults residing in a state that continues to experience high rates of opioid and stimulant use, and where the risks associated with use are well-known (Havens et al., 2011; SAMHSA, 2016). Kentucky is an ideal state for such exploration. Many corrections-involved adults in Kentucky with a history of drug use have likely experienced or observed adverse consequences related to high-risk drugs first-hand. As noted earlier, the region that a person lives in can influence cognition, behavior, and drug choices. Regional differences in Kentucky can be stark, meaning that the area that a person resides in has the potential to shape both choice and behavior. People who reside in Central Appalachia, the Easternmost part of the state, may be considered to live in a "harsh" region compared to people who live in Central and Western parts of Kentucky outside of Central Appalachia. Given the insular nature of Central Appalachia, the persistently high rates of morbidities and disabilities, and the region's greater rurality, it is likely that drug markets within this area of Kentucky also influence drug-related decision-making and behavior.

Using behavioral economics as a molar framework for exploring high-risk drug use among corrections-involved adults in Kentucky prior and subsequent to incarceration, several questions can be addressed: What endogenous and exogenous factors with the potential to influence decision-making about 
high risk drugs prior and subsequent to incarceration can be identified using available data sources? What differences are observed between groups who use different types of high-risk drugs during these periods, including the geographic areas in which they reside? What factors are associated with an increased likelihood of observing high-risk drug use during these periods?

\section{Specific Study Aims}

The primary aim of this study is to explore high-risk drug use among a sample of correctionsinvolved adults in Kentucky and to identify and discuss endogenous and exogenous factors with the potential to have influenced drug-related decision-making prior and subsequent to incarceration. This will be accomplished with three specific study aims.

\footnotetext{
Aim One

Provide a descriptive profile of a sample of adults in Kentucky with a history of drug use prior and subsequent to incarceration using self-report data collected during intake into a corrections-based drug treatment program and self-report data collected during the one-year period subsequent to incarceration for years 2012-2017. Determine the prevalence of concomitant opioid/stimulant use, opioid use, stimulant use, and other and/or no drug use that occurred during the 30-day period prior to incarceration and the one-year period subsequent to incarceration. Provide a description of these drug use groups and identify betweengroup differences. This includes identifying differences in the use of specific opioid and stimulant drugs by participant residence across an urban-rural continuum, as well as differences in the use of specific opioid and stimulant drugs by participant residence within or outside of Central Appalachia.
}

\section{Aim Two}

Identify factors associated with a change in likelihood of observing concomitant use, opioid use, and stimulant use during the 30-day period prior to incarceration and the one-year period subsequent to incarceration, compared to an outcome with less risk, such as the use of other drugs and/or no drug use.

\section{Aim Three}

Because heroin was the drug associated with the highest risk in the US during the one-year postrelease period for participants in this sample (2012-2017), it is of interest to also determine the prevalence of heroin use that occurred during the one-year period subsequent to incarceration, the between-group 
differences among those who did and did not report heroin use, and to identify factors associated with an increased likelihood of heroin use. 


\section{CHAPTER III}

\section{METHOD}

Chapter 1 provided an overview of some of the well-known risks associated with opioids and stimulants. It also introduced behavioral economics as a conceptual approach for understanding drugrelated decision-making. In Chapter 2, many endogenous and exogenous factors with the potential to influence drug-related decision-making were discussed and situated within a molar view of choice and behavior. For instance, scarcity might contribute to a person to becoming more present-biased, more prone to discount the future, and more likely to evidence poorer decision-making. Stress and living in a harsh environment can contribute to greater reliance on more automatic, habituated, lower-level cognitive systems, rather than higher-level, deliberative cognitive systems. Prior opioid use can increase the likelihood of continued opioid use. Residing in an area that is more rural, may restrict access to a wider variety of drugs, compared to urban drug markets.

A simplified way to think about the previously discussed factors (and others not discussed here), is to consider that they have the potential to influence decision-making in roughly one of two ways: 1) Influencing a person's cognitive capacity to make decisions that maximize overall utility (versus choosing immediately rewarding options with higher local, but lower overall utility); and/or 2) Influencing the set of drug and non-drug choices available or accessible to people. This simplified conceptual model of how endogenous and exogenous factors have the potential to influence drug-related decision-making and drug use is displayed in Figure 1. 
Figure 1. Concept model of endogenous and exogenous factors with potential to influence drugrelated decision-making by constraining cognitive capacities and by constraining choice.

\section{Exogenous}

\section{Contextual Factors:}

Has the potential to range from proximate conditions (i.e., immediate environment, e.g., house) to the region a person lives in, to broader economic and sociocultural conditions.
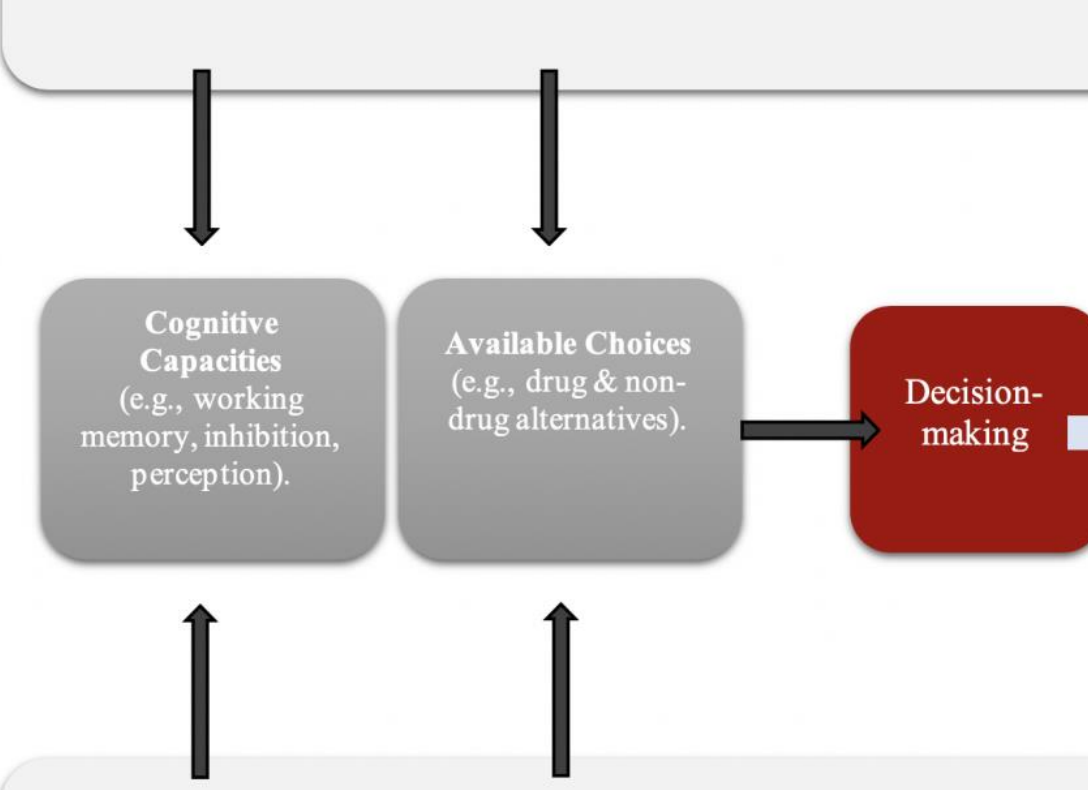

Individual Factors:

Has the potential to range from the genome to observable or measurable traits (i.e., phenotypes; e.g., sex), states (e.g., being low-income, being in pain), and history (e.g., abuse, head trauma, drug use).

\section{Endogenous}

Importantly, both types of influence can occur at the level of the person or at the level of the person's context or environment. When Bickel et al. (2014) describes the "yin and yang of addiction" as high levels of endogenous factors and low level of exogenous factors, this is, in part, what they are referring to. Behavioral economics understands that endogenous factors can also be influenced by exogenous factors so as to influence cognition, affect, and decision-making. This general idea, that organisms are influenced by their environment, spans many disciplines. That context can influence decision-making and behavior was hopefully made apparent in literature reviewed in the previous chapter. 
Ideally, that overview also made more apparent that the "system of constraint" in which people make decisions is not unidimensional (Bickel \& Marsch, 2000; Bickel et al., 1995). Rather, people are constrained across levels. People are constrained by their capacities (traits and states), by their contexts, and by the choices for behavior available to them. The specific configuration of endogenous and exogenous factors that constrain people's decision-making in relation to opioids and stimulants differs by person. At the group level, some endogenous and exogenous factors with demonstrated potential to influence decisionmaking may be observed for given time periods. In this chapter, methods are proposed for identifying some of these factors among a sample of drug users prior and contemporaneous to incarceration and participation in corrections-based treatment. Discussion of findings and their implications will be considered in subsequent chapters.

\section{Data Sources and Sample}

\section{Data Sources}

In order to address the aims of this exploratory study, secondary data analyses were conducted using individual-level and county-level data. All individual-level data were collected as part of the Criminal Justice Kentucky Treatment Outcome Study (CJKTOS). CJKTOS is ongoing evaluation of the KY DOC Substance Abuse Treatment Program (SAP). CJKTOS was initiated in April 2005 with the primary objectives of better understanding the population served by SAP and assessing the effectiveness of SAP in reducing drug use and recidivism. CJKTOS is conducted by the University of Kentucky's Center on Drug and Alcohol Research (CDAR) and is funded on an annual basis through a contract with the KY DOC. SAP is available to adults involved with the KY DOC who have a history drug use or who are currently KY DOC-involved due to a drug-related offense. People may voluntarily enroll in SAP and SAP aftercare, or may be mandated by a judge or parole board. While the majority of SAP participants are mandated (approximately 75\%), even those who volunteer are incentivized, in that SAP participation may be regarded favorably by the parole board. Moreover, all SAP participants who successfully complete the program receive a 90-day sentence reduction. SAP is delivered by trained clinicians in Kentucky jails, prisons, or in secure DOC-affiliated community residential facilities that constitute controlled environments and correctional custody. 
SAP lasts approximately six months. During this time, it is expected that participants will indicate therapeutic progress in a variety of ways. Progress indicators include demonstrating a willingness to change, completing recovery-related assignments, engaging in group activities, adhering to institutional and SAP regulations, and holding themselves and other SAP participants accountable for attitudes and behaviors considered antithetical to the broad therapeutic goals of SAP. These goals include reducing or extinguishing drug use through cognitive, spiritual, and behavioral modification. Although there is variability across correctional institutions in terms of how SAP is delivered (e.g., amount of individual time with a counselor versus time spent in groups), all programs are styled as a residential therapeutic community grounded in 12-Step principles and cognitive behavioral modalities, and each uses structured materials (e.g., workbooks, readings) to facilitate treatment.

Subsequent to release, SAP participants who still reside in Kentucky are either paroled onto community supervision or are released having "served out" their time, such that they are not mandated to community supervision. On any given year, a majority of CJKTOS participants are on community supervision subsequent to release. Some of the terms of community supervision include abstaining from alcohol and drug use, not associating with known felons, monthly reporting to a parole officer, random drug monitoring (e.g., urine analyses, breathalyzer), and mandated participation in recommended services (e.g., aftercare) and/or completion of reentry tasks (e.g., obtaining employment, driver's license, etc.).

CJKTOS data includes data collected by CDAR research staff, data collected by SAP clinicians, and institutional data provided by the KY DOC and community supervision officers. Parole officers, KY DOC officials, and social service clinicians enter information about offenders (e.g., supervision notes, program referrals, drug screen results, parole violation reports, reincarceration dates, institution location etc.) electronically into the web-based Kentucky Offender Management System (KOMS).

Individual-level CJKTOS data include several different types of information (e.g., age, employment status) and also include the counties that participants resided in prior and subsequent to incarceration. County-level self-report data was used to determine the relative rurality of participants' county of residence by examining urban influence designations, as well as the proportion of participants who resided in one of fifty-four counties in Kentucky that fall within Central Appalachia. In addition to CJKTOS data, publicly available data measured at the county-level were obtained for this study from state 
and federal entities for all 120 Kentucky counties. These entities include: Alcohol Beverage Control, Appalachian Regional Commission, the Federal Communications Commission, the Kentucky Cabinet for Health and Human Services, the Social Security Administration, and the U.S. Census Bureau. Using participants' counties of residence prior and subsequent to incarceration allowed for the possibility of matching individual cases with corresponding county data, thus potentially providing an additional level of measurement.

Individual-level and county-level data have been successfully integrated and analyzed by many researchers, and are included among investigations examining county or regional influence on a variety of outcomes. Such outcomes include drug use, drug-related health outcomes, depression, recidivism, obesity, self-rated health, quality of life, and mortality (Blakely, Lochner, \& Kawachi, 2002; Goodman, Huang, Wade, \& Kahn, 2003; Jia, Moriarty, \& Kanarek, 2009; Kim, Subramanian, Gortmaker, \& Kawachi, 2006; Lochner, Pamuk, Makuc, Kennedy, \& Kawachi, 2001; Muramatsu, 2003; Orrick et al., 2011; Patrick et al., 2019; Subramanian, Kawachi, \& Kennedy, 2001). The growth of social epidemiological methodology and the expanded awareness of multi-level social determinants of health have increased the availability of publications describing how the integration and analysis of multiple data sources and types can be accomplished, and how data may be examined within and across levels (Blakely \& Woodward, 1999; DiezRoux, 2000; Duncan, Jones, \& Moon, 1996; Galea, Hall, \& Kaplan, 2009; Jia, Muennig, \& Borawski, 2004; Soobader, Cubbin, Gee, Rosenbaum, \& Laurenson, 2006; Macintyre, Ellaway, \& Cummins, 2002; Pickett \& Peral, 2001).

\section{Data Collection}

In implementing CJKTOS, the KY DOC and CDAR worked to develop a baseline instrument that functions as a psychosocial assessment of incoming SAP participants as well as a data collection tool. Information self-reported by participants is used by SAP clinicians to help evaluate participants' psychosocial and drug use histories. All SAP baseline data are de-identified, aggregated, and analyzed as part of CJKTOS (e.g., outcome evaluation reports). Every SAP participant completes a baseline survey "assessment" as part of the standard enrollment process into the treatment program. 


\section{Baseline Data Collection}

Baseline data are collected after a person has been referred to SAP, but prior to treatment initiation. Baseline data collection is conducted by a trained SAP provider at their respective location (e.g., jail, prison, community-based DOC facility). Baseline surveys are completed in one of two ways: 1) A trained SAP provider administers the survey by reading items aloud to participants and then recording responses into the secure, university-operated web-based Client Information System (CIS); 2) Participants self-administer the baseline survey electronically into the CIS online survey platform, seeking assistance from the SAP provider as necessary. The total number of baseline survey questions varies between years, and between participants within years, given that certain responses may prompt automatic follow-up questions, thereby increasing the number of questions asked. Across years 2012-2017, the maximum number of possible individual baseline survey items ranged from approximately $232-261$, though this is likely far more questions than participants typically respond to. The baseline survey takes approximately 1 hour to complete.

\section{Follow-up Data Collection}

Follow-up data are collected approximately 12 months subsequent to a participant's release. To be eligible for follow-up, the person must have participated in SAP, consented to participate in the CJKTOS follow-up portion of the study, and have a release date from a KY DOC institution following SAP participation that is verified using KOMS. Consent for study participation is typically over half of all SAP participants. For instance, between $2010-2018,57.3 \%$ of people who completed a CJKTOS baseline survey as part of their SAP assessment $(\mathrm{N}=43,390)$ consented to be contacted in the one-year post-release period as part of the CJKTOS follow-up study. CJKTOS uses randomized proportionate sampling, whereby SAP participants who consented to be contacted for follow-up interviews are randomly selected for inclusion into a given FY's follow-up sample. This method is utilized to help ensure that each FY the follow-up sample reflects the entire SAP population released for the same FY period for characteristics such as gender, race, SAP program type (e.g., jail, prison, community-based), so that findings from the follow-up sample can be generalized (Shadish et al., 2002). The final FY follow-up sample is thus an approximate representation of the total state population of SAP participants (Lavrakas, 2008b). Participants included in the follow-up pool become eligible for contact and data collection 10 months from their release date. The 
follow-up window closes 14 months from participants' release date. The CJKTOS follow-up rate for FYs 2013-2017 is as follows: FY13 $=82.0 \%$, FY14=81.0\%, FY15=80.0\%, FY16=83.0\%, FY17=84.0\%. This means that of the total follow-up sample pool, 16-20\% of participants for which contact attempts were made did not complete interviews, either because contact attempts were unsuccessful, or because the participant chose not to complete the interview. Follow-up refusal rates for FYs 2013-17 range from 4\%$7 \%$.

During the follow-up data collection window, trained CDAR research staff use information provided by participants at baseline, in conjunction with more current contact information obtained from KOMS, to facilitate contact. KOMS also provides contact information for relatives and associates of participants (e.g., mother, fiancé, children) and information about the known location of participants who are on community supervision or who have been reincarcerated. Facebook and VINELink, an online inmate search tool, are also used to search for participants who cannot be readily located using self-reported contact information or KOMS. The use of social media, including Facebook, for locating and contacting research participants is increasingly common and well supported, particularly among rural, drug-using, transient, or otherwise difficult-to-reach populations (Bolanos et al., 2012; Dickson et al., 2017; Staton et al., 2018).

Research staff make repeated attempts to contact participants via telephone, direct mail, and social media until successful contact is made, or until the 14-month follow-up contact window closes. When making contact through these means, the study is referred to as a "UK Health Study" so that sensitive information related to correctional involvement and drug use is not inadvertently disclosed. Participants must verify their date of birth prior to study specifics being disclosed. At follow-up, participants are reminded of the voluntary nature of the study and asked if they would like to participate in the follow-up interview, which is conducted via telephone at their convenience. Approximately 30-35\% of CJKTOS follow-up interviews are conducted with participants who are currently in jail or prison, meaning that subsequent to release they either violated the terms of their community supervision or were arrested for a new offense. Prison and jail interviews are also conducted via telephone and are scheduled by coordinating with correctional facility staff. 
Before beginning any follow-up interview, the identity of participants is verified using birthdate, release date, and the SAP institution of record. Research staff read questions from the follow-up instrument aloud and enter participant responses into CIS. The number of follow-up survey items also varies between FYs and between participants within FYs based on response patterns. Between 2012-2017, the maximum possible number of unique follow-up survey items ranged from approximately 201-232. Again, it is unlikely that all questions would be asked. The follow-up interview takes approximately 30 minutes to complete. Compensation, in the form of a $\$ 20$ check, is provided via direct mail only to participants who complete both baseline and follow-up surveys. Some information self-reported by participants (e.g., release or rearrest dates, program completion, recidivism, parole status) is cross-referenced and verified by CDAR research staff using KOMS and KY DOC records.

\section{Study Inclusion Criteria}

Cases were included in the sample if they met the following four criteria: 1) Verified SAP participation; 2) Completed CJKTOS baseline interview; 3) Completed CJKTOS follow-up interview for 2012-2017; 4) Reported residing in a Kentucky county for the majority of months (>6) out of the one-year period prior and subsequent to incarceration. Kentucky residency for participants who became reincarcerated after release was determined using the county that participants self-reported that they would be residing in if not incarcerated, versus designating the county of incarceration as the county of residence; 5) Reported alcohol or illicit drug use for the one-year period prior to incarceration. The latter criterion was included due to the fact that some SAP participants are enrolled in SAP due to drug-related charges (e.g., drug trafficking), but not necessarily due to drug use. As lifetime history of drug use is not assessed as part of CJKTOS, but rather only past-year and past 30-day use, no satisfactory determination could be reached as to what the nature of participants' drug use history entailed. Such ambiguity would hinder conceptualization of the sample and interpretation of findings. Further, even for cases where some history of drug use could be discerned using the variable of "age of illicit drug use initiation", the inclusion of such cases into the sample was still untenable due to the numerous drug-related variables that were missing for the time periods of interest. 


\section{Final Sample and Missing Data}

The sample is comprised of men and women age $>18$ with a history of drug use who participated in KY DOC SAP between 2012-2017. As displayed in Figure 2, a total of 1,740 CJKTOS participants completed follow-up interviews for FYs 2013-2017. It was determined that the first three study inclusion criteria (i.e., SAP participation, completion of both baseline and follow-up surveys) were met for all cases. Using zip code or county residence self-reported by participants, a total of 102 cases were identified as residing in a county outside of Kentucky for the majority of months $(>6)$ of the one-year period prior and subsequent to incarceration. Removing these cases resulted in a sample size of $\mathrm{N}=1,638$. Cases were then examined to see if the last inclusion criterion of past-year alcohol and/or illicit drug use was met. This was accomplished by examining responses for the baseline survey item "I did not use any illegal drugs in the 12 months prior to incarceration" ( $0=$ "no" vs. $1=$ "yes"), in conjunction with the dichotomized responses for the baseline survey item "During the 12 months before you were incarcerated, how many months did you use any alcohol?" ( $0=$ "no alcohol use" vs. 1-12= "alcohol use"). A total of 148 participants reported no past-year illicit drug use. Of these, 63 also reported no past-year alcohol use. These 63 cases with no pastyear illicit drug use and no past-year alcohol use were removed, resulting in a sample size of $\mathrm{N}=1,575$.

Two variables were identified as having missing data for unexplained reasons, as these variables appeared in all FY 2013-17 instruments. These included "age of illicit drug use" and "preferred drug of choice" for the past-year period prior to incarceration. Ninety-eight cases were missing values for "age of illicit drug use initiation". This variable was analyzed to rule out the possibility of data missing completely at random (MCAR; Little, 1988). Tests indicated that data were not MCAR ( $\mathrm{p}<.001)$. Upon further inspection, it was determined that missing values may have been due to the fact that these participants only had a history of alcohol use, not alcohol and/or illicit drug use. However, examining past-year and past 30day use rates indicated that some, but not all, of these participants had used illicit drugs. In order to partially address this issue, the variable, "age of drug use initiation" was created to reflect the estimated age at which participants began using any psychoactive drug (see "Independent Variables" subsection, pg. 149). 
Figure 2. CJKTOS sample size for FYs 2013-2017, removal of cases, and final sample size.

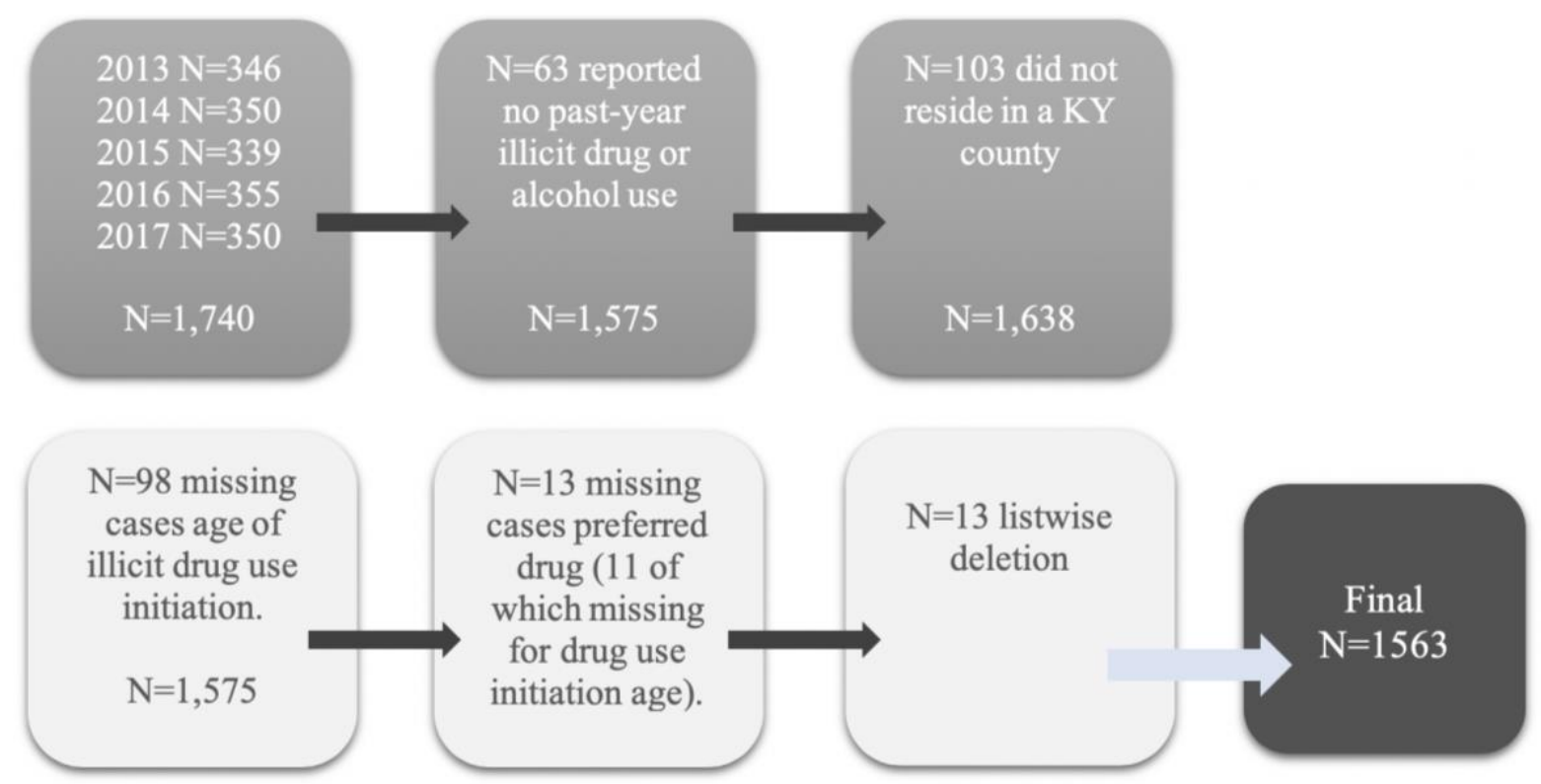

For this variable, missing values for age of illicit drug use initiation were imputed with age of alcohol use initiation. Then, the lowest age for either alcohol or drug use initiation was used in creating the final variable. This combined variable allows for approximation of the age that participants first used a psychoactive drug. One limitation of the method is that the missing value for age of illicit drug use initiation could be significantly lower than age of alcohol use initiation. However, evidence suggests that alcohol use often predates use of illicit drugs (Beman, 1995; McGue, Iacono, Legrand, Malone, \& Elkins, 2001). Still, there are exceptions (Mackesy-Amiti, Fendrich, \& Goldstein, 1997). Therefore, this variable has some limitations, but still adequately represents the underlying phenomenon of interest (e.g., approximate age of psychoactive drug use initiation).

Even after combining alcohol and illicit drug use into a single variable, 11 cases remained that had missing values for "preferred drug of choice", which was missing for 13 total cases. When analyzed, "preferred drug of choice" MCAR could not be ruled out $(\mathrm{p}<.001)$. Examining the merged data set, and copies of original baseline datasets, the reason for missingness could not be determined. All cases for which this variable were missing had affirmative responses for some past-year drug use, meaning that this question should have been applicable to them. As there is a meaningful difference between "preferred drug 
of choice" and past-year use of a drug (even if it is frequent use), missing values for this variable were not imputed with the drug most frequently used during this time period, even though this single imputation method was considered.

Several methods for handling missing data, (e.g., single imputation, multiple imputation, or pairwise deletion) were considered; however, it was determined that listwise deletion would be the most reasonable method, because missingness accounted for less than $1.0 \%$ of cases and because deleting these cases would result in the least disturbance to the data integrity, by not manipulating the raw data (Kang, Rowe, Barreira, Robinson, \& Mahar, 2009; McKnight \& McNight, 2011; McKnight, McKnight, Sidani, \& Figueredo, 2007; Rubin, 2004; Schafer, 1999; Schafer \& Graham, 2002). Mean substitution was considered for age of illicit drug use, but for the same reasons, was not performed. Removing the 13 cases that were missing data for past-year drug preference resulted in a complete dataset with 1,563 intact cases (shown in Figure 2).

This exploratory study was approved by the University of Louisville Institutional Review Board (IRB) and the University of Kentucky IRB via an Institutional Authorization Agreement between the two university IRBs under the study name "Criminal Justice Treatment Outcome Study" (see Appendix A). The University of Kentucky served as the IRB of record. Permission to analyze CJKTOS data and disseminate findings was granted by the KY DOC.

\section{Measures}

Many CJKTOS survey questions, including those used as part of dependent variable measurement described below, were adapted for use by CDAR from the Addiction Severity Index (ASI) (5 $5^{\text {th }}$ edition) "Alcohol/Drugs" (McLellan et al., 1992). The ASI is a public domain clinical and research instrument that assesses drug use, drug treatment history, and drug-related problems across life domains (e.g., employment, criminal justice involvement) (McLellan et al., 1985). The ASI has been used as a data collection tool for a variety of drug-using populations, including corrections-involved drug users (Haas \& Peters, 2000; Jaffe, Du, Huang, \& Hser, 2012; Wahler, 2015; Zanis, McLellan, \& Corse, 1997). The “Alcohol/Drugs" section of the ASI was adapted for use in CJKTOS instruments due to the fact that this section has demonstrated adequate criterion validity, convergent validity, and discriminant validity, as well as adequate internal consistency and test-retest reliability (Calsyn et al., 2004; Chermack et al., 2000, Mäkelä, 2004; McLellan 
et al., 1985; Wertz et al., 1995; Zanis, McLellan, \& Randall, 1994). ASI questions were adapted by CDAR for use as part of the CJKTOS surveys to serve as a standardized question format. Due to slight modifications, they are not utilized by CDAR, or in this study, as a validated measure, or as a measure based on calculating ASI substance use scores or perceived severity ratings; the latter of which is subjective and intended for clinical use. Use of the ASI as part of the CJKTOS surveys is noted so as to make clear the standardized formatting of drug-related questions across baseline and follow-up surveys and across FYs. Other adaptations of standardized instruments for inclusion into the CJKTOS surveys will be subsequently discussed.

\section{Dependent Variables}

Three dependent variables were examined in this study: Drug use outcomes that occurred during the 30-day period prior to incarceration, drug use outcomes that occurred during the one-year period subsequent to incarceration, and heroin use that occurred during the one-year period subsequent to incarceration.

Detailed measures of these dependent variables are described in the following subsections. First, it should be noted that dependent variables were created using ASI "Alcohol/Drugs" section items that comprise part of the larger CJKTOS surveys. These include responses provided for past-year and past 30-

day drug use. Past-year drug use and past 30-day drug use was self-reported by participants at baseline and follow-up. At baseline, participants were asked to list the total number of months out of the past $12(0-12)$, and number of days out of the past 30 (0-30), that they used alcohol or an illicit drug (e.g., "During the past 12 months before you were incarcerated, how many months did you use marijuana/hashish, pot?"; "During the past 30 days before you were incarcerated, how many days did you use marijuana/hashish, pot?’). Including alcohol, participants reported use for a total of 13 drugs/drug classes. At follow-up, participants were asked to self-report drug use for these same drugs, with use pertaining to the one-year post-release period (e.g., "During the past 12 months, how many months did you use cocaine/crack?") in an identical manner.

\section{Drug Use Outcomes Prior to Incarceration}

For the 30-day period prior to incarceration, drug use outcomes were measured using of a categorical variable comprised of four mutually exclusive categories: past 30-day: 1) concomitant use of 
both opioids and stimulants, 2) opioid, but not stimulant use, 3), stimulant, but not opioid, use, and 4) other drug use, that excludes opioid and stimulant use. As discussed earlier, opioids and stimulants can be considered as high-risk drugs. Concomitant use of these drugs may increase risk further. Use of opioids, stimulants, and concomitant use of both during the 30 days prior to incarceration represent decisional outcomes involving high-risk drugs for this period. Use of other drugs, while still entailing risk, can be considered to have comparatively less risk in many, but certainly not all, circumstances. Therefore, the drug outcome of "other drug use", that excludes opioid and stimulant use, can be conceptualized as less risky relative to the other drug use outcomes examined. Because uncertainty is greatest, and the probability of adverse outcomes higher with opioid use, any outcome involving opioids can be considered as entailing greater risk than outcomes not involving opioids. Additional considerations in conceptualizing risk will be discussed in Chapter 5.

The past 30-day period prior to incarceration was chosen for examination (versus past-year), because it provided a narrower time frame and because it could facilitate examination of outcomes in relation to other measures reflecting time periods prior to or contemporaneous with the 30-day period (e.g., past-year, past 30-day, and 7-days). For instance, it is logical to suggest that endogenous and exogenous factors of a person's life in the past-year had the potential to influence past 30-day outcomes, but not the reverse.

Past 30-day opioid use prior to incarceration was measured using data collected at baseline and was defined as use of any opioid agonist drug. These include heroin, prescription opioids (e.g., Oxycontin, Lortab, Demerol), methadone, and buprenorphine. For opioid drugs that might have been used licitly by prescription, participants were asked to affirm only illicit use (e.g., "In the 30 days prior to this incarceration, have you used methadone not prescribed for you?"). Use of any opioid drug for the this time period was summed in order to create a dichotomized variable (e.g., $0=$ "no use" vs. 1="use").

Past 30-day stimulant use prior to incarceration was measured using data collected at baseline and was defined as use of any psychostimulant drug, which included powder cocaine, crack cocaine, amphetamines (e.g., Adderall, Dexedrine), MDMA, and methamphetamines. For stimulant drugs that might have been used licitly by prescription, participants were asked to affirm only illicit use (e.g., "In the 30 days prior to this incarceration, have you used stimulants (e.g., Adderall, Dexedrine, Ritalin, etc.) not 
prescribed for you?"). Use of any stimulant drug for the this time period was summed in order to create a dichotomized variable (e.g., $0=$ "no use" vs. $2=$ "use").

Past 30-day concomitant use of opioids and stimulants prior to incarceration was measured by summing the two dichotomized variables for past 30-day opioid and stimulant use in order to create a categorical variable that consisted of four categories (e.g., $0=$ "no opioid/stimulant use", $1=$ "opioid but not stimulant use", $2=$ "stimulant, but not opioid use", and 3="concomitant opioid and stimulant use"). Concomitant use of both opioids and stimulants was coded so as to include only cases for which both opioid and stimulant drugs were used within the same 30-day period prior to incarceration.

Past 30-day other drug use is defined as use of any non-opioid/non-stimulant drugs (e.g., alcohol, cannabis, hallucinogens, sedatives, etc.) during this time period. For drugs that might have been used licitly by prescription, participants were asked during the baseline interview to affirm only illicit use (e.g., "In the 30 days prior to this incarceration, have you used benzodiazepines (e.g., Xanax, Valum, etc.) not prescribed for you?"). Drugs excluding opioids and stimulants were summed and then a dichotomous variable was created by recoding all non-zero values equal to 4 (e.g., $0=$ "no other drug use" vs. 4="other drug use"). This variable was then combined with the prior categorical variable described above. The result was a single polytomous dependent variable, reflecting the four decisional outcomes of interest for the 30-day period prior to incarceration (e.g., 1=“opioid, but not stimulant use", 2="stimulant, but not opioid use", $3=$ "concomitant opioid and stimulant use", 4="other drug use").

\section{Drug Use Outcomes Subsequent to Incarceration}

Subsequent to incarceration, drug use outcomes were examined for the one-year post-release period. This was done for four reasons. First, rates of drug use are significantly lower for the one-year and past 30-day periods subsequent to incarceration, compared to these respective periods prior to incarceration, and past-year use subsequent to incarceration is typically higher than past 30-day use subsequent to incarceration. Second, examining outcomes for the one-year post-release period broadens the timeframe during which a drug use occurred, permitting examination of drug use contemporaneous with other reported states (e.g., depression, cognitive difficulties) that were measured for the entire year. Many of the measures used in the CJKTOS follow-up interview pertain to the past year, rather than the past 30days or past 7-days. Third, looking at this longer period permits some discussion about the possibility that 
reentry, as a process, influenced participants' drug-related decision-making. Finally, examining this longer time period allowed the community supervision status (e.g., parole) of participants to be examined as a potential influence on decision-making (e.g., behavioral reinforcer). Some participants who reentered the community on probation/parole, and who may have been under supervision for the majority of the year, may not have still be on parole in the 30-day period prior to the follow-up interview. Therefore, for the purposes of this study, it was more important to examine the past-year period subsequent to incarceration rather than the 30-day period prior to follow-up interview completion.

For this one-year post-release period, drug use outcomes were measured using of a categorical variable comprised of four mutually exclusive categories: past year: 1) concomitant use of both opioids and stimulants, 2) opioid, but not stimulant use, 3), stimulant, but not opioid, use, 4) other drug or/no drug use. Questions pertaining to past-year drug use were asked at follow-up in an identical manner to how questions pertaining to past 30-day drug use were asked at baseline. The final outcome variable was summed using the same process described above for the outcome variable of "past 30-day drug use outcomes" (see above subsection "Drug Use Outcomes Prior to Incarceration").

\section{Past-year Heroin Use Subsequent to Incarceration}

Past-year heroin use was measured at follow-up, by having participants respond to the question, "During the past 12 months, how many months did you use did you use heroin?". Responses were then dichotomized ( $0=$ no use vs. $1=$ use).

\section{A Note on Fentanyl}

It is important to note that, at baseline, participants reported drug use for the one-year and 30-day periods prior to incarceration, not simply prior to $S A P$ entry. This means that there is some variance among the time periods of use reported by some, but not most, participants. For instance, a minority of participants may have been describing drug use that occurred in the late 1990s or early 2000s, while others may have described drug use that occurred in 2014 or 2016 . This is due to the fact that some participants may have been incarcerated for several years prior to entering SAP, due to having longer sentences, whereas other participants, with shorter sentences, may have entered into SAP within a matter of months following their conviction. At follow-up, however, differences in the time period reported for is no longer a concern, making it possible to examine drug use outcomes for the same time period (2012-2017). This is noteworthy 
insofar as fentanyl was introduced into the US drug supply during this time period (Daniulaityte et al., 2019; O’Donnell, Halpin, Mattson, Goldberger, \& Gladden, 2017; Peterson, 2016; Seth, Scholl, Rudd, \& Bacon, 2018). In addition to fentanyl-adulterated heroin, stimulant products and counterfeit prescription drugs are increasingly identified as containing fentanyl analogues (Dai et al., 2019; Gladden, 2016; Green \& Gilbert, 2016; Nolan et al., 2019; O’Donnell et al., 2017). It is likely that many people in this sample would not have known definitively that they were using fentanyl and that, prior to 2014, people would have been far less likely to encounter fentanyl-adulterated products. One limitation is that participants were not asked to report on use of fentanyl specifically. It may be that some participants intended to use fentanyl or were aware that they may be using fentanyl (Kenney, Anderson, Conti, Bailey, \& Stein, 2018). This confounds an already challenging process of conjecture about the risks potentially known by participants and of assessing objective risk independent of user knowledge. The drugs that will be examined, (e.g., heroin, prescription opioids, buprenorphine, cocaine/crack cocaine, amphetamines) still reflect what is of interest: decisional outcomes involving high-risk drugs. Fentanyl, in some ways, may in future years become an equalizer, in the sense that greater uncertainty surrounding both opioid and stimulant use will likely only grow. The significance of fentanyl's introduction into the drug supply during the time period will be considered further in Chapter 5.

\section{Independent Variables}

\section{Drug-related Influences}

Age of drug use initiation was measured at baseline by having participants report the age at which they first used an illicit drug (e.g., "How old were you when you first began to use illicit drugs like marijuana, cocaine, heroin, tranquilizers, stimulants, sedatives, barbiturates, inhalants or prescription painkillers not prescribed for you?"). For participants who did not report any illicit drug use, or for which age of first illicit drug use was missing $(\mathrm{N}=54)$, the age of first alcohol intoxication was used (e.g., "How old were you when you had your first alcoholic drink, other than a few sips?"). In cases where there were responses for both alcohol and illicit drugs, the younger age was retained (see also, "Final Sample and Missing Data” subsection, pg. 138). Combining responses for age of first alcohol use or age of first illicit drug use and using the younger age reported, while not precise, does provide an estimate of the age that participants first used a psychoactive drug. As discussed earlier, age of drug use initiation indicates several 
possibilities, any one of which, or any combination of which, has the potential to influence drug-related decision-making so as to bias a person toward further drug use. Response options were unlimited.

Past 30-day drug use prior to incarceration was measured at baseline by summing the number of days that ten specific drugs (alcohol, cannabis, prescription sedatives, hallucinogens, synthetic drugs, cocaine/crack cocaine, amphetamines, heroin, prescription opioids, and buprenorphine) were used in the 30-day period prior to incarceration (0-30). Ten dichotomous variables were then generated from these to reflect "use" or "no use" of a given drug for the 30-day period prior to incarceration. These items were examined as a possible influence on drug outcomes for the one-year period subsequent to incarceration.

Drug use severity was measured using data collected at baseline by creating a composite variable. This variable consists of three questions pertaining to past 30-day drug problems contained in the "Alcohol/Drugs" subsection of the ASI-5 (range 1-40). At baseline, participants were first asked to report the total number of days out of the past 30 prior to incarceration that they experienced drug problems, "such as craving, withdrawal, wanting to quit but being unable to". Participants then ranked on a 5-point Likert scale how troubled or bothered they were by these drug problems and how important receiving treatment for these drug problems was to them ( $1=$ Not at all $-5=$ Extremely). Internal consistency of this composite variable was sufficient $(\alpha=0.76)$. Drug craving, withdrawal, and persistent problematic use despite a desire to moderate or abstain comprise some of the DSM-5 diagnostic criteria for SUD, meaning that this composite variable may be considered as an estimated, but inexact, indicator of drug use severity (APA, 2015). Including participants' self-rated importance of receiving treatment for their problematic use also provides indication that the problems associated with use were significant enough so as to make them consciously registered and contemplated, rather than wholly unrecognized or dismissed (DiCemente, 2018).

Past-year drug use versatility prior to incarceration was measured by summing the number of different drugs that a participant reported using during the one-year period prior to incarceration (1-13). This was explored due to the fact that polydrug use is a high-risk form of drug use. Versatile drug use, wherein a greater number of different drugs are used during a given time period, versus fewer, may influence the likelihood that drugs such as opioids and stimulants are used concomitantly. It may also indicate the possibility the co-use of opioids and/or stimulants with other drugs (e.g., alcohol, synthetic 
drugs, prescription sedatives) is occurring, which can be conceived as a higher-risk form of use (Smith \& Staton, 2018). Using a greater number of drugs during a given time period may also indicate noveltyseeking and higher tolerance for risk, uncertainty, and adverse consequences.

Abstinence self-efficacy prior to treatment was measured at baseline by asking participants to respond on a 5 -point Likert scale ( $0=$ very poor, $2=$ uncertain, $5=$ very good) the degree to which they believed they could refrain from alcohol and illicit drug use (e.g., "Based upon what you know about yourself and your situation, how good are the chances that you can get off and stay off of drugs/alcohol?"). Responses were recoded to reflect the presence or absence/ambivalence of abstinence self-efficacy (e.g., “moderately poor, very poor, uncertain"=0 vs. "moderately good, very good"=1).

Past-year Drug Use Subsequent to Incarceration was measured using data collected at followup pertaining to past-year drug use for the following drugs: Alcohol, cannabis, prescription sedatives, hallucinogens, synthetic drugs, cocaine/crack cocaine, amphetamines, heroin, prescription opioids, and buprenorphine. For each of the 10 drugs, the number of months out of the 12 months subsequent to incarceration was summed and then dichotomized to reflect past-year use ( $0=$ "no use" vs. $1=$ "past-year use"), resulting in ten separate drug use variables that were then dichotomized. These were used as independent variables for the outcome of past-year heroin use subsequent to incarceration.

\section{Demographic Characteristics}

Age was measured at baseline in years using participants' dates of birth. Birthdates were confirmed for all participants at follow-up. Sex was measured at baseline by asking participants to select which gender they identified with (e.g., male, female, transgender). For baseline surveys prior to 2016, the "transgender" option did not include a specifier (e.g., "male to female" vs. "female to male"). However, no participants selected "transgender" for these years. In 2016, a specifier was included with the transgender option. However, because sex, rather than gender, is being considered as a potential influence on decisionmaking, cases for which "transgender" was selected $(\mathrm{N}=3)$ were recoded to reflect biological sex which was cross-referenced by examining the correctional institution in which they were housed (i.e., all-male or all-female prison). More information would have been needed in order to determine if trans male or trans female cases were appropriate for this study's conceptualization of sex. For instance, no information was available to clarify if these participants had undergone hormone replacement therapies or other elective 
procedures. As the majority of the sample was male, a dummy variable was created ("not male" $=0$ vs.

"male"=1).

\section{Indirect Indicators of Cognitive Functioning}

To measure the presence of some potential indirect indicators of cognitive functioning, three variables were examined, these included one composite variable denoting learning disorder diagnosis and/or prior special education involvement, as well as two dichotomous variables, past-year cognitive difficulties, and lifetime history of head trauma.

The first variable, learning disorder (LD) diagnosis and/or special education (SE) involvement included summing responses for two questions asked at baseline pertaining to LD and SE enrollment. LD was measured by asking participants, "Have you ever been told that you have learning disability?". Participants could respond "no" or "yes" for each item. SE enrollment was measured at baseline by asking participants, "Have you ever been in special education?". Participants could respond "no" or "yes" for each item. Responses were dichotomized to reflect the presence or absence of these conditions $(0=$ no LD diagnosis/no SE enrollment vs. 1=LD diagnosis and/or SE enrollment).

The second indicator of cognitive functioning, past-year cognitive difficulties was measured by identifying the presence of cognitive deficits during the one-year post-release period with the potential to influence decision-making. For this item, participants were asked to report the number of months out of the past 12 that they experienced "trouble understanding, concentrating, or remembering, not as a direct result of alcohol and drug intoxication" (0-12). Responses were summed and recoded to reflect the presence or absence of past-year cognitive difficulties ( $0=$ "none" vs. 1="some past-year cognitive difficulties").

The third indicator of cognitive functioning, lifetime history of head trauma, was measured at baseline by asking participants to self-report the number of times in their life that they experienced head trauma (e.g., "How many times have you ever had a head injury from being hit, having an auto accident, or another incident that resulted in being knocked out/unconscious?"). Response options were unlimited. History of head trauma was measured again at follow-up by asking participants to report any head trauma that occurred during the one-year post-release period. This response was summed with the number of head injuries reported at baseline in order to create a new head trauma variable with an updated lifetime head trauma incident total. However, this variable does not reflect head injuries sustained between a participant's 
completion of the baseline survey and their release date. For instance, head trauma could have occurred while incarcerated, meaning that the actual number of times that head trauma was sustained could be underestimated. Additionally, participants may have sustained head trauma as young children, but may be unaware of this history. Accordingly, a dichotomous variable was created for both time periods $(0=$ "no reported history of head trauma" vs. 1="reported of head trauma").

\section{Scarcity and Social Influences}

As discussed in Chapter 2, scarcity, economic hardship, and lower social status can influence cognition and decision-making. Additionally, income can influence drug purchasing behaviors. These potential influences were explored using economic hardship, past-month income, past-year homelessness, and subjective socioeconomic status.

Past-year economic hardship, defined as difficulty in meeting basic living (e.g., food, shelter) and healthcare needs (Beverly, 1999) was measured at baseline by combining two subscales in the CJKTOS survey. These subscales were adapted from the Survey of Income and Program Participation (SIPP), a longitudinal nationally representative survey of non-institutionalized adults conducted by the U.S. Census Bureau (Beverly, 2001; Iceland \& Bauman, 2004; She \& Livermore, 2007). The first subscale consists of five dichotomous questions ("no" vs. "yes") and measures difficulty meeting basic living needs during the one-year period prior to incarceration (e.g., "In the 12 months prior to this incarceration, did you and/or your family have difficulty paying the full amount of rent or mortgage?"). Due to the increased use and cost of cellular telephones, one SIPP question pertaining to telephone disconnection was modified by CDAR to also reflect cell phones in addition to landlines. The second subscale consists of three dichotomous questions and measures difficulty meeting basic healthcare needs during the one-year period prior to incarceration (e.g., "In the twelve months prior to this incarceration was there a time when you or someone in your household needed to see a doctor or go to the hospital but wasn't able to because of financial reasons?"). Because of the rising prescription drug costs in the U.S., a question about accessing prescription medication was included by CDAR as one of the three subscale items measuring difficulties in meeting basic healthcare needs (e.g., "In the twelve months prior to this incarceration was there a time when you or someone in your household needed to fill a prescription for medication but was unable to because of cost?'), though this item does not appear in SIPP. Among a clinical sample of drug users in 
Kentucky $(\mathrm{N}=5,273)$ this revised economic hardship scale (range 0-8) demonstrated good internal consistency reliability ( $\alpha=0.83$ ), as did the subscales measuring difficulties in meeting basic living needs $(\alpha=0.76)$ and health care needs $(\alpha=0.83$; Cole, Logan, Miller, $\&$ Scrivner, 2016). The combined scale among the current study sample demonstrated adequate internal consistency $(\alpha=0.85)$.

Past-month income was measured at follow-up using self-reported income in US dollars from all past 30-day income sources. As discussed in the previous chapter, lower-income is believed to reflect a type of scarcity and uncertainty, whereas higher income is believed to reflect a state of that enables (comparatively) enhanced cognitive functioning, greater, choice, and potentially more purchasing power for drugs (or non-drug commodities). Questions pertaining to employment and income used as part of CJKTOS surveys were adapted from the ASI-5 "Employment/Support Status" subsection. Participants were asked at follow-up to report the income received from licit work (e.g., payroll, odd jobs), illicit work (e.g., drug dealing), alternative sources (e.g., gambling winnings), public assistance and benefits (e.g., Supplemental Nutrition Assistance Program, Social Security Disability Insurance), and money received from friends and family for the past 30 days. Amounts from all sources were summed for a total past 30day income amount.

Past-year homelessness was measured at baseline by asking participants to respond ("yes" vs. "no") to the question, "In the 12 months prior to this incarceration, did you consider yourself to be homeless?".

Subjective socioeconomic status was measured at baseline using an adapted version of the Subjective Socioeconomic Scale (Adler et al., 2000). This one-item measure is comprised of a 10-rung ladder, displayed vertically in the form of a drawing, that participants used to help respond to the accompanying question: "Overall, on a scale of 1 to 10 , with 1 representing the people who are worst off, those who have the least money, least education, and worst jobs or no job, and 10 representing the people who are best off, have the most money, most education and best jobs, how would you rate yourself on that scale?". Here, the ladder was used to help visualize the lowest status (e.g., 1) versus highest status (e.g., 10). Due to instrument changes, the ladder only accompanied the question for 3 out of the 5 baseline years examined as part of this study. However, the wording of the question remained consistent across years. Lower scores for this item (range, 1-10) indicate lower perceived social and economic status. 


\section{Community-wide Scarcity and Inequality}

As discussed earlier, regional and household poverty, scarcity, and inequality can influence decision making and high-risk drug use in several ways. To examine this potential influence, county-level poverty and county-level wealth inequality were considered for examination.

County-level poverty was measured at the county-level using Small Area Income and Poverty Estimates (SAIPE) for years 2013-2017 by using the median value for the proportion of households per county considered by the U.S. Department of Health and Human (HHS) to be living at or below the poverty threshold, which is calculated using household size and income (U.S. Census Bureau, 2018). For example, in 2019, a household size of one is considered to be living in poverty if annual income does not exceed $\$ 12,490$, whereas a household size of five is considered to be living in poverty if annual income does not exceed $\$ 30,170$ (HHS, 2019). Although median household income for counties provides an indicator of typical income, examining the proportion of households per county living in poverty provides an indication of how thinly spread annual income may be per household.

County-level wealth inequality was operationalized using the GINI coefficient, a measure of income and wealth distribution that indicates financial resource inequality, whereby a value of 0 reflects perfect equality and a value of 1 reflects the maximum value of inequality (Atkinson, 1970; U.S. Census Bureau, 2019a). Using ACS data for years 2013-2017, GINI coefficients for each Kentucky county were considered for examination. As there are not significant fluctuations between years, the median coefficient value for the five-year period was selected for possible use.

\section{Psychiatric Symptoms, Stress, Pain and Misregulation}

As noted earlier, mood, affect, and symptoms related to psychiatric conditions, such as depression or anxiety, can influence drug-related decision-making and behavior. The potential influence of psychiatric symptoms was considered by examining past-year depressive symptoms, past-year suicidal ideation, and past-year anxiety symptoms.

Past-year depressive symptoms was measured at baseline using items adapted from the 9-item Patient Health Questionnaire-9 (PHQ-9), an instrument that measures depression symptom severity using DSM-IV major depressive disorder (MDD) diagnostic criteria (Kroenke, Spitzer, Williams, 2001). Participants responded ("no/absent" versus "yes/present") to questions pertaining to any two-week period 
in the past-year prior to incarceration (e.g., "In the 12 months prior to incarceration, did you have two weeks in a row when you were consistently down or depressed most of the day, nearly every day?"). Overall, the PHQ-9 has demonstrated good criterion and convergent validity, with strong positive correlations observed between higher PHQ-9 scores and lower scores on measures of mental health, social functioning, overall functioning, and role functioning (Kroenke et al., 2001; Kroenke, Spitzer, Williams, \& Löwe, 2010). Here, PHQ-9 items were adapted for use on the CJKTOS baseline survey by CDAR by dichotomizing response options, instead of using Likert scales (0-3), thus resulting in a range of 0-9 rather than the 0-27 PHQ-9 range. Good internal consistency reliability for this adapted PHQ-9 was found in a clinical sample of drug users in Kentucky ( $\mathrm{N}=5,273 ; \alpha=0.95$; Cole et al., 2016), as well as in the current sample $(\alpha=0.94)$. A dichotomous variable was created using a cutoff point between those evidencing symptoms of MDD and those not, whereby participants were classified as having MDD symptoms if they responded "yes" to 5 out of 9 questions. At follow-up, the presence of past-year depressive symptoms was measured by asking participants to report the number of months out of the past 12 that any depressive symptoms were experienced (e.g., "In the past 12 months how many months have you experienced serious depression, that was not a direct result of drug/alcohol use?"). Responses can range from 0-12. Responses were dichotomized (e.g., zero months=0 vs. $>1$ month=1) so as to reflect the presence or absence of depressive symptoms for this period.

Past-year suicidal ideation was measured at baseline by asking participants to respond ("yes" vs. "no") to the question, "During the 12 months prior to this incarceration, did you have thoughts about ending your life or committing suicide?". At follow-up, suicidal ideation was measured by asking participants to respond the question, "In the past 12 months how many months have you experienced serious thoughts of suicide that was not a direct result of drug/alcohol use?" for which they could report 012 range. Responses were recoded to reflect the presence or absence of past-year suicidal ideation ("no suicidal ideation"=0 vs. "suicidal ideation" $=1$ ).

Past-year anxiety symptoms was measured at baseline using an adapted version of the Generalized Anxiety Disorder scale (GAD-7), a 7-item measure designed to identify and assess symptoms of GAD using DSM-IV diagnostic criteria (Spitzer, Kroenke, Williams, \& Löwe, 2006). The GAD-7 has demonstrated adequate convergent and criterion validity as well as good internal consistency (Delgadillo et 
al., 2012; Löwe et al., 2008; Spitzer et al., 2006). Items were adapted by CDAR researchers by dichotomizing response options ("no/absent" versus "yes/present"), instead of using 4-point Likert scales to measure frequency of symptoms, resulting in a 0-7 range. Among a clinical sample of drug users in Kentucky, good internal consistency was found ( $\mathrm{N}=5273 ; \alpha=0.98$; Cole et al., 2016). In this sample, good internal consistency was also observed using this modified version of the GAD-7 ( $\alpha=0.92)$. A cutoff point between those evidencing symptoms of GAD and those not was created whereby participants were classified as having GAD symptoms if they responded "yes" to 4 out of 7 questions, including the screener question: "In the 12 months before you were incarcerated, did you have a time period lasting 6 months or longer where you worried excessively or were anxious about multiple things on more days than not for all 6 months?". At follow-up, the presence of past-year anxiety symptoms were measured by asking participants to report the number of months out of the past 12 that any symptoms associated with anxiety were experienced (e.g., "In the past 12 months how many months have you experienced serious anxiety or tension that was not a direct result of drug/alcohol use?"). Responses can range from 0-12. Responses were dichotomized (e.g., zero months $=0$ vs. $>1$ month=1) so as to reflect the presence or absence of anxiety symptoms for this period.

As noted earlier, increased stress response can impair decision-making in a variety of ways. Accordingly, past-week stress-related health effects was measured with data collected at baseline using 11 items contained on the 15-item Stress-related Health Consequences Scale (HCS), developed to measure past-week stress indicators and stress-related health consequences (Logan \& Walker, 2010). Participants were asked to respond using a 4-point Likert scale (1=None of the time $-4=$ All of the time) the degree to which they experienced indicators of stress in the seven days prior to incarceration (e.g., "In 7 days prior to incarceration, have you experienced anxiety and/or panic attacks?"; "In 7 days prior to incarceration, have you felt stressed out?"). For a total scale score, all 15 scale items are be summed, with higher scores indicating a greater degree of physical and psychological stress (15-60). Among a clinical sample of drug users $(\mathrm{N}=5,273)$, the scale demonstrated good internal consistency reliability ( $\alpha=0.90$; Cole et al., 2016). The initial consistency for all 15 items was acceptable in this sample $(\alpha=0.88)$.

However, because the content of the HCS is a multidimensional construct, seeking to measure states and experiences that are somatic or physiological (e.g., "experienced unexplained aches and pains", 
“increased heart rate"), psychological (e.g., "experienced stress, anxiety, worry, or fear"), and behavioral (e.g., "used illicit drugs to reduce stress, anxiety, worry, or fear") principal component analysis (PCA) was used to examine scale items in order to further scrutinize item correlations and to determine if subscales could be formed. Three components were extracted $\left(\mathrm{KMO}=0.93 ; \mathrm{X}^{2}=9410.79, \mathrm{df}=105, \mathrm{p}<.001\right)$. Component one, comprised of seven items pertaining to affect, sleep, fatigue, and changes in eating patterns, accounted for $40.2 \%$ of the total variance; component two, comprised of five items pertaining to somatic disruptions and the re-experiencing of trauma, accounted for $8.8 \%$ of the variance; component three, comprised of the three items pertaining to drug-related stress coping behaviors, explained $7.7 \%$ of the variance.

Upon closer examination, one of the items comprising component two was identified as potentially invalid in this sample due to self-report limitations, as it asked participants to rate the degree to which they experienced high blood pressure, a physiological state that is not always subjectively felt or consciously recognized as high blood pressure (Greenstadt, Shapiro, \& Whitehead,1986; Kollenbaum, Dahme, \& Kirchner, 1996 Koroboki, 2010). For younger participants, high blood pressure, both conceptually and phenomenologically, may be less understood, even as interoceptive capacity may be greater in younger individuals (Khalsa, Rudrauf, \& Tranel, 2009). Removing this item did not reduce the internal consistency $(\alpha=0.88)$.

The three items pertaining to drug-related stress coping behaviors, that comprised component three were removed and retained for use as a composite variable indicating misregulation. Removing these component three items, along with the HBP item, resulted in a final 11-item measure (range, 11-44) of recent stress-related health effects with good internal consistency $(\alpha=0.89)$.

Chronic pain, defined as pain lasting three months or longer (Treede et al., 2015), was measured by asking participants at baseline and follow-up to report ("no" vs. "yes") any chronic pain experienced (e.g., "Do you have any chronic physical pain, that is, pain that has lasted three or more months?") so as to reflect the presence or absence of chronic pain experienced during each period.

Misregulation, defined as exerting control over one's actions in a manner that achieves a shortterm end (e.g., reducing stress) at the expense of a long-term end via instrumental coping (Baumeister, Heatherton, \& Tice, 1994; Tice, Bratslavsky, \& Baumeister, 2018), was measured using the three 
behavioral items contained on the HCS (e.g., "In 7 days prior to incarceration, have you used alcohol to reduce stress, anxiety, worry or fear?"). This question was asked in reference to alcohol, prescription drugs, and illicit drugs. Participants could respond using a 5-point Likert scale $(0=$ None of the time-5=All of the time). Although these items differ in kind (i.e., drinking alcohol to cope with stress versus using illicit drugs) and, when grouped, did not demonstrate robust internal consistency $(\alpha=0.64)$, these behaviors all nevertheless indicate some propensity toward misregulation in that a non-zero response to any of these questions means that the participant has engaged in instrumental coping by using a psychoactive drug as an attempt to reduce stress. Responses were dichotomized where values of $>1$ were coded as $1=$ "misregulation occurred" and values of $0=$ "no misregulation occurred". One limitation in using this variable is that participants who did not report any of the three behaviors indicative of misregulation during this 7-day period, either due to the fact they did not experience "stress, anxiety, worry, or fear" during this time or because they did not use drugs in this time (even as they used drugs within the larger 30-day period), may still have some proclivity toward misregulation, despite the fact that it was not captured for the week in question. As such, this variable should be considered as an approximation of misregulation that occurred contemporaneous to past 30-day drug use, indicating some propensity toward misregulation or "self-medication" during this time period.

\section{Access to Drug Markets}

As discussed earlier, the area that a person lives in and the access they have to drug markets can influence drug-related decision making and high-risk drug use by restricting the range of choices and/or influencing the cost and difficulty with which some drugs may be acquired. To examine potential influences, county Internet connectivity, county disability rates, county opioid and buprenorphine prescribing rates, and county alcohol availability were considered for examination.

Internet connectivity was measured using the percentage the households per Kentucky county with internet access, 25 minimum broadband speed (i.e., the slowest speed option) for 2017 (Federal Communications Commission, 2018). This was examined due to the fact that Kentucky counties vary in their internet access, with some counties far more connected than others. Since the Internet is increasingly used as an avenue for accessing drug markets, the ease or difficulty for accessing these online markets is important to consider. 
County disability rates were measured by creating a five-year average for the proportion of residents per Kentucky county receiving Social Security Disability Insurance benefits for years 2013-17 (Social Security Administration, 2019). As noted earlier, areas with a higher density of people who are ill and/or are receiving prescription medications may influence local drug markets in terms of access to diverted medications.

County opioid prescribing rates were measured using data collected from the Kentucky All Schedule Prescription Electronic Reporting (KASPER) surveillance program, which consists of an online database used to document and monitor the prescription and distribution of all Schedule II-V Controlled Substances in Kentucky (Kentucky Cabinet for Health and Family Services [CHFS], 2019). Prescribing medical professionals and pharmacists are mandated to enter data into KASPER and may utilize the system to generate reports and make clinical decisions. KASPER is also utilized by state government and law enforcement to monitor prescribing trends and to investigate potential prescription drug diversion (CHFS, 2019). County opioid prescribing rates were measured by first summing all individual opioid prescriptions (e.g., Oxycontin, Hydrocodone, Methadone, and Tramadol) written and filled per county, creating five-year averages for years 2013-17, and then by calculating per 1,000 prescribing rates based on the 2013-17 fiveyear county adult population ASC averages (U.S. Census Bureau, 2019b). County buprenorphine prescribing rates were calculated similarly, but instead by summing all Suboxone/Subutex prescriptions written and filled.

\section{Non-drug Alternative Reinforce}

As noted previously, many non-drug alternatives can reinforce behavior. Some of these are important to consider as possible influences on drug-related decision-making prior and subsequent to incarceration. Here, they were measured using close relationships, social interaction, perceived value of social interaction, social worth, leisure time, educational and vocational involvement, and post-release correctional status.

Close relationships were measured using data collected at baseline and follow-up. Participants were asked to indicate from a 5-item list important people, such as family, romantic partners, with whom they had a relationship with (e.g., "Would you say you have had a close, long-lasting relationship with your children?"). Participants then had the opportunity to report ("yes" vs. "no") if they had other people with 
whom they had a close, long-lasting relationship with that were not already listed. Lastly, participants were asked "How many close friends do you have?", for which there was an unlimited response option. The final number of close relationships was calculated by summing the dichotomous close relationship responses (06) and then summing this total with the number of close friends, resulting in a variable with unrestricted range.

Social interaction was measured at baseline and follow-up by asking participants to select one of three response options to the following question "In the past 12 months, with whom did you spend most of your free time?". Participants could select "Friends", "Family", or “Alone.” Responses were recoded to reflect the absence ( $0=$ "alone") or presence ( $1=$ "friends, family") of past-year social interaction.

Perceived value of social interaction was also considered. To assess if social interaction was rewarding, participants were asked to rate their satisfaction with how their free time was spent (e.g., "Were you satisfied spending your time this way?"). Participants could respond "No", "Yes", "Indifferent". Responses were recoded into a dichotomous variable reflecting the presence or absence of reward (0=“no/indifferent" vs. 1="satisfied").

Some of the rewarding aspects of non-drug alternatives that are social in nature, such as close relationships, extend beyond the experiential pleasure that can come from being with others. One aspect is that, when relating to others, a person not only comes to value other people, but comes to be valued and supported by other people. A sense of worth and support can be experientially pleasurable and directly rewarding, which has the potential to reinforce behavior accordingly; but it can also, under the right conditions, buffer stress response, pain, and other negatively valanced states, which also has the potential to reinforce behavior (Cutrona \& Russell, 1987; Hagerty \& Williams, 1999; Heinrichs, Baumgartner, Kirschbaum, \& Ehlert, 2003; Langford, Bowsher, Maloney, \& Lillis, 1997; Yoshida et al., 2009). Further, social worth and support may also be in keeping with the idea of "having a stake in the conventional life", insofar as a person may be more likely to invest in people and activities for which their presence matters, and is encouraged through supportive gestures. In other words, reciprocity of rewarding prosocial patterns might be established (Cropanzano \& Mitchell, 2005). Social worth was measured at follow-up by asking participants to rate on a 5 -point Likert scale ( $1=$ not at all $-5=$ Extremely) the degree to which they felt valued and supported by others (e.g., "How much do you feel cared about or supported by the important 
people in your life?"). Responses were recoded to reflect the presence or absence of feeling valued ( $0=$ "“did not feel cared about/supported" vs. 1="felt cared about and supported").

Leisure time was measured at baseline by asking participants to report the average number of days per week that they could pursue activities of personal interest (e.g., "In the 12 months prior to this incarceration, how many days per week, on average, were you involved in hobbies or activities that you liked to do?"). This item was considered because it indicates the presence of the flexible resource of time which is often needed to pursue potentially rewarding activities of interest.

Past-year educational and vocational involvement was measured using data collected at baseline and follow-up. To first assess vocational involvement, participants choose from a list the employment designation that best reflected their status for the majority of months (>6) out of the 12months prior and subsequent to incarceration (e.g., unemployed, retired, disabled, working part-time, working, full-time, seasonal/irregular work, retired, student, etc.). Responses were dichotomized to reflect the presence or absence of regular vocational involvement (e.g., $0=$ "unemployed, retired, disabled, irregular employment" vs. 1="full- or part-time employment"). For people who reported being unemployed, they could also specify that they were a student, thus indicating educational involvement even in the absence of vocational involvement ( $2=$ student). In a separate question, participants were also asked at baseline and follow-up to report any involvement in educational/vocational training, irrespective of employment $(0=$ no training vs. 1=educational training). These two variables were summed and then recoded into a dichotomized variable to reflect the presence or absence of educational and/or vocational involvement $(0=$ no educational/vocational involvement vs. $1=$ educational/vocational involvement $)$ in the one-year period prior and subsequent to incarceration.

Post-release correctional status was measured at follow-up by asking participants to self-report

if they were on community supervision (i.e., probation, parole) at any point since their release. Post-release correctional status is considered as an additional behavioral reinforcer.

\section{Central Appalachia}

As described earlier, residing in a harsh region can have a profound impact decision-making and behavior. It can accomplish this by influencing cognition and one's beliefs about their future, but also by narrowing choices and opportunities for pursuing rewarding non-drug alternatives. For people in Eastern 
Kentucky, the portion of the state that falls within Central Appalachia, opportunities for accessing rewarding non-drug alternatives are more limited. Historically and presently, Central Appalachia is a region in which there are excess morbidities---including high rates of disability and psychiatric health disorders-premature death, resource scarcity, and economic insecurity. Relative to other areas in the state, Central Appalachia portion of Kentucky may be considered as a harsh environment, even other regions in the state may have smaller, concentrated areas that might also be considered harsh. To explore this possible influence of residing in a "harsh" region, Central Appalachia was measured at baseline and follow-up using the zip code and/or county of residence that participants reported residing in for the majority of months (>6) out of the past 12, prior and subsequent to incarceration. At baseline, U.S. postal zip codes were converted into counties and coded as either "Central Appalachia" or "non-Appalachian" using Appalachian Regional Commission (ARC) designations (ARC, 2019). At follow-up, participants reported the county in which they resided in and similar codes were created. For participants reincarcerated at follow-up, the county of residence, rather than county of incarceration, was used to code responses.

\section{Control Variables}

In addition to the independent variables described above, which are believed to influence decisionmaking in respect to opioids and stimulants, several additional participant characteristics with the potential to influence the outcomes of interest were also measured.

High school diploma (HSD)/General Equiveillance Degree (GED) was measured using data collected at baseline. Participants were asked to select from a list the highest level of education they completed. Categories ranged from " $1=$ never attended" to " $21=$ doctoral degree". Responses were summed and a cut-off point reflecting the attainment of HSD/GED was used to create a dichotomized variable (e.g., $0=$ no high school diploma/GED vs. $1=$ high school diploma/GED or greater).

Race/ethnicity was measured using data collected at baseline. Participants were asked to select from a list the race/ethnicity with which they identified themselves as (e.g., African American, Alaskan, Hispanic-Mexican, Hispanic-Cuban, Native American, White, etc.) or to write-in “other" for categories not already provided. Responses were dummy coded into a dichotomized variable (e.g., "non-white" $=0$ vs. "white"=1). This was done due to the small group sizes for all race/ethnic groups other than African American and White. State-wide, both Kentucky's non-institutionalized population and KY DOC 
population are majority White, making this category as a dummy variable an appropriate choice.

Limitations for dummy coding race/ethnicity will be discussed in Chapter 6.

Lifetime drug treatment episodes were measured using data collected at baseline by summing the number of times that participants reported having ever received any of the following substance use services: Medical detox, outpatient treatment, inpatient/residential treatment, for which the response range was unlimited.

Ever IDU was measured at baseline by asking participants to respond to the question "Before this incarceration, did you ever inject any drugs?" for which they could respond "no" or "yes".

Nights spent in a controlled environment was measured at baseline for the 30-day period prior to incarceration (0-30). At follow-up, the nights spent in a controlled environment was measured for the entire one-year post-release period (i.e., the time period beginning the date of participants' release and concluding the date of follow-up interview completion) (0-365).

Mandated to SAP was measured using KOMS data (which comprises the broader CJKTOS datasets). Cases were coded $0=$ "voluntary participation" vs. 1=“mandated participation".

SAP completion was also measured using KOMS data, which provides a documented date of SAP graduation or termination. Participants who were terminated from SAP were coded as 0="terminated" and participants who completed SAP were coded as 1="graduated".

\section{Descriptive Variables}

In addition to the independent and control variables described above, the below variables were also examined in order to provide a fuller description of the entire study sample and as well as richer descriptions of all drug use outcome groups, including how characteristics that differed between groups.

Marital status was measured using data collected at baseline. Participants were asked to select from five categories (e.g., "married”, “widowed”, "separated”, “divorced”, and "never married”), the status that best reflected their marital situation.

In addition to exploring past-year drug use versatility as an independent variable, past 30-day drug use versatility prior to incarceration was included for descriptive purposes. This item was measured by summing the number of different drugs that a participant reported using during the 30-day period prior to incarceration (1-13). 
In addition to examining the dichotomized variable of past 30-day drug use prior to incarceration as an independent variable, past 30-day drug use prior to incarceration and past 30-day drug use frequency prior to incarceration are presented for descriptive purposes for all drug use outcomes. These items were measured at baseline by summing the number of days that ten specific drugs (alcohol, cannabis, prescription sedatives, hallucinogens, synthetic drugs, cocaine/crack cocaine, amphetamines, heroin, prescription opioids, and buprenorphine) were used in the 30-day period prior to incarceration (0-30). Responses were then dichotomized so that proportions for "use" versus "no use" of specific drugs could also be presented.

\section{Past-year drug use prior to incarceration and past-year drug use frequency prior to} incarceration were measured using data collected at baseline pertaining to past-year drug use for the following drugs: Alcohol, cannabis, prescription sedatives, hallucinogens, synthetic drugs, cocaine/crack cocaine, amphetamines, heroin, prescription opioids, and buprenorphine. For each of the 10 drugs, the number of months out of the 12 months prior to incarceration was summed, resulting in ten separate drug use frequency variables (range 0-12). New variables were generated from these to produce an additional dichotomous variable of past-year use ( $0=$ no use vs. $1=$ past-year use).

\section{Past-year drug use subsequent to incarceration and past-year drug use frequency subsequent}

to incarceration were measured using data collected at follow-up in a manner identical to past-year drug use prior to incarceration, with the only difference being the time period (range 0-12). Although the 10 "past-year drug use subsequent to incarceration variables" are considered as independent variables for the outcome of past-year heroin use subsequent to incarceration, they are also presented as means and proportions to help describe the sample and drug use groups during the one-year period subsequent to incarceration.

Past-year preferred drug of choice was measured at baseline by asking participants to select from a list of 13 drugs the one which they most preferred using during the year prior to incarceration (e.g., "In the 12 months prior to this incarceration, what was your primary substance of choice?"). Responses were recoded into 9 categories for the most commonly reported drugs: alcohol, marijuana, cocaine/crack cocaine, amphetamine-based stimulants, heroin, prescription opioids, buprenorphine, prescription sedatives/barbiturates, and inhalants/hallucinogens/synthetic drugs. 
Urban proximity was measured using the 2013 Urban Influence Codes, a 12-part classification scheme that categorizes counties by metropolitan (i.e., counties in metro areas with 20,000 to $>1$ million residents) and non-metropolitan (i.e., counties adjacent/not adjacent to metro areas, but not in metro areas) status into two metro and ten nonmetro designations (U.S. Department of Agriculture, 2013). Metropolitan counties are differentiated by population size of their metro area and nonmetropolitan counties are differentiated by size of largest city and proximity to metro and micropolitan areas (U.S. Department of Agriculture, 2013). Counties designations range from 1 (e.g., "Large-in a metro area with at least $\geq 1$ million residents") to 12 (e.g., "Noncore-not adjacent to a metro/micro area and <2,500 population (Department of Agriculture, 2013).

Unrestricted alcohol sales was measured using data obtained from the Kentucky Alcohol Beverage Control (KY ABC) (KY ABC, 2017). A dichotomous variable was created that coded counties as either $1=$ "restricted" (i.e., alcohol is significantly restricted or completely prohibited) or $0=$ "unrestricted" (i.e., alcohol sales are unrestricted county-wide). Because alcohol is widely consumed, may be used as a substitute for or compliment to various illicit drugs, and because its use more difficult to detect among people on community supervision, it is important to consider how access to this licit, and overall less risky, drug may influence drug use outcomes.

Days until post-release alcohol and/or illicit drug use was measured at follow-up by asking participants who reported alcohol use subsequent to incarceration to respond to the following question, "In 12 months since your release, how many days were you on the street before you first used alcohol?". For participants who reported illicit drug use subsequent to incarceration the same question was asked (range 0$365)$.

Intoxicated during offense was measured at baseline by asking participants to respond ("yes" vs. "no") to the question, "Were you under the influence of alcohol and/or drugs when you committed the offense that led to your current incarceration?".

In addition to the independent variable of "chronic pain" described above, a description of chronic pain severity, for participants who reported experiencing chronic prior to incarceration, was measured by creating a composite variable composed of three scale items adapted from the Brief Pain Inventory (BPI; Cleeland \& Ryan, 1994), a widely used instrument for assessing clinical pain caused by a variety of health 
conditions that has demonstrated good validity and reliability (Cleeland, 2009). Participants were asked to rate the intensity of their chronic pain when the pain is at its "least", "average", and "worst" using a 10poing Likert scale (e.g., "Please rate the intensity your chronic at its worst in the 30 days prior to this incarceration"). Higher composite scores indicated overall greater pain severity $(3-30 ; \alpha=0.95)$.

\section{Analytic Plan Overview}

\section{Data Handling and Preparation}

A total of ten datasets were obtained from CDAR along with four codebooks and all original survey instruments. Datasets included five baseline survey datasets and five follow-up data survey datasets for FYs 2013-17. Methods proposed by MacInnes (2017) for handling and preparing (e.g., inspecting, cleaning, merging) data obtained from secondary sources for preliminary examination and analyses were followed, along with other sources, as needed (Kiecolt \& Nathan, 1985; Trzesniewski, Donnellan, \& Lucas, 2011). Variables and variable names were reviewed prior to merging datasets, as were all codebooks.

Small instrument changes between FYs resulted in some survey versions containing slightly more or fewer questions. Although instrument changes were not extensive, they nevertheless resulted in some variables only being collected for one or two FYs. To include variables that were only collected for one or two years would have resulted in significant non-random missing data for many cases $(>900)$. Rather than deleting a significant percentage of cases and drastically reducing the sample size, a decision was made to exclude variables that were not measured across all FYs, particularly if the same the same construct could be represented using a different variable or combination of variables, or if it was not relevant to the current study (e.g., military history, criminal offenses) (Cook \& Campbell, 1979; MacInnes, 2017; Trzesniewski et al., 2011). Variables of interest to this study were renamed such that all variable names across datasets were uniform. Codebooks were annotated and dated with each change. Variables that were not of possible interest were not renamed and not included during merging. The final merged baseline and follow-up CJKTOS dataset contained 696 variables.

In addition to visually inspecting the data, preliminary analyses such as frequency distributions and descriptive statistics were produced in order to preliminarily identify any potential data entry or merging errors, improperly coded variables, and missing data that was not expected (e.g., if participants 
responded "no" to lifetime incidence of head trauma, then there should be no value for "number of times" that head trauma was experienced).

\section{Statistical Software}

Data cleaning, merging, and preliminary analyses were conducted using IBM-SPSS version 27 (IBM Corp., 2018). All subsequent analyses were conducted using Stata/SE version 15 (StataCorp LLC, 2017).

\section{Analytic Plan Development}

One of the conceptual assumptions underlying this study is that people and their decision-making cannot be divorced from the contexts in which they live. In this sense, they can be conceptualized as nested within and across contextual layers. In acknowledging that people are nested within larger contexts and conditions, it cannot be assumed that observations observed at the individual-level are truly independent. This is important, in that it violates assumptions for many families of statistical tests, including ordinarily least squares (OLS) regression (Robson \& Pevalin, 2016). This can result in incorrect inference and inflated statistical significance based on too many degrees of freedom that are not truly independent (Tabachnick \& Fidell, 2013). To help illustrate, a commonly used example is to consider outcome measures for students. Outcomes for a population of students who live in the same city, but who reside in different neighborhoods and attend different schools may vary not only due to student-level differences, but also due to contextual factors that influence learning and development. Here, variance might be observed at more one than one level. Student performance may differ as a result not only of student-level differences (e.g., measures of general intelligence), but also due to broader conditions, such the different classrooms they learn in, schools they attend, and neighborhoods they live in. In other words, observed effects or outcomes may vary as a function of individual characteristics and shared group characteristics identifiable at other levels. Even if study aims were not concerned with exploring possible contextual influences on drug use, the nature of the data used in this study (i.e., cases clustered at higher levels, such as counties) may violate the assumption of independence of errors, potentially contributing to incorrect estimations of the standard errors (Robinson \& Pevalin, 2016; Tabachnick \& Fidell, 2013).

Because of this possibility, mixed-effects (random-intercept) multilevel regression models (MLMs) was explored first, prior to developing the final analytic plan, using the exploratory strategy 
proposed by Hox (2002). Mixed-effect MLMs include fixed and random effects, needed to "nest" one subset of data in another. MLMs are an extension of OLS regression and can be highly versatile and customizable (Hox, Moerbeek, \& Van de Schoot, 2017). As noted above, in some instances, individuallevel outcomes examined without taking into account the hierarchical structure of the data can produce inaccurate estimates. MLM addresses such an issue by permitting intercepts (means) and slopes (IV-DV relationship) to vary between levels (Hox et al., 2017). For example, the relationship between cocaine use and, say, misregulation would be allowed to vary between different second-level groups (Tabachnick \& Fidell, 2013). Stata enables MLM model building and customization, with many available commands and online resources that can be utilized as models are built (Acock, 2016; Robson \& Pevalin, 2016; StataCorp LLC, 2017).

\section{Power analysis}

Because MLM uses maximum likelihood estimation (MLE) and because there are numerous variables of interest in this study, it was important to determine that the final sample size of 1,563 would provide adequate statistical power (Maas \& Hox, 2005). Although Long (1997) notes that >500 cases are likely sufficient be able to obtain reliable estimates of variance, that does not necessarily take into consideration the number of covariates across levels, of which there are several in this study, nor the power needed to detect smaller effect sizes (Acock, 2016; Hox, et al., 2017).

In areas of exploratory, non-critical research, a power of 0.80 may be minimally adequate (Jones, Carley, \& Harrison, 2003). Even though the current study is exploratory and heavily conceptual in nature, ideally it is desired that there will be sufficient power for accurately detecting a small effect size in terms of explained variance $\left(\mathrm{f}^{2}=0.02 ; \mathrm{R}^{2}=0.02\right)$. However, it would be acceptable for the purposes of this study if power is sufficient for accurately detecting a medium effect size $\left(\mathrm{f}^{2}=0.15 ; \mathrm{R}^{2}=0.13\right)$. A priori estimates of the required sample size, conducted using the software G*Power (Faul, Erdfelder, Buchner, \& Lang, 2009), determined that for a small effect size $\mathrm{f}^{2}=0.02$, alpha $=0.05$, power $=0.85$, tested predictors $=40$, a sample size $\mathrm{N}=1,545$ was required $^{9}$. Setting the power $=0.90$ or 0.95 would have necessitated increasing the sample size to $\mathrm{N}=1,726$ and 2,005 , respectively. In order to be able to accurately detect a medium effect size $\mathrm{f}^{2}=0.15$ at

\footnotetext{
${ }^{9}$ Note that 40 variables was used to err on the side of caution. While there were many IVs proposed for examination, not all of them were included into the models, as some did not demonstrate any correlation to outcome variables and others were not included in order to avoid overfitting the model.
} 
alpha $=0.05$, power $=0.85$, tested predictors $=40$, a sample size of $\mathrm{N}=232$ would be required. Increasing this to power $=0.90$ or 0.95 would have necessitated increasing the sample size to $\mathrm{N}=256$ or $\mathrm{N}=293$, respectively. Preliminary analyses using the current study sample size $(\mathrm{N}=1,564)$ with $\mathrm{f}^{2}=0.02$, alpha $=0.05$, tested predictors $=40$, calculated achieved power $=0.86$.

However, with MLM, needed sample size varies between levels. Although level-one had sufficient power at $\mathrm{N}=1,643$, the overall power needed for each MLM varies based on the groupings and sample sizes for groupings across levels (Hox \& Maas, 2002). Typically, MLE methods, which produce parameter estimates and asymptotic standard errors, is used in MLM as well as logistic and multinomial regression, in which only fixed effects are modeled (Hox et al., 2017). MLE is used to test the significance of specific parameters and to establish confidence intervals (CI). This means that there are assumptions of normal distribution of error, not just at level-1, but at level-2 and so on. (Maas \& Hox, 2004). Small sample sizes $(<50)$ for groups at higher levels can lead to biased estimates; specifically, the random effects at the second level may be inaccurate due to distribution assumptions at the second-level (or third-level, etc.) not being satisfied (Maas \& Hox, 2004). It has been suggested that the number of level-2 or level-3 groups is generally more important than the number of observations within each grouping (Hox et al., 2017).

In this study, variables measured at the level of the county were spread over $>100$ county groupings ( $\mathrm{N}=119)$, meaning that there was sufficient sample size of counties to produce reliable error estimates (see Maas \& Hox, 2005). Although the recommended sample size for groups varies from to 2030, the general consensus is that power increases as the number of groups increase (Duncan, 1998; Maas \& Hox, 2005; Mox, 1995; Snijders, 2005). As noted above, the number of higher-level clusters is typically considered more important for conducting MLM than the number of observations within each cluster, with a recommendation of 20 clusters minimum (Hox et al., 2017). There is growing consensus that as the number of clusters increases the number of observations needed per cluster may decease without biasing estimates (Hox, 1998, 2010; McNeish \& Stapleton, 2014; Snijders \& Bosker, 2012). For instance, 50 clusters with 20 observations or 100 clusters with 10 observations may produce a stable MLM (Hox 1998, 2010). Dedrick et al. (2009) have proposed a "30/30" guideline based on a review of MLMs in which they found that just over $20 \%$ of reviewed studies did not have adequate clusters and observations per cluster to perform MLM or MLM that produced unbiases estimates. 


\section{Exploratory MLM Building Results}

With this recommendation in mind, the distribution of participant counties was examined to determine if the number of observations per group within the county-level cluster would permit MLM of county-level variables (e.g., household poverty, Internet access, etc.). When baseline data were collected participants reported living in 118 counties; at the time follow-up data were collected, participants reported living in 119 counties (out of a possible 120 counties). Although there were >100 groups at the countylevel, there were $>10$ observations for only $33.1 \%$ of the counties for the period prior to incarceration and for only $32.6 \%$ of the counties for the period subsequent to incarceration. Prior to incarceration, observations were identified as primarily clustered within Jefferson $(\mathrm{N}=228,14.6 \%)$, Fayette $(\mathrm{N}=71$, 4.5\%), Kenton ( $\mathrm{N}=56,3.6 \%)$, Hardin $(\mathrm{N}=54,3.5 \%)$, and Daviess $(\mathrm{N}=51,3.3 \%)$ counties. Subsequent to incarceration, observations were identified as still primarily clustered within Jefferson $(\mathrm{N}=259,16.6 \%)$, Fayette $(\mathrm{N}=82,5.2 \%)$, Kenton $(\mathrm{N}=53,3.4 \%)$, Daviess $(\mathrm{N}=53,3.4 \%)$, and, Hardin $(\mathrm{N}=48,3.1 \%)$. In Appendix B, Figures 3.0 and 3.1 display frequencies of all counties with $<10$ observations per county. Frequencies for all counties with $>10$ observations are presented in Figures 4.0 and 4.1 in Appendix B. In order to demonstrate that MLM could not be pursued due to insufficient observations at the level-two grouping variable, models were built following the general sequence outlined by Hox (2002), which involved several steps. The first step included analyzing the intercept-only (null) model and examining the interclass correlation (ICC), (i.e., ratio of the variance between groups at higher levels and variance within groups at these levels), with high values indicating that the assumption of independence of errors had been violated. This was done for five drug outcomes. These included heroin (ICC=0.055, random intercept=0.0723; between-subject $\mathrm{SD}=0.2986$, chibar=59.6, $\mathrm{p}<.001$ ), prescription opioids ( $\mathrm{ICC}=0.003$, random intercept $=0.0194$, between-subjects $\mathrm{SD}=0.3492$, chibar $=0.24, \mathrm{p}=.313$ ), buprenorphine $(\mathrm{ICC}=0.0142$, random intercept=0.0348; between-subject $\mathrm{SD}=0.2900$, chibar=1.74, $\mathrm{p}=.094)$, cocaine/crack cocaine $(\mathrm{ICC}=0.000$, random intercept=0.0348; between-subject $\mathrm{SD}=0.2610$, chibar=0.0, $\mathrm{p}=1.00)$, amphetamines $(\mathrm{ICC}=0.0476$, random intercept=0.0785; between-subject $\mathrm{SD}=0.3513$, chibar=21.6, $\mathrm{p}<.001)$. Additionally, counties were ranked in order of random intercepts on outcomes to create confidence intervals (CIs) around the estimates of the random intercepts. Standard error bar charts were then used to graph the relative random intercept values. Tabachnick \& Fidell (2013) note that if the ICC is sufficiently 
small that a single-level regression analysis is appropriate. Still, as Robson \& Pevalin (2016) note, it is not always clear when a non-zero ICC indicates a clear cut-off point.

Second, models were analyzed using all level-1 IVs fixed, assessing contribution of each IV and model differences. IVs were selected for inclusion based on bivariate statistics indicating statistically significant correlation between an IV and outcome variables. Next, a MLM for heroin was built, using "county" as the higher-level group. This outcome was chosen because it had the largest ICC value. Here, the slope for each IV was permitted to be random, adding county-level IVs individually. Though the model converged after 10 iterations, SEs and CIs were not produced, indicating that the Hessian calculation had become unstable. Further, when comparing the saved fixed- and random-effects estimates, output reported that levels were "not nested". It was therefore concluded there were insufficient cases for MLM in general and that, for some of the other drug outcomes explored in preliminary analyses, there was insufficient variance at the individual-level explained by the county-level to justify the use of MLM. Ultimately, due to insufficient cases, MLM was excluded as a means for examining drug use outcomes of interest. A revised analytic plan was created and is outlined below.

\section{Final Analytic Plan}

\section{Aim One}

To address this aim, (described on pg. 126) descriptive statistics, such as means and proportions, were generated for the entire sample and for all four drug use groups (e.g., concomitant use, opioid use, stimulant use, other drug use and other drug/no drug use) using data collected at baseline and follow-up. Chi-square test of independence and the Kruskal-Wallis analysis of variance by ranks were used to determine if groups were statistically significantly different $(\mathrm{p}<.05)$ (Everitt, 1977; Meyers et al., 2013; Siegel \& Castellan, 1988).

\section{Aim Two}

To address this aim (described on pg 126), multinomial logistic regression was used to examine the association between independent variables and concomitant, opioid, and stimulant use for each time period. Independent and control variables that differed significantly $(\mathrm{p}<.05)$ across drug use outcome groups in non-parametric tests were chosen for inclusion into regression models. Multinomial logistic regression was selected due to the polytomous outcome variable (Luke, 2004; Skrondal \& Rabe-Hesketh, 
2003). For the 30-day period prior to incarceration, the drug use outcome group of "other drug use" was used as the reference category, meaning that the coefficients for all other drug use outcome groups describe how the IVs are related to the probability of being in one of the drug use outcome groups, versus the reference group of other drug use. Because all participants reported some drug use in the 30-day period prior to incarceration, "no drug use" was not also used to define that group, but rather only "other drug use". For the one-year post-release period, the drug use group of "other/no drug use" was used as the reference category. This is because, during the one-year post-release period, some participants reported using drugs other than opioids and stimulants while other participants reported not using any drugs, unlike the 30-day period prior to incarceration, in which all participants reported some drug use. These drug use outcome groups of "other drug use" and "other/no drug use" were selected to serve as the reference categories because they reflect decisions with the less relative risk compared to decisions involving opioids and stimulants.

\section{Aim Three}

To address this aim, (described on pg. 127) descriptive statistics, chi-square, and t-test were used to describe and examine differences between participants who reported heroin use during the one-year postrelease period and those who did not. Binary logistic regression was used to examine the relationship between independent variables and heroin use during the one-year post-release period. Independent variables that differed significantly $(\mathrm{p}<.05)$ between the heroin-use group and the non-heroin use group in bivariate analyses were selected for inclusion into regression model. 


\section{CHAPTER IV}

\section{RESULTS}

\section{Aim One Results \\ Drug Outcomes During the 30-day Period Prior to Incarceration}

Table 1 displays means and proportions for the final sample $(\mathrm{N}=1,563)$ and for each drug use group for the period prior to incarceration. For the 30-day period prior to incarceration, $29.0 \%$ of participants reported concomitant use of opioid and stimulants, $28.5 \%$ reported opioid, but not stimulant, use; $18.0 \%$ reported stimulant, but not opioid use; and $24.4 \%$ reported use of drugs other than opioids and stimulants.

The majority of the sample was White (81.5\%), with the remainder African American (17.1\%), Hispanic (0.8\%), and other (e.g., Asian, American Indian, Middle Eastern; 0.6\%). There was a higher proportion of White participants among those who reported past 30-day opioid (93.9\%) and concomitant use $(90.1 \%)$, compared to past 30 -day stimulant $(70.6 \%)$ and other drug use $\left(64.7 \%, X^{2}=161.71, \mathrm{df}=3\right.$, $\mathrm{p}<.001$ ). Approximately $71 \%$ of the sample had earned a high school diploma (HSD)/GED. The proportion of participants with a HSD/GED were equivalent across groups $\left(X^{2}=2.27, \mathrm{df}=3, \mathrm{p}=.519\right)$, with rates highest among participants who reported past 30-day concomitant use (73.3\%) and lowest among participants who reported past 30-day opioid use (68.8\%). Twenty percent of the sample was married and $47.6 \%$ reported never having been married, with the remainder widowed (1.6\%), separated (7.5\%), or divorced (23.3\%). Marital status was similar across groups $\left(X^{2}=14.13, \mathrm{df}=12, \mathrm{p}=.292\right)$. Participants who reported past 30-day opioid use had slightly higher rates of 
being currently married (22.0\%) as well as divorced (25.8\%). Participants who reported past 30-day other drug use had the highest rates of never being married $(52.6 \%)$.

Although the mean number of nights spent in a controlled environment out of the 30-day period prior to incarceration was low sample-wide $(\bar{x}=1.3, S D=3.5)$, participants who reported past 30 -day other drug use were incarcerated for 1.6 nights, whereas those who reported past 30-day stimulant use were incarcerated $<1$ night $(0.9)(\mathrm{p}=.011)$. For the entire sample, the mean number of lifetime drug treatment episodes was $2.2(S D=3.8$, range 0-62). Past 30-day concomitant use was associated with a greater average number of lifetime treatment episodes (2.8) compared to use of opioids (2.1), stimulants (2.2), and other drugs $(1.8)(\mathrm{p}<.001)$. Participants who reported past 30-day concomitant use had a higher rate of IDU history $\left(64.2 \% ; X^{2}=238.35, \mathrm{df}=3, \mathrm{p}<.001\right)$, whereas history of IDU for the entire sample was $42.9 \%$. Participants who reported past 30-day opioid use also had a higher rate of IDU history (53.1\%) compared to participants who reported past 30-day stimulant (29.1\%) and other drug use (16.0\%). 
Table 1

Sample Characteristics and Drug Use Group Means, Proportions, and Between-group Differences Prior to Incarceration

\begin{tabular}{|c|c|c|c|c|c|c|}
\hline $\begin{array}{l}\mathrm{N}= \\
\%\end{array}$ & $\begin{array}{c}\text { Sample } \\
1,563\end{array}$ & $\begin{array}{c}\text { Concomitant } \\
453 \\
29.0 \\
\end{array}$ & $\begin{array}{c}\text { Opioid } \\
446 \\
28.5 \\
\end{array}$ & $\begin{array}{c}\text { Stimulant } \\
282 \\
18.0 \\
\end{array}$ & $\begin{array}{c}\text { Other } \\
\\
382 \\
24.4 \\
\end{array}$ & $\begin{array}{c}\mathbf{p} \\
\text { value }\end{array}$ \\
\hline \multicolumn{7}{|l|}{ Racelethnicity } \\
\hline White & $81.5 \%$ & $90.1 \%$ & $93.9 \%$ & $70.6 \%$ & $64.7 \%$ & .001 \\
\hline African American & $17.1 \%$ & $7.9 \%$ & $4.9 \%$ & $27.7 \%$ & $34.6 \%$ & \\
\hline Hispanic & $0.8 \%$ & $1.3 \%$ & $0.9 \%$ & $0.7 \%$ & $0.3 \%$ & \\
\hline Other & $0.6 \%$ & $0.7 \%$ & $0.2 \%$ & $1.1 \%$ & $0.5 \%$ & \\
\hline HSD/GED & $71.3 \%$ & $73.3 \%$ & $68.8 \%$ & $72.0 \%$ & $71.2 \%$ & .519 \\
\hline Marital status & $20.0 \%$ & $19.4 \%$ & $22.0 \%$ & $20.9 \%$ & $17.8 \%$ & .292 \\
\hline Married & $1.6 \%$ & $2.0 \%$ & $1.1 \%$ & $0.7 \%$ & $2.1 \%$ & \\
\hline Widowed & $7.5 \%$ & $8.4 \%$ & $8.3 \%$ & $5.7 \%$ & $6.8 \%$ & \\
\hline Separated & $23.3 \%$ & $23.0 \%$ & $25.8 \%$ & $23.4 \%$ & $20.7 \%$ & \\
\hline Divorced & $47.6 \%$ & $47.0 \%$ & $42.8 \%$ & $49.3 \%$ & $52.6 \%$ & \\
\hline \multicolumn{7}{|l|}{ Never married } \\
\hline $\begin{array}{l}\text { Nights in controlled } \\
\text { environment }\end{array}$ & 1.3 & 1.4 & 1.3 & 0.9 & 1.6 & .011 \\
\hline $\begin{array}{l}\text { Lifetime drug treatment } \\
\text { episodes }\end{array}$ & 2.2 & 2.8 & 2.1 & 2.2 & 1.8 & .001 \\
\hline Ever IDU & $42.9 \%$ & $64.2 \%$ & $53.1 \%$ & $29.1 \%$ & $16.0 \%$ & .001 \\
\hline Age & 33.9 & 32.2 & 34.0 & 36.9 & 33.8 & .001 \\
\hline Male & $80.9 \%$ & $77.3 \%$ & $76.5 \%$ & $85.8 \%$ & $86.9 \%$ & .001 \\
\hline $\begin{array}{l}\text { LD diagnosis/SE } \\
\text { enrollment }\end{array}$ & $28.6 \%$ & $29.4 \%$ & $28.0 \%$ & $28.7 \%$ & $28.3 \%$ & .974 \\
\hline Head trauma & $41.9 \%$ & $47.1 \%$ & $44.2 \%$ & $37.9 \%$ & $36.4 \%$ & .007 \\
\hline $\begin{array}{l}\text { Mean head injuries } \\
(\mathrm{N}=655)\end{array}$ & 2.5 & 2.3 & 2.5 & 2.8 & 2.7 & .009 \\
\hline Anxiety symptoms & $44.5 \%$ & $57.0 \%$ & $44.6 \%$ & $41.1 \%$ & $32.2 \%$ & .001 \\
\hline Depressive symptoms & $46.2 \%$ & $59.6 \%$ & $49.1 \%$ & $42.2 \%$ & $29.8 \%$ & .001 \\
\hline Suicidality & $11.6 \%$ & $15.7 \%$ & $12.1 \%$ & $11.7 \%$ & $6.3 \%$ & .001 \\
\hline Chronic pain & $24.8 \%$ & $27.6 \%$ & $28.7 \%$ & $20.6 \%$ & $19.9 \%$ & .004 \\
\hline $\begin{array}{l}\text { Chronic pain severity } \\
(\mathrm{N}=387 ; 3-30)\end{array}$ & 15.6 & 15.6 & 16.2 & 15.1 & 15.2 & .003 \\
\hline $\begin{array}{l}\text { Stress-related health } \\
\text { effects }(11-44)\end{array}$ & 23.5 & 26.3 & 23.6 & 22.7 & 20.1 & .001 \\
\hline Economic hardship (0-8) & 1.6 & 2.2 & 1.7 & 1.4 & 1.1 & .001 \\
\hline Homelessness & $14.8 \%$ & $19.4 \%$ & $16.1 \%$ & $12.1 \%$ & $9.9 \%$ & .001 \\
\hline $\begin{array}{l}\text { Subjective } \\
\text { socioeconomic status } \\
(1-10)\end{array}$ & 5.0 & 4.7 & 4.8 & 5.3 & 5.3 & .001 \\
\hline Misregulation & $83.1 \%$ & $91.4 \%$ & $86.8 \%$ & $83.7 \%$ & $68.8 \%$ & .001 \\
\hline Close relationships & 7.1 & 6.7 & 6.5 & 7.3 & 7.9 & .010 \\
\hline Social interaction & $88.9 \%$ & $88.1 \%$ & $89.2 \%$ & $86.5 \%$ & $91.4 \%$ & .228 \\
\hline Social satisfaction & $70.8 \%$ & $61.8 \%$ & $71.3 \%$ & $73.4 \%$ & $79.8 \%$ & .001 \\
\hline
\end{tabular}




\begin{tabular}{|c|c|c|c|c|c|c|}
\hline $\begin{array}{l}\mathrm{N}= \\
\%\end{array}$ & $\begin{array}{c}\text { Sample } \\
1,563\end{array}$ & $\begin{array}{c}\text { Concomitant } \\
453 \\
29.0\end{array}$ & $\begin{array}{c}\text { Opioid } \\
\\
446 \\
28.5 \\
\end{array}$ & $\begin{array}{c}\text { Stimulant } \\
\\
282 \\
18.0\end{array}$ & $\begin{array}{c}\text { Other } \\
\\
382 \\
24.4 \\
\end{array}$ & $\begin{array}{c}\mathbf{p} \\
\text { value }\end{array}$ \\
\hline \multicolumn{7}{|l|}{ Table 1 (continued) } \\
\hline $\begin{array}{l}\text { Leisure time }(0-7) \\
\text { Educational/vocational } \\
\text { involvement }\end{array}$ & $\begin{array}{c}2.5 \\
65.8 \%\end{array}$ & $\begin{array}{c}2.0 \\
66.2 \%\end{array}$ & $\begin{array}{c}2.2 \\
62.8 \%\end{array}$ & $\begin{array}{c}2.5 \\
70.2 \%\end{array}$ & $\begin{array}{c}3.3 \\
65.7 \%\end{array}$ & $\begin{array}{l}.001 \\
.232\end{array}$ \\
\hline $\begin{array}{l}\text { Age of drug use } \\
\text { initiation }\end{array}$ & 14.7 & 13.7 & 15.5 & 15.3 & 14.5 & .001 \\
\hline $\begin{array}{l}\text { Severity of drug use, } \\
\text { past } 30 \text {-days } \\
(0-40)\end{array}$ & 23.2 & 30.0 & 27.3 & 22.3 & 11.2 & .001 \\
\hline $\begin{array}{l}\text { Intoxicated during } \\
\text { offense }\end{array}$ & $78.2 \%$ & $89.0 \%$ & $81.8 \%$ & $79.4 \%$ & $60.2 \%$ & .001 \\
\hline $\begin{array}{l}\text { Past-year drug use } \\
\text { versatility }\end{array}$ & 3.8 & 6.1 & 3.6 & 2.6 & 2.1 & .001 \\
\hline $\begin{array}{l}\text { Past } 30 \text {-day drug use } \\
\text { versatility }\end{array}$ & 3.0 & 5.3 & 2.9 & 2.3 & 1.1 & .001 \\
\hline \multicolumn{7}{|l|}{$\begin{array}{l}\text { Past-year drug prior to } \\
\text { incarceration }\end{array}$} \\
\hline Alcohol & $63.5 \%$ & $68.7 \%$ & $54.0 \%$ & $63.1 \%$ & $68.8 \%$ & .001 \\
\hline Cannabis & $61.4 \%$ & $72.0 \%$ & $52.0 \%$ & $56.0 \%$ & $63.6 \%$ & .001 \\
\hline Sedatives & $39.0 \%$ & $66.9 \%$ & $42.6 \%$ & $14.2 \%$ & $19.9 \%$ & .001 \\
\hline Hallucinogens & $6.1 \%$ & $13.0 \%$ & $3.1 \%$ & $5.0 \%$ & $2.1 \%$ & .001 \\
\hline Synthetics & $10.1 \%$ & $19.6 \%$ & $7.0 \%$ & $7.8 \%$ & $4.2 \%$ & .001 \\
\hline Cocaine/crack cocaine & $33.5 \%$ & $63.8 \%$ & $12.1 \%$ & $47.5 \%$ & $12.3 \%$ & .001 \\
\hline Amphetamines & $36.7 \%$ & $72.0 \%$ & $7.4 \%$ & $62.4 \%$ & $10.2 \%$ & .001 \\
\hline Heroin & $21.8 \%$ & $40.0 \%$ & $30.9 \%$ & $0.4 \%$ & $5.5 \%$ & .001 \\
\hline Prescription opioids & $55.8 \%$ & $91.4 \%$ & $86.5 \%$ & $4.6 \%$ & $15.4 \%$ & .001 \\
\hline Buprenorphine & $24.9 \%$ & $44.4 \%$ & $35.9 \%$ & $1.8 \%$ & $6.0 \%$ & .001 \\
\hline \multicolumn{7}{|l|}{$\begin{array}{l}\text { Past } 30 \text {-day drug use } \\
\text { prior to incarceration }\end{array}$} \\
\hline Alcohol & 7.3 & 8.4 & 4.7 & 8.1 & 8.3 & .001 \\
\hline Cannabis & 11.2 & 14.9 & 9.1 & 11.1 & 10.1 & .001 \\
\hline Sedatives & 5.2 & 9.9 & 6.1 & 1.1 & 1.6 & .001 \\
\hline Hallucinogens & 0.3 & 0.5 & 0.1 & 0.4 & 0.0 & .001 \\
\hline Synthetic drugs & 1.1 & 2.2 & 0.5 & 1.0 & 0.6 & .001 \\
\hline Cocaine/crack cocaine & 3.3 & 7.4 & 0.0 & 6.6 & 0.0 & - \\
\hline Amphetamines & 6.2 & 12.9 & 0.0 & 13.6 & 0.0 & - \\
\hline Heroin & 3.7 & 6.6 & 6.1 & 0.0 & 0.0 & - \\
\hline Prescription opioids & 10.4 & 18.1 & 18.2 & 0.0 & 0.0 & - \\
\hline Buprenorphine & 2.7 & 5.1 & 4.2 & 0.0 & 0.0 & - \\
\hline \multicolumn{7}{|l|}{$\begin{array}{l}\text { Past-year preferred drug } \\
\text { of choice }\end{array}$} \\
\hline Alcohol & $13.1 \%$ & $5.5 \%$ & $7.2 \%$ & $11.3 \%$ & $30.4 \%$ & .001 \\
\hline Cannabis & $21.3 \%$ & $9.7 \%$ & $14.1 \%$ & $19.9 \%$ & $44.5 \%$ & \\
\hline Sedatives & $2.9 \%$ & $2.0 \%$ & $3.8 \%$ & $1.8 \%$ & $3.9 \%$ & \\
\hline Hallucinogens/synthetics & $1.3 \%$ & $2.2 \%$ & $0.4 \%$ & $1.4 \%$ & $1.0 \%$ & \\
\hline Cocaine/crack cocaine & $8.7 \%$ & $11.0 \%$ & $0.4 \%$ & $25.2 \%$ & $3.4 \%$ & \\
\hline Amphetamines & $15.2 \%$ & $23.4 \%$ & $0.7 \%$ & $38.7 \%$ & $5.0 \%$ & \\
\hline
\end{tabular}




\begin{tabular}{|c|c|c|c|c|c|c|}
\hline $\begin{array}{l}\mathrm{N}= \\
\%\end{array}$ & $\begin{array}{c}\text { Sample } \\
1,563\end{array}$ & $\begin{array}{c}\text { Concomitant } \\
453 \\
29.0\end{array}$ & $\begin{array}{c}\text { Opioid } \\
\\
446 \\
28.5 \\
\end{array}$ & $\begin{array}{c}\text { Stimulant } \\
\\
282 \\
18.0\end{array}$ & $\begin{array}{c}\text { Other } \\
\\
382 \\
24.4 \\
\end{array}$ & $\begin{array}{c}\mathbf{p} \\
\text { value }\end{array}$ \\
\hline \multicolumn{7}{|l|}{ Table 1 (continued) } \\
\hline $\begin{array}{l}\text { Heroin } \\
\text { Prescription opioids } \\
\text { Buprenorphine }\end{array}$ & $\begin{array}{c}9.8 \% \\
25.2 \% \\
2.6 \%\end{array}$ & $\begin{array}{l}13.9 \% \\
29.8 \% \\
2.4 \%\end{array}$ & $\begin{array}{l}17.3 \% \\
50.7 \% \\
5.4 \%\end{array}$ & $\begin{array}{l}0.0 \% \\
1.8 \% \\
0.0 \%\end{array}$ & $\begin{array}{l}3.4 \% \\
7.1 \% \\
1.3 \%\end{array}$ & \\
\hline $\begin{array}{l}\text { Central Appalachia } \\
\text { Urban influence }\end{array}$ & $\begin{array}{l}31.8 \% \\
4.6\end{array}$ & $\begin{array}{l}35.3 \% \\
4.7\end{array}$ & $\begin{array}{c}43.9 \% \\
5.1\end{array}$ & $\begin{array}{c}18.4 \% \\
4.3\end{array}$ & $\begin{array}{c}23.3 \% \\
4.0\end{array}$ & $\begin{array}{l}.001 \\
.001\end{array}$ \\
\hline
\end{tabular}

\section{Age and Sex}

Participants were 33.9 years old on average $(S D=9.0$, range=18-74) prior to entering SAP.

Participants who reported past 30-day stimulant use were relatively older $(36.9 ; \mathrm{p}<.001)$ compared to participants who reported past 30-day opioid (34.0), concomitant (32.2), and other drug use (33.8). While the sample was majority male (80.9\%), the proportion of males was highest among participants who reported past 30-day other drug (86.9\%) and stimulant use (85.8\%), whereas the proportion of males among participants reporting past 30-day opioid and concomitant use was lower, and more similar, at $76.5 \%$ and $77.3 \%$, respectively $\left(X^{2}=22.95, \mathrm{df}=3, \mathrm{p}<.001\right)$.

\section{LD diagnosis, SE enrollment and History of Head Trauma}

Over a quarter of participants (28.6\%) reported a history of LD diagnosis and/or SE enrollment. The proportion of participants reporting LD diagnosis and/or SE enrollment was similar across groups, with rates ranging from $28.3 \%$ among participants who reported using other drugs to $29.4 \%$ among participants who reported concomitant use $\left(X^{2}=0.22, \mathrm{df}=3, \mathrm{p}=.974\right)$. Nearly $42.0 \%$ of participants reported lifetime history of head trauma. Among those who did $(\mathrm{N}=655)$, the average number of head injuries reported was $2.5(S D=3.3$, range $=1-40, \mathrm{p}=.009)$. Rates for any history of head trauma were highest among participants reporting past 30-day concomitant use and lowest among participants who reported past 30-day other drug use $\left(X^{2}=11.99, \mathrm{df}=3,47.1 \%\right.$ vs $\left.36.4 \% ; \mathrm{p}=.007\right)$.

\section{Psychiatric Symptoms}

During the year prior to incarceration, $44.5 \%$ of participants met or surpassed the GAD cutoff point, meaning that they experienced $\geq 4$ anxiety symptoms during the one- year period prior to incarceration at a level of clinical significance. Approximately $46.0 \%$ of participants met or surpassed the 
MDD cutoff point, meaning that they experienced $\geq 5$ symptoms associated with major depressive disorder during the year prior to incarceration at a level of clinical significance. Groups significantly differed in terms of the proportion of participants who met these thresholds for anxiety $\left(X^{2}=53.14, \mathrm{df}=3, \mathrm{p}<.001\right)$ and depressive symptoms during this pre-incarceration time period $\left(X^{2}=77.19, \mathrm{df}=3, \mathrm{p}<.001\right)$. Rates of past-year anxiety and depressive symptoms were highest among participants who reported past 30-day concomitant use, with $57.0 \%$ reporting anxiety symptoms and $59.0 \%$ reporting depressive symptoms. Rates of anxiety symptoms were lower among participants reporting past 30-day opioid (44.6\%) and stimulant use (41.1\%), as were rates of depressive symptoms, with $49.1 \%$ of participants who reported past 30 -day opioid use and $42.2 \%$ of participants who reported past 30-day stimulant use reporting depressive symptoms. Participants who reported past 30-day other drug use had the lowest rates of both depressive and anxiety symptoms, $32.2 \%$ and $29.8 \%$, respectively. Suicidal ideation for this same time period was reported by $11.6 \%$ of the sample. For participants who reported past 30-day concomitant use, however, 15.7\% reported past-year suicidal ideation, the highest among all groups $\left(X^{2}=17.92, \mathrm{df}=3, \mathrm{p}<.001\right)$. Approximately $12 \%$ of participants who reported past 30-day opioid and stimulant use also reported past-year suicidal ideation, still relatively high at nearly double the rates of those who reported past 30-day other drug use (6.3\%).

\section{Chronic Pain}

Just under a quarter of the sample reported experiencing chronic pain in the one-year period prior to incarceration (24.8\%). Of those who experienced chronic pain $(\mathrm{N}=387)$, the mean BPI chronic pain severity score was 15.6 out of a possible $30(S D=5.5$, range=3-30, $\mathrm{p}=.003)$. For subscales $(0-10)$, pain "at its worst" was ranked 7.0, pain "on average" was ranked 4.8, and pain "at its least" was ranked 3.8, meaning that pain severity ranged from approximately 4-7 on a 10-point scale. Participants who reported past 30-day opioid and concomitant use evidenced the highest rates of chronic pain, $28.7 \%$ and $27.6 \%$, respectively, whereas rates of chronic pain among participants who reported past 30-day stimulant and other drug use were slightly lower, $20.6 \%$ and $19.9 \%$, respectively $\left(X^{2}=13.82, \mathrm{df}=3, \mathrm{p}=.004\right)$.

\section{Stress-related Health Effects}

In terms of past-week stress-related health effects reported for the 7-day period prior to incarceration, the mean score on the revised HCS was 23.5 ( $S D=8.1$, range=11-44), indicating that, on average, moderate stress-related health effects were experienced during this time period by the majority of 
participants (93.0\% reported at least one stress-related health effect). Participants who reported past 30-day concomitant use scored the highest on the HCS, whereas participants who reported past 30-day other drug use scored the lowest (26.3 vs. 20.1; p<.001). Participants who reported past 30-day opioid and stimulant use had similar HCS scores, at 23.6 and 22.7, respectively.

\section{Economic Hardship, Homelessness, and Subjective Socioeconomic Status}

The mean economic hardship score for the entire sample during the one-year period prior to incarceration was $1.6(S D=2.2$, range $=0-8)$, meaning that, on average, participants experienced fewer than 2 episodes of economic hardship during the year prior to their incarceration. Measures of past-year economic hardship were highest among participants reporting past 30-day opioid (1.7) and concomitant use (2.2), the latter approximately double that of participants who reported other drug use (1.1) $(\mathrm{p}<.001)$. A total of $14.8 \%$ of participants reported experiencing homelessness during this same time period. Experiencing pastyear homelessness was highest among participants who reported past 30-day concomitant use (19.4\%), nearly double that of participants who reported past 30-day other drug use $\left(9.9 \%, X^{2}=17.01, \mathrm{df}=3, \mathrm{p}<.001\right)$. Participants who reported past 30-day opioid use also reported relatively higher rates of past-year homelessness $(16.1 \%)$.

The average subjective social comparison rating was $5.0(S D=2.0$, range 1-10). This means that perceived socioeconomic status for the sample was, on average, one in which participants' subjective social comparison equated to being "worse off" than approximately one-half of people in US society in terms of social and economic status (or "better off", depending on how one wishes to interpret this). Among participants who reported past 30-day opioid and concomitant use, the average rank of perceived socioeconomic status was just under the sample mean of 5.0, at 4.8 and 4.7 respectively, differing from participants who reported past 30-day stimulant and other drug use, which had the same mean rank of 5.3 $(\mathrm{p}<.001)$.

\section{Misregulation}

In the 7-day period prior to incarceration, $83.1 \%$ of participants reported using drugs (e.g., alcohol, prescription drugs, illicit drugs) to reduce stress, anxiety, worry or fear. This means that not only did a majority of participants report experiencing stress, anxiety, worry, or fear for a portion of the 30-day period prior to incarceration, but also that a majority sought out drugs as a means of coping with these states. 
Using a psychoactive drug to ameliorate fear, stress, worry, or anxiety in the week prior to incarceration was highest among participants who reported past 30-day concomitant use and lowest among participants reporting other drug use $\left(91.4 \%\right.$ vs. $\left.68.8 \%, X^{2}=82.05, \mathrm{df}=3, \mathrm{p}<.001\right)$. Nearly $87.0 \%$ of participants who reported past 30-day opioid use also engaged in this form of coping or "self-medication", as did slightly fewer, but still a majority (83.7\%), of participants who reported past 30-day stimulant use.

\section{Non-drug Alternative Reinforcers}

On average, participants reported having fewer than 10 close relationships $(\bar{x}=7.1)$ with people such as family members, friends, children, intimate partners, and other people with whom they felt close ( $S D=5.2$, range=0-56). Participants who reported past 30-day concomitant and opioid use reported having fewer close relationships on average during the one-year year period prior to incarceration (6.7 and 6.5, respectively), compared to participants who reported past 30-day stimulant use (7.3). Participants who reported past 30-day other drug use had the highest average number of close relationships 7.9 ( $\mathrm{p}=.010$ ).

Compared to spending the majority of time alone, $88.9 \%$ of the sample reported some social interaction (i.e., spending time friends or family) during the one-year period prior to incarceration. Participants who reported past 30-day other drug use had the highest rates of social interaction, though groups did not differ significantly overall $\left(X^{2}=4.33, \mathrm{df}=3, \mathrm{p}=.228\right)$. Specifically, $91.4 \%$ of participants who reported past 30-day other drug use reported spending time with friends or family, versus alone, whereas rates of spending time with friends or family were lowest among participants who reported past 30-day stimulant use (86.5\%). Participants who reported opioid and concomitant use had similar rates at $88.1 \%$ and $89.2 \%$, respectively. Compared to being indifferent to or not enjoying spending their time this way, $70.8 \%$ of the sample reported that they received satisfaction from these social interactions. Participants who reported past 30-day concomitant use had the lowest rates of receiving satisfaction from past-year social interaction, whereas participants who reported past 30-day other drug use had the highest (61.8\% vs. $\left.79.8 \% ; X^{2}=31.30, \mathrm{df}=3, \mathrm{p}<.001\right)$. Rates of satisfaction were similar among participants who reported past 30-day opioid (71.3\%) and stimulant use (73.4\%).

In the year prior to incarceration, the average number of leisure days per week, in which hobbies or enjoyable activities could be freely pursued, was 2.5 for the entire sample $(S D=2.4$, range $=0-7)$. The mean number of leisure days was highest among participants who reported past 30-day other drug use 
(3.3), but slightly lower among participants who reported past 30-day stimulant (2.5), opioid (2.2), and concomitant use (2.0, $\mathrm{p}=.002)$.

Approximately $66 \%$ of participants reported being involved in educational and/or vocational pursuits in the one-year period prior to incarceration. Past-year educational/vocational involvement did not differ between groups $\left(X^{2}=4.29, \mathrm{df}=3, \mathrm{p}=.232\right)$, but was highest among participants who reported past 30 day stimulant use (70.2\%), followed by concomitant (66.2\%), other drug (65.7\%), and opioid use (62.8\%).

\section{Age of Drug Use Initiation}

The mean age of drug use initiation for the entire sample was just over 14 years old $(\bar{x}=14.7$, $S D=4.9$, range=4-56). Participants who reported past 30-day opioid and stimulant use were similar in terms of the average age at which drug use was initiated, at just over 15 years of age; however, age of first drug use occurred at a younger age among participants who reported other drug (14.5) and concomitant use $(13.7 ; \mathrm{p}<.001)$.

\section{Drug Use Severity}

In the 30-day period prior to incarceration, the mean drug use severity score was 23.2 ( $S D=15.8$, range=2-40). Severity scores, which indicate higher degree of being bothered by drug-related problems and more frequent instances of craving, withdrawal, and/or unsuccessful attempts to quit, were highest among participants who reported past 30-day concomitant (30.0) and opioid use (27.3). Participants who reported past 30-day stimulant use had lower scores at 22.3, though even this was still approximately double that of the average severity score for participants who reported past 30-day other drug use (11.2; $\mathrm{p}<.001)$.

Similarly, a greater proportion of participants who reported past 30-day concomitant use also reported being intoxicated at the time that they committed their offense, compared to participants who reported past 30-day other drug use ( $89.0 \%$ vs. $\left.60.2 \%, X^{2}=106.96, \mathrm{df}=3, \mathrm{p}<.001\right)$.

\section{Drug Use Versatility}

Across the entire sample, the average number of drugs used during the one-year period prior to incarceration was $3.8(S D=2.3$ range=1-13). However, among participants who reported past 30-day concomitant use, the average was 6.1, higher than participants who reported past 30-day opioid (3.6) and stimulant use (2.6), and nearly three times more than participants who reported past 30-day other drug use $(2.1 ; \mathrm{p}<.001)$. Sample-wide, the average number of drugs used during the 30-day period prior to 
incarceration was $3.0(S D=2.1$, range $=0-13)$. However, among participants who reported past 30-day concomitant use, the average was 5.3, higher than participants who reported past 30-day opioid (2.9) and stimulant use (2.3), and nearly three times more than participants who reported past 30-day other drug use $(1.1 ; \mathrm{p}<.001)$.

\section{Past-year Drug Use Prior to Incarceration}

Among non-opioid and non-stimulant drugs, alcohol was used in the one-year period prior to incarceration by $63.5 \%$ of the sample, with rates similar among participants who reported past 30-day concomitant and other drug use, approximately $68 \%$. Rates were lowest among participants who reported past 30-day opioid use $\left(54.0 \%, X^{2}=27.2, \mathrm{df}=3, \mathrm{p}<.001\right)$. Just over $60 \%$ of the sample reported past-year cannabis use. Participants who reported past 30-day concomitant use had the highest rates of past-year cannabis use (72.0\%), followed by participants who reported past 30-day other drug use (63.6\%). This differed from participants who reported past 30-day opioid and stimulant use, among whom just over half reported past-year cannabis use $\left(X^{2}=42.10, \mathrm{df}=3, \mathrm{p}<.001\right)$. Thirty-nine percent of the sample reported pastyear prescription sedative use, but only $14.2 \%$ of participants who reported past 30-day stimulant use and $19.9 \%$ of participants who reported past 30-day other drug use reported past-year prescription sedative use; rates were highest among participants who reported past 30 -day concomitant use $\left(66.9 \%, X^{2}=282.28, \mathrm{df}=3\right.$, $\mathrm{p}<.001)$. Past-year hallucinogen use was relatively low sample-wide (6.1\%), with rates among groups lowest among participants who reported past 30-day other drug use (2.1\%) and highest among those who reported past 30-day concomitant use $\left(13.0 \%, X^{2}=26.27, \mathrm{df}=3, \mathrm{p}<.001\right)$. Synthetic drugs were also not widely used across the sample during the year prior to incarceration (10.1\%), but similar to past-year hallucinogen use, rates were lowest among participants reporting past 30-day other drug use (4.2\%) and highest among those reporting past 30 -day concomitant use $\left(19.6 \%, X^{2}=66.63, \mathrm{df}=3, \mathrm{p}<.001\right)$. Still, between 7-8\% of participants who reported past 30-day opioid and stimulant use reported past-year synthetic drug use.

Although the rates of past-year use of stimulant and opioid drugs would necessarily differ as a function of past 30-day use of opioids and stimulants, it was important to determine differences for particular types of drugs used during the one-year period prior to incarceration, as it may be that 
participants who reported past 30-day stimulant use used cocaine/crack cocaine, but not amphetamines and vice versa. Here, the proportions of use for a specific drug are more informative than the test statistic itself. For stimulant drugs, $33.5 \%$ of the sample reported using cocaine/crack cocaine during the oneyear prior to incarceration. Rates of cocaine/crack cocaine use were highest among participants who reported past 30-day concomitant use (63.8\%), followed by past 30-day stimulant use $\left(47.5 \%, X^{2}=380.04\right.$, $\mathrm{df}=3, \mathrm{p}<.001$ ). Participants who reported past 30-day opioid and other drug use had similar rates, at just over $12.0 \%$. Nearly $37.0 \%$ of the sample reported past-year amphetamine use. Rates were highest among participants who reported past 30-day concomitant (72.0\%) and stimulant use (62.5\%) and lowest among participants who reported past 30-day other drug $(10.2 \%)$ and opioid use $\left(7.4 \%, X^{2}=602.79, \mathrm{df}=3, \mathrm{p}<.001\right)$.

For opioid drugs, $21.8 \%$ of the sample reported using heroin during the one-year period prior to incarceration. Rates were highest among participants who reported past 30-day concomitant use (40.0\%), followed by participants who reported past 30-day opioid use (30.9\%). Only 5.5\% of participants who reported past 30-day other drug use and only $0.4 \%$ of participants who reported past 30 -day stimulant use reported past-year heroin use prior to incarceration $\left(X^{2}=244.95, \mathrm{df}=3, \mathrm{p}<.001\right)$. Compared to heroin, a greater proportion of the sample reported past-year prescription opioid use (55.8\%). Participants who reported past 30-day concomitant use had the highest rates of past-year prescription opioid use (91.4\%), followed by participants reporting past 30-day opioid use (86.5\%). These rates differed from those who reported past 30-day other drug (15.4\%) and stimulant use $\left(4.6 \%, X^{2}=955.41, \mathrm{df}=3, \mathrm{p}<.001\right)$. Sample-wide, past-year use of buprenorphine was also higher, albeit slightly, compared with heroin, with $24.9 \%$ reporting use. While only $1.8 \%$ of participants who reported past 30-day stimulant use reported past-year buprenorphine use, $44.4 \%$ of participants who reported past 30-day concomitant use and $35.9 \%$ of participants who reported past 30-day opioid use reported past-year buprenorphine use; only $6.0 \%$ of participants who reported past 30-day other drug use reported past-year buprenorphine use $\left(X^{2}=274.12\right.$, $\mathrm{df}=3, \mathrm{p}<.001)$.

\section{Past-year Drug Use Frequency Prior to Incarceration}

Frequency, measured in months (results not shown in table), that a particular drug was used during the one-year period prior to incarceration, varied from an average of 0.2 month to 6.2 months. Included among non-opioid and non-stimulant drugs was alcohol, used an average of 4.6 months, cannabis, used an 
average of 5.1 months, prescription sedatives, used an average of 2.7 months, hallucinogens, used an average of 0.2 months, and synthetic drugs, used an average of 0.5 months. The average number of months out of the one-year period prior to incarceration that alcohol was used was fairly uniform across groups, ranging from 3.4 months among participants who reported past 30-day opioid use and 5.1 months among participants who reported past 30-day other drug use $(\mathrm{p}=.306)$. The average number of months that cannabis was used was highest among participants who reported past 30-day concomitant use (6.1), followed by participants who reported other drug use (5.2), though groups did not differ overall $(\mathrm{p}=.938)$. For prescription sedatives, the average number of months that these were used was highest among participants who reported past 30-day concomitant (4.8) and opioid use (3.2) compared to other groups $(\mathrm{p}<.001)$. Hallucinogens, which were less frequently used sample-wide, were used a greater number of months on average among participants who reported past 30-day concomitant use (1.1), whereas all other groups used hallucinogens $1<$ month on average (range= $0.2-0.4 ; \mathrm{p}<.001$ ). Synthetic drugs, also less frequently used, were used a greater number of months among participants who reported past 30-day concomitant use (2.2), compared to participants who reported past 30-day opioid (0.5), stimulant (0.9) and other drug use $(0.6 ; \mathrm{p}<001)$.

For stimulant drugs, amphetamines were used on average 6.2 months, making it the most frequently used drug for this time period as measured in months. Cocaine/crack cocaine was used an average of 1.8 months. Unsurprisingly, the average number of months that cocaine/crack cocaine was used was highest among participants who reported past 30-day stimulant and concomitant use, 3.2 and 3.5, respectively $(\mathrm{p}<.001)$. Participants who reported past 30-day opioid use used cocaine/crack cocaine on average $1<$ month (0.3), as did participants who reported past 30-day other drug use (0.5). Similarly, amphetamine use was highest among participants who reported past 30-day stimulant and concomitant use, 5.3 and 5.4, respectively $(\mathrm{p}<.001)$. Participants who reported past 30-day opioid use also used amphetamines on average $<1$ month $(0.2)$ out of the one-year period prior to incarceration, as did participants who reported past 30-day other drug use (0.4).

For opioid drugs, heroin was used for 1.4 months on average, prescription opioids 4.8 months, and buprenorphine 1.4 months. The average number of months that heroin was used was similar across participants who reported past 30-day opioid and concomitant use, at 2.2 and 2.4 months, respectively; 
however, no participants who reported past 30-day stimulant use reported heroin use, and among participants who reported past 30-day other drug use, the average number of months heroin was used was $<1(0.2 ; \mathrm{p}<.001)$. Prescription opioids were used an average of 7.9 months among participants who reported both past 30-day opioid use as well as past 30-day concomitant use. Among participants who reported past 30-day stimulant use, the average number of months that prescription opioids were used was $<1$ ( 0.1$)$, and among participants who reported past 30-day other drug use, was $0.7(\mathrm{p}=206)$. The average number of months that buprenorphine was used was highest among participants who reported past 30-day concomitant (2.5) and opioid use (2.0). The average number of months that buprenorphine was used among participants who reported past 30-day stimulant or other drug use was $<1$ month, 0.4 and 0.2 , respectively ( $<<.001$ ).

\section{Past 30-day Drug Use Frequency Prior to Incarceration}

Frequency of drug use that occurred during the 30-day period prior to incarceration also varied significantly from an average of 0.3 days to 11.2 days. Included among non-opioid and non-stimulant drugs was alcohol, used an average of 7.3 days, cannabis, used an average of 11.2 days, prescription sedatives, used an average of 5.2 days, hallucinogens, used an average of 0.3 days, and synthetic drugs, used an average of 1.1 days.

Alcohol was used, on average, for approximately the same number of days out of the 30-day period prior to incarceration among participants who reported past 30-day stimulant (8.1), concomitant (8.4), and other drug use (8.3), whereas participants who reported past 30-day opioid use used alcohol for approximately 4.7 days $(\mathrm{p}<.001)$. The average number of days that cannabis was used during the 30 -day period prior to incarceration was highest among participants who reported past 30-day concomitant use (14.9), followed by stimulant (11.1), other drug (10.1), and opioid use (9.1; $p<.001)$. Prescription sedatives were used nearly 10 days on average by participants who reported past 30-day concomitant use, far higher than participants who reported past 30-day opioid (6.1), stimulant (1.1), and other drug use (1.6; $\mathrm{p}<.001)$. Hallucinogens were used $<1$ day on average across groups (range= $=0-0.5$ ), with participants who reported past 30-day other drug use reporting zero days of hallucinogen use for this time period $(\mathrm{p}<.001)$. Synthetic drugs were used 2.2 days on average among participants who reported past 30-day concomitant use and approximately 1.0 day among participants who reported past 30-day stimulant use, still significantly more often than participants who reported past 30-day opioid and other drug use ( $p<.001)$. 
Among stimulant drugs, amphetamines were used an average 6.4 days and cocaine/crack cocaine was used an average of 1.8 days. Between the two groups who reported past 30-day use of stimulants (i.e., stimulants and concomitant use), cocaine/crack cocaine was used for more days on average among participants who reported past 30-day concomitant use compared to stimulant use (7.4 vs. 6.6).

Amphetamine use among these same groups was slightly more frequent among participants who reported past 30-day stimulant versus concomitant use (13.6 vs. 12.9).

Among opioid drugs, heroin was used for 3.7 days on average, prescription opioids 10.4 days, and buprenorphine 2.7 days. Between the two groups who reported past 30-day use of opioids (i.e., opioids and concomitant use), heroin was used approximately the same number of days on average among participants who reported past 30-day opioid (6.1) and concomitant use (6.6). The frequency of prescription opioid use among these same groups was nearly equal, an average of 18 days out of the 30 days prior to incarceration. Among these two groups, buprenorphine was used slightly more days on average by participants who reported past 30-day concomitant use (5.1), compared to participants who reported past 30-day opioid use (4.2).

\section{Past-year Preferred Drug of Choice}

The most commonly preferred drugs across the sample were prescription opioids $(25.2 \%)$, cannabis $(21.3 \%)$, and amphetamines $(15.2 \%)$. Heroin was preferred by $9.8 \%$, and buprenorphine was preferred by $2.6 \%$ of participants. Cocaine was preferred at a comparable rate to heroin at $8.7 \%$. Additional non-opioid and non- stimulant drugs included alcohol, preferred by $13.1 \%$ of participants, prescription sedatives, preferred by $2.9 \%$, and less frequently used drugs such as inhalants, hallucinogens, and synthetic drugs, preferred by $1.3 \%$ collectively. Unsurprisingly, groups differed in terms of their preferred drug of choice for the one-year period prior to incarceration $\left(X^{2}=945.73, \mathrm{df}=24, \mathrm{p}<.001\right)$. Among participants who reported past 30-day concomitant use, prescription opioids (29.8\%), heroin (13.9\%), amphetamines (14.1\%), and cocaine $(11.0 \%)$ were the most preferred drugs. Among participants who reported past 30-day opioid use, prescription opioids (50.7\%), heroin $(17.3 \%)$, cannabis $(14.1 \%)$ and alcohol $(7.2 \%)$ were the most preferred drugs. Among participants who reported past 30-day stimulant use, amphetamines (38.7\%), cocaine/crack cocaine $(25.5 \%)$, cannabis $(19.9 \%)$ and alcohol $(11.3 \%)$ were the most preferred drugs. 
Lastly, among participants who reported past 30-day other drug use, cannabis (50.7\%), alcohol (44.5\%), prescription opioids, (7.1\%), and amphetamines (5.0\%) were the most preferred drugs.

\section{Central Appalachia}

Approximately one-third of participants (31.8\%) reported residing within Central Appalachian, the Easternmost region of Kentucky, for the majority of the one-year period prior to incarceration. A greater proportion of participants who reported past 30-day opioid resided in Central Appalachia (43.9\%), followed by participants who reported concomitant use (35.3\%). These rates differed from the $18.4 \%$ of participants who reported past 30 -day stimulant use and the $23.3 \%$ of participants who reported past 30 -day other drug use $\left(X^{2}=68.90, \mathrm{df}=3, \mathrm{p}<.001\right)$.

Specific opioid and stimulant drug use by region was also examined (chi-square results not shown in-table). Proportions of past 30-day opioid and stimulant drug use by region (Central Appalachia vs. nonAppalachia) are displayed in Figure 5. For past 30-day heroin use, rates were slightly lower among participants who resided in Central Appalachia compared to those who resided outside of Central Appalachia ( $16.3 \%$ vs. $\left.18.4 \%, X^{2}=0.88, \mathrm{df}=1, \mathrm{p}=.314\right)$. Groups by region differed, however, for rates of past 30-day prescription opioid ( $64.8 \%$ vs. $\left.41.8 \%, X^{2}=71.44, \mathrm{df}=1, \mathrm{p}<.001\right)$ and buprenorphine $(30.0 \%$ vs. $\left.13.4 \%, X^{2}=61.23, \mathrm{df}=1, \mathrm{p}<.001\right)$ use, with a higher proportion of participants residing in Central Appalachia for both groups. A greater proportion of participants who resided outside of Central Appalachia reported past 30-day cocaine/crack cocaine use 
Figure 5. Opioid and stimulant use during the 30-day pre-incarceration period by Appalachia and Non-Appalachia Residence.

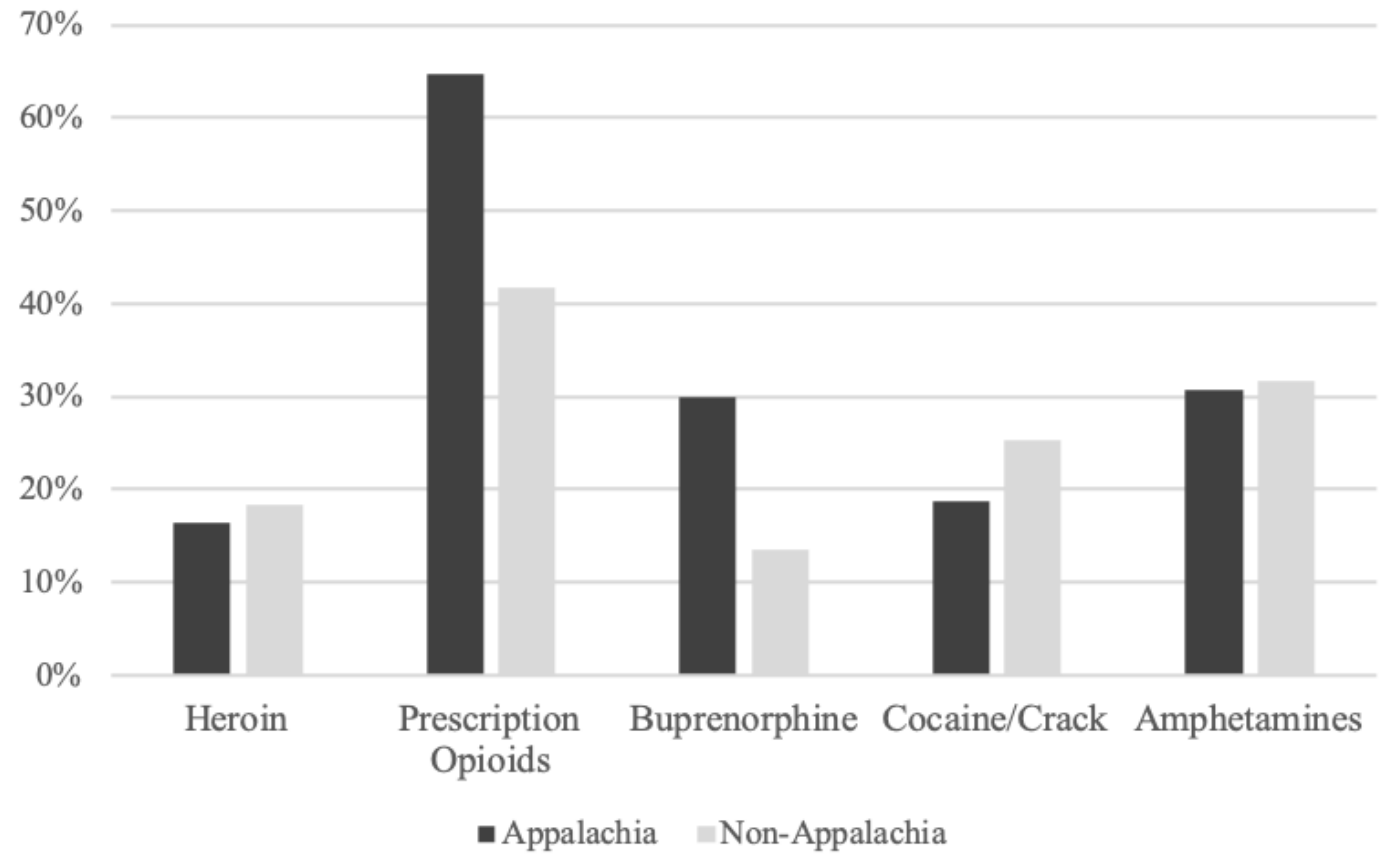

( $18.7 \%$ vs. $\left.25.3 \%, X^{2}=8.31, \mathrm{df}=1, \mathrm{p}=.005\right)$, though no difference was observed for past 30-day amphetamine use ( $30.6 \%$ vs. $\left.31.6 \%, X^{2}=0.18, \mathrm{df}=1, \mathrm{p}=.726\right)$. In addition to these drugs, past 30 -day use of alcohol $\left(41.2 \%\right.$ vs. $\left.53.4 \%, X^{2}=19.47, \mathrm{df}=1, \mathrm{p}<.001\right)$ cannabis $\left(45.9 \%\right.$ vs. $\left.55.6 \%, X^{2}, 12.54, \mathrm{df}=1, \mathrm{p}<.001\right)$ occurred at higher rates for participants residing outside of Appalachia. Past 30-day prescription sedative use also differed by region, with a slightly greater proportion of users residing within Appalachia (36.8\% vs. $\left.30.2 \%, X^{2}=6.78, \mathrm{df}=1, \mathrm{p}=.011\right)$. Because past 30 -day use does not necessarily reflect use over a longer time period, use of specific opioid and stimulant drugs for the one-year period prior to incarceration was also examined. Proportions are displayed in Figure 6. 
Figure 6. Opioid and stimulant use during the one-year pre-incarceration period by Appalachia and Non-Appalachia Residence.

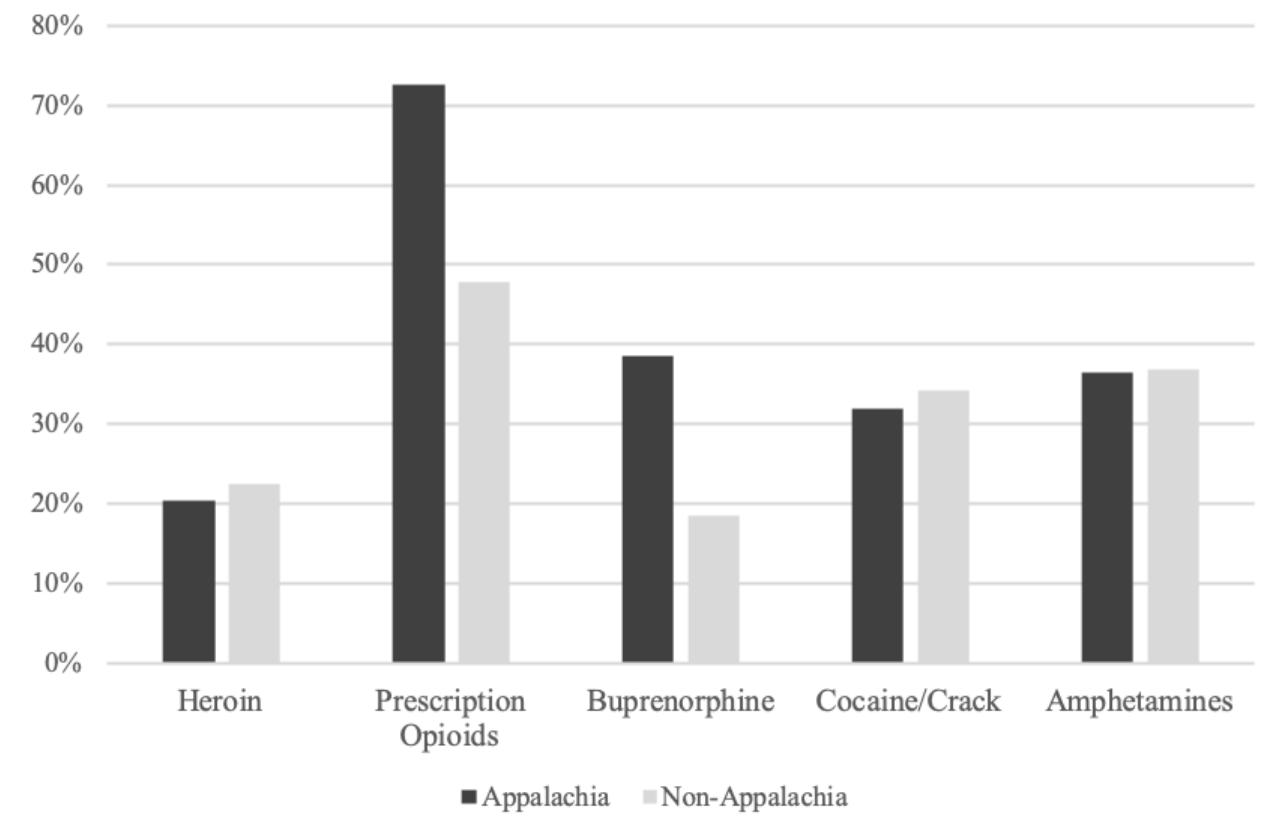

Among participants who reported past-year heroin use, 20.5\% resided in Central Appalachia and $22.4 \%$ resided outside of Central Appalachia $\left(X^{2}=0.72, \mathrm{df}=1, \mathrm{p}=.435\right)$. For the one-year period prior to incarceration, differences were observed for past-year prescription opioid $\left(72.6 \%\right.$ vs. $47.9 \%, X^{2}=82.84$, $\mathrm{df}=1, \mathrm{p}<.001)$ and buprenorphine ( $38.6 \%$ vs. $\left.18.5 \%, X^{2}=72.56, \mathrm{df}=1, \mathrm{p}<.001\right)$ use, with a higher proportion of participants residing in Central Appalachia. Groups by region did not differ significantly for past-year cocaine/crack cocaine use ( $32.0 \%$ vs. $\left.34.2 \%, X^{2}=0.67, \mathrm{df}=1, \mathrm{p}=.413\right)$ or past-year amphetamine use $(36.4 \%$ vs $\left.36.9 \%, X^{2}=0.29, \mathrm{p}=.864\right)$.

\section{Urban Influence}

In keeping with this finding, the mean urban influence for the entire sample, with 1 denoting the greatest urban influence and 12 denoting the least (i.e., more rural, less populated), was 4.6, indicating that the past-year residence of the sample was skewed toward metropolitan and urban areas, rather than toward rural, non-metropolitan areas. The average urban influence designation was higher among participants who reported past 30-day opioid (5.1) and concomitant use (4.7), and lower among participants who reported past 30-day other drug (4.0) and stimulant use (4.3; $\mathrm{p}<.001)$. Figure 7 displays the proportion past 30 -day drug use prior to incarceration for specific opioid and stimulant drugs by urban influence designation. 
Although higher proportions for all drugs were associated with greater urban and metro influence, the highest rate of use by urban influence designation for any specific drug was prescription opioids $(60.0 \%)$, and this was associated with greater rurality. Apart from the two designations indicating greatest urban influence (1 and 2), the next two urban influence designations with the largest proportion of concentrated use were found among designations 8 and 9 (more rural, less populated areas), as evidenced by the modest uptick for all drugs other than heroin, which increased within these designations only slightly. Between designations of 7 and 10 is where rates of prescription opioid use, as noted before, substantially increase. Overall, higher rates of heroin and cocaine use were more likely to be found in designations indicating greater urban influence, whereas prescription opioid, buprenorphine, and amphetamine use showed greater overall dispersion across designations.

Figure 7. Opioid and stimulant use during the 30-day pre-incarceration period by urban influence designation.

$70 \%$

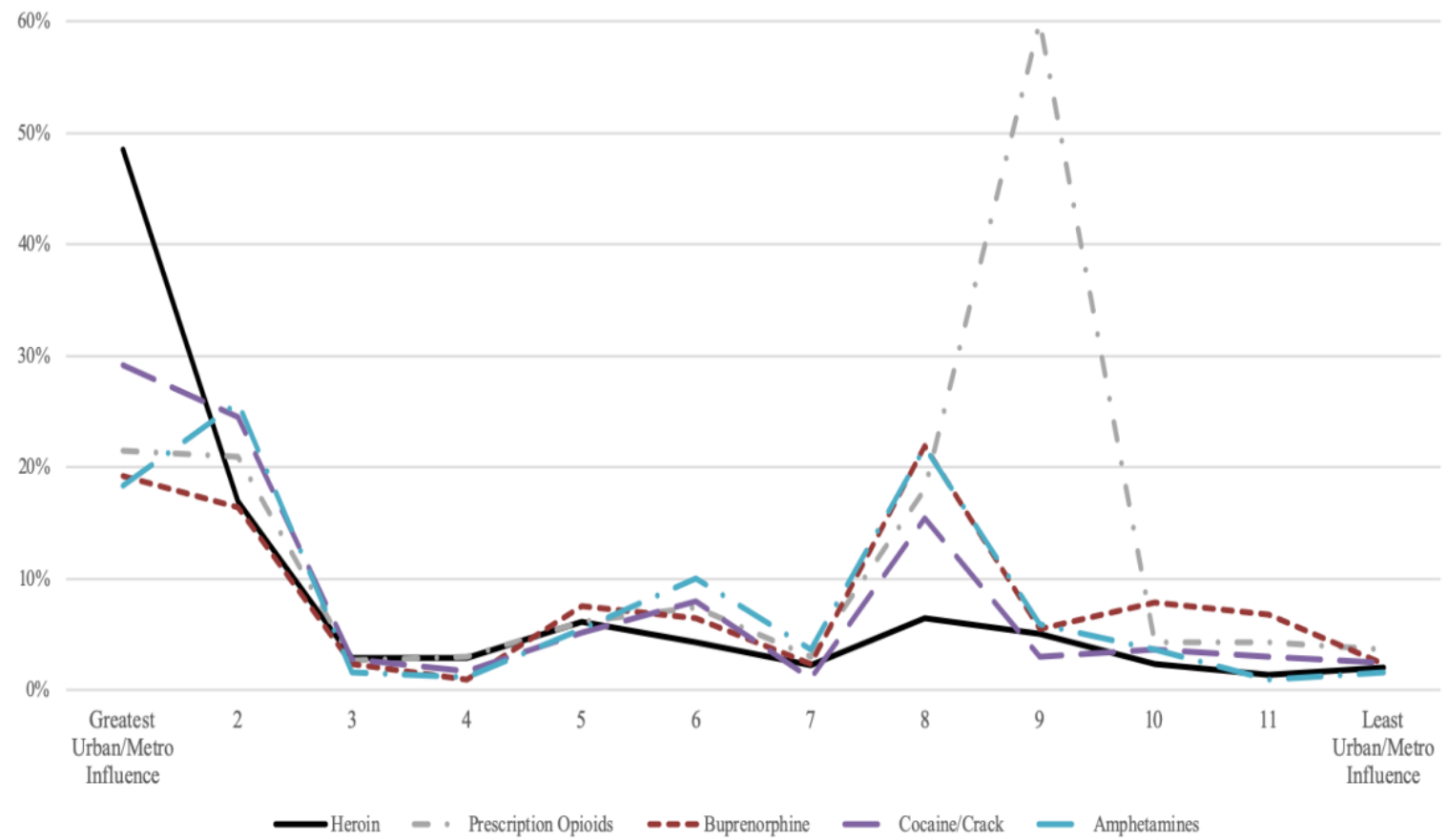




\section{Drug Outcomes During the One-year Period Subsequent to Incarceration}

Table 2 displays means and proportions for the entire sample and for each drug use group for the one-year period subsequent to incarceration. For this post-release period, $11.9 \%$ of participants reported concomitant use of opioid and stimulants, $12.5 \%$ reported opioid, but not stimulant, use; $8.3 \%$ reported stimulant, but not opioid use; $22.9 \%$ reported use of drugs other than opioids and stimulants, and $44.4 \%$ reported no past-year use, a total of $67.3 \%$ for the other/no drugs outcome group.

During the one-year post-release period, the largest proportion of White participants were found among those who reported past-year concomitant (95.2\%) and opioid use (91.5\%), compared to participants who reported past-year stimulant $(77.7 \%)$ and other/no drug use $\left(77.8 \%, X^{2}=56.46, \mathrm{df}=3\right.$, $\mathrm{p}<.001)$. The proportion of participants with a HSD/GED were equivalent across groups $\left(X^{2}=1.67, \mathrm{df}=3\right.$, $\mathrm{p}=.643$ ), with the highest proportion of HSD/GED among participants who reported past-year opioid use (74.4\%), and the lowest among participants reporting past-year stimulant use (68.5\%). Seventy-three percent of the sample was mandated (i.e., parole board ordered) to complete SAP, and 78.3\% completed SAP. Participants who reported past-year opioid use had slightly higher rates of mandated SAP participation (76.4\%), whereas participants who reported past-year stimulant use had the lowest $(66.2 \%)$; though groups did not significantly differ overall $\left(X^{2}=5.10, \mathrm{df}=3, \mathrm{p}=.164\right)$. Rates of SAP completion did not differ between groups either $\left(X^{2}=3.35, \mathrm{df}=3, \mathrm{p}=.341\right)$.

During the one-year post-release period, the mean number of nights spent in a controlled environment was $43.6(S D=77.6$, range $=0-351)$, however, participants who reported past-year other/no drug use reported fewer nights in a controlled environment on average, particularly compared to participants who reported past-year concomitant use (31.7 vs. 78.2; $\mathrm{p}<.001)$. Compared to the sample-wide rate of $42.9 \%$, approximately $65.0 \%$ of participants who reported past-year opioid and concomitant use had a history of IDU, nearly double that of participants who reported past-year stimulant (35.4\%) and other/no drug use $\left(36.0 \%, X^{2}=96.29, \mathrm{df}=3, \mathrm{p}<.001\right)$. 
Table 2

Sample Characteristics and Drug Use Group Means, Proportions, and Between-group Differences Subsequent to Incarceration

\begin{tabular}{|c|c|c|c|c|c|c|}
\hline $\begin{array}{l}\mathrm{N}= \\
\%\end{array}$ & $\begin{array}{r}\text { Sample } \\
1,563\end{array}$ & $\begin{array}{c}\text { Concomitant } \\
186 \\
11.9\end{array}$ & $\begin{array}{c}\text { Opioids } \\
195 \\
12.5\end{array}$ & $\begin{array}{c}\text { Stimulants } \\
\\
130 \\
8.3\end{array}$ & $\begin{array}{c}\begin{array}{c}\text { Other } \\
\text { /none }\end{array} \\
\\
\mathbf{1 , 0 5 2} \\
67.3 \\
\end{array}$ & $\begin{array}{c}\mathbf{p} \\
\text { value }\end{array}$ \\
\hline White & $81.5 \%$ & $95.2 \%$ & $91.5 \%$ & $77.7 \%$ & $77.8 \%$ & .001 \\
\hline HSD/GED & $71.2 \%$ & $72.6 \%$ & $74.4 \%$ & $68.5 \%$ & $70.8 \%$ & .643 \\
\hline Mandated to SAP & $73.0 \%$ & $70.4 \%$ & $76.4 \%$ & $66.2 \%$ & $73.7 \%$ & .164 \\
\hline SAP completion & $78.3 \%$ & $73.7 \%$ & $81.0 \%$ & $77.7 \%$ & $78.7 \%$ & .341 \\
\hline $\begin{array}{l}\text { Nights in a controlled } \\
\text { environment, past-year }\end{array}$ & 43.6 & 78.2 & 54.4 & 75.1 & 31.7 & .001 \\
\hline $\begin{array}{l}\text { Lifetime drug treatment } \\
\text { episodes }\end{array}$ & 2.2 & 3.1 & 3.2 & 2.7 & 1.9 & $\begin{array}{l}.001 \\
.001\end{array}$ \\
\hline Ever IDU & $42.9 \%$ & $64.5 \%$ & $64.6 \%$ & $35.4 \%$ & $36.0 \%$ & \\
\hline $\begin{array}{l}\text { Age } \\
\text { Male }\end{array}$ & $\begin{array}{c}34.6 \\
80.9 \%\end{array}$ & $\begin{array}{c}31.6 \\
79.0 \%\end{array}$ & $\begin{array}{c}32.1 \\
81.0 \%\end{array}$ & $\begin{array}{c}35.6 \\
88.5 \%\end{array}$ & $\begin{array}{c}35.3 \\
80.3 \%\end{array}$ & $\begin{array}{l}.001 \\
.141\end{array}$ \\
\hline Past-year cognitive difficulties & $26.4 \%$ & $38.8 \%$ & $25.6 \%$ & $32.3 \%$ & $23.7 \%$ & .001 \\
\hline $\begin{array}{l}\text { Lifetime history of head } \\
\text { trauma }\end{array}$ & $43.5 \%$ & $49.5 \%$ & $41.0 \%$ & $41.5 \%$ & $43.0 \%$ & .321 \\
\hline Past-year anxiety symptoms & $40.7 \%$ & $58.1 \%$ & $43.1 \%$ & $50.0 \%$ & $36.1 \%$ & .001 \\
\hline Past-year depressive symptoms & $32.7 \%$ & $52.2 \%$ & $35.4 \%$ & $45.5 \%$ & $27.3 \%$ & .001 \\
\hline Past-year suicidal ideation & $4.5 \%$ & $11.3 \%$ & $5.6 \%$ & $6.9 \%$ & $2.8 \%$ & .001 \\
\hline Chronic pain & $32.5 \%$ & $39.8 \%$ & $32.8 \%$ & $31.5 \%$ & $31.4 \%$ & .160 \\
\hline Past-month income & $\$ 1,290$ & $\$ 1,056$ & $\$ 1,311$ & $\$ 1,227$ & $\$ 1,336$ & .001 \\
\hline Close relationships & 6.1 & 5.5 & 5.8 & 6.2 & 6.4 & .001 \\
\hline Social interaction & $88.4 \%$ & $86.6 \%$ & $88.2 \%$ & $89.2 \%$ & $88.7 \%$ & .851 \\
\hline Social satisfaction & $88.9 \%$ & $75.1 \%$ & $84.6 \%$ & $79.2 \%$ & $93.3 \%$ & .001 \\
\hline Perceived social worth & $92.3 \%$ & $87.5 \%$ & $90.3 \%$ & $90.0 \%$ & $93.7 \%$ & .013 \\
\hline $\begin{array}{l}\text { Past-year } \\
\text { educational/vocational } \\
\text { involvement }\end{array}$ & $67.1 \%$ & $60.8 \%$ & $65.6 \%$ & $57.7 \%$ & $69.6 \%$ & .008 \\
\hline On community supervision & $87.0 \%$ & $92.5 \%$ & $84.1 \%$ & $92.3 \%$ & $85.9 \%$ & .014 \\
\hline Age of drug use initiation & 14.7 & 13.6 & 14.3 & 13.7 & 15.3 & .001 \\
\hline \multicolumn{7}{|l|}{$\begin{array}{l}\text { Past } 30 \text {-day drug use prior to } \\
\text { incarceration }\end{array}$} \\
\hline Alcohol & $49.5 \%$ & $50.5 \%$ & $39.0 \%$ & $51.4 \%$ & $51.0 \%$ & .019 \\
\hline Cannabis & $52.5 \%$ & $55.4 \%$ & $53.3 \%$ & $61.5 \%$ & $50.8 \%$ & 102 \\
\hline Sedatives & $32.3 \%$ & $52.2 \%$ & $40.5 \%$ & $24.6 \%$ & $28.2 \%$ & .001 \\
\hline Hallucinogens & $3.2 \%$ & $7.0 \%$ & $3.1 \%$ & $3.1 \%$ & $2.6 \%$ & .001 \\
\hline Synthetics & $7.7 \%$ & $11.8 \%$ & $8.7 \%$ & $10.8 \%$ & $6.4 \%$ & .001 \\
\hline Cocaine/crack cocaine & $23.2 \%$ & $32.8 \%$ & $17.9 \%$ & $30.0 \%$ & $21.7 \%$ & .001 \\
\hline Amphetamines & $31.3 \%$ & $48.4 \%$ & $22.6 \%$ & $50.0 \%$ & $27.6 \%$ & .001 \\
\hline Heroin & $17.7 \%$ & $27.4 \%$ & $35.4 \%$ & $3.8 \%$ & $14.4 \%$ & .001 \\
\hline
\end{tabular}




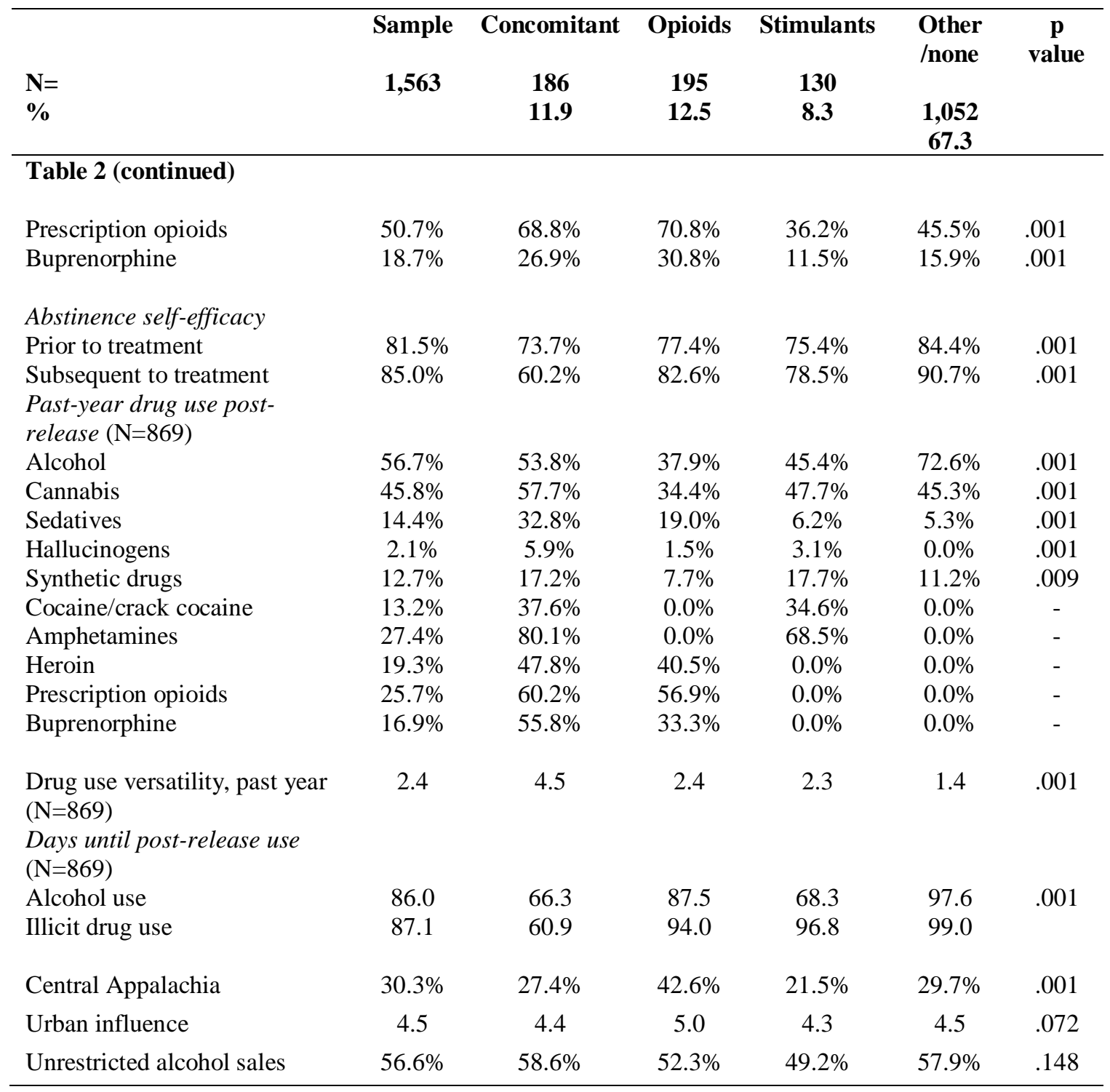




\section{Age and Sex}

During the one-year post-release period, the sample was 34.5 years old $(S D=9.1$, range $=19-75)$ on average. Participants who reported past-year stimulant and other drug/no drug use were relatively older, 35.6 and 35.3, respectfully, compared to participants who reported past-year opioid (32.1) and concomitant use (31.6; $\mathrm{p}<.001)$. The proportion of males did not differ by group $\left(X^{2}=6.07, \mathrm{df}=3, \mathrm{p}=.141\right)$, but was highest among participants who reported past-year stimulant use and lowest among participants reporting past-year concomitant use (88.5\% vs. $79.0 \%)$.

\section{Past-year Cognitive Difficulties and History of Head Trauma}

For the one-year post-release period, $26.4 \%$ of participants reported experiencing past-year cognitive difficulties (i.e., trouble understanding, concentrating, or remembering). Rates of past-year cognitive difficulties varied by group. Participants who reported past-year concomitant use had the highest rates $(38.8 \%)$, followed by participants who reported past-year stimulant (32.3\%), opioid (25.6\%), and other/no drug use $\left(23.7 \% ; X^{2}=20.92, \mathrm{df}=3, \mathrm{p}<.001\right)$. Subsequent to incarceration, approximately $44 \%$ of participants reported a history of head trauma. Rates of head trauma history were highest among participants who reported concomitant use and lowest among participants who reported other/no drug use (49.5\% vs $43.0 \%)$, though groups did not differ significantly overall $\left(X^{2}=3.50, \mathrm{df}=3, \mathrm{p}=.321\right)$.

\section{Psychiatric Symptoms}

Anxiety and depressive symptoms during the one-year post-release period were experienced by $40.7 \%$ and $32.7 \%$ of participants, respectively. Groups differed in the proportion of participants who reported experiencing past-year depressive symptoms $\left(X^{2}=56.10, \mathrm{df}=3, \mathrm{p}<.001\right)$ as well as past-year anxiety symptoms $\left(X^{2}=37.47, \mathrm{df}=3, \mathrm{p}<.001\right)$. Experiencing past-year anxiety symptoms was more common among participants who reported past-year concomitant use and less common among participants who reported past-year other/no drug use (58.1.\% vs. 36.1\%). Over half of participants who reported past-year concomitant use reported past-year depressive symptoms (52.2\%), nearly double that of participants who reported past-year other drug/no drug use (27.3\%). However, over one-third of participants who reported past-year opioid and stimulant use also reported depressive symptoms, $35.4 \%$ and $45.5 \%$, respectively. Past-year suicidal ideation was reported by $4.5 \%$ of the sample during the one-year post-release period. Past-year suicidal ideation was highest among participants who reported past-year concomitant use 
(11.3\%), followed by participants who reported past-year stimulant use (6.9\%.). Participants who reported past-year other/no drug use had the lowest rates (2.8\%), followed by those who reported past-year opioid use $\left(5.6 \% ; X^{2}=29.91, \mathrm{df}=3, \mathrm{p}<.001\right)$.

\section{Chronic Pain}

During the one-year post-release period, $32.5 \%$ of the sample reported experiencing chronic pain. Groups were similar for rates of chronic pain $\left(X^{2}=5.17, \mathrm{df}=3, \mathrm{p}=.160\right)$, with rates highest among participants who reported past-year concomitant use (39.8\%). Among participants who reported past-year opioid use, $32.8 \%$ reported experiencing chronic pain.

\section{Past-month Income}

During the one-year post-release period, the average past-month income from all income sources (e.g., disability benefits, earned income, money received from friends and family) among participants was $\$ 1,290$, meaning that all groups might be considered as low-income, when not taking into account the potential contributions of other household members' income (which was not asked as part of this question). Participants who reported past-year other/no drug use had a higher average past-month income, whereas participants who reported past-year concomitant use had the lowest $(\$ 1,336$ vs. $\$ 1,056, p<.001)$, a modest practical difference.

\section{Non-drug Alternative Reinforcers}

During the one-year post-release period, the mean number of close relationships that participants reported having was 6.1 ( $S D=5.5$, range=0-78). The average number of close relationships ranged from 5.56.4 across groups. Participants who reported past-year stimulant and other/no drug use reported more close relationships on average, 6.2 and 6.4, respectively. Participants who reported past-year concomitant use reported having the fewest close relationships $(5.5, \mathrm{p}<.001)$.

Compared to spending the majority of time alone, $88.4 \%$ of the sample reported some social interaction with friends or family during the one-year post-release period. Groups were similar in terms of reported social interaction $\left(X^{2}=0.80, \mathrm{df}=3, \mathrm{p}=.851\right)$, with $>85.0 \%$ of all groups reporting some social interaction. Compared to being indifferent to or not enjoying spending their time in this way, $88.9 \%$ of participants reported that they received satisfaction from these social interactions. Groups differed, however, in terms of the social satisfaction they received from this past-year social interaction $\left(X^{2}=72.22\right.$, 
$\mathrm{df}=3, \mathrm{p}<.001)$. Specifically, $75.1 \%$ of participants who reported past-year concomitant use received satisfaction from past-year social interaction, whereas $79.2 \%$ of participants who reported past-year stimulant use, and $84.6 \%$ of participants who reported past-year opioid use, reported receiving satisfaction from past-year social interaction. Among participants who reported past-year other drug/no drug use, 93.3\% reported receiving satisfaction from past-year social interaction, the highest rate of all groups.

The majority of the sample (92.3\%) reported feeling as though they had some social worth, meaning that they reported feeling cared about and supported by people in their lives. Rates were highest, however, among participants who reported past-year other/no drug use and lowest among those who reported past-year concomitant use $\left(93.7 \%\right.$ vs. $\left.87.5 \%, X^{2}=10.79, \mathrm{df}=3, \mathrm{p}=.013\right)$. Participants who reported past-year opioid and stimulant use had similar rates of approximately $90.0 \%$.

Approximately $67 \%$ of the sample reported being involved in either educational or vocational pursuits during the one-year post-release period, though such involvement was lowest among participants who reported past-year stimulant (57.7\%) and concomitant use (60.8\%), and highest among participants who reported past-year other drug/no drug use $\left(69.6 \%, X^{2}=11.72, \mathrm{df}=3 \mathrm{p}=.008\right)$.

Finally, while the majority of participants across all groups (87.0\%) were on community supervision at some point during the one-year post-release period, rates were highest for participants who reported past-year concomitant (92.5\%) and stimulant use (92.3\%), and lowest among participants who reported past-year opioid $(84.1 \%)$ and other/no drug use $\left(85.9 \% ; X^{2}=10.68, \mathrm{df}=3 ; \mathrm{p}=.014\right)$.

\section{Age of Drug Use Initiation}

The mean age of drug use initiation was 14.7 ( $S D=4.9$, range=4-56). Drug use groups for the oneyear period subsequent to incarceration differed, though, with older drug use initiation observed among participants who reported past-year other/no drug use (15.3), followed by participants who reported pastyear opioid use (14.3; $\mathrm{p}<.001)$. Age of drug use initiation was $<14$ for participants who reported past-year stimulant and concomitant use, 13.7 and 13.6 years of old, respectively.

\section{Past 30-day Drug Use and Drug Use Frequency Prior to Incarceration}

During the one-year post-release period, groups differed for both rates of use during the 30-day period prior to incarceration as well as frequency of use (frequency of use not shown in table), measured in days, for this same pre-incarceration time period. 
For non-opioid and non-stimulant drugs used during the 30-day period prior to incarceration, rates of pre-incarceration alcohol use were similar across all groups examined (approximately 50.0\%), except among those who reported past-year opioid use $\left(39.0 \%, X^{2}=9.94 ; \mathrm{df}=3, \mathrm{p}=.019\right)$. Pre-incarceration alcohol use was more frequent among participants who reported past-year other drug/non-drug (7.7) and stimulant use (7.6), whereas participants who reported past-year opioid use drank alcohol an average of only 5.5 days for this same time period ( $\mathrm{p}<.001)$. Rates of pre-incarceration cannabis use did not differ between groups $\left(X^{2}=6.21, \mathrm{df}=3, \mathrm{p}=.102\right)$, however, cannabis was used prior to incarceration most often among participants who reported past-year stimulant use (13.9), whereas all other groups reported cannabis use for approximately 11 days prior to incarceration $(\mathrm{p}<.001)$. Participants who reported past-year concomitant and opioid use reported higher rates of pre-incarceration prescription sedative use, 52.2\% and 40.5\%, respectively $\left(X^{2}=51.1, \mathrm{df}=3, \mathrm{p}<.001\right)$. These groups used prescription sedatives an average of 7.5 and 6.9 days, respectively $(\mathrm{p}<.001)$. Hallucinogens were used $<1$ day on average prior to incarceration across groups, though the average number of days was highest among participants who reported past-year concomitant use $(0.6, \mathrm{p}=.019)$; this group also had the highest rate of use $\left(7.0 \%, X^{2}=10.1, \mathrm{df}=3, \mathrm{p}<.001\right)$. Participants who reported past-year concomitant use also reported using synthetic drugs prior to incarceration at higher rates $(11.8 \%)$ followed by participants who reported past-year stimulant use $(10.8 \%$, $\left.X^{2}=9.12, \mathrm{df}=3, \mathrm{p}<.001\right)$.

For stimulant drugs, rates of cocaine/crack cocaine use prior to incarceration were highest among participants who reported past-year concomitant (32.8\%) and stimulant use (30.0\%), though $17.9 \%$ of participants who reported past-year opioid use and $21.7 \%$ of participants who reported past-year other/no drug use also reported pre-incarceration cocaine/crack cocaine use $\left(X^{2}=17.37, \mathrm{df}=3, \mathrm{p}<.001\right)$. The average number of days that cocaine/crack cocaine was used prior to incarceration was greatest among participants who reported past-year stimulant (5.3) and concomitant use (5.0), differing from participants who reported past-year opioid (2.2) and past-year other drug/no drug use (3.0; $\mathrm{p}<.001)$. Groups also differed for rates $\left(X^{2}=60.15, \mathrm{df}=3, \mathrm{p}<.001\right)$ and average number of days $(\mathrm{p}<.001)$ of pre-incarceration amphetamine use. Fifty percent of participants who reported past-year stimulant use reported using amphetamines prior to incarceration, for 11.6 days on average. Nearly the same proportion of participants who reported past-year concomitant use (48.4\%) also reported pre-incarceration amphetamine use, for 8.5 days on average. Still, 
approximately a quarter of participants who reported past-year opioid and other/no drug use also reported pre-incarceration amphetamine use, using 3.9 and 5.6 days, respectively.

For opioid drugs, rates of pre-incarceration heroin use were highest among participants who reported past-year opioid (35.4\%) and concomitant use $\left(27.4 \%, X^{2}=78.61, \mathrm{df}=3, \mathrm{p}<.001\right)$. These groups also reported using heroin a greater number of days prior to incarceration, 8.4 and 5.4, respectively, compared to participants who reported past-year stimulant (0.9) and other/no drug use (2.8; $\mathrm{p}<.001)$. Similarly, participants who reported past-year opioid (70.8\%) and concomitant use (68.8\%) reported higher rates of pre-incarceration prescription opioid use compared to participants who reported past-year stimulant (36.2\%) and other/no drug use $\left(45.5 \%, X^{2}=70.09, \mathrm{df}=3, \mathrm{p}<.001\right)$. Participants who reported past-year opioid and concomitant use reported using prescription opioids more days on average prior to incarceration, 16.2 and 14.9, respectively, compared to participants who reported past-year other/no drug (9.8) and stimulant use (5.3; $\mathrm{p}<.001)$. Buprenorphine was used prior to incarceration by all groups, though rates were higher among participants who reported past-year opioid (30.8\%) and concomitant use $\left(26.9 \%, X^{2}=36.81, \mathrm{df}=3\right.$, $\mathrm{p}<.001$ ). These groups also reported using buprenorphine more days on average (4.8 and 3.8, respectively), followed by participants who reported past-year other/no drug (2.2) and stimulant use (1.4, p<.001).

\section{Abstinence Self-efficacy Prior and Subsequent to Treatment}

Prior to entering SAP, $81.5 \%$ of the sample reported that they perceived their ability to abstain from alcohol and other drugs as "moderately good" or "very good". Participants who reported past-year other/no drug use had the highest rates of pre-treatment abstinence self-efficacy (84.4\%), compared to participants who reported concomitant (73.7\%), stimulant (75.4\%), and opioid use $\left(77.4 \% ; X^{2}=18.87\right.$, $\mathrm{p}<.001)$. Interestingly, this was also the case for abstinence self-efficacy reported during the one-year postrelease period. At this time, participants who reported past-year other/no drug use had the highest rates of abstinence self-efficacy $(90.7 \%)$ and participants who reported concomitant use had the lowest $(60.2 \%$; $\left.X^{2}=121.5, \mathrm{p}<.001\right)$

\section{Past-year Drug Use Subsequent to Incarceration}

Among participants who reported any drug use during the one-year post-release period $(\mathrm{N}=869)$, non-opioid and non-stimulant drug use varied. Alcohol was used during the one-year post-release period at a fairly high rate sample-wide (56.7\%), with rates of use higher among participants who reported past-year 
other drug $(72.6 \%)$ and concomitant use $\left(53.8 \%, X^{2}=72.36, \mathrm{df}=3, \mathrm{p}<.001\right)$. Participants who reported pastyear opioid use had the lowest rates of past-year alcohol use (37.9\%). Rates of past-year cannabis use were highest among participants who reported past-year concomitant use $(57.7 \%)$, followed by participants who reported past-year stimulant $(47.7 \%)$ and other drug use $\left(45.3 \%, X^{2}=20.82, \mathrm{df}=3, \mathrm{p}<.001\right)$. For this time period, prescription sedatives were used at higher rates among participants who reported past-year concomitant use, with rates lowest among participants who reported past-year other drug use (32.8\% vs. $\left.5.3 \%, X^{2}=85.34, \mathrm{df}=3, \mathrm{p}<.001\right)$, though nearly one-fifth of participants who reported past-year opioid use also reported past-year prescription sedative use. Hallucinogens were used by $<10 \%$ of all groups, but were used at higher rates among participants who reported past-year concomitant use $\left(5.9 \%, X^{2}=22.03, \mathrm{df}=3\right.$, $\mathrm{p}<.001)$. Synthetic drug use was approximately equivalent between participants who reported past-year stimulant and concomitant use, at just above 17\%. This differed from participants who reported past-year opioid $(7.7 \%)$ and other drug use $\left(11.2 \%, X^{2}=11.52, \mathrm{df}=3, \mathrm{p}=.009\right)$.

Among this subsample that reported any drug use during the one-year post-release period, $13.2 \%$ reported past-year cocaine/crack cocaine use. This was divided among participants who reported past-year concomitant and stimulant use, with $37.6 \%$ of participants who reported past-year concomitant use and $34.6 \%$ who reported past-year stimulant use reporting cocaine/crack cocaine use specifically. Nearly $28 \%$ of this subsample reported past-year amphetamine use, with $80.1 \%$ of participants who reported past-year concomitant use and $68.5 \%$ who reported past-year stimulant use reporting amphetamine use specifically. Heroin was used by $19.3 \%$ of this subsample, with $47.8 \%$ of participants who reported past-year concomitant use and $40.5 \%$ of participants who reported past-year opioid use reporting heroin use specifically. Prescription opioids were used by $25.7 \%$ of this subsample, with $60.2 \%$ of participants who reported past-year concomitant use and $56.9 \%$ of participants who reported past-year opioid use reporting prescription opioid use specifically. Buprenorphine was used by $16.9 \%$ of this subsample, with $55.8 \%$ of participants who reported past-year concomitant use and $33.3 \%$ who reported past-year opioid use reporting buprenorphine use specifically.

Among this subsample who reported drug use for the one-year post-incarceration period, the average number of different drugs used was 2.4. The highest rates were among participants who reported 
past-year concomitant use (4.5). This differed from participants who reported past-year opioid and stimulant use, who used approximately 2.3 different drugs on average $(\mathrm{p}<.001)$.

\section{Days Until Post-Release Use}

Among this subsample, the number of days between participants' release date and their first incidence of alcohol or other drug use was approximately the same, with 86.0 days out before the first incidence of alcohol use and 86.7 days out before the first incidence of illicit drug use. Participants who reported past-year concomitant use used alcohol and drugs earlier than participants in all other groups, with use occurring between 60-70 days on average subsequent to release. Participants who reported past-year other drug use went approximately 97-98 days subsequent to release before using alcohol or other drugs, the longest for all groups. This was followed by participants who reported past-year opioid use, who did not report using alcohol until nearly 88 days after their release and who did not report using illicit drugs until 94 days after their release.

\section{Central Appalachia}

During the one-year post-release period, $30.3 \%$ of participants reported residing in Central Appalachia for the majority of time. A greater proportion of participants who reported past-year opioid resided in Central Appalachia $\left(42.6 \%, X^{2}=19.41, \mathrm{df}=3, \mathrm{p}<.001\right)$. This was followed by participants who reported past-year other/no drug (29.7\%), concomitant (27.4\%), and stimulant (22.0\%). Specific opioid and stimulant drug use by region were also examined. Proportions of past-year specific opioid and stimulant drug use by region (Central Appalachia vs. non-Appalachia) are displayed in Figure 8.

For past-year heroin use, rates were lower among participants who resided in Central Appalachia compared to those who resided outside of Appalachia (6.1\% vs. $\left.12.8 \%, X^{2}=15.20, \mathrm{df}=1, \mathrm{p}<.001\right)$. Groups by region also differed for rates of past-year prescription opioid $\left(17.9 \%\right.$ vs. $\left.12.7 \%, X^{2}=7.47, \mathrm{df}=1, \mathrm{p}=.008\right)$ and buprenorphine $\left(13.3 \%\right.$ vs. $\left.7.7 \%, X^{2}=12.06, \mathrm{df}=1, \mathrm{p}<.001\right)$ use, with a higher proportion of participants residing in Central Appalachia. Groups by region differed for rates of past-year cocaine/crack 
Figure 8. Opioid and stimulant use during the one-year post-release period by Appalachia and Non-Appalachia residence.

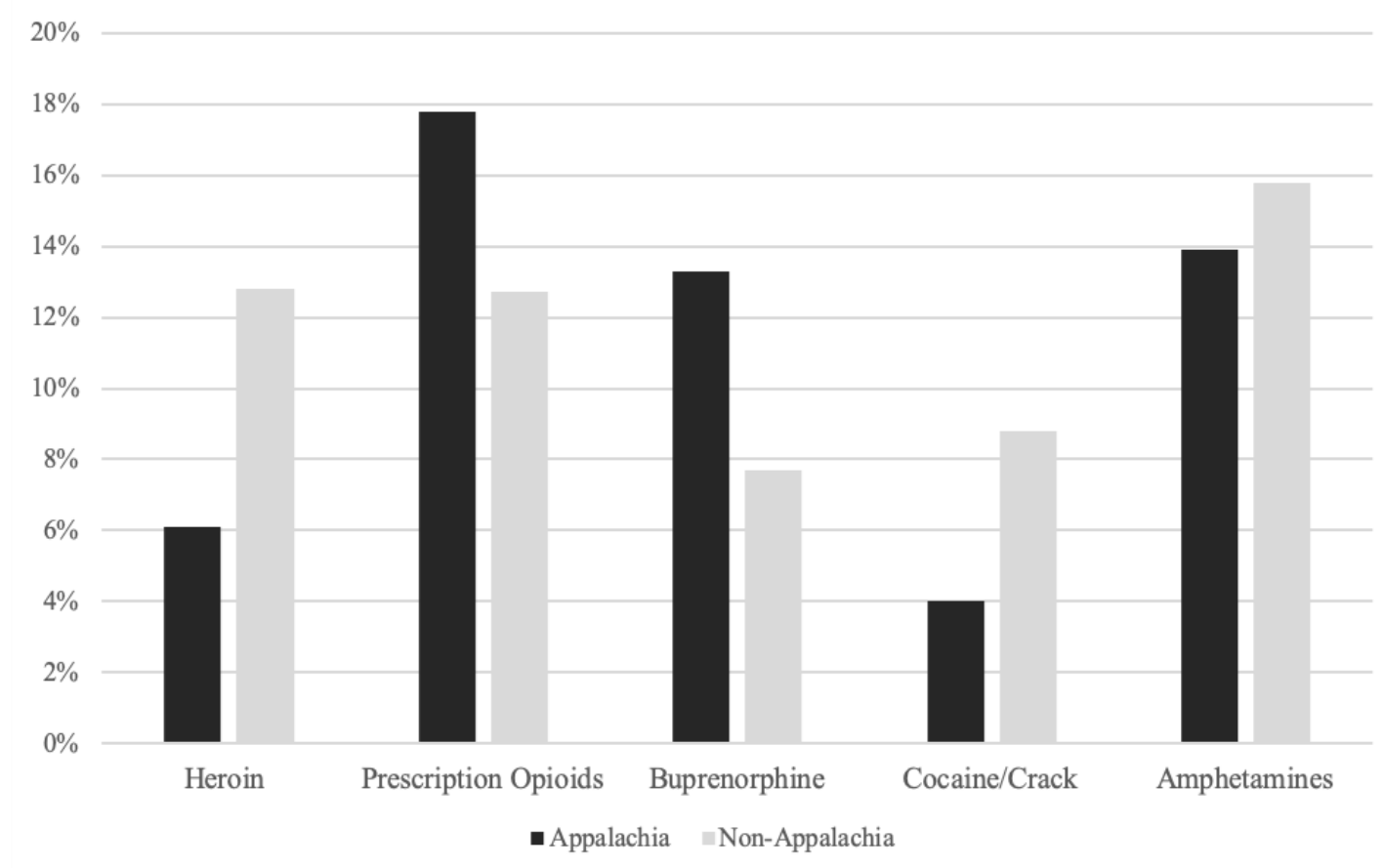

cocaine use $\left(4.0 \%\right.$ vs. $\left.8.8 \%, X^{2}=11.20, \mathrm{df}=1, \mathrm{p}<.001\right)$, but not past-year amphetamine use $(13.9 \%$ vs. $\left.15.8 \%, X^{2}=0.90, \mathrm{p}=.385\right)$. In addition to these drugs, past-year alcohol $\left(23.8 \%\right.$ vs. $34.9 \%, X^{2}=18.69, \mathrm{df}=1$ p<.001) and synthetic drug use ( $4.2 \%$ vs. $8.3 \%$,

$\left.X^{2}=8.26, \mathrm{df}=1, \mathrm{p}=.006\right)$ also differed by region.

\section{Urban Influence}

The mean urban influence for the sample during the one-year period subsequent to incarceration $(\bar{x}=4.5)$ indicated that, similar to participant residence reported for the one-year period prior to incarceration, the sample was comprised of a greater proportion of people living in a metro or urban area. As displayed in Table 2, the average urban influence designation was higher among participants who reported past-year opioid (5.0) and other drug/no drug use (4.5), indicating greater rurality and less population density, but was slightly lower among participants who reported past-year stimulant (4.3) and concomitant use $(4.4 ; \mathrm{p}<.001)$, indicating greater population and urban influence.

Similar to drug use prior to incarceration, higher proportions for all drugs were associated with greater urban and metro influence. However, instead of prescription opioids showing the highest use rates 
in association with less urban influence, the highest rate of use for any drug by urban influence designation during the one-year period subsequent to incarceration was heroin, which was associated with the designation indicating greatest urban influence (56.0\%). Apart from the two designations indicating greatest urban influence (1 and 2), the next two urban influence designations with the largest proportion of concentrated use were found in 6 and 8. Overall, only prescription opioids, buprenorphine, and amphetamines had rates of use across more rural areas that, while still significantly less than those found in areas with greater urban influence, approached something comparable. As shown in Figure 10, cocaine/crack cocaine and heroin use in designations 1 and 2 are over four- and five-fold compared to other designations.

\section{Figure 9. Opioid and stimulant use during the one-year post-release period by urban influence designation.}

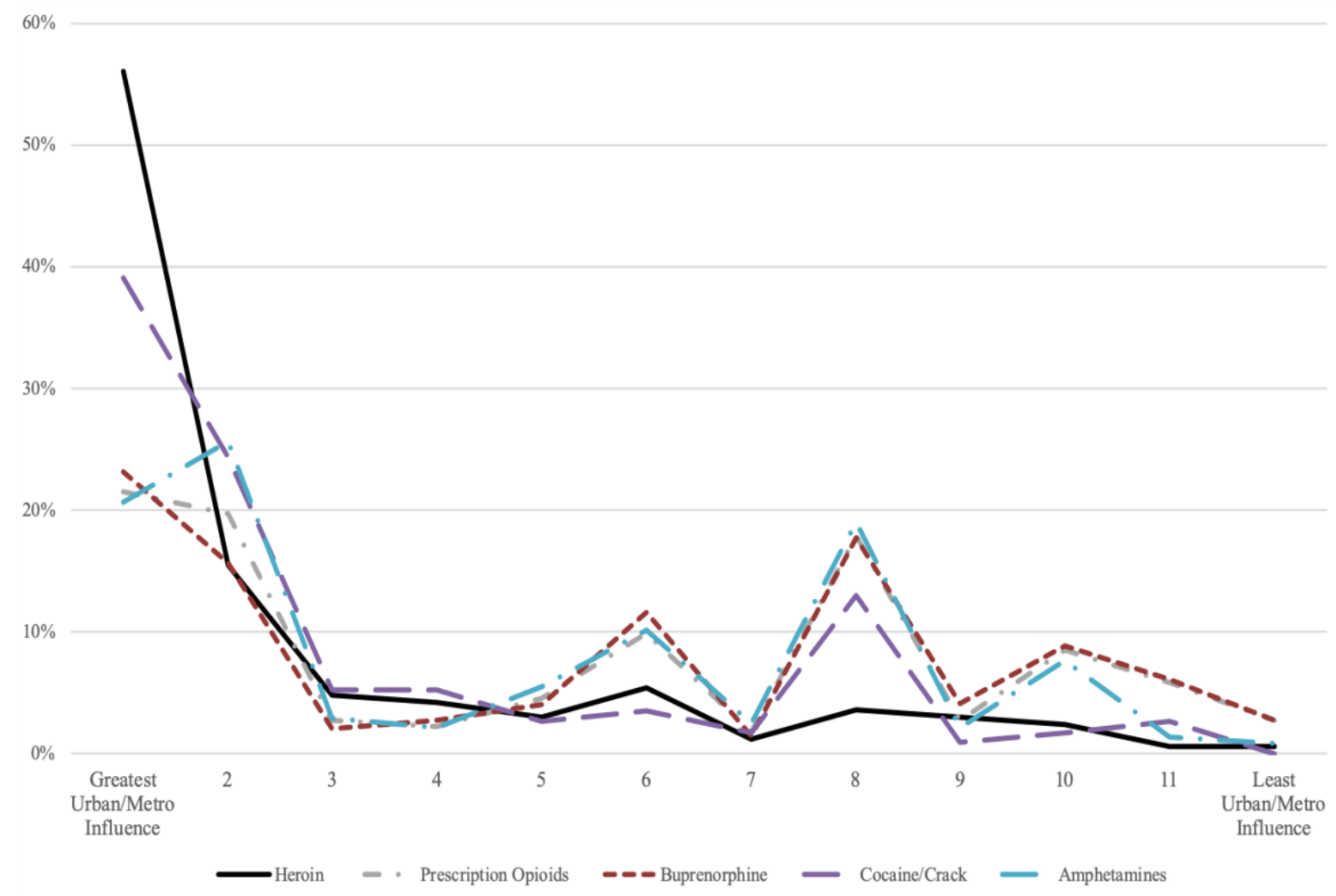




\section{Access to Alcohol}

During the one-year post-release period, $56.6 \%$ of participants resided in a county with unrestricted alcohol sales for the majority of time. Groups were similar in the proportion of participants who resided in a county with unrestricted alcohol sales $\left(X^{2}=5.35, \mathrm{df}=3, \mathrm{p}=.148\right)$, with participants who reported past-year concomitant use having the highest rate $(58.6 \%)$ and participants who reported past-year stimulant use having the lowest rate $(49.2 \%)$

\section{Aim Two Results \\ Multinomial Logistic Regression Results for Drug Outcomes Prior to Incarceration}

Table 3 displays the results of the multinomial logistic regression examining opioid and stimulant outcomes for the 30-day period prior to incarceration, with "other drug use" as the reference group. Independent and control variables (as opposed to variables used for description purposes only; see Measures subsection “Descriptive Variables” pg. 171) which differed significantly by group when examined using nonparametric tests were included into the regression model. The only exception was the variable measuring past-year depressive symptoms, which was omitted due to collinearity with the variable "stress-related health effects". Collinearity was assessed via variance inflation factor (VIF) and 1/VIF (1 minus the $\mathrm{R}^{2}$ obtained with IV regressed on the set of other IVs); an approximate VIF $<1.5$ or $1 / \mathrm{VIF}>0.85$ was considered acceptable (Hair, Anderson, Tatham, \& Black, 1995). VIFs for different models were calculated using different variable configurations (i.e., singularly removing or adding past-year depressive symptoms, stress-related health effects, suicidal ideation, and past-year anxiety symptoms to the model). A decision was made to remove the variable of past-year depressive symptoms, rather than the stress-related health effects variable. This decision was made for three reasons. First, stress-related health effects (measured for the 7-day period prior to incarceration) may have served as a potentially more proximate influence on past 30-day drug-related decision-making and is important in this regard. Second, depressive symptoms were considered as a stress-related health effect, as measured by one question contained in the revised $\mathrm{HCS}$, meaning that by using the variable of stress-related health effects, depression is not entirely ruled out as still possibly exerting some, albeit less discernable, influence on drug use for this time period. That depression and, to a lesser extent, anxiety, were both positively correlated with stress-related health effects (i.e., revised HCS scores) may actually provide some oblique and partial support for the modified 
scale's construct validity. Lastly, past-year suicidality was retained as an independent variable. Though suicidality is not synonymous with depression, it is positively correlated with and conceptually related to it such that it may provide an indirect indicator of past-year depression even with the variable of past-year depressive symptoms removed. Model fit statistics, displayed at the bottom of Table 3, indicated acceptable model fit.

In the first column of Table 3, robust standard errors are displayed. These were calculated to address the fact that some cases were clustered by county and to help establish a more "model-agnostic" model. The specific correction-method that produces robust errors (versus asymptotic standard errors) used was the Huber/White robust estimator of variance (Huber, 1967; Gutierrez \& Drukker, 2019; White, 1982). The Huber/White estimator for obtaining robust variance estimates is available as a command in Stata, and has been utilized to address clustered or nested data, such as cases clustered by county or some other grouping (Froot, 1989; Gutierrez \& Drukker, 2019; Rogers, 1993; Williams, 2000; Wooldridge, 2002). In the second column, corresponding confidence intervals are displayed. The third column displays regression coefficients calculated as relative risk ratios (RRR), which can be conceptualized as increases or decreases in $\log$ odds of being in one of the opioid or stimulant outcome groups (i.e., the ratio of the probability of one of the three outcome groups being chosen over the probability of the reference group of "other drug use" being chosen). By specifying that linear equations be exponentiated, regression coefficients that are the relative risk ratio for a unit change in the independent variables can be produced and displayed. This can be termed as relative risk, or odds. In column four, $\mathrm{p}$ values are displayed.

Table 3

Results of Multinomial Logistic Regression Examining Opioid and Stimulant Use During the 30-day Pre-incarceration Period

\begin{tabular}{lcccc}
\hline & Robust SE & 95\% CI & RRR & p value \\
Concomitant & & & & .607 \\
White & 0.304 & $0.68-1.92$ & 1.15 & .893 \\
Controlled environment, past 30-days & 0.262 & $0.89-0.99$ & 0.94 & .865 \\
Lifetime drug treatment episodes & 0.300 & $0.94-1.05$ & 0.99 & .001 \\
Ever IDU & 0.879 & $2.43-5.88$ & 3.81 & .015 \\
Age & 0.012 & $1.00-1.05$ & 1.03 & .363 \\
Male & 0.383 & $0.74-2.32$ & 1.31 & .996 \\
Lifetime history of head trauma & 0.208 & $0.66-1.50$ & 0.99 & .286 \\
Past-year economic hardship & 0.057 & 0.951 .17 & 1.06 & .122 \\
Past-year homelessness & 0.196 & $0.32-1.11$ & 0.64 & .588 \\
Subjective socioeconomic status & 0.056 & $0.93-1.14$ & 1.03 & .929 \\
Past-week stress related health effects & 0.016 & $0.97-1.03$ & 1.00 & .716 \\
Past-year anxiety symptoms & 0.219 & $0.57-1.46$ & 0.92 & \\
\hline
\end{tabular}




\begin{tabular}{|c|c|c|c|c|}
\hline \multicolumn{5}{|l|}{ Table 3 (continued) } \\
\hline Past-year suicidal ideation & 0.365 & $0.54-2.08$ & 1.06 & .858 \\
\hline Chronic pain & 0.261 & $0.64-1.70$ & 1.04 & .874 \\
\hline Misregulation & 0.524 & $0.94-3.12$ & 1.72 & .077 \\
\hline Close relationships & 0.017 & $0.97-1.03$ & 1.00 & .953 \\
\hline Social satisfaction & 0.195 & $0.52-1.31$ & 0.82 & .422 \\
\hline Leisure time, in days & 0.038 & $0.86-1.01$ & 0.93 & .084 \\
\hline Age of drug use initiation & 0.024 & $1.03-1.12$ & 1.08 & .001 \\
\hline Severity of use & 0.007 & $1.04-1.07$ & 1.06 & .001 \\
\hline Drug use versatility, past-year & 0.310 & $2.59-3.84$ & 3.15 & .001 \\
\hline Central Appalachia & 0.284 & $0.79-1.94$ & 1.24 & .344 \\
\hline \multicolumn{5}{|l|}{ Opioids } \\
\hline White & 0.941 & $2.19-6.01$ & 3.64 & .001 \\
\hline Controlled environment, past 30-days & 0.022 & $0.92-1.00$ & 0.96 & .065 \\
\hline Lifetime drug treatment episodes & 0.027 & $0.93-1.03$ & 0.98 & .509 \\
\hline Ever IDU & 0.569 & $1.90-4.08$ & 2.81 & .001 \\
\hline Age & 0.010 & $0.99-1.03$ & 1.01 & .177 \\
\hline Male & 0.308 & $0.75-1.90$ & 1.23 & .425 \\
\hline Lifetime history of head trauma & 0.204 & $0.81-1.61$ & 1.14 & .465 \\
\hline Past-year economic hardship & 0.050 & $0.91-1.10$ & 1.00 & .959 \\
\hline Past-year homelessness & 0.277 & $0.51-1.62$ & 0.91 & .744 \\
\hline Subjective socioeconomic status & 0.048 & $0.91-1.10$ & 1.00 & .917 \\
\hline Past-week stress related health effects & 0.013 & $0.96-1.00$ & 0.98 & .193 \\
\hline Past-year anxiety symptoms & 0.189 & $0.60-1.35$ & 0.90 & .618 \\
\hline Past-year suicidal ideation & 0.361 & $0.66-2.14$ & 1.19 & .574 \\
\hline Chronic pain & 0.282 & $0.85-1.97$ & 1.30 & .236 \\
\hline Misregulation & 0.427 & $1.21-2.90$ & 1.89 & .005 \\
\hline Close relationships & 0.015 & $0.94-0.99$ & 0.97 & .040 \\
\hline Social satisfaction & 0.244 & $0.80-1.78$ & 1.19 & .399 \\
\hline Leisure time, in days & 0.033 & $0.86-0.96$ & 0.92 & .019 \\
\hline Age of drug use initiation & 0.021 & $1.05-1.13$ & 1.09 & .001 \\
\hline Severity of use & 0.006 & $1.04-1.07$ & 1.06 & .001 \\
\hline Drug use versatility, past-year & 0.161 & $1.51-2.14$ & 1.80 & .001 \\
\hline Central Appalachia & 0.316 & $1.13-2.39$ & 1.64 & .010 \\
\hline \multicolumn{5}{|l|}{ Stimulants } \\
\hline White & 0.209 & $0.72-1.55$ & 1.06 & .774 \\
\hline Controlled environment, past 30-days & 0.023 & $0.89-0.98$ & 0.94 & .014 \\
\hline Lifetime drug treatment episodes & 0.030 & $0.93-1.03$ & 1.00 & .947 \\
\hline Ever IDU & 0.869 & $1.09-2.56$ & 1.68 & .019 \\
\hline Age & 0.012 & $1.03-1.06$ & 1.05 & .001 \\
\hline Male & .0383 & $0.78-2.34$ & 1.35 & .279 \\
\hline Lifetime history of head trauma & 0.208 & $0.73-1.48$ & 1.04 & .834 \\
\hline Past-year economic hardship & 0.057 & $0.91-1.10$ & 1.00 & .930 \\
\hline Past-year homelessness & 0.285 & $0.51-1.62$ & 0.94 & .842 \\
\hline Subjective socioeconomic status & 0.047 & $0.97-1.15$ & 1.06 & .179 \\
\hline Past-week stress-related health effects & 0.013 & $0.97-1.01$ & 0.99 & 657 \\
\hline Past-year anxiety symptoms & 0.198 & $0.60-1.35$ & 0.98 & .923 \\
\hline Past-year suicidal ideation & 0.466 & $0.79-2.74$ & 1.48 & .217 \\
\hline Chronic pain & 0.172 & $0.50-1.18$ & 0.76 & .234 \\
\hline Misregulation & 0.376 & $1.14-2.65$ & 1.74 & .010 \\
\hline Close relationships & 0.012 & $0.97-1.01$ & 0.99 & .452 \\
\hline Social satisfaction & 0.202 & $0.65-1.46$ & 0.98 & .921 \\
\hline
\end{tabular}




\begin{tabular}{lllll}
\hline Table 3 (continued) & & & \\
& & & \\
Leisure time, in days & 0.033 & $0.86-0.99$ & 0.92 & .025 \\
Age of drug use initiation & 0.020 & $1.00-1.08$ & 1.04 & .034 \\
Severity of use & 0.007 & $1.03-1.06$ & 1.05 & .001 \\
Drug use versatility, past-year & 0.109 & $1.13-1.56$ & 1.33 & .002 \\
Central Appalachia & 0.135 & $1.13-2.39$ & 0.63 & .032 \\
& & \\
Intercept-only: -2141.458 Model: -1522.240 & Pseudo R ${ }^{2}=0.289$ & \\
Wald Chi2 (df=66) $=630.810, \mathrm{p}<.001$ & Cox-Snell/ML=0.547 & \\
& Cragg-Uhler/Nagelkerke=0.585 & \\
\hline
\end{tabular}

\section{Past 30-day Concomitant Use Prior to Incarceration}

Among participants who reported past 30-day concomitant use, having a history of IDU was associated with the greatest increased odds for observing the outcome of past 30-day concomitant use $(\mathrm{RRR}=3.81, \mathrm{p}<.001)$. Younger age $(\mathrm{RRR}=1.03, \mathrm{p}=0.15)$ was also associated with increased likelihood of concomitant use for this period. Younger age of drug use initiation $(R R R=1.08, p<.001)$, greater severity of use $(\mathrm{RRR}=1.06, \mathrm{p}<.001)$, and greater past-year drug use versatility, indicated by a greater number of drugs used $(\mathrm{RRR}=3.15, \mathrm{p}<.001)$, were all associated with an increased likelihood of past 30-day concomitant use. Residing in Central Appalachia for the one-year period prior to incarceration was not associated with a statistically significant increase in likelihood of past 30-day concomitant use ( $R R R=1.24, p=.344)$. For the entire model, the overall effect of residing in Central Appalachia was significant $\left(X^{2}=25.01, \mathrm{p}<.001\right)$. The effect of residing in Central Appalachia on predicting the probability of past 30-day concomitant did not differ from the effect of residing in Central Appalachia on predicting the probability of past 30-day opioid use $\left(X^{2}=2.84, \mathrm{p}=.0918\right)$, but did differ modestly from past 30-day stimulant use $\left(X^{2}=9.37, \mathrm{p}=.022\right)$.

Figure 10 displays the percent change in odds for a one unit increase among binary independent variables and the percent change in odds for a one standard deviation increase among continuous or ranked independent variables (Long \& Freese, 2005, 2014). Only independent variables significantly associated with changes in the likelihood of observing concomitant use for the 30-day period prior to incarceration are presented. 
Figure 10. Percent change in odds of past 30-day concomitant use prior to incarceration.

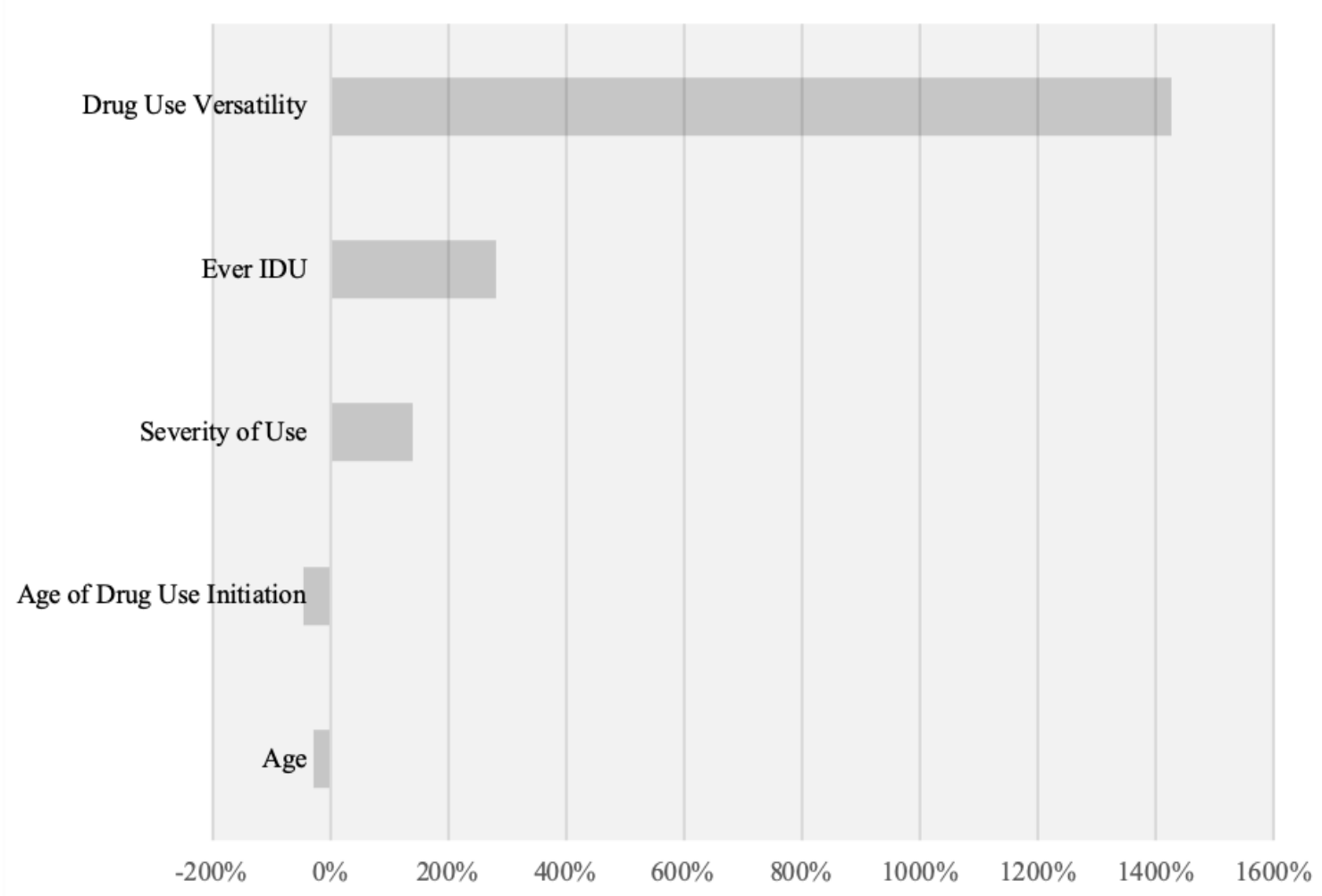

Drug use versatility (i.e., the number of drugs used in the year prior to incarceration) increased the odds of observing past 30-day concomitant use the most, with the likelihood of past 30-day concomitant use increasing $1,426.7 \%$ for every SD increase ( $S D=2.4)$; an approximate $215.2 \%$ increase in odds for every 1 additional drug used. The likelihood of observing past 30-day concomitant use increased by $281.1 \%$ when history of IDU was also reported. For every SD increase in severity of use ( $S D=15.8)$, past 30-day concomitant use was $140.2 \%$ more likely (i.e., a 5.7\% increase for every one-digit severity score increase). Observing past 30-day concomitant use was $44.7 \%$ less likely for every SD increase in age of drug use initiation $(S D=5.0)$; an approximate $7.7 \%$ decrease for every one-year increase. Odds decreased $29.6 \%$ for every SD increase in age ( $S D=9.0)$; an approximate $2.9 \%$ decrease for every year.

\section{Past 30-day Opioid Use Prior to Incarceration}

Being White $(\mathrm{RRR}=3.64, \mathrm{p}<.001)$ and having a history of IDU $(\mathrm{RRR}=2.81, \mathrm{p}<.001)$ were both associated with an increased likelihood of using opioids during the 30-day period prior to incarceration. Misregulation was also associated with an increased likelihood of using opioids for this time period 
$(R R R=1.89, p<.005)$. Having fewer close relationships $(R R R=0.97, p=.040)$ and fewer number of leisure days per week $(R R R=0.92, p=.019)$ also increased the likelihood of past 30-day opioid use. Older age of drug use initiation $(R R R=1.09, p<.001)$, greater severity of use $(R R R=1.06, p<.001)$, and greater drug use versatility during the one-year period prior to incarceration $(R R R=1.80, p<.001)$, were all associated with an increased likelihood that opioids would be used during the 30-day period prior to incarceration. Finally, residing the majority of the year prior to incarceration in Central Appalachia also increased the likelihood that past 30-day opioid use would be observed, with participants who resided in this region 1.64 times more likely to report past 30-day opioid use $(\mathrm{p}=.010)$. Figure 11 displays the percent change in odds for independent variables significantly associated with changes in the likelihood of observing past 30-day opioid use prior to incarceration.

Figure 11. Percent change in odds of past 30-day opioid use prior to incarceration.

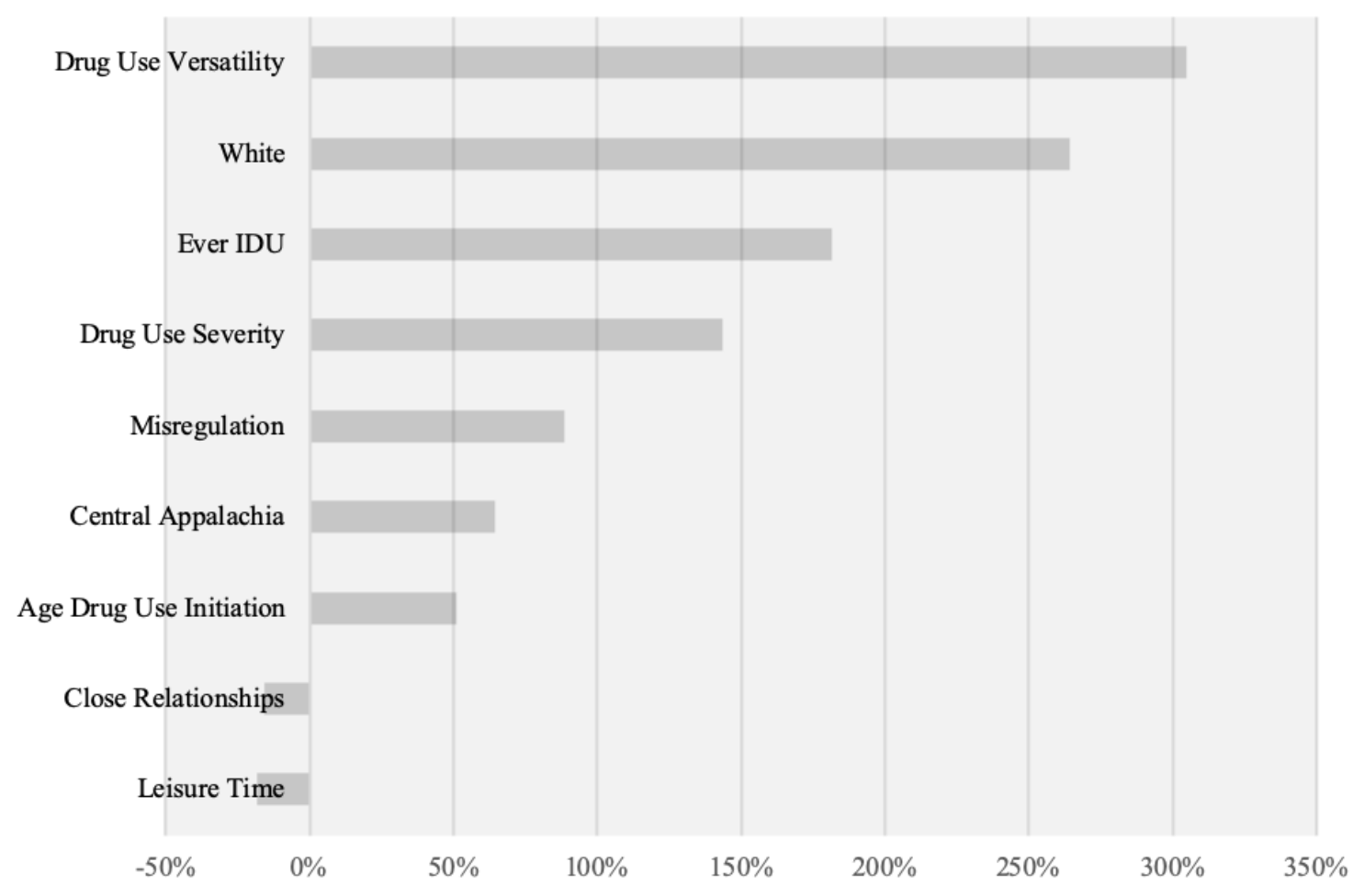

Drug use versatility increased the odds of observing past 30-day opioid use by $305.0 \%$ for every SD increase $(S D=2.4)$; an approximate increase of $80.3 \%$ for every 1 drug used. The likelihood of observing past 30 -day opioid use increased by $264.1 \%$ when the person was also White and by $181 \%$ if 
history of IDU was reported. For every SD increase in severity of use ( $S D=15.8)$ the likelihood of observing past 30-day opioid use increased by $143.6 \%$ (i.e., a 5.8\% increase for every one-digit severity score increase). Past-week misregulation increased the likelihood of observing past 30-day opioid use by 88.6\%. Residing in Central Appalachia during the one-year period prior to incarceration increased the odds of observing past 30-day opioid use by $64.2 \%$. Past 30-day opioid use was 51.0\% more likely for every SD increase in age of drug use initiation $(S D=5.0)$; an approximate $8.6 \%$ increase for every year. Close relationships modestly decreased the likelihood of observing past 30-day opioid use by $15.6 \%$ for every SD increase $(S D=4.1)$; an approximate decrease of $3.3 \%$ in odds per relationship. Finally, for every SD increase in leisure days $(S D=2.4)$, the odds of observing past 30-day opioid use decreased by $18.1 \%$, about an $8.1 \%$ decrease for per day.

\section{Past 30-day Stimulant Use Prior to Incarceration}

Fewer number of nights spent in a controlled environment during the 30-day period prior to incarceration was associated with an increased likelihood of stimulant use for the 30-day period prior to incarceration $(\mathrm{RRR}=0.94, \mathrm{p}=.014)$. History of IDU was also associated with and increased likelihood of past 30-day stimulant use $(R R R=1.68, p=.019)$, as was older age $(R R R=1.05, p<.001)$. Similar to past 30day opioid use prior to incarceration, misregulation was associated with increased likelihood of past 30-day simulant use $(\mathrm{RRR}=1.74, \mathrm{p}=.010)$. The likelihood of past 30-day stimulant use also decreased in association with an increase in the number of leisure days per week ( $R R R=0.92, p=.025)$. Older age of drug use initiation ( $R R R=1.04, p=0.34)$, greater severity of use $(R R R=1.05, p<.001)$, and greater number of drugs use versatility prior to incarceration $(R R R=1.33, p<.002)$, were all associated with an increased likelihood that stimulants would be used during the 30-day period prior to incarceration. Unlike past 30-day opioid use, residing in Central Appalachia for the majority of time out of the year prior to incarceration slightly decreased the likelihood of stimulant use $(\mathrm{RRR}=0.63, \mathrm{p}=0.32)$. The effect of residing in Central Appalachia on predicting the probability of past 30-day use differed between participants who reported past 30 -day opioid and past 30-day stimulant use $\left(X^{2}=24.24, \mathrm{p}<.001\right)$. Figure 12 displays the percent change in odds for independent variables significantly associated with changes in the likelihood of observing past 30day stimulant use prior to incarceration. 
Figure 12. Percent change in odds of past 30-day stimulant use prior to incarceration.

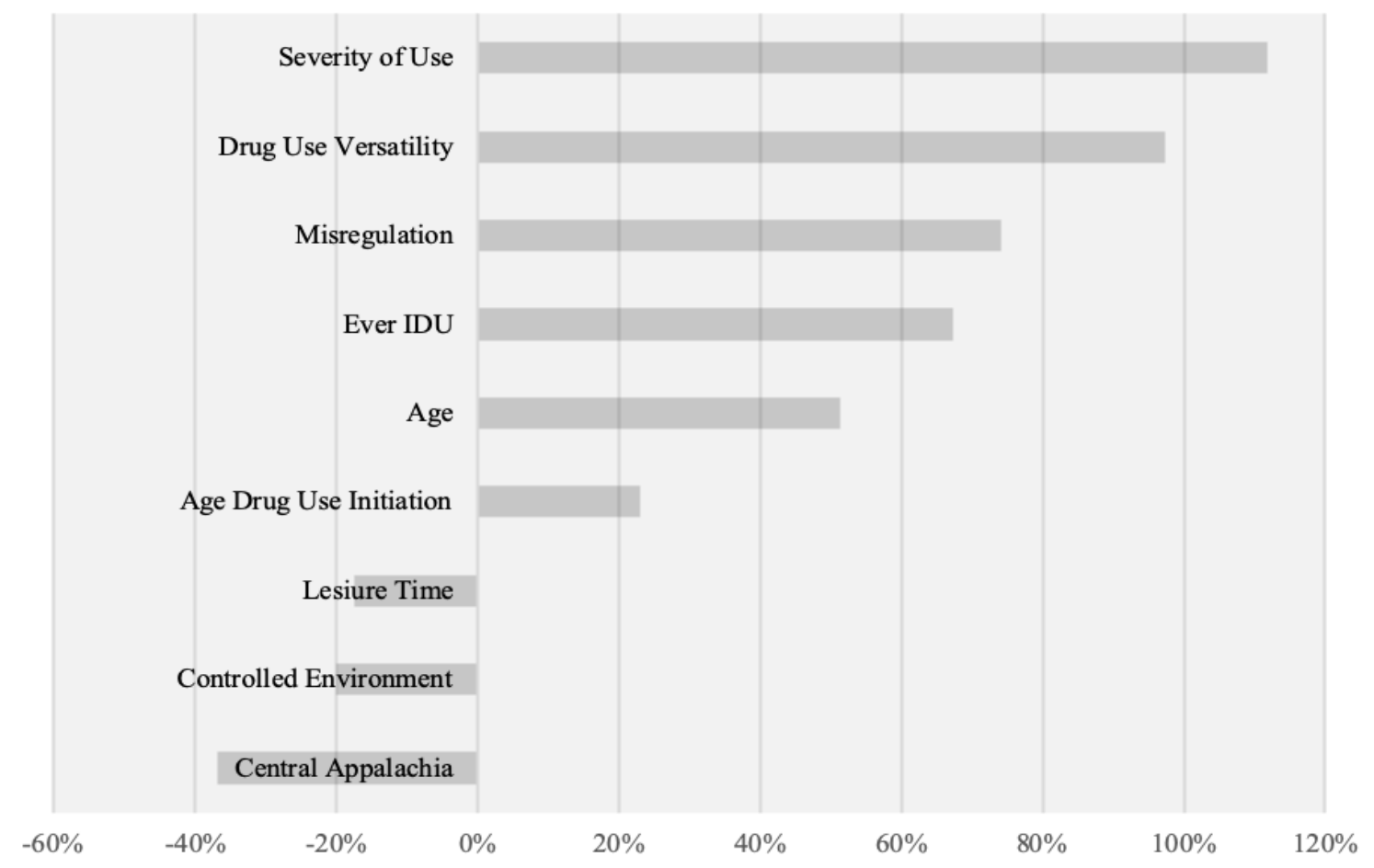

For every SD increase in drug use severity $(S D=15.8)$, the likelihood of observing past 30-day stimulant use increased by $111.8 \%$ (i.e., a $4.9 \%$ increase for every one-digit severity score increase), and for every SD increase in drug use versatility $(S D=2.4)$ the likelihood increased 97.4\%; an approximate $33.2 \%$ increase for every one drug used. Past-week misregulation was associated with a $74.1 \%$ increase in the likelihood of observing past 30-day stimulant use. The odds of observing past 30-day stimulant use increased by $67.3 \%$ if history of IDU was reported and by $51.3 \%$ for every SD increase in age ( $S D=9.0)$, an approximate increase of $4.7 \%$ for each year. The likelihood of observing past 30-day stimulant use increased modestly by $22.9 \%$ for every SD increase for age of drug use initiation ( $S D=5.0)$; an approximate increase of $4.2 \%$ for each year. For every SD increase in leisure days $(S D=2.4)$, the odds of observing past 30-day stimulant use decreased by $17.4 \%$, about a $7.7 \%$ decrease for per day. The likelihood of observing past 30-day stimulant use decreased by $20.0 \%$ for every SD increase in nights spent in a controlled environment ( $S D=3.6)$; an approximate $6.0 \%$ decrease for each night. Lastly, residing in Central Appalachia was associated with a $36.9 \%$ decrease in the likelihood of observing stimulant use for the 30day period prior to incarceration. 


\section{Multinomial Logistic Regression Results for Drug Outcomes Subsequent to Incarceration}

Tables 5.0 and 5.1 display the results of the multinomial logistic regression models examining opioid and stimulant outcomes for the one-year period subsequent to incarceration, with "other/no drug use" as the reference group. Variables considered as control variables and independent variables (as opposed to variables used for description only) which differed significantly by group when examined using nonparametric tests were included into the regression models.

Due to the overall number of independent variables which differed significantly by group in nonparametric analyses, two separate models were built to avoid model overfitting, as the events (i.e., taken to be the smaller number of cases reporting the outcome of interest, versus the number of cases for which the outcome was not reported; e.g., past-year opioid use rather than no use) for each outcome variable were smaller for the one-year post-release period compared to the 30-day pre-incarceration period. Specifically, $\mathrm{N}=186$ for past-year concomitant use, $\mathrm{N}=195$ for past-year opioid use, and $\mathrm{N}=130$ for past-year stimulant use). The events per variable (EPV), the number of events divided by the number of independent variables, thus translates to approximately 19,20 , and 13 , covariates respectively. A decision was made to examine the relationship between pre-incarceration drug use and opioid and stimulant outcomes that occurred during the one-year post-release period in a separate model. As prior drug use was not included in the multinomial regression model examining opioid and stimulant use outcomes for the 30-day period prior to incarceration, this decision allowed for some continuity between sets of analyses and related discussion. Examining the relationship between pre-incarceration drug use on opioid and stimulant outcomes subsequent to incarceration also permitted a narrower examination of premise discussed in earlier chapters that prior exposure to, or use of, drugs can significantly influence and constrain future decision-making about drugs, and therefore future drug behaviors, independent of other factors. Prior to examining the relationship between pre-incarceration use of specific drugs and opioid and stimulant outcomes for the one-year period subsequent to incarceration, it was expected (both for conceptual reasons as well as due to non-parametric test results) that specific opioid and stimulant drugs (e.g., heroin, buprenorphine, cocaine, etc.) used during the 30-day period prior to incarceration would demonstrate a positive correlation with drug outcomes for the one-year post-release period by drug class (e.g., opioids, stimulants) or combination of classes (e.g., concomitant) of which they are constituents. It was unclear, however, if all specific drugs for each drug 
class would demonstrate a positive and statistically significant relationship with expected outcomes, or if instead, only certain drugs would. Further, it was uncertain what, if any drugs, might be associated with a decreased likelihood of observing a given outcome.

For the first model examining opioid and stimulant outcomes during the one-year period subsequent to incarceration, VIF indicated that collinearity was not an issue for either model. Model fit statistics for the first model, displayed at the bottom of Table 4.0, indicated acceptable model fit.

Table 4

Results of Multinomial Logistic Regression Examining Opioid and Stimulant Use During the One-year Post-release Period

\begin{tabular}{|c|c|c|c|c|}
\hline & Robust SE & $95 \% \mathrm{CI}$ & RRR & $\underset{\text { value }}{\mathbf{p}}$ \\
\hline \multicolumn{5}{|l|}{ Concomitant } \\
\hline White & 1.730 & $2.15-10.07$ & 4.67 & .001 \\
\hline Nights controlled environment, past-year & 0.011 & $1.00-1.08$ & 1.01 & .001 \\
\hline Lifetime drug treatment episodes & 0.022 & $1.00-1.09$ & 1.05 & .016 \\
\hline Ever IDU & 0.440 & $1.61-3.37$ & 2.32 & .001 \\
\hline Age & 0.010 & $0.93-0.97$ & 0.95 & .001 \\
\hline Past-month income & 0.007 & $0.99-1.01$ & 1.00 & .188 \\
\hline Past-year cognitive difficulties & 0.316 & $1.00-2.26$ & 1.50 & .058 \\
\hline Past-year anxiety symptoms & 0.251 & $0.73-1.74$ & 1.13 & .583 \\
\hline Past-year depressive symptoms & 0.473 & $1.33-3.25$ & 2.09 & .001 \\
\hline Past-year suicidal ideation & 0.898 & $1.23-5.05$ & 2.49 & .011 \\
\hline Close relationships & 0.017 & $0.97-1.03$ & 1.00 & .846 \\
\hline Social satisfaction & 0.085 & $0.22-0.55$ & 0.36 & .001 \\
\hline Perceived social worth & 0.266 & $0.46-1.57$ & 0.86 & .627 \\
\hline $\begin{array}{l}\text { Past-year educational/vocational } \\
\text { involvement }\end{array}$ & 0.327 & $1.00-2.31$ & 1.53 & .046 \\
\hline On community supervision & 0.610 & $1.01-3.55$ & 1.89 & .049 \\
\hline Age of drug use initiation & 0.024 & $0.95-1.03$ & 0.99 & .556 \\
\hline Abstinence self-efficacy prior to treatment & 0.142 & $0.45-1.02$ & 0.68 & .066 \\
\hline Central Appalachia & 0.163 & $0.58-1.23$ & 0.84 & .376 \\
\hline \multicolumn{5}{|l|}{ Opioids } \\
\hline White & 0.594 & $1.08-3.33$ & 1.90 & .026 \\
\hline Nights controlled environment, past-year & 0.001 & $1.00-1.01$ & 1.00 & .001 \\
\hline Lifetime drug treatment episodes & 0.020 & $1.02-1.10$ & 1.07 & .001 \\
\hline Ever IDU & 0.450 & $1.69-3.42$ & 2.42 & .001 \\
\hline Age & 0.011 & $0.94-0.98$ & 0.96 & .001 \\
\hline Income, past-month & 0.005 & $0.99-1.00$ & 1.00 & .834 \\
\hline Past-year cognitive difficulties & 0.209 & $0.70-1.54$ & 1.04 & .847 \\
\hline Past-year anxiety symptoms & 0.181 & $0.62-1.33$ & 0.91 & .618 \\
\hline Past-year depressive symptoms & 0.281 & $0.87-2.00$ & 1.32 & .187 \\
\hline Past-year suicidal ideation & 0.677 & $0.82-3.73$ & 1.75 & .154 \\
\hline Close relationships & 0.021 & $0.95-1.03$ & 0.99 & .640 \\
\hline Social satisfaction & 0.117 & $0.27-0.75$ & 0.45 & .002 \\
\hline Perceived social worth & 0.232 & $0.44-1.40$ & 0.78 & .405 \\
\hline $\begin{array}{l}\text { Past-year educational/vocational } \\
\text { involvement }\end{array}$ & 0.255 & $0.83-1.81$ & 1.25 & .289 \\
\hline
\end{tabular}




\section{Table 4 (continued)}

On community supervision

$\begin{array}{llll}0.176 & 0.51-1.23 & 0.80 & .304 \\ 0.019 & 0.96-1.02 & 1.00 & .855 \\ 0.169 & 0.55-1.22 & 0.82 & .334 \\ 0.279 & 1.12-2.24 & 1.60 & .008\end{array}$

Abstinence self-efficacy prior to treatment

Central Appalachia

0.279

$0.55-1.22$
$1.12-2.24$

.855

Stimulants

White

0.303

$0.72-1.95$

1.18

.520

Nights controlled environment, past-year

0.001

1.00-1.01

.001

Lifetime drug treatment episodes

0.025

$1.00-1.10$

1.06

.055

Ever IDU

0.183

$0.55-1.28$

1.05

.420

Age

$0.99-1.03$

0.84

.163

Income, past-month

0.011

$0.99-1.00$

1.01

.650

0.259

$0.66-1.71$

1.00

.796

Past-year anxiety symptoms

$0.69-1.80$

1.07

.635

Past-year depressive symptoms

$1.11-2.98$

1.13

.018

Past-year suicidal ideation

0.460

$0.66-3.46$

1.82

.310

Close relationships

0.666

0.98-1.04

1.54

.360

Social satisfaction

0.111

0.23-0.69

1.01

.001

Perceived social worth

0.383

$0.53-2.16$

0.40

.844

Past-year educational/vocational

0.246

$0.66-1.66$

1.01

.852 involvement

On community supervision

0.652

0.027

$0.93-3.62$

1.05

8

Age of drug use initiation

0.140

$0.90-1.00$

1.86

.078

Abstinence self-efficacy prior to treatment

Central Appalachia

0.179

$0.38-0.94$

0.95

.072

$0.45-1.17$

0.59

.027

Intercept-only: -1538.692/Model: -1342.738

Pseudo $\mathrm{R}^{2}=0.127$

Wald Chi $2(\mathrm{df}=54)=300.538, \mathrm{p}<.001$

Cox-Snell/ML=0.222

Cragg-Uhler/Nagelkerke $=0.25$

\section{Past-year Concomitant Use Subsequent to Incarceration}

Being White was significantly associated with past-year concomitant use $(R R R=4.68, p<001)$.

Greater number of nights spent in a controlled environment during the one-year post-release period $(R R R=1.01, p<.001)$, greater number of lifetime drug treatment episodes $(R R R=1.05, p=0.16)$, and history of IDU $(\mathrm{RRR}=2.32, \mathrm{p}<.001)$ were all associated with an increased likelihood of observing past-year concomitant use. While experiencing past-year cognitive difficulties approached statistical significance $(\mathrm{RRR}=1.50, \mathrm{p}=.053)$, experiencing past-year depressive symptoms $(\mathrm{RRR}=2.09, \mathrm{p}<.001)$ and past-year suicidal ideation $(\mathrm{RRR}=2.49, \mathrm{p}=.011)$ were both associated with an increased likelihood of past-year concomitant use. Receiving satisfaction from past-year social interaction, as opposed to being indifferent to or not receiving satisfaction, decreased the likelihood of past-year concomitant use $(R R R=0.37, p<.001)$. Past-year concomitant use was also significantly associated with educational and/or vocational involvement $(\mathrm{RRR}=1.53, \mathrm{p}=.046)$, as well as being on community supervision during the one-year post-release period 
$(\mathrm{RRR}=1.89, \mathrm{p}=.049)$. Residing in Central Appalachia during the one-year post-release period was not associated with changes in the likelihood of observing past-year concomitant use ( $R R=0.84, p=3.76)$. The overall effect of residing in Central Appalachia for the entire model was significant $\left(X^{2}=11.52 \mathrm{p}=.0092\right)$. The effect of residing in Central Appalachia on predicting the probability of past-year concomitant use differed from the effect on predicting the probability of past-year opioid use $\left(X^{2}=7.73, \mathrm{p}=.0051\right)$, but not past-year stimulant use $\left(X^{2}=0.23, \mathrm{p}=.6352\right)$. Figure 13 displays the percent change in odds for independent variables significantly associated with changes in the likelihood of observing concomitant use during the one-year period subsequent to incarceration.

Figure 13. Percent change in odds of concomitant use during one-year post-release period.

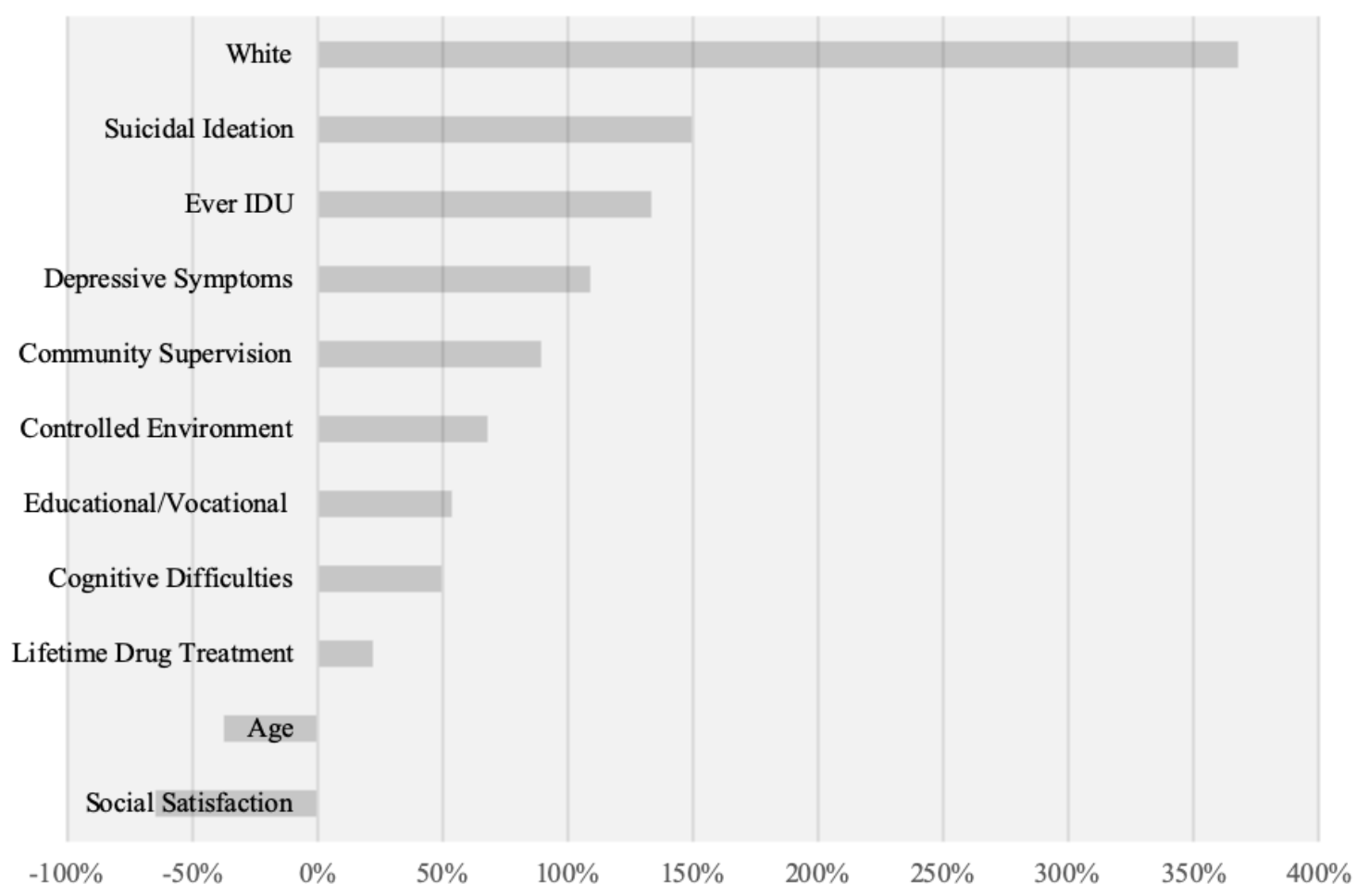

Being White increased the likelihood that concomitant use during the one-year post-release period would be observed by $367.4 \%$. The likelihood of observing past-year concomitant use increased by $149.3 \%$ with past-year suicidal ideation. History of IDU increased the likelihood of observing past-year concomitant use by $133.0 \%$, whereas past-year depressive symptoms increased the likelihood by $109.0 \%$. 
Being on community supervision for this same time period increased odds by $89.0 \%$. For every SD increase in nights spent in a controlled environment during the past year ( $S D=77.7)$, the odds of observing past-year concomitant use increased by $67.6 \%$; an approximate increase of $0.8 \%$ per night. Past-year educational/vocational involvement was associated increased 53.2\% increased odds of observing past-year concomitant use. Past-year cognitive difficulties were associated with a $49.3 \%$ increase in odds of observing past-year concomitant use. The likelihood of observing concomitant use also increased by $22.0 \%$ for each SD increase in lifetime drug treatment episodes ( $S D=3.9)$; an approximate increase of $5.6 \%$ per treatment episode. For age, the likelihood of observing past-year concomitant use decreased by $37.3 \%$ for every SD increase $(S D=9.1)$; an approximate 5\% decrease for every 1 year. Lastly, receiving satisfaction from past-year social interaction decreased the odds of observing past-year concomitant use by $64.8 \%$.

\section{Past-year Opioid Use Subsequent to Incarceration}

As with past-year concomitant use, being White $(R R R=1.90, p=.026)$ was associated with increased odds of past-year opioid use. Greater number of nights spent in a controlled environment during the one-year post-release period $(\mathrm{RRR}=1.00, \mathrm{p}<.001)$, greater number of lifetime drug treatment episodes $(\mathrm{RRR}=1.07, \mathrm{p}=<.001)$, and history of IDU $(\mathrm{RRR}=2.42, \mathrm{p}<.001)$ were all associated with an increased likelihood of observing past-year opioid use. Participants who reported past-year opioid use were also more likely to be younger $(\mathrm{RRR}=0.96, \mathrm{p}<.001)$. Receiving satisfaction from past-year social interaction was associated with decreased odds of past-year opioid use ( $R R R=0.46, p=.002)$. Finally, residing in Central Appalachia for the majority of the year subsequent to incarceration was associated with an increased likelihood of past-year opioid use $(\mathrm{RRR}=1.60, \mathrm{p}=.008)$. The effect of residing in Central Appalachia for the one-year post-release period on predicting the probability of past-year opioid use differed from the effect on predicting the probability of past-year stimulant use $\left(X^{2}=7.57, \mathrm{p}=.0051\right)$ as well as concomitant use $\left(X^{2}=7.73, \mathrm{p}=.0058\right)$.

Figure 14 displays the percent change in odds for independent variables significantly associated with changes in the likelihood of observing opioid use during the one-year subsequent to incarceration. 
Figure 14. Percent change in odds of opioid use during one-year post-release period.

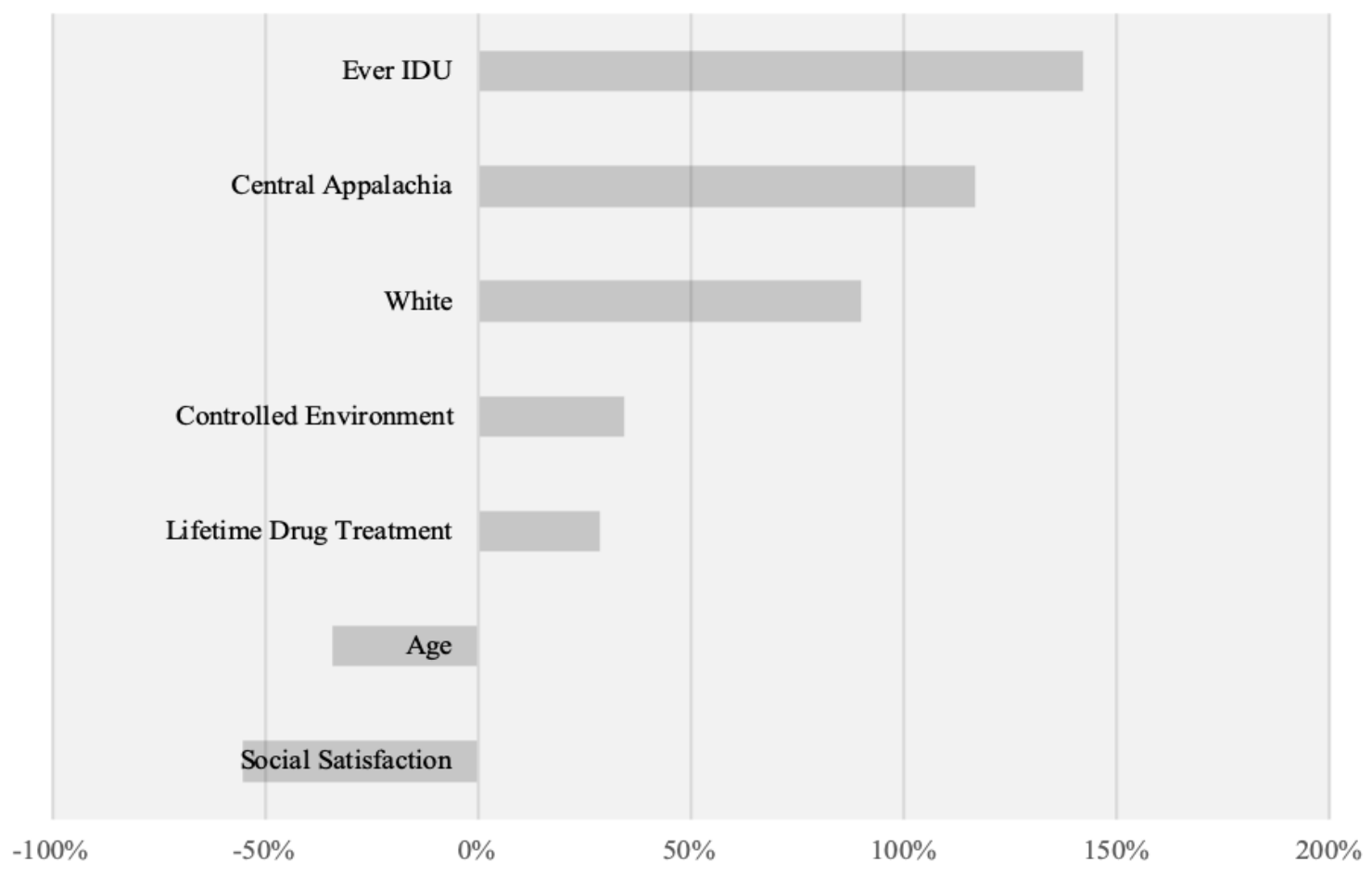

History of IDU increased the odds of observing past-year opioid use by $142.2 \%$. Residing in Central Appalachia for the majority of time out of the past year increased the odds of observing past-year opioid use by $116.8 \%$. Being White increased the likelihood of observing past-year opioid use by $90.1 \%$. For every SD increase in nights spent in a controlled environment during the past year ( $S D=77.7)$, the odds of observing past-year opioid use increased by $34.3 \%$, just $0.4 \%$ for each night. For each SD increase $(S D=3.9)$ in lifetime drug treatment episodes, there was a $28.8 \%$ increase in the likelihood of observing past-year opioid use; an approximate 6.7\% increase for each treatment episode. For every SD increase in age $(S D=9.0)$, the likelihood of past-year opioid use decreased by $34.2 \%$; an approximate $4.5 \%$ decrease for every 1 year. Lastly, receiving satisfaction from past-year social interaction decreased the odds of observing past-year opioid use by $55.1 \%$.

\section{Past-year Stimulant Use Subsequent to Incarceration}

Unlike concomitant and opioid use for the one-year post-release period, being White did not increase the likelihood that past-year stimulant use would be observed $(R R R=1.18, p=.520)$. Similar to past-year concomitant and opioid use, though, greater number of nights spent in a controlled environment 
in the year following release was associated with an increased likelihood of past-year stimulant use $(R R R=1.06, p<.001)$. Greater number of prior drug treatment episodes $(R R R=1.05, p=.055)$ approached statistical significance. Experiencing past-year depressive symptoms was associated with an increased likelihood of past-year stimulant use $(\mathrm{RRR}=1.82, \mathrm{p}=.018)$, whereas receiving satisfaction from social interaction was associated with decreased likelihood $(\mathrm{RRR}=0.40, \mathrm{p} .<001)$. Reporting "moderately good" or "very good" abstinence self-efficacy prior to treatment was associated with decreased odds of past-year stimulant $(\mathrm{RRR}=0.59, \mathrm{p}=.027)$. Residing in Central Appalachia for the majority of the one-year postrelease period was not associated with a change in odds of stimulant use for this same time period $(\mathrm{RRR}=0.74, \mathrm{p}=.206)$.

Figure 15 displays the percent change in odds for independent variables significantly associated with changes in the likelihood of observing stimulant use during the one-year period subsequent to incarceration.

\section{Figure 15. Percent change in odds of stimulant use during one-year post-release period.}

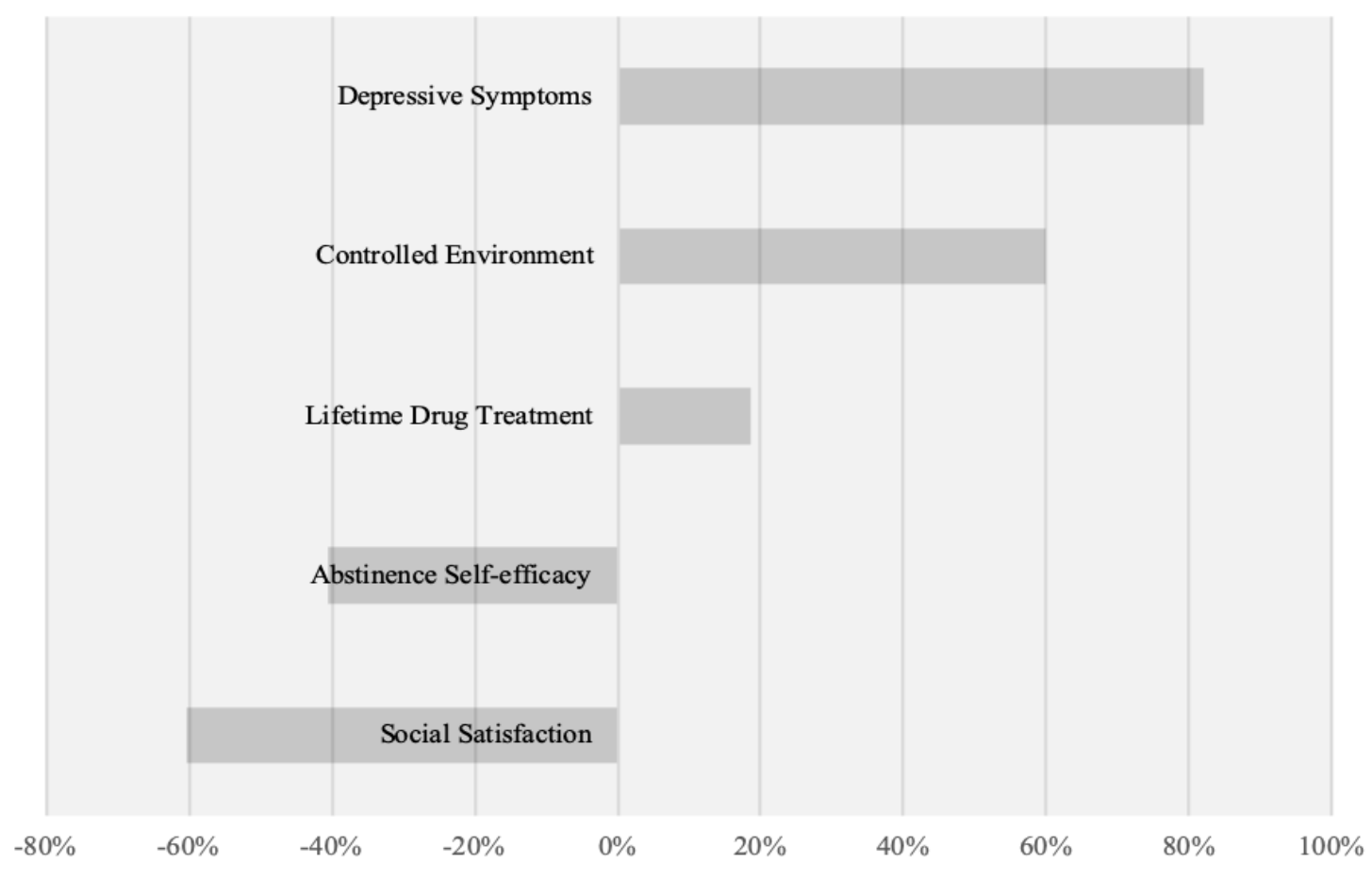

The likelihood of observing past-year stimulant use increased $82.1 \%$ when past-year depressive symptoms were also reported. For every SD increase in nights spent in a controlled environment during the 
year subsequent to incarceration $(S D=77.7)$, the odds of observing past-year stimulant use increased by $59.9 \%$, just $0.6 \%$ for each night. Lifetime drug treatment was associated with a $18.7 \%$ increase in the likelihood of observing past-year stimulant use for each SD increase $(S D=3.9)$; an approximate $4.5 \%$ increase for each treatment episode. Having reported "moderately good" or "very good" abstinence selfefficacy prior to treatment was associated with a $40.5 \%$ decrease in odds of observing stimulant use during the one-year post-release period. Lastly, receiving satisfaction from social interaction was associated with a $60.5 \%$ decrease in the odds of observing stimulant use for this time period, similar to the percentage change in odds observed for past-year concomitant and opioid use.

For the second model examining the relationship between specific drug use that occurred during the 30-day period prior to incarceration and opioid and stimulant outcomes that occurred during the oneyear post-release period, collinearity was not an issue and model fit statistics, displayed at the bottom of Table 4.1., indicated acceptable fit.

For concomitant use during the one-year period subsequent to incarceration, drugs used during the 30-day period prior to incarceration that were significantly associated with an increased likelihood of pastyear concomitant use included prescription sedatives $(R R R=1.69, p=.006)$, heroin $(R R R=1.52, p=.043)$, prescription opioids $(R R R=1.74, p=.006)$ and amphetamines $(R R R=1.97, p<.001)$. For opioid use during the one-year period subsequent to incarceration, drugs used during the 30-day period prior to incarceration that were significantly associated with an increased likelihood of past-year opioid use included heroin $(\mathrm{RRR}=2.55, \mathrm{p}<.001)$ and prescription opioids $(\mathrm{RRR}=2.19, \mathrm{p}<.001)$. Alcohol $(\mathrm{RRR}=0.64, \mathrm{p}=.009)$, cocaine/crack cocaine $(R R R=0.63, p=.034)$, and amphetamine use $(R R R=0.62, p=.022)$ during the 30-day period prior to incarceration were all associated with a decreased likelihood of opioid use during the oneyear period subsequent to incarceration. For stimulant use during the one-year period subsequent 
Table 4.1 Results of Multinomial Logistic Regression Examining Past 30-day Pre-incarceration Drug Use and Past-year Post-release Drug Use

\begin{tabular}{|c|c|c|c|c|}
\hline & Robust SE & $95 \% \mathrm{CI}$ & RRR & p value \\
\hline \multicolumn{5}{|l|}{ Concomitant } \\
\hline Alcohol & 0.141 & $0.60-1.16$ & 0.83 & .241 \\
\hline Sedatives & 0.330 & $1.18-2.50$ & 1.69 & .006 \\
\hline Hallucinogens & 0.583 & $0.72-3.22$ & 1.49 & .291 \\
\hline Synthetic drugs & 0.306 & $0.58-1.84$ & 1.01 & .954 \\
\hline Cocaine/crack cocaine & 0.260 & $1.01-2.44$ & 1.37 & .101 \\
\hline Amphetamines & 0.343 & $0.95-2.00$ & 1.97 & .001 \\
\hline Heroin & 0.312 & $1.02-2.27$ & 1.52 & .043 \\
\hline Prescription opioids & 0.489 & $1.18-2.57$ & 1.74 & .006 \\
\hline Buprenorphine & 0.229 & $0.70-1.62$ & 1.07 & .757 \\
\hline \multicolumn{5}{|l|}{ Opioids } \\
\hline Alcohol & 0.106 & $0.46-0.88$ & 0.64 & .009 \\
\hline Sedatives & 0.237 & $0.84-1.80$ & 1.25 & .241 \\
\hline Hallucinogens & 0.572 & $0.42-3.01$ & 1.16 & .763 \\
\hline Synthetic drugs & 0.375 & $0.64-2.20$ & 1.19 & .554 \\
\hline Cocaine/crack cocaine & 0.137 & $0.40-0.95$ & 0.63 & .034 \\
\hline Amphetamines & 0.127 & $0.41-0.93$ & 0.62 & .022 \\
\hline Heroin & 0.495 & $1.74-3.72$ & 2.55 & .001 \\
\hline Prescription opioids & 0.423 & $1.51-3.01$ & 2.19 & .001 \\
\hline Buprenorphine & 0.293 & $0.98-2.15$ & 1.45 & .066 \\
\hline \multicolumn{5}{|l|}{ Stimulants } \\
\hline Alcohol & 0.171 & $0.61-1.29$ & 0.92 & .704 \\
\hline Sedatives & 0.209 & $1.99-1.30$ & 0.87 & .570 \\
\hline Hallucinogens & 0.471 & $0.80-3.03$ & 0.79 & .726 \\
\hline Synthetic drugs & 0.531 & $0.81-3.03$ & 1.65 & .144 \\
\hline Cocaine/crack cocaine & 0.420 & $1.19-2.89$ & 1.92 & .004 \\
\hline Amphetamines & 0.574 & $1.98-4.23$ & 3.0 & .001 \\
\hline Heroin & 0.107 & $0.09-0.57$ & 0.23 & .002 \\
\hline Prescription opioids & 0.152 & $0.44-1.06$ & 0.68 & .082 \\
\hline Buprenorphine & 0.255 & $0.40-1.46$ & 0.77 & .438 \\
\hline \multicolumn{5}{|c|}{ Intercept-only: -1541.577/Model: -1423.094 Pseudo $\mathrm{R}^{2}=.078$} \\
\hline \multicolumn{5}{|c|}{ Wald Chi2 $(\mathrm{df}=27)=214.211, \mathrm{p}<.001$} \\
\hline
\end{tabular}

to incarceration, the only two drugs used in the 30-day period prior to incarceration significantly associated with an increased likelihood of past-year stimulant use during the one-year period subsequent to incarceration included cocaine/crack cocaine $(R R R=1.92, p=.004)$ and amphetamines $(R R R=3.0, p<.001)$. Heroin use during the 30-day period prior to incarceration was associated with a decreased likelihood of stimulant use during the one-year period subsequent to incarceration ( $R R R=0.23, p=.002)$. Buprenorphine was the only opioid drug used during the 30-day period prior to incarceration not associated with statistically significant change in odds for any of the outcomes of interest. 
Figures 16, 17, and 18 display the percent change in odds for independent variables significantly associated with changes in the likelihood of observing concomitant, opioid, and stimulant use during the one-year period subsequent to incarceration, respectively.

Figure 16. Percent change in odds of concomitant use during one-year post-release period by preincarceration drug use.

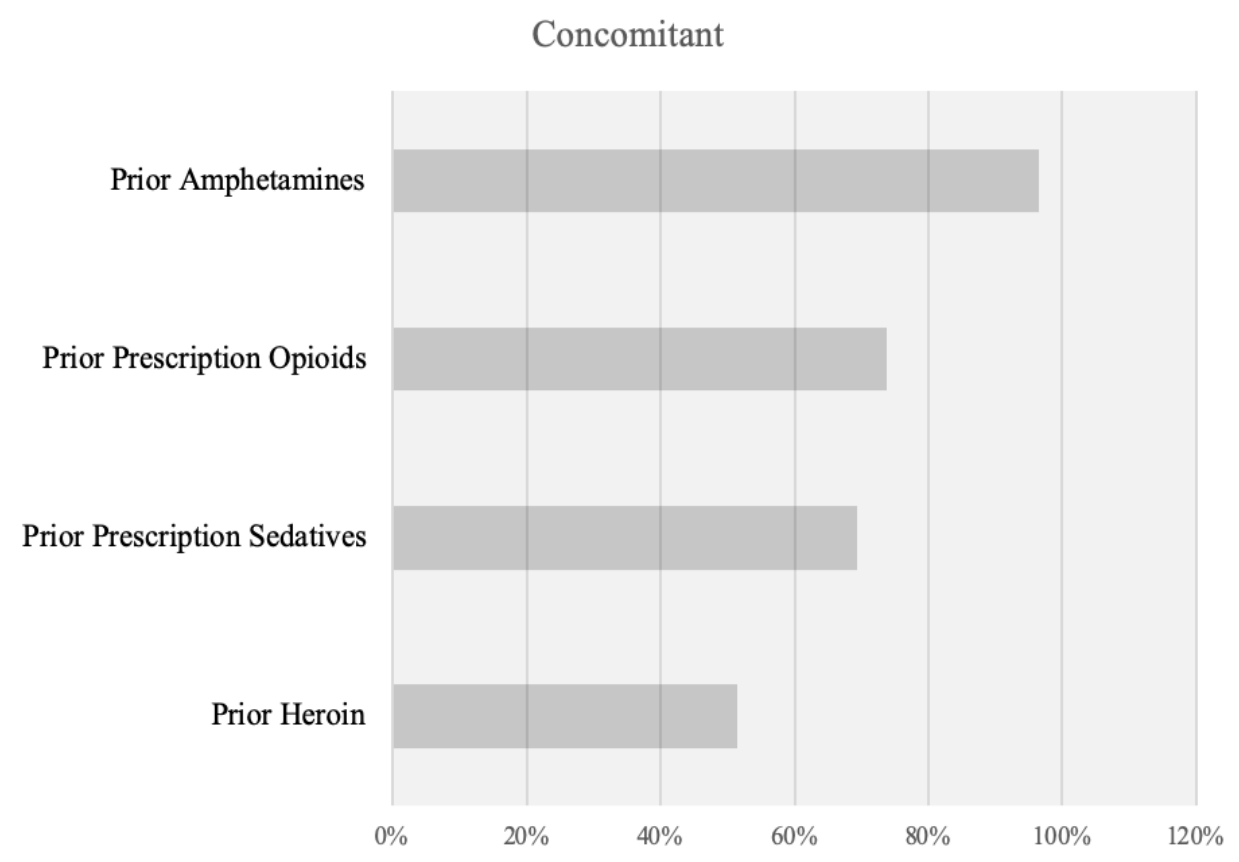


Figure 17. Percent change in odds of opioid use during one-year post-release period by preincarceration drug use.

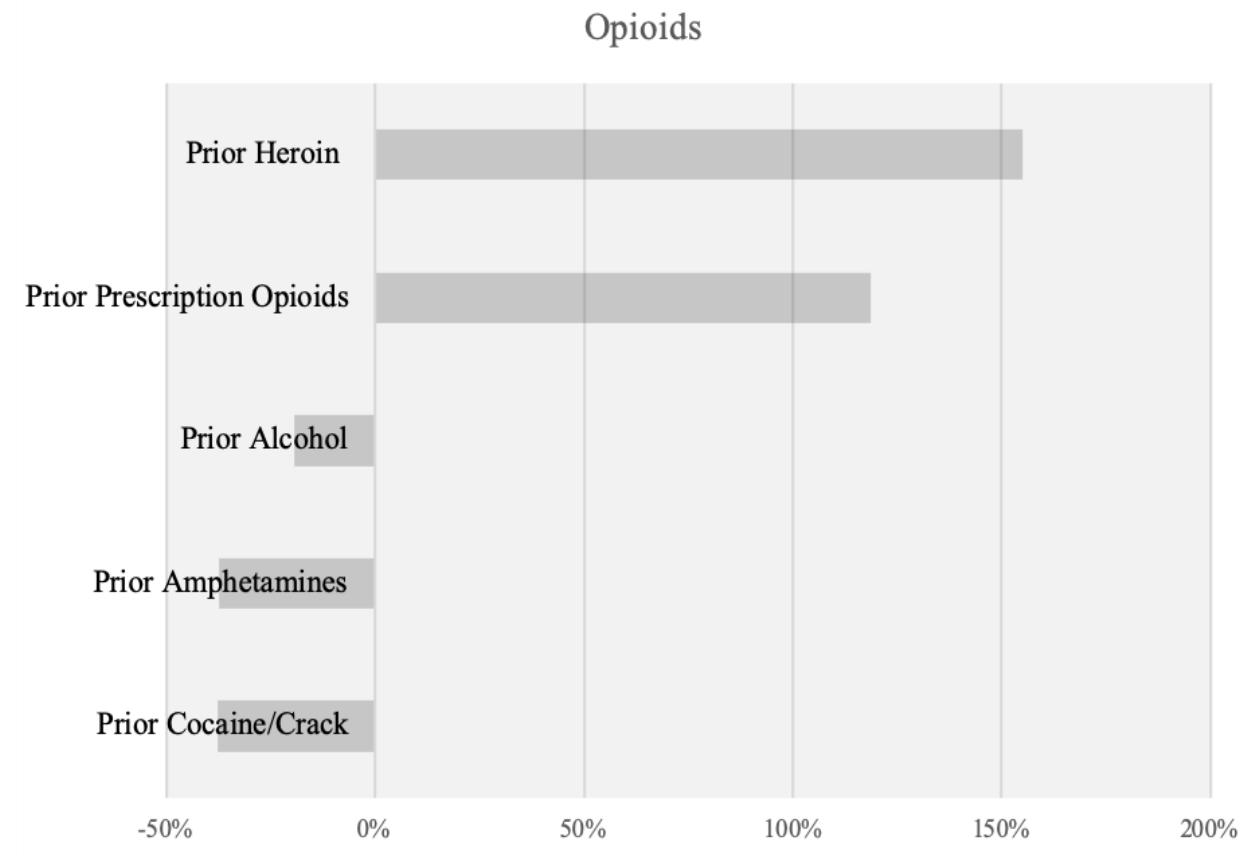

Figure 18. Percent change in odds of stimulant use during one-year post-release period by preincarceration drug use.

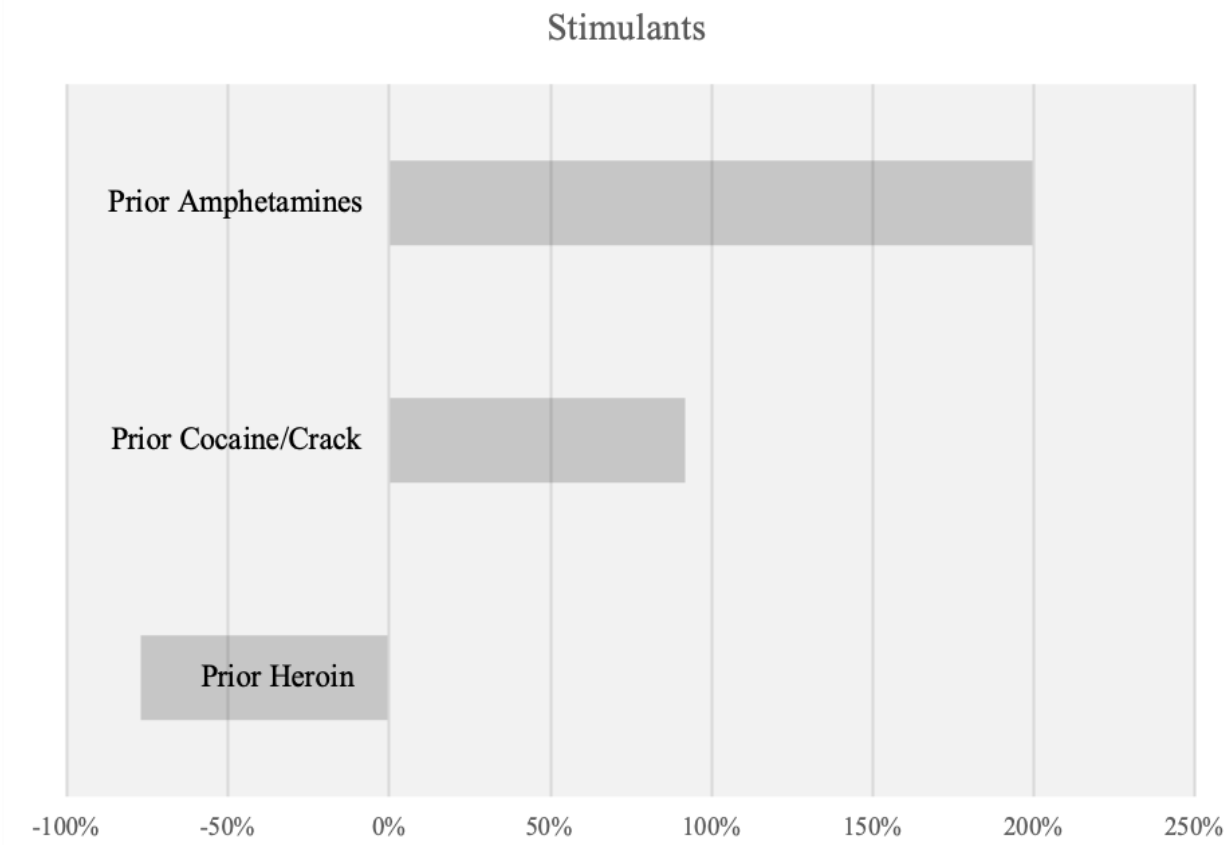

Amphetamine use during the 30-day period prior to incarceration was associated with the largest increase in odds for past-year concomitant use, with participants who reported using amphetamines during 
the 30 -day period prior to incarceration $96.5 \%$ more likely to have reported concomitant use subsequent to incarceration. This was followed by past 30-day prescription opioid (73.8), prescription sedative (69.3\%), and heroin use (51.4\%), which all increased the likelihood that concomitant use would be observed for the one-year period subsequent to incarceration.

Heroin and prescription opioid use during the 30-day period prior to incarceration were associated with the largest increase in odds for past-year opioid use, with participants who reported past 30-day heroin use prior to incarceration $155.1 \%$ more likely to have reported past-year opioid use subsequent to incarceration and participants who reported past 30-day prescription opioid use $118.8 \%$ more likely. Past 30-day use of alcohol, cocaine/crack cocaine, and amphetamines prior to incarceration decreased the odds of observing past-year opioid use subsequent to incarceration by approximately $20-40 \%$.

Amphetamine use during the 30-day period prior to incarceration was associated with the largest increase in odds for observing past-year stimulant use for the one-year period subsequent to incarceration, with participants who reported using amphetamines just prior to incarceration $199.6 \%$ more likely to have reported stimulant use subsequent to incarceration. This was followed by past 30-day cocaine/crack cocaine use prior to incarceration, which increased odds by $91.7 \%$. Past 30-day heroin use decreased odds by $77.1 \%$.

\section{Aim Three Results}

Table 5 displays means and proportions of participants who reported using heroin during the oneyear period subsequent to incarceration. For this time period, 10.7\% $(\mathrm{N}=168)$ of the sample reported pastyear heroin use. Only $2.1 \%$ of the entire sample reported heroin use within the 30 -day period prior to follow-up data collection (approximately 10-14 months subsequent to release).

Compared to the non-use group, the majority of the heroin-use group was White $(96.4 \%$, $\left.X^{2}=26.86, \mathrm{df}=1, \mathrm{p}<.001\right)$. Groups were similar in terms of rates for HSD/GED $\left(76.2 \%\right.$ vs. $70.6 \%, X^{2}=2.21$, $\mathrm{df}=1, \mathrm{p}=.161)$, mandated SAP participation $\left(67.9 \%\right.$ vs. $\left.73.6 \%, X^{2}=2.24, \mathrm{df}=1, \mathrm{p}=.133\right)$, and SAP completion $\left(73.8 \%\right.$ vs. $\left.78.9 \%, X^{2}=1.96, \mathrm{df}=1, \mathrm{p}=.160\right)$. However, during the one-year period subsequent to incarceration, the heroin-use group spent more nights on average in a controlled environment (68.7, $S D=78.0$ vs. $40.6, S D=77.0, t=-4.40, \mathrm{p}<.001)$. This group also reported a higher number of lifetime drug 
treatment episodes on average $(3.3, S D=4.8$ vs. $2.1, S D=3.7, t=-3.10, \mathrm{p}=.002)$ and higher rates for IDU history compared to the non-use group $\left(66.1 \%\right.$ vs. $\left.39.9 \%, X^{2}=41.12, \mathrm{df}=1, \mathrm{p}<.001\right)$.

\section{Age and Sex}

The heroin-use group was slightly younger than the non-use group (31.5, $S D=7.3$ vs. 34.9 , $S D=9.1, t=5.4, \mathrm{p}<.001)$, though did not differ significantly from the non-use group in the proportion of males $\left(76.8 \%\right.$ vs. $\left.81.4 \%, X^{2}=1.81, \mathrm{df}=1, \mathrm{p}=.179\right)$.

\section{Past-year Cognitive Difficulties and History of Head Trauma}

Approximately $30 \%$ of the heroin-use group reported experiencing past-year cognitive difficulties, not significantly higher than the non-use group $\left(29.8 \%\right.$ vs. $\left.26.0 \%, X^{2}=0.89, \mathrm{df}=1, \mathrm{p}=.341\right)$. Rates for lifetime incidence of trauma history were essentially identical across groups, at approximately $43.0 \%$ $\left(X^{2}=0.00, \mathrm{df}=1, \mathrm{p}=1.00\right)$.

Table 5

Group Means, Proportions, and Between-group Differences Among Heroin and Non-heroin users for One-year Post-release Period

\begin{tabular}{|c|c|c|c|c|}
\hline & Sample & Heroin & No heroin & p value \\
\hline $\begin{array}{l}\mathrm{N}= \\
\%\end{array}$ & 1,563 & $\begin{array}{r}168 \\
10.7 \\
\end{array}$ & $\begin{array}{c}1,395 \\
89.3 \\
\end{array}$ & \\
\hline White & $81.5 \%$ & $96.4 \%$ & $79.7 \%$ & .001 \\
\hline High School Diploma/GED & $71.2 \%$ & $76.2 \%$ & $70.6 \%$ & .161 \\
\hline Mandated SAP & $73.0 \%$ & $67.9 \%$ & $73.6 \%$ & .133 \\
\hline Graduated SAP & $78.3 \%$ & $73.8 \%$ & $78.9 \%$ & .160 \\
\hline $\begin{array}{l}\text { Nights controlled environment, } \\
\text { past-year }\end{array}$ & 43.6 & 68.7 & 40.6 & .001 \\
\hline Lifetime drug treatment episodes & 2.2 & 3.3 & 2.1 & .002 \\
\hline Ever IDU & $42.9 \%$ & $66.1 \%$ & $39.9 \%$ & .001 \\
\hline Age & 33.9 & 31.5 & 34.9 & .001 \\
\hline Male & $80.9 \%$ & $76.8 \%$ & $81.4 \%$ & .179 \\
\hline Cognitive difficulties, past-year & $26.4 \%$ & $29.8 \%$ & $26.0 \%$ & .341 \\
\hline History of head injury & $43.4 \%$ & $43.5 \%$ & $43.4 \%$ & 1.00 \\
\hline Past-month income & $\$ 1,290$ & $\$ 1,128$ & $\$ 1,310$ & .144 \\
\hline Anxiety symptoms & $40.7 \%$ & 45.8 & 40.1 & .180 \\
\hline Depressive symptoms & $32.7 \%$ & 45.2 & 31.2 & .001 \\
\hline Suicidal ideation & $4.5 \%$ & $9.5 \%$ & $3.9 \%$ & .002 \\
\hline Chronic pain & $32.5 \%$ & $31.5 \%$ & $32.7 \%$ & .838 \\
\hline Close relationships & 6.1 & 5.8 & 6.2 & .379 \\
\hline Social interaction & $88.4 \%$ & $84.5 \%$ & $88.9 \%$ & .122 \\
\hline
\end{tabular}




\begin{tabular}{|c|c|c|c|c|}
\hline & Sample & Heroin & No heroin & p value \\
\hline $\begin{array}{l}\mathrm{N}= \\
\%\end{array}$ & 1,563 & $\begin{array}{l}168 \\
10.7\end{array}$ & $\begin{array}{c}1,395 \\
89.3\end{array}$ & \\
\hline \multicolumn{5}{|l|}{ Table 5 (continued) } \\
\hline Social satisfaction & $88.9 \%$ & $76.2 \%$ & $90.5 \%$ & .001 \\
\hline $\begin{array}{l}\text { Educational/vocational } \\
\text { involvement }\end{array}$ & $67.1 \%$ & $65.5 \%$ & $67.3 \%$ & .705 \\
\hline Perceived social worth & $92.3 \%$ & $86.3 \%$ & $93.0 \%$ & .004 \\
\hline On community supervision & $87.0 \%$ & $88.1 \%$ & $86.8 \%$ & .732 \\
\hline Age of drug use initiation & 14.7 & 13.6 & 14.8 & .010 \\
\hline $\begin{array}{l}\text { Abstinence self-efficacy prior to } \\
\text { treatment }\end{array}$ & $81.5 \%$ & $73.2 \%$ & $82.5 \%$ & .005 \\
\hline \multicolumn{5}{|l|}{$\begin{array}{l}\text { Past 30-day drug use prior to } \\
\text { incarceration }\end{array}$} \\
\hline Alcohol & $49.5 \%$ & $44.6 \%$ & $50.1 \%$ & .209 \\
\hline Cannabis & $52.5 \%$ & $50.6 \%$ & $52.8 \%$ & .653 \\
\hline Sedatives & $32.3 \%$ & $45.2 \%$ & $30.8 \%$ & .001 \\
\hline Hallucinogens & $3.2 \%$ & $5.4 \%$ & $2.9 \%$ & .147 \\
\hline Synthetics & $7.7 \%$ & $10.1 \%$ & $7.4 \%$ & .269 \\
\hline Cocaine/crack cocaine & $23.2 \%$ & $32.1 \%$ & $22.2 \%$ & .005 \\
\hline Amphetamines & $31.3 \%$ & $31.0 \%$ & $31.3 \%$ & .992 \\
\hline Heroin & $17.7 \%$ & $54.2 \%$ & $13.3 \%$ & .001 \\
\hline Prescription opioids & $50.7 \%$ & $71.4 \%$ & $48.2 \%$ & .001 \\
\hline Buprenorphine & $18.7 \%$ & $25.0 \%$ & $17.9 \%$ & .034 \\
\hline \multicolumn{5}{|l|}{ Past-year drug use, post-release } \\
\hline Alcohol & $31.5 \%$ & $42.3 \%$ & $30.3 \%$ & .002 \\
\hline Cannabis & $25.5 \%$ & $45.8 \%$ & $23.0 \%$ & .001 \\
\hline Sedatives & $8.0 \%$ & $25.6 \%$ & $5.9 \%$ & .001 \\
\hline Hallucinogens & $1.2 \%$ & $5.4 \%$ & $0.6 \%$ & .001 \\
\hline Synthetic drugs & $7.0 \%$ & $8.9 \%$ & $6.8 \%$ & .393 \\
\hline Cocaine/crack cocaine & $7.4 \%$ & $28.6 \%$ & $4.8 \%$ & .001 \\
\hline Amphetamines & $15.2 \%$ & $37.5 \%$ & $12.5 \%$ & .001 \\
\hline Prescription opioids & $14.3 \%$ & $38.7 \%$ & $11.3 \%$ & .001 \\
\hline Buprenorphine & $9.4 \%$ & $33.3 \%$ & $6.5 \%$ & .001 \\
\hline Central Appalachia & $30.3 \%$ & $17.3 \%$ & $31.9 \%$ & .001 \\
\hline Urban proximity & 4.5 & 2.7 & 4.7 & .001 \\
\hline Unrestricted alcohol sales & $56.6 \%$ & $74.4 \%$ & $54.4 \%$ & .001 \\
\hline
\end{tabular}

\section{Psychiatric Symptoms and Chronic Pain}

Just under half of the heroin-use group reported experiencing past-year anxiety symptoms, similar to the rates among the non-use group $\left(45.8 \%\right.$ vs. $\left.40.1 \%, X^{2}=1.78, \mathrm{df}=1, \mathrm{p}=.180\right)$. Groups differed, though, in rates at which past-year depressive symptoms were experienced $\left(45.2 \%\right.$ vs $31.2 \%, X^{2}=12.68, \mathrm{df}=1$, $\mathrm{p}<.001)$. Past-year suicidal ideation was nearly three-times higher among the heroin-use group (9.5\% vs. 
$\left.3.1 \%, X^{2}=9.92, \mathrm{df}=1, \mathrm{p}=.002\right)$. Because of the high rates of past year suicidal ideation, the relationship between past 30-day heroin use and suicidal ideation was also explored (results not displayed). Among participants who reported heroin use during the 30 -day period prior to follow-up data collection ( $\mathrm{N}=33$ ), $24.1 \%$ also reported past-year suicidal ideation, compared to $4.1 \%$ of participants who did not report heroin use during this same time period $\left(X^{2}=26.3, \mathrm{df}=1, \mathrm{p}<.001\right)$. For rates of chronic pain, groups were nearly identical ( $31.5 \%$ vs. $\left.32.7 \%, X^{2}=0.45, \mathrm{df}=1, \mathrm{p}=.838\right)$.

\section{Past-month Income}

Past-month income did not differ between groups. Reflecting the generally low past-month income for the entire sample, both groups reported past-month income $<\$ 1,500$. The heroin use group reported an average past-month income of $\$ 1,128$, whereas the non-use group reported an average pastmonth income of $\$ 1,320(t=1.5, \mathrm{p}=.144)$.

\section{Non-drug Alternative Reinforcers}

Groups were similar in terms of the average number of close relationships reported (5.8, $S D=6.6$ vs. $6.2, S D=5.3, t=0.87, \mathrm{p}=.379)$, rates of past-year social interaction $\left(84.5 \%\right.$ vs. $88.9 \%, X^{2}=2.30, \mathrm{df}=1$, $\mathrm{p}=.122)$, past-year educational/vocational involvement ( $65.5 \%$ vs. $\left.67.3 \%, X^{2}=0.14, \mathrm{df}=1, \mathrm{p}=.705\right)$, and being on community supervision $\left(88.1 \%\right.$ vs. $\left.86.8 \%, X^{2}=0.11, \mathrm{df}=1, \mathrm{p}=.732\right)$. However, groups differed for rates of receiving satisfaction from their past-year social interactions as well as for rates of perceived social worth, as measured by feelings of being cared about and supported by others. Specifically, the heroin-use group had far lower rates of receiving satisfaction for past-year social interaction, with $76.2 \%$ of the heroinuse group reporting satisfaction compared to $90.5 \%$ of the non-use group $\left(X^{2}=29.61, \mathrm{df}=1, \mathrm{p}<.001\right)$.

Likewise, $86.3 \%$ of the heroin-use group reported feeling cared about and supported by others, compared to 93.0\% of the non-use group $\left(X^{2}=8.42, \mathrm{df}=1, \mathrm{p}=.004\right)$.

\section{Age of Drug Use Initiation and Abstinence Self-efficacy Prior to Treatment}

On average, the heroin-use group was younger at the time they first used alcohol or illicit drugs compared to the non-use group $(13.5, S D=4.1$ vs. $14.8, S D=5.1, t=2.6, \mathrm{p}=.010)$. This group also had lower rates of "moderately good" or "very good" abstinence self-efficacy prior to treatment $(73.2 \%$ vs. $82.5 \%$, $\left.X^{2}=79.9, \mathrm{df}=1, \mathrm{p}=.005\right)$. 


\section{Past 30-day Drug Use and Drug Use Frequency Prior to Incarceration}

Groups differed for both pre-incarceration drug use rates as well as frequency of use, as measured in days, for the 30-day period prior to incarceration.

For non-opioid and non-stimulant drugs used during the 30-day period prior to incarceration, rates of pre-incarceration alcohol use were similar across groups, with $44.6 \%$ of the heroin-use group and $50.1 \%$ of the non-use group reporting use $\left(X^{2}=1.58, \mathrm{df}=1, \mathrm{p}=.209\right)$. The number of days that alcohol was used also differed slightly from an average of $5.7(S D=9.6)$ for the heroin-use group and $7.5(S D=11.0)$ for the nonuse group $(t=1.9, \mathrm{p}=.034)$. Rates of pre-incarceration cannabis use did not differ between groups $\left(X^{2}=0.20\right.$, $\mathrm{df}=1, \mathrm{p}=.653$ ), with approximately half of both groups reporting use, nor did frequency of days used, with each group reporting 10-11 days of use on average ( $S D=13.0$ for both groups) $(t=1.1, \mathrm{p}=.261)$. The heroinuse group did report higher rates of prescription sedative use prior to incarceration (45.2\% vs. $30.8 \%$, $\left.X^{2}=13.73, \mathrm{df}=1, \mathrm{p}<.001\right)$, reporting an average of $6.6(S D=10)$ days of use, compared to $5.0(S D=9)$ days for the non-use group $(t=-1.9, \mathrm{p}<.001)$. Hallucinogens were used $<1$ day on average prior to incarceration for both groups ( $t=-1.2, \mathrm{p}=.346)$, with rates of use higher among the heroin-use group (5.4\% vs. $2.9 \%$, $\left.X^{2}=2.10, \mathrm{df}=1, \mathrm{p}=.147\right)$. Similarly, the heroin-use group reported using synthetic drugs prior to incarceration at slightly higher rates $(10.1 \%$ vs. $7.4 \%)$, though groups did not differ significantly $\left(X^{2}=1.22\right.$, $\mathrm{df}=1, \mathrm{p}=.269)$. Both groups used synthetic drugs $<2$ days on average $(t=-0.9, \mathrm{p}=.385)$.

For stimulant drugs, rates of past 30-day cocaine/crack cocaine use prior to incarceration were higher among the heroin-use group ( $32.1 \%$ vs. $\left.22.2 \%, X^{2}=7.85, \mathrm{df}=1, \mathrm{p}=.005\right)$. The average number of days that cocaine/crack cocaine was used prior to incarceration was also slightly greater among the heroin-use group (4.9, $S D=9.6$ vs. $3.2, S D=8.1, t=2.1, \mathrm{p}=.013)$. For past 30-day amphetamine use prior to incarceration, rates between groups were nearly equivalent, approximately $31.0 \%\left(X^{2}=0.10, \mathrm{df}=1, \mathrm{p}=.922\right)$. However, the non-use group reported using amphetamines for slightly more days on average $(6.4, S D=11.3$ vs. $4.6, S D=10.1, t=2.2 \mathrm{p}=.032)$.

For opioid drugs, rates of past 30-day heroin use prior to incarceration were, as anticipated, higher among the heroin-use group $\left(54.2 \%\right.$ vs. $\left.13.3 \%, X^{2}=168.67, \mathrm{df}=1, \mathrm{p}<.001\right)$, as was the average number of days that heroin was used (13.0, $S D=14.0$ vs. $2.5, S D=7.9, t=-9.4, \mathrm{p}<.001)$. Similarly, the heroin-use group reported using prescription opioids at a higher rate $\left(71.4 \%\right.$ vs. $\left.48.2 \%, X^{2}=31.52, \mathrm{df}=1, \mathrm{p}<.001\right)$ and, again, 
for a greater number of days on average (15.3, $S D=13.4$ vs. 10.3, $S D=13.0, t=-4.6, \mathrm{p}<.001)$. For past 30 day buprenorphine use prior to incarceration, the heroin use group reported higher rates than the non-use group, but by a narrower margin ( $25.0 \%$ vs. $\left.17.9 \%, X^{2}=4.49, \mathrm{df}=1, \mathrm{p}=.034\right)$ compared to the other opioid drugs examined. The heroin-use group did report using buprenorphine for slightly more days on average (3.3, $S D=8.2$ vs. $2.6, S D=7.4, t=-1.2, \mathrm{p}=.245)$.

\section{Past-year Drug Use Subsequent to Incarceration}

During the one-year period subsequent to incarceration, the heroin-use group reported higher rates of use for all drugs, used 3.8 drugs on average, and went 71.7 days from the date of their release to the date that they first used illicit drugs.

Among non-opioid and non-stimulant drugs, alcohol was most commonly used sample-wide (31.5\%). Just over $40 \%$ of the heroin-use group reported past-year alcohol use compared to $30.3 \%$ of the non-use group $\left(X^{2}=9.47, \mathrm{df}=1, \mathrm{p}=.002\right)$. Cannabis was the next most commonly used drug during the postrelease period (25.5\%). The heroin-use group had higher rates of cannabis use compared to the non-use group ( $45.8 \%$ vs. $\left.23.0 \%, X^{2}=39.96, \mathrm{df}=1, \mathrm{p}<.001\right)$. For this time period, prescription sedatives, used by $8.0 \%$ of the entire sample, were used at higher rates among the heroin-use group $\left(X^{2}=76.57, \mathrm{df}=1,25.6 \%\right.$ vs. $5.9 \%$, p<.001). Hallucinogens and synthetic drugs were used by $<10 \%$ of both groups. Higher rates of use were reported by the heroin-use group for both hallucinogens $\left(5.4 \%\right.$ vs. $\left.0.6 \%, X^{2}=25.25, \mathrm{df}=1, \mathrm{p}<.001\right)$ and synthetic drugs ( $8.9 \%$ vs. $6.8 \%)$, though groups did not significantly differ for the latter $\left(X^{2}=0.73, \mathrm{df}=1\right.$, $\mathrm{p}=.393)$.

For stimulant drugs, cocaine/crack cocaine, used by $7.4 \%$ of the entire sample, was used during the one-year post-release period at a far higher rate by the heroin-use group $\left(28.6 \%\right.$ vs. $4.5 \%, X^{2}=120.81$, $\mathrm{df}=1, \mathrm{p}<.001)$, as were amphetamines $\left(37.5 \%\right.$ vs. $\left.12.5 \%, X^{2}=70.42, \mathrm{df}=1, \mathrm{p}<.001\right)$, which were used by $15.2 \%$ of the entire sample. For opioid drugs, prescription opioids, used by $14.3 \%$ of the entire sample, were used by the heroin-use group during the one-year post-release period at a rate similar to that of amphetamines (38.7\%), whereas $11.3 \%$ of the non-use group reported prescription opioid use during this time period $\left(X^{2}=89.57, \mathrm{df}=1, \mathrm{p}<.001\right)$. Sample-wide, buprenorphine was used during the one-year postrelease period by $9.4 \%$ of participants, nearly the same proportion that reported heroin use for this same 
time period. Among the heroin-use group, however, 33.3\% reported past-year buprenorphine use, nearly five times that of the non-use group $\left(6.5 \%, X^{2}=123.36, \mathrm{df}=1, \mathrm{p}<.001\right)$.

\section{Central Appalachia, Urban Influence, and Access to Alcohol}

The heroin-use group had a smaller proportion of participants who resided in Central Appalachia for the majority of time during the one-year period subsequent to incarceration $(17.3 \%$ vs. $31.9 \%$, $\left.X^{2}=15.21, \mathrm{df}=1, \mathrm{p}<.001\right)$. Accordingly, the mean urban influence designation for the heroin-use group was lower, indicating greater urban and metro area influence, compared to the non-use group (2.7 vs. 4.7, $\mathrm{p}<.001)$. Accordingly, the heroin-use group had a higher proportion of participants residing in a county with unrestricted alcohol sales $\left(74.4 \%\right.$ vs. $\left.54.4 \%, X^{2}=23.6, \mathrm{df}=1, \mathrm{p}<.001\right)$, as urban and metro areas have overall fewer alcohol prohibitions.

\section{Binary Logistic Regression Results for Heroin Use Subsequent to Incarceration}

Tables 7.0 and 7.1 display the results of the binary logistic regression models examining heroin use (versus "other/no drug use") for one-year period subsequent to incarceration. Variables considered as control variables and independent variables (as opposed to variables used for description only) which differed significantly by group in bivariate analyses were included into the regression models. Due to the overall number of variables which differed significantly by group, two separate models were used to examine the outcome, so as to avoid model overfitting. The first model examined factors correlated with heroin use that occurred during the one-year post-release period along with drug use that occurred during the 30-day period prior to incarceration. A second model examined the relationship between heroin and other drugs used during the one-year post-release period in order to identify what other drugs were significantly associated with a change in the probability that heroin use would be observed.

A displayed in Table 6.0, participants who reported heroin use for the one-year period subsequent to incarceration were more likely to be White $(\mathrm{AOR}=4.84, \mathrm{p}<.001)$. They were also more likely to have spent a greater number of nights in a controlled environment since their release $(A O R=1.00, p=.005)$ and to be younger $(\mathrm{AOR}=0.96, \mathrm{p}=.002)$. Though past-year depressive symptoms were not associated with increased odds of observing past-year heroin use ( $\mathrm{AOR}=1.42, \mathrm{p}=.089)$, past-year suicidal ideation was $(\mathrm{AOR}=2.55, \mathrm{p}=.017)$. Receiving satisfaction from past-year social interaction was also associated with a slight, but significant, decreased likelihood of past-year heroin use (AOR=0.42, $\mathrm{p}<.001)$. Among drugs 
used during the 30-day period prior to incarceration, amphetamines ( $\mathrm{AOR}=0.58, \mathrm{p}=.008)$, heroin $(\mathrm{AOR}=5.32, \mathrm{p}<.001)$, and prescription opioids $(\mathrm{AOR}=2.05, \mathrm{p}=.002)$ were all associated with an increased likelihood that heroin use would be observed for the one-year period subsequent to incarceration, whereas use of prescription sedatives $(\mathrm{AOR}=0.95, \mathrm{p}=.812)$, cocaine/crack cocaine $(\mathrm{AOR}=1.43, \mathrm{p}=0.80)$, and buprenorphine $(\mathrm{AOR}=0.72, \mathrm{p}=.154)$ prior to incarceration were not. Residing in Central Appalachia for the majority of time during the one-year post-release period was associated with a decreased likelihood of pastyear heroin use $(\mathrm{AOR}=0.38, \mathrm{p}<.001)$.

Table 6.0

Results from Binary Logistic Regression Examining Heroin Use for the One-year Post-release Period

\begin{tabular}{llccc}
\hline & Robust & 95\% CI & AOR & p value \\
& SE & & & .001 \\
White & & & & \\
Nights spent in controlled environment & 2.177 & $2.00-11.06$ & .84 & .005 \\
Lifetime drug treatment episodes & 0.001 & $1.00-1.01$ & 1.00 & .285 \\
Ever IDU & 0.022 & $0.98-1.06$ & .1 .02 & .180 \\
Age & 0.296 & $0.87-2.07$ & 1.34 & .002 \\
Past-year depressive symptoms & 0.011 & $0.94-0.99$ & 0.96 & .089 \\
Past-year suicidal ideation & 0.290 & $0.95-2.11$ & 1.42 & .017 \\
Social satisfaction & 0.996 & $1.17-5.42$ & 2.55 & .001 \\
Perceived social worth & 0.101 & $0.25-0.67$ & 0.42 & .096 \\
Age of drug use initiation & 0.175 & $0.36-1.08$ & 0.62 & .903 \\
Abstinence self-efficacy prior to treatment & 0.022 & $0.95-1.04$ & 0.99 & .654 \\
30-day drug use prior to incarceration & 0.192 & $0.60-1.37$ & 0.90 & \\
Prescription sedatives & & & & .812 \\
Cocaine/crack cocaine & 0.203 & $0.62-1.44$ & 0.95 & .080 \\
Amphetamines & 0.297 & $0.96-2.15$ & 1.43 & .008 \\
Heroin & 0.121 & $0.38-0.87$ & 0.58 & .001 \\
Prescription opioids & 1.191 & $3.54-8.25$ & 5.32 & .002 \\
Buprenorphine & 0.468 & $1.31-3.21$ & 2.05 & .154 \\
Central Appalachia & 0.168 & $0.46-1.13$ & 0.72 & .001 \\
Intercept-only: -533.336/Model: -405.742 & 0.091 & $0.24-0.60$ & 0.38 & \\
Wald Chi2 (df=18) 220.306 & & & \\
Hosmer-Lemeshow Chi2=2.39, p=0.966 & Cragg-Uhler/Nagelkerke=0.305 & & \\
\hline
\end{tabular}

Figure 19 displays the percent change in odds for independent variables significantly associated with changes in the likelihood of observing heroin use during the one-year period subsequent to incarceration. 
Figure 19. Percent change in odds for heroin use during the one-year post-release period.

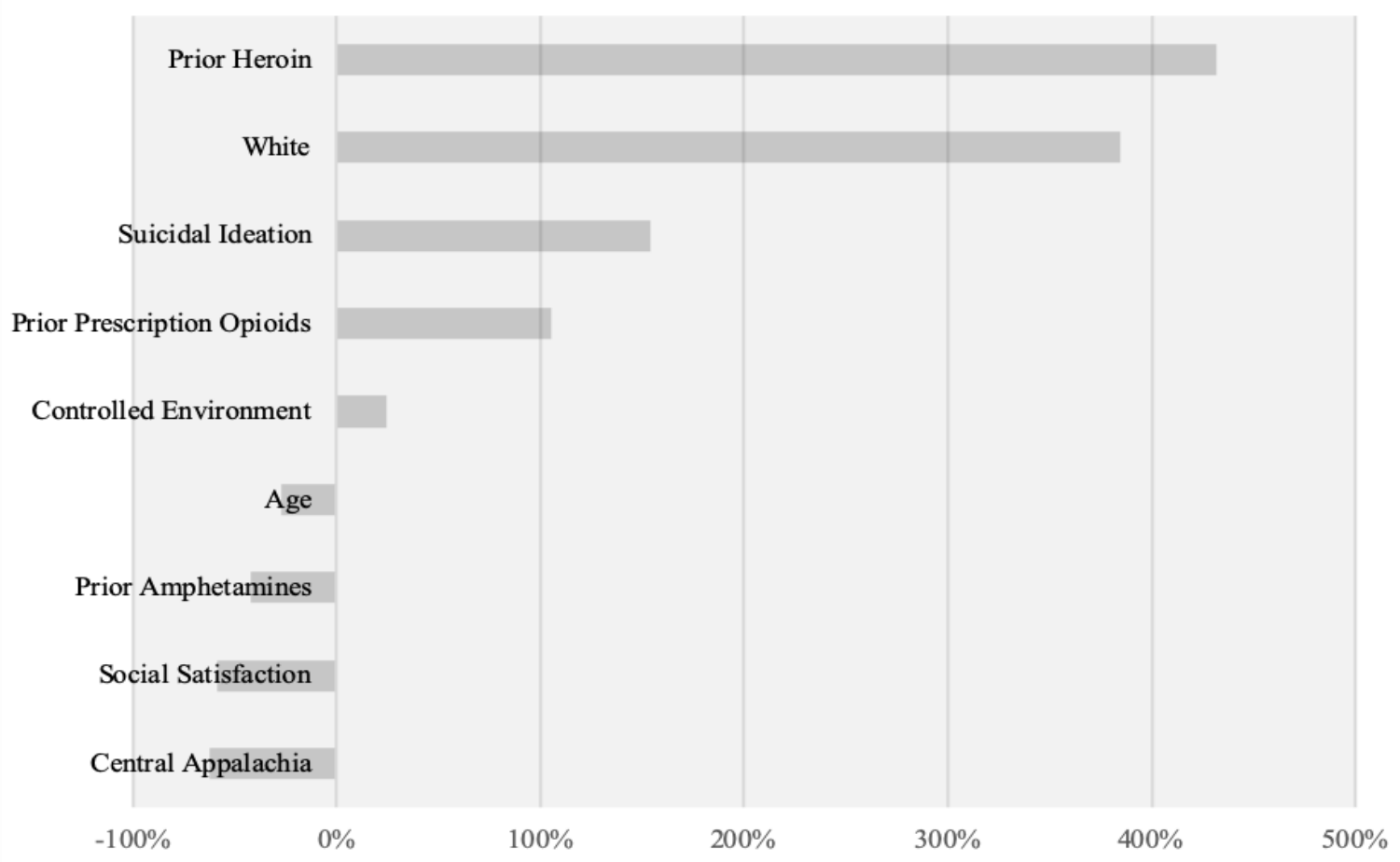

Heroin use during the 30-day period prior to incarceration increased the odds of observing pastyear heroin use for the one-year period subsequent to incarceration by $432.1 \%$. Being White increased the likelihood of past-year heroin use by $384.4 \%$. Past-year suicidal ideation increased the odds of observing past-year heroin use by $154.2 \%$. Use of prescription opioids prior to incarceration increased the odds of past-year heroin use subsequent to incarceration by $105.3 \%$. For every SD increase for nights spent in a controlled environment ( $S D=77.7$ ) the odds of observing past-year heroin use increased by $24.2 \%$, just $0.4 \%$ for each night. The likelihood of observing past-year heroin use decreased by $27.0 \%$ for every SD increase in age $(S D=9.1)$; an approximate $3.4 \%$ decrease for every year increase. Amphetamine use during the 30-day period prior to incarceration decreased the likelihood of observing heroin-use subsequent to incarceration by $42.4 \%$. Receiving satisfaction from past-year social interaction decreased the likelihood by 58.7\%. Finally, residing in Central Appalachia for the majority of the one-year period subsequent to incarceration decreased the likelihood of heroin use by $62.2 \%$.

Results of the second model are displayed in Table 6.1. Prescription sedatives (AOR=1.82, $\mathrm{p}=.043)$, cocaine/crack cocaine $(\mathrm{AOR}=5.70, \mathrm{p}<.001)$, amphetamines $(\mathrm{AOR}=1.71, \mathrm{p}=.029)$, prescription 
opioids (AOR=1.72, $\mathrm{p}=.030$ ), and buprenorphine ( $\mathrm{AOR}=3.66, \mathrm{p}<.001)$ used during the one-year period subsequent to incarceration were all associated with an increased likelihood of heroin use for this same time period. Alcohol (AOR=0.85, $\mathrm{p}=.446)$, cannabis $(\mathrm{AOR}=1.40, \mathrm{p}=.119)$, and hallucinogens $(\mathrm{AOR}=1.61$, $\mathrm{p}=.397$ ) used during the one-year period prior to incarceration did not significantly influence the likelihood of past-year heroin use.

\section{Table 6.1}

Results of Binary Logistic Regression Examining Past-year Other Drug use and Past-year Heroin Use During One-year Post-release Period

\begin{tabular}{|c|c|c|c|c|}
\hline & Robust SE & $95 \% \mathrm{CI}$ & AOR & p value \\
\hline Alcohol & 0.179 & $0.56-1.28$ & 0.85 & .446 \\
\hline Cannabis & 0.302 & $0.92-2.13$ & 1.40 & .119 \\
\hline Prescription sedatives & 0.540 & $1.01-3.22$ & 1.82 & .043 \\
\hline Hallucinogens & 0.908 & $0.53-4.66$ & 1.61 & .397 \\
\hline Cocaine/crack cocaine & 1.44 & $3.48-9.34$ & 5.70 & .001 \\
\hline Amphetamines & 0.421 & $1.06-2.76$ & 1.71 & .029 \\
\hline Prescription opioids & 0.431 & $1.06-2.81$ & 1.72 & .030 \\
\hline Buprenorphine & 0.907 & $2.25-5.91$ & 3.66 & .001 \\
\hline \multicolumn{2}{|c|}{ Intercept-only: -533.336/Model: -436.689 } & \multicolumn{3}{|c|}{ Pseudo $R^{2}=0.181$} \\
\hline \multicolumn{2}{|c|}{ Wald Chi2 (df=8) 189.581} & \multicolumn{3}{|c|}{ Cox-Snell/ML= 0.116} \\
\hline \multicolumn{2}{|c|}{ Hosmer-Lemeshow Chi $2=15.56, p=0.082$} & \multicolumn{3}{|c|}{ Cragg-Uhler/Nagelkerke $=0.235$} \\
\hline
\end{tabular}

Figure 20 displays the percent change in odds for drugs used during the one-year period subsequent to incarceration significantly associated with changes in the likelihood of observing heroin use during the same time period. 
Figure 20. Percent change in odds for heroin use during the one-year post-release period by co-used drug.

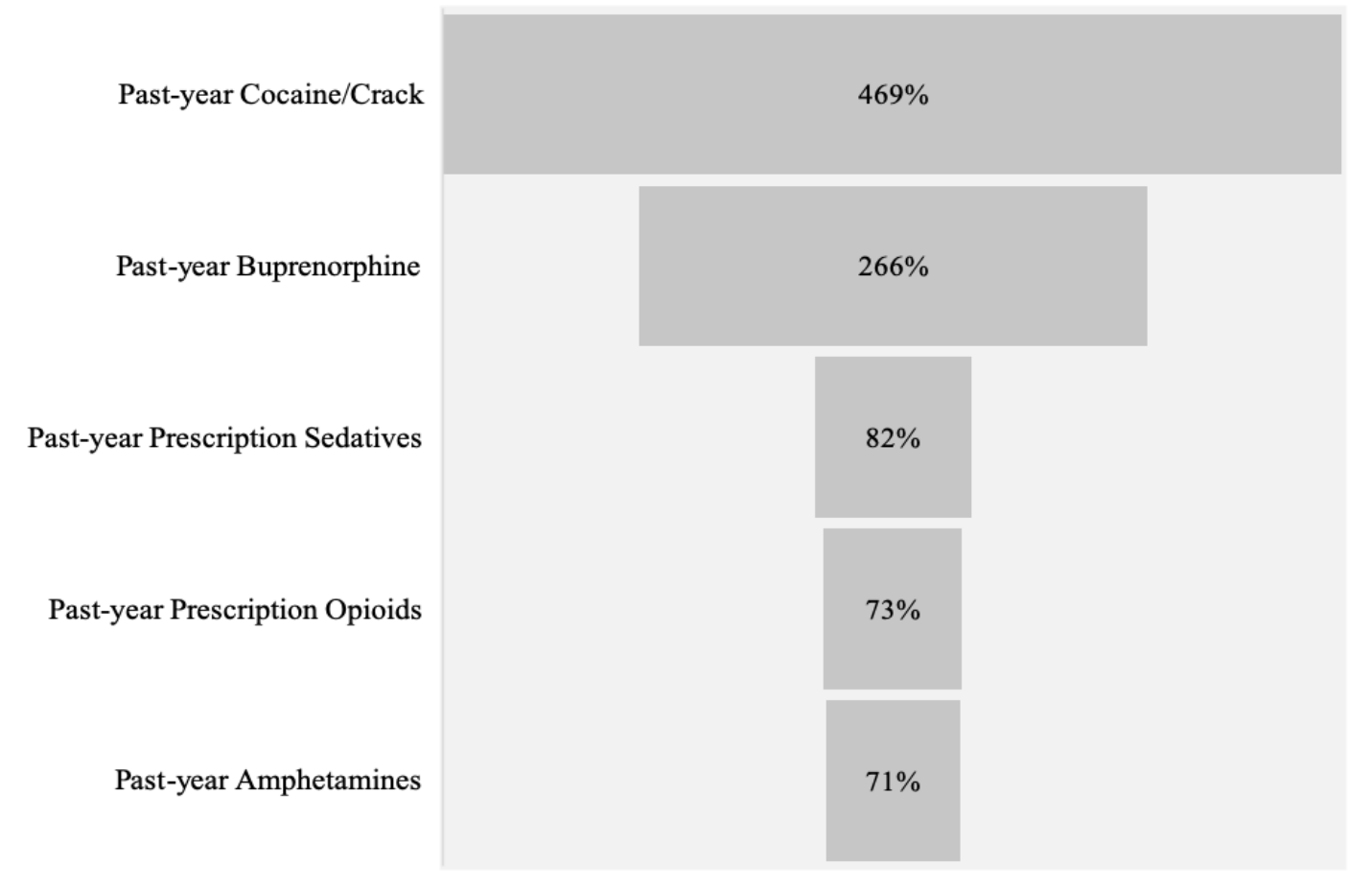

For all drugs used during the one-year period subsequent to incarceration associated with a change in odds for past-year heroin use, past-year cocaine/crack cocaine use showed the most influence, increasing the odds that heroin use would be observed by $469.1 \%$. This was followed by past-year buprenorphine use, associated with $265.9 \%$ increased odds of observing past-year heroin use. Past-year prescription sedative use increased the likelihood of observing past-year heroin use by $82.3 \%$, whereas past-year prescription opioid use increased the likelihood by of $72.9 \%$ and past-year amphetamine use increased the likelihood by $70.9 \%$. 


\section{CHAPTER V}

\section{DISCUSSION}

The use of high-risk drugs, such as opioids and stimulants, constitutes a significant economic, social, legal, and medical problem in the US (Degenhardt et al., 2014; Schuchat, Houry, \& Guy, 2017; Volkow \& Collins, 2017). Kentucky is a state that continues to experience high rates of opioid and stimulant use and related problems, and is also a state that incarcerates a disproportionate number of people who use opioids and stimulants (CDC 2017a, CDCb; Faryar et al. 2017; Prison Policy Initiative, 2018). It is important to better understand decisions to use high-risk drugs among corrections-involved people in Kentucky, in part because the adverse consequences of continued use for this population can be extreme (e.g., becoming reincarcerated, increased odds of overdose following release from correctional custody) (Merrall et al. 2010).

Accordingly, this study's primary aim was to explore the social problem of high-risk drug use among a sample of corrections-involved adults in Kentucky and to identify and discuss endogenous and exogenous factors with the potential to have influenced drug-related decision-making prior and subsequent to incarceration. This was accomplished with three specific study aims. First, using self-report data, a descriptive profile of a sample of corrections-involved adults in Kentucky with a history of drug use was provided. The prevalence of concomitant opioid/stimulant use, opioid use, stimulant use, and other and/or no drug use during the 30-day period prior to incarceration and the one-year period subsequent to incarceration was determined. A description of these drug use outcome groups was presented, and differences between groups was determined. Differences in the use of specific opioid and stimulant drugs (e.g., heroin, prescription opioids, buprenorphine, cocaine/crack cocaine, and amphetamines) by participant 
residence across an urban-rural continuum and by participant residence within or outside of Central Appalachia were also established. Second, factors associated with a change in likelihood of observing concomitant use, opioid use, and stimulant use during the 30-day period prior to incarceration and the oneyear period subsequent to incarceration, compared to an outcome with less risk (e.g., other/no drug use), were identified. Third, the prevalence of heroin use that occurred during the one-year period subsequent to incarceration was determined and the between-group differences among participants who reported heroin use and those who did not were identified. Factors associated with a change in the likelihood that heroin use would be observed were also identified.

Behavioral economics, a molar view of human choice and behavior, was used in earlier chapters to first conceptualize the problem of high-risk drug use and then to help situate and better understand the problem in relation to a large and growing body of empirical research. While only a small portion of research relevant to understanding how people's decision-making and behavior can be influenced was reviewed in Chapter 2, the overview hopefully demonstrated the important point that many endogenous and exogenous factors have the ability to influence decision-making and behavior, not only in respect to highrisk drug use, but in respect to all choice and action. It also hopefully articulated the idea that while many of these factors are not always knowable, observable, or readily modeled, they are nonetheless recognizable (and worth recognizing) using a molar paradigm.

Because of the vast number of possible endogenous and exogenous factors exerting influence on human behavior, it is important to at times seek a broad understanding of specific behaviors, such as highrisk drug use, as an expansive view acknowledges the inherent complexity and dynamic nature of choice and action (Sapolsky, 2017). Opioid and stimulant use specifically are important to understand, as use of these drugs constitutes a threat to both the people using them as well as to those around them. That opioid and stimulant use simultaneously represents the potential for pleasure and pain, and that only a small fraction of people will ever in their lifetimes pursue such risky choice, suggests the possibility that certain unique factors present among high-risk drug users, and among corrections-involved drug users in particular, are influencing decision-making and behavior. Why and how people choose to do something that they know is potentially detrimental, and then continue to choose it even after experiencing adverse outcomes, are important empirical questions generally. It is necessary, though, to explore this phenomenon 
both conceptually and empirically; the latter helping to guide empirical exploration, and the former helping to refine conceptualizations of choice and behavior within a system of constraint. This chapter seeks to achieve limited accomplishment of this in respect to study findings in several ways.

First, by revisiting the puzzling phenomenon of high-risk drug use, with attention paid to how participants might continue to choose to use opioids and stimulants even after problems related to using have become apparent. In earlier chapters, the conflict that can arise between a person during intertemporal choice was discussed along with the idea that people can have time-inconsistent preferences (Shane, Loewenstein, \& O'Donoghue, 2002). Also discussed was how a person can want, and choose, what they don't actually like, and which does not produce experienced utility (Berreidge \& O’Doherty 2014). People can also prefer a particular outcome, but then act in a discordant manner by choosing to undertake a behavior that will bring about its opposite. Recall that while drugs have a high local utility, their repeated use over time decreases their overall utility (Herrnstein \& Prelec, 1992). A person might prefer to not get arrested, overdose, or become dependent on heroin, yet may still repeatedly decide to use heroin due to its high local utility, thus increasing the probability that such unwelcome outcomes will occur. These ideas will be briefly revisited using information self-reported by participants as an example. This will be followed by considering how drug-related decision-making prior and subsequent to incarceration may differ. Not all people who use opioids and stimulants experience adverse outcomes, and certainly do not all experience an outcome as extreme or adverse as incarceration; however, many in this sample did. This makes the phenomenon of incarceration itself a point of interest. Among participants in this sample, it reflects time spent in a controlled environment between two periods of decision-making for which selfreport information was available for examination.

Second, several key study findings will be discussed, giving attention to some of the ways that they relate to a behavioral economic account of choice and behavior. Of the many findings presented in Chapter 4, those most illustrative of the concepts described in earlier chapters will be discussed. It will hopefully be made clear by the nature of the discussion that all findings, that is, all factors identified and explored in this study as having the potential to influence drug-related decision-making and high-risk drug use, are important--as are many other factors not explored as part of this study. An omission of a particular study finding is not meant to suggest its irrelevance or unimportance. As this is an exploratory study, 
priority will be given to highlighting broad take-aways from study findings. In the examples that follow, it will be apparent that all factors with the potential to influence decision-making and the complex behavior of high-risk drug use are potentially relevant, even if it is impractical to enumerate, and impossible to measure, all such influences. Referring back to Figure 1 provides the "big picture" view that is of interest and which is applicable to study findings. For findings not specifically discussed, or not discussed at a length equal to others, the idea of choice and behavior as operating within a system of constraint can still be applied. The broader take-away when this conceptual framework is applied to any or all findings is unchanged; a testimony to its parsimony and flexibility. A summary of future directions, limitations, and concluding thoughts will be presented at the chapter's end.

\section{Some Potential Differences in Drug-related Decision-making Prior and Subsequent to Incarceration}

As described earlier, the many endogenous and exogenous factors of a person's life comprise part of the system of constraint within which they make decisions and behave (Bickel et al., 2014; 2016). These factors influence people's cognition, choice, and capacity for action. Better recognition of these factors can help make sense of decisions to use opioids and stimulants despite known risks, and decisions to continue using despite adverse consequences. It can also be extended to help make sense of why use could continue despite a person's desire or intention to stop. People who choose to undertake the steps necessary to acquire and self-administer drugs with the potential to kill them are an extreme example of how choice and behavior can be influenced in profound and counterintuitive ways. This is most poignant when a person at one moment desires to use opioids and then undertakes the actions required to use them, while a moment before desired not to use opioids and may have even resolved not to use them, indicating that people, their preferences, and their intentions are not necessarily consistent over time, or even within the same moment of time (Ainsile, 2001; Bratmann, 1999; Mann, 2004; Melrose et al., 2015; Monterosso \& Lou, 2010; O'Donoghue \& Rabin,1999). In part, self-control conflict involves tension between preference at different scales, the molecular (e.g., desire for crack cocaine right now) and the molar (e.g., desire not to be addicted to crack cocaine) (Rachlin, 1995).

This idea may be applicable to some participants in this sample and indirectly reflected among some survey responses. For instance, some participants made a decision to use opioids or stimulants subsequent to incarceration, but may have, during their incarceration, contemplated doing otherwise. 
Examination of some of the constituent elements of the "drug use severity" variable used in this study may be illustrative Some of its specific qualities are considered here for the purposes of thinking about how discrepancies in utility, or preference, can occur between a person's current and future self. For instance, the majority of participants who reported using opioids (74.2\%) and stimulants (70.3\%) during the one-year period subsequent to incarceration also reported being "moderately, considerably, or extremely troubled or bothered" by their drug use during the 30-day period prior to incarceration.

Further, a majority of participants who reported using opioids (95.8\%) and stimulants (94.9\%) during the one-year period subsequent to incarceration also reported believing that receiving drug treatment for their drug problems was "moderately, considerably, or extremely important to them" at the time of treatment intake, which occurred weeks, months, and in some cases, years after their arrest. This means that participants had some time since their last period of regular using to possibly consider whether receiving drug treatment was a low or high priority. When answering this question, participants were presumably not intoxicated, nor were they presumably at their drug-using nadir--in a state of withdrawal or acute crisis common among regular opioid and stimulant users. In other words, a response to this question was likely provided during a period of relative stability. If responses are considered valid, it means that $>70 \%$ participants who later used opioids and stimulants felt at these earlier times not only bothered by their drug problems, but also felt that receiving drug treatment was important in helping them address these problems. For the latter, rates were significantly higher among participants who reported opioid and stimulant use subsequent to incarceration, compared to those who reported other drug or no drug use $(\mathrm{p}<.05$; results not displayed). Thus, despite acknowledgment of drug problems, despite an articulated belief that drug treatment was important, and despite receiving severe consequences as a result of drug use, opioid and stimulant use continued.

That people who once used opioids and stimulants experienced adverse consequences as a result of use, expressed some interest in treatment, participated in treatment, and then subsequently chose to use opioids and stimulants, is not shocking. Rates of lapses and relapses subsequent to a variety of drug interventions or after periods of abstinence are well documented (Dodge, Sindelar, \& Sinha, 2005; McLellan, Lewis, O'brien, \& Kleber, 2000; Sinha, 2001). Indeed, the conceptualization of drug addiction as a chronic condition or "disease" relies in part on the fact that many people who once misused opioids and 
stimulants go on to use them again even after intervention of periods of abstinence (Denis \& Scott, 2007; McLellan, et al., 2000).

There is, of course, no way to know whether participants who used opioids and stimulants both prior and subsequent to incarceration at any point developed in the interim a desire or intention to not use these drugs, but it is possible that many at some point did. It is possible for two reasons. First, participants who used opioids and stimulants during the one-year period subsequent to incarceration experienced 16 days of drug-related problems on average during the 30-day period prior to incarceration. These problems included craving, withdrawal, and a desire but inability to quit using. It is possible that during this time period in which some very frustrating and painful symptoms were frequently occurring that an intention to modulate or discontinue use was formed by at least some portion of participants; though there are insufficient data to clearly support this proposition. This possibility is nonetheless noted to raise the point that people are capable of deciding to engage in a behavior that they previously indicated was problematic for them and after experiencing significant consequences, reflective of time-inconsistent preferences and the diachronic model of conflict (DiClemente, 2018; Melrose et al., 2015). Second, this 30-day period when frequent drug-related problems occurred climaxed with incarceration, which is itself comprised of multiple humiliations and punishments and involved, for participants in this sample, a period of confinement lasting many months or longer. This means that there were many occasions for these participants to possibly consider some of the benefits of not using opioids and stimulants upon release and, possibly, of developing some desire or intention not to use following release. Yet, many still used. Trying to better understand desire and intention related to high-risk drug use among people with history of highrisk drug use will be important to explore in future work concerned with drug-related decision-making.

\section{Incarceration and Community Supervision}

The possibility that cognition and behavior in respect to opioids and stimulants was influenced by incarceration and treatment makes exploring high-risk drug use among this sample interesting, as there is a demarcation (pre- and post-incarceration) between two periods of decision-making. These periods are distinctive in several ways. For example, participants could have been influenced to use or not use following a period of incarceration by a variety of factors that could have materialized as part of the incarceration experience itself. On the one hand, these might include generally stabilizing experiences, such 
as rebuilding relationships with family members, receiving treatment, engaging in spiritual, religious, or mutual-aid programs, exercising, developing a routine, etc. (Buckaloo, Krug, \& Nelson, 2009;

Freudenberg, Wilets, Greene, \& Richie, 1998; Stringer, 2009; Tasca, Mulvey, \& Rodriguez, 2016). On the other hand, they could include experiences such as sexual and physical violence, social isolation, family estrangement, and overall decreased psychological health due to jail and prison conditions (Alarid, 2000; Bonner \& Rich, 1990; Brinkley-Rubinstein, 2013; Kaba et al., 2014; Rokach, 1997). This study was not concerned with investigating highly personal and varied experiences that might influence a person's overall cognitive health prior to re-entry, though they warrant investigation. Rather, the idea that incarceration may have influenced later decision-making about opioids and stimulants is noted because it applies to findings for the post-incarceration period when considered using a molar paradigm, such as behavioral economics.

First, by having experienced the adverse outcome of incarceration as a direct or indirect result of use and by being under DOC supervision upon release, participants may have been influenced to modify their behavior so as to reduce the likelihood of their experiencing incarceration again. Experienced punishment and the threat of additional punishment could have influenced decision-making about opioids and stimulants subsequent to incarceration in several ways. For instance, whereas prior to incarceration the risks associated with opioid and stimulant use may have only been considered in terms of outcome uncertainty in the abstract--the known potential for adverse outcomes with contingent future states discounted as a function of time or probability (or both)--subsequent to incarceration, risks associated with opioid and stimulant use could have been partially reflected upon in terms of experienced adverse outcomes (Bickel et al., 2007; Rachlin, Raineri, \& Cross, 1991; Vuchinich \& Simpson, 1998). Future states would still be abstract and uncertain, and therefore have the potential to be discounted as a function of time and probability, but the salience associated with the experience of adverse outcomes from opioid and stimulant (and their memories) might have served as a behaviorally motivating influence on decision-making subsequent to incarceration for some, but certainty not all, participants (Blair, Morton, Leonard, \& Blair, 2006; He, Cassaday, Howard, Khalifa, \& Bonardi, 2011; Newman, 1987). Just as reward can facilitate learning and reinforce behavior, so too can punishment; fear of threat and anticipation of reward both involve memory, outcome expectations, prospection, etc. (Bohnert et al., 2018; Herry \& Johansen, 2014; Sigurdsson, Doyère, Cain, \& LeDoux, 2007). For example, having overdosed once or having been arrested 
once, a person would develop a clearer understanding and appreciation of what the possible future outcome of overdose or arrest entail (Warner-Smith, Darke, \& Day, 2002). However, people who experience adverse events related to drugs may differ in terms of how future behaviors are influenced by past experiences, as threat (of punishment) detection, fear processing, and risk-aversion may differ across people, drug use histories, and contexts (Hoppenbrouwers, Bulten, \& Brazil, 2016; Mcgregor, Darke, Ali, \& Christie, 1998; Wood \& Anagnostaras, 2009). Relevant, too, is that some memories associated with drug pleasure may also be highly salient (Stacy, Ames, Wiers, \& Krank, 2010). There is no way to know which may have been more influential at any given moment subsequent to incarceration: memories of drug-related pleasure or memories of drug-related pain; and, likewise, prospection about possible future drug-related reward or prospection about possible future drug-related punishment.

Community supervision following release, which includes drug monitoring and the threat of sanctions, may have served as one specific influence on decision-making and behavior (Burdon, Roll, Prendergast, \& Rawson, 2001; Franken et al., 2003; Marlowe \& Wong, 2008). However, while rates of being on community supervision subsequent to incarceration differed by drug use outcome group, differences were $<10 \%$; not substantial. Further, a greater proportion of participants who reported concomitant and stimulant use subsequent to incarceration were on community supervision, meaning that being on probation/parole did not necessarily influence a decision not to use or to use less dangerous drugs in all instances. This is evidenced further by the fact that being on community supervision was associated with an $89 \%$ increase in odds that concomitant use would be observed.

Experiencing incarceration and being on community supervision may partially explain why rates of opioid and stimulant use subsequent to incarceration decreased from rates of use prior to incarceration, but it does less to explain why use would continue. It is possible that participants on community supervision who chose to use may have discounted future outcomes not only as a function of time, but also probability (Rachlin, 2006; Rachlin et al., 1991). While some interaction with a probation or parole officer while on community supervision is an eventuality, the particulars of those interactions, such as the frequency of drug monitoring, are often irregular. One potential appeal of opioids and stimulants is that their metabolites are cleared from the body fairly rapidly, depending on the person's age, weight, dosing, and drug type, meaning that it is possible that a portion of participants who used these drugs while on 
community supervision did so with an expectation that they would be able to avoid detection via drug monitoring (Moeller, Kissack, Atayee, \& Lee, 2017). The purposeful selection of drugs while on community supervision as a means of circumnavigating drug monitoring has been documented elsewhere (Smith \& Stoops, 2019, Smith \& Staton, 2019).

Second, having spent time in a controlled correctional facility means that participants went from an environment where there were few restrictions to accessing drugs to a facility where access to drugs was significantly restricted. Even if it cannot be assumed that the facilities that participants resided in were $100 \%$ drug-free $100 \%$ of the time, it can be assumed that the accessibility of alcohol and illicit drugs was still significantly less than the accessibility of alcohol and illicit drugs in the free world. This means that for many in this sample opioid and stimulant use either stopped or decreased significantly during their incarceration. Even if use only became irregular, rather than ceasing entirely, it still means that the frequent and regular use of drugs, characteristic of the 30-day period prior to incarceration, was moderated. This is relevant insofar as a period of intermittent use or complete abstinence, would have permitted the opportunity for some modest and heterogenic, but nevertheless potentially important, neurobiological and behavioral changes (Galinato et al., 2018; Hollander \& Carelli, 2005; Pitel et al., 2009; Schulte et al., 2014; Volkow et al., 2001).

There would have also likely been fewer drug-related cues in jail or prison capable of producing a conditioned-response, compared to places where participants spent time prior to incarceration, meaning that attention would be directed less frequently to drug stimuli. Even if participants' attention was still largely pre-occupied with drugs during incarceration, by virtue of just thinking about drugs, these cognitions would not have had behavioral traction in most cases due to the decreased drug availability. Thus, for this sample, drug-related decision-making subsequent to incarceration could not have been undertaken with the exact same set of endogenous factors (i.e., not the same brain) that drug-related decision-making prior to incarceration was undertaken with. However subtle and ambiguous the changes were, there was still some change. This is important, because it means that at the time of participants' release, drug-related visceral states, cognitions, and behavioral patterns would have be different than at the time of arrest. Put differently, their cognitive capacities would be, holding other factors constant, less constrained than they were prior to incarceration. Although a participant's regular cocaine use prior to incarceration would still have the 
potential to bias them toward a decision to use cocaine subsequent to incarceration, this prior regular cocaine use would not necessarily be influencing decision-making subsequent to incarceration in the same manner or to the same degree as it did prior to incarceration. Consider how decision-making about cocaine might look for a person who had used cocaine every day for the past month compared to the same person who used cocaine for 30 days one year and one month ago. It is easy to envision how more or less constrained a person would be in their decision-making about cocaine between these two time periods. That prior decisions to use can still bias a person toward future use of the same drug is reflected in the findings that use of specific opioids and stimulants prior to incarceration increased the likelihood of the same, or similar, drug use subsequent to incarceration. But that many participants also did not use subsequent to incarceration reflects the possibility that they were potentially less constrained in their drug-related decision-making than they had been during the 30-day period prior to incarceration. These ideas will be described in more detail below.

Upon re-entering the community, participants would have become exposed to significantly more actionable choices than they were while incarcerated. Greater access to drugs is, unsurprisingly, associated with greater likelihood of use and continued use. The mere knowledge that opioids and stimulants are again accessible would have been another factor with the potential to influence participants' visceral, affective, and cognitive states subsequent to incarceration, including those associated with craving, which can be a profound influence on drug decision-making and behavior (Badger et al., 2007; Berridge \& O’Doherty, 2014; Paliwal, Hyman, \& Sinha, 2008; Robinson \& Berridge, 1993; Tiffany, Warthen, \& Goedeker, 2008; Tsui, et al., 2016). Although access to rewarding non-drug choices would also have increased significantly upon reentry, not all participants would have had equitable access to the same types of non-drug alternatives. Ultimately, the context into which participants were released would have had significant influence on decisions to use and to continue using (Marlatt, 1996).

Lastly, and perhaps most importantly, because reentry into the community following incarceration involves significant transition across all aspects of daily living means that it is likely that some participants experienced stress following their release (Bahr, Armstrong, Gibbs, Harris, \& Fisher, 2005; Phillips \& Lindsay, 2011; Shivy et al., 2007). Stress is toxic for higher-level cognitive functioning, such as deliberative decision-making, planning, prospection, and inhibition, meaning that participants may have 
been generally less capable of engaging in adaptive decision-making in relation to drugs during this period of transition and possible stress (Kosten \& George, 2002; Weiss, 2005; McEwen, 2006; McEwen \& Sapolsky, 1995). That reentry-related stress or other daily life challenges would have occurred contemporaneous with a significant increase in potential exposure to drug-related cues suggests that community reentry should be considered a time period of heightened risk, insofar as stress + drug-related cue exposure is associated with craving and drug use among abstinent and current drug users (Fox, Bergquist, Hong, \& Sinha, 2007; Hyman, Fox, Hong, Doebrick, \& Sinha, 2007; Phillips \& Lindsay, 2011; Sinha, Catapano, \& O’Malley, 1999). Among participants who prefer opioids, a return to opioid use following a period of decreased use or abstinence would have the potential to be life-threatening, due to diminished tolerance, meaning that risk among this group may be greatest (Davidson, Wagner, Tokar, \& Scholar, 2019).

\section{Drug-related Influences on Decision-making}

A person's ability to make decisions that maximize overall utility are, even in the best of circumstances, not superb. People are generally biased toward the present more so than the future and can have time-inconsistent preferences (Loewenstein \& Prelec, 1993; Monterosso \& Lou, 2010). Certain endogenous and exogenous conditions can hinder decision-making capacities further, by either influencing cognition and executive function and/or by restricting available choices and opportunities (Bickel et al., 2014). For participants in this sample with a history of opioid and stimulant use, the capacity for making decisions that maximize overall utility, instead of repeatedly selecting choices with higher local utility, but greater cost or risk, may be diminished compared to people without a history of such drug use (Badiani et al. 2011; Heil et al., 2006; Kirby \& Petry, 2004; Kirby, Petry, \& Bickel, 1999; Petry, 2001; Petry et al., 1998; Reynolds, 2006; Rogers et al., 1999). This may be partially due to the presence of certain factors that may have pre-dated opioid and stimulant use, and which are found disproportionately among people who elect to use opioids and stimulants, or due to characteristics and states that developed as a result of opioid and stimulant use (Badiani et al. 2011; Petry, 2001; Potvin et al., 2018; Verdejo-García, et al., 2008). In either case, there is an implication that all participants who used opioids and stimulants during their lifetime may have been less able to engage in adaptive decision-making compared to people without such history. 
The rates of opioid and stimulant use during the year prior to incarceration among this sample indicate fairly regular use. Although it is possible that participants who used opioids and stimulants during the 30-day period prior to incarceration did so regularly, but in a controlled manner not suggestive of disordered use, it is not likely, given the high rates of drug use severity, IDU history, and having been intoxicated during the commission of a crime. Overall, evidence suggests that the high rates of opioid and stimulant use during the one-year period prior to incarceration, and any use prior to that, would have served to influence participants' decision-making capacities in respect to these drugs during the 30-day period prior to incarceration, insofar as neurocognitive changes associated with regular opioid and stimulant use correspond to impairments in impulse control, future-oriented cognition and behavior, metacognition, and working memory capacity, among other things (Bechara, 2005; Nichols \& Wilson, 2015; Koob \& Volkow, 2010). Recall that a behavioral economic account of drug use and addiction describes changes in the person as a result of drug use over time, as well as changes in available choices as a result of drug use over time, with the person's overall set of motivations informing the development and perpetuation of use (Bickel et al., 2011, 2014). While a person's brain changes as a result of regular using, so too does their economic situation, their social standing, their health, the quality of their relationships, and their priorities, preferences, and goals, etc., all of which, in turn, change their brain, cognition, and behavior still further (Adophs, 1999; Cacioppo \& Hawkley, 2009; Fiske \& Taylor, 2013; Volkow, Baler, \& Goldstein, 2011). These neurocognitive and social conditions, some more long-lasting than others, can have profound effects, including altering how drug and non-drug choices are perceived, valued, and acted upon (Heilig et al., 2016; Kosten \& George, 2002).

During the 30-day period prior to incarceration, participants, by virtue of their prior regular use, may have already reached the point where opioids and stimulants were significantly overvalued and nondrug choices, which would have likely already declined in number and quality for many, were significantly undervalued; this imbalance would have helped to further reinforce continued decisions to use (Bickel et al., 2001). Crucially, too, the very type of cognitive capacities (e.g., flexibly, ability for abstract, counterfactual, or prospective thinking, top-down cortical control to inhibit reflexive or habituated responding) that participants would have most needed to "do otherwise" would have been either more diminished or impaired during the 30-day period prior to incarceration than at earlier times, due to the fact 
that opioids and stimulants had, by that point, been used over a longer period of time and used fairly recently (Di Sclafani, Tolou-Shams, Price, \& Fein, 2002; Jovanovski, Erb, Zakzanis, 2005; Potvin et al., 2018; Verdejo-García, 2011; Verdejo-García, Bechara, Recknor, \& Perez-Garcia, 2006).

One possible result of regular opioid and stimulant use in the months leading up the 30-day period prior to incarceration is that degrees of freedom for the development of preference, intention, choice, and action would have decreased, rather than increased or remained in a state of equipoise, due to neurophysiological and psychosocial changes that developed as a result of continued drug taking (Bratman, 1999; Brower, Guilfoy, 2004; Dennett, 2015; Mann, 2004; O’Connor, 2009; Volkow \& Li, 2004). In this way, there would have been fewer mechanisms by which participants could have overridden the many conditions biasing them toward continued decisions to use during that 30-day period. These mechanisms might be cognitive, but they may also consist of resources and other tools external to the person that became degraded after a period of regular use (e.g., decrease in the number of avenues for pursuing nondrug opportunities, decrease in amount of available help or support) (Peirce et al., 2000; Skinner, Feather, Freeman, \& Roche, 2007). This means, in part, that the opportunity costs associated with continued use may have been fewer, the non-drug options slimmer, and understanding and assistance from others limited or difficult to access (Ahmed, 2013; Bickel et al., 2014).

Participants who used opioids and stimulants during the 30-day period prior to incarceration who had a history of opioid and stimulant use can be considered to have already been at a disadvantage by the time they reached this point, in that their past use was likely exerting some influence on decision-making and behavior in relation to opioids and stimulants, and through whatever conditions of their lives were shaped as a result of prior using. This would have occurred to greater or lesser degrees depending on the nature of their respective past-year or lifetime using history. Because participants' lifetime drug use history could not be examined as part of this exploratory study, due to the fact such data were not collected as part of the SAP clinical assessment and the CJKTOS evaluation, there are many limits to understanding the degree to which participants may have been influenced in their decision-making about opioids and stimulants. However, several pieces of information related to participants' drug use history were able to be examined. These may help to further contextualize decisions about opioids and stimulants that were made prior and subsequent to incarceration. 


\section{Prior Drug Use}

Rates of drug use decreased between the one-year period prior to incarceration, the 30-day period prior to incarceration, and again between the 30-day period prior to incarceration and the one-year period subsequent to incarceration. ${ }^{10}$ Considered together, these basic findings convey several possibilities. For one, prior exposure to a drug does not guarantee continued use, but rather may only confer increased likelihood of use compared to people who have never previously used these drugs. This is evidenced by the fact that rates of use decreased over time, but did not decrease to zero. Some participants who used an opioid during the year prior to incarceration did not use an opioid during the 30 days prior to incarceration; fewer still used opioids and stimulants subsequent to incarceration. Thus, a history of use, while informative, is not a marker of some later fate. Other factors besides prior opioid and stimulant use may have also influenced the likelihood that participants would decide to use these drugs subsequent to incarceration, as will be discussed below in subsequent sections.

Differences were found, though, between participants who reported concomitant, opioid, and stimulant use during the 30 days prior to incarceration for the number of months out of the year prior to incarceration that specific opioid and stimulant drugs were used. For example, participants who reported past 30-day concomitant use or past 30-day opioid use reported using heroin, prescription opioids, and buprenorphine more months on average during the year prior to incarceration compared to past 30-day stimulant users, even though the latter group did report some, albeit less, opioid use during this same oneyear period. Heroin was the only opioid drug that wasn't used during the one-year period prior to incarceration among participants who reported stimulant use during the 30 days prior to incarceration. As was anticipated, participants who reported past 30-day concomitant use prior to incarceration used opioids and stimulants, and several other drugs, at higher rates, and for more months on average, than the other

\footnotetext{
${ }^{10}$ During the one-year period prior to incarceration $37.0 \%$ of participants reported concomitant use, and for the 30-day period prior to incarceration $29.0 \%$ reported concomitant use; for the one-year period subsequent to incarceration $11.9 \%$ reported concomitant use. For the one-year period prior to incarceration, $63.7 \%$ of participants reported using opioids, and for the 30-day period prior to incarceration, $28.5 \%$ used opioids; for the one-year subsequent to incarceration $12.5 \%$ reported using opioids. For the one-year period prior to incarceration $56.7 \%$ reported using stimulants, and for the 30-day period prior to incarceration $18.0 \%$ reported using stimulants; for the one-year subsequent to incarceration $8.3 \%$ reported using stimulants.
} 
groups. The only exception was for alcohol, which was used by all groups at approximately the same rate and frequency.

During the one-year period subsequent to incarceration, a portion of participants for all drug use outcome groups reported prior use of opioids and stimulants. The post-release rates of use for opioids and stimulants disproportionately fell along pharmacological lines, though. For instance, a greater proportion of participants who reported stimulant use subsequent to incarceration reported greater stimulant use during the 30 days prior to incarceration; likewise, participants who reported opioid use subsequent to incarceration had higher rates of opioid use prior to incarceration. Between $17.9 \%-22.6 \%$ of participants who reported opioid use subsequent to incarceration had used cocaine or amphetamines prior incarceration, but within this same group, between 30.8\%-70.8\% had used heroin, prescription opioids, or buprenorphine prior to incarceration. Similarly, between $3.8 \%-36.2 \%$ of participants who reported stimulant use subsequent to incarceration had used heroin, prescription opioids, or buprenorphine prior incarceration, but between $30.0 \%-50.0 \%$ had used cocaine/crack cocaine or amphetamines prior to incarceration. In other words, groups used across drug classes, though pre-incarceration rates of use for specific opioid and stimulant drugs corresponded to participants' later use of either opioids, stimulants, or both.

Results from the regression model examining the association between drug use during the 30-day period prior to incarceration and concomitant, opioid, and stimulant use during the one-year period subsequent to incarceration further articulate these relationships. For instance, heroin use and prescription opioid use during the 30-day period prior to incarceration significantly increased the likelihood that opioids would be used subsequent to incarceration, whereas alcohol, amphetamine, and cocaine/crack cocaine use during the 30-day period prior to incarceration decreased the likelihood of later opioid use. For stimulant use during the one-year period subsequent to incarceration, the converse was observed; amphetamine and cocaine/crack cocaine use during the 30-day period prior to incarceration substantially increased the likelihood of stimulant use subsequent to incarceration, whereas heroin use during the 30-day period prior to incarceration decreased the likelihood of later stimulant use. The odds of observing concomitant use for the one-year period subsequent to incarceration did not decrease in association with prior use of any drug; rather, amphetamines, prescription opioids, heroin, and prescription sedative use during the 30-day period prior to incarceration increased the likelihood of later concomitant use. Participants who reported 
concomitant use subsequent to incarceration did have the highest rates of preferring prescription sedatives prior to incarceration, even though the proportion of concomitant users with this preference was low (3.8\%) compared to preferences reported for other drugs.

When examining heroin use for the one-year period subsequent to incarceration, similar findings were observed. Participants who reported heroin use subsequent to incarceration also reported having used both stimulants and opioids during the 30-day period prior to incarceration, with rates far higher for opioid drugs; the only exception being buprenorphine. For all drugs used during the 30-day period prior to incarceration, prescription opioids had the highest rates of use among participants who reported heroin use subsequent to incarceration. Heroin was the next most frequently used drug during the 30-day period prior to incarceration for this group. Approximately $11 \%$ of participants $(\mathrm{N}=158)$ did not report any heroin use subsequent to incarceration, but did report other opioid use. This group had lower rates of heroin, prescription opioid, and buprenorphine use during the 30-day period prior to incarceration compared to participants who used heroin subsequent to incarceration. Rates of use ranged from $13.3 \%$ for heroin, $17.9 \%$ for buprenorphine, and $48.2 \%$ for prescription opioids. This indicates that prior use of opioids did not ensure later heroin use, even as this prior use was still positively correlated with later prescription opioid or buprenorphine use.

Differences in heroin use subsequent to incarceration may be partially attributable to the frequency with which heroin and other drugs had previously been used. For instance, participants who reported using heroin subsequent to incarceration used prescription opioids 15 days on average out of the past 30 days that they were last on the street and used heroin for 13 days on average during this same time; buprenorphine was used only 3.3 days on average. Among this group, amphetamines and cocaine/crack cocaine were used at comparatively lower rates during the 30-day period prior to incarceration, but were still used at rates higher than buprenorphine. Both amphetamines and cocaine/crack cocaine were used less than a week on average during the 30-day period prior to incarceration, far less than the number of days that prescription opioids and heroin were used. Participants who did not report past-year heroin use subsequent to incarceration used prescription opioids and heroin fewer days on average during the 30-day period prior to incarceration, 10 and 2.5 days respectively. The relationship between drug use at an earlier time and later use of similar drugs was highlighted further by findings from the regression model showing that heroin use 
and prescription opioid use during the 30-day period prior to incarceration significantly increased the likelihood of observing later heroin use; the relationship was strongest between heroin use prior to incarceration and heroin use subsequent to incarceration.

These findings illustrate some of the concepts discussed in earlier chapters describing how past drug use, particularly when it constituted a regular behavior, and certainly when it constituted a habituated behavior, has the capacity to influence people's ability to think and behave differently in respect to the same drugs in the future. Put simply, prior opioid and stimulant use can lead to continued or increased use (Ahmed, Walker, \& Koob, 2000). While it is possible that anyone might decide to use heroin, it is far more probable that people who have previously used heroin would make such a decision. Even for drugs with extreme danger or stigma associated with them, use may become ritualized and normalized (Brown, 2015; Mars, Bourgois, Karandinos, Montero, \& Ciccarone, 2014; Waldorf et al., 1992; Zinberg, 1984). That drug use subsequent to incarceration was associated with frequent use of similar drugs prior to incarceration is not surprising. Decisions and behaviors that have been previously made or preformed are easier to persist in than initiation of novel decisions and behaviors (Ronis, Yates, \& Kirscht, 1989). Recall that habits can be strengthened or weakened over time, with behaviors preformed repeatedly instantiated in the brain in a manner that an infrequently preformed behavior would not be, potentially making choice and behavior cognitively "easier", particularly amidst the same or similar environmental conditions, and particularly if the behavioral outcome is highly rewarding (e.g., greater automaticity and less deliberation) (Everitt \& Robbins, 2016; Gardner, 2015; Graybiel, 2008; Robbins \& Everitt, 1999; Sebold et al., 2017; Wood \& Rünger, 2016). Conversely, decisions and behaviors that were dissimilar to previously made decisions and previously performed behaviors would require more deliberation and more effort.

Even if time spent in a controlled environment disrupted participants' ability to perform a behavior pertaining to opioids, upon release that behavior would have the potential to be resumed and would be more likely to be resumed if participants were exposed to the same or very similar environments in which they previously obtained and used opioids, and certainly more likely if they were exposed to the drugs themselves (Childress et al., 1993; Crombag, Bossert, Koya, \& Shaham, 2008; de Wit, 1996). That most participants returned to the same county that they resided in prior to incarceration means that it is not only possible, but probable, that some participants were exposed to places and people which would have had the 
potential to produce a conditioned-response (Childress, et al., 1993; Lee, Milton, \& Everitt, 2006; Leverentz,, 2010). Among participants who had regularly used opioids and stimulants, exposure to drugrelated cues, both before and after incarceration, would have had the potential to influence decisionmaking about these drugs, in part by constraining the cognitive and behavioral repertoire with which participants might have flexibly responded (Bechara, 2005; Monti, Schoenbaum et al., 2006; Tiffany, 1999; Rohsenow, \& Hutchison, 2000). Exposure to cues associated with opioids and stimulants could have influenced not only the frequency with which these drugs were thought about explicitly, meaning that greater cognitive attention is directed toward drugs, but also could have produced visceral states (e.g., craving, stress, arousal, etc.) and further biased attention to drug-related cues in the environment (Field, Munafò, \& Franken, 2009; Franken, Kroon, Wiers, \& Jansen, 2000; Lubman, Peters, Mogg, Bradley, \& Deakin, 2000). Craving and attentional bias to drug-related cues have been shown to predict use (Franken, 2003; Marissen et al., 2005, 2006).

Visceral states, even if people are not always fully aware of their presence and are not able to articulate their exact nature or origin, may nevertheless influence mood, affect, cognition, and decisionmaking (Cooney, Litt, Morse, Bauer, \& Gaupp, 1997; Critchley, Wiens, Rotshtein, Öhman, \& Dolan, 2004; Drummond, 2001; Monteroso \& Luo, 2010; Wiers, Field, \& Stacy, 2016). Recall that a racing heart, sweaty palms, and queasy stomach might be as easily attributable to fear as it is to excitement or happy anticipation (Garfinkel \& Critchley, 2016). Recall, too, that disruptions in somatic signaling and interoceptive awareness have been associated with drug use, meaning that it may have been difficult for some participants to have adaptively navigated somatic, affective, and cognitive states during times in which it would have been most crucial (Bechara \& Damasio, 2002, de la Fuente et al., 2019; Paulus \& Stewart, 2014; Sönmez, Kahyacı Kılıç, Ateş Çöl, Görgülü, \& Köse, 2017). Irrespective of any disruption, interceptive awareness, necessary for adaptive human functioning, can compel a person's attention and potentiate action, potentially exacerbating visceral sensations of craving, desire, or temptation (Naqvi, Rudrauf, Damasio, \& Bechara, 2007; Turel et al. 2014; Van den Bergh et al., 2018). When interoceptive awareness is activated, top-down cognitive capacities needed for deliberation and inhibition may be weakened, while bottom-up impulses and urges may be excited, thus potentially enabling action without forethought (Noël, Brevers, \& Bechara, 2013; Turel \& Bechara, 2016). 
In summary, opioid and stimulant drugs used with regularity prior to incarceration were associated with use of similar drugs subsequent to incarceration. During both time periods, drug-related cues in the environment, along with other stressors that may have been present, would have had the potential to influence participants' physiological, cognitive, and affective states, making higher-level cognition needed for impulse control and adaptive decision-making more challenging (Sinha et al., 2003; 2005; Snyder \& Hankin, 2016; Tice et al. 2001). Some of these additional influences will be discussed further below.

\section{Versatile Drug Use, Preference, and Drug Use Severity}

Many participants in this sample can be considered as polydrug users. Drug use versatility, indicated by the total number of different drugs used during the one-year periods prior and subsequent to incarceration, was higher among participants who reported concomitant and opioid use for the 30-day period prior to incarceration and the one-year period subsequent to incarceration. Concomitant use during the 30-day period, rather than separate use of either opioids or stimulants, might lead one to intuit that the total number of drugs used for the entire one-year period prior to incarceration would be greater compared to other groups, and this was indeed the case. But this was not a foregone conclusion. Participants who reported past 30-day opioid, stimulant, or other drug use could have used alcohol, cannabis, sedatives, hallucinogens, or synthetic drugs during that year, meaning that they would have used five or more drugs. Participants who reported past 30-day concomitant use could have only used opioids and stimulants for that year, making them less versatile users by comparison. That this wasn't the case, but instead that concomitant use of two high-risk drugs was associated with overall greater drug use versatility, hints at the possibility that there is a subgroup of participants who are less discriminant in their drug selection and possibly more risk-prone.

This idea is partially supported by the finding that participants who reported concomitant use had the highest rates of drug use severity followed by participants who reported past 30-day opioid use. This means that during the 30-day period prior to incarceration, participants who co-used opioids and stimulants, or who used opioids (either alone or contemporaneously with non-stimulant drugs), more often experienced drug-related problems, craving, a desire but inability to quit using, and withdrawal. The presence of craving and withdrawal among some participants is especially significant, as these qualities indicate that 
participants were possibly dependent on certain drugs, which would imply that the local utility of these drugs was high, the discount rate of future, non-drug rewards was steep, and that decision-making may have been more impulsive and less deliberative (Bickel, Koffarnus, Moody, \& Wilson, 2014; Giordano et al., 2002; Mitchell, 2004; Franken, 2003).

While higher rates and greater frequency of drug use across many classes does not necessarily correspond to drug use severity in a linear way, using more drugs more often does mean that a person is exposed to the rewarding, and behaviorally reinforcing, effects of drugs with greater regularity.

Participants' ability to flexibly make intertemporal decisions, particularly about higher-risk drugs, would likely be impaired with regular use of opioids, stimulants, and other drugs, but so too would their ability to make adaptive, less-risky decisions in general due to any direct effects of drug intoxication (Cromer, Cromer, Maruff, \& Snyder, 2010; Gilman, Smith, Ramchandani, Momenan, \& Hommer, 2012; Goldstein et al., 2009; Ramaekers et al., 2006; Rogers et al., 1999). The initial use of one drug may precipitate further drug use and, among all drugs used, those associated with the greater perceived value or preference may continue to be used at higher rates (Aharonovich et al., 2005; Greenwald, 2008). This is indirectly evidenced in this sample by the correspondence between preferred drugs and drug use outcomes, with greater preference for heroin, prescription opioids, and buprenorphine associated with opioid use, greater preference for cocaine/crack cocaine and amphetamine use associated with stimulant use, and some preference for all of these drugs associated with concomitant use. Although participants who reported other drug use during the 30-day period prior to incarceration did prefer opioid and stimulant drugs, rates were lower compared to other groups based on drug type.

Drug use versatility was greatest among the concomitant use group, both before incarceration and after. However, in the regression model examining concomitant, opioid, and stimulant use during the 30day period prior to incarceration, the odds of observing an outcome of concomitant, opioid, or stimulant use increased across all groups as a function of drug use versatility. In other words, the more drugs that were used, the more likely it was that a participant would use opioids or stimulants, or to co-use them, during this 30-day period prior to incarceration, rather than use non-opioid and non-stimulant drugs such as alcohol, cannabis, prescription sedatives, etc. Polydrug use, and particularly use of a greater number of different drugs across drug classes, may indicate a willingness to try new drug experiences even if regular 
use of all drugs is not adopted (Smith \& Staton, 2019; Smith \& Stoops, 2019; Sutherland et al., 2016; Wagner et al., 2014). Regular, versatile, and overall more severe drug use that was more common among participants who reported concomitant and opioid use during the 30-day period prior to incarceration may indirectly indicate the possible presence of traits and/or states that would incline a person toward more diverse experimentation, such as greater impulsivity, higher propensity for novelty- or sensation-seeking, and less risk-aversion which, as previously discussed, are all strongly associated with opioid and stimulant use specifically and less adaptive decision-making generally (Bardo, Donohew, \& Harrington, 1996; Butler \& Montgomery, 2004; Galizio \& Stein, 1983; Jentsch et al., 2014; Kirby \& Petry, 2004; Verdejo-García, Perales, \& Pérez-García, 2007; Wingo et al., 2016). Being more versatile and less discriminate in respect to drug experiences would also influence decision-making about opioids or stimulants by virtue of the fact that all drug options remain open for selection, thereby increasing the probability that opioids and stimulants would be used, or used concomitantly, irrespective of overall preference.

Worth emphasizing is that drug use severity was measured so as not to pertain to any one drug. Had severity of use for each drug type been assessed, it would have likely helped to clarify drug selection among participants who reported concomitant use prior and subsequent to incarceration, though doing so would not have explicated the overall greater propensity toward versatile drug use. For instance, a person could have a higher drug use severity score, but also ever only use crack cocaine, meaning that severity itself cannot explain the phenomenon a using a more diverse range of drugs. Of additional interest is the higher average number of previous drug treatment episodes among participants who reported concomitant use for the 30-day period prior to incarceration and among participants who reported past-year concomitant and opioid use for the one-year period subsequent to incarceration, as this may further indicate drug use severity. For instance, participants in these groups may have experienced psychosocial or medical problems that precipitated or otherwise motivated treatment entry or may have engaged in problematic drug behaviors that resulted in treatment being mandated (Cacciola, Dugosh, Foltz, Leahy, \& Stevens; 2005; Cosden et al., 2006; DiClemente, 2018; Hser, Grella, Chou, \& Anglin,1998).

\section{Abstinence Self-efficacy}

Interestingly, participants who reported concomitant use subsequent to incarceration also had the lowest rates of reporting moderately or very good abstinence self-efficacy prior to treatment initiation. This 
group also had the lowest proportion of participants reporting "moderately" or "very good" abstinence selfefficacy approximately one-year post-release. It is important to note that the abstinence self-efficacy question (e.g., "Based on what you know about yourself and your situation, how good do you think the chances are that you can stay off drugs and alcohol?") was phrased in a manner that may confuse interpretation of findings. Specifically, the question implies that it is the intention of participants to "stay off drugs and alcohol". It may be that some participants had an intention to abstain from some drugs but use others in moderation and that others had no intention of trying to abstain from any drug and were uncommitted to treatment (Kelly \& Greene, 2014; Laudet \& Stanick, 2010). Other information obtained from the survey could not clarify this ambiguity. This limitation notwithstanding, the fact that abstinence self-efficacy at two different time periods was lowest among participants who reported concomitant use and highest among participants who used other/no drugs may indicate that one possible factor influencing later drug-related decision-making was participants' belief that abstinence was in fact possible. Selfefficacy beliefs are considered cognitively instrumental in intention formation, decision-making, and behavioral control (Ajzen, 2002; Bandura, 1982; Bandura, 1999). Participants who believed that abstinence was both possible and likely to be achieved may have been primed and enabled to later actualize those selfefficacy beliefs. These participants may have believed that they were capable of making different decisions, controlling their behavior, or engaging in recovery or non-drug behaviors (Bandura, 1989, 1991; Marlatt, Baer, \& Quigley, 1997). For participants who may not have intended to abstain from all drugs, and therefore may have been more likely to respond "uncertain", "moderately poor", or" very poor" to this question (as opposed to responses of "moderately good" or "very good"), findings of greater drug use among these participants would still be in keeping with the idea that earlier ambivalence about using or earlier intention to keep using would have had the potential to later influence decisions to use, rather than abstain. In other words, decisions to use could reflect both lower perceived abstinence self-efficacy or the absence of intention to quit using.

\section{Drug Substitutes, Compliments, and Risk Amidst Changing Markets}

Concomitant use of opioids and stimulants during the one-year period prior to incarceration was most common among participants who reported concomitant use during the 30-day period prior to incarceration, but was also found among participants that used opioids-only during the 30-day period prior 
to incarceration. Other than participants who reported concomitant use prior and subsequent to incarceration, participants who reported using opioids during the 30-day period prior to incarceration and the one-year period subsequent to incarceration used more drugs across drug classes compared to participants who used stimulants-only for these same time periods.

Although greater drug use versatility was also associated with increased odds that stimulant-only use would be observed during the 30-day period prior to incarceration, participants who used stimulants, but not opioids, during this time period had the lowest rates of past-year opioid use, even when compared to participants who reported past 30-day other drug use. Stimulant users in this and other samples, while still predominantly polydrug users, may be less likely to use opioids as either compliments or substitutes, or to use opioids only infrequently (Harrell, Mancha, Petras, Trenz, \& Latimer, 2012). Older stimulant users in this sample may have been using stimulants for a longer period of time and, as a result, may have transitioned from use to dependence, or may have simply had more well-established drug preferences and use patterns (Booth et al., 2006; Brecht et al., 2008; Lee, Vivier, \& Diercks, 2009; Lopez-Quintero et al., 2011; Wu et al., 2009). Alcohol or cannabis may have been treated as compliments among stimulant users in this sample, as evidenced by past-year rates of use for these drugs that were comparable to those of other groups, unlike rates of past-year opioid use, which were far lower among participants who reported past 30day stimulant use. Alcohol and cannabis were also preferred by a greater proportion of participants who reported past 30-day stimulant use, compared to participants who reported past 30-day concomitant or opioid use. Alcohol and cannabis have been documented as compliments to cocaine and are often co-used with cocaine (John \& Wu, 2017; Lindsay, Stotts, Green, Herin, \& Schmitz, 2009; Midanik, Tam, \& Weisner, 2007; McCance-Katz et al., 1993; Williams \& Parker, 2001).

Contemporaneous use of alcohol and cannabis is not uncommon among people who use multiple drugs, even among people who prefer drugs other than alcohol and cannabis (Barrett, Darredeau, \& Pihl, 2006; Smith and Lawson, 2017; Yurasek, Aston, \& Metrik, 2017). This study produced similar findings, with alcohol and cannabis not overwhelmingly preferred, but still used, by a majority of participants. This phenomenon may be due in part to the greater availability and affordability of alcohol and cannabis compared to other drugs. Cannabis is increasingly legal in some form throughout the US and has fewer social and legal risks associated with it than it did during previous decades (Carliner, Brown, Sarvet, \& 
Hasin, 2017; Hall \& Lynskey, 2016; Hathaway, Mostaghim, Erickson, Kolar, \& Osborne, 2018).

Interestingly, a slightly higher proportion of participants who reported past-year stimulant use subsequent to incarceration resided in a county with restricted alcohol sales, though differences in alcohol accessibility did not differ appreciably by drug use outcome group.

Alcohol, cannabis, prescription sedatives, cocaine/crack cocaine, and amphetamines were used at fairly high rates among participants who also used opioid drugs, which raises the possibility that these drugs may have been treated as compliments or as substitutes among participants who co-used them (Lucas et al., 2016; Petry \& Bickel, 1999; Reiman, 2009; Sumnal et al., 2004). As previously discussed, cocaine/crack cocaine and amphetamines are co-used with heroin and prescription opioids at fairly high rates (e.g., "speedball", "goofball") among polydrug users due to their mutually reinforcing effects, meaning that for some participants they may have been more often treated as compliments (Bickel et al., 1995; Jasinski \& Preston, 1986; Guzman \& Ettenberg, 2004; Leri, Bruneau, \& Stewart, 2003; Lorvick, Browne, Lambdin, \& Comfort, 2018; Wade-Galuska, Winger, \& Woods, 2007). Due to their distinct subjective psychoactive effects, it may be less likely that regular drug users, and drug users with greater preference for either opioids or stimulants, but not both, would treat drugs within these two classes as substitutes.

\section{Heroin and...Buprenorphine}

That approximately one-third of participants who reported past-year heroin use subsequent to incarceration also reported using prescription opioids and buprenorphine for this same time period raises the possibility that drug substitution within the same drug class occurred or that during this time period some participants made a decision to transition from prescription opioids to heroin (Evans et al., 2018; Monti, 2019; Peavy et al., 2012). Many people who currently use heroin in the US transitioned to heroin as a substitute for prescription opioids, and it has been shown elsewhere that opioid users are willing to substitute preferred opioids with other, less preferred opioids, such as buprenorphine (Compton et al., 2016; Kanouse \& Compton, 2015; Lankenau et al., 2012; Smith et al., 2017). This was partially evidenced in this study by high rates of multiple opioid drug use among participants who reported concomitant and opioid use prior and subsequent to incarceration. It could be that at various times during the one-year post-release period that prescription opioids were substituted for heroin or vice versa, due to periods of greater or lesser 
availability of either drug, increases or decreases in price, changes in drug quality, or fluctuations in participant income (Goudie, Sumnall, Field, Clayton, \& Cole, 2007; Greenwald \& Hursh, 2006; Chalmers et al., 2010; Petry, 2000).

While use of heroin, prescription opioids, amphetamines, and cocaine during the 30-day period prior to incarceration were all associated with changes in the likelihood of opioid, stimulant, and concomitant use during the one-year period subsequent to incarceration, buprenorphine was not significantly associated with these post-release outcomes. Similarly, buprenorphine use during the 30-day period prior to incarceration was not associated with a change in odds for observing heroin use during the one-year period subsequent to incarceration. For the 30-day period prior to incarceration, buprenorphine was used by $25.0 \%$ of participants who reported using heroin during the one-year period subsequent to incarceration. Subsequent to incarceration, however, buprenorphine use increased to $33.3 \%$ among participants who reported heroin use, far higher than rates found among participants who did not report heroin use subsequent to incarceration (only $6.5 \%$ reported buprenorphine use). Buprenorphine use during the one-year period subsequent to incarceration was associated with a $266 \%$ increase in odds of observing heroin use for this same time period, whereas use of prescription opioids was associated with a $73 \%$ increase in odds of heroin use. Although prior to incarceration buprenorphine was used at higher rates among participants who resided in rural counties, subsequent to incarceration rates of buprenorphine use were slightly higher in urban areas. Rates of heroin use both prior and subsequent to incarceration were higher among participants who resided in urban areas, increasing during the 2012-2017 post-release period. It may be that proliferation of MAT providers in urban areas provided greater access to diverted buprenorphine during a time when heroin was also becoming more ubiquitous in urban areas.

For some participants, buprenorphine could have served as a substitute and may have been chosen by heroin-using participants for purposes such as self-adopted harm-reduction or informal OUD selftreatment, as such use has been documented elsewhere (Bazazi, Yokell, Fu, Rich, \& Zaller, 2011; Daniulaityte, Falck, Wang, \& Carlson, 2009; Furst, 2013; Gwin-Mitchell et al., 2009; Jayadeva et al. 2017; Schuman-Olivier et al., 2010). Buprenorphine may have also been selected by some participants to achieve pleasant subjective effects, though evidence remains mixed as to whether buprenorphine should be regarded as a serious drug of abuse and as a preferred drug among polysubstance users given its ceiling 
effects (Cicero, Surratt, \& Inciardi, 2007; Smith et al., 2019). Because buprenorphine was preferred by only $2.6 \%$ of the entire sample, and $1.2 \%$ of participants who reported using heroin subsequent to incarceration, it may be less likely that participants decided to use buprenorphine primarily as a means of achieving states of intense euphoria or pleasure. That the survey question pertaining to buprenorphine included both Suboxone and/or Subutex, but made no distinction between them, makes speculation difficult due to the fact that these drugs differ in their effects. Buprenorphine, an agonist and/or antagonist at different opioid receptors, is found in both brand-name drugs; Suboxone also contains naloxone, an opioid antagonist, whereas Subutex contains only buprenorphine (Lobmaier, Gossop, Waal, \& Bramness, 2010; Lufty \& Cowan, 2004).

Although buprenorphine might not ordinarily have high local utility for many polydrug users, its perceived value might increase significantly among participants who regularly used heroin or prescription opioids, but then encountered a period of supply disruption. It may also have been more valued among participants using buprenorphine to self-treat OUD. This relationship between heroin and buprenorphine may also reflect broader changes in the illicit drug market. Over the past eight years, opioid prescribing has decreased and prescription opioid monitoring has increased, driving up the price of prescription opioids in illicit drug markets (Lebin et al., 2019; Mallatt, 2018). Conversely, buprenorphine prescribing has increased significantly in the US, with prescribing rates higher among states that adopted Medicaid expansion under the Patient Protection Affordable Care Act (ACA), Kentucky being one such state (CHFS, 2019; Clemans-Cope, Epstein, \& Kenney, 2017; Wen, Hockenberry, Borders, \& Druss, 2017). Prescription drug diversion is a perennial problem in the US and there is often a positive relationship between rates of drug prescribing and prescription drug diversion (Cicero et al., 2007; Daniulaityte et al., 2006; Kurtz, Buttram, Margolin, \& Wogenstahl, 2019). Use of diverted buprenorphine has been found among many samples, including among other corrections-involved samples in Kentucky, (Cicero et al., 2018; Lofwall \& Havens, 2012; Molfenter et al., 2019; Smith et al., 2019).

Given the high rates of any opioid use prior to incarceration, it is worth considering two related questions. First, why did so many participants decide not to use heroin subsequent to incarceration? Nearly $22 \%$ of the sample reported using heroin during the one-year period prior to incarceration, but this declined to $10.7 \%$ during the one-year period subsequent to incarceration. Participants were released during a time 
when prescription opioids were becoming more expensive and heroin was becoming more ubiquitous, cheaper, and potent, making it a potentially more attractive opioid substitute. Second, why did so many participants in this sample decide to use heroin subsequent to incarceration? Participants were released during a time when heroin was becoming more adulterated, dangerous, and deadly (Jalal et al., 2018; Marshall et al., 2017; Ruhm, 2018). The penalties for selling even small quantities of heroin, which is one way that heroin users can help generate the funds needed to support their own heroin use, increased during this time (Biernaki, 1979; Duncan, 2013; Offices of the United States Attorneys, 2017). These increased sanctions, coupled with the increased risk of heroin-related overdose and death, still did not deter about $10 \%$ of participants. Heroin is a drug associated with great reward as well as great risk. That heroin was more available in Kentucky during the 2012-2017 post-release period than in any previous decade (thus including any pre-incarceration period), but that use declined among participants in this sample, suggests that, for some participants, the risks associated with use may have been perceived as too great (Ciccarone, Ondocsin, \& Mars, 2017; Votaw, Wittenauer, Connery, Weiss, \& McHugh, 2017).

\section{Greatest Risk}

One disquieting finding is that the use of prescription sedatives, cocaine/crack cocaine, prescription opioids, and amphetamines during the one-year period subsequent to incarceration was associated with increased likelihood of also observing heroin use for this same time. Put differently, the single most dangerous drug that could be used during this time period was significantly associated with contemporaneous use of drugs most likely to result in an acute adverse event when used with it (Clinton, Hunter, Logan, \& Lapidus, 2019; Hobelmann et al., 2016; Mital, Windle, Cooper, \& Crawford, 2017; Yamamoto et al., 2019). That rates of opioid and stimulant use among participants reporting past-year heroin use were higher both prior and subsequent to incarceration means that these 168 participants may be appropriately considered as polydrug users across time periods and arguably the highest-risk users of the sample. Participants who did not use heroin, but who used prescription opioids contemporaneously or concomitantly with other drugs were still engaging in high-risk form of drug use, in that co-use of opioids with stimulants, benzodiazepines, or alcohol is associated with increased likelihood of adverse outcomes, including overdose and death (Calcaterra et al., 2013; Hobelmann \& Clark, 2016; Jones et al., 2012; Kaye \& Darke, 2004; Warner et al., 2016). 


\section{Intravenous Administration}

High-risk drug decisions can also be reflected by the route of administration. Approximately $40 \%$ of participants in this sample had some history of IDU. Participants who reported past 30-day concomitant and opioid use prior to incarceration had the highest rates of IDU history, with rates highest among participants who reported concomitant use. During the one-year period subsequent to incarceration, rates of IDU history were approximately equal between concomitant and opioid users. While history of IDU was associated with increased odds of concomitant, opioid, and stimulant use during the 30-day period prior to incarceration, it was associated only with increased likelihood of observing past-year concomitant and opioid use subsequent to incarceration. This means that opioids, either used in isolation or in combination with other drugs, were associated with history of IDU, a relationship that has been observed elsewhere for both prescription opioids and heroin (Al-Tayyib, Koester, \& Riggs, 2017; Warner-Smith, Darke, Lynskey, \& Hall, 2001).

Differences in history of IDU between participants who reported past-year heroin use and those who did not were stark. In separate analyses (results not displayed), among participants who reported any drug use during the one-year period subsequent to incarceration $(\mathrm{N}=869)$, participants who reported using heroin were significantly more likely than participants who reported other drug use to report IDU during the one-year period subsequent to incarceration $\left(17.2 \%\right.$ vs. $\left.6.9 \%, X^{2}=14.6 ; \mathrm{p}<.001\right)$. This may be partially attributable to preferred routes of administration that includes intravenous injection. A person who prefers this method may be influenced in their decision-making, being inclined to choose more readily injectable drugs, such as heroin, and less inclined to choose drugs that involve more preparatory steps, such as prescription opioids (Ponton \& Scott, 2004).

In illicit drug markets, there has been a decrease in availability of many popular prescription opioids that were once commonly injected (e.g., original formulation OxyContin) (Alpert et al., 2018). These prescription opioids, while not eliminated from illicit drug markets, are nevertheless being increasingly replaced with tamper-resistant prescription opioid formulations intended to deter abuse, meaning that they are far more difficult to prepare for injection (Alpert, et al., 2018; Chilcoat, Coplan, Harikrishnan, \& Alexander, 2016; Patrick, Fry, Jones, \& Buntin, 2016). Although earlier generation prescription opioids required several steps of preparation prior to injection, and certainly more steps than 
heroin, participants who may have attempted injecting formulations brought to market after 2010 would have needed peer-based instruction on how to prepare these pills for injection or would have needed to undertake extensive research and trial-and-error (Duxbury, 2018; Rönkä \& Katainen, 2017; Vosburg, Haynes, Besharat, \& Green, 2017). They may have also needed to acquire additional chemical compounds required for the refinement of pills into injectable form. In short, while abuse-deterrent prescription opioids might be less expensive than original formula prescription opioids (though still more expensive than heroin), they would cost more in effort and time (Lebin et al., 2019). The potential for wasted product or weaker effects, in cases where pills were not prepared properly or were unable to be injected successfully, coupled with the increased cost in time, could have made heroin, rather than prescription opioids, a more attractive drug among participants with a preference for this route of administration (Vosburg et al., 2013). For people who inject drugs, time might be considered a cost due to the fact that one of the appeals of IDU is that drug effects are extreme and felt nearly instantaneously (3-7 seconds), particularly when compared to swallowing or even insufflation (Zering et al., 2003). However, increased tolerance and variability in potency may have made heroin less desirable over the long-term among some participants (Monico \& Mitchell, 2018).

Cocaine and amphetamines are also more readily injectable than other drugs, one reason why concomitant use of heroin and cocaine or heroin and amphetamine remain popular among some IV polydrug users (Al-Tayyib, Koester, Langegger, \& Raville, 2017; Dolan et al., 1991; Harrell, Mancha, Petras, Trenz, \& Latimer, 2012; Sanders, Lankenau, Jackson-Bloom, \& Hathazi, 2008). Crack cocaine may also be injected, though requires more steps to prepare, meaning that some participants may have preferred to smoke rather than inject crack cocaine (Lankenau, Clatts, Goldsamt, \& Welle, 2004; Waninger, Gotsch, Watts, \& Thuahnai, 2008).

Although high-risk drug use was common across this sample, participants with a with a history of IDU may be considered as even less risk-averse compared to participants without such history. Riskaversion may be less pronounced still among people with a history of IV heroin administration, versus IV prescription opioid administration, as IV heroin administration entails the highest risk of all for this generation of drug users. Though heroin and prescription opioids are more often associated with IV administration and, to a lesser extent, cocaine and amphetamines, IV buprenorphine use is an increasingly 
well-documented phenomenon that warrants closer examination (Brecht, O'Brien, Von Mayrhauser, \& Anglin, 2004; Fischer et al., 2018; Kelly et al., 2017; Novak \& Kral, 2002; Yokell et al., 2011).

\section{Additional Endogenous Factors with the Potential to Influence Decision-making Age of Drug Use Initiation}

Participants who reported concomitant use prior and subsequent to incarceration were $<14$ years old on average when they first used drugs. The relationship remained significant in the regression model, with younger age associated with slightly increased odds of use. Participants who reported using heroin during the one-year period subsequent to incarceration were 13.8 years-old on average when drug use was initiated, a full year younger than the average age of drug use initiation among participants who did not report heroin use, though this association did not remain significant in the regression model. Participants who reported other drug use prior to incarceration were on average 14.5 years old when they first used drugs and those who reported other/no drug use during the one-year period subsequent to incarceration were 15.3.

These average ages associated with other drug and/or no drug use, while all >14 years old, are still young. Even though age 14 is regarded as a critical neurodevelopmental juncture, with drug use and other insults to the brain prior to age 14 having more enduring effects, there does not exist any clear line; what can be considered normative development still allows for variation. It may be that, for some, the neurodevelopmental implications of drug use initiation at age 15 differs little than use which began at age 13. It may be more important to understand that, overall, a positive relationship between earlier age of drug use inhiation and problems associated with drug use in later adolescence and adulthood is commonly observed (Chen et al., 2009; King \& Chassin, 2007; Labouvie \& White, 2002; Walters \& Urban, 2014). Even this understanding should be contextualized by other factors, such as the frequency of use at young ages, social conditions (e.g., trauma, poverty, and structural inequality), or by a number of other endogenous or exogenous factors with demonstrated capacity to adversely influence neurodevelopment and impair later cognitive-behavioral functioning (Bergen, Gardner, \& Kendler, 2007; Champagne, 2010; Champagne \& Curley, 2005; Charach, Yeung, Climans, \& Lillie, 2011; Dube et al., 2003 Perry et al., 1995; Walker et al., 2011). Information about participants' childhood and adolescence was not available for 
examination in this study, meaning that some important details pertaining to early drug use initiation will need to be more fully considered in future work.

In separate analyses (results not displayed), differences in age of drug use initiation were examined by sex, history of head injury, and LD diagnosis and/or SE enrollment, as all of these factors might confer the possibility of less adaptive cognitive function, including poorer inhibitory control and working memory, and, in some cases, less risk-aversion (APA, 2013; Cottle et al., 2001; Mcallister, Flashman, Sparling, \& Saykin, 2004; Van der Meere, Marzocchi, \& De Meo, 2005). On average, participants who were male, had a history of head trauma, or LD diagnosis/SE enrollment initiated drug use at an earlier age. Males were 14.4 years-old on average, compared to females, who were 15.9 years-old on average $(t=4.52 ., \mathrm{p}<.001)$, reflecting possible sex-based differences in early life for impulsivity, risk-taking, and dose-dependent drug reward (Cross, Copping, Campbell, 2011; Eckel \& Grossman, 2008; HosseiniKamkar \& Morton, 2014; Vansickel, Stoops, \& Rush, 2010). Participants with a history of head injury were 14.2 years-old on average when drug use was initiated, compared to those without, who were 15.1 yearsold on average $(t=3.51, \mathrm{p}<.001)$. Participants with a history of LD diagnosis and/or SE enrollment were 14.2 years-old on average when they initiated drug use, compared to those without this history, who were 14.9 years-old on average $(\mathrm{t}=2.22, \mathrm{p}=0.27)$. Thus, there is some limited evidence to suggest that participants who initiated drug use at an earlier age may have had traits and states that biased them toward riskier decision-making and/or decreased their ability to inhibit acting on the urges which precipitated these decisions. However, because temporal order cannot be established, these associations cannot be considered past the point of conjecture and are presented primarily to draw attention to the idea that any initial decision to use a drug is not made in isolation and that many factors beyond the age of initiation are important to examine with greater sophistication in future work.

That the average age of drug use initiation for the entire sample was 14.7 years, does mean that drug use occurred for most participants at a fairly young age in terms of neurolocognitive and psychosocial development (Gogtay et al., 2004; Shaw et al., 2008; Sorrells et al., 2018). Early age of drug use may indirectly indicate that some participants in this sample may be higher in trait impulsivity compared to people who either do not elect to use drugs, or who initiate use later in life (Petry, 2001; Verdejo-García, Lawrence, \& Clark, 2008). Though as discussed previously, novelty-seeking, impulsiveness, risk-taking, 
and poorer intertemporal decision-making are more prominent in childhood and adolescence, meaning that early drug experimentation cannot be taken as a clear indicator of the presence of traits often associated with greater risk-taking, impulsiveness, or poorer decision-making at later times. Findings should be interpreted with that in mind.

\section{Age and Sex}

Overall, younger age was most often associated with concomitant and opioid use both prior and subsequent to incarceration, including among participants who reported past-year heroin-use. That participants who reported other/no drug use subsequent to incarceration were slightly older is in keeping with previous research documenting that drug use and other high-risk behaviors are more prominent in early adulthood, decreasing or moderating with age (Gfroerer \& Brodsky, 1992; Fleming, White, Catalano, 2010). It may be that slightly older participants had other factors, such as a spouse, children, and other investments in everyday life that helped establish greater stability, serving to reinforce less risky behavior; though this trend is not always uniform, with minority groups sometimes continuing to use drugs in adulthood due to inequitable access to non-drug alternatives and other stabilizing resources (Duncan, Wilkerson, \& England, 2006; Sampson, Laub, \& Wimer, 2006; Visher, Knight, Chalfin, \& Roman, 2009; Vogt Yuan, 2010; Zapolski, Baldwin, Banks, \& Stump, 2017). For participants who were younger than 27 years-old during the time periods examined, and particularly those who were younger than 23 years-old, frontocortical areas crucial for deliberative decision-making and inhibition would have been less developed, meaning that they would have had comparatively less capacity for adaptive decision-making during intertemporal choice (Gogtay et al., 2004; Spear, 2007).

Although drug use for the 30-day period prior to incarceration differed by sex, with slightly more males found among the stimulant use and other drug use groups, the proportion of males among drug use groups did not differ subsequent to incarceration, including for heroin use. That a greater proportion of females were found among the higher risk drug using groups (concomitant, opioid, and heroin), compared to stimulant and other/no drug use groups, suggests that greater propensity for risk-taking based on sex was not a meaningful influence among participants in this sample, even though elsewhere in Kentucky males have found to use a greater variety and quantity of drugs (Shannon, Havens, Mateyoke-Scrivner, \& Walker, 2009). 
It may also be that other factors influenced the use of these generally higher risk opioid drugs among female participants. Some factors that might have influenced cognitive capacity and drug-related decision-making include higher rates of prior or ongoing sexual and physical violence, childhood adversity and trauma, physical and psychological health symptoms, chronic pain, disability, and poverty, as these characteristics have been found at high rates among corrections-involved women with a history of drug use, including samples in Kentucky (Binswanger, Krueger, \& Steiner, 2009; Champagne \& Curley, 2005; Bronson, Maruschak, \& Berzofsky, 2015; Golder, Engstrom, Hall, Higgins, \& Logan, 2015; Hall et al., 2016; Logan, Walker, Jordan, \& Leukefeld, 2006; Lynch, DeHart, Belknap, \& Green, 2012; Staton, Leukefeld, \& Logan, 2001; Staton-Tindall et al., 2015; Smith et al., 2019). Not only have many stressrelated states and conditions been shown to influence drug-taking among women differently, and in some ways disproportionally, then men, but psychological problems associated with drug use are typically greater among women than men (Fox \& Sinha, 2009).

Additional analyses (results not displayed) showed that during the one-year period prior to incarceration female participants experienced higher rates of depressive $\left(68.8 \%\right.$ vs. $40.9 \%, X^{2}=74.5$, $\mathrm{p}<.001)$ and anxiety symptoms $\left(60.1 \%\right.$ vs. $40.9 \%, X^{2}=35.2$, p<.001), suicidal ideation $(17.4 \%$ vs. $10.3 \%$, $\left.X^{2}=11.4, \mathrm{p}<.001\right)$, unemployment $\left(47.3 \%\right.$ vs. $\left.31.1 \%, X^{2}=31.3, \mathrm{p}<.001\right)$, chronic pain (34.2\% vs. $22.5 \%$, $\left.X^{2}=17.1, \mathrm{p}<.001\right)$, and higher stress-related health symptom scores (27.1 vs. $\left.22.6, t=8.81, \mathrm{p}<.001\right)$. Perhaps unsurprisingly, rates of misregulation were slightly higher among women $\left(87.9 \%\right.$ vs. $82.0 \%, X^{2}=5.2$, $\mathrm{p}=0.19)$. Though, fewer women than men reported LD diagnosis/SE enrollment $\left(22.8 \%\right.$ vs. $30.0 \%, X^{2}=5.7$, $\mathrm{p}=.017$ ). During the one-year period subsequent to incarceration, female participants experienced higher rates of depressive ( $38.9 \%$ vs. $\left.31.3 \%, X^{2}=6.02, \mathrm{p}=.014\right)$ and anxiety symptoms $\left(56.7 \%\right.$ vs. $37.0 \%, X^{2}=38.0$, $\mathrm{p}<.001)$, cognitive difficulties ( $35.9 \%$ vs. $\left.24.2 \%, X^{2}=16.4, \mathrm{p}<.001\right)$, and unemployment $(42.6 \%$ vs. $34.2 \%$, $\left.X^{2}=7.02, \mathrm{p}=.008\right)$, though rates of chronic pain $\left(36.6 \%\right.$ vs. $\left.31.6 \%, X^{2}=2.48, \mathrm{p}=.116\right)$ and suicidal ideation ( $5.0 \%$ vs. $\left.4.3 \%, X^{2}=0.13, p=.719\right)$ did not differ significantly between men and women subsequent to incarceration. These findings may help to explain why rates of high-risk drug use were similar among male and female participants. While men tend to be less risk-averse then women, certain conditions in the lives of corrections-involved women, including greater stress, may have contributed to decisions to use drugs with high risk, either by influencing cognitive capacities needed for adaptive decision-making or by 
increasing the likelihood that drugs would be used in an attempt to help reduce stress, anxiety, fear, or other unpleasant states (Logan et al., 2006; Zlotnick, Najavits, Rohsenow, \& Johnson, 2003). It is important to keep in mind that responses for some survey questions (e.g., anxiety, pain, suicidal ideation) may reflect gender-based differences in self-report (Herbert et al., 1997; Sigmon et al., 2005). Ultimately, continued work is needed to better understand sex-based differences in high-risk drug-related decision-making among corrections-involved people.

\section{Psychiatric Symptoms, Stress, Misregulation}

Past-year anxiety and depressive symptoms were higher among participants who reported concomitant use during the 30-day period prior to incarceration, as were rates of suicidal ideation. Participants who reported concomitant use also had significantly higher stress-related health effects scores, whereas scores were lowest among participants who reported other drug use. Higher stress-related health effects served as an indirect measure of stress among participants prior to incarceration. Higher scores would be indicative of greater psychological stress, as well as heightened stress response, meaning that both the manifestation and phenomenology of stress would have likely varied considerably between participants.

That participants who evidenced the greatest drug use versatility and severity and higher rates of psychiatric symptoms (e.g., the concomitant group) also experienced greater stress-related health effects, but that participants who evidenced the least drug use versatility and severity and lowest rates of psychiatric symptoms (e.g., other drug use group) evidenced the fewest, may not be surprising. For one, two stress-related health effects items measured the presence or absence of anxiety and depressive symptoms, and the relationship between stress and psychiatric symptoms is well-established, meaning there should be positive relationship between these measures (Turner, Wheaton, \& Lloyd, 1995). Moreover, while subjective stress, stress response, and depressive and anxiety symptoms all have the potential to contribute to drug craving, seeking, and use (though not always uniformly), they can also develop as a result of these and other drug-related conditions (e.g., withdrawal, exposure to drug-related cues) (Epstein et al., 2009; Mantsch, Baker, Funk, Lê, \& Shaham, 2016; Shaham \& Stewart, 1995; Sinha, Fuse, Aubin, \& O'Malley, 2000; Sinha \& Li, 2007). Regular drug use, and certainly addiction, can be conceptualized as a 
protracted state of chronic stress (i.e., allostasis), the body working to maintain homeostasis and the person taking more drugs in response (George, Le Moal, \& Koob, 2012; Koob, 2008; 2009).

Whereas rates of misregulation were lowest among participants who reported other drug use, rates were highest among participants who reported concomitant use prior to incarceration and were associated with increased odds of concomitant, opioid, and stimulant use during this same time. This suggests that among these groups, at least one decision to use drugs during the 30-day period prior to incarceration involved a specific intention of trying to mitigate negatively valanced states, as the variable “misregulation" reflected the use of drugs as an attempt to "reduce stress, anxiety, worry or fear", possibly because participants believed these drugs would produce an ameliorating effect, despite overall risk of use (Baumeister \& Heatherton, 1996; Doweiko, 1999). The association between stress, craving, and drug use, including relapse, is well documented (Mulia et al., 2008; Hyman et al., 2007; Preston \& Epstein, 2011; Sinha, 2008, 2012). Decisions to use drugs as a form of "self-medication" are not uncommon and may not necessarily be considered, under the right conditions, as irrational, per se, even if, over time, the utility associated with a drug has the potential to decrease, the states that the drug was used to alleviate have the potential to intensify, and additional drug-related problems have the potential to develop as a result of continued "self-medication" (for differing viewpoints on this topic see Becker \& Murphy 1988, Darke, 2013; Khantzian, 1997, 2013; Tomer, 2001). Because costs of drug use are often delayed and uncertain, but because unpleasant anticipatory states, such as anxiety or fear, are certain, experienced in the moment, means that opioids and stimulants would likely have been weighted differently compared to non-drug choices for which ameliorating or otherwise rewarding effects may not have been either immediate or certain (Bickel et al., 2007; Green, Myerson, \& McFadden, 1997). For these participants experiencing negatively valanced states, the local utility of opioids and stimulants may have simply been higher than other options. With drugs selected in an attempt to reduce unpleasant states, use might be considered utilitarian rather than hedonic (O'Curry \& Strahilevitz, 2001).

Subsequent to incarceration, rates of psychiatric symptoms were still most prevalent among participants who reported concomitant use, followed by participants who reported stimulant use. For both groups, depressive symptoms were associated with increased odds of use. Among participants who reported past-year heroin use specifically, rates of past-year depressive symptoms were far higher than those of 
participants who did not use heroin. Suicidal ideation was still highest among those who reported concomitant use, followed by those who reported stimulant use. Interestingly, past-year suicidal ideation was over twice as high among participants who reported heroin use, with suicidal ideation associated with a $154 \%$ increase in odds of observing heroin use. Again, participants who reported other drug/no drug use subsequent to incarceration had the lowest rates for all symptoms, including lowest rates of suicidal ideation.

As noted in Chapter 2, depression and anxiety symptoms can influence cognition, present-bias, risk assessment, goal pursuit, intertemporal choice, and the degree to which outcomes are rewarding or punishing (Dias-Ferreira et al., 2009; Furman et al., 2013; Gohier et al., 2009; Harvey et al., 2004; Lerner, Li, \& Weber, 2012; Marazziti et al., 2010; Nebes et al., 2000; Schulz \& Vögele, 2015; Pulcu et al., 2013; Schwabe \& Wolf, 2013; Scott et al., 2015; Shackman et al., 2006; Wittmann \& Paulus, 2008). However, there is insufficient evidence to suggest that anxiety or depressive symptoms influenced opioid and stimulant use among participants in this sample in any definitive way. Still, one idea of interest is that depression may contribute to a person being more present-biased due to the fact that they perceive time passing more slowly, have diminished capacity to feel sufficiently attached to notions of their future self, or to believe that affective states experienced during depressive episodes will ever be other than they currently are (Bschor et al., 2004; Dombrovski et al., 2011). This unpleasant myopic orientation to the present has been described poignantly among people who have experienced depression (Soloman, 2014; Styron, 2010). That suicidal ideation was highest among participants who reported concomitant use prior and subsequent to incarceration, and who reported heroin use subsequent to incarceration, suggests that for a portion of opioid and stimulant users in this sample the idea of a future self might have been not only less salient and less behaviorally motivating during decision-making, in that delayed outcomes of use may not have been expected to be cashed in. Choosing to use drugs may in some circumstances be regarded as an act of willful destruction, in which the potential for adverse consequences in the short- and long-term is fully known; in the extreme, this may extend to self-harm and suicide (Flanagan, 2016; Pickard \& Ahmed, 2016; Oquendo \& Volkow, 2018). If future consequences are not only more difficult to envision, but also something that a person might not expect to be alive long enough to experience, then risk and utility beyond the present moment lose coherent meaning. For a small minority of participants, use of these high-risk drugs may have 
even been considered as a possible means to an end, as intentional drug overdoses are a real, if all-too underexplored, phenomena (Austin, Proescholdbell, Creppage, \& Asbun, 2017; Brådvik, Frank, Hulenvik, Medvedeo, \& Berglund, 2007; Heale, Dietze, \& Fry, 2003; Roxburgh et al., 2017).

\section{Indirect Indicators of Cognitive Functioning}

Just under one-third of the entire sample reported a LD diagnosis or SE enrollment history, with no observed difference between groups. Rates of self-reported cognitive difficulties (e.g., difficulties understanding, concentrating, and remembering) that occurred during the one-year period subsequent to incarceration were highest among participants who reported concomitant use and lowest among participants who reported other/no drug use. Past-year cognitive difficulties increased the likelihood of concomitant use during the one-year post-release period by approximately $50 \%$. As neurocognitive impairments and indicators of executive dysfunction commonly associated with poorer decision-making and inhibitory control are found among people with a history of opioid and stimulant use, it is unsurprising that participants who reported concomitant use had higher rates of poorer cognitive functioning compared to participants who reported less drug use. Unfortunately, the question pertaining to past-year cognitive difficulties asked as part of the follow-up survey did not appear on the baseline survey. It is possible that participants who reported concomitant use prior to incarceration would also have had high rates of selfreported cognitive difficulties.

Participants who reported concomitant use both prior and subsequent to incarceration had the highest rates for history of head trauma as well, whereas rates were again lowest among participants who reported other drug use prior to incarceration. That both history of head trauma and past-year cognitive difficulties were represented at higher rates among participants who reported more versatile and severe drug use provides further indirect support for the idea that indicators of poorer cognitive function may correspond to greater impulsiveness and risk-taking, and decreased capacity for advantageous decisionmaking during intertemporal choice. However, findings cannot explicate causality, meaning that poorer cognitive functioning could have pre-dated opioid and stimulant use, or developed as a result of use.

\section{Chronic Pain}

Chronic pain rates were highest among participants who reported concomitant and opioid use prior and subsequent to incarceration. As discussed in earlier chapters, chronic pain can significantly alter and 
impair cognitive functioning and contribute to less adaptive decision-making (Apkarian et al., 2004; Elvemo, et al., 2017; Landrø et al., 2013; Walteros et al., 2011). Experiencing chronic pain may also increase the perceived value of opioid drugs due to their analgesic properties (Trescot, Datta, Lee, \& Hansen, 2008). Although rates of chronic pain were higher among participants who used opioids, including concomitantly with stimulants, chronic pain was, somewhat unexpectedly, not associated with increased odds of opioid or concomitant use either prior or subsequent to incarceration. No differences in chronic pain rates were observed between participants who reported heroin use subsequent to incarceration and those who did not. It is possible, then, that chronic pain may correspond to use of specific types of opioids, but not opioid use more generally. Additional examination (results not displayed) of differences in chronic pain by heroin, prescription opioid, and buprenorphine use revealed that chronic pain rates differed for prescription opioids, with slightly more participants who reported chronic pain prior (64.2\% vs. $53.1 \%$, $\left.X^{2}=13.0, \mathrm{p}<.001\right)$ and subsequent to incarceration $\left(17.7 \%\right.$ vs. $\left.12.7 \%, X^{2}=6.3, \mathrm{p}=.014\right)$ reporting prescription opioid use; though no statistically significant differences were found for heroin or buprenorphine.

People who experience chronic pain experience pain symptoms that can increase or decrease in severity over a period of time, with some non-cancer chronic pain conditions having the potential to continue for years or an entire lifetime (Fine, 2011; Goldberg \& McGee, 2011). Given this, it is possible that participants who reported chronic pain had previously been prescribed opioids for pain symptoms. Particularly for pain that was treated prior to revised opioid prescribing guidelines and prior to 2012, when opioid prescriptions per 100 persons peaked in the US (CDC, 2019). Subsequent analysis revealed that $4.2 \%$ of participants who reported illicit drug use prior to incarceration also reported that they were introduced to drugs by a doctor or physician, meaning that illicit opioid use, dependence, or addiction may have been iatrogenic for some participants (Ballantyne \& LaForge, 2007; Keller et al., 2012). Prior exposure to prescription opioids, even if initially used as prescribed and not misused, has the potential to contribute to dependence or OUD (Cerdá, Santaella, Marshall, Kim, \& Martins, 2015; Edlund et al., 2014; Volkow \& McLellan, 2016). In general, people with OUD are often undertreated for their pain symptoms and routinely encounter stigma, discrimination, and other obstacles in accessing medical care (Baldacchino, et al.2010, Dunn et al., 2014; Hines et al., 2008; Karasz et al., 2004; Rosenblum et al., 2003; van Boekel, Brouwers, van Weeghel, \& Garrestsen, 2013; van Boekel, van Weeghel, \& Garrestsen, 2014). Under such 
conditions, participants with OUD who experience chronic pain might continue to use prescription opioids illicitly to self-treat their pain symptoms in addition to other reasons (e.g., producing a euphoric effect, relieving withdrawal symptoms) (Cicero, Lynskey, Todorov, Inciardi, \& Surratt, 2008; Leukefeld, Walker, Havens, 2007). The local utility of opioids for such participants would likely be extremely high, and the long-term risks of use not weighted similarly.

\section{Scarcity and Economic Hardship}

Participants who reported past 30-day concomitant and opioid use prior to incarceration experienced a greater number of economic hardships on average during the one-year period prior to incarceration, with the former reporting double that of participants who reported other drug use. Similarly, rates of having experienced past-year homelessness were twice as high among participants who reported concomitant use compared to those who reported other drug use. This association may not be surprising, insofar as homelessness is associated with psychiatric health symptoms, history of head injury, and greater drug use severity (Galea \& Vlahov, 2002; Lafferty, 2010; Oddy, Moir, Fortescue, \& Chadwick; 2012; Watson Crawley, \& Kane, 2016). While disruption in economic stability can produce a scarcity mentality, in which cognition, valuation, and decision-making abilities become impaired, homelessness may be considered an extreme form of scarcity, as it involves protracted uncertainty and hardship in addition to having fewer tangible resources and social supports (Mani et al., 2013; Phelan, Link, Moore, \& Stueve, 1997; Hwang et al., 2009; Shah et al., 2015). Homelessness is associated with a low social rank and stigmatized status, which can influence psychiatric functioning among people experiencing homelessness (Caton, Wilkins, \& Anderson, 2007; Link, Struening, Rahav, Phelan, \& Nuttbrock,1997; Roschelle \& Kaufman, 2004; Skosireva et al., 2014). Additionally, homelessness reflects a state in which basic needs are unmet, or under threat of not being met, and in which existential uncertainty is elevated due to greater exposure to unsafe conditions. Taken together, experiencing homelessness has similar characteristics of living in a harsh environment, and has the potential to not only produce an acute stress response, but also to keep states of arousal sustained at elevated levels over a period of time (Frankenhuis et al., 2016). Presentbias and reflexive decision-making would likely increase under these uncertain conditions, with cognition directed toward the capture of certain and immediate, versus uncertain and delayed, reward (Frankenhuis et 
al., 2016; see also optimal foraging theory: Abarca \& Fantino, 1982; Brown, Laundré, \& Gurung, 1999; Kagel et al., 1986).

During the one-year period subsequent to incarceration, participants reported their total income (before taxes) from all sources for the 30-day period prior to completing the follow-up interview. This means that most participants would have been out of jail or prison for approximately 10-14 months when this income was reported. Past 30-day income was low sample-wide, with $\$ 1,290$ the average income before taxes; approximately $\$ 15,480$ annually. Assuming that this was taxable income, which is not the case for some types of reported income (e.g., SNAP benefits, Social Security Insurance), average monthly take-home pay was $\$ 1,049$, or approximately $\$ 12,588$ annually, just $\$ 448$ above the Federal Poverty Guideline for a household of one (U.S. Department of Health and Human Services, 2019). However, the mean number of people who were listed as being dependent on this income was 2.1 (range 0-6), meaning that some participants may have been living below the Federal Poverty line. Because participants were not asked to report income from other household members, such as a spouse or parent, a more precise estimate could not be made. It may be that other family members had high monthly earnings. What is known, is that, participant earnings were low overall and unemployment rates were high. These conditions would have had the potential to produce stress and a scarcity mentality, both of which can disrupt adaptive decision-making (Bratanova, Loughnan, Klein, Claassen, \& Wood, 2016; Mani et al., 2013; Pearlin, Menaghan, Lieberman, \& Mullan, 1981). Lower income subsequent to incarceration could have also influenced drug purchasing, by potentially restricting the overall flexibility with which participants could make purchasing decisions about preferred drugs and non-drug goods, as well as respond to short- and long-term changes in drug markets related to perceived drug quality, drug risk, and drug cost (Becker, Murphy, \& Grossan, 2004; Carroll \& Rodefer, 1993; Goudie et al., 2007; Ornstein \& Levy, 1983; Petry, 2002). Though past-month income was lowest among participants who reported past-year concomitant use subsequent to incarceration and highest among those who reported other/no drug use, the low income across groups makes it difficult to discern the extent to which income may have influenced drug-related decision-making differentially across groups, given that the lower income associated with concomitant drug use was marginal. 


\section{Subjective Socioeconomic Status}

The average perceived subjective socioeconomic rank that participants reported was 5.0, though for participants who reported past 30-day concomitant or opioid use prior to incarceration, it was slightly below that. Differences in rank were statistically significant, but it is unclear if there are substantive differences between groups, as lower perceived subjective socioeconomic status was not associated with changes in odds for any outcome. Considering that participants in this sample believed, on average, that they were ranked--in terms of education, money, desirable employment-- below approximately one-half of the rest of society, but above approximately another one-half, means that, similar to all people, participants' stress response and cognition likely varied dynamically as a function of context, engaging in downward or upward social comparison, increased or decreased vigilance, and arousal or ease depending on the social context (Collins, 1996; Fiske \& Taylor, 2013; Kraus, Horberg, Goetz, \& Keltner, 2011 Taylor \& Lobel, 1989). Examining the frequencies of responses for this variable showed that $35.3 \%$ of the sample reported a rank $4<$, meaning that a subgroup of participants perceived themselves as having a lower perceived socioeconomic status ${ }^{11}$.

Across the life course, lower actual and perceived social and/or economic status is associated with poor physical (e.g., increased cortisol, impaired immunological function) and psychological health (e.g., anxiety), higher stress, and impairments in executive function (Abbott et al., 2003; Adler et al., 2000; Derry et al., 2013; Hackman, Farah, \& Meaney, 2010; McEwen \& Gianaros, 2010; Ursache, Noble, \& Blair, 2015; Wilkinson \& Pickett, 2003). Though differing somewhat between stable and unstable social hierarchies and populations, being and/or perceiving oneself as lower-ranking (or subordinate) in a social hierarchy is often associated with increased stress response and less adaptive neurocognitive functioning, whereas being higher ranking (or superordinate) is associated with decreased stress response and more adaptive neurocognitive functioning (Gesquiere et al., 2011; Noonan et al., 2014; Sapolsky, 2017; Sallet et al., 2011). Even a rank of 5, indicating perception of oneself as lower than half of all others in a society where higher socioeconomic status is associated with increased power, opportunity, prestige, and social worth, would have had the potential to contribute to negatively valanced states and increased stress

\footnotetext{
${ }^{11}$ A breakdown of this $35.3 \%$ of participants: $6.7 \%$ reported a rank of $1,5.0 \%$ reported a rank of $2,10 \%$ reported a rank of 3 , and $13.6 \%$ reported a rank of 4 .
} 
response, particularly during interaction with a supraordinate, all of which could have influenced cognition and behavior related to drugs, including increased drug taking and relapse (Caldwell \& Riccio, 2010; Covington et al., 2005; Covington \& Miczek, 2001; Cruz, Quadros, Hogenelst, Planeta, \& Miczek, 2011; Do Couto et al., 2006, 2009; Weiss, 2005).

That the entire sample is comprised of corrections-involved people with a history of drug use means that some participants may have experienced social, affective, cognitive, and physiological effects associated with lower status in respect to these stigmatized characteristics, as both groups are openly castigated and routinely experience discrimination, rejection, and marginalization (Fiske, 2005; Moore, Tangney, \& Stuewig, 2016; Harris \& Fiske, 2007; Major, Dovidio, \& Link, 2017; Pachankis et al., 2017). It is possible that had participants been asked to rank their perceived socioeconomic status during the oneyear period subsequent to incarceration that mean ranks would have decreased across groups as a result of having a stigmatized "felon" status, which was not necessarily the case for all participants prior to incarceration (Behrens, 2004; Copenhaver, Edwards-Willey, \& Byers, 2007; Moore, Stuewig, \& Tangney, 2013, 2016).

\section{Non-drug Alternative Reinforcers}

\section{Social Satisfaction and Social Worth}

Participants did not differ either prior or subsequent to incarceration in terms of social interaction, with $>80 \%$ participants reporting spending the majority of their free time in some social capacity (e.g., with friends or family). Participants who used other drugs and stimulants during the 30-day period prior to incarceration had, on average, a greater number of close relationships and also reported receiving greater satisfaction from these relationships. It may be of some interest that stimulants, alcohol, and cannabis, used at high rates among these groups, are more often conceptualized as social or "party" drugs compared to opioids, meaning that some drug use among these groups may have been social (Freese, Miotto, \& Reback, 2002; Green, Pickering, Foster, Power, \& Stimson; 1994; Waldorf et al.,1992).

Reported social satisfaction was lower among participants who reported past 30-day concomitant and opioid use, with both groups also having slightly fewer close relationships on average. Although receiving satisfaction from social interaction was not associated with a change in odds of observing concomitant, opioid, or stimulant use prior to incarceration, receiving satisfaction from social interaction 
was associated with a 50-60\% decrease in the likelihood that concomitant, opioid, and stimulant use during the one-year post-release period would be observed. This was found when examining past-year heroin use as well; the odds of heroin use decreased by approximately $59 \%$ when social satisfaction was reported.

Sample-wide, the average number of close relationships decreased from 7.1 prior to incarceration to 6.1 subsequent to incarceration. While it is possible that fewer available non-drug choices, such as close relationships, may have contributed to drug use initiation and continued use, it is also possible that as a result of continued drug use and incarceration the real and perceived number of non-drug alternatives needed to help reinforce behavior away from continued drug use declined (Ahmed et al., 2010; Bickel et al., 2014; Lenoir \& Ahmed, 2008; Staton-Tindall, Royse, \& Leukfeld, 2007). As noted earlier, one possible result of continued drug use is that the number of avenues for pursuing non-drug opportunities decreases, as does the degree of support received from others. It can be challenging to be in a relationship with a person who continues to use high-risk drugs, in part due to the higher level of social dysfunction found among regular drug users (Cunha, Bechara, de Andrade, \& Nicastri, 2011). As use persists, relationships, which may in some cases have already been defined by dysfunction or discord, can become strained further, less satisfying, and possibly terminated (Collins et al., 2007; Mowen \& Visher, 2015; Wallace et al., 2016). Fewer, and poorer quality, relationships would decrease some of the opportunity costs associated with continued use. This idea is partially reflected by the negative relationship observed between receiving satisfaction from social interactions and high-risk drug use that occurred during the one-year period subsequent to incarceration.

In addition to having the highest number of relationships on average subsequent to incarceration, participants who reported other/no drug use had the highest rates of social worth, meaning that a greater proportion of this group reported feeling "valued, supported, and cared about" by other people in their life compared to other groups. Conversely, participants who reported concomitant use subsequent to incarceration, who had the fewest close relationships on average, reported the lowest rates of social satisfaction. Although a majority of all groups reported feeling at least "somewhat" cared about or supported by people in their lives, the fact that rates differed by groups who reported more or less use helps to articulate the idea that family and friends may be less willing to remain invested in and supportive of people who continue to use high-risk drugs and also the idea that people who feel less cared about and 
supported by others may come to believe that they have fewer reasons to moderate opioid and stimulant use and fewer avenues for accessing help. As noted earlier, positive social interaction can have a powerful influence on a person's perceived sense of well-being, psychological health, and cognitive functioning, whereas social isolation, social exclusion, withdraw of social support, and other forms of social stress can produce cognitively damaging effects along with increases in drug craving and use (Abbott et al., 2003; Cole et al., 2011; Stein, van Honk, Ipser, Solms, \& Panksepp, 2007; also see Heilig et al., 2016 for further discussion).

\section{Education, Employment, and Leisure Time}

Past-year educational and/or vocational involvement was similar across all drug use outcome groups prior and subsequent to incarceration. Although post-incarceration rates of employment are often higher than pre-incarceration rates among corrections-involved samples, educational/vocational involvement increased by only $2.0 \%$ between time periods, even though alcohol and other drug use decreased substantially, perhaps suggesting that participants' felony status made obtaining employment and entry into post-secondary education more challenging (Copenhaver et al., 2007; Duwe \& Clark, 2017; Flake, 2015; Halkovic et al., 2013; Lam \& Harcourt, 2003; Pager, 2003). This is a particularly compelling possibility given that the period subsequent to incarceration was after the 2007 economic crisis had stabilized, a time when the unemployment rate in Kentucky decreased annually from 8.4\% in January 2012 to $4.4 \%$ in December 2017 (U.S. Bureau of Labor Statistics, 2019).

That a majority of participants who reported concomitant, opioid, and stimulant use both prior and subsequent to incarceration were employed or otherwise engaged in educational pursuits during the same time period raises the important point that drug use, licit or illicit, is not always mutually exclusive with other behaviors (Kowalski-McGraw, Green-McKenzie, Pandalai, \& Schulte, 2017; Tiesman, Konda, Cimineri, \& Castillo, 2019). Potentially rewarding non-drug alternatives, such as work and education, comprise activities, as does drug use, meaning that they are extended over time and are comprised of many temporally connected choices and behaviors (Baum, 2004). Many behaviors must be executed in a particular order prior to and during an activity, such as "going to work" and "being employed" or "using amphetamines" (Baum, 2004; Rachlin, 1995). Drug use, over a day or year, can be chosen or not chosen at different intervals, meaning that it can be avoided or approached in terms of trade-offs with family 
obligations, employment, school, or other roles (Waldorf et al., 1992). A person may therefore choose to use amphetamines to excess, miss work or school, but then subsequently choose to put in extra hours or effort at work or school to offset this earlier decision to binge. For some participants, opioid and stimulant use might have continued to the point where the loss off a job or academic suspension from school became a credible threat or probable outcome, making them, at that point, mutually exclusive choices. That such patterns could not be discerned in this study, makes it an important avenue of investigation in future work.

\section{Labor as Labor, and the Indignity of Work}

An important possibility not to overlook is the fact that work may influence a person's willingness or desire to use opioids or stimulants. Although employment and educational involvement have been framed in terms of potentially rewarding non-drug alternatives, it may be that for some participants in this sample neither work nor school were associated with anything rewarding, and not contemplated of in terms of feeling more vested in their everyday lives and future. Instead, it may be that employment was simply an unpleasant means to achieving some meager economic end or fulfilling a stipulation of community supervision (Gurusami, 2017; Travis \& Stacey, 2010). Because jobs are demanding and can be perceived, particularly by people with lower socioeconomic status, as something over which they can exert little control, means that employment can also be considered a possible source of stress (Kunz-Ebrecht, Kirschbaum, \& Steptoe, 2004). That work is hard and not always rewarding should also not be overlooked. It is possible that the labor expended by participants in this sample was disproportionate to social and economic gains. Rather than contributing to participants' feeling as though they have "stake in the conventional life", disparities between effort and compensation may have, for some, produced a sense of disillusionment (Buck, 2001; Kahn, 2018; Shipler, 2005; Waldorf et al., 1992).

The histories of drug use and labor are indeed closely linked. The term "skid row" originates from logs being "skidded" toward lumber mills along stretches of road in the Pacific Northwest, where the supply of alcohol, opium, and other drugs proliferated to serve the many timber laborers who resided in the area and who routinely worked themselves to exhaustion under difficult conditions (Keniston-Longrie, 2009). Prior to incarceration, participants who reported past 30-day other drug use reported having more leisure days on average on a given week in which they could pursue hobbies or other activities of interest to them, whereas participants who reported past 30-day concomitant use had the fewest, even though the rate 
of educational and/or vocational involvement between these two groups was similar. This means that having fewer leisure days was associated with overall greater drug use. Stimulant and opioid use as a form of misregulation may not be uncommon among employed people, particularly among people employed in physically and psychologically taxing, or otherwise tedious and dangerous, occupations, in which reducing pain and increasing stamina can be central to a person's ability to work or to work longer hours (Anglin, Burke, Perrochet, Stamper, \& Dawud-Noursi, 2000; Dalla, Xia, \& Kennedy, 2003; Gibson, Leamon, \& Flynn, 2002). Indeed, some decisions to use opioids and stimulants may have been influenced by a perceived need to function or enhance performance in vocational or educational roles (Ahmad \& Aziz, 2012; Arria et al., 2018; Dalla et al., 2003; Liu, Elliott, Striley, Gurka, \& Cottler, 2019).

For participants without a high school or college degree, and particularly after a felony record was established, it is possible that many of the occupations that they were engaged in were challenging, exhausting, stressful, and associated with lower prestige, compared to people with high school and college degrees and no criminal record (Bridgeland, Dilulio, \& Morison, 2006; Liker, 1982). Labor exploitation (e.g., forced day labor, temporary employment, percentage cut of hourly pay) among people with criminal histories, including among people on community supervision, is not uncommon (Elcioglu, 2010; Purser, 2012) and is reflected in some routine practices among US companies (e.g., PeopleReady, LaborReady) (Capstone Law, 2019; Theirman Buck Law Firm, 2019). It is not inconceivable, then, that some participants may have found it difficult to feel a sense of reward from working at Wal-Mart, picking up garbage, or toiling in a factory, let alone to achieve the feeling of having "a stake in the conventional life", and therefore more vested in their everyday life. The idea of working at Wal-Mart for the rest of one's life, assuming that working at Wal-Mart is not a person's life ambition, may contribute to depression or anxiety, feelings of resentment and disenfranchisement from the wealth of society in which they do not equitably share despite their hard work, and feelings of estrangement from their own future (Kraus et al., 2011; Shipler, 2005). Having a job in which one knows that they are easily replaceable, as is the case with almost all menial work in the US, also has the potential to create a sense of uncertainty. A belief that a bleak present will not give way to a brighter future may have the capacity to increase decisions to use high-risk drugs in the present (Buer et al., 2016). "Deaths of despair", which include premature mortalities associated 
with drugs, have increased in the US, particularly among men without college degrees in economically depressed areas with high drug accessibility (Ruhm, 2019; Stein et al., 2017).

\section{Central Appalachia}

Central Appalachia is a geographic region spanning four states, Western North Carolina, Eastern Tennessee, Eastern Kentucky, and all of West Virginia. Fifty-four of Kentucky’s 120 counties comprise part of this Central Appalachian region (ARC, 2019). Central Appalachia, and Eastern Kentucky in particular, have experienced historic disadvantage compared to other areas of the US and continue to experience economic and social hardship and collective uncertainty about the future (Billings \& Blee, 2000; Eller, 2008; Greenberg, 2018; Hayes, 2018; Mather, 2004). There remain significant psychological and physical health disparities between Eastern Kentucky and the rest of the state, as well as the rest of the country (Crosby, Wendel, Vanderpool, \& Casey, 2012; Hendryx, 2010). These are reflected by significantly higher rates psychological, developmental, and learning disorders, SUDs (including for nicotine), chronic illness, chronic pain conditions, cancer, obesity, and disability (Herath \& Brown, 2013; Lane et al., 2012; Pugh, 2014; Rodriguez, Vanderford, Huang, \& Vanderpool, 2018; Schoenberg, Huang, Seshadri, \& Tucker, 2015). In Eastern Kentucky counties, the life expectancy is 9-12 years younger than counties in other parts of the state; between 2014 and 2017 the average life expectancy actually decreased, despite Kentucky's adoption of Medicaid expansion under the ACA, which increased access to care for many in the region (University of Wisconsin Population Health Institute, 2019). These disparities are partially attributable to greater difficulties in accessing preventative medical services or interventions, including for SUD (Hill, Cantrell, Edwards, \& Dalton Sexton, Carlson, 2016; Leukefeld, Booth, 2008; Patrick et al., 2018; Staton-Tindall et al., 2015).

However, some disparities may also be attributable to economic conditions in the region, as 37 out of the 54 Kentucky counties within Central Appalachia are economically distressed (ARC, 2018). These counties rank in the bottom $10 \%$ of all counties in the US in terms of poverty and employment (ARC, 2018). Nationally, the poverty rate between $2011-2016$ was approximately $16 \%$, whereas for Central Appalachian Kentucky counties during this same time period, it was approximately $26 \%$; in 2015, per capita income in these counties was $\$ 19,204$, sharply lower than the national per capita income of $\$ 39,778$. 
For these reasons, Appalachian Kentucky may be conceptualized as a "harsh" environment (Frankenhuis et al., 2016; Meit et al., 2017).

\section{Carpe Diem}

Beyond the fact that these conditions may contribute to a scarcity mentality, residing in a harsh environment, such as Central Appalachia, has the potential to decrease opportunities for accessing rewarding non-drug alternatives, particularly when they require more effort or cost to obtain (e.g., needing to drive long distances, but not having a vehicle). Residing in a more rural part of Central Appalachia may present unique barriers for accessing non-drug alternatives, such as higher education, employment and economic advancement, civic organizations, sports clubs, mutual aid groups, and many types of leisure activities ubiquitous in urban and suburban areas (Buck, 2001; Denk, 2019; Nelson \& Smith, 1999; Petry et al., 2001). Residing in a harsh region characterized by wide-spread hardship, premature mortality, restricted opportunity, and overall greater uncertainty about the future, would have had the potential to influence participants by increasing present-bias and decreasing risk-aversion in order to facilitate the immediate capture of rewards despite risk (Frankenhuis et al., 2016; Pepper \& Nettle, 2013; Smith \& Tickamyer, 2011). Though the future is always uncertain, and therefore less motivating when making decisions in the present, for participants in Central Appalachia, this uncertainty may be perceived as even greater. Why wait to enjoy something that may never materialize, when relief can be found in the present? In this way, living in a harsh environment might also engender propensity toward misregulation, with opioids and stimulants a possibly appealing anesthetic to bleak conditions (Buer et al., 2016). ${ }^{12}$

\footnotetext{
${ }^{12}$ Interestingly, factors that might be expected to differ between participants who resided in Central Appalachian and those who did not (e.g., chronic pain, misregulation, depressive symptoms, suicidal ideation) evidenced very few statistically significant between-group differences when examined (results not displayed). One difference, though, included higher unemployment among participants who resided in Central Appalachia prior ( $41.4 \%$ vs. $\left.30.8 \%, X^{2}=18.0, p<.001\right)$, but not subsequent to incarceration $(38.7 \%$ vs. $\left.34.6 \%, X^{2}=2.1, \mathrm{p}=.146\right)$. Other modest differences, though not statistically significant, were reflected by the fact that past 30-day income among participants who resided in Central Appalachia was slightly lower $(\$ 1,194$ vs. $\$ 1,332, t=1.43, \mathrm{p}=.109)$ and that a greater average portion of income was received through Social Security Disability Insurance benefits ( $\$ 862$ vs. $\$ 792, t=.671, \mathrm{p}=.503$ ). Participants who resided in Central Appalachia reported having more close relationships subsequent to incarceration (6.7 vs. 5.9, $t=2.30, \mathrm{p}=.022)$, though groups were approximately equal for the year prior to incarceration (7.0 vs. 7.1, $t=1.79, \mathrm{p}=.858$ ). While there were no between-group differences for rates of feeling cared about or supported by others, there were differences for social interaction subsequent to incarceration, with a larger proportion of participants who resided in Central Appalachia reporting that they spent more free time with others, versus alone (91.1\% vs. 87.2\%, $\left.X^{2}=4.5, \mathrm{p}=.033\right)$. Participants who resided in Central Appalachia during this same time also reported higher rates of social satisfaction $\left(92.8 \%\right.$ vs. $\left.87.0 \%, X^{2}=9.93, p=.002\right)$.
} 
Additional analyses (results not displayed) showed that participants differed across several drugrelated variables depending on whether they resided within or outside of Central Appalachia. Participants who resided in Central Appalachia were more versatile users, using a greater number of different drugs on average during the one-year periods prior (4.1 vs. 3.6, $t=2.7, \mathrm{p}=.007)$ and subsequent $(1.5$ vs. $1.2, t=2.44$, $\mathrm{p}=.011)$ to incarceration, had higher drug use severity scores ( 25.2 vs. $22.3, t=3.4, \mathrm{p}<.001)$, and had higher rates of being intoxicated at the time that they committed their offense $\left(81.5 \%\right.$ vs. $\left.76.6 \%, X^{2}=4.39, p=.036\right)$. Older average age of drug use initiation, however, was found among participants who resided in Central Appalachia (15.1 vs. 14.5, $t=2.31, \mathrm{p}=0.20)$. Participants who resided in Central Appalachia had higher rates of IDU history $\left(56.6 \%\right.$ vs. $\left.37.0 \%, X^{2}=50.6, \mathrm{p}<.001\right)$, though rates of IDU reported subsequent to incarceration were equivalent $\left(7.2 \%\right.$ vs. $\left.10.8 \%, X^{2}=2.64, \mathrm{p}=.881\right)$. Because of the difference in IDU history by Central Appalachia residence, infectious disease was also explored. Participants who resided in Central Appalachia prior to incarceration were significantly more likely to report having Hepatitis C (15.3\% vs. $\left.7.1 \%, X^{2}=22.6, \mathrm{p}<.001\right)$, though no participants who resided in Central Appalachia reported having HIV. As presented in Chapter 4, a greater proportion of participants who reported opioid use prior and subsequent to incarceration resided in Central Appalachia, whereas a greater proportion of participants who reported stimulant use resided outside of Central Appalachia. This relationship was evidenced further by the finding that Central Appalachian residence prior to incarceration was associated with a $64 \%$ increase in odds of past 30-day opioid use and a $37 \%$ decrease in odds of past 30-day stimulant use. Subsequent to incarceration, Central Appalachian residence was associated with a nearly $117 \%$ increase in the likelihood of past-year prescription opioid use. Heroin and cocaine/crack cocaine were used at lower rates among participants residing in Central Appalachia, potentially reflecting overall greater availability and use of these drugs in urban areas (Habecker, Welch-Lazoritz, \& Dombrowski, 2018; Jalal et al., 2018). Odds of observing past-year heroin use decreased by approximately $62 \%$ subsequent to incarceration by Central Appalachian residence. At both time periods, rates of prescription opioid and buprenorphine use were higher among participants residing in Central Appalachia, potentially attributable to the significantly higher per 1,000 rates of opioid and buprenorphine prescribing found in Eastern Kentucky and wider-spread acceptability of prescription opioid use found in rural areas of Kentucky (Havens, Talbert, Robert, Cynthia, \& Leukefeld, 2006; CHFS, 2019; Havens, Young, Havens, 2011). Amphetamine use did not differ 
significantly between Appalachian and non-Appalachian regions, suggesting that this drug may be equally accessible, or manufacturable, across regions (Weisheit \& Brownstein, 2016; Sexton, Carlson, Leukefeld, \& Booth, 2006; Stoops et al., 2011). That amphetamine drugs include both "street meth" as well as prescription stimulants means that it is also possible that some amphetamine use among participants who resided in Central Appalachia was attributable to diverted prescription stimulants, though this may have only accounted for a small portion of stimulant use and may have been more common among younger participants (Cassidy et al., 2015; Kroutil et al., 2006).

Kentucky counties in Central Appalachia are predominately rural, meaning that they are not proximate to major urban drug markets, such as Louisville or Cincinnati, which borders Kentucky and continues to be a source of heroin for Northern and Central Kentucky (Stewart, Cao, Hsu, Artigiani, \& Wish, 2017) ${ }^{13}$ Accessibility to drugs based on urban influence was highlighted by some of the findings in this study. For instance, prior to incarceration, rates of prescription opioid use were highest among participants who resided in more rural counties compared to heroin, which was used at higher rates among participants who resided in urban counties. Subsequent to incarceration, rates of heroin use were still highest among participants who resided in urban counties and prescription opioids, while still used at high rates in rural areas, decreased from pre-incarceration rates, likely reflecting decreases in opioid prescribing that began in 2012. Buprenorphine was used at higher rates in rural areas during the period prior to incarceration, but not subsequent. Heroin availability and use continues to increase in rural Appalachia, but during the time that data were collected was simply less available than prescription opioids and other drugs (Cloud, Ibragimov, Prood, Young, \& Cooper, 2019; Moody, Satterwhite, \& Bickel, 2017).

As noted in earlier chapters, accessibility has the potential to shape drug preferences, not only because it exposes a person to a drug more regularly, thus increasing the probability of use and later possible dependence, but also because it decreases the effort needed to obtain the drug. Driving 80 miles to buy heroin costs more in time, effort, energy, and risk for contact with law enforcement then driving 10 miles to buy prescription opioids. It may be that among participants who resided in Central Appalachia, proximity to drugs was an influence on decision-making and the development of drug preferences. This is

\footnotetext{
${ }^{13}$ The mean urban influence for non-Appalachian counties was 3.1, whereas the mean urban influence for Appalachian counties was 7.7 (range=1-12).
} 
partially reflected by the fact that rates of county-wide opioid and buprenorphine prescribing were higher in Central Appalachia during the time periods examined in this study, and that rates of prescription opioid and buprenorphine use were higher among participants who resided in these counties (CHFS, 2019). It is further reflected by the fact that prescription opioids ( $39.6 \%$ vs. $18.4 \%$ ), buprenorphine ( $6.5 \%$ vs. $0.8 \%$ ), and prescription sedatives (4.4\% vs. $2.3 \%$ ) were more often preferred among participants who resided in Central Appalachia, whereas heroin (4.8\% vs. $12.1 \%$ ), cocaine (5.2\% vs. $10.3 \%)$, and alcohol (8.5\% vs 15.3\%), were more often preferred among participants who resided outside of Central Appalachia $\left(X^{2}=164.2, \mathrm{p}<.001\right)$. Unrestricted alcohol sales did differ significantly by residence within or outside of Central Appalachia, with the latter having overall greater access to unrestricted alcohol sales (72.8\% vs. $\left.19.6 \%, X^{2}=375.78, \mathrm{df}=1, \mathrm{p}<.001\right)$. This may account for slightly lower preference for alcohol. Amphetamines were preferred approximately equally across regions (15.1\% vs. $15.2 \%)$ and used at similar rates across regions.

\section{Ambiguous Risk}

It is unclear, though, the extent to which participants' decisions to use high-risk drugs meaningfully differed according to living within a harsh region, such as the Central Appalachian region of Kentucky. However, other studies have documented risky drug practices among people in Kentucky who reside in rural Appalachia and have found associations between high-risk drug use in this region and economic distress (Cloud et al., 2019; Havens, Oser, \& Leukefeld, 2011; Staton-Tindall, Webster, Oser, Havens, \& Leukefeld, 2015). Though Central Appalachia and other distressed rural areas continue to be associated with "deaths of despair", hardship, and widespread uncertainty, there were also other factors present among participants who resided in Central Appalachia that could have influenced drug-related decision-making (Erwin, 2017; Meit et al., 2017; Segal et al., 2017; Woolf, Schoomaker, Hill, \& Orndahl, 2019). For instance, many participants who resided in Central Appalachia reported having close relationships, social interaction, and social satisfaction, which could all be considered as rewarding nondrug alternatives.

Still, drug use and involvement with family, friends, work, or other activities is not mutually exclusive, and it could be that some participants were interacting with friends, family, or intimate partners who also used high-risk drugs (Fleming et all., 2010; Havassy, Hall, \& Wasserman, 1991; Mayock, Cronly, 
\& Clatts, 2015; McCrady, 2004; Staton et al., 2018). Family-wide drug use and greater cultural acceptance of drug misuse is not uncommon in the Central Appalachian region of Kentucky (Jonas et al., 2012; Keyes et al., 2014; Leukefeld et al., 2007; Young, Havens, \& Leukefeld, 2010). In fact, some rural, drug-using women in Appalachian Kentucky have reported feeling "stuck" to the region, other people within the region, and to prescription opioids as a fixture of the region and relationships, with myriad constraints on their ability to make different drug-related decisions (Buer, Leukefeld, \& Havens, 2016).

It should not be overlooked that some participants who resided outside of Central Appalachia resided in regions that, while not broadly and diffusely harsh, nevertheless may have contained concentrated harsh locales that influenced decisions to use opioids and stimulants (e.g., blocks, neighborhoods, zip codes) (Boardman, Finch, Ellison, Williams, \& Jackson, 2001; Dear \& Wolch, 2014; Schroeder et al., 2001; Stull et al., 2019). That participants did not differ by region for characteristics such as psychiatric symptoms, chronic pain, suicidal ideation, stress-related health effects, and financial distress may not discredit the notion that Central Appalachia constitutes a harsh region with the potential to influence cognition and choice, so much as lend support to the idea that participants who resided outside of Central Appalachia may have also resided in harsh urban and suburban areas. This is reflected by the fact that, compared to the general population, the entire sample had higher rates for many factors with the potential to unfavorably influence decision-making in relation to high-risk drugs.

Overall, findings indicate that drug use may not have been necessarily or straightforwardly risker among participants who resided in Central Appalachia, but rather that it had a different tenor. Participants who resided in Central Appalachia did evidence overall greater drug use severity and versatility, using more drugs on average compared to participants who resided outside of Central Appalachia. These participants not only used prescription opioids with or contemporaneous to amphetamines, alcohol, prescription sedatives, buprenorphine, and to a lesser extent cocaine and heroin, but also reported significantly higher rates of IDU. That said, participants who resided outside of Central Appalachia also reported high rates of opioid and stimulant use, with heroin, perhaps the riskiest drug that could be used during the time periods explored in this study, used at higher rates. Considered together, and in combination with findings previously discussed, it may be reasonable to suggest that high-risk drug use is 
endemic across the sample, even as it varied by time period, specific drug type, residence in or outside of Central Appalachia, and other factors already highlighted.

\section{Broader Implications and Future Directions}

\section{The Road Back to Hell is Still Paved with Good Intentions}

The idea that guided this exploratory study is that there are many endogenous and exogenous factors with the ability to influence and constrain drug-related decision-making and behavior. This pertains to people before they choose to take their first drug and at all points thereafter. As prior decisions to use drugs accumulate, a person's capacity to choose to do otherwise in relation to drugs becomes more, not less, difficult. In the extreme, and under the right conditions, choosing drugs over all other choices is not unthinkable, meaning that it is imperative to understand what motivates continued drug use despite great risk and great cost.

Part of the behavioral economic perspective of drug use includes an understanding that many psychological, affective, and neurophysiological states and changes occur when addictive drugs such as opioids and stimulants are used. Brain changes are, after all, endogenous to the person and can contribute to the person's pathological overvaluing of drugs and their continued use despite costs (Heyman \& Mims, 2015). In this way, there is some harmony between the behavioral economic/neuroeconomic conceptualization of drug use that persists despite adverse consequences and the BDMA conceptualization, suggesting possible common ground for advancing a more nuanced understanding of the nature of the problem (Fenton \& Wiers, 2017). But, in order to better make sense of and intervene on these endogenous factors, and to better understand how decisions to use high-risk drugs can continue despite adverse consequences, it may also be necessary to identify and examine people's overall set of available capacities and choices prior to and during a period of problematic use, along with the contexts in which drug-related decisions are being made (Bickel et al., 2014).

This study used a molar view of choice (Baum, 2004; Vuchinich \& Heather, 2003) to identify and explore several factors with the potential to influence decision-making about high-risk drugs among a sample of corrections-involved adults using available survey data. In so doing, it was able to help articulate the idea that endogenous and exogenous conditions have the capacity to constrain choice and behavior. 
Broadly and indirectly, some study findings helped to illustrate the important idea that influences on decision-making and behavior are not always in the direct awareness or control of people. These ideas may be extended in future work to consider how endogenous and exogenous conditions also have the capacity to influence and constrain the formation of desires and intentions, both of which are important for understanding high-risk drug use. As discussed earlier, a person's preferences may be ordered:

1) Not be addicted to heroin.

2) Inject heroin.

3) Do not inject heroin.

This could also be reflected as conflicting desires that constrain a person's will, in which a firstorder desire constrains a second-order desire, making it impossible for a person to act on their desire $(\text { Mann, 2004) })^{14}$ :

1) Wants to inject heroin.

2) Does not want to inject heroin.

A better understanding of the ranked preferences and desires of people who use drugs would help to clarify choice and behavior in respect to use. There is an important clinical difference between:

1) Continued drug-taking despite a person's desire to stop

2) Continued drug-taking because of a person's desire to continue.

There is also an important clinical difference between:

1) Remitted drug-taking despite a person's desire to take drugs.

2) Remitted drug-taking because of a person's desire to not take drugs.

If facilitating a state of remission or achieving a state of abstinence is a principal goal when addressing SUD, then both cases of the latter example might be considered treatment success. But it is easy to see why the differences between the two matter. Achieving a behavioral change without also achieving a change in desire or intention, assuming it is even possible, may be missing the bigger point: most of us

\footnotetext{
14 "One has freedom of will if one is free to want what one wants to want; if, that is, there is harmony between one's first-order desires and one's second-order desires. A narcotics addict who takes drugs because he wants to has freedom of action. Nonetheless, he lacks freedom of will if his second-order desire not to have a first order-desire for narcotics is powerless over the first-order desire" (Mann, 2004, pg. 286).
} 
would rather live in a world in which people's lives are of a quality that they no longer want to choose to take drugs with significant risks and at tremendous cost.

A person's primary and secondary intentions in relation to drugs are also of interest and warrant investigation. Future study of people's ranked intentions is a potentially useful area of exploration, insofar as intention precedes purposeful and planned behavior and insofar as intentions cannot be formed except by a broader set of conditions which, in theory, have the potential to be manipulated or improved (Bratman, 1987, 1999). For instance, a person may be able to form a primary intention to quit using drugs if sufficiently appealing services or non-drug options were offered. Even with this primary intention formed, secondary intentions (e.g., not calling the drug dealer, throwing away syringes and not buying new ones, developing non-drug routines etc.) would still need to be reinforced over time. Because the formation of intent is conditional and temporally situated, it is important that future work not only examine intentions related to drug-related choice and behavior, but also seek to understand what conditions influence their development and ability to be acted upon (Bratman, 1987, 1990). Further examination of intentions related to drugs may also help bridge some of the conceptual divide between the BDMA and behavioral economic/neuroeconomic model. While the BDMA does emphasize desire and behavior, choice and intention are de-emphasized.

Detailed study of discrepancies between desires, intentions, choice, and behavior in relation to drug use, moderation, and cessation may begin to reduce some of the assumptions made during research and practice. For instance, it cannot be assumed that any participant in this sample wanted to use opioids or wanted to stop using opioids, or that they intended to keep using cocaine subsequent to incarceration or intended to quit using cocaine subsequent to incarceration. Even though participants' drug-related decisions were implied by their behaviors, participants' desires and intentions, and the exogenous and endogenous conditions which may have influenced and constrained their development, could not be inferred from any study data. Nor could any conflict or agreement between ordered preferences, desires, and intentions be discerned, leaving many fundamental questions to be addressed in future studies. This is important to work towards, because, ultimately, rates of drug use or abstinence subsequent to any intervention lose much of their meaning if the degree of concordance or discordance between the goals of treatment and the goals of the people receiving treatment are not also known. Insofar as there is discordance between the two, policies 
and practices may continue to fall short, and people who may otherwise benefit from tailored treatment or from harm-reduction intervention may not been given access to potentially beneficial services that take into account what it is that they as people want and intend.

\section{Additional Considerations for Future Work}

This study identified many factors with the potential to have influenced decisions about opioids and stimulants, but because of this broadness of scope, both conceptually and from a measurement standpoint, there was no way define what was of greatest influence on decisions to use. Because there are in reality even more factors with the potential to influence drug-related decision-making then were discussed or examined here, it will be important moving forward to develop a more parsimonious study of what remains a complex phenomenon.

This may be accomplished, in part, by significantly narrowing the scope of future investigation and improving data collection methods, but also by examining a few select aspects of drug-related decision-making from different angles. For instance, the use of ecological momentary assessment and other sophisticated data collection methods that allow for passive and active data collection, coupled with structured interviewing, would facilitate measurement of some proximate influences over a period of time, but also more nuanced exploration of participants' desires and intentions (Buckner, Crosby, Silgado, Wonderlich, \& Schmidt, 2012; Epstein et al., 2009; Moore et al., 2014; Onnela \& Rauch, 2016; Roth, et al., 2017; Shiffman, Stone, \& Hufford, 2008). Despite some of the inherent limitations of using qualitative data, this type of information has the potential to reduce the burden of interpretation for some findings (or rather add an additional layer of data for interpretation, depending on how one wishes to view this). Had such data been available for examination in this preliminary study, they would have likely helped to provide a fuller picture of drug-related decision-making than was able to be established. In addition to EMA and structured interviewing, neuroimaging, biosensors, and biological measures could also be used in a limited capacity, helping to provide a fuller picture of how people's decision-making may be influenced during a particular period of time. Longitudinal studies (particularly those beginning during childhood and adolescence), though more challenging and costly to conduct, are particularly needed to help move this area of research forward. Aiming for data collection across multiple levels and time scales would help to establish a line of research that gives primacy to understanding endogenous and exogenous factors that can 
constrain choice and behavior in real-time and over time, and may permit study of how people's desires and intentions can vary as a function of broader conditions.

Of particular interest would be to investigate if the conditions of incarceration, which often include enforced abstinence, change cognition in respect to drugs. People with a history of opioid and stimulant use who are on community supervision or who are participating in drug court programs would be more likely to become incarcerated than other drug-using populations. These populations might be studied over time to see how cognition, desires, and intentions change as a function of being in a controlled environment where abstinence is enforced. Confinement in jail or prison might conceivably have the potential to decrease present-bias and increase future-oriented cognition. For some, time spent in a controlled environment may help achieve, at least temporarily, greater consonance between what their current self and future self value. Presently, these possibilities are offered here as speculation. However, should a study of drug-related cognition and temporal perception among people residing for long periods of time in a controlled environment be developed, it may be able to inform how interventions for correctionsinvolved populations are developed. Measuring present-bias, craving intensity, attentional bias, and temporal perception, along with ranked desires and intentions, during periods of incarceration might help to explain differences in lapses and relapses following release. As noted by Epstein et al. (2006), there is an important difference between abstinence that is imposed by external circumstances and abstinence that occurs under conditions which permit volitional drug use as well as volitional abstinence. Focusing on preferences, motivations, and intentions across contexts during future research will be crucial for developing a more well-rounded understanding of drug use that occurs subsequent to people's release from controlled environments.

Future work would also benefit from more diverse sample, not just in terms of race, sex, and age, but also in terms of educational status, income, and geographic location of participant residence. Greater heterogeneity of residence would permit further exploration of the idea that residing in harsh environment might influence drug-related decision-making. Such work might be undertaken in Tennessee, West Virginia, and Kentucky to examine differences between people who reside within or outside of Central Appalachia, but might also be undertaken in highly urban areas, such as Chicago or Baltimore, based on the block, neighborhood, or zip code (Furr-Holden et al., 2010; Stockdale et al., 2007; Stull et al., 2019). 


\section{Finding Common Ground?}

Regardless of whether addiction is ultimately conceptualized as a reinforcer pathology or as a brain disease, if the major approaches for understanding drug use and addiction can agree on the premise that people who use high-risk drugs do not have direct awareness or control over many of the factors that influence their ultimate behavior, and that as a result of repeated use they have fewer degrees of freedom in thought and action in respect drugs, then there may be agreement enough to move forward in collectively asking and answering the question of how intervention of high-risk drug use may be improved ethically in light of shared belief in this premise (Hyman, 2017). Presently, bioethical frameworks for clinical decisionmaking in SUD intervention are not well-developed and may be wholly absent within some settings, such as peer-delivered recovery services and mandated programs affiliated with 12-Step models of recovery (Bassuk, Hanson, Greene, Richard, \& Laudet, 2016; Bøg, Filges, Brännström, Jørgensen, \& Fredriksson, 2017; Logan, Miller, Cole, \& Scrivner, 2018). This means that certain practices, such as collaborative treatment planning or obtaining informed consent that is truly informed, cannot be taken for granted (Walker, Logan, Clark, \& Leukefeld, 2005).

Significant heterogeneity among SUD interventions is not necessarily a bad thing, as the factors that influence drug use differ across people. However, across mandated and voluntary SUD interventions there remain shortfalls in evidence-based practice (with some research-informed interventions unable to be implemented with fidelity to the underlying science), and continued reliance on non-professional services (Alcoholics Anonymous, 1994; Bøg et al., 2017; Butler, Chapman, Forman, \& Beck, 2006; De Leon, Perfas, Joseph, \& Bunt, 2015; Logan, Miller, Cole, \& Scrivner, 2018; Oser, Harp, O'Connell, Martin, \& Leukefeld, 2012; Walker, Godlaski, \& Staton-Tindall, 2013). Beyond this, evidence-based practice constitutes a process that includes taking into account not only scientific findings, but also the goals, preferences, and needs of people on an individual basis (Elwyn et al., 2012; Gambrill, 2006; Sackett, 1997).

The wide-spread awareness of the BDMA and the lack of awareness about the behavioral economic/neuroeconomic model of addiction across many service providers may, however unintentionally, encourage an overly simplistic view of and morally ambiguous response to the problem of high-risk drug use (Hall, Carter, \& Forlini, 2015; Hammersley, 2019 Kirby, Benishek, Dugosh, \& Kerwin, 2006). This may be partially due to the BDMA's narrower scope, but also due to the language commonly associated 
with this perspective that de-emphasizes the roles of intention, choice, and agency (NIDA, 2018). Because the BDMA, however unintentionally, finds itself in some rough conceptual agreement with 12-Step models of addiction (i.e., alcoholics and addicts have "an allergy" to alcohol and drugs, but continue to use despite this allergy because of spiritual estrangement), an overly simplistic, and still not quite clear, view of the problem might continue to persist among treatment providers and treatment-seekers alike (Alcoholics Anonymous, 2008; 2009, 2014; NIDA, 2018). Worryingly, the BDMA's de-emphasis of choice, intention, and agency does not yet seem to have resulted in fundamental transformations in how people with SUD are treated socially or within systems of intervention (Carter \& Hall, 2017). This is partially reflected by the continued practice of incarcerating people for using drugs, even though drug use is, by definition, part of the disease (Bureau of Justice Statistics, 2018). That addiction is considered to be a disease that is both chronic and relapsing may also inadvertently influence the degree to which the public, policy makers, clinicians, and people who use drugs believe that meaningful change is possible, even though evidence suggests it is (Heyman \& Mims, 2016; Wiens \& Walker, 2015).

Seeking to better understand drug use within a system of constraint, in which constraints on desire, intention, choice, and behavior are all more unequivocally considered, may permit, perhaps a bit paradoxically, some exoneration, while also better allowing for the person's humanity and dignity to be present in a way that the BDMA less readily permits. By directly acknowledging and purposefully studying people's capacities for forming desires and intentions, making decisions, and undertaking action, the lines of their personhood may become more evident and the factors which influence their decision-making about drugs more readily addressed. The degree to which investigation of and discussion about drug use involves or omits the idea of a decision-making agent is the degree to which people's values, preferences, and goals may be neglected during intervention. Omitting some of these central ideas may incline treatment providers to aim primarily for the problem behavior, without also aiming to identify and better understand the conditions that make that behavior possible. It also means that, in theory, decrease in substance use might be sought as an isolated target outcome without ever treating the person who decided to use drugs and without asking them what they desire and intend, and under what conditions they believe fulfilling those desires and intentions are possible. Many SUD interventions and recovery services in the US, especially mandated ones, do not commonly take into account what the person using drugs actually wants or desires, 
or how those desires may be at odds. It may be that because of the externalizing nature of SUD there is little sympathy for what the person using drugs wants or intends. Or it may be that, due to the increasingly accepted notion that drug users have a chronic and relapsing brain disease, there is a belief that drug users cannot be trusted to know what it is they want or intend, or that there is little point in asking due to the putative permeance of the condition.

It is in some ways easier to talk about, research, and moderate certain behaviors (e.g., "using" versus "not using") than it is to talk about, research, and target cognitive states (e.g., desire, intention), even though the two are inextricably linked (Bandura, 1986; Beck \& Haigh, 2014; Bratman, 1999). But it may be unwise to think that behaviors can be understood and moderated in the long-term, versus achieving a short period of remitted abstinence, without first seeking to better understand, clarify, and moderate people's desires, intentions, cognitive capacities, and conditions prior to choice. Aiming to better understand and address people's desires, intentions, and cognitive capacities in relation to drug-related decision-making, and aiming to improve the conditions in which these states and decisions are formed, has the potential to produce outcomes that are more expansive and enduring, as well as more faithful to the concept of person-centered care, compared to efforts that seek primarily to achieve just the behavioral outcome (e.g., remission or abstinence). It would also imply that prevention is equally if not more important than intervention.

\section{Limitations}

There were many important limitations to this exploratory study. First, because this sample was a comprised of corrections-involved participants in Kentucky, generalizability of any finding is limited. Second, using data from a survey that was not designed with the primary concern of establishing the intercorrelation of variables, or their relation to the outcomes examined in this study, poses a threat to validity in terms of temporal ambiguity (Langley, 1999; Shadish et al., 2002). Here, the temporal order of some factors and their influence on opioid and stimulant use outcomes cannot be established within or across given time periods (e.g., past-year, past 30-days, past 7-days). For instance, if someone experienced depressive symptoms during the past year, the relationship to an outcome of past 30-day use is far from direct. This is related to the fact that the CJKTOS surveys used to collect data contained measures created for other purposes and were unconcerned with theoretically-informed causal modeling. This may not be a 
limitation, per se, as this study was exploratory and sought principally to identify and describe some approximate contiguity or coincidence between endogenous and exogenous factors and high-risk drug use, making it agnostic about which constructs were "crucial" for causation (Bagozzi \& Yi, 2012; Hume, 2007; Shadish, 2002). This is noted simply as an acknowledgement that the measures used to collect data were not developed or implemented for purposes other than clinical use and program evaluation. This is a fact that may be applied to several other study limitations discussed in this and in previous sections.

Third, opioid and/or stimulant use (or no use) during a particular time period represents not just one decision, but many. It is likely that most in this sample did not decide to use a given drug only one time per day or month during the 30 days prior to incarceration or the one-year period subsequent to incarceration, but rather multiple times over these time periods, potentially dosing one or more drugs dozens of times per day, or even hour. For powder and crack cocaine, it is likely that the number of the decisions made to use the drug is greater than other drugs, given the pharmacological differences of cocaine relative to other drugs whose subjective psychoactive effects and half-life are relatively longer (Cone, 1995; Gawin, 1991, Jenkins, Oyler, \& Cone, 1995). It should be understood, then, that many drug-related decisions were being made contemporaneous to each other, in relation to proximate factors with the potential to influence decision-making (e.g., anxiety symptoms, chronic pain, living in Central Appalachia), and in relation to other, more distal factors (e.g., age of drug use initiation) with the potential to influence decision-making.

Fourth, random error is an inevitable part of any measurement process, but in this study, there are potentially several sources of nonrandom error, wherein instruments do not measure what they purport to measure, either in regard to states of the world at certain time points, or in regard to abstract concepts (Althauser \& Heberlein, 1970, Carmines \& Zeller, 1979; Werts \& Linn, 1970). For example, participant self-report in the form of discrete descriptions, that can only be one particular way or another (e.g., sex, age), or in the form of descriptions of their life during a given time period (e.g., nights spent in a controlled environment), can result in measurement error by only allowing participants to select from prefigured categories. These categories may not provide a full range of response options that accurately reflect the participant's lived experience (Coughlan, Cronin, \& Ryan, 2009). For instance, powder cocaine and crack cocaine could not be examined as separable decisional outcomes, as the CJKTOS survey combines the two 
in both baseline and follow-up surveys. It is likely that different factors would influence the likelihood of observing a decisional outcome for powder cocaine versus crack cocaine (Guindalini, Vallada, Breen, \& Laranjeira, 2006; Hatsukami \& Fischman,1996).

Additionally, measurement at other levels, such as the availability of alcohol within counties, was imprecise given the heterogeneity of alcohol prohibitions at the city and county-level across Kentucky. Although alcohol was considered as either "restricted" or "unrestricted", there was inevitably some variation between restricted counties, due to within-county differences in city statutes for the conditions of permissible alcohol sales, including by type of alcohol and type of establishment in which alcohol may or may not be distributed. The question pertaining to "current marital status" did not capture current relationship status, making it impossible to know if participants were in a committed relationship outside of legal marriage.

Nonrandom error was also possible, and perhaps more probable, in the measurement of variables indirectly indicating abstract concepts (e.g., impulsiveness), variables indicating the presence or absence of psychiatric constructs (e.g., anxiety symptoms), or variables indicating severity of somatic states (e.g., chronic pain). Nonrandom error may have also been introduced by the ways survey questions were worded. For instance, participants' response to items with ambiguous terminology (e.g., the number of close relationships participants believe they had, for which the concept of "close" is utterly subjective) and for which the surveys did not attempt to establish greater objectivity or linguistic coherence by defining terms (Searle, 2008). That the question pertaining to abstinence self-efficacy did not permit a response that clarified if participants intended to remain abstinent makes interpretation of that variable oblique at best.

Fifth, this exploratory study used single-item indicators and composite variables. Both have their limitations. Although the validity and reliability of single-item indicators have been questioned, and their use cautioned against in some instances, there is growing evidence and support for their limited use (AbdelKhalek, 2006; Bergkvist \& Rossiter, 2007; Nagy, 2002; Sarstedt \& Wilczynski, 2009). Some studies have found that single-item indicators have acceptable performance for reliability and construct and criterion validity (Gardner, Cummings, Dunham, \& Pierce, 1998; Elo, Leppänen, \& Jahkola, 2003; Ruekert \& Churchill, 1984; Wanous \& Hudy, 2001; Wanus \& Reichers, 1996; Youngblut \& Casper, 1993). After consulting various sources, it is reasonable to state that single-item indicators--while not always preferable 
and not equal to multi-item measures in predictive validity-- may still be used with caution and with understanding of their limitations. Single-item indicators can be helpful, and are sometimes necessary to bridge theoretical demands and problematic data collection methods that arise out of practical necessity (e.g., to reduce survey length, secondary data analyses; institutional and clinical considerations) (Bergkvist, 2015; Diamantopoulos, Sarstedt, Fuchs, Wilczynski, \& Kaiser, 2012; Fuchs \& Diamantopoulos, 2009; Loo, 2002; Wanous, Reichers, \& Hudy, 1997). Use of single-item indicators in this study was therefore undertaken with appropriate caution and with an understanding for how they may have the potential to weaken conceptual claims made upon the data and hinder interpretation.

Although composite variables created for use in this study were evaluated for internal consistency reliability, there is no way to measure criterion validity, just as criterion validity could not be assessed for the single-item indicators used (Carmines \& Zeller, 1979; Messick, 1975). It is also important to note that while some validated instruments, or items from validated instruments, were adapted for use as part of the CJKTOS surveys, these changes did not undergo validity or reliability testing. Other composite variables used consisted of CKTOS survey questions that were created by CDAR researchers and the KY DOC Substance Use Treatment Director during survey development. It is important to emphasize the fact that CJKTOS surveys are intended for use both clinically and as an outcome study evaluation tool. Therefore, there is no established validity for CJKTOS surveys nor survey sections, even as they are standardized in their content format during a given FY so as to minimize burden on participants (Dillman, Smyth, \& Christian, 2014; Tourangeau, Couper, \& Conrad, 2004).

Sixth, additional potential threats to validity due to other issues. Some of these issues include the possibility of: 1) social desirability bias or interviewer effects, 2) test fatigue/survey length, 3) instrument changes between baseline and follow-up, 4) possible participant difficulties with verbal and reading comprehension, mendacity, or poor recall, and 5) other conditions present during data collection and testing that cannot be known or accounted for (Cook \& Cshadish, 1994; Davis, Couper, Janz, Caldwell, \& Resnicow, 2009; Lavrakas, 2008a; Pedhazur, \& Pedhazur-Schmelkin, 1991; Holbrook, Green, \& Krosnick, 2003; Richman, Kiesler, Weisband, \& Drasgow, 1999; Shadish, Cook, \& Campbell, 2002). However, among samples of drug users, including those who are corrections-involved, self-report of drug use and drug-related behaviors has been found to be reliable (Del Boca \& Darkes, 2003; Del Boca \& Noll, 2000; 
Denis et al., 2012; Zanis, McLellan, \& Randall, 1994). In this study, the probability of nonrandom error being introduced was likely higher at baseline, as there were a greater number of different SAP clinicians collecting baseline data across multiple institutional settings, compared to the fewer number of different CDAR research staff collecting follow-up data via telephone in a non-clinical capacity (Dillman, 1978; Fink, 2015; Holbrook et al., 2003). Further, at baseline, some participants may have self-administered the survey, whereas others provided verbal responses to survey items read by SAP clinicians. This variation in survey administration and response format, within baseline data collection and between baseline and follow-up data collection, is one potential source of error (Carmines \& Zeller, 1979).

Seventh, despite that this study was developed with the idea of exploring the possible influence of participant residence as an important exogenous factor with the potential to influence decision-making about high-risk drugs, it was unable to be investigated with the depth or precision needed due to insufficient sample size, and in some cases insufficient variance. Accordingly, county-level measures initially proposed for examination (e.g., wealth inequality, poverty, opioid prescribing rates) were unable to be explored. However limited, urban influence and residence in Central Appalachia did reveal some important differences in opioid and stimulant use.

Eighth, using a behavioral economic approach for thinking about drug-related decision-making and drug use was a necessarily expansive approach, as it explicates drug use from a molar perspective of choice. The potential expansiveness of behavioral economics and a molar account of choice reflects, among other things, a conceptual sensitivity to the complexities involved, including context and time. This expansiveness opens the door to narrow and broad investigation alike (Tomer, 2007). Conceptual breadth permits researchers a fair degree of empirical latitude, as well as use of a variety of methodologies and data sources, including survey data (Katona, 1951, 1975; Tomer, 2007). But with breadth and inclusiveness, comes the potential for loss of specificity of inquiry, decreased likelihood for incremental advancement of parochial subareas, and, in some cases, an inability to reproduce or replicate methods or findings. Given that this exploratory study was not designed with reproducibility or replicability in mind, but rather with conceptual breadth in mind (even at the expense of specificity), some allowances for investigating the problem of high-risk drug use in a coarse-grained manner might be made. This coarse-grained approach may be considered as a starting point for future work. 
Because the outcome variables used in this study are understood from a molar view of choice as the behavioral indicator of a drug-related decision, but because participants were not asked specific questions about some of the important factors that may have influenced decision-making, such as drug price, drug availability, intent, perceived risks, etc., important gaps between conceptualization and measurement remain. Opioid and stimulant use explored in this study reflect the fact that a drug-related decision occurred prior to the behavior (e.g., heroin use during the one-year period subsequent to incarceration is the behavioral referent of a heroin-related decision that occurred during that one-year period). One limitation already noted is that the frequency of decisions could not be quantified. Another is that many other non-drug choices that would have been of interest to explore were likely made during these time periods, but were not able to be indicated with available data. Again, due to temporal ambiguity, it was not possible to discern the temporal relation between "an influence" and "that which was influenced", but rather only possible to understand that there was co-occurrence during a given time period (30 days, one year, or a lifetime) of potential influences and decisions involving high-risk drugs.

Finally, in seeking improved understanding of choice and behavior in relation to high-risk drugs, the identification of an appropriate stopping point for data collection, statistical modeling, and discussion was not always clear. The premise underlying this exploratory study, that endogenous and exogenous factors of a participants' lives influenced and constrained drug-related decision-making and behavior, paved the road generously toward narrow and precise investigation, as well as and broad and coarse investigation. One potential strength of this study, its broadness in conceptual scope, was also a limitation in terms of how data were selected for examination and in terms of how such broad conception could be statistically modeled. Using institutional survey data, many self-reported factors of participants' lives were able to be explored and, to a limited extent, indirectly discerned, thus illustrating some important concepts. Doing this, though, did not encourage recognition or emphasis of what factors mattered most; nor could it have. The absence of focus on any one factor or set of factors stemmed partially from measurement limitations, but also from the fact that from this study's outset nothing was presumed to be irrelevant. With this study's conclusion, this presumption has not changed.

What has changed is understanding that the inherent tension between theory and measurement will need to be approached differently, and more parsimoniously, in future research, with greater emphasis 
placed on streamlining study aims, questions, and data collection and scaling these differently than the conceptual model itself. The dynamic, self-organizing complexity of human cognition and behavior that is of most interest when seeking to better describe and understand high-risk use within a system of constraint is also what is least measurable by its nature, requiring appropriate use of simple mental models in places where elaborate data collection methods and statistical models are not yet developed. While a small degree of complexity was approachable in this exploratory study conceptually, it was not approachable from a measurement standpoint. This study can therefore be considered, in part, as a conceptual gesture, undertaken in the spirit and recognition of the need to move toward greater integration of information and theory, as well as more rigorous methods and more fine-grained measurement. Study methodology did permit a coarse-grained snapshot of some of the factors that may have influenced decision-making about high-risk drugs among this corrections-involved sample. Study limitations notwithstanding, findings may still have value when considered from a behavioral economic perspective, insofar as they may help facilitate a discussion about the complicated and challenging lives of people who choose to use opioids and stimulants despite associated risks and who continue to use despite adverse consequences.

\section{Conclusion}

This exploratory study provided a descriptive profile of a sample of adults in Kentucky with a history of drug use using self-report data collected prior and subsequent to incarceration and established the prevalence of opioid, stimulant, and concomitant opioid and stimulant use at both time periods. Description of and differences between groups who reported opioid, stimulant, and concomitant use was presented. Endogenous and exogenous factors that demonstrated an association with opioid and stimulant use were explored in order to further understand the relationship between these factors and outcomes involving opioids and stimulants. These factors were described in terms of their potential to influence and constrain cognition and choice related to high-risk drug use.

Overall, opioids and stimulants were used at high rates among participants in this sample and were often used contemporaneously with other drugs. While polydrug use in was a feature of this sample generally, greater drug use versatility was observed primarily among people who reported using opioids, and using them concomitantly with stimulants. This is concerning due to the fact opioids used in combination with other drugs entails significant risk. Concomitant use and heroin use, both associated with 
greater drug use versatility, were positively correlated with many factors with the potential to adversely influence cognition and constrain choice. Similar relationships between many of these factors and outcomes involving other/no drug use were not observed. The primary take-away is that risker use was associated with a greater number of factors with the potential to constrain choice and behavior, whereas other/no drug use was less frequently associated with these factors. Still, these relationships were not unambiguous and should be interpreted cautiously. More work is required to better understand when the presence of certain factors has the capacity to influence choice in respect to opioids and stimulants, including the strength of any observed effect.

Although use of specific opioid and stimulant drugs was found to vary based on participants' residence within or outside of Central Appalachia and by urban proximity, ongoing investigation of these influences is needed as drug markets continually change. For instance, it may be that heroin is now more available in Central Appalachia and rural locations than it was when follow-up data were collected. The increased presence of fentanyl in the drug supply also means that many of the drugs used by participants prior and subsequent to incarceration are possibly risker now than when data were collected.

Despite differences in opioid and stimulant use among participants, there was some sample-wide homogeneity that may be reflective of the overall greater disadvantage found among corrections-involved people in the US. Seeking to understand high-risk drug use among a sample of corrections-involved people is in some ways synonymous with seeking to understand high-risk drug-use among a vulnerable and stigmatized population. Because of this population's marginalized and disempowered status in the US, it is of particular importance to better understand how certain conditions influence desire, intention, choice, and behavior in relation to opioids and stimulants, as the potential consequences of such high-risk use may be disproportionate among this population and less easy to reverse, compared to populations with greater access to flexible resources (Phelan et al., 2010).

Through examination of available survey data and broad review of related literature this exploratory study identified many factors with the potential to influence decision-making about high-risk drugs. It left many other factors unexplored. Given the preponderance of possible influences on drugrelated decision-making, policy-makers and clinicians might better consider how they can aim for two moving targets simultaneously: advance interventions that assist people in critical moments as they decide 
to use drugs or not (interventions could include decreasing craving or decreasing present-bias, pharmacotherapies, etc.), but also better develop and expand access to interventions that may help people acquire capacities and competencies across multiple life domains (e.g., mindfulness-based interventions, cognitive-behavioral interventions, working-memory training, intensive case management, memory reconsolidation, etc.), stabilizing them over time by increasing opportunities for engaging with non-drug alternative reinforcers and by helping them reduce stress while habits of daily living are strengthened (Bickel et al., 2011; Bowen et al., 2006; D’Onofrio et al., 2015; Fjorback, Arendt, Ørnbøl, Fink, \& Walach, 2011; Gorelick, Zangen, \& George, 2014; Himelstein, Saul, \& Garcia-Romeu, 2015; Jarlais et al., 2015; Laudet, 2011; Peiper et al., 2019; Tobin, Davey, \& Latkin, 2005; Volkow, Frieden, Hyde, \& Cha, 2014; Wheeler, Davidson, Jones, \& Irwin, 2019; Witkiewitz, Marlatt, \& Walker, 2005). Longer-term stabilizing forces (i.e., improved cognitive capacities, greater choice) can, in part, develop as a result of aggregated instances of inhibiting urges to use opioids and stimulants to excess. As stability increases, these critical moments of craving would become less frequent, and more cognitively and behaviorally manageable. Successful intervention would need to gain purchase on both developments, at both time scales.

Ultimately, the idea that people choose to use high-risk drugs, but that their capacities for decision-making are constrained by many endogenous and exogenous factors, is not only conceptually coherent, but also optimistic. It is coherent insofar as intention and choice precede action. It is optimistic because many of the conditions of people's lives before, during, and after drug use have the potential to be changed. That the idea of constraint is in some way represented in both the BDMA and the behavioral economic/neuroeconomic model may be an important bit of common ground to exploit moving forward, even if, for now, there remains disagreement about what specifically is being constrained. This point of modest agreement, though, indicates that in seeking to change people's use of high-risk drugs, it is also necessary to change the conditions, the constraints, that give rise to the problem. If all organisms operate within a system of constraint, then there are practical and moral reasons for investing in efforts aimed at making that system of constraint one in which people are more likely flourish and less likely to suffer as a result of the decisions that they make. 


\section{REFERENCES}

Aarts, H., Verplanken, B., \& Van Knippenberg, A. (1998). Predicting behavior from actions in the past: Repeated decision making or a matter of habit?. Journal of Applied Social Psychology, 28(15), $1355-1374$.

Abarca, N., \& Fantino, E. (1982). Choice and foraging. Journal of the Experimental Analysis of Behavior, 38(2), 117-123.

Abbott, D. H., Keverne, E. B., Bercovitch, F. B., Shively, C. A., Mendoza, S. P., Saltzman, W., ... \& Sapolsky, R. M. (2003). Are subordinates always stressed? A comparative analysis of rank differences in cortisol levels among primates. Hormones and Behavior, 43(1), 67-82.

Abdel-Khalek, A. M. (2006). Measuring happiness with a single-item scale. Social Behavior and Personality: An International Journal, 34(2), 139-150.

Abraham, A., \& Bubic, A. (2015). Semantic memory as the root of imagination. Frontiers in Psychology, 6, 325.

Acock, A. C. (2008). A gentle introduction to Stata. College Station, TX: Stata press.

Adler, N. E., Epel, E. S., Castellazzo, G., \& Ickovics, J. R. (2000). Relationship of subjective and objective social status with psychological and physiological functioning: Preliminary data in healthy, White women. Health Psychology, 19(6), 586.

Adolphs, R. (1999). Social cognition and the human brain. Trends In Cognitive Sciences, 3(12), 469-479.

Agan, A., \& Starr, S. (2017). The effect of criminal records on access to employment. American Economic Review, 107(5), 560-64.

Aharonovich, E., Hasin, D. S., Brooks, A. C., Liu, X., Bisaga, A., \& Nunes, E. V. (2006). Cognitive deficits predict low treatment retention in cocaine dependent patients. Drug and Alcohol Dependence, 81(3), 313-322.

Aharonovich, E., Liu, X., Samet, S., Nunes, E., Waxman, R., \& Hasin, D. (2005). Postdischarge cannabis use and its relationship to cocaine, alcohol, and heroin use: A prospective study. American Journal of Psychiatry, 162(8), 1507-1514.

Ahern, J., Stuber, J., \& Galea, S. (2007). Stigma, discrimination and the health of illicit drug users. Drug and Alcohol Dependence, 88(2-3), 188-196.

Ahmed, S. H., Walker, J. R., \& Koob, G. F. (2000). Persistent increase in the motivation to take heroin in rats with a history of drug escalation. Neuropsychopharmacology, 22(4), 413.

Alcoholics Anonymous: A.A. in Treatment Settings. (1994). Retrieved from http://www.aa.org/assets/en_US/treatment-committees/p-27-aa-in-treatment-settings 
Alcoholics Anonymous. (2009). Alcoholics Anonymous. New York: AA World Services.

Alcoholics Anonymous. (2008). Came to believe: The spiritual adventure of A.A. as experienced by individual members. New York, NY: AA World Services, Inc.

Alcoholics Anonymous. (2014). Twelve steps and twelve traditions. New York: Alcoholics Anonymous World Services, Inc.

Al-Tayyib, A. A., Koester, S., \& Riggs, P. (2017). Prescription opioids prior to injection drug use: Comparisons and public health implications. Addictive Behaviors, 65, 224-228.

Ainslie, G. (2001). Breakdown of will. Cambridge, England: Cambridge University Press.

Al-Tayyib, A., Koester, S., Langegger, S., \& Raville, L. (2017). Heroin and methamphetamine injection: An emerging drug use pattern. Substance Use \& Misuse, 52(8), 1051-1058.

Ahmad, K., \& Aziz, Z. (2012). Mitragyna speciosa use in the northern states of Malaysia: A cross-sectional study. Journal of Ethnopharmacology, 141(1), 446-450.

Ahmed, S. H. (2010). Validation crisis in animal models of drug addiction: Beyond non-disordered drug use toward drug addiction. Neuroscience \& Biobehavioral Reviews, 35(2), 172-184.

Ahmed, S. H., Lenoir, M., \& Guillem, K. (2013). Neurobiology of addiction versus drug use driven by lack of choice. Current Opinion in Neurobiology, 23(4), 581-587.

Ahn, W. Y., Vasilev, G., Lee, S. H., Busemeyer, J. R., Kruschke, J. K., Bechara, A., \& Vassileva, J. (2014). Decision-making in stimulant and opiate addicts in protracted abstinence: Evidence from computational modeling with pure users. Frontiers in Psychology, 5, 849.

Ainslie, G. (1992). Picoeconomics: The strategic interaction of successive motivational states within the person. Cambridge, England. Cambridge University Press.

Ainslie, G., \& Haslam, N. (1992). Hyperbolic discounting. In G. Loewenstein \& J. Elster (Eds.), Choice over time (pp. 57-92). New York, NY, US: Russell Sage Foundation.

Ajzen, I. (2002). Perceived behavioral control, self-efficacy, locus of control, and the theory of planned behavior 1. Journal of Applied Social Psychology, 32(4), 665-683.

Alarid, L. F. (2000). Sexual assault and coercion among incarcerated women prisoners: Excerpts from prison letters. The Prison Journal, 80(4), 391-406.

Albertson, D. N., Schmidt, C. J., Kapatos, G., \& Bannon, M. J. (2006). Distinctive profiles of gene expression in the human nucleus accumbens associated with cocaine and heroin abuse. Neuropsychopharmacology, 31(10), 2304.

Alcohol and Tobacco Tax and Trade Bureau. (2018, February 14). TTB | Consumer | Product Safety. Retrieved February 19, 2019, from https://www.ttb.gov/consumer/safety_testing.shtml

Alford, D. P., German, J. S., Samet, J. H., Cheng, D. M., Lloyd-Travaglini, C. A., \& Saitz, R. (2016). Primary care patients with drug use report chronic pain and self-medicate with alcohol and other drugs. Journal of General Internal Medicine, 31(5), 486-491.

Alexander, M., Barbieri, M., \& Kiang, M. (2017, April). Opioid deaths by race in the United States, 20002015. In Population Association of America Meetings, Chicago (pp. 27-29). 
Alexander, C., Piazza, M., Mekos, D., \& Valente, T. (2001). Peers, schools, and adolescent cigarette smoking. Journal of Adolescent Health, 29(1), 22-30.

Allen, C., \& Alberici, A. M. (2018). “Once a junkie, always a junkie”: A narrative analysis 2 of cinematic representations of the attribution of 3 criminality and deviancy to heroin users 4. Criminology, 22, 23.

Allard, S. W. (2017). Places in need: The changing geography of poverty. Russell Sage Foundation.

Allison, K. W., Crawford, I., Leone, P. E., Trickett, E., Perez-Febles, A., Burton, L. M., \& Le Blanc, R. (1999). Adolescent substance use: Preliminary examinations of school and neighborhood context. American Journal of Community Psychology, 27(2), 111-141.

Alpert, A., Powell, D., \& Pacula, R. L. (2018). Supply-side drug policy in the presence of substitutes: Evidence from the introduction of abuse-deterrent opioids. American Economic Journal: Economic Policy, 10(4), 1-35.

Altarac, M., \& Saroha, E. (2007). Lifetime prevalence of learning disability among US children. Pediatrics, 119(Supplement 1), S77-S83.

Althauser, R. P., \& Heberlein, T. A. (1970). Validity and the multitrait-multimethod matrix. Sociological Methodology, 2, 151-169.

Amato, P. R., \& Rogers, S. J. (1997). A longitudinal study of marital problems and subsequent divorce. Journal of Marriage and the Family, 612-624.

American Psychiatric Association. (2013). Diagnostic and statistical manual of mental disorders (DSM5®). Washington, DC. American Psychiatric Pub.

Amlung, M., \& MacKillop, J. (2014). Understanding the effects of stress and alcohol cues on motivation for alcohol via behavioral economics. Alcoholism: Clinical and Experimental Research, 38(6), 1780-1789.

Amlung, M., Vedelago, L., Acker, J., Balodis, I., \& MacKillop, J. (2017). Steep delay discounting and addictive behavior: A meta-analysis of continuous associations. Addiction, 112(1), 51-62.

Andersen, S. L., \& Navalta, C. P. (2004). Altering the course of neurodevelopment: A framework for understanding the enduring effects of psychotropic drugs. International Journal of Developmental Neuroscience, 22(5-6), 423-440.

Andresen, E., Catlin, T., Wyrwich, K., \& Jackson-Thompson, J. (2003). Retest reliability of surveillance questions on health related quality of life. Journal of Epidemiology and Community Health, 57 , 339-343.

Andersson, M., Persson, M., \& Kjellgren, A. (2017). Psychoactive substances as a last resort-A qualitative study of self-treatment of migraine and cluster headaches. Harm Reduction Journal, 14(1), 60.

Anglin, M. D., Burke, C., Perrochet, B., Stamper, E., \& Dawud-Noursi, S. (2000). History of the methamphetamine problem. Journal of Psychoactive Drugs, 32(2), 137-141.

Anokhin, A. P., Golosheykin, S., Grant, J. D., \& Heath, A. C. (2011). Heritability of delay discounting in adolescence: A longitudinal twin study. Behavior Genetics, 41(2), 175-183.

Anthony, J. C., \& Petronis, K. R. (1995). Early-onset drug use and risk of later drug problems. Drug and Alcohol Dependence, 40(1), 9-15. 
Anthony, J. C., Warner, L. A., \& Kessler, R. C. (1994). Comparative epidemiology of dependence on tobacco, alcohol, controlled substances, and inhalants: Basic findings from the National Comorbidity Survey. Experimental and Clinical Psychopharmacology, 2(3), 244.

Apkarian, A. V., Sosa, Y., Krauss, B. R., Thomas, P. S., Fredrickson, B. E., Levy, R. E., ... \& Chialvo, D. R. (2004). Chronic pain patients are impaired on an emotional decision-making task. Pain, 108(12), 129-136.

Appalachian Regional Commission. (2019). Research, maps, and data - Appalachian Regional Commission. Retrieved February 1, 2019, from https://www.arc.gov/research/RegionalDataandResearch.asp

Arciniegas, D. B., Topkoff, J., \& Silver, J. M. (2000). Neuropsychiatric aspects of traumatic brain injury. Current Treatment Options in Neurology, 2(2), 169-186.

Armenian, P., Vo, K. T., Barr-Walker, J., \& Lynch, K. L. (2018). Fentanyl, fentanyl analogs and novel synthetic opioids: A comprehensive review. Neuropharmacology, 134, 121-132.

Arndt, S., Clayton, R., \& Schultz, S. K. (2011). Trends in substance abuse treatment 1998-2008: Increasing older adult first-time admissions for illicit drugs. The American Journal of Geriatric Psychiatry, 19(8), 704-711.

Arnsten, A. F. (2000). Stress impairs prefrontal cortical function in rats and monkeys: role of dopamine D1 and norepinephrine $\alpha-1$ receptor mechanisms. In Progress in Brain Research (Vol. 126, pp. 183192). Elsevier.

Aronowitz, S. V., \& Laurent, J. (2016). Screaming behind a door: The experiences of individuals incarcerated without medication-assisted treatment. Journal of Correctional Health Care, 22(2), 98-108.

Artiles, A. J., \& Trent, S. C. (1994). Overrepresentation of minority students in special education: A continuing debate. The Journal of Special Education, 27(4), 410-437.

Arria, A. M., Geisner, I. M., Cimini, M. D., Kilmer, J. R., Caldeira, K. M., Barrall, A. L., ... \& Lee, C. M. (2018). Perceived academic benefit is associated with nonmedical prescription stimulant use among college students. Addictive Behaviors, 76, 27-33.

Asendorpf, J., Conner, M., De Fruyt, F., De Houwer, J., \& Denissen, J. (2016). Recommendations for increasing replicability in psychology. In Methodological issues and strategies in clinical research (pp. 607-622). American Psychological Association.

Ash, I. K., \& Wiley, J. (2006). The nature of restructuring in insight: An individual-differences approach. Psychonomic Bulletin \& Review, 13(1), 66-73.

Ashrafioun, L. (2016). Prescription opioid craving: Relationship with pain and substance use-related characteristics. Substance Use \& Misuse, 51(11), 1512-1520.

Assanangkornchai, S., Muekthong, A., Sam-Angsri, N., \& Pattanasattayawong, U. (2007). The use of mitragynine speciosa ("Krathom"), an addictive plant, in Thailand. Substance Use \& Misuse, 42(14), 2145-2157.

Associated Press. (1982, May 29). Death rate soars in cocaine cases. New York Times. Retrieved from https://www.nytimes.com/1982/05/29/us/death-rate-soars-in-cocaine-cases.html

Atkinson, A. B. (1970). On the measurement of inequality. Journal of Economic Theory, 2(3), 244-263. 
Audrain-McGovern, J., Rodriguez, D., Epstein, L. H., Cuevas, J., Rodgers, K., \& Wileyto, E. P. (2009). Does delay discounting play an etiological role in smoking or is it a consequence of smoking?. Drug and Alcohol Dependence, 103(3), 99-106.

Austin, A. E., Proescholdbell, S. K., Creppage, K. E., \& Asbun, A. (2017). Characteristics of self-inflicted drug overdose deaths in North Carolina. Drug and Alcohol Dependence, 181, 44-49.

Aviram, H., Bragg, A., \& Lewis, C. (2017). Felon Disenfranchisement. Annual Review of Law and Social Science, 13, 295-311.

A Yokell, M., D Zaller, N., C Green, T., \& D Rich, J. (2011). Buprenorphine and buprenorphine/naloxone diversion, misuse, and illicit use: An international review. Current Drug Abuse Reviews, 4(1), 2841.

Badger, G. J., Bickel, W. K., Giordano, L. A., Jacobs, E. A., Loewenstein, G., \& Marsch, L. (2007). Altered states: The impact of immediate craving on the valuation of current and future opioids. Journal of Health Economics, 26(5), 865-876.

Back, S. E., Hartwell, K., DeSantis, S. M., Saladin, M., McRae-Clark, A. L., Price, K. L., ... \& Brady, K. T. (2010). Reactivity to laboratory stress provocation predicts relapse to cocaine. Drug and Alcohol Dependence, 106(1), 21-27.

Baldacchino, A., Balfour, D. J. K., Passetti, F., Humphris, G., \& Matthews, K. (2012). Neuropsychological consequences of chronic opioid use: a quantitative review and meta-analysis. Neuroscience \& Biobehavioral Reviews, 36(9), 2056-2068.

Baldacchino, A., Balfour, D. J. K., \& Matthews, K. (2015). Impulsivity and opioid drugs: Differential effects of heroin, methadone and prescribed analgesic medication. Psychological Medicine, 45(6), $1167-1179$.

Baldacchino, A., Gilchrist, G., Fleming, R., \& Bannister, J. (2010). Guilty until proven innocent: A qualitative study of the management of chronic non-cancer pain among patients with a history of substance abuse. Addictive Behaviors, 35(3), 270-272.

Badiani, A. (2013). Substance-specific environmental influences on drug use and drug preference in animals and humans. Current Opinion in Neurobiology, 23(4), 588-596.

Badiani, A., Belin, D., Epstein, D., Calu, D., \& Shaham, Y. (2011). Opiate versus psychostimulant addiction: The differences do matter. Nature Reviews Neuroscience, 12(11), 685.

Bachi, K., Sierra, S., Volkow, N. D., Goldstein, R. Z., \& Alia-Klein, N. (2017). Is biological aging accelerated in drug addiction?. Current Opinion in Behavioral Sciences, 13, 34-39.

Baglivio, M. T., Wolff, K. T., Piquero, A. R., \& Epps, N. (2015). The relationship between adverse childhood experiences (ACE) and juvenile offending trajectories in a juvenile offender sample. Journal of Criminal Justice, 43(3), 229-241.

Bagozzi, R. P., \& Yi, Y. (2012). Specification, evaluation, and interpretation of structural equation models. Journal of the Academy of Marketing Science, 40(1), 8-34.

Bahr, S. J., Armstrong, A. H., Gibbs, B. G., Harris, P. E., \& Fisher, J. K. (2005). The reentry process: How parolees adjust to release from prison. Fathering: A Journal of Theory, Research \& Practice about Men as Fathers, 3(3). 
Bak, P. (2013). How nature works: The science of self-organized criticality. Springer Science \& Business Media. New York, NY.

Baldo, B. A. (2016). Prefrontal cortical opioids and dysregulated motivation: A network hypothesis. Trends in Neurosciences, 39(6), 366-377.

Baldwin, M. L., Marcus, S. C., \& De Simone, J. (2010). Job loss discrimination and former substance use disorders. Drug and Alcohol Dependence, 110(1-2), 1-7.

Ballantyne, J. C., \& LaForge, S. K. (2007). Opioid dependence and addiction during opioid treatment of chronic pain. Pain, 129(3), 235-255.

Bali, A., Randhawa, P. K., \& Jaggi, A. S. (2015). Stress and opioids: Role of opioids in modulating stressrelated behavior and effect of stress on morphine conditioned place preference. Neuroscience \& Biobehavioral Reviews, 51, 138-150.

Bandura, A. (1982). Self-efficacy mechanism in human agency. American Psychologist, 37(2), 122.

Bandura, A. (1989). Regulation of cognitive processes through perceived self-efficacy. Developmental Psychology, 25(5), 729.

Bandura, A. (1991). Social cognitive theory of self-regulation. Organizational Behavior and Human Decision Processes, 50(2), 248-287.

Bandura, A. (1999). A sociocognitive analysis of substance abuse: An agentic perspective. Psychological Science, 10(3), 214-217.

Banks, D. E., Rowe, A. T., Mpofu, P., \& Zapolski, T. C. (2017). Trends in typologies of concurrent alcohol, marijuana, and cigarette use among US adolescents: An ecological examination by sex and race/ethnicity. Drug and Alcohol Dependence, 179, 71-77.

Bardo, M. T., Neisewander, J. L., \& Kelly, T. H. (2013). Individual differences and social influences on the neurobehavioral pharmacology of abused drugs. Pharmacological Reviews, 65(1), 255-290.

Bardo, M. T., Donohew, R. L., \& Harrington, N. G. (1996). Psychobiology of novelty seeking and drug seeking behavior. Behavioural Brain Research, 77(1-2), 23-43.

Barkley, R. A., Edwards, G., Laneri, M., Fletcher, K., \& Metevia, L. (2001). Executive functioning, temporal discounting, and sense of time in adolescents with attention deficit hyperactivity disorder (ADHD) and oppositional defiant disorder (ODD). Journal of Abnormal Child Psychology, 29(6), 541-556.

Barkley, R. A., Edwards, G., Laneri, M., Fletcher, K., \& Metevia, L. (2001). The efficacy of problemsolving communication training alone, behavior management training alone, and their combination for parent-adolescent conflict in teenagers with ADHD and ODD. Journal of Consulting and Clinical Psychology, 69(6), 926.

Barnard, M., \& McKeganey, N. (2004). The impact of parental problem drug use on children: What is the problem and what can be done to help?. Addiction, 99(5), 552-559.

Barrett, S. P., Darredeau, C., \& Pihl, R. O. (2006). Patterns of simultaneous polysubstance use in drug using university students. Human Psychopharmacology: Clinical and Experimental, 21(4), 255263. 
Barry, C. L., McGinty, E. E., Pescosolido, B. A., \& Goldman, H. H. (2014). Stigma, discrimination, treatment effectiveness, and policy: Public views about drug addiction and mental illness. Psychiatric Services, 65(10), 1269-1272.

Bassuk, E. L., Hanson, J., Greene, R. N., Richard, M., \& Laudet, A. (2016). Peer-delivered recovery support services for addictions in the United States: A systematic review. Journal of Substance Abuse Treatment, 63, 1-9.

Baum, W. M. (2004). Molar and molecular views of choice. Behavioural Processes, 66(3), 349-359.

Baum, W. M. (2004). Choice in free-ranging wild pigeons. Science, 185(4145), 78-79.

Baumeister, R. F. (2002a). Yielding to temptation: Self-control failure, impulsive purchasing, and consumer behavior. Journal of Consumer Research, 28(4), 670-676.

Baumeister, R. F. (2002b). Ego depletion and self-control failure: An energy model of the self's executive function. Self and Identity, 1(2), 129-136.

Baumeister, R. F. (2014). Self-regulation, ego depletion, and inhibition. Neuropsychologia, 65, 313-319.

Baumeister, R. F., Heatherton, T. F., \& Tice, D. M. (1994). Losing control: How and why people fail at selfregulation. Academic press. San Diego, CA.

Baumeister, R. F., \& Heatherton, T. F. (1996). Self-regulation failure: An overview. Psychological Inquiry, 7(1), 1-15.

Baumeister, R. F., \& Leary, M. R. (1995). The need to belong: Desire for interpersonal attachments as a fundamental human motivation. Psychological Bulletin, 117(3), 497.

Baumeister, R. F., \& Vohs, K. D. (2007). Self-Regulation, ego depletion, and motivation. Social and Personality Psychology Compass, 1(1), 115-128.

Baumeister, R. F., \& Vonasch, A. J. (2015). Uses of self-regulation to facilitate and restrain addictive behavior. Addictive Behaviors, 44, 3-8.

Baumeister, R. F., Zell, A. L., \& Tice, D. M. (2007). How emotions facilitate and impair selfregulation. Handbook of Emotion Regulation, 408-426.

Bazazi, A. R., Yokell, M., Fu, J. J., Rich, J. D., \& Zaller, N. D. (2011). Illicit use of buprenorphine/naloxone among injecting and noninjecting opioid users. Journal of Addiction Medicine, 5(3), 175.

Bechara, A. (2003). Risky business: Emotion, decision-making, and addiction. Journal of Gambling Studies, 19(1), 23-51.

Bechara, A. (2005). Decision making, impulse control and loss of willpower to resist drugs: A neurocognitive perspective. Nature Neuroscience, 8(11), 1458.

Bechara, A., Dolan, S., Denburg, N., Hindes, A., Anderson, S. W., \& Nathan, P. E. (2001). Decision-making deficits, linked to a dysfunctional ventromedial prefrontal cortex, revealed in alcohol and stimulant abusers. Neuropsychologia, 39(4), 376-389.

Bechara, A., Dolan, S., \& Hindes, A. (2002). Decision-making and addiction (part II): Myopia for the future or hypersensitivity to reward?. Neuropsychologia, 40(10), 1690-1705. 
Bechara, A., \& Damasio, H. (2002). Decision-making and addiction (part I): Impaired activation of somatic states in substance dependent individuals when pondering decisions with negative future consequences. Neuropsychologia, 40(10), 1675-1689.

Bechara, A., \& Damasio, A. R. (2005). The somatic marker hypothesis: A neural theory of economic decision. Games and Economic Behavior, 52(2), 336-372.

Bechara, A., Damasio, H., Tranel, D., \& Damasio, A. R. (2005). The Iowa Gambling Task and the somatic marker hypothesis: Some questions and answers. Trends in Cognitive Sciences, 9(4), 159-162.

Bechara, A., \& Martin, E. M. (2004). Impaired decision making related to working memory deficits in individuals with substance addictions. Neuropsychology, 18(1), 152.

Bechara, A., Noel, X., \& Crone, E. A. (2006). Loss of willpower: Abnormal neural mechanisms of impulse control and decision making in addiction. Handbook of implicit cognition and addiction, 1, 215232.

Bechara, A., \& Van Der Linden, M. (2005). Decision-making and impulse control after frontal lobe injuries. Current Opinion in Neurology, 18(6), 734-739.

Beck, A. T., \& Haigh, E. A. (2014). Advances in cognitive theory and therapy: The generic cognitive model. Annual Review of Clinical Psychology, 10, 1-24.

Becker, G. S. (2013). The economic approach to human behavior. Chicago, IL: University of Chicago press.

Becker, G. S., Murphy, K. M., \& Grossman, M. (2004). The economic theory of illegal goods: The case of drugs (No. w10976). National Bureau of Economic Research.

Becker, G. S., \& Murphy, K. M. (1988). A theory of rational addiction. Journal of Political Economy, 96(4), 675-700.

Becker, H. S. (2017). Knowledge, Power, and Drug Effects. In Drugs and Politics (pp. 167-190). Abingdonon-Thames, UK. Routledge.

Becton, A. B., Chen, R. K., \& Paul, T. (2017). A second chance: Employers' perspectives in hiring individuals in addiction recovery. Journal of Applied Rehabilitation Counseling, 48(1), 6-15.

Behrens, A. (2004). Less than the average citizen': Stigma, role transition and the civic reintegration of convicted felons. After crime and punishment: Pathways to offender reintegration, 261. Cullompton, Devon, UK: Willian Publishing.

Belanger, H. G., Vanderploeg, R. D., Curtiss, G., \& Warden, D. L. (2007). Recent neuroimaging techniques in mild traumatic brain injury. The Journal of Neuropsychiatry and Clinical Neurosciences, 19(1), 5-20.

Beletsky, L., LaSalle, L., Newman, M., Paré, J., Tam, J., \& Tochka, A. (2015). Fatal re-entry: Legal and programmatic opportunities to curb opioid overdose among individuals newly released from incarceration. NEULJ, 7, 149.

Belin, D., Mar, A. C., Dalley, J. W., Robbins, T. W., \& Everitt, B. J. (2008). High impulsivity predicts the switch to compulsive cocaine-taking. Science, 320(5881), 1352-1355.

Beman, D. S. (1995). Risk factors leading to adolescent substance abuse. Adolescence, 30(117), 201.

Bender, W. N., Rosenkrans, C. B., \& Crane, M. K. (1999). Stress, depression, and suicide among students with learning disabilities: Assessing the risk. Learning Disability Quarterly, 22(2), 143-156. 
Benéitez, M. C., \& Gil-Alegre, M. E. (2017). Opioid addiction: Social problems associated and implications of both current and possible future treatments, including polymeric therapeutics for giving up the habit of opioid consumption. BioMed Research International, 2017.

Benjamin, D. J., Choi, J. J., \& Strickland, A. J. (2010). Social identity and preferences. American Economic Review, 100(4), 1913-28.

Bentham, J. (1996). The collected works of Jeremy Bentham: An introduction to the principles of morals and legislation. Oxford, England, UK. Clarendon Press.

Benthin, A., Slovic, P., \& Severson, H. (1993). A psychometric study of adolescent risk perception. Journal of Adolescence, 16(2), 153-168.

Bergen, S. E., Gardner, C. O., \& Kendler, K. S. (2007). Age-related changes in heritability of behavioral phenotypes over adolescence and young adulthood: A meta-analysis. Twin Research and Human Genetics, 10(3), 423-433.

Bergkvist, L., \& Rossiter, J. R. (2007). The predictive validity of multiple-item versus single-item measures of the same constructs. Journal of Marketing Research, 44(2), 175-184.

Bergkvist, L. (2015). Appropriate use of single-item measures is here to stay. Marketing Letters, 26(3), 245255.

Bernheim, B. D., Ray, D., \& Yeltekin, Ş. (2015). Poverty and self-control. Econometrica, 83(5), 1877-1911.

Berns, G. S., Laibson, D., \& Loewenstein, G. (2007). Intertemporal choice-toward an integrative framework. Trends in Cognitive Sciences, 11(11), 482-488.

Bernstein, B. E., Meissner, A., \& Lander, E. S. (2007). The mammalian epigenome. Cell, 128(4), 669-681.

Bernstein, I. H., Rush, A. J., Yonkers, K., Carmody, T. J., Woo, A., McConnell, K., \& Trivedi, M. H. (2008). Symptom features of postpartum depression: Are they distinct?. Depression and Anxiety, 25(1), 20-26.

Berridge, K. C. (2007). The debate over dopamine's role in reward: The case for incentive salience. Psychopharmacology, 191(3), 391-431.

Berridge, K. C. (2017). Is addiction a brain disease?. Neuroethics, 10(1), 29-33.

Berridge, K. C., \& O’Doherty, J. P. (2014). From experienced utility to decision utility. In Neuroeconomics (pp. 335-351). Academic Press.

Berridge, K. C., Robinson, T. E., \& Aldridge, J. W. (2009). Dissecting components of reward: 'liking', 'wanting', and learning. Current Opinion in Pharmacology, 9(1), 65-73.

Bertrand, M., Mullainathan, S., \& Shafir, E. (2004). A behavioral-economics view of poverty. American Economic Review, 94(2), 419-423.

Beverly, S. G. (1999). Economic Poverty Reconsidered: The Case for" Direct" Measures.

Beverly, S. G. (2001). Material hardship in the United States: Evidence from the survey of income and program participation. Social Work Research, 25(3), 143-151.

Biernacki, P. (1979). Junkie work, "hustles" and social status among heroin addicts. Journal of Drug Issues, 9(4), 535-551. 
Biernacki, K., Terrett, G., McLennan, S. N., Labuschagne, I., Morton, P., \& Rendell, P. G. (2018). Decisionmaking, somatic markers and emotion processing in opiate users. Psychopharmacology, 235(1), 223-232.

Bridgeland, J. M., DiIulio Jr, J. J., \& Morison, K. B. (2006). The silent epidemic: Perspectives of high school dropouts. Civic Enterprises.

Buck, P. D. (2001). Worked to the bone: Race, class, power, and privilege in Kentucky. Monthly Review Press.

Bubier, J. L., \& Drabick, D. A. (2008). Affective decision-making and externalizing behaviors: The role of autonomic activity. Journal of Abnormal Child Psychology, 36(6), 941.

Buer, L. M., Leukefeld, C. G., \& Havens, J. R. (2016). “I'm stuck”: Women's navigations of social networks and prescription drug misuse in central Appalachia. North American Dialogue, 19(2), 70-84.

Buhle, J. T., Silvers, J. A., Wager, T. D., Lopez, R., Onyemekwu, C., Kober, H., ... \& Ochsner, K. N. (2014). Cognitive reappraisal of emotion: A meta-analysis of human neuroimaging studies. Cerebral Cortex, 24(11), 2981-2990.

Bullmore, E., \& Sporns, O. (2009). Complex brain networks: Graph theoretical analysis of structural and functional systems. Nature Reviews Neuroscience, 10(3), 186.

Burling, T. A., Reilly, P. M., Moltzen, J. O., \& Ziff, D. C. (1989). Self-efficacy and relapse among inpatient drug and alcohol abusers: A predictor of outcome. Journal of Studies on Alcohol, 50(4), 354-360.

Bush, G., Luu, P., \& Posner, M. I. (2000). Cognitive and emotional influences in anterior cingulate cortex. Trends in Cognitive Sciences, 4(6), 215-222.

Biernacki, P. (1979). Junkie work, "hustles" and social status among heroin addicts. Journal of Drug Issues, 9(4), 535-551.

Bickel, W. K., Koffarnus, M. N., Moody, L., \& Wilson, A. G. (2014). The behavioral-and neuro-economic process of temporal discounting: A candidate behavioral marker of addiction. Neuropharmacology, 76, 518-527.

Bickel, W. K., Crabbe, J. C., \& Sher, K. J. (2019). What is addiction? How can animal and human research be used to advance research, diagnosis, and treatment of alcohol and other substance use disorders?. Alcoholism: Clinical and Experimental Research, 43(1), 6-21.

Bickel, W. K., DeGrandpre, R. J., \& Higgins, S. T. (1995). The behavioral economics of concurrent drug reinforcers: a review and reanalysis of drug self-administration research. Psychopharmacology, 118(3), 250-259.

Bickel, W. K., Giordano, L. A., \& Badger, G. J. (2004). Risk-sensitive foraging theory elucidates risky choices made by heroin addicts. Addiction, 99(7), 855-861.

Bickel, W. K., Green, L., \& Vuchinich, R. E. (1995). Behavioral economics. Journal of the Experimental Analysis of Behavior, 64(3), 257.

Bickel, W. K., Hughes, J. R., De Grandpre, R. J., Higgins, S. T., \& Rizzuto, P. (1992). Behavioral economics of drug self-administration. Psychopharmacology, 107(2-3), 211-216. 
Bickel, W. K., Johnson, M. W., Koffarnus, M. N., MacKillop, J., \& Murphy, J. G. (2014). The behavioral economics of substance use disorders: Reinforcement pathologies and their repair. Annual Review of Clinical Psychology, 10, 641-677.

Bickel, W. K., Madden, G. J., \& Petry, N. M. (1998). The price of change: The behavioral economics of drug dependence. Behavior Therapy, 29(4), 545-565.

Bickel, W. K., \& Marsch, L. A. (2001). Toward a behavioral economic understanding of drug dependence: Delay discounting processes. Addiction, 96(1), 73-86.

Bickel, W. K., Marsch, L. A., \& Carroll, M. E. (2000). Deconstructing relative reinforcing efficacy and situating the measures of pharmacological reinforcement with behavioral economics: A theoretical proposal. Psychopharmacology, 153(1), 44-56.

Bickel, W. K., Mellis, A. M., Snider, S. E., Moody, L., Stein, J. S., \& Quisenberry, A. J. (2016). Novel therapeutics for addiction: Behavioral economic and neuroeconomic approaches. Current Treatment Options in Psychiatry, 3(3), 277-292.

Bickel, W. K., Miller, M. L., Yi, R., Kowal, B. P., Lindquist, D. M., \& Pitcock, J. A. (2007). Behavioral and neuroeconomics of drug addiction: Competing neural systems and temporal discounting processes. Drug and Alcohol Dependence, 90, S85-S91.

Bickel, W. K., Mueller, E. T., MacKillop, J., \& Yi, R. (2016). 12 Behavioral-economic and neuroeconomic perspectives on addiction. The Oxford Handbook of Substance Use and Substance Use Disorders, 1, 422.

Bickel, W. K., Jarmolowicz, D. P., Mueller, E. T., \& Gatchalian, K. M. (2011). The behavioral economics and neuroeconomics of reinforcer pathologies: Implications for etiology and treatment of addiction. Current Psychiatry Reports, 13(5), 406.

Bickel, W. K., Miller, M. L., Yi, R., Kowal, B. P., Lindquist, D. M., \& Pitcock, J. A. (2007). Behavioral and neuroeconomics of drug addiction: Competing neural systems and temporal discounting processes. Drug and Alcohol Dependence, 90, S85-S91.

Bickel, W. K., Moody, L., \& Higgins, S. T. (2016). Some current dimensions of the behavioral economics of health-related behavior change. Preventive Medicine, 92, 16-23.

Bickel, W. K., \& Vuchinich, R. E. (2000). Reframing health behavior change with behavioral economics. Psychology Press. Mahwah, NJ.

Bickel, W. K., \& Yi, R. (2008). Temporal discounting as a measure of executive function: Insights from the competing neuro-behavioral decision system hypothesis of addiction. In Neuroeconomics (pp. 289-309). Bingley, UK: Emerald Group Publishing Limited.

Bickel, W. K., Yi, R., Landes, R. D., Hill, P. F., \& Baxter, C. (2011). Remember the future: Working memory training decreases delay discounting among stimulant addicts. Biological Psychiatry, 69(3), 260-265.

Bickel, W. K., Yi, R., Mueller, E. T., Jones, B. A., \& Christensen, D. R. (2010). The behavioral economics of drug dependence: Towards the consilience of economics and behavioral neuroscience. In Behavioral neuroscience of Drug Addiction (pp. 319-341). New York, NY: Springer, Berlin, Heidelberg.

Biederman, J., Monuteaux, M. C., Doyle, A. E., Seidman, L. J., Wilens, T. E., Ferrero, F., ... \& Faraone, S. V. (2004). Impact of executive function deficits and attention-deficit/hyperactivity disorder 
(ADHD) on academic outcomes in children. Journal of Consulting and Clinical Psychology, 72(5), 757.

Biernacki, K., Terrett, G., McLennan, S. N., Labuschagne, I., Morton, P., \& Rendell, P. G. (2018). Decisionmaking, somatic markers and emotion processing in opiate users. Psychopharmacology, 235(1), 223-232.

Billings, D. B., \& Blee, K. M. (2000). The road to poverty: The making of wealth and hardship in Appalachia. Cambridge, England: Cambridge University Press.

Binswanger, I. A., Kral, A. H., Bluthenthal, R. N., Rybold, D. J., \& Edlin, B. R. (2000). High prevalence of abscesses and cellulitis among community-recruited injection drug users in San Francisco. Clinical Infectious Diseases, 30(3), 579-581.

Binswanger, I. A., Krueger, P. M., \& Steiner, J. F. (2009). Prevalence of chronic medical conditions among jail and prison inmates in the United States compared with the general population. Journal of Epidemiology \& Community Health, jech-2009.

Binswanger, I. A., Stern, M. F., Deyo, R. A., Heagerty, P. J., Cheadle, A., Elmore, J. G., \& Koepsell, T. D. (2007). Release from prison - a high risk of death for former inmates. New England Journal of Medicine, 356(2), 157-165.

Binswanger, I. A., Nowels, C., Corsi, K. F., Glanz, J., Long, J., Booth, R. E., \& Steiner, J. F. (2012). Return to drug use and overdose after release from prison: A qualitative study of risk and protective factors. Addiction Science \& Clinical Practice, 7(1), 3.

Birtel, M. D., Wood, L., \& Kempa, N. J. (2017). Stigma and social support in substance abuse: Implications for mental health and well-being. Psychiatry Research, 252, 1-8.

Black, J. L., \& Macinko, J. (2008). Neighborhoods and obesity. Nutrition Reviews, 66(1), 2-20.

Blackford, L. (2018, July 25). Kentucky drug overdose deaths hit record high in 2017. More than half used this drug. Lexington Herald Leader. Retrieved from https://www.kentucky.com/news/state/article215507955.html

Blackman, S., \& Bradley, R. (2017). From niche to stigma-Headshops to prison: Exploring the rise and fall of synthetic cannabinoid use among young adults. International Journal of Drug Policy, 40, 7077.

Blaine, S. K., \& Sinha, R. (2017). Alcohol, stress, and glucocorticoids: From risk to dependence and relapse in alcohol use disorders. Neuropharmacology, 122, 136-147.

Blair, K. S., Morton, J., Leonard, A., \& Blair, R. J. R. (2006). Impaired decision-making on the basis of both reward and punishment information in individuals with psychopathy. Personality and Individual Differences, 4l(1), 155-165.

Blakely, T. A., Lochner, K., \& Kawachi, I. (2002). Metropolitan area income inequality and self-rated health-A multi-level study. Social Science \& Medicine, 54(1), 65-77.

Blakely, T. A., \& Woodward, A. J. (2000). Ecological effects in multi-level studies. Journal of Epidemiology \& Community Health, 54(5), 367-374.

Blanchard, T. C., Hayden, B. Y., \& Bromberg-Martin, E. S. (2015). Orbitofrontal cortex uses distinct codes for different choice attributes in decisions motivated by curiosity. Neuron, 85(3), 602-614. 
Blanton, D. J., \& Dagenais, P. A. (2007). Comparison of language skills of adjudicated and nonadjudicated adolescent males and females. Language, Speech, and Hearing Services in Schools.

Blazer, D. G., \& Wu, L. T. (2009). The epidemiology of substance use and disorders among middle aged and elderly community adults: National survey on drug use and health. The American Journal of Geriatric Psychiatry, 17(3), 237-245.

Blum, J., Gerber, H., Gerhard, U., Schmid, O., Petitjean, S., Riecher-Rössler, A., ... \& Walter, M. (2013). Acute effects of heroin on emotions in heroin-dependent patients. The American Journal on Addictions, 22(6), 598-604.

Blumstein, A. (1995). Youth violence, guns, and the illicit-drug industry. Journal of Criminal Law and Criminology. 86,10.

Boardman, J. D., Finch, B. K., Ellison, C. G., Williams, D. R., \& Jackson, J. S. (2001). Neighborhood disadvantage, stress, and drug use among adults. Journal of Health and Social Behavior, 151-165.

Bøg, M., Filges, T., Brännström, L., Jørgensen, A. M. K., \& Fredriksson, M. K. (2017). 12-step programs for reducing illicit drug use. Campbell Systematic Reviews, 13.

Bogod, N. M., Mateer, C. A., \& Macdonald, S. W. (2003). Self-awareness after traumatic brain injury: A comparison of measures and their relationship to executive functions. Journal of the International Neuropsychological Society, 9(3), 450-458

Bohnert, A. S., Walton, M. A., Cunningham, R. M., Ilgen, M. A., Barry, K., Chermack, S. T., \& Blow, F. C. (2018). Overdose and adverse drug event experiences among adult patients in the emergency department. Addictive Behaviors, 86, 66-72.

Bolanos, F., Herbeck, D., Christou, D., Lovinger, K., Pham, A., Raihan, A., ... \& Brecht, M. L. (2012). Using Facebook to maximize follow-up response rates in a longitudinal study of adults who use methamphetamine. Substance Abuse: Research and Treatment, 6, SART-S8485.

Bonner, R. L., \& Rich, A. R. (1990). Psychosocial vulnerability, life stress, and suicide ideation in a jail population: A cross-validation study. Suicide and Life-Threatening Behavior, 20(3), 213-224.

Booth, B. M., Leukefeld, C., Falck, R., Wang, J., \& Carlson, R. (2006). Correlates of rural methamphetamine and cocaine users: Results from a multistate community study. Journal of Studies on Alcohol, 67(4), 493-501.

Borders, T. F., \& Wen, H. (2018). Illicit drug and opioid use disorders among non-metropolitan residents Lexington, KY: Rural and Underserved Health Research Center; 2018.

Borders, T. F., Booth, B. M., Han, X., Wright, P., Leukefeld, C., Falck, R. S., \& Carlson, R. G. (2008). Longitudinal changes in methamphetamine and cocaine use in untreated rural stimulant users: Racial differences and the impact of methamphetamine legislation. Addiction, 103(5), 800-808.

Borders, T. F., Booth, B. M., Stewart, K. E., Cheney, A. M., \& Curran, G. M. (2015). Rural/urban residence, access, and perceived need for treatment among African American cocaine users. The Journal of Rural Health, 31(1), 98-107.

Borg, C., Emond, F. C., Colson, D., Laurent, B., \& Michael, G. A. (2015). Attentional focus on subjective interoceptive experience in patients with fibromyalgia. Brain and Cognition, 101, 35-43.

Bornovalova, M. A., Daughters, S. B., Hernandez, G. D., Richards, J. B., \& Lejuez, C. W. (2005).

Differences in impulsivity and risk-taking propensity between primary users of crack cocaine and 
primary users of heroin in a residential substance-use program. Experimental and Clinical Psychopharmacology, 13(4), 311.

Boulding, K. E. (1956). General systems theory—the skeleton of science. Management Science, 2(3), 197208.

Boutwell, B. B., Nelson, E. J., Emo, B., Vaughn, M. G., Schootman, M., Rosenfeld, R., \& Lewis, R. (2016). The intersection of aggregate-level lead exposure and crime. Environmental Research, 148, 79-85.

Bowen, M. T., \& Neumann, I. D. (2017). Rebalancing the addicted brain: Oxytocin interference with the neural substrates of addiction. Trends in Neurosciences.

Bowen, S. K., \& Rude, H. A. (2006). Assessment and students with disabilities: Issues and challenges with educational reform. Rural Special Education Quarterly, 25(3), 24-30.

Bowen, S., Witkiewitz, K., Dillworth, T. M., Chawla, N., Simpson, T. L., Ostafin, B. D., ... \& Marlatt, G. A. (2006). Mindfulness meditation and substance use in an incarcerated population. Psychology of Addictive Behaviors, 20(3), 343.

Bowser, B., Fullilove, R., \& Word, C. (2017). Is the new heroin epidemic really new? Racializing heroin. Journal of the National Medical Association, 109(1), 28-32.

Brådvik, L., Frank, A., Hulenvik, P., Medvedeo, A., \& Berglund, M. (2007). Heroin addicts reporting previous heroin overdoses also report suicide attempts. Suicide and Life-Threatening Behavior, 37(4), 475-481.

Brady, K. T., McCauley, J. L., \& Back, S. E. (2015). Prescription opioid misuse, abuse, and treatment in the United States: An update. American Journal of Psychiatry, 173(1), 18-26.

Brand, M., Roth-Bauer, M., Driessen, M., \& Markowitsch, H. J. (2008). Executive functions and risky decision-making in patients with opiate dependence. Drug and Alcohol Dependence, 97(1-2), 6472 .

Bratanova, B., Loughnan, S., Klein, O., Claassen, A., \& Wood, R. (2016). Poverty, inequality, and increased consumption of high calorie food: Experimental evidence for a causal link. Appetite, 100, 162171.

Bratman, M. E. (1999). Faces of intention: Selected essays on intention and agency. Cambridge, Rngland: Cambridge University Press.

Bratman, M. (1987). Intention, plans, and practical reason (Vol. 10). Cambridge, MA: Harvard University Press.

Bratman, M. E. (1990). What is intention. Intentions in communication, 15-31. Cambridge, MA: MIT Press

Brecht, M. L., Huang, D., Evans, E., \& Hser, Y. I. (2008). Polydrug use and implications for longitudinal research: Ten-year trajectories for heroin, cocaine, and methamphetamine users. Drug and Alcohol Dependence, 96(3), 193-201.

Brecht, M. L., O'Brien, A., Von Mayrhauser, C., \& Anglin, M. D. (2004). Methamphetamine use behaviors and gender differences. Addictive Behaviors, 29(1), 89-106.

Breese, G. R., Chu, K., Dayas, C. V., Funk, D., Knapp, D. J., Koob, G. F., ... \& Sinha, R. (2005). Stress enhancement of craving during sobriety: A risk for relapse. Alcoholism: Clinical and Experimental Research, 29(2), 185-195. 
Bretteville-Jensen, A. L., \& Sutton, M. (1996). Under the influence of the market: An applied study of illicitly selling and consuming heroin. York, England: University of York, Centre for Health Economics.

Bretteville-Jensen, A. L., \& Biørn, E. (2003). Heroin consumption, prices and addiction: Evidence from selfreported panel data. The Scandinavian Journal of Economics, 661-679.

Brinkley-Rubinstein, L. (2013). Incarceration as a catalyst for worsening health. Health \& Justice, 1(1), 3.

Brinkley-Rubinstein, L., Zaller, N., Martino, S., Cloud, D. H., McCauley, E., Heise, A., \& Seal, D. (2018). Criminal justice continuum for opioid users at risk of overdose. Addictive Behaviors.

Brocas, I., \& Carrillo, J. D. (2014). Dual-process theories of decision-making: A selective survey. Journal of Economic Psychology, 41, 45-54.

Bronson, J., Maruschak, L. M., \& Berzofsky, M. (2015). Disabilities among prison and jail inmates, 201112. US Department of Justice Bureau of Justice Statistics.

Brook, J., \& McDonald, T. (2009). The impact of parental substance abuse on the stability of family reunifications from foster care. Children and Youth Services Review, 31(2), 193-198.

Brook, J., McDonald, T. P., Gregoire, T., Press, A., \& Hindman, B. (2010). Parental substance abuse and family reunification. Journal of Social Work Practice in the Addictions, 10(4), 393-412.

Brooks, S. J., Lochner, C., Shoptaw, S., \& Stein, D. J. (2017). Using the research domain criteria (RDoC) to conceptualize impulsivity and compulsivity in relation to addiction. In Progress in Brain Research (Vol. 235, pp. 177-218). Elsevier.

Brower, J. E., \& Guilfoy, K. (Eds.). (2004). The Cambridge Companion to Abelard. Cambridge, England: Cambridge University Press

Brown, S. A. (2015). Stigma towards marijuana users and heroin users. Journal of Psychoactive Drugs, 47(3), 213-220.

Brown, D. L., \& Hirschl, T. A. (1995). Household poverty in rural and metropolitan-core areas of the United States 1. Rural sociology, 60(1), 44-66.

Brown, J. S., Laundré, J. W., \& Gurung, M. (1999). The ecology of fear: Optimal foraging, game theory, and trophic interactions. Journal of Mammalogy, 80(2), 385-399.

Brown, R., \& Madsen, H. (2018). Reframing addiction: Habit, choice, cues and compulsivity. Progress in Neuro-psychopharmacology \& Biological Psychiatry, 87(PA), 1-2.

Brownstein, H. H., Mulcahy, T. M., Fernandes-Huessy, J., Taylor, B. G., \& Woods, D. (2012). The organization and operation of illicit retail methamphetamine markets. Criminal Justice Policy Review, 23(1), 67-89.

Brownstein, H. H. (1996). The rise and fall of a violent crime wave: Crack cocaine and the social construction of a crime problem. Guilderland, NY: Harrow and Heston.

Broz, D., Zibbell, J., Foote, C., Roseberry, J. C., Patel, M. R., Conrad, C., ... \& Duwve, J. M. (2018). Multiple injections per injection episode: High-risk injection practice among people who injected pills during the 2015 HIV outbreak in Indiana. International Journal of Drug Policy, 52, 97-101. 
Bruneau, J., Roy, É., Arruda, N., Zang, G., \& Jutras-Aswad, D. (2012). The rising prevalence of prescription opioid injection and its association with hepatitis $\mathrm{C}$ incidence among street-drug users. Addiction, 107(7), 1318-1327.

Brunswick, E. (1955). Representative design and probabilistic theory in functional psychology. Psychological Review, 62, 193-217.

Bryan, K., Freer, J., \& Furlong, C. (2007). Language and communication difficulties in juvenile offenders. International Journal of Language \& Communication Disorders, 42(5), 505-520.

Bschor, T., Ising, M., Bauer, M., Lewitzka, U., Skerstupeit, M., Müller-Oerlinghausen, B., \& Baethge, C. (2004). Time experience and time judgment in major depression, mania and healthy subjects. A controlled study of 93 subjects. Acta Psychiatrica Scandinavica, 109(3), 222-229.

Buchanan, D. R., \& Wallack, L. (1998). This is the partnership for a drug-free America: Any questions? Journal of Drug Issues, 28(2), 329-356.

Buckaloo, B. J., Krug, K. S., \& Nelson, K. B. (2009). Exercise and the low-security inmate: Changes in depression, stress, and anxiety. The Prison Journal, 89(3), 328-343.

Buckner, R. L., \& Carroll, D. C. (2007). Self-projection and the brain. Trends in Cognitive Sciences, 11(2), 49-57.

Buckner, J. D., Crosby, R. D., Silgado, J., Wonderlich, S. A., \& Schmidt, N. B. (2012). Immediate antecedents of marijuana use: An analysis from ecological momentary assessment. Journal of Behavior Therapy and Experimental Psychiatry, 43(1), 647-655.

Bulley, A., Henry, J., \& Suddendorf, T. (2016). Prospection and the present moment: The role of episodic foresight in intertemporal choices between immediate and delayed rewards. Review of General Psychology, 20(1), 29.

Burdon, W. M., Roll, J. M., Prendergast, M. L., \& Rawson, R. A. (2001). Drug courts and contingency management. Journal of Drug Issues, 31(1), 73-90.

Bureau of Justice Statistics. (2018). Probation and parole in the United States, 2016. Retrieved from U.S. Department of Justice website: https://www.bjs.gov/content/pub/pdf/ppus16.pdf

Butler, S. F., Black, R. A., Severtson, S. G., Dart, R. C., \& Green, J. L. (2018). Understanding abuse of buprenorphine/naloxone film versus tablet products using data from ASI-MV® substance use disorder treatment centers and RADARS® System Poison Centers. Journal of Substance Abuse Treatment, 84, 42-49.

Butler, A. C., Chapman, J. E., Forman, E. M., \& Beck, A. T. (2006). The empirical status of cognitivebehavioral therapy: A review of meta-analyses. Clinical Psychology Review, 26(1), 17-31.

Butler, G. K. L., \& Montgomery, A. M. J. (2004). Impulsivity, risk taking and recreational 'ecstasy'(MDMA) use. Drug and Alcohol Dependence, 76(1), 55-62.

Byrnes, J. P., Miller, D. C., \& Schafer, W. D. (1999). Gender differences in risk taking: A metaanalysis. Psychological Bulletin, 125(3), 367.

Cacciola, J. S., Dugosh, K., Foltz, C., Leahy, P., \& Stevens, R. (2005). Treatment outcomes: First time versus treatment-experienced clients. Journal of Substance Abuse Treatment, 28(2), S13-S22.

Cacioppo, J. T., \& Hawkley, L. C. (2009). Perceived social isolation and cognition. Trends in Cognitive Sciences, 13(10), 447-454. 
Cadet, J. L. (2016). Epigenetics of stress, addiction, and resilience: therapeutic implications. Molecular Neurobiology, 53(1), 545-560.

Cahill, C. M., Xue, L., Grenier, P., Magnussen, C., Lecour, S., \& Olmstead, M. C. (2013). Changes in morphine reward in a model of neuropathic pain. Behavioural Pharmacology, 24(3), 207-213.

Caldwell, E. E., \& Riccio, D. C. (2010). Alcohol self-administration in rats: Modulation by temporal parameters related to repeated mild social defeat stress. Alcohol, 44(3), 265-274.

Calcaterra, S., Glanz, J., \& Binswanger, I. A. (2013). National trends in pharmaceutical opioid related overdose deaths compared to other substance related overdose deaths: 1999-2009. Drug and Alcohol Dependence, 131(3), 263-270.

Camerer, C. F., Loewenstein, G., \& Prelec, D. (2004). Neuroeconomics: Why economics needs brains. Scandinavian Journal of Economics, 106(3), 555-579.

Cantin, L., Lenoir, M., Augier, E., Vanhille, N., Dubreucq, S., Serre, F., ... \& Ahmed, S. H. (2010). Cocaine is low on the value ladder of rats: Possible evidence for resilience to addiction. PloS One, 5(7), e11592.

Capstone Law. (2019). Some Labor Ready Lawsuits. Retrieved June 7, 2019, from http://www.naffe.net/lr/legal.htm

Caton, C. L., Wilkins, C., \& Anderson, J. (2007, September). People who experience long-term homelessness: Characteristics and interventions. In Toward understanding homelessness: The 2007 national symposium on homelessness research. Washington, DC: US Department of Health and Human Services and US Department of Housing and Urban Development.

Cape, G. S. (2003). Addiction, stigma and movies. Acta Psychiatrica Scandinavica, 107(3), 163-169.

Caprioli, D., Celentano, M., Dubla, A., Lucantonio, F., Nencini, P., \& Badiani, A. (2009). Ambience and drug choice: Cocaine-and heroin-taking as a function of environmental context in humans and rats. Biological Psychiatry, 65(10), 893-899.

Caprioli, D., Celentano, M., Paolone, G., \& Badiani, A. (2007). Modeling the role of environment in addiction. Progress in Neuro-psychopharmacology and Biological Psychiatry, 31(8), 1639-1653.

Caprioli, D., Zeric, T., Thorndike, E. B., \& Venniro, M. (2015). Persistent palatable food preference in rats with a history of limited and extended access to methamphetamine self-administration. Addiction Biology, 20(5), 913-926.

Carliner, H., Brown, Q. L., Sarvet, A. L., \& Hasin, D. S. (2017). Cannabis use, attitudes, and legal status in the US: A review. Preventive Medicine, 104, 13-23.

Carmines, E. G., \& Zeller, R. A. (1979). Reliability and validity assessment (Vol. 17). Thousand Oaks, CA. Sage Publications.

Carroll, J. J., Marshall, B. D., Rich, J. D., \& Green, T. C. (2017). Exposure to fentanyl-contaminated heroin and overdose risk among illicit opioid users in Rhode Island: A mixed methods study. International Journal of Drug Policy, 46, 136-145.

Carroll, J. J., Rich, J. D., \& Green, T. C. (2018). The more things change: Buprenorphine/naloxone diversion continues while treatment remains inaccessible. Journal of Addiction Medicine, 12(6), 459-465 
Carroll, M. E., \& Rodefer, J. S. (1993). Income alters choice between drug and an alternative nondrug reinforcer in monkeys. Experimental and Clinical Psychopharmacology, 1(1-4), 110.

Carroll, M. E. (1993). The economic context of drug and non-drug reinforcers affects acquisition and maintenance of drug-reinforced behavior and withdrawal effects. Drug and Alcohol Dependence, 33(2), 201-210.

Carter, A. \& Hall W. (2017) Chapter 25 Looking to the future: Clinical and policy implications of the brain disease model of addiction. (pp 497-514). In Neuroethics: Anticipating the future. Oxford University Press.

Case, A., \& Deaton, A. (2017). Mortality and morbidity in the 21 st century. Brookings Papers on Economic Activity, 2017, 397.

Cassidy, T. A., McNaughton, E. C., Varughese, S., Russo, L., Zulueta, M., \& Butler, S. F. (2015). Nonmedical use of prescription ADHD stimulant medications among adults in a substance abuse treatment population: Early findings from the NAVIPPRO surveillance system. Journal of Attention Disorders, 19(4), 275-283.

Caulkins, J. P. (1995). Estimating Elasticities of Demand for Cocaine and Heroin with Data from the Drug Use Forecasting System.

Caulkins, J. P. (2001). Drug prices and emergency department mentions for cocaine and heroin. American Journal of Public Health, 91(9), 1446-1448.

Caulkins, J. P., \& Reuter, P. (2006). Illicit drug markets and economic irregularities. Socio-Economic Planning Sciences, 40(1), 1-14.

Cecil, K. M., Brubaker, C. J., Adler, C. M., Dietrich, K. N., Altaye, M., Egelhoff, J. C., ... \& Lanphear, B. P. (2008). Decreased brain volume in adults with childhood lead exposure. PLoS Medicine, 5(5), e112.

Cella, M., Dymond, S., \& Cooper, A. (2010). Impaired flexible decision-making in major depressive disorder. Journal of Affective Disorders, 124(1-2), 207-210.

Centers for Disease Control and Prevention. (1982, May 28). National Surveillance of Cocaine Use and Related Health Consequences. Retrieved from https://www.cdc.gov/mmwr/preview/mmwrhtml/00001101.htm

Centers for Disease Control and Prevention (2019). Alcohol and public health: Alcohol-related disease impact (ARDI). Average for United States 2006-2010 Alcohol-attributable deaths due to excessive alcohol use. Available at: https://nccd.cdc.gov/DPH_ARDI/Default/Report.aspx?T=AAM\&P=f6d7eda7-036e-45539968-9b17ffad620e\&R=d7a9b303-48e9-4440-bf47-070a4827e1fd\&M=8E1C5233-5640-4EE89247-1ECA7DA325B9\&F=\&D=. Accessed 1/18/19.

Centers for Disease Control and Prevention. (2018a). Quality improvement and care coordination: Implementing the CDC guideline for prescribing opioids for chronic pain. National Center for Injury Prevention and Control, Division of Unintentional Injury Prevention, Atlanta, GA.

Centers for Disease Control and Prevention. (2018b, December 19). Heroin overdose data | Drug Overdose | CDC Injury Center. Retrieved February 4, 2019, from https://www.cdc.gov/drugoverdose/data/heroin.html

Centers for Disease Control and Prevention. Annual surveillance report of drug-related risks and outcomes — United States, 2019. (2019). Surveillance Special Report 1. Centers for Disease Control and 
Prevention, U.S. Department of Health and Human Services. Accessed April 1, 2018 from https://www.cdc.gov/ drugoverdose/pdf/pubs/2017 cdc-drug-surveillance-report.pdf.

Centers for Disease Control and Prevention. (2017b). Drug overdose death data $\mid$ Drug Overdose | CDC Injury Center. Retrieved April 11, 2018, from

https://www.cdc.gov/drugoverdose/data/statedeaths.html.

Centers for Disease Control and Prevention. (1993). Healthy days core module: HRQOL-14 measure| HRQOL | CDC. Retrieved February 1, 2019, from https://www.cdc.gov/hrqol/hrqol14_measure.htm

Centers for Disease Control and Prevention. (2000). Measuring Healthy Days. Atlanta, GA: CDC. Retrieved from https://www.cdc.gov/hrqol/pdfs/mhd.pdf

Cerdá, M., Gaidus, A., Keyes, K. M., Ponicki, W., Martins, S., Galea, S., \& Gruenewald, P. (2017). Prescription opioid poisoning across urban and rural areas: Identifying vulnerable groups and geographic areas. Addiction, 112(1), 103-112.

Cerdá, M., Santaella, J., Marshall, B. D., Kim, J. H., \& Martins, S. S. (2015). Nonmedical prescription opioid use in childhood and early adolescence predicts transitions to heroin use in young adulthood: A national study. The Journal of Pediatrics, 167(3), 605-612.

Chalmers, J., Bradford, D., \& Jones, C. (2010). The effect of methamphetamine and heroin price on polydrug use: A behavioural economics analysis in Sydney, Australia. International Journal of Drug Policy, 21(5), 381-389.

Chalmers, J., Lancaster, K., \& Hughes, C. (2016). The stigmatisation of 'ice' and under-reporting of meth/amphetamine use in general population surveys: A case study from Australia. International Journal of Drug Policy, 36, 15-24.

Chaloupka, F. J., \& Warner, K. E. (2000). The economics of smoking. Handbook of health economics, 1, 1539-1627.

Chaloupka, F. J., Emery, S., \& Liang, L. (2003). Evolving models of addictive behavior: From neoclassical to behavioral economics. In Choice, Behavioural Economics and Addiction(pp. 71-94). Pergamon.

Chambers, R. A., Taylor, J. R., \& Potenza, M. N. (2003). Developmental neurocircuitry of motivation in adolescence: A critical period of addiction vulnerability. American Journal of Psychiatry, 160(6), 1041-1052.

Champagne, F. A. (2010). Early adversity and developmental outcomes: Interaction between genetics, epigenetics, and social experiences across the life span. Perspectives on Psychological Science, 5(5), 564-574.

Champagne, F. A., \& Curley, J. P. (2005). How social experiences influence the brain. Current opinion in neurobiology, 15(6), 704-709.

Charach, A., Yeung, E., Climans, T., \& Lillie, E. (2011). Childhood attention-deficit/hyperactivity disorder and future substance use disorders: comparative meta-analyses. Journal of the American Academy of Child \& Adolescent Psychiatry, 50(1), 9-21.

Chattarji, S., Tomar, A., Suvrathan, A., Ghosh, S., \& Rahman, M. M. (2015). Neighborhood matters: Divergent patterns of stress-induced plasticity across the brain. Nature Neuroscience, 18(10), 1364. 
Chavkin, C., \& Ehrich, J. M. (2014). How does stress-induced activation of the kappa opioid system increase addiction risk?. Biological Psychiatry, 76(10), 760-762.

Chen, C. Y., Storr, C. L., \& Anthony, J. C. (2009). Early-onset drug use and risk for drug dependence problems. Addictive Behaviors, 34(3), 319-322.

Cheng, E. R., \& Kindig, D. A. (2012). Disparities in premature mortality between high-and low-income US counties. Preventing chronic disease, 9.

Chermack, S. T., Roll, J., Reilly, M., Davis, L., Kilaru, U., \& Grabowski, J. (2000). Comparison of patient self-reports and urinalysis results obtained under naturalistic methadone treatment conditions. Drug and Alcohol Dependence, 59(1), 43-49.

Chetty, R. (2015). Behavioral economics and public policy: A pragmatic perspective. American Economic Review, 105(5), 1-33.

Childress, A. R., Hole, A. V., Ehrman, R. N., Robbins, S. J., McLellan, A. T., \& O’Brien, C. P. (1993). Cue reactivity and cue reactivity interventions in drug dependence. NIDA research monograph, 137, 73-73.

Chilcoat, H. D., Coplan, P. M., Harikrishnan, V., \& Alexander, L. (2016). Decreased diversion by doctorshopping for a reformulated extended release oxycodone product (OxyContin). Drug and Alcohol Dependence, 165, 221-228.

Churchwell, J. C., Lopez-Larson, M., \& Yurgelun-Todd, D. A. (2010). Altered frontal cortical volume and decision making in adolescent cannabis users. Frontiers in Psychology, 1, 225.

Ciccarone, D. (2017). Fentanyl in the US heroin supply: Rapidly changing risk environment. International Journal of Drug Policy, 46, 107-111.

Ciccarone, D., Ondocsin, J., \& Mars, S. G. (2017). Heroin uncertainties: Exploring users' perceptions of fentanyl-adulterated and-substituted 'heroin'. International Journal of Drug Policy, 46, 146-155.

Cicerone, K. D. (1996). Attention deficits and dual task demands after mild traumatic brain injury. Brain Injury, 10(2), 79-90.

Cicero, T. J., Ellis, M. S., Surratt, H. L., \& Kurtz, S. P. (2014). The changing face of heroin use in the United States: A retrospective analysis of the past 50 years. JAMA Psychiatry, 71(7), 821-826.

Cicero, T. J., Ellis, M. S., \& Kasper, Z. A. (2017a). Relative preferences in the abuse of immediate-release versus extended-release opioids in a sample of treatment-Seeking opioid abusers. Pharmacoepidemiology and Drug Safety, 26(1), 56-62.

Cicero T. J., Ellis M. S., Kasper Z. A. (2017b). Increased use of heroin as an initiating opioid of abuse. Addictive Behaviors, 74:63-6.

Cicero, T. J., Ellis, M. S., \& Surratt, H. L. (2012). Effect of abuse-deterrent formulation of OxyContin. New England Journal of Medicine, 367(2), 187-189.

Cicero, T. J., Lynskey, M., Todorov, A., Inciardi, J. A., \& Surratt, H. L. (2008). Co-morbid pain and psychopathology in males and females admitted to treatment for opioid analgesic abuse. Pain, 139(1), 127-135.

Cicero, T. J., Surratt, H. L., \& Inciardi, J. (2007). Use and misuse of buprenorphine in the management of opioid addiction. J Opioid Manag, 3(6), 302-308. 
Cicero, T. J., Surratt, H., Inciardi, J. A., \& Munoz, A. (2007). Relationship between therapeutic use and abuse of opioid analgesics in rural, suburban, and urban locations in the United States. Pharmacoepidemiology and Drug Safety, 16(8), 827-840.

Clark, L., Cools, R., \& Robbins, T. W. (2004). The neuropsychology of ventral prefrontal cortex: Decisionmaking and reversal learning. Brain and Cognition, 55(1), 41-53.

Cleeland, C. (2009). Brief Pain Inventory (BPI). Retrieved from https://www.mdanderson.org/research/departments-labs-institutes/departmentsdivisions/symptom-research/symptom-assessment-tools/brief-pain-inventory.html

Cleeland, C. S., \& Ryan, K. M. (1994). Pain assessment: Global use of the Brief Pain Inventory. Annals, Academy of Medicine, Singapore.

Clemans-Cope, L., Epstein, M., \& Kenney, G. M. (2017). Rapid growth in Medicaid spending on medications to treat opioid use disorder and overdose. The Urban Institute.

Clinchy, M., Sheriff, M. J., \& Zanette, L. Y. (2013). Predator-induced stress and the ecology of fear. Functional Ecology, 27(1), 56-65.

Clinton, H. A., Hunter, A. A., Logan, S. B., \& Lapidus, G. D. (2019). Evaluating opioid overdose using the National Violent Death Reporting System, 2016. Drug and Alcohol Dependence, 194, 371-376.

Cloninger, C. R. (1987). Neurogenetic adaptive mechanisms in alcoholism. Science, 236(4800), 410-416.

Cloninger, C. R., Sigvardsson, S., Gilligan, S. B., von Knorring, A. L., Reich, T., \& Bohman, M. (1988). Genetic heterogeneity and the classification of alcoholism. Advances in Alcohol \& Substance Abuse, 7(3-4), 3-16.

Clotfelter, C. T., Cook, P. J., Edell, J. A., \& Moore, M. (1999). State lotteries at the turn of the century: Report to the national gambling impact study commission (pp. 1-2). Washington, DC: National Gambling Impact Study Commission.

Cloud, D. H., Ibragimov, U., Prood, N., Young, A. M., \& Cooper, H. L. (2019). Rural risk environments for hepatitis c among young adults in appalachian kentucky. International Journal of Drug Policy.

Cohn, L. D., Macfarlane, S., Yanez, C., \& Imai, W. K. (1995). Risk-perception: differences between adolescents and adults. Health Psychology, 14(3), 217.

Cohen, J. D. (2005). The vulcanization of the human brain: A neural perspective on interactions between cognition and emotion. Journal of Economic Perspectives, 19(4), 3-24.

Cohen, J. D., \& Blum, K. I. (2002). Reward and decision. Neuron, 36(2), 193-198.

Cohen, K., \& Weinstein, A. M. (2018). Synthetic and non-synthetic cannabinoid drugs and their adverse effects-a review from public health prospective. Frontiers in Public Health, 6, 162.

Coffin, P. O., Galea, S., Ahern, J., Leon, A. C., Vlahov, D., \& Tardiff, K. (2003). Opiates, cocaine and alcohol combinations in accidental drug overdose deaths in New York City, 199098. Addiction, 98(6), 739-747.

Cole, J., \& Logan, T. K. (2010). Nonmedical use of sedative-hypnotics and opiates among rural and urban women with protective orders. Journal of Addictive Diseases, 29(3), 395-409.

Cole, J., Logan, TK, Miller, J., \& Scrivner, A. (2016). Adult Kentucky treatment outcome study 2016 annual report. Lexington, KY: University of Kentucky, Center on Drug and Alcohol Research. 
Cole, J., Logan, T. K., \& Walker, R. (2011). Social exclusion, personal control, self-regulation, and stress among substance abuse treatment clients. Drug and Alcohol Dependence, 113(1), 13-20.

Coleman, J. J. (2012). The supply chain of medicinal controlled substances: addressing the Achilles heel of drug diversion. Journal of Pain \& palliative Care Pharmacotherapy, 26(3), 233-250.

Coles, C. D., Platzman, K. A., Raskind-Hood, C. L., Brown, R. T., Falek, A., \& Smith, I. E. (1997). A comparison of children affected by prenatal alcohol exposure and attention deficit, hyperactivity disorder. Alcoholism: Clinical and Experimental Research, 21(1), 150-161.

Collins, R. L. (1996). For better or worse: The impact of upward social comparison on selfevaluations. Psychological Bulletin, 119(1), 51.

Collins, R. L., Ellickson, P. L., \& Klein, D. J. (2007). The role of substance use in young adult divorce. Addiction, 102(5), 786-794.

Collins, F. S., Koroshetz, W. J., \& Volkow, N. D. (2018). Helping to end addiction over the long-term: The research plan for the NIH HEAL initiative. Jama, 320(2), 129-130.

Comer, S. D., Collins, E. D., Wilson, S. T., Donovan, M. R., Foltin, R. W., \& Fischman, M. W. (1998). Effects of an alternative reinforcer on intravenous heroin self-administration by humans. European Journal of Pharmacology, 345(1), 13-26.

Compton, W. M., Jones, C. M., \& Baldwin, G. T. (2016). Relationship between nonmedical prescriptionopioid use and heroin use. New England Journal of Medicine, 374(2), 154-163.

Cone, E. J. (1995). Pharmacokinetics and pharmacodynamics of cocaine. Journal of Analytical Toxicology, 19(6), 459-478.

Conklin, C. A., \& Perkins, K. A. (2005). Subjective and reinforcing effects of smoking during negative mood induction. Journal of Abnormal Psychology, 114(1), 153.

Cook, T. D., \& Campbell, D. T. (1979). Quasi-experimentation: Design and analysis for field settings (Vol. 3). Chicago, IL: Rand McNally.

Cook, T. D., \& Shadish, W. R. (1994). Social experiments: Some developments over the past fifteen years. Annual Review of Psychology, 45(1), 545-580.

Cooney, N. L., Litt, M. D., Morse, P. A., Bauer, L. O., \& Gaupp, L. (1997). Alcohol cue reactivity, negativemood reactivity, and relapse in treated alcoholic men. Journal of Abnormal Psychology, 106(2), 243.

Coplan, P. M., Chilcoat, H. D., Butler, S. F., Sellers, E. M., Kadakia, A., Harikrishnan, V., ... \& Dart, R. C. (2016). The effect of an abuse-deterrent opioid formulation (OxyContin) on opioid abuse-related outcomes in the postmarketing setting. Clinical Pharmacology \& Therapeutics, 100(3), 275-286.

Copenhaver, A., Edwards-Willey, T. L., \& Byers, B. D. (2007). Journeys in social stigma: The lives of formerly incarcerated felons in higher education. Journal of Correctional Education, 268-283.

Cornish, J. W., \& O'Brien, C. P. (1996). Crack cocaine abuse: An epidemic with many public health consequences. Annual Review of Public Health, 17(1), 259-273.

Corrigan, P. W. (2000). Mental health stigma as social attribution: Implications for research methods and attitude change. Clinical Psychology: Science and Practice, 7(1), 48-67. 
Corrigan, P. W. (2005). Changing stigma through contact. Advances in Schizophrenia and Clinical Psychiatry, 1, 614-625.

Corrigan, P. W., \& Wassel, A. (2008). Understanding and influencing the stigma of mental illness. Journal of Psychosocial Nursing and Mental Health Services, 46(1), 42-48.

Correia, C. J. (2004). Behavioral economics: Basic concepts and clinical applications. Handbook of Motivational Counseling, 49.

Correia, C. J., Murphy, J. G., Irons, J. G., \& Vasi, A. E. (2010). The behavioral economics of substance use: Research on the relationship between substance use and alternative reinforcers. Journal of Behavioral Health and Medicine, 1(3), 216.

Cosden, M., Basch, J. E., Campos, E., Greenwell, A., Barazani, S., \& Walker, S. (2006). Effects of motivation and problem severity on court-based drug treatment. Crime \& Delinquency, 52(4), 599-618.

Cottle, C. C., Lee, R. J., \& Heilbrun, K. (2001). The prediction of criminal recidivism in juveniles: A metaanalysis. Criminal justice and behavior, 28(3), 367-394.

Coughlan, M., Cronin, P., \& Ryan, F. (2009). Survey research: Process and limitations. International Journal of Therapy and Rehabilitation, 16(1), 9-15.

Covey, R. (2007). Reconsidering the relationship between cognitive psychology and plea bargaining. Marquvette Law Review, 91, 213.

Covington III, H. E., Kikusui, T., Goodhue, J., Nikulina, E. M., Hammer Jr, R. P., \& Miczek, K. A. (2005). Brief social defeat stress: Long lasting effects on cocaine taking during a binge and zif268 mRNA expression in the amygdala and prefrontal cortex. Neuropsychopharmacology, 30(2), 310.

Covington, H. E., \& Miczek, K. A. (2001). Repeated social-defeat stress, cocaine or morphine. Psychopharmacology, 158(4), 388-398.

Covington, H. E., \& Miczek, K. A. (2005). Intense cocaine self-administration after episodic social defeat stress, but not after aggressive behavior: Dissociation from corticosterone activation. Psychopharmacology, 183(3), 331-340.

Cox, E. R., Motheral, B. R., Henderson, R. R., \& Mager, D. (2003). Geographic variation in the prevalence of stimulant medication use among children 5 to 14 years old: Results from a commercially insured US sample. Pediatrics, 111(2), 237-243.

Cox, W. M., Klinger, E., \& Fadardi, J. S. (2017). Free will in addictive behaviors: A matter of definition. Addictive Behaviors Reports, 5, 94-103.

Cramer, D., \& Howitt, D. L. (2004). The Sage dictionary of statistics: A practical resource for students in the social sciences. Sage.

Camerer, C. F., Loewenstein, G., \& Prelec, D. (2004). Neuroeconomics: Why economics needs brains. Scandinavian Journal of Economics, 106(3), 555-579.

Camerer, C., Loewenstein, G., \& Prelec, D. (2005). Neuroeconomics: How neuroscience can inform economics. Journal of Economic Literature, 43(1), 9-64.

CDC, WONDER. (2018). Multiple cause of death data on CDC WONDER. Atlanta, GA: US Department of Health and Human Services. Retrieved January 3, 2019, from https://wonder.cdc.gov/mcd.html 
Crisp, A. H., Gelder, M. G., Rix, S., Meltzer, H. I., \& Rowlands, O. J. (2000). Stigmatisation of people with mental illnesses. The British Journal of Psychiatry, 177(1), 4-7.

Critchfield, T. S., \& Kollins, S. H. (2001). Temporal discounting: Basic research and the analysis of socially important behavior. Journal of Applied Behavior Analysis, 34(1), 101-122.

Critchley, H. D., Wiens, S., Rotshtein, P., Öhman, A., \& Dolan, R. J. (2004). Neural systems supporting interoceptive awareness. Nature Neuroscience, 7(2), 189.

Croft, T., Foppe, K., Huffines, R., \& Subedi, B. (2018). Consumption Profiles of Illicit and Neuropsychiatric Drugs in Urban and Rural Communities in Kentucky Using Sewage Epidemiology.

Cromer, J. R., Cromer, J. A., Maruff, P., \& Snyder, P. J. (2010). Perception of alcohol intoxication shows acute tolerance while executive functions remain impaired. Experimental and Clinical Psychopharmacology, 18(4), 329.

Crombag, H. S., Bossert, J. M., Koya, E., \& Shaham, Y. (2008). Context-induced relapse to drug seeking: A review. Philosophical Transactions of the Royal Society: Biological Sciences, 363(1507), 32333243.

Crone, E. A., Somsen, R. J., Beek, B. V., \& Van Der Molen, M. W. (2004). Heart rate and skin conductance analysis of antecendents and consequences of decision making. Psychophysiology, 41(4), 531-540.

Cropanzano, R., \& Mitchell, M. S. (2005). Social exchange theory: An interdisciplinary review. Journal of Management, 31(6), 874-900.

Crosby, R. A., Wendel, M. L., Vanderpool, R. C., \& Casey, B. R. (2012). Rural populations and health: Determinants, disparities, and solutions. John Wiley \& Sons.

Cross, C. P., Copping, L. T., \& Campbell, A. (2011). Sex differences in impulsivity: A metaanalysis. Psychological Bulletin, 137(1), 97.

Cruz, F. C., Quadros, I. M., Hogenelst, K., Planeta, C. S., \& Miczek, K. A. (2011). Social defeat stress in rats: Escalation of cocaine and "speedball" binge self-administration, but not heroin. Psychopharmacology, 215(1), 165-175.

Cullen, F. T., \& Wilcox, P. (Eds.). (2015). The Oxford handbook of criminological theory. Oxford, England, UK. Oxford University Press.

Cunha, P. J., Bechara, A., de Andrade, A. G., \& Nicastri, S. (2011). Decision-making deficits linked to reallife social dysfunction in crack cocaine-dependent individuals. The American Journal on Addictions, 20(1), 78-86.

Cunningham, J. K., \& Liu, L. M. (2003). Impacts of federal ephedrine and pseudoephedrine regulations on methamphetamine-related hospital admissions. Addiction, 98(9), 1229-1237.

Cunningham, J. K., \& Liu, L. M. (2008). Impact of methamphetamine precursor chemical legislation, a suppression policy, on the demand for drug treatment. Social Science \& Medicine, 66(7), 14631473.

Cunningham, J. K., \& Liu, L. M. (2005). Impacts of federal precursor chemical regulations on methamphetamine arrests. Addiction, 100(4), 479-488.

Cunningham, J. K., Liu, L. M., \& Callaghan, R. (2009). Impact of US and Canadian precursor regulation on methamphetamine purity in the United States. Addiction, 104(3), 441-453. 
Curtis, V., De Barra, M., \& Aunger, R. (2011). Disgust as an adaptive system for disease avoidance behaviour. Philosophical Transactions of the Royal Society B: Biological Sciences, 366(1563), 389-401.

Cuthbert, B. N. (2015). Research domain criteria: Toward future psychiatric nosologies. Dialogues in Clinical Neuroscience, 17(1), 89.

Cutrona, C. E., \& Russell, D. W. (1987). The provisions of social relationships and adaptation to stress. Advances in Personal Relationships, 1(1), 37-67.

Czermainski, F. R., Willhelm, A. R., Santos, Á. Z., Pachado, M. P., \& de Almeida, R. M. M. (2017). Assessment of inhibitory control in crack and/or cocaine users: A systematic review. Trends in Psychiatry and Psychotherapy, 39(3), 216-225.

D’Onofrio, G., O’Connor, P. G., Pantalon, M. V., Chawarski, M. C., Busch, S. H., Owens, P. H., ... \& Fiellin, D. A. (2015). Emergency department-initiated buprenorphine/naloxone treatment for opioid dependence: A randomized clinical trial. Jama, 313(16), 1636-1644.

Dai, Z., Abate, M. A., Smith, G. S., Kraner, J. C., \& Mock, A. R. (2019). Fentanyl and fentanyl-analog involvement in drug-related deaths. Drug and Alcohol Dependence, 196, 1-8.

Dalla, R. L., Xia, Y., \& Kennedy, H. (2003). "You just give them what they want and pray they don't kill you" street-level sex workers' reports of victimization, personal resources, and coping strategies. Violence Against Women, 9(11), 1367-1394.

Dallman, M. F. (2010). Stress-induced obesity and the emotional nervous system. Trends in Endocrinology \& Metabolism, 21(3), 159-165.

Daly, M. \& Wilson, M. (2001). Risk-taking, intrasexual competition, and homicide. Nebraska Symposium on Motivation (Vol. 47, pp. 1-36).

Damasio, A. R. (2006). Descartes' error. Random House Tower, NYC. Random House.

Damasio’s, A. R. (2012). 11 Emotional, social and moral intuition. Inside Intuition, 271.

Daniulaityte, R., Falck, R. S., Wang, J., \& Carlson, R. G. (2009). Illicit use of pharmaceutical opioids among young polydrug users in Ohio. Addictive Behaviors, 34(8), 649-653.

Daniulaityte R., Falck R., \& Carlson R. G., 2012. Illicit use of buprenorphine in a community sample of young adult non-medical users of pharmaceutical opioids. Drug and Alcohol Dependence 122: 201-207.

Daniulaityte, R., Carlson, R. G., \& Kenne, D. R. (2006). Initiation to pharmaceutical opioids and patterns of misuse: Preliminary qualitative findings obtained by the Ohio Substance Abuse Monitoring Network. Journal of Drug Issues, 36(4), 787-808.

Daniulaityte, R., Falck, R., \& Carlson, R. G. (2012). "I'm not afraid of those ones just 'cause they've been prescribed": Perceptions of risk among illicit users of pharmaceutical opioids. International Journal of Drug Policy, 23(5), 374-384.

Daniulaityte, R., Carlson, R., Brigham, G., Cameron, D., \& Sheth, A. (2015). "Sub is a weird drug:" A webbased study of lay attitudes about use of buprenorphine to self-treat opioid withdrawal symptoms. The American Journal on Addictions, 24(5), 403-409. 
Daniulaityte, R., Juhascik, M. P., Strayer, K. E., Sizemore, I. E., Zatreh, M., Nahhas, R. W., ... \& Carlson, R. R. (2019). Trends in fentanyl and fentanyl analogue-related overdose deaths-Montgomery County, Ohio, 2015-2017. Drug and Alcohol Dependence.

Darke, S. (2013). Pathways to heroin dependence: Time to re-appraise self-medication. Addiction, 108(4), 659-667.

Darke, S. (1988). Anxiety and working memory capacity. Cognition and Emotion, 2(2), 145-154

Dart, R. C., Surratt, H. L., Cicero, T. J., Parrino, M. W., Severtson, S. G., Bucher-Bartelson, B., \& Green, J. L. (2015). Trends in opioid analgesic abuse and mortality in the United States. New England Journal of Medicine, 372(3), 241-248.

Dasgupta, N., Freifeld, C., Brownstein, J. S., Menone, C. M., Surratt, H. L., Poppish, L., ... \& Dart, R. C. (2013). Crowdsourcing black market prices for prescription opioids. Journal of Medical Internet Research, 15(8).

Davidson, P. J., Wagner, K. D., Tokar, P. L., \& Scholar, S. (2019). Documenting need for naloxone distribution in the Los Angeles County jail system. Addictive Behaviors, 92, 20-23.

Davis, N. (2014). The Journey to Recovery in Eastern Kentucky: A Symbolic Interactionist Assessment of Drug Addicts' Relationships. (2014) Honors Thesis. Paper 184

Davis, R. E., Couper, M. P., Janz, N. K., Caldwell, C. H., \& Resnicow, K. (2009). Interviewer effects in public health surveys. Health Education Research, 25(1), 14-26.

Dawe, S., \& Loxton, N. J. (2004). The role of impulsivity in the development of substance use and eating disorders. Neuroscience \& Biobehavioral Reviews, 28(3), 343-351.

Dawson, M. E., Schell, A. M., \& Courtney, C. G. (2011). The skin conductance response, anticipation, and decision-making. Journal of Neuroscience, Psychology, and Economics, 4(2), 111.

Day, C., Conroy, E., Lowe, J., Page, J., \& Dolan, K. (2006). Patterns of drug use and associated harms among rural injecting drug users: Comparisons with metropolitan injecting drug users. Australian Journal of Rural Health, 14(3), 120-125

Dayan, P., \& Balleine, B. W. (2002). Reward, motivation, and reinforcement learning. Neuron, 36(2), 285298.

Dear, M. J., \& Wolch, J. R. (2014). Landscapes of despair: From deinstitutionalization to homelessness (Vol. 823). Princeton, NJ: Princeton University Press.

De Kloet, E. R., Joëls, M., \& Holsboer, F. (2005). Stress and the brain: From adaptation to disease. Nature Reviews Neuroscience, 6(6), 463.

de la Fuente, A., Sedeño, L., Vignaga, S. S., Ellmann, C., Sonzogni, S., Belluscio, L., ... \& Torralva, T. (2019). Multimodal neurocognitive markers of interoceptive tuning in smoked cocaine. Neuropsychopharmacology, 1.

De Leon, G., Perfas, F. B., Joseph, A., \& Bunt, G. (2015). Therapeutic communities for addictions: Essential elements, cultural, and current issues. Textbook of Addiction Treatment: International Perspectives, 1033-1047.

De Tocqueville, A. (2003). Democracy in America (Vol. 10). Regnery Publishing. 
de Wit, H. (1996). Priming effects with drugs and other reinforcers. Experimental and Clinical Psychopharmacology, 4(1), 5.

de Wit, S. (2018). The balance between goal-directed and habitual action control in disorders of compulsivity. In Goal-Directed Decision Making (pp. 331-365). Academic Press.

de Wit, H. (2009). Impulsivity as a determinant and consequence of drug use: A review of underlying processes. Addiction Biology, 14(1), 22-31.

de Wit H. \& Richards J. B. (2004). Dual determinants of drug use in humans: Reward and impulsivity in Bevins, R. A. \& Bardo, M. T. (Eds.), Motivational factors in the etiology of drug abuse Vol. 50, p19. University of Nebraska Press.

DeYoung, C. G. (2016). Impulsivity as a personality trait. Handbook of self-regulation: Research, theory, and applications, 3, 345-363.

Del Boca, F. K., \& Darkes, J. (2003). The validity of self-reports of alcohol consumption: State of the science and challenges for research. Addiction, 98, 1-12.

Del Boca, F. K., \& Noll, J. A. (2000). Truth or consequences: The validity of self-report data in health services research on addictions. Addiction, 95(11s3), 347-360.

Deering, D. E., Adamson, S. J., Sellman, J. D., Henderson, C., Sheridan, J., Pooley, S., ... \& Frampton, C. M. (2018). Potential risk for fatal drug overdose perceived by people using opioid drugs. Drug and Alcohol Review, 37, S309-S313.

Degenhardt, L., Charlson, F., Mathers, B., Hall, W. D., Flaxman, A. D., Johns, N., \& Vos, T. (2014). The global epidemiology and burden of opioid dependence: Results from the global burden of disease 2010 study. Addiction, 109(8), 1320-1333.

Delgadillo, J., Payne, S., Gilbody, S., Godfrey, C., Gore, S., Jessop, D., \& Dale, V. (2012). Brief case finding tools for anxiety disorders: Validation of GAD-7 and GAD-2 in addictions treatment. Drug and Alcohol Dependence, 125(1-2), 37-42.

Denis, C., Fatséas, M., Beltran, V., Bonnet, C., Picard, S., Combourieu, I., ... \& Auriacombe, M. (2012). Validity of the self-reported drug use section of the Addiction Severity Index and associated factors used under naturalistic conditions. Substance Use \& Misuse, 47(4), 356-363.

Denk, E. (2019). The Relationship Between Prime-age Male Labor Force Participation and Opiod Overdoses in Appalachia. (Doctoral dissertation, Georgetown University).

Dennett, D. C. (2015). Elbow room: The varieties of free will worth wanting. Cambridge, MA. MIT Press.

Dennis, M., \& Scott, C. K. (2007). Managing addiction as a chronic condition. Addiction Science \& Clinical Practice, 4(1), 45.

Derry, H. M., Fagundes, C. P., Andridge, R., Glaser, R., Malarkey, W. B., \& Kiecolt-Glaser, J. K. (2013). Lower subjective social status exaggerates interleukin-6 responses to a laboratory stressor. Psychoneuroendocrinology, 38(11), 2676-2685.

Des Jarlais, D. C., Arasteh, K., Perlis, T., Hagan, H., Heckathorn, D. D., Mcknight, C., ... \& Friedman, S. R. (2007). The transition from injection to non-injection drug use: Long-term outcomes among heroin and cocaine users in New York City. Addiction, 102(5), 778-785. 
Desrochers, T. M., Jin, D. Z., Goodman, N. D., \& Graybiel, A. M. (2010). Optimal habits can develop spontaneously through sensitivity to local cost. Proceedings of the National Academy of Sciences, 107(47), 20512-20517.

Dew, B., Elifson, K., \& Dozier, M. (2007). Social and environmental factors and their influence on drug use vulnerability and resiliency in rural populations. The Journal of Rural Health, 23, 16-21.

DiClemente, C. C. (1986). Self-efficacy and the addictive behaviors. Journal of Social and Clinical Psychology, 4(3), 302-315.

DiClemente, C. C. (2018). Addiction and change: How addictions develop and addicted people recover. New York City, NY. Guilford Publications.

Di Chiara, G., \& Bassareo, V. (2007). Reward system and addiction: What dopamine does and doesn't do. Current Opinion in Pharmacology, 7(1), 69-76.

Di Lernia, D., Serino, S., \& Riva, G. (2016). Pain in the body. Altered interoception in chronic pain conditions: A systematic review. Neuroscience \& Biobehavioral Reviews, 71, 328-341.

Di Sclafani, V., Tolou-Shams, M., Price, L. J., \& Fein, G. (2002). Neuropsychological performance of individuals dependent on crack-cocaine, or crack-cocaine and alcohol, at 6 weeks and 6 months of abstinence. Drug and Alcohol Dependence, 66(2), 161-171.

Diamantopoulos, A., Sarstedt, M., Fuchs, C., Wilczynski, P., \& Kaiser, S. (2012). Guidelines for choosing between multi-item and single-item scales for construct measurement: A predictive validity perspective. Journal of the Academy of Marketing Science, 40(3), 434-449.

Dias-Ferreira, E., Sousa, J. C., Melo, I., Morgado, P., Mesquita, A. R., Cerqueira, J. J., ... \& Sousa, N. (2009). Chronic stress causes frontostriatal reorganization and affects decisionmaking. Science, 325(5940), 621-625.

Dickman, S. J. (1993). Impulsivity and information processing. In W. G. McCown, J. L. Johnson, \& M. B. Shure (Eds.), The impulsive client: Theory, research, and treatment (pp. 151-184). Washington, DC, US: American Psychological Association. http://dx.doi.org/10.1037/10500-010

Dickson, M. F., Staton-Tindall, M., Smith, K. E., Leukefeld, C., Webster, J. M., \& Oser, C. B. (2017). A Facebook Follow-up Strategy for Rural Drug-using Women. The Journal of Rural Health, 33(3), 250-256.

DiClemente, C. C., Schlundt, D., \& Gemmell, L. (2004). Readiness and stages of change in addiction treatment. The American Journal on Addictions, 13(2), 103-119.

Diddle, G., \& Denham, S. A. (2010). Spirituality and its relationships with the health and illness of Appalachian people. Journal of Transcultural Nursing, 21(2), 175-182.

Diez-Roux, A. V. (2000). Multilevel analysis in public health research. Annual review of public health, 2l(1), 171-192.

Dillman, D. A. (1978). Mail and telephone surveys: The total design method (Vol. 19). New York, NY: Wiley.

Dillman, D. A., Smyth, J. D., \& Christian, L. M. (2014). Internet, phone, mail, and mixed-mode surveys: The tailored design method. Hoboken, NJ. John Wiley \& Sons. 
Do Couto, B. R., Aguilar, M. A., Manzanedo, C., Rodriguez-Arias, M., Armario, A., \& Minarro, J. (2006). Social stress is as effective as physical stress in reinstating morphine-induced place preference in mice. Psychopharmacology, 185(4), 459-470.

Do Couto, B. R., Aguilar, M. A., Lluch, J., Rodríguez-Arias, M., \& Miñarro, J. (2009). Social experiences affect reinstatement of cocaine-induced place preference in mice. Psychopharmacology, 207(3), 485-498.

Dobkin, C., \& Nicosia, N. (2009). The war on drugs: Methamphetamine, public health, and crime. American Economic Review, 99(1), 324-49.

Dockree, P. M., Bellgrove, M. A., O’Keeffe, F. M., Moloney, P., Aimola, L., Carton, S., \& Robertson, I. H. (2006). Sustained attention in traumatic brain injury (TBI) and healthy controls: Enhanced sensitivity with dual-task load. Experimental Brain Research, 168(1-2), 218-229.

Dodge, N. C., Jacobson, J. L., \& Jacobson, S. W. (2016). Alcohol Dehydrogenase Alleles and Impact on Neuropathology. In Neuropathology of Drug Addictions and Substance Misuse (pp. 510-519). Academic Press.

Dodge, R., Sindelar, J., \& Sinha, R. (2005). The role of depression symptoms in predicting drug abstinence in outpatient substance abuse treatment. Journal of Substance Abuse Treatment, 28(2), 189-196.

Dolan, M. P., Black, J. L., Malow, R. M., \& Penk, W. E. (1991). Clinical differences among cocaine, opioid, and speedball users in treatment. Psychology of Addictive Behaviors, 5(2), 78.

Donlin, W. D., Knealing, T., \& Silverman, K. (2008). Employment-based reinforcement in the treatment of drug addiction. Contingency management in substance abuse treatment, 314-333.

Dombrovski, A. Y., Szanto, K., Siegle, G. J., Wallace, M. L., Forman, S. D., Sahakian, B., ... \& Clark, L. (2011). Lethal forethought: delayed reward discounting differentiates high-and low-lethality suicide attempts in old age. Biological Psychiatry, 70(2), 138-144.

Dombrowski, K., Crawford, D., Khan, B., \& Tyler, K. (2016). Current rural drug use in the US Midwest. Journal of Drug Abuse, 2(3).

Donnermeyer, J. F., Jobes, P., \& Barclay, E. (2006). Rural crime, poverty, and community. Advancing critical criminology: Theory and application, 199-218.

Doweiko, H. E. (1999). Concepts of chemical dependency . Pacific Grove, CA: Brooks.

Dowell, D., Haegerich, T. M., \& Chou, R. (2016). CDC guideline for prescribing opioids for chronic painUnited States, 2016. Jama, 315(15), 1624-1645.

Dowell, D., Noonan, R. K., \& Houry, D. (2017). Underlying factors in drug overdose deaths. Jama, 318(23), 2295-2296.

Draus, P. J., \& Carlson, R. G. (2009). "The Game Turns on You” Crack, Sex, Gender, and Power in SmallTown Ohio. Journal of Contemporary Ethnography, 38(3), 384-408.

Dreer, L. E., DeVivo, M. J., Novack, T. A., Krzywanski, S., \& Marson, D. C. (2008). Cognitive predictors of medical decision-making capacity in traumatic brain injury. Rehabilitation Psychology, 53(4), 486.

Drummond, D. C. (2001). Theories of drug craving, ancient and modern. Addiction, 96(1), 33-46. 
DuPaul, G. J., \& Volpe, R. J. (2009). ADHD and learning disabilities: Research findings and clinical implications. Current Attention Disorders Reports, 1(4), 152.

Dube, S. R., Felitti, V. J., Dong, M., Chapman, D. P., Giles, W. H., \& Anda, R. F. (2003). Childhood abuse, neglect, and household dysfunction and the risk of illicit drug use: The adverse childhood experiences study. Pediatrics, 111(3), 564-572.

Dublin, S., \& Von Korff, M. (2018). Prescription opioids and infection risk: research and caution needed. Annals of internal medicine, 168(6), 444-445.

Dugas, M. J., Gagnon, F., Ladouceur, R., \& Freeston, M. H. (1998). Generalized anxiety disorder: A preliminary test of a conceptual model. Behaviour Research and Therapy, 36(2), 215-226.

Duncan, I. The Baltimore Sun. (2013, August 27). Heroin dealer gets mandatory term of life without parole. Retrieved from https://www.baltimoresun.com/news/maryland/crime/bs-md-ci-heroin-dealersentence-20130827-story.html

Duncan, C., Jones, K., \& Moon, G. (1996). Health-related behaviour in context: A multilevel modelling approach. Social Science \& Medicine, 42(6), 817-830.

Duncan, G. J., Wilkerson, B., \& England, P. (2006). Cleaning up their act: The effects of marriage and cohabitation on licit and illicit drug use. Demography, 43(4), 691-710.

Dunn, B. D., Galton, H. C., Morgan, R., Evans, D., Oliver, C., Meyer, M., ... \& Dalgleish, T. (2010). Listening to your heart: How interoception shapes emotion experience and intuitive decision making. Psychological Science, 21(12), 1835-1844.

Duncan, G. J., Wilkerson, B., \& England, P. (2006). Cleaning up their act: The effects of marriage and cohabitation on licit and illicit drug use. Demography, 43(4), 691-710.

Dunn, K. E., Barrett, F. S., Yepez-Laubach, C., Meyer, A. C., Hruska, B. J., Petrush, K., ... \& Bigelow, G. E. (2016). Opioid overdose experience, risk behaviors, and knowledge in drug users from a rural versus an urban setting. Journal of Substance Abuse Treatment, 71, 1-7.

Duschek, S., Montoro, C. I., \& Reyes Del Paso, G. A. (2015). Diminished interoceptive awareness in fibromyalgia syndrome. Behav Med, 4289, Oct-2.

Dutra, L., Stathopoulou, G., Basden, S. L., Leyro, T. M., Powers, M. B., \& Otto, M. W. (2008). A metaanalytic review of psychosocial interventions for substance use disorders. American Journal of Psychiatry, 165(2), 179-187.

Duwe, G., \& Clark, V. A. (2017). Nothing will work unless you did: The predictors of postprison employment. Criminal Justice and Behavior, 44(5), 657-677.

Duxbury, S. W. (2018). Information creation on online drug forums: How drug use becomes moral on the margins of science. Current Sociology, 66(3), 431-448.

Ebstein, R. P., \& Belmaker, R. H. (2002). Genetics of sensation or novelty seeking and criminal behavior. In The Neurobiology of Criminal Behavior (pp. 51-78). Boston, MA: Springer.

Eckel, C. C., \& Grossman, P. J. (2008). Men, women and risk aversion: Experimental evidence. Handbook of experimental economics results, 1, 1061-1073.

Edlund, M. J., Martin, B. C., Russo, J. E., DeVries, A., Braden, J. B., \& Sullivan, M. D. (2014). The role of opioid prescription in incident opioid abuse and dependence among individuals with chronic noncancer pain: The role of opioid prescription. The Clinical Journal of Pain, 30(7), 557. 
Ehrlich, I. (1996). Crime, punishment, and the market for offenses. Journal of Economic Perspectives, 10(1), 43-67.

Eide, E., Rubin, P. H., \& Shepherd, J. M. (2006). 2 The economics of criminal behavior. Foundations and Trends in Microeconomics, 2(3), 207-207.

Eidelman, A. I., Hoffmann, N. W., \& Kaitz, M. A. R. S. H. A. (1993). Cognitive deficits in women after childbirth. Obstetrics and Gynecology, 81, 764-764.

Eisenberger, N. I., \& Lieberman, M. D. (2004). Why rejection hurts: A common neural alarm system for physical and social pain. Trends in Cognitive Sciences, 8(7), 294-300.

Eller, R. (2008). Uneven ground: Appalachia since 1945. Lexington, KY: University Press of Kentucky.

Elliott, R., Sahakian, B. J., McKay, A. P., Herrod, J. J., Robbins, T. W., \& Paykel, E. S. (1996). Neuropsychological impairments in unipolar depression: the influence of perceived failure on subsequent performance. Psychological Medicine, 26(5), 975-989.

Elo, A. L., Leppänen, A., \& Jahkola, A. (2003). Validity of a single-item measure of stress symptoms. Scandinavian Journal of Work, Environment \& Health, 444-451.

Elvemo, N. A., Nilsen, K. B., Landrø, N. I., Borchgrevink, P. C., \& Håberg, A. K. (2014). Patients with chronic pain lack somatic markers during decision-making. Journal of Pain Research, 7, 425.

Elwyn, G., Frosch, D., Thomson, R., Joseph-Williams, N., Lloyd, A., Kinnersley, P., ... \& Edwards, A. (2012). Shared decision making: a model for clinical practice. Journal of General Internal Medicine, 27(10), 1361-1367.

Engle, R. W., \& Kane, M. J. (2004). Executive attention, working memory capacity, and a two-factor theory of cognitive control. Psychology of Learning and Motivation, 44, 145-200.

Epstein, D. H., Heilig, M., \& Shaham, Y. (2018). Science-based actions can help address the opioid crisis. Trends in Pharmacological Sciences, 39(11), 911-916.

Epstein, D. H., Preston, K. L., \& Jasinski, D. R. (2006). Abuse liability, behavioral pharmacology, and physical-dependence potential of opioids in humans and laboratory animals: Lessons from tramadol. Biological Psychology, 73(1), 90-99.

Epstein, D. H., Preston, K. L., Stewart, J., \& Shaham, Y. (2006). Toward a model of drug relapse: An assessment of the validity of the reinstatement procedure. Psychopharmacology, 189(1), 1-16.

Epstein, D. H., Willner-Reid, J., Vahabzadeh, M., Mezghanni, M., Lin, J. L., \& Preston, K. L. (2009). Realtime electronic diary reports of cue exposure and mood in the hours before cocaine and heroin craving and use. Archives of General Psychiatry, 66(1), 88-94.

Erickson, W. A., VanLooy, S., von Schrader, S., \& Bruyère, S. M. (2018). Disability, income, and rural poverty. In Disability and vocational rehabilitation in rural settings (pp. 17-41). Springer, Cham.

Ersche, K. D., Clark, L., London, M., Robbins, T. W., \& Sahakian, B. J. (2006). Profile of executive and memory function associated with amphetamine and opiate dependence. Neuropsychopharmacology, 31(5), 1036.

Ersche, K. D., Fletcher, P. C., Lewis, S. J. G., Clark, L., Stocks-Gee, G., London, M., ... \& Sahakian, B. J. (2005). Abnormal frontal activations related to decision-making in current and former amphetamine and opiate dependent individuals. Psychopharmacology, 180(4), 612-623. 
Ersche, K. D., Roiser, J. P., Clark, L., London, M., Robbins, T. W., \& Sahakian, B. J. (2005). Punishment induces risky decision-making in methadone-maintained opiate users but not in heroin users or healthy volunteers. Neuropsychopharmacology, 30(11), 2115.

Ersche, K. D., Roiser, J. P., Robbins, T. W., \& Sahakian, B. J. (2008). Chronic cocaine but not chronic amphetamine use is associated with perseverative responding in humans. Psychopharmacology, 197(3), 421-431.

Ersche, K. D., Turton, A. J., Pradhan, S., Bullmore, E. T., \& Robbins, T. W. (2010). Drug addiction endophenotypes: Impulsive versus sensation-seeking personality traits. Biological Psychiatry, 68(8), 770-773.

Erwin, P. C. (2017). Despair in the American heartland? A focus on rural health. American Journal of Public Health, 107(10), 1533-1534.

Eslami-Shahrbabaki, M., Fekrat, A., \& Mazhari, S. (2015). A study of the prevalence of psychiatric disorders in patients with methamphetamine-induced psychosis. Addiction \& health, 7(1-2), 37.

Etten, M. L. V., Higgins, S. T., Budney, A. J., \& Badger, G. J. (1998). Comparison of the frequency and enjoyability of pleasant events in cocaine abusers vs. non-abusers using a standardized behavioral inventory. Addiction, 93(11), 1669-1680.

Evans, J. S. B. (2008). Dual-processing accounts of reasoning, judgment, and social cognition. Annual Review of Psychology, 59, 255-278.

Evans, J. S. B., \& Stanovich, K. E. (2013). Dual-process theories of higher cognition: Advancing the debate. Perspectives on Psychological Science, 8(3), 223-241.

Evans, C. J., \& Cahill, C. M. (2016). Neurobiology of opioid dependence in creating addiction vulnerability. F1000Research, 5.

Evans, W. N., Lieber, E., \& Power, P. (2018). How the reformulation of OxyContin ignited the heroin epidemic. Review of Economics and Statistics, (00).

Everitt, B. S. (1992). The analysis of contingency tables. Boca Raton, FL. Chapman and Hall/CRC.

Everitt, B. J., \& Robbins, T. W. (2005). Neural systems of reinforcement for drug addiction: From actions to habits to compulsion. Nature Neuroscience, 8(11), 1481.

Everitt, B. J., \& Robbins, T. W. (2016). Drug addiction: Updating actions to habits to compulsions ten years on. Annual Review of Psychology, 67, 23-50.

Fagan, J. (1994). Women and drugs revisited: Female participation in the cocaine economy. Journal of Drug Issues, 24(2), 179-225.

Fagan, J., \& Chin, K. L. (1989). Initiation into crack and cocaine: A tale of two epidemics. Contemporart Drug Problems, 16, 579.

Fagiolini, M., Jensen, C. L., \& Champagne, F. A. (2009). Epigenetic influences on brain development and plasticity. Current Opinion in Neurobiology, 19(2), 207-212.

Falck, R. S., Wang, J., \& Carlson, R. G. (2007). Crack cocaine trajectories among users in a midwestern American city. Addiction, 102(9), 1421-1431. 
Falck, R. S., Siegal, H. A., Wang, J., Carlson, R. G., \& Draus, P. J. (2005). Nonmedical drug use among stimulant-using adults in small towns in rural Ohio. Journal of Substance Abuse Treatment, 28(4), 341-349.

Farrington, D. P. (2018). The need to include biological variables in prospective longitudinal studies of the development of criminal behavior. JAMA pediatrics, 172(2), 118-120.

Felitti, V. J., Anda, R. F., Nordenberg, D., Williamson, D. F., Spitz, A. M., Edwards, V., \& Marks, J. S. (1998). Relationship of childhood abuse and household dysfunction to many of the leading causes of death in adults: The Adverse Childhood Experiences (ACE) Study. American Journal of Preventive Medicine, 14(4), 245-258.

Fellows, L. K. (2006). Deciding how to decide: Ventromedial frontal lobe damage affects information acquisition in multi-attribute decision making. Brain, 129(4), 944-952.

Felson, R. B., \& Staff, J. (2017). Committing economic crime for drug money. Crime \& Delinquency, 63(4), 375-390.

Feng, Y., He, X., Yang, Y., Chao, D., H Lazarus, L., \& Xia, Y. (2012). Current research on opioid receptor function. Current Drug Targets, 13(2), 230-246.

Fenton, T., \& Wiers, R. W. (2017). Free will, black swans and addiction. Neuroethics, 10(1), 157-165.

Farrell, M., \& Marsden, J. (2008). Acute risk of drug-related death among newly released prisoners in England and Wales. Addiction, 103(2), 251-255.

Faul, F., Erdfelder, E., Buchner, A., \& Lang, A.-G. (2009). Statistical power analyses using G*Power 3.1: Tests for correlation and regression analyses. Behavior Research Methods, 41, 1149-1160.

Ferguson, C. J. (2009). An effect size primer: A guide for clinicians and researchers. Professional Psychology: Research and Practice, 40(5), 532.

Fernández-Serrano, M. J., Lozano, Ó., Pérez-García, M., \& Verdejo-García, A. (2010). Impact of severity of drug use on discrete emotions recognition in polysubstance abusers. Drug and Alcohol Dependence, 109(1-3), 57-64.

Fernandez-Serrano, M. J., Pérez-García, M., Schmidt Río-Valle, J., \& Verdejo-Garcia, A. (2010). Neuropsychological consequences of alcohol and drug abuse on different components of executive functions. Journal of Psychopharmacology, 24(9), 1317-1332.

Field, M., Munafò, M. R., \& Franken, I. H. (2009). A meta-analytic investigation of the relationship between attentional bias and subjective craving in substance abuse. Psychological Bulletin, 135(4), 589.

Fink, A. (2015). How to conduct surveys: A step-by-step guide. Thousand Oaks, CA: Sage Publications.

Figner, B., Knoch, D., Johnson, E. J., Krosch, A. R., Lisanby, S. H., Fehr, E., \& Weber, E. U. (2010). Lateral prefrontal cortex and self-control in intertemporal choice. Nature Neuroscience, 13(5), 538.

Figner, B., Mackinlay, R. J., Wilkening, F., \& Weber, E. U. (2009). Affective and deliberative processes in risky choice: Age differences in risk taking in the Columbia Card Task. Journal of Experimental Psychology: Learning, Memory, and Cognition, 35(3), 709.

Figner, B., \& Weber, E. U. (2011). Who takes risks when and why? Determinants of risk taking. Current Directions in Psychological Science, 20(4), 211-216. 
Finch, K. A., Ramo, D. E., Delucchi, K. L., Liu, H., \& Prochaska, J. J. (2013). Subjective social status and substance use severity in a young adult sample. Psychology of Addictive Behaviors, 27(3), 901.

Fine, P. G. (2011). Long-term consequences of chronic pain: Mounting evidence for pain as a neurological disease and parallels with other chronic disease states. Pain Medicine, 12(7), 996-1004.

Fiske, S. T., \& Taylor, S. E. (2013). Social cognition: From brains to culture. Thousand Oaks, CA: Sage Publications.

Fischer, B., Fischer, B., Patra, J., Fischer, B., Patra, J., Firestone Cruz, M., ... \& Fischer, B. (2008). Comparing heroin users and prescription opioid users in a Canadian multi-site population of illicit opioid users. Drug and Alcohol Review, 27(6), 625-632.

Fjorback, L. O., Arendt, M., Ørnbøl, E., Fink, P., \& Walach, H. (2011). Mindfulness-based stress reduction and mindfulness-based cognitive therapy-A systematic review of randomized controlled trials. Acta Psychiatrica Scandinavica, 124(2), 102-119.

Flake, D. F. (2015). When any sentence is a life sentence: Employment discrimination against exoffenders. Washington University Law Review, 93, 45.

Flanagan, O. (2016). Willing Addicts? Drinkers, dandies, druggies and other Dionysians. Addiction and Choice: Rethinking the Relationship, 66-81.

Fleming, C. B., White, H. R., \& Catalano, R. F. (2010). Romantic relationships and substance use in early adulthood: An examination of the influences of relationship type, partner substance use, and relationship quality. Journal of Health and Social Behavior, 5l(2), 153-167.

Florence, C., Luo, F., Xu, L., \& Zhou, C. (2016). The economic burden of prescription opioid overdose, abuse and dependence in the United States, 2013. Medical Care, 54(10), 901.

Foucault, M. (2003). Madness and civilization. Routledge.

Foucault, M. (2012). Discipline and punish: The birth of the prison. Vintage.

Fox, H. C., Axelrod, S. R., Paliwal, P., Sleeper, J., \& Sinha, R. (2007). Difficulties in emotion regulation and impulse control during cocaine abstinence. Drug and Alcohol Dependence, 89(2-3), 298-301.

Fox, H. C., Bergquist, K. L., Hong, K. I., \& Sinha, R. (2007). Stress-induced and alcohol cue-induced craving in recently abstinent alcohol-dependent individuals. Alcoholism: Clinical and Experimental Research, 31(3), 395-403.

Fox, H. C., Hong, K. A., \& Sinha, R. (2008). Difficulties in emotion regulation and impulse control in recently abstinent alcoholics compared with social drinkers. Addictive Behaviors, 33(2), 388-394.

Fox, A. D., Maradiaga, J., Weiss, L., Sanchez, J., Starrels, J. L., \& Cunningham, C. O. (2015). Release from incarceration, relapse to opioid use and the potential for buprenorphine maintenance treatment: A qualitative study of the perceptions of former inmates with opioid use disorder. Addiction Science \& Clinical Practice, 10(1), 2.

Fox, H. C., \& Sinha, R. (2009). Sex differences in drug-related stress-system changes: Implications for treatment in substance-abusing women. Harvard Review of Psychiatry, 17(2), 103-119.

Franken, I. H. (2003). Drug craving and addiction: Integrating psychological and neuropsychopharmacological approaches. Progress in Neuro-Psychopharmacology and Biological Psychiatry, 27(4), 563-579. 
Franken, I. H., Kroon, L. Y., Wiers, R. W., \& Jansen, A. (2000). Selective cognitive processing of drug cues in heroin dependence. Journal of Psychopharmacology, 14(4), 395-400.

Frankenhuis, W. E., Panchanathan, K., \& Nettle, D. (2016). Cognition in harsh and unpredictable environments. Current Opinion in Psychology, 7, 76-80.

Frazer, K. M., Richards, Q., \& Keith, D. R. (2018). The long-term effects of cocaine use on cognitive functioning: A systematic critical review. Behavioural brain research.

Franzini, L., Ribble, J., \& Spears, W. (2001). The effects of income inequality and income level on mortality vary by population size in Texas counties. Journal of health and Social Behavior, 373387.

Freudenberg, N., Wilets, I., Greene, M. B., \& Richie, B. E. (1998). Linking women in jail to community services: Factors associated with rearrest and retention of drug-using women following release from jail. Jamwa, 53(2), 89-93.

Fry, C. E., Langley, K., \& Shelton, K. H. (2017). A systematic review of cognitive functioning among young people who have experienced homelessness, foster care, or poverty. Child Neuropsychology, 23(8), 907-934.

Frederick, S. (2005). Cognitive reflection and decision making. Journal of Economic Perspectives, 19(4), $25-42$.

Frederick, S., Loewenstein, G., \& O'donoghue, T. (2002). Time discounting and time preference: A critical review. Journal of Economic Literature, 40(2), 351-401.

Freedman, E. M. (2007). Introduction: Re-stating the standard of practice for death penalty counsel: The supplementary guidelines for the mitigation function of defense teams in death penalty cases. Hofstra L. Rev., 36, 663.

Freeman, S. F., \& Alkin, M. C. (2000). Academic and social attainments of children with mental retardation in general education and special education settings. Remedial and Special Education, 21(1), 3-26.

Freeman, P. R., Goodin, A., Troske, S., \& Talbert, J. (2015). Kentucky House Bill 1 impact evaluation. Institute for Pharmaceutical Outcomes and Policy: University of Kentucky.

Freese, T. E., Miotto, K., \& Reback, C. J. (2002). The effects and consequences of selected club drugs. Journal of Substance Abuse Treatment, 23(2), 151-156.

Friedmann, P. D., Rhodes, A. G., Taxman, F. S., \& Step'n Out Research Group of CJ-DATS. (2009). Collaborative behavioral management: Integration and intensification of parole and outpatient addiction treatment services in the Step'n Out study. Journal of Experimental Criminology, 5(3), 227-243.

Fritz, M., El Rawas, R., Salti, A., Klement, S., Bardo, M. T., Kemmler, G., ... \& Zernig, G. (2011). Reversal of cocaine-conditioned place preference and mesocorticolimbic Zif268 expression by social interaction in rats. Addiction Biology, 16(2), 273-284.

Fu, L. P., Bi, G. H., Zou, Z. T., Wang, Y., Ye, E. M., Ma, L., \& Yang, Z. (2008). Impaired response inhibition function in abstinent heroin dependents: An fMRI study. Neuroscience Letters, 438(3), 322-326. 
Fuchs, C., \& Diamantopoulos, A. (2009). Using single-item measures for construct measurement in management research: Conceptual issues and application guidelines. Die

Betriebswirtschaft, 69(2), 195.

Furnari, M., Epstein, D. H., Phillips, K. A., Jobes, M. L., Kowalczyk, W. J., Vahabzadeh, M., ... \& Preston, K. L. (2015). Some of the people, some of the time: Field evidence for associations and dissociations between stress and drug use. Psychopharmacology, 232(19), 3529-3537.

Furr-Holden, C. D. M., Campbell, K. D. M., Milam, A. J., Smart, M. J., Ialongo, N. A., \& Leaf, P. J. (2010). Metric properties of the Neighborhood Inventory for Environmental Typology (NIfETy): An environmental assessment tool for measuring indicators of violence, alcohol, tobacco, and other drug exposures. Evaluation Review, 34(3), 159-184.

Furst, R. T. (2013). Suboxone misuse along the opiate maintenance treatment pathway. Journal of Addictive Diseases, 32(1), 53-67.

Furst, R. T., D. Johnson, B., Dunlap, E., \& Curtis, R. (1999). The stigmatized image of the "crack head": A sociocultural exploration of a barrier to cocaine smoking among a cohort of youth in New York City. Deviant Behavior, 20(2), 153-181.

Füstös, J., Gramann, K., Herbert, B. M., \& Pollatos, O. (2012). On the embodiment of emotion regulation: Interoceptive awareness facilitates reappraisal. Social Cognitive andAaffective Neuroscience, $8(8)$, 911-917.

Galea, S., Ahern, J., \& Vlahov, D. (2003). Contextual determinants of drug use risk behavior: A theoretic framework. Journal of Urban Health, 80(3), iii50-iii58.

Galea, S., Hall, C., \& Kaplan, G. A. (2009). Social epidemiology and complex system dynamic modelling as applied to health behaviour and drug use research. International Journal of Drug Policy, 20(3), 209-216.

Galea, S., \& Vlahov, D. (2002). Social determinants and the health of drug users: Socioeconomic status, homelessness, and incarceration. Public Health Reports, 117(Suppl 1), S135.

Galinato, M. H., Takashima, Y., Fannon, M. J., Quach, L. W., Silva, R. J. M., Mysore, K. K., ... \& Mandyam, C. D. (2018). Neurogenesis during abstinence is necessary for context-driven methamphetamine-related memory. Journal of Neuroscience, 38(8), 2029-2042.

Galizio, M., \& Stein, F. S. (1983). Sensation seeking and drug choice. International Journal of the Addictions, 18(8), 1039-1048.

Gallet, C. A. (2014). Can price get the monkey off our back? A meta-analysis of illicit drug demand. Health Economics, 23(1), 55-68.

Gardner, B. (2015). A review and analysis of the use of 'habit'in understanding, predicting and influencing health-related behaviour. Health Psychology Review, 9(3), 277-295.

Gardner, D. G., Cummings, L. L., Dunham, R. B., \& Pierce, J. L. (1998). Single-item versus multiple-item measurement scales: An empirical comparison. Educational and Psychological Measurement, 58(6), 898-915.

Gardner, M., \& Steinberg, L. (2005). Peer influence on risk taking, risk preference, and risky decision making in adolescence and adulthood: An experimental study. Developmental Psychology, 41(4), 625 . 
Garfinkel, S. N., \& Critchley, H. D. (2016). Threat and the body: How the heart supports fear processing. Trends in Cognitive Sciences, 20(1), 34-46.

Garland, E. L., Franken, I. H., \& Howard, M. O. (2012). Cue-elicited heart rate variability and attentional bias predict alcohol relapse following treatment. Psychopharmacology, 222(1), 17-26.

Garland, E. L., Froeliger, B., Zeidan, F., Partin, K., \& Howard, M. O. (2013). The downward spiral of chronic pain, prescription opioid misuse, and addiction: Cognitive, affective, and neuropsychopharmacologic pathways. Neuroscience \& Biobehavioral Reviews, 37(10), 25972607.

Garland, E. L., Brown, S. M., \& Howard, M. O. (2016). Thought suppression as a mediator of the association between depressed mood and prescription opioid craving among chronic pain patients. Journal of Behavioral Medicine, 39(1), 128-138.

Garoupa, N. (2003). Behavioral economic analysis of crime: A critical review. European Journal of Law and Economics, 15(1), 5-15.

Garriott, W. (2016). Methamphetamine and the changing rhetoric of drugs in the United States. Routledge International Handbook of Rural Criminology, 275-284.

Gasior, M., Bond, M., \& Malamut, R. (2016). Routes of abuse of prescription opioid analgesics: A review and assessment of the potential impact of abuse-deterrent formulations. Postgraduate Medicine, 128(1), 85-96.

Gawin, F. H. (1991). Cocaine addiction: Psychology and Neurophysiology. Science, 251(5001), 15801586.

Gawin, F. H., Allen, D., \& Humblestone, B. (1989). Outpatient treatment of 'crack' cocaine smoking with flupenthixol decanoate: A preliminary report. Archives of General Psychiatry, 46(4), 322-325.

Geisler, F. C., Kleinfeldt, A., \& Kubiak, T. (2016). Restrained eating predicts effortful self-control as indicated by heart rate variability during food exposure. Appetite, 96, 502-508.

George, O., Le Moal, M., \& Koob, G. F. (2012). Allostasis and addiction: Role of the dopamine and corticotropin-releasing factor systems. Physiology \& Behavior, 106(1), 58-64.

Gershon, J., \& Gershon, J. (2002). A meta-analytic review of gender differences in ADHD. Journal of Attention Disorders, 5(3), 143-154.

Gerst, K. R., Gunn, R. L., \& Finn, P. R. (2017). Delay discounting of losses in alcohol use disorders and antisocial psychopathology: Effects of a working memory load. Alcoholism: Clinical and Experimental Research, 41(10), 1768-1774.

Gesquiere, L. R., Learn, N. H., Simao, M. C. M., Onyango, P. O., Alberts, S. C., \& Altmann, J. (2011). Life at the top: Rank and stress in wild male baboons. Science, 333(6040), 357-360.

Gfroerer, J., \& Brodsky, M. (1992). The incidence of illicit drug use in the United States, 1962 1989. British Journal of Addiction, 87(9), 1345-1351.

Gfroerer, J. C., Larson, S. L., \& Colliver, J. D. (2007). Drug use patterns and trends in rural communities. The Journal of Rural Health, 23, 10-15.

Gibson, D. R., Leamon, M. H., \& Flynn, N. (2002). Epidemiology and public health consequences of methamphetamine use in California's Central Valley. Journal of psychoactive drugs, 34(3), 313319. 
Giordano, L. A., Bickel, W. K., Loewenstein, G., Jacobs, E. A., Marsch, L., \& Badger, G. J. (2002). Mild opioid deprivation increases the degree that opioid-dependent outpatients discount delayed heroin and money. Psychopharmacology, 163(2), 174-182.

Gilbert, M., \& Dasgupta, N. (2017). Silicon to syringe: Cryptomarkets and disruptive innovation in opioid supply chains. International Journal of Drug Policy, 46, 160-167.

Gilman, J. M., Smith, A. R., Ramchandani, V. A., Momenan, R., \& Hommer, D. W. (2012). The effect of intravenous alcohol on the neural correlates of risky decision making in healthy social drinkers. Addiction Biology, 17(2), 465-478.

Giordano, L. A., Bickel, W. K., Loewenstein, G., Jacobs, E. A., Marsch, L., \& Badger, G. J. (2002). Mild opioid deprivation increases the degree that opioid-dependent outpatients discount delayed heroin and money. Psychopharmacology, 163(2), 174-182.

Gladden, R. M. (2016). Fentanyl law enforcement submissions and increases in synthetic opioid-involved overdose deaths-27 states, 2013-2014. MMWR. Morbidity and Mortality Weekly Report, 65.

Gliem, J. A., \& Gliem, R. R. (2003). Calculating, interpreting, and reporting Cronbach's alpha reliability coefficient for Likert-type scales. Midwest Research-to-Practice Conference in Adult, Continuing, and Community Education.

Gluhoski, V. L. (1995). A cognitive perspective on bereavement: Mechanisms and treatment. Journal of Cognitive Psychotherapy, 9(2), 75.

Gogtay, N., Giedd, J. N., Lusk, L., Hayashi, K. M., Greenstein, D., Vaituzis, A. C., ... \& Rapoport, J. L. (2004). Dynamic mapping of human cortical development during childhood through early adulthood. Proceedings of the National Academy of Sciences, 101(21), 8174-8179.

Gohier, B., Ferracci, L., Surguladze, S. A., Lawrence, E., El Hage, W., Kefi, M. Z., ... \& Le Gall, D. (2009). Cognitive inhibition and working memory in unipolar depression. Journal of Affective Disorders, 116(1-2), 100-105.

Goldberg, D. S., \& McGee, S. J. (2011). Pain as a global public health priority. BMC Public Health, 11(1), 770.

Golder, S., Engstrom, M., Hall, M. T., Higgins, G. E., \& Logan, T. K. (2015). Psychological distress among victimized women on probation and parole: A latent class analysis. American Journal of Orthopsychiatry, 85(4), 382.

Golder, S., Hall, M. T., Logan, T. K., Higgins, G. E., Dishon, A., Renn, T., \& Winham, K. M. (2014). Substance use among victimized women on probation and parole. Substance Use \& Misuse, 49(4), 435-447.

Goldstein, R. Z., Bechara, A., Garavan, H., Childress, A. R., Paulus, M. P., \& Volkow, N. D. (2009). The neurocircuitry of impaired insight in drug addiction. Trends in Cognitive Sciences, 13(9), 372-380.

Gonzalez, R., Bechara, A., \& Martin, E. M. (2007). Executive functions among individuals with methamphetamine or alcohol as drugs of choice: Preliminary observations. Journal of Clinical and Experimental Neuropsychology, 29(2), 155-159.

Gonzalez, J. M. R., Walters, S. T., Lerch, J., \& Taxman, F. S. (2015). The relationship between drug use, drug-related arrests, and chronic pain among adults on probation. Journal of Substance Abuse Treatment, 53, 33-38. 
Goodlad, J. K., Marcus, D. K., \& Fulton, J. J. (2013). Lead and attention-deficit/hyperactivity disorder (ADHD) symptoms: A meta-analysis. Clinical Psychology Review, 33(3), 417-425.

Goodman, E., Huang, B., Wade, T. J., \& Kahn, R. S. (2003). A multilevel analysis of the relation of socioeconomic status to adolescent depressive symptoms: Does school context matter?. The Journal of Pediatrics, 143(4), 451-456.

Gorelick, D. A., Zangen, A., \& George, M. S. (2014). Transcranial magnetic stimulation in the treatment of substance addiction. Annals of the New York Academy of Sciences, 1327(1), 79-93

Goudie, A. J., Sumnall, H. R., Field, M., Clayton, H., \& Cole, J. C. (2007). The effects of price and perceived quality on the behavioural economics of alcohol, amphetamine, cannabis, cocaine, and ecstasy purchases. Drug and Alcohol Dependence, 89(2-3), 107-115.

Gourley, S. L., \& Taylor, J. R. (2016). Going and stopping: Dichotomies in behavioral control by the prefrontal cortex. Nature Neuroscience, 19(5), 656.

Goymann, W., \& Wingfield, J. C. (2004). Allostatic load, social status and stress hormones: The costs of social status matter. Animal Behaviour, 67(3), 591-602.

Graybiel, A. M. (2008). Habits, rituals, and the evaluative brain. Annual Review of Neuroscience.,31, 359387.

Graham, M. H. (2003). Confronting multicollinearity in ecological multiple regression. Ecology, 84(11), 2809-2815.

Graham, K. (2010). Sorry seems to be the hardest word: The Fair Sentencing Act of 2010, crack, and methamphetamine. University of Richmond Law Review, 45, 765.

Graif, C., Gladfelter, A. S., \& Matthews, S. A. (2014). Urban poverty and neighborhood effects on crime: Incorporating spatial and network perspectives. Sociology Compass, 8(9), 1140-1155.

Grant, J. (2007). Rural women's stories of recovery from addiction. Addiction Research \& Theory, 15(5), 521-541.

Grant, B. F., Stinson, F. S., Dawson, D. A., Chou, S. P., Dufour, M. C., Compton, W., ... \& Kaplan, K. (2004). Prevalence and co-occurrence of substance use disorders and independentmood and anxiety disorders: Results from the national epidemiologic survey on alcohol and relatedconditions. Archives of General Psychiatry, 61(8), 807-816.

Grant, B. F., \& Dawson, D. A. (1998). Age of onset of drug use and its association with DSM-IV drug abuse and dependence: Results from the National Longitudinal Alcohol Epidemiologic Survey. Journal of Substance Abuse, 10(2), 163-173.

Grant, S., Contoreggi, C., \& London, E. D. (2000). Drug abusers show impaired performance in a laboratory test of decision making. Neuropsychologia, 38(8), 1180-1187.

Grant, S., Contoreggi, C., \& London, E. D. (2000). Drug abusers show impaired performance in a laboratory test of decision making. Neuropsychologia, 38(8), 1180-1187.

Gray, J. C., MacKillop, J., Weafer, J., Hernandez, K. M., Gao, J., Palmer, A. A., \& de Wit, H. (2018). Genetic analysis of impulsive personality traits: Examination of a priori candidates and genomewide variation. Psychiatry Research, 259, 398-404.

Green, A., Pickering, H., Foster, R., Power, R., \& Stimson, G. V. (1994). Who uses cocaine? Social profiles of cocaine users. Addiction Research, 2(2), 141-154. 
Green, T. C., Bowman, S. E., Ray, M., Zaller, N., Heimer, R., \& Case, P. (2013). Collaboration or coercion? Partnering to divert prescription opioid medications. Journal of Urban Health, 90(4), 758-767.

Green, T. C., \& Gilbert, M. (2016). Counterfeit medications and fentanyl. JAMA Internal Medicine, 176(10), 1555-1557.

Green, L., Myerson, J., \& Ostaszewski, P. (1999). Discounting of delayed rewards across the life span: Age differences in individual discounting functions. Behavioural Processes, 46(1), 89-96.

Green, L., Myerson, J., Lichtman, D., Rosen, S., \& Fry, A. (1996). Temporal discounting in choice between delayed rewards: The role of age and income. Psychology and Aging, 11(1), 79.

Greenberg, P. (2018). Coal waste, socioeconomic change, and environmental inequality in appalachia: Implications for a just transition in coal country. Society \& Natural Resources, 31(9), 995-1011.

Greenstadt, L., Shapiro, D., \& Whitehead, R. (1986). Blood pressure discrimination. Psychophysiology, 23(5), 500-509.

Greenwald, M. K. (2008a). Behavioral economic analysis of drug preference using multiple choice procedure data. Drug and Alcohol Dependence, 93(1-2), 103-110.

Greenwald, M. K. (2008b). Opioid abstinence reinforcement delays heroin lapse during buprenorphine dose tapering. Journal of Applied Behavior Analysis, 41(4), 603-607.

Greenwald, M. K. (2010). Effects of experimental unemployment, employment and punishment analogs on opioid seeking and consumption in heroin-dependent volunteers. Drug and Alcohol Dependence, 111(1-2), 64-73.

Greenwald, M. K., \& Hursh, S. R. (2006). Behavioral economic analysis of opioid consumption in heroindependent individuals: Effects of unit price and pre-session drug supply. Drug and Alcohol Dependence, 85(1), 35-48.

Greenwald, M. K., \& Steinmiller, C. L. (2009). Behavioral economic analysis of opioid consumption in heroin-dependent individuals: Effects of alternative reinforcer magnitude and post-session drug supply. Drug and Alcohol Dependence, 104(1-2), 84-93.

Greenwald, M. K., \& Steinmiller, C. L. (2014). Cocaine behavioral economics: From the naturalistic environment to the controlled laboratory setting. Drug and Alcohol Dependence, 141, $27-33$.

Grella, C. E., Stein, J. A., \& Greenwell, L. (2005). Associations among childhood trauma, adolescent problem behaviors, and adverse adult outcomes in substance-abusing women offenders. Psychology of Addictive Behaviors, 19(1), 43.

Greely, H., Sahakian, B., Harris, J., Kessler, R. C., Gazzaniga, M., Campbell, P., \& Farah, M. J. (2008). Towards responsible use of cognitive-enhancing drugs by the healthy. Nature, 456(7223), 702.

Greve, K. W., Sherwin, E., Stanford, M. S., Mathias, C., Love, J., \& Ramzinski, P. (2001). Personality and neurocognitive correlates of impulsive aggression in long-term survivors of severe traumatic brain injury. Brain Injury, 15(3), 255-262.

Griskevicius, V., Ackerman, J. M., Cantú, S. M., Delton, A. W., Robertson, T. E., Simpson, J. A., ... \& Tybur, J. M. (2013). When the economy falters, do people spend or save? Responses to resource scarcity depend on childhood environments. Psychological Science, 24(2), 197-205. 
Griskevicius, V., Tybur, J. M., Delton, A. W., \& Robertson, T. E. (2011). The influence of mortality and socioeconomic status on risk and delayed rewards: A life history theory approach. Journal of Personality and Social Psychology, 100(6), 1015.

Gruber, E., DiClemente, R. J., Anderson, M. M., \& Lodico, M. (1996). Early drinking onset and its association with alcohol use and problem behavior in late adolescence. Preventive Medicine, 25(3), 293-300.

Guan, Y., \& He, J. (2018). The effect of state self-control on the intertemporal decisions made by individuals with high and low trait self-control. PloS One, 13(4), e0195333.

Guerri, C., \& Pascual, M. (2010). Mechanisms involved in the neurotoxic, cognitive, and neurobehavioral effects of alcohol consumption during adolescence. Alcohol, 44(1), 15-26.

Guindalini, C., Vallada, H., Breen, G., \& Laranjeira, R. (2006). Concurrent crack and powder cocaine users from Sao Paulo: Do they represent a different group?. BMC Public Health, 6(1), 10.

Gunn, R. L., \& Finn, P. R. (2013). Impulsivity partially mediates the association between reduced working memory capacity and alcohol problems. Alcohol, 47(1), 3-8.

Gullone, E., Moore, S., Moss, S., \& Boyd, C. (2000). The adolescent risk-taking questionnaire: Development and psychometric evaluation. Journal of Adolescent Research, 15(2), 231-250.

Gupta, A., Mayer, E. A., Fling, C., Labus, J. S., Naliboff, B. D., Hong, J. Y., \& Kilpatrick, L. A. (2017). Sex-based differences in brain alterations across chronic pain conditions. Journal of Neuroscience Research, 95(1-2), 604-616.

Gurusami, S. (2017). Working for redemption: Formerly incarcerated Black women and punishment in the labor market. Gender \& Society, 31(4), 433-456.

Guzman, D., \& Ettenberg, A. (2004). Heroin attenuates the negative consequences of cocaine in a runway model of self-administration. Pharmacology Biochemistry and Behavior, 79(2), 317-324.

Gwin-Mitchell, S., Kelly, S. M., Brown, B. S., Schacht Reisinger, H., Peterson, J. A., Ruhf, A., ... \& Schwartz, R. P. (2009). Uses of diverted methadone and buprenorphine by opioid-addicted individuals in Baltimore, Maryland. American Journal on Addictions, 18(5), 346-355.

Haas, A. L., \& Peters, R. H. (2000). Development of substance abuse problems among drug-involved offenders: Evidence for the telescoping effect. Journal of Substance Abuse, 12(3), 241-253.

Habecker, P., Welch-Lazoritz, M., \& Dombrowski, K. (2018). Rural and Urban Differences in Nebraskans' Access to Marijuana, Methamphetamine, Heroin, and Prescription Pills. Journal of Drug Issues, 48(4), 608-624.

Hackman, D. A., \& Farah, M. J. (2009). Socioeconomic status and the developing brain. Trends in Cognitive Sciences, 13(2), 65-73.

Hackman, D. A., Farah, M. J., \& Meaney, M. J. (2010). Socioeconomic status and the brain: mechanistic insights from human and animal research. Nature Reviews Neuroscience, 11(9), 651.

Hagerty, B. M., \& Williams, A. (1999). The effects of sense of belonging, social support, conflict, and loneliness on depression. Nursing Research, 48(4), 215-219.

Hair, J. F. Jr., Anderson, R. E., Tatham, R. L. \& Black, W. C. (1995). Multivariate data analysis (3rd ed). New York: Macmillan. 
Halkovic, A., Fine, M., Bae, J., Campbell, L., Evans, D., Gary, C., ... \& Tebout, R. (2013). Higher Education and Reentry: The Gifts They Bring. Reentry Research in the First Person. Prisoner Reentry Institute.

Hall, M. T., Ball, D., Sears, J., Higgins, G. E., Logan, T. K., \& Golder, S. (2018). Past-year nonmedical use of prescription drugs among women on probation and parole: A cross-sectional study. Substance Abuse, 1-6.

Hall, M. T., Golder, S., Higgins, G. E., \& Logan, T. K. (2016). Nonmedical prescription opioid use among victimized women on probation and parole. Addictive Behaviors, 53, 113-119.

Hall M. T., Leukefeld C. G., \& Havens J. R., 2013. Factors associated with high-frequency illicit methadone use among rural Appalachian drug users. American Journal of Drug and Alcohol Abuse, 39(4), 241-246.

Hall, W., \& Lynskey, M. (2016). Evaluating the public health impacts of legalizing recreational cannabis use in the United States. Addiction, 111(10), 1764-1773.

Hall, W. (2015). What has research over the past two decades revealed about the adverse health effects of recreational cannabis use? Addiction, 110(1), 19-35.

Hall, W., Carter, A., \& Forlini, C. (2015). The brain disease model of addiction: Is it supported by the evidence and has it delivered on its promises? The Lancet Psychiatry, 2(1), 105-110.

Hammersley, R. (2018) Sociology of Addiction In Pickard, H., \& Ahmed, S. H. (Eds.), The Routledge handbook of philosophy and science of addiction. Routledge (220-228). New York, NY.

Han, B., Gfroerer, J. C., Colliver, J. D., \& Penne, M. A. (2009). Substance use disorder among older adults in the United States in 2020. Addiction, 104(1), 88-96.

Han, G., Klimes-Dougan, B., Jepsen, S., Ballard, K., Nelson, M., Houri, A., ... \& Cullen, K. (2012). Selective neurocognitive impairments in adolescents with major depressive disorder. Journal of Adolescence, 35(1), 11-20.

Haney, C. (1994). Social context of capital murder: Social histories and the logic of mitigation. Santa Clara L. Rev., 35, 547.

Haney, C. (2007). Evolving standards of decency: Advancing the nature and logic of capital mitigation. Hofstra L. Rev., 36, 835.

Harding, D. J., Wyse, J. J., Dobson, C., \& Morenoff, J. D. (2014). Making ends meet after prison. Journal of Policy Analysis and Management, 33(2), 440-470.

Hardisty, D. J., Appelt, K. C., \& Weber, E. U. (2013). Good or bad, we want it now: Fixed-cost present bias for gains and losses explains magnitude asymmetries in intertemporal choice. Journal of Behavioral Decision Making, 26(4), 348-361.

Harlé, K. M., Allen, J. J., \& Sanfey, A. G. (2010). The impact of depression on social economic decision making. Journal of Abnormal Psychology, 119(2), 440.

Harris, L. T., \& Fiske, S. T. (2007). Social groups that elicit disgust are differentially processed in mPFC. Social Cognitive and Affective Neuroscience, 2(1), 45-51.

Harrell, P. T., Mancha, B. E., Petras, H., Trenz, R. C., \& Latimer, W. W. (2012). Latent classes of heroin and cocaine users predict unique HIV/HCV risk factors. Drug and Alcohol Dependence, 122(3), 220-227. 
Harris, A. M. (2016). Increases in acute hepatitis B virus infections-Kentucky, Tennessee, and West Virginia, 2006-2013. MMWR. Morbidity and Mortality Weekly Report, 65.

Harvey, P. O., Le Bastard, G., Pochon, J. B., Levy, R., Allilaire, J. F., Dubois, B. E. E. A., \& Fossati, P. (2004). Executive functions and updating of the contents of working memory in unipolar depression. Journal of Psychiatric Research, 38(6), 567-576.

Hartman, D. M., \& Golub, A. (1999). The social construction of the crack epidemic in the print media. Journal of Psychoactive Drugs, 31(4), 423-433.

Hartigan, J. (2013). Who are these White people?: "rednecks," "hillbillies," and "white trash" as marked racial subjects. In White out (pp. 100-116). Routledge.

Hathaway, A. D., Mostaghim, A., Erickson, P. G., Kolar, K., \& Osborne, G. (2018). "It's really no big deal": The role of social supply networks in normalizing use of cannabis by students at Canadian universities. Deviant Behavior, 39(12), 1672-1680.

Hatsukami, D. K., \& Fischman, M. W. (1996). Crack cocaine and cocaine hydrochloride: Are the differences myth or reality? Jama, 276(19), 1580-1588.

Hatzenbuehler, M. L. (2017). Structural stigma and health. The Oxford Handbook of Stigma, Discrimination, and Health, 105.

Haushofer, J., Schunk, D., \& Fehr, E. (2013). Negative income shocks increase discount rates. University of Zurich Working Paper.[aGVP].

Havassy, B. E., Hall, S. M., \& Wasserman, D. A. (1991). Social support and relapse: Commonalities among alcoholics, opiate users, and cigarette smokers. Addictive Behaviors, 16(5), 235-246.

Havens, J. R., Leukefeld, C. G., DeVeaugh-Geiss, A. M., Coplan, P., \& Chilcoat, H. D. (2014). The impact of a reformulation of extended-release oxycodone designed to deter abuse in a sample of prescription opioid abusers. Drug and Alcohol Dependence, 139, 9-17.

Havens, J. R., Oser, C. B., Knudsen, H. K., Lofwall, M., Stoops, W. W., Walsh, S. L., ... \& Kral, A. H. (2011). Individual and network factors associated with non-fatal overdose among rural Appalachian drug users. Drug and Alcohol Dependence, 115(1-2), 107-112.

Havens, J. R., Oser, C. B., Leukefeld, C. G., Webster, J. M., Martin, S. S., O'Connell, D. J., ... \& Inciardi, J. A. (2007). Differences in prevalence of prescription opiate misuse among rural and urban probationers. The American Journal of Drug and Alcohol Abuse, 33(2), 309-317.

Havens, J. R., Oser, C. B., \& Leukefeld, C. G. (2011). Injection risk behaviors among rural drug users: Implications for HIV prevention. AIDS Care, 23(5), 638-645.

Havens, J. R., Stoops, W. W., Leukefeld, C. G., Garrity, T. F., Carlson, R. G., Falck, R., ... \& Booth, B. M. (2009). Prescription opiate misuse among rural stimulant users in a multistate community-based study. The American Journal of Drug and Alcohol Abuse, 35(1), 18-23.

Havens, J. R., Talbert, J. C., Robert, W., Cynthia, L., \& Leukefeld, C. G. (2006). Trends in controlledrelease oxycodone (OxyContin $\left.{ }^{\circledR}\right)$ prescribing among medicaid recipients in Kentucky, 19982002. The Journal of Rural Health, 22(3), 276-278.

Havens, J. R., Young, A. M., \& Havens, C. E. (2011). Nonmedical prescription drug use in a nationally representative sample of adolescents: Evidence of greater use among rural adolescents. Archives of Pediatrics \& Adolescent Medicine, 165(3), 250-255. 
Havens, J. R., Walker, R., \& Leukefeld, C. G. (2007). Prevalence of opioid analgesic injection among rural nonmedical opioid analgesic users. Drug and Alcohol Dependence, 87(1), 98-102.

Hawdon, J. E. (2001). The role of presidential rhetoric in the creation of a moral panic: Reagan, Bush, and the war on drugs. Deviant Behavior, 22(5), 419-445.

Hawkins, D. M. (1980). Identification of outliers (Vol. 11). London: Chapman and Hall.

Hayes, L. A. (2018). Mobile and temporary: Women and workplace precarity in Appalachian Kentucky. Journal of Appalachian Studies, 24(1), 26-44.

He, Z., Cassaday, H. J., Howard, R. C., Khalifa, N., \& Bonardi, C. (2011). Impaired Pavlovian conditioned inhibition in offenders with personality disorders. The Quarterly Journal of Experimental Psychology, 64(12), 2334-2351.

Heale, P., Dietze, P., \& Fry, C. (2003). Intentional overdose among heroin overdose survivors. Journal of Urban Health, 80(2), 230-237.

Heather, N., \& Vuchinich, R. E. (Eds.). (2003). Choice, behavioural economics and addiction. Oxford, UK. Elsevier.

Hedegaard, H., Warner, M., \& Miniño, A. M. (2017). Drug overdose deaths in the United States, 19992016.

Heil, S. H., Johnson, M. W., Higgins, S. T., \& Bickel, W. K. (2006). Delay discounting in currently using and currently abstinent cocaine-dependent outpatients and non-drug-using matched controls. Addictive Behaviors, 31(7), 1290-1294.

Heilig, M., Epstein, D. H., Nader, M. A., \& Shaham, Y. (2016). Time to connect: Bringing social context into addiction neuroscience. Nature Reviews Neuroscience, 17(9), 592.

Heinrichs, M., Baumgartner, T., Kirschbaum, C., \& Ehlert, U. (2003). Social support and oxytocin interact to suppress cortisol and subjective responses to psychosocial stress. Biological Psychiatry, 54(12), 1389-1398.

Hendryx, M. (2010). Poverty and mortality disparities in central Appalachia: Mountaintop mining and environmental justice. Journal of Health Disparities Research and Practice, 4(3), 6.

Henry, B. L., Minassian, A., \& Perry, W. (2010). Effect of methamphetamine dependence on everyday functional ability. Addictive Behaviors, 35(6), 593-598.

Hebert, J. R., Ma, Y., Clemow, L., Ockene, I. S., Saperia, G., Stanek III, E. J., ... \& Ockene, J. K. (1997). Gender differences in social desirability and social approval bias in dietary self-report. American Journal of Epidemiology, 146(12), 1046-1055.

Herath, J., \& Brown, C. (2013). An analysis of adult obesity and hypertension in Appalachia. Global Journal of Health Science, 5(3), 127.

Hernandez, Y., Meyers-Ohki, S., Farkas, S., \& Rotrosen, J. (2018). How Massachusetts, Vermont, and New York are taking action to address the opioid epidemic. American Journal of Public Health, 108(12), 1621-1622.

Herrnstein, R. J., \& Prelec, D. (1991). Melioration: A theory of distributed choice. Journal of Economic Perspectives, 5(3), 137-156. 
Herrnstein, R. J. \& Prelec, D. (1992). In Loewenstein, G., \& Elster, J. (Eds.). (1992). Choice over time. 331-360New York: Russell Sage Foundation.

Herry, C., \& Johansen, J. P. (2014). Encoding of fear learning and memory in distributed neuronal circuits. Nature Neuroscience, 17(12), 1644.

Heshmat, S. (2015). Addiction: A behavioral economic perspective. Abingdon-on-Thames, UK. Routledge.

Hestad, K., Updike, M., Selnes, O. A., \& Royal III, W. (1995). Cognitive sequelae of repeated head injury in a population of intravenous drug users. Scandinavian Journal of Psychology, 36(3), 246-255.

Heyman, G. M. (2003). Consumption dependent changes in reward value: A framework for understanding addiction. In Choice, Behavioural Economics and Addiction (pp. 95-127). Pergamon.

Heyman, G. M. (1996). Resolving the contradictions of addiction. Behavioral and Brain Sciences, 19(4), 561-574.

Heyman, G. M. (2009). Addiction: A disorder of choice. Cambridge, MA. Harvard University Press.

Heyman, G. M. (2013a). Addiction and choice: Theory and new data. Frontiers in Psychiatry, 4, 31.

Heyman, G. M. (2013b). Quitting drugs: Quantitative and qualitative features. Annual Review of Clinical Psychology, 9, 29-59.

Heyman, G. M., \& Dunn, B. (2002). Decision biases and persistent illicit drug use: An experimental study of distributed choice and addiction. Drug and Alcohol Dependence, 67(2), 193-203.

Heyman, G. M., \& Mims, V. (2016). What addicts can teach us about addiction: A natural history approach. Addiction and Choice: Rethinking the Relationship, 386-408.

Higgins, S. T., Bickel, W. K., \& Hughes, J. R. (1994). Influence of an alternative reinforcer on human cocaine self-administration. Life Sciences, 55(3), 179-187.

Higgins, S. T. (1997). The influence of alternative reinforcers on cocaine use and abuse: A brief review. Pharmacology Biochemistry and Behavior, 57(3), 419-427.

Higgins, S. T., Silverman, K., \& Heil, S. H. (Eds.). (2007). Contingency Management in Substance Abuse Treatment. New York City, NY. Guilford Press.

Hill, S. K., Cantrell, P., Edwards, J., \& Dalton, W. (2016). Factors influencing mental health screening and treatment among women in a rural south central Appalachian primary care clinic. The Journal of Rural Health, 32(1), 82-91.

Himelstein, S., Saul, S., \& Garcia-Romeu, A. (2015). Does mindfulness meditation increase effectiveness of substance abuse treatment with incarcerated youth? A pilot randomized controlled trial. Mindfulness, 6(6), 1472-1480.

Hines, S., Hines, S., Theodorou, S., Hines, S., Theodorou, S., Williamson, A., ... \& Hines, S. (2008). Management of acute pain in methadone maintenance therapy in-patients. Drug and Alcohol Review, 27(5), 519-523.

Hinson, J. M., Jameson, T. L., \& Whitney, P. (2003). Impulsive decision making and working memory. Journal of Experimental Psychology: Learning, Memory, and Cognition, 29(2), 298.

Hobelmann, J. G., \& Clark, M. R. (2016). Benzodiazepines, alcohol, and stimulant use in combination with opioid use. In Controlled Substance Management in Chronic Pain (pp. 75-86). Springer, Cham. 
Hoffmann, J., \& Larison, C. (1999). Drug use, workplace accidents and employee turnover. Journal of Drug Issues, 29(2), 341-364.

Hofford, R. S., Chow, J. J., Beckmann, J. S., \& Bardo, M. T. (2017). Effects of environmental enrichment on self-administration of the short-acting opioid remifentanil in male rats. Psychopharmacology, 234(23-24), 3499-3506.

Hofmann, D. A., \& Gavin, M. B. (1998). Centering decisions in hierarchical linear models: Implications for research in organizations. Journal of Management, 24(5), 623-641.

Hofmann, W., Schmeichel, B. J., \& Baddeley, A. D. (2012). Executive functions and selfregulation. Trends in Cognitive Sciences, 16(3), 174-180.

Hofmann, W., Vohs, K. D., \& Baumeister, R. F. (2012). What people desire, feel conflicted about, and try to resist in everyday life. Psychological Science, 23(6), 582-588.

Hofmann, W., \& Vohs, K. D. (2016). Desire and self-regulation in (Eds.) Vohs, K. D., \& Baumeister, R. F. Handbook of Self-Regulation: Research, Theory, and Applications, 76.

Hojat, M., \& Xu, G. (2004). A visitor's guide to effect sizes-statistical significance versus practical (clinical) importance of research findings. Advances in Health Sciences Dducation, 9(3), 241-249.

Holbrook, A. L., Green, M. C., \& Krosnick, J. A. (2003). Telephone versus face-to-face interviewing of national probability samples with long questionnaires: Comparisons of respondent satisficing and social desirability response bias. Public Opinion Quarterly, 67(1), 79-125.

Holgate, J. Y., Garcia, H., Chatterjee, S., \& Bartlett, S. E. (2017). Social and environmental enrichment has different effects on ethanol and sucrose consumption in mice. Brain and Behavior, 7(8), e00767.

Hollander, J. A., \& Carelli, R. M. (2005). Abstinence from cocaine self-administration heightens neural encoding of goal-directed behaviors in the accumbens. Neuropsychopharmacology, 30(8), 1464.

Holstege, C. P., Ferguson, J. D., Wolf, C. E., Baer, A. B., \& Poklis, A. (2004). Analysis of moonshine for contaminants. Journal of Toxicology: Clinical Toxicology, 42(5), 597-601.

Holt, M., \& Treloar, C. (2008). Pleasure and drugs. International Journal of Drug Policy, 19(5), 349-352.

Homish, G. G., Leonard, K. E., Kozlowski, L. T., \& Cornelius, J. R. (2009). The longitudinal association between multiple substance use discrepancies and marital satisfaction. Addiction, 104(7), 12011209.

Hoppenbrouwers, S. S., Bulten, B. H., \& Brazil, I. A. (2016). Parsing fear: A reassessment of the evidence for fear deficits in psychopathy. Psychological Bulletin, 142(6), 573.

Hosmer, D. W., Lemeshow, S., Sturdivant, R. X. (2013) Applied logistic regression. Third edition. New Jersey: John Wiley \& Sons.

Hosseini-Kamkar, N., \& Morton, J. B. (2014). Sex differences in self-regulation: An evolutionary perspective. Frontiers in Neuroscience, 8, 233.

Hox, J. J., Moerbeek, M., \& Van de Schoot, R. (2017). Multilevel analysis: Techniques and applications. Abingdon-on-Thames, UK: Routledge. 
Hox, J. J., \& Maas, C. J. (2002). Sample sizes for multilevel modeling. Methodology European Journal of Research Methods for the Behavioral and Social Sciences 1(3) · November 2004.

Hox, J. J., Moerbeek, M., \& Van de Schoot, R. (2017). Multilevel analysis: Techniques and applications. Abingdon-on-Thames, UK. Routledge.

Hsieh, C. C., \& Pugh, M. D. (1993). Poverty, income inequality, and violent crime: A meta-analysis of recent aggregate data studies. Criminal Justice Review, 18(2), 182-202.

Hsieh, F. Y., Bloch, D. A., \& Larsen, M. D. (1998). A simple method of sample size calculation for linear and logistic regression. Statistics in Medicine, 17(14), 1623-1634.

Hser, Y. I., Grella, C., Chou, C. P., \& Anglin, M. D. (1998). Relationships between drug treatment careers and outcomes: Findings from the National Drug Abuse Treatment Outcome Study. Evaluation Review, 22(4), 496-519.

Hu, T., \& Sung, S. Y. (2004). A trimmed mean approach to finding spatial outliers. Intelligent Data Analysis, 8(1), 79-95.

Huhn, A. S., Harris, J., Cleveland, H. H., Lydon, D. M., Stankoski, D., Cleveland, M. J., ... \& Bunce, S. C. (2016). Ecological momentary assessment of affect and craving in patients in treatment for prescription opioid dependence. Brain Research Bulletin, 123, 94-101.

Hume, D. (2007). A treatise of human nature: Volume, (eds. Norton D. F. \& Norton, M. J). New York, NY: Oxford University Press.

Hunt, E. (2010). Human intelligence. Cambridge, England. Cambridge University Press.

Hursh, S. R., \& Silberberg, A. (2008). Economic demand and essential value. Psychological Review, 115(1), 186.

Huynh, M., Parker, J. D., Harper, S., Pamuk, E., \& Schoendorf, K. C. (2005). Contextual effect of income inequality on birth outcomes. International Journal of Epidemiology, 34(4), 888-895.

Hwang, S. W., Kirst, M. J., Chiu, S., Tolomiczenko, G., Kiss, A., Cowan, L., \& Levinson, W. (2009). Multidimensional social support and the health of homeless individuals. Journal of Urban Health, 86(5), 791-803.

Hyman, S. E. (2005). Addiction: A disease of learning and memory. American Journal of Psychiatry, 162(8), 1414-1422.

Hyman, S. E. (2007). The neurobiology of addiction: Implications for voluntary control of behavior. The American Journal of Bioethics, 7(1), 8-11.

Hyman, S. M., Fox, H., Hong, K. I. A., Doebrick, C., \& Sinha, R. (2007). Stress and drug-cue-induced craving in opioid-dependent individuals in naltrexone treatment. Experimental and Clinical Psychopharmacology, 15(2), 134.

IBM Corp. Released 2018. IBM SPSS Statistics for Windows, Version 26.0. Armonk, NY: IBM Corp.

Iceland, J., \& Bauman, K. J. (2007). Income poverty and material hardship: How strong is the association?. The Journal of Socio-Economics, 36(3), 376-396.

Ikink, I., Engelmann, J. B., van den Bos, W., Roelofs, K., \& Figner, B. (2019). Time ambiguity during intertemporal decision-making is aversive, impacting choice and neural value coding. NeuroImage, 185, 236-244. 
Imperio, C. G., McFalls, A. J., Hadad, N., Blanco-Berdugo, L., Masser, D. R., Colechio, E. M., ... \& Grigson, P. S. (2018). Exposure to environmental enrichment attenuates addiction-like behavior and alters molecular effects of heroin self-administration in rats. Neuropharmacology, 139, 26-40.

Inagaki, T. K. (2018). Opioids and social connection. Current Directions in Psychological Science, 27(2), 85-90.

Inciardi, J. A., \& Cicero, T. J. (2009). Black beauties, gorilla pills, footballs, and hillbilly heroin: Some reflections on prescription drug abuse and diversion research over the past 40 years. Journal of Drug Issues, 39(1), 101-114.

Inciardi, J. A., Surratt, H. L., Kurtz, S. P., \& Burke, J. J. (2006). The diversion of prescription drugs by health care workers in Cincinnati, Ohio. Substance Use \& Misuse, 41(2), 255-264.

Inciardi, J. A., Surratt, H. L., Lugo, Y., \& Cicero, T. J. (2007, November). The diversion of prescription opioid analgesics. In Law enforcement executive forum (Vol. 7, No. 7, p. 127). NIH Public Access.

Inciardi, J. A., Surratt, H. L., Cicero, T. J., Kurtz, S. P., Martin, S. S., \& Parrino, M. W. (2009). The "black box" of prescription drug diversion. Journal of Addictive Diseases, 28(4), 332-347.

Inciardi, J. A., Surratt, H. L., Cicero, T. J., \& Beard, R. A. (2009). Prescription opioid abuse and diversion in an urban community: The results of an ultrarapid assessment. Pain Medicine, 10(3), 537-548.

Iudicello, J. E., Woods, S. P., Vigil, O., Cobb Scott, J., Cherner, M., Heaton, R. K., ... \& HIV Neurobehavioral Research Center (HNRC) Group. (2010). Longer term improvement in neurocognitive functioning and affective distress among methamphetamine users who achieve stable abstinence. Journal of Clinical and Experimental Neuropsychology, 32(7), 704-718.

Jabs, J., \& Devine, C. M. (2006). Time scarcity and food choices: An overview. Appetite, 47(2), 196-204.

Jacobs, E. A., \& Bickel, W. K. (1999). Modeling drug consumption in the clinic using simulation procedures: Demand for heroin and cigarettes in opioid-dependent outpatients. Experimental and Clinical Psychopharmacology, 7(4), 412.

Jaffe, A., Du, J., Huang, D., \& Hser, Y. I. (2012). Drug-abusing offenders with comorbid mental disorders: Problem severity, treatment participation, and recidivism. Journal of Substance Abuse Treatment, 43(2), 244-250.

Jakubczyk, A., Skrzeszewski, J., Trucco, E. M., Suszek, H., Zaorska, J., Nowakowska, M., ... \& Kopera, M. (2019). Interoceptive accuracy and interoceptive sensibility in individuals with alcohol use disorder-Different phenomena with different clinical correlations?. Drug and Alcohol Dependence.

Jalal, H., Buchanich, J. M., Roberts, M. S., Balmert, L. C., Zhang, K., \& Burke, D. S. 2018. Changing dynamics of the drug overdose epidemic in the United States from 1979 through 2016. Science, 361(6408), eaau1184.

James, W. L., \& Cossman, J. S. (2006). Does regional variation affect ecological mortality research? An examination of mortality, income inequality and health infrastructure in the Mississippi Delta. Population Research and Policy Review, 25(2), 175-195.

Janulis, P., Ferrari, J. R., \& Fowler, P. (2013). Understanding public stigma toward substance dependence. Journal of Applied Social Psychology, 43(5), 1065-1072. 
Jarlais, D. C. D., Nugent, A., Solberg, A., Feelemyer, J., Mermin, J., \& Holtzman, D. (2015). Syringe service programs for persons who inject drugs in urban, suburban, and rural areas-United States, 2013. Morbidity and Mortality Weekly Report, 64(48), 1337-1341.

Jarvis, B. P., Holtyn, A. F., Subramaniam, S., Tompkins, D. A., Oga, E. A., Bigelow, G. E., \& Silverman, K. (2018). Extended-release injectable naltrexone for opioid use disorder: a systematic review. Addiction, 113(7), 1188-1209.

Jasinski, D. R., \& Preston, K. L. (1986). Evaluation of mixtures of morphine and d-amphetamine for subjective and physiological effects. Drug and Alcohol Dependence, 17(1), 1-13.

Jayadeva, V., Bunnag, A., Meyen, R., \& Fernando, I. (2017). Kratom (Mitragyna speciosa) Use in a Veteran With Chronic Pain. American Journal of Psychiatry Residents' Journal, 12(3), 13-15.

Jeffers, A. J., Benotsch, E. G., Green, B. A., Bannerman, D., Darby, M., Kelley, T., \& Martin, A. M. (2015). Health anxiety and the non-medical use of prescription drugs in young adults: A crosssectional study. Addictive Behaviors, 50, 74-77.

Jenkins, P. (1994). “The ice age" the social construction of a drug panic. Justice Quarterly, 11(1), 7-31.

Jenkins, A. J., Oyler, J. M., \& Cone, E. J. (1995). Comparison of heroin and cocaine concentrations in saliva with concentrations in blood and plasma. Journal of Analytical Toxicology, 19(6), 359-374.

Jentsch, J. D., Ashenhurst, J. R., Cervantes, M. C., Groman, S. M., James, A. S., \& Pennington, Z. T. (2014). Dissecting impulsivity and its relationships to drug addictions. Annals of the New York Academy of Sciences, 1327(1), 1-26.

Jia, H., Moriarty, D. G., \& Kanarek, N. (2009). County-level social environment determinants of healthrelated quality of life among US adults: a multilevel analysis. Journal of Community Health, 34(5), 430-439.

Jia, H., Muennig, P., \& Borawski, E. (2004). Comparison of small-area analysis techniques for estimating county-level outcomes. American Journal of Preventive Medicine, 26(5), 453-460.

Jinjuvadia, R., \& Liangpunsakul, S. (2015). Trends in alcoholic hepatitis related hospitalizations, financial burden, and mortality in the United States. Journal of Clinical Gastroenterology, 49(6), 506.

Jofre-Bonet, M., \& Petry, N. M. (2008). Trading apples for oranges?: Results of an experiment on the effects of heroin and cocaine price changes on addicts' polydrug use. Journal of Economic Behavior \& Organization, 66(2), 281-311.

John, W. S., \& Wu, L. T. (2017). Trends and correlates of cocaine use and cocaine use disorder in the United States from 2011 to 2015. Drug and Alcohol Dependence, 180, 376-384.

Johnson, M. W., \& Bickel, W. K. (2006). Replacing relative reinforcing efficacy with behavioral economic demand curves. Journal of the Experimental Analysis of Behavior, 85(1), 73-93.

Johanson, C. E., \& Fischman, M. W. (1989). The pharmacology of cocaine related to its abuse. Pharmacological Reviews, 41(1), 3-52.

Jonas, A. B., Young, A. M., Oser, C. B., Leukefeld, C. G., \& Havens, J. R. (2012). OxyContin® as currency: OxyContin ${ }^{\circledR}$ use and increased social capital among rural Appalachian drug users. Social Science \& Medicine, 74(10), 1602-1609.

Jones, E. B. (2018). Medication-assisted opioid treatment prescribers in federally qualified health centers: Capacity lags in rural areas. The Journal of Rural Health, 34(1), 14-22. 
Jones, J. D., Mogali, S., \& Comer, S. D. (2012). Polydrug abuse: A review of opioid and benzodiazepine combination use. Drug and Alcohol Dependence, 125(1-2), 8-18.

Jovanovski, D., Erb, S., \& Zakzanis, K. K. (2005). Neurocognitive deficits in cocaine users: A quantitative review of the evidence. Journal of Clinical and Experimental Neuropsychology, 27(2), 189-204.

Julian, M. M., Rosenblum, K. L., Doom, J. R., Leung, C. Y., Lumeng, J. C., Cruz, M. G., ... \& Miller, A. L. (2018). Oxytocin and parenting behavior among impoverished mothers with low vs. high early life stress. Archives of Women's Mental Health, 21(3), 375-382.

Juster, R. P., McEwen, B. S., \& Lupien, S. J. (2010). Allostatic load biomarkers of chronic stress and impact on health and cognition. Neuroscience \& Biobehavioral Reviews, 35(1), 2-16.

Kaba, F., Lewis, A., Glowa-Kollisch, S., Hadler, J., Lee, D., Alper, H., ... \& Venters, H. (2014). Solitary confinement and risk of self-harm among jail inmates. American Journal of Public Health, 104(3), $442-447$.

Kadam, P., \& Bhalerao, S. (2010). Sample size calculation. International Journal of Ayurveda Research, 1(1), 55.

Kagel, J. H., Green, L., \& Caraco, T. (1986). When foragers discount the future: Constraint or adaptation?. Animal Behaviour, 34, 271-283.

Kalechstein, A. D., Newton, T. F., \& Green, M. (2003). Methamphetamine dependence is associated with neurocognitive impairment in the initial phases of abstinence. The Journal of Neuropsychiatry and Clinical Neurosciences, 15(2), 215-220.

Kahneman, D. (2003). A psychological perspective on economics. American Economic Review, 93(2), 162168.

Kahneman, D. (2003). Experienced utility and objective happiness: A moment-based approach. In (Eds. Kahneman, D., \& Tversky, A.) The Psychology of Economic Decisions, 1, 187-208.

Kahneman, D. (2011). Thinking, fast and slow. New York: Farrar, Straus and Giroux.

Kahneman, D., \& Deaton, A. (2010). High income improves evaluation of life but not emotional wellbeing. Proceedings of the National Academy of Sciences, 107(38), 16489-16493.

Kahneman, D., \& Tversky, A. (2013). Prospect theory: An analysis of decision under risk. In Handbook of the fundamentals of financial decision making: Part I (pp. 99-127).

Kahneman, D., Wakker, P. P., \& Sarin, R. (1997). Back to Bentham? Explorations of experienced utility. The Quarterly Journal of Economics, 112(2), 375-406.

Kalivas, P. W. (2007). Cocaine and amphetamine-like psychostimulants: Neurocircuitry and glutamate neuroplasticity. Dialogues in Clinical Neuroscience, 9(4), 389.

Kandel, D., Chen, K., Warner, L. A., Kessler, R. C., \& Grant, B. (1997). Prevalence and demographic correlates of symptoms of last year dependence on alcohol, nicotine, marijuana and cocaine in the US population. Drug and Alcohol Dependence, 44(1), 11-29.

Kandel, D. B., Yamaguchi, K., \& Chen, K. (1992). Stages of progression in drug involvement from adolescence to adulthood: Further evidence for the gateway theory. Journal of Studies on Alcohol, 53(5), 447-457. 
Kang, M., Rowe, D. A., Barreira, T. V., Robinson, T. S., \& Mahar, M. T. (2009). Individual informationcentered approach for handling physical activity missing data. Research Quarterly for Exercise and Sport, 80(2), 131-137.

Kanouse, A. B., \& Compton, P. (2015). The epidemic of prescription opioid abuse, the subsequent rising prevalence of heroin use, and the federal response. Journal of Pain \& Palliative Care Pharmacotherapy, 29(2), 102-114

Karasz, A., Zallman, L., Berg, K., Gourevitch, M., Selwyn, P., \& Arnstein, J. (2004). The experience of chronic severe pain in patients undergoing methadone maintenance treatment. Journal of Pain and Symptom Management, 28(5), 517-525.

Kassab, H. S., \& Rosen, J. D. (2019). Illicit markets and the internet age. In illicit markets, organized crime, and global security (pp. 155-175). Palgrave Macmillan, Cham.

Katona, G. (1951). Psychological analysis of economic behavior. New York, NY, US: McGraw-Hill.

Katona, G. (1975). Psychological economics. Oxford, England: Elsevier.

Katz, J. (2017, February 22). Drug deaths in america are rising faster than ever. Retrieved from https://www.nytimes.com/interactive/2017/06/05/upshot/opioid-epidemic-drug-overdose-deathsare-rising-faster-than-ever.html

Katz, J. L. (1990). Models of relative reinforcing efficacy of drugs and their predictive utility. Behav Pharmacol, 1(4), 283-301.

Kautt, P., \& Spohn, C. (2002). Crack-ing down on black drug offenders? Testing for interactions among offenders' race, drug type, and sentencing strategy in federal drug sentences. Justice Quarterly, 19(1), 1-35

Kavanagh, L., Rowe, D., Hersch, J., Barnett, K. J., \& Reznik, R. (2010). Neurocognitive deficits and psychiatric disorders in a NSW prison population. International Journal of Law and Psychiatry, 33(1), 20-26.

Kaye, S., \& Darke, S. (2004). Non-fatal cocaine overdose among injecting and non-injecting cocaine users in Sydney, Australia. Addiction, 99(10), 1315-1322.

Keller, C. E., Ashrafioun, L., Neumann, A. M., Van Klein, J., Fox, C. H., \& Blondell, R. D. (2012). Practices, perceptions, and concerns of primary care physicians about opioid dependence associated with the treatment of chronic pain. Substance Abuse, 33(2), 103-113.

Kelly, J. F., \& Greene, M. C. (2014). Where there'sa will there'sa way: A longitudinal investigation of the interplay between recovery motivation and self-efficacy in predicting treatment outcome. Psychology of Addictive Behaviors, 28(3), 928.

Kelly, P. J., Robinson, L. D., Baker, A. L., Deane, F. P., McKetin, R., Hudson, S., \& Keane, C. (2017). Polysubstance use in treatment seekers who inject amphetamine: Drug use profiles, injecting practices and quality of life. Addictive Behaviors, 71, 25-30.

Kelly, J. F., \& Westerhoff, C. M. (2010). Does it matter how we refer to individuals with substance-related conditions? A randomized study of two commonly used terms. International Journal of Drug Policy, 21(3), 202-207.

Kelley, A. E., \& Berridge, K. C. (2002). The neuroscience of natural rewards: Relevance to addictive drugs. Journal of Neuroscience, 22(9), 3306-3311. 
Keniston-Longrie, Joy (2009) Seattle's Pioneer Square. Chicago, San Francisco, \& Charleston, SC: Arcadia Publishing. p. 128-162

Kenney, S. R., Anderson, B. J., Conti, M. T., Bailey, G. L., \& Stein, M. D. (2018). Expected and actual fentanyl exposure among persons seeking opioid withdrawal management. Journal of Substance Abuse Treatment, 86, 65-69.

Kentucky alcohol beverage control. (2017, May 1). Retrieved from https://abc.ky.gov/LocalInformation/Documents/Wet-Dry\%20Map\%206-17.pdf

Kentucky Cabinet for Health and Family Services. 2018. Kentucky syringe exchange programs - Cabinet for Health and Family Services. Retrieved from https://chfs.ky.gov/agencies/dph/dehp/hab/Pages/kyseps.aspx

Kentucky Cabinet for Health and Family Services. (2019). Kentucky all schedule prescription electronic reporting - Cabinet for Health and Family Services. Retrieved January 9, 2019, from https://chfs.ky.gov/agencies/os/oig/dai/deppb/Pages/kasper.aspx

Kentucky Housing Corporation. 2018. Recovery Kentucky - Kentucky Housing Corporation. Retrieved from http://www.kyhousing.org/Specialized-Housing/Pages/Recovery-Kentucky.aspx

Kentucky Office of Drug Control Policy. 2016 Overdose fatality report. Retrieved from https://odcp.ky.gov/Documents/2016\%20ODCP\%20Overdose\%20Fatality\%20Report\%20Final.pd f. 2016.

Kennedy-Hendricks, A., Barry, C. L., Gollust, S. E., Ensminger, M. E., Chisolm, M. S., \& McGinty, E. E. (2017). Social stigma toward persons with prescription opioid use disorder: Associations with public support for punitive and public health-oriented policies. Psychiatric Services, 68(5), 462469.

Kepler, K. L., Kest, B., Kiefel, J. M., Cooper, M. L., \& Bodnar, R. J. (1989). Roles of gender, gonadectomy and estrous phase in the analgesic effects of intracerebroventricular morphine in rats. Pharmacology Biochemistry and Behavior, 34(1), 119-127.

Kessler, R. C., \& Cleary, P. D. (1980). Social class and psychological distress. American Sociological Review, 463-478.

Keyes, K. M., Cerdá, M., Brady, J. E., Havens, J. R., \& Galea, S. (2014). Understanding the rural-urban differences in nonmedical prescription opioid use and abuse in the United States. American Journal of Public Health, 104(2), e52-e59.

Khalsa, S. S., Rudrauf, D., \& Tranel, D. (2009). Interoceptive awareness declines with age. Psychophysiology, 46(6), 1130-1136.

Khantzian, E. J. (1997). The self-medication hypothesis of substance use disorders: A reconsideration and recent applications. Harvard Review of Psychiatry, 4(5), 231-244.

Khantzian, E. J. (2013). Addiction as a self-regulation disorder and the role of selfmedication. Addiction, 108(4), 668-669.

Khurana, A., Romer, D., Betancourt, L. M., Brodsky, N. L., Giannetta, J. M., \& Hurt, H. (2013). Working memory ability predicts trajectories of early alcohol use in adolescents: The mediational role of impulsivity. Addiction, 108(3), 506-515. 
Khwaja, A., Silverman, D., \& Sloan, F. (2007). Time preference, time discounting, and smoking decisions. Journal of Health Economics, 26(5), 927-949.

Kiecolt, K. J., \& Nathan, L. E. (1985). Secondary analysis of survey data (Vol. 53). Sage.

Kilmer, B., Everingham, S., Caulkins, J. P., Midgette, G., Reuter, P., Pacula, R. L., ... \& Lundberg, R. (2014). What America's users spend on illicit drugs: 2000-2010. Office of National Drug Control Policy.

Kim, E. (2002). Agitation, aggression, and disinhibition syndromes after traumatic brain injury. NeuroRehabilitation, 17(4), 297-310.

Kim, D., Subramanian, S. V., Gortmaker, S. L., \& Kawachi, I. (2006). US state-and county-level social capital in relation to obesity and physical inactivity: A multilevel, multivariable analysis. Social Science \& Medicine, 63(4), 1045-1059.

Kim, S., Arora, M., Fernandez, C., Landero, J., Caruso, J., \& Chen, A. (2013). Lead, mercury, and cadmium exposure and attention deficit hyperactivity disorder in children. Environmental Research, 126, 105-110.

King, K. M., \& Chassin, L. (2007). A prospective study of the effects of age of initiation of alcohol and drug use on young adult substance dependence. Journal of Studies on Alcohol and Drugs, 68(2), 256-265.

Kirby, K. C., Benishek, L. A., Dugosh, K. L., \& Kerwin, M. E. (2006). Substance abuse treatment providers' beliefs and objections regarding contingency management: Implications for dissemination. Drug and Alcohol Dependence, 85(1), 19-27.

Kirby, K. N., \& Petry, N. M. (2004). Heroin and cocaine abusers have higher discount rates for delayed rewards than alcoholics or non-drug-using controls. Addiction, 99(4), 461-471.

Kirby, K. N., Petry, N. M., \& Bickel, W. K. (1999). Heroin addicts have higher discount rates for delayed rewards than non-drug-using controls. Journal of Experimental Psychology: General, 128(1), 78

Kistner, J., Haskett, M., White, K., \& Robbins, F. (1987). Perceived competence and self-worth of LD and normally achieving students. Learning Disability Quarterly, 10(1), 37-44.

Kjome, K. L., Lane, S. D., Schmitz, J. M., Green, C., Ma, L., Prasla, I., ... \& Moeller, F. G. (2010). Relationship between impulsivity and decision making in cocaine dependence. Psychiatry Research, 178(2), 299-304.

Klapproth, F. (2012). The date-delay framing effect in temporal discounting depends on substance abuse. Behavioural Processes, 90(3), 420-423.

Kleiman, M. (1992). Against excess: Drug policy for results (p. 360). New York, NY: BasicBooks.

Koechlin, E., \& Hyafil, A. (2007). Anterior prefrontal function and the limits of human decisionmaking. Science, 318(5850), 594-598.

Kohtz, A. S., Lin, B., Smith, M. E., \& Aston-Jones, G. (2018). Attenuated cocaine-seeking after oxytocin administration in male and female rats. Psychopharmacology, 235(7), 2051-2063.

Kollenbaum, V. E., Dahme, B., \& Kirchner, G. (1996). 'Interoception'of heart rate, blood pressure, and myocardial metabolism during ergometric work load in healthy young subjects. Biological Psychology, 42(1-2), 183-197. 
Koob, G. F. (1992, April). Dopamine, addiction and reward. In Seminars in Neuroscience (Vol. 4, No. 2, pp. 139-148). Academic Press.

Koob, G. F. (1996). Drug addiction: The yin and yang of hedonic homeostasis. Neuron, 16(5), 893-896.

Koob, G. F. (2008). A role for brain stress systems in addiction. Neuron, 59(1), 11-34.

Koob, G. F. (2009). Brain stress systems in the amygdala and addiction. Brain Research, 1293, 61-75.

Koob, G. F., Buck, C. L., Cohen, A., Edwards, S., Park, P. E., Schlosburg, J. E., ... \& George, O. (2014). Addiction as a stress surfeit disorder. Neuropharmacology, 76, 370-382.

Koob, G. F., Everitt, B. J., \& Robbins, T. W. (2014). Reward, motivation, and addiction. In Fundamental Neuroscience (Fourth Edition) (pp. 871-898).

Koob, G. F., \& Vokow, N. D. (2010). Neurocircuitry of addiction. Neurophsychopharmacology,35, 217.

Kopetz, C. E., Woerner, J. I., \& Briskin, J. L. (2018). Another look at impulsivity: Could impulsive behavior be strategic?. Social and Personality Psychology Compass, 12(5), e12385.

Koroboki, E., Zakopoulos, N., Manios, E., Rotas, V., Papadimitriou, G., \& Papageorgiou, C. (2010). Interoceptive awareness in essential hypertension. International Journal of Psychophysiology, 78(2), 158-162.

Korponay, C., Pujara, M., Deming, P., Philippi, C., Decety, J., Kosson, D. S., ... \& Koenigs, M. (2017). Impulsive-antisocial psychopathic traits linked to increased volume and functional connectivity within prefrontal cortex. Social Cognitive and Affective Neuroscience, 12(7), 1169-1178.

Kosten, T. R., \& George, T. P. (2002). The neurobiology of opioid dependence: Implications for treatment. Science \& Practice Perspectives, 1(1), 13.

Kowalczyk, W. J., Moran, L. M., Bertz, J. W., Phillips, K. A., Ghitza, U. E., Vahabzadeh, M., ... \& Preston, K. L. (2018). Using ecological momentary assessment to examine the relationship between craving and affect with opioid use in a clinical trial of clonidine as an adjunct medication to buprenorphine treatment. The American Journal of Drug and Alcohol Abuse, 1-10.

Kowalski-McGraw, M., Green-McKenzie, J., Pandalai, S. P., \& Schulte, P. A. (2017). Characterizing the interrelationships of prescription opioid and benzodiazepine drugs with worker health and workplace hazards. Journal of Occupational and Environmental Medicine, 59(11), 1114-1126.

Kramer, T. L., Han, X., \& Booth, B. M. (2009). Young adult, rural, African American stimulant users: Antecedents and vulnerabilities. Journal of Ethnicity in Substance Abuse, 8(4), 378-399.

Kraus, M. W., Horberg, E. J., Goetz, J. L., \& Keltner, D. (2011). Social class rank, threat vigilance, and hostile reactivity. Personality and Social Psychology Bulletin, 37(10), 1376-1388.

Kreek, M. J. (1996). Opiates, opioids and addiction. Molecular Psychiatry, 1(3), 232-254.

Kreek, M. J., Bart, G., Lilly, C., Laforge, K. S., \& Nielsen, D. A. (2005). Pharmacogenetics and human molecular genetics of opiate and cocaine addictions and their treatments. Pharmacological Reviews, 57(1), 1-26.

Kreek, M. J., Levran, O., Reed, B., Schlussman, S. D., Zhou, Y., \& Butelman, E. R. (2012). Opiate addiction and cocaine addiction: Underlying molecular neurobiology and genetics. The Journal of Clinical Investigation, 122(10), 3387-3393. 
Kreek, M. J., Nielsen, D. A., Butelman, E. R., \& LaForge, K. S. (2005). Genetic influences on impulsivity, risk taking, stress responsivity and vulnerability to drug abuse and addiction. Nature Neuroscience, 8(11), 1450.

Kreft, I. G., \& De Leeuw, J. (1998). Introducing multilevel modeling. Thousand Oaks, CA. Sage.

Kroenke, K., Spitzer, R. L., \& Williams, J. B. (2001). The PHQ-9: Validity of a brief depression severity measure. Journal of General Internal Medicine, 16(9), 606-613.

Kroenke, K., Spitzer, R. L., Williams, J. B., \& Löwe, B. (2010). The patient health questionnaire somatic, anxiety, and depressive symptom scales: A systematic review. General Hospital Psychiatry, 32(4), 345-359.

Kroutil, L. A., Van Brunt, D. L., Herman-Stahl, M. A., Heller, D. C., Bray, R. M., \& Penne, M. A. (2006). Nonmedical use of prescription stimulants in the United States. Drug and Alcohol Dependence, 84(2), 135-143.

Krueger Jr, N., \& Dickson, P. R. (1994). How believing in ourselves increases risk taking: Perceived selfefficacy and opportunity recognition. Decision Sciences, 25(3), 385-400.

Kuhar, M. J. (2002). Social rank and vulnerability to drug abuse. Nature Neuroscience, 5(2), 88.

Kurth-Nelson, Z., Bickel, W., \& Redish, A. D. (2012). A theoretical account of cognitive effects in delay discounting. European Journal of Neuroscience, 35(7), 1052-1064.

Kunz-Ebrecht, S. R., Kirschbaum, C., \& Steptoe, A. (2004). Work stress, socioeconomic status and neuroendocrine activation over the working day. Social Science \& Medicine, 58(8), 1523-1530.

Kurtz, S. P., Buttram, M. E., Margolin, Z. R., \& Wogenstahl, K. (2019). The diversion of nonscheduled psychoactive prescription medications in the United States, 2002 to 2017. Pharmacoepidemiology and Drug Safety.

Kutlu, M. G., \& Gould, T. J. (2016). Effects of drugs of abuse on hippocampal plasticity and hippocampusdependent learning and memory: Contributions to development and maintenance of addiction. Learning \& Memory, 23(10), 515-533.

Kwako, L. E., Momenan, R., Litten, R. Z., Koob, G. F., \& Goldman, D. (2016). Addictions neuroclinical assessment: A neuroscience-based framework for addictive disorders. Biological Psychiatry, 80(3), 179-189.

Labouvie, E., \& White, H. R. (2002). Drug sequences, age of onset, and use trajectories as predictors of drug abuse/dependence in young adulthood. Stages and pathways of drug involvement: Examining the gateway hypothesis, 19-41. Cambridge, England: Cambridge University Press

Lacey, N., \& Pickard, H. (2012). From the consulting room to the court room? Taking the clinical model of responsibility without blame into the legal realm. Oxford Journal of Legal Studies, 33(1), 1-29.

Lafferty, B. (2010). Traumatic brain injury: A factor in the causal pathway to homelessness?. The Journal for Nurse Practitioners, 6(5), 358-362.

Lake, S., Wood, E., Buxton, J., Dong, H., Montaner, J., \& Kerr, T. (2015). Prescription opioid use and nonfatal overdose in a cohort of injection drug users. The American Journal of Drug and Alcohol Abuse, 41(3), 257-263. 
Lakens, D. (2013). Calculating and reporting effect sizes to facilitate cumulative science: A practical primer for t-tests and ANOVAs. Frontiers in Psychology, 4, 863.

Lally, P., Van Jaarsveld, C. H., Potts, H. W., \& Wardle, J. (2010). How are habits formed: Modelling habit formation in the real world. European Journal of Social Psychology, 40(6), 998-1009.

Lam, H., \& Harcourt, M. (2003). The use of criminal record in employment decisions: The rights of exoffenders, employers and the public. Journal of Business Ethics, 47(3), 237-252.

Lambert, D., Gale, J. A., \& Hartley, D. (2008). Substance abuse by youth and young adults in rural America. The Journal of Rural Health, 24(3), 221-228.

Landrø, N. I., Fors, E. A., Våpenstad, L. L., Holthe, Ø., Stiles, T. C., \& Borchgrevink, P. C. (2013). The extent of neurocognitive dysfunction in a multidisciplinary pain centre population. Is there a relation between reported and tested neuropsychological functioning?. PAIN®, 154(7), 972-977.

Lane, N. M., Lutz, A. Y., Baker, K., Konrad, T. R., Ricketts, T. C., Randolph, R., ... \& Beadles, C. A. (2012). Health care costs and access disparities in Appalachia. PDA, Incorporated.

Langford, C. P. H., Bowsher, J., Maloney, J. P., \& Lillis, P. P. (1997). Social support: a conceptual analysis. Journal of Advanced Nursing, 25(1), 95-100.

Langley, A. (1999). Strategies for theorizing from process data. Academy of Management Review, 24(4), 691-710.

Lankenau, S. E., Clatts, M. C., Goldsamt, L. A., \& Welle, D. (2004). Crack cocaine injection practices and HIV risk: Findings from New York and Bridgeport. Journal of Drug Issues, 34(2), 319-332.

Lankenau, S. E., Teti, M., Silva, K., Bloom, J. J., Harocopos, A., \& Treese, M. (2012). Initiation into prescription opioid misuse amongst young injection drug users. International Journal of Drug Policy, 23(1), 37-44.

Laorden, M. L., Fuertes, G., González-Cuello, A., \& Milanés, M. V. (2000). Changes in catecholaminergic pathways innervating paraventricular nucleus and pituitary-adrenal axis response during morphine dependence: Implication of $\alpha 1$-and $\alpha 2$-adrenoceptors. Journal of Pharmacology and Experimental Therapeutics, 293(2), 578-584.

Larance, B., Lintzeris, N., Bruno, R., Peacock, A., Cama, E., Ali, R., ... \& Degenhardt, L. (2015). The characteristics of a cohort who tamper with prescribed and diverted opioid medications. Journal of Substance Abuse Treatment, 58, 51-61.

Latkin, C. A., Edwards, C., Davey-Rothwell, M. A., Yang, C., \& Tobin, K. E. (2018). The relationship between drug use settings, roles in the drug economy, and witnessing a drug overdose in Baltimore, Maryland. Substance Abuse, 1-6.

Lau, S., Hiemisch, A., \& Baumeister, R. F. (2015). The experience of freedom in decisions-Questioning philosophical beliefs in favor of psychological determinants. Consciousness and Cognition, 33, $30-46$.

Laudet, A. B., \& White, W. L. (2008). Recovery capital as prospective predictor of sustained recovery, life satisfaction, and stress among former poly-substance users. Substance Use \& Misuse, 43(1), 2754.

Laudet, A. B. (2011). The case for considering quality of life in addiction research and clinical practice. Addiction Science \& Clinical Practice, 6(1), 44. 
Laudet, A. B., \& Stanick, V. (2010). Predictors of motivation for abstinence at the end of outpatient substance abuse treatment. Journal of Substance Abuse Treatment, 38(4), 317-327.

Lavrakas, P. J. (2008a). Social desirability - SAGE research methods. In SAGE Research Methods: Find resources to answer your research methods and statistics questions. Retrieved from http://methods.sagepub.com/reference/encyclopedia-of-survey-research-methods/n537.xml

Lavrakas, P. J. (2008b). Encyclopedia of survey research methods. Thousand Oaks, CA. Sage Publications.

Lawton-Craddock, A., Nixon, S. J., \& Tivis, R. (2003). Cognitive efficiency in stimulant abusers with and without alcohol dependence. Alcoholism: Clinical and Experimental Research, 27(3), 457-464.

Lebin, J. A., Murphy, D. L., Severtson, S. G., Bau, G. E., Dasgupta, N., \& Dart, R. C. (2019). Scoring the best deal: Quantity discounts and street price variation of diverted oxycodone and oxymorphone. Pharmacoepidemiology and Drug Safety, 28(1), 25-30.

Lee, M. R., Maume, M. O., \& Ousey, G. C. (2003). Social isolation and lethal violence across the metro/nonmetro divide: The effects of socioeconomic disadvantage and poverty concentration on homicide. Rural Sociology, 68(1), 107-131.

Lee, M. O., Vivier, P. M., \& Diercks, D. B. (2009). Is the self-report of recent cocaine or methamphetamine use reliable in illicit stimulant drug users who present to the emergency department with chest pain?. The Journal of Emergency Medicine, 37(2), 237-241.

Lee, D. (2013). Decision making: From neuroscience to psychiatry. Neuron, 78(2), 233-248.

Lee, C. M., Geisner, I. M., Patrick, M. E., \& Neighbors, C. (2010). The social norms of alcohol-related negative consequences. Psychology of Addictive Behaviors, 24(2), 342.

Lee, J. L., Milton, A. L., \& Everitt, B. J. (2006). Cue-induced cocaine seeking and relapse are reduced by disruption of drug memory reconsolidation. Journal of Neuroscience, 26(22), 5881-5887.

Lejuez, C. W., Magidson, J. F., Mitchell, S. H., Sinha, R., Stevens, M. C., \& De Wit, H. (2010). Behavioral and biological indicators of impulsivity in the development of alcohol use, problems, and disorders. Alcoholism: Clinical and Experimental Research, 34(8), 1334-1345.

Leknes, S., \& Tracey, I. (2008). A common neurobiology for pain and pleasure. Nature Reviews Neuroscience, 9(4), 314.

Leland, D. S., Arce, E., Feinstein, J. S., \& Paulus, M. P. (2006). Young adult stimulant users' increased striatal activation during uncertainty is related to impulsivity. Neuroimage, 33(2), 725-731.

Leland, D. S., \& Paulus, M. P. (2005). Increased risk-taking decision-making but not altered response to punishment in stimulant-using young adults. Drug and Alcohol Dependence, 78(1), 83-90.

Lemoine, D. (2018). Age-induced acceleration of time: Implications for intertemporal choice. Journal of Economic Behavior \& Organization, 153, 143-152.

Lempert, K. M., \& Phelps, E. A. (2016). The malleability of intertemporal choice. Trends in Cognitive Sciences, 20(1), 64-74.

Lempert, K. M., \& Pizzagalli, D. A. (2010). Delay discounting and future-directed thinking in anhedonic individuals. Journal of Behavior Therapy and Experimental Psychiatry, 4l(3), 258-264.

Lenoir, M., \& Ahmed, S. H. (2008). Supply of a nondrug substitute reduces escalated heroin consumption. Neuropsychopharmacology, 33(9), 2272. 
Leri, F., Bruneau, J., \& Stewart, J. (2003). Understanding polydrug use: Review of heroin and cocaine couse. Addiction, 98(1), 7-22.

Lerner, J. S., Li, Y., \& Weber, E. (2012). Sadder, but not wiser: The myopia of misery. ACR North American Advances.

Leshner, A. I. (1999). Science-based views of drug addiction and its treatment. Jama, 282(14), 1314-1316.

Leshner, A. I. (2001). Addiction is a brain disease. Issues in Science and Technology, 17(3), 75-80.

Leuchs, L., Schneider, M., Czisch, M., \& Spoormaker, V. I. (2017). Neural correlates of pupil dilation during human fear learning. Neuroimage, 147, 186-197.

Leukefeld, C., Walker, R., Havens, J., Leedham, C. A., \& Tolbert, V. (2007). What does the community say: Key informant perceptions of rural prescription drug use. Journal of Drug Issues, 37(3), 503524.

Levandowski, M. L., Tractenberg, S. G., de Azeredo, L. A., De Nardi, T., Rovaris, D. L., Bau, C. H., ... \& Grassi-Oliveira, R. (2016). Crack cocaine addiction, early life stress and accelerated cellular aging among women. Progress in Neuro-psychopharmacology and Biological Psychiatry, 71, 83-89.

Leverentz, A. (2010). People, places, and things: How female ex-prisoners negotiate their neighborhood context. Journal of Contemporary Ethnography, 39(6), 646-681.

Levine, M. D., Perkins, K. A., Kalarchian, M. A., Cheng, Y., Houck, P. R., Slane, J. D., \& Marcus, M. D. (2010). Bupropion and cognitive behavioral therapy for weight-concerned women smokers. Archives of Internal Medicine, 170(6), 543-550

Levine, T. A., \& Woodward, L. J. (2018). Early inhibitory control and working memory abilities of children prenatally exposed to methadone. Early Human Development, 116, 68-75.

Lewis, M. (2015). The biology of desire: Why addiction is not a disease. Abingdon, UK. Hachette UK.

Lewis, M. (2017). Addiction and the brain: Development, not disease. Neuroethics, 10(1), 7-18.

Leys, C., Ley, C., Klein, O., Bernard, P., \& Licata, L. (2013). Detecting outliers: Do not use standard deviation around the mean, use absolute deviation around the median. Journal of Experimental Social Psychology, 49(4), 764-766.

Li, Y., Baldassi, M., Johnson, E. J., \& Weber, E. U. (2013). Complementary cognitive capabilities, economic decision making, and aging. Psychology and Aging, 28(3), 595.

Li, D. H., Depoortere, R. Y., \& Emmett-Oglesby, M. W. (1994). Tolerance to the reinforcing effects of cocaine in a progressive ratio paradigm. Psychopharmacology, 116(3), 326-332.

Li, X., Zhang, F., Zhou, Y., Zhang, M., Wang, X., \& Shen, M. (2013). Decision-making deficits are still present in heroin abusers after short-to long-term abstinence. Drug and Alcohol Dependence, 130(1-3), 61-67.

Liker, J. K. (1982). Wage and status effects of employment on affective well-being among exfelons. American Sociological Review, 264-283.

Lindsay, J. A., Stotts, A. L., Green, C. E., Herin, D. V., \& Schmitz, J. M. (2009). Cocaine dependence and concurrent marijuana use: A comparison of clinical characteristics. The American Journal of Drug and Alcohol Abuse, 35(3), 193-198. 
Link, B. G., Struening, E. L., Rahav, M., Phelan, J. C., \& Nuttbrock, L. (1997). On stigma and its consequences: Evidence from a longitudinal study of men with dual diagnoses of mental illness and substance abuse. Journal of Health and Social Behavior, 38, 177-190.

Linnemann, T., \& Wall, T. (2013). 'This is your face on meth': The punitive spectacle of 'white trash 'in the rural war on drugs. Theoretical Criminology, 17(3), 315-334.

Liston, C., Miller, M. M., Goldwater, D. S., Radley, J. J., Rocher, A. B., Hof, P. R., ... \& McEwen, B. S. (2006). Stress-induced alterations in prefrontal cortical dendritic morphology predict selective impairments in perceptual attentional set-shifting. Journal of Neuroscience, 26(30), 7870-7874.

Listwan, S. J., Cullen, F. T., \& Latessa, E. J. (2006). How to prevent prisoners re-entry programs from failing: Insights from evidence-based corrections. Federal Probation, 70, 19.

Little, R. J. (1988). A test of missing completely at random for multivariate data with missing values. Journal of the American Statistical Association, 83(404), 1198-1202.

Liu, S., Lane, S. D., Schmitz, J. M., Waters, A. J., Cunningham, K. A., \& Moeller, F. G. (2011). Relationship between attentional bias to cocaine-related stimuli and impulsivity in cocainedependent subjects. The American Journal of Drug and Alcohol Abuse, 37(2), 117-122.

Liu, S., Heitz, R. P., \& Bradberry, C. W. (2009). A touch screen based stop signal response task in rhesus monkeys for studying impulsivity associated with chronic cocaine self-administration. Journal of Neuroscience Methods, 177(1), 67-72.

Liu, Y., Elliott, A. L., Striley, C. W., Gurka, K. K., \& Cottler, L. B. (2019). Motives for prescription stimulant use by patterns of non-medical use. Journal of Substance Use, 24(4), 455-460.

Lloyd, M. H., \& Akin, B. A. (2014). The disparate impact of alcohol, methamphetamine, and other drugs on family reunification. Children and Youth Services Review, 44, 72-81.

Lobmaier, P., Gossop, M., Waal, H., \& Bramness, J. (2010). The pharmacological treatment of opioid addiction- A clinical perspective. European Journal of Clinical Pharmacology, 66(6), 537-545.

Lochner, K., Pamuk, E., Makuc, D., Kennedy, B. P., \& Kawachi, I. (2001). State-level income inequality and individual mortality risk: A prospective, multilevel study. American Journal of Public health, 91(3), 385.

Loewenstein, G. (2000). Willpower: A decision-theorist's perspective. Law and Philosophy, 19(1), 51-76.

Loewenstein, G. (2000). Emotions in economic theory and economic behavior. American Economic Review, 90(2), 426-432.

Loewenstein, G., \& Elster, J. (Eds.). (1992). Choice over time. New York, NY: Russell Sage Foundation.

Loewenstein, G., \& Elster, J. (1992). Utility from memory and anticipation. In (Eds. Loewenstein, G., \& Elster, J.) Choice over time, 213-234. New York, NY: Russell Sage Foundation.

Loewenstein, G., Read, D., \& Baumeister, R. F. (Eds.). (2003). Time and decision: Economic and psychological perspectives of intertemporal choice. New York, NY: Russell Sage Foundation.

Loewenstein, G. F., \& Prelec, D. (1993). Preferences for sequences of outcomes. Psychological Review, 100(1), 91. 
Logan, T. K., Walker, R., Jordan, C. E., \& Leukefeld, C. G. (2006). Women and victimization:

Contributing factors, interventions, and implications. American Psychological Association.

Loibl, C. (2017). Living in poverty: Understanding the financial behaviour of vulnerable groups. Economic Psychology, 421-434.

Lloyd, C. (2013). The stigmatization of problem drug users: A narrative literature review. Drugs: Education, Prevention and Policy, 20(2), 85-95.

Lofwall, M. R., \& Havens, J. R. (2012). Inability to access buprenorphine treatment as a risk factor for using diverted buprenorphine. Drug and Alcohol Dependence, 126(3), 379-383.

Lofwall, M. R., Schuster, A., \& Strain, E. C. (2008). Changing profile of abused substances by older persons entering treatment. The Journal of Nervous and Mental Disease, 196(12), 898.

London, E. D., Berman, S. M., Voytek, B., Simon, S. L., Mandelkern, M. A., Monterosso, J., ... \& Hayashi, K. M. (2005). Cerebral metabolic dysfunction and impaired vigilance in recently abstinent methamphetamine abusers. Biological Psychiatry, 58(10), 770-778.

London, E. D., Ernst, M., Grant, S., Bonson, K., \& Weinstein, A. (2000). Orbitofrontal cortex and human drug abuse: Functional imaging. Cerebral Cortex, 10(3), 334-342.

Loo, R. (2002). A caveat on using single-item versus multiple-item scales. Journal of Managerial Psychology, 17(1), 68-75.

Lopez-Quintero, C., de los Cobos, J. P., Hasin, D. S., Okuda, M., Wang, S., Grant, B. F., \& Blanco, C. (2011). Probability and predictors of transition from first use to dependence on nicotine, alcohol, cannabis, and cocaine: Results of the National Epidemiologic Survey on Alcohol and Related Conditions (NESARC). Drug and Alcohol Dependence, 115(1-2), 120-130.

Lorvick, J., Browne, E. N., Lambdin, B. H., \& Comfort, M. (2018). Polydrug use patterns, risk behavior and unmet healthcare need in a community-based sample of women who use cocaine, heroin or methamphetamine. Addictive Behaviors, 85, 94-99.

Löwe, B., Decker, O., Müller, S., Brähler, E., Schellberg, D., Herzog, W., \& Herzberg, P. Y. (2008). Validation and standardization of the Generalized Anxiety Disorder Screener (GAD-7) in the general population. Medical Care, 266-274.

Lubman, D. I., Peters, L. A., Mogg, K., Bradley, B. P., \& Deakin, J. F. W. (2000). Attentional bias for drug cues in opiate dependence. Psychological Medicine, 30(1), 169-175.

Lucas, P., Walsh, Z., Crosby, K., Callaway, R., Belle-Isle, L., Kay, R., ... \& Holtzman, S. (2016). Substituting cannabis for prescription drugs, alcohol and other substances among medical cannabis patients: The impact of contextual factors. Drug and Alcohol Review, 35(3), 326-333.

Lutfy, K., \& Cowan, A. (2004). Buprenorphine: A unique drug with complex pharmacology. Current Neuropharmacology, 2(4), 395-402.

Luke, D. A. (2004). Multilevel modeling (Vol. 143). Sage.

Luoma, J. B., O'Hair, A. K., Kohlenberg, B. S., Hayes, S. C., \& Fletcher, L. (2010). The development and psychometric properties of a new measure of perceived stigma toward substance users. Substance Use \& Misuse, 45(1-2), 47-57. 
Lundqvist, T. (2005). Cognitive consequences of cannabis use: Comparison with abuse of stimulants and heroin with regard to attention, memory and executive functions. Pharmacology Biochemistry and Behavior, 81(2), 319-330.

Lupien, S. J., Maheu, F., Tu, M., Fiocco, A., \& Schramek, T. E. (2007). The effects of stress and stress hormones on human cognition: Implications for the field of brain and cognition. Brain and Cognition, 65(3), 209-237.

Lupien, S. J., McEwen, B. S., Gunnar, M. R., \& Heim, C. (2009). Effects of stress throughout the lifespan on the brain, behaviour and cognition. Nature Reviews Neuroscience, 10(6), 434.

Luu, H., Slavova, S., Freeman, P. R., Lofwall, M., Browning, S., \& Bush, H. (2018). Trends and patterns of opioid analgesic prescribing: Regional and rural-urban variations in kentucky from 2012 to 2015. The Journal of Rural Health.

Lykken, D. T., \& Venables, P. H. (1971). Direct measurement of skin conductance: A proposal for standardization. Psychophysiology, 8(5), 656-672.

Lynch, S., DeHart, D., Belknap, J., \& Green, B. (2012). Women's pathways to jail: The roles and intersections of serious mental illness and trauma. Washington, DC: US Department of Justice, Bureau of Justice Assistance.

Lyons, P., \& Rittner, B. (1998). The construction of the crack babies phenomenon as a social problem. American Journal of Orthopsychiatry, 68(2), 313-320.

Mani, A., Mullainathan, S., Shafir, E., \& Zhao, J. (2013). Poverty impedes cognitive function. Science, 341(6149), 976-980.

Maas, C. J., \& Hox, J. J. (2005). Sufficient sample sizes for multilevel modeling. Methodology, 1(3), 86-92.

MacArthur, G. J., Jacob, N., Pound, P., Hickman, M., \& Campbell, R. (2017). Among friends: A qualitative exploration of the role of peers in young people's alcohol use using Bourdieu's concepts of habitus, field and capital. Sociology of Health \& Illness, 39(1), 30-46.

McCabe, D. P., Roediger III, H. L., McDaniel, M. A., Balota, D. A., \& Hambrick, D. Z. (2010). The relationship between working memory capacity and executive functioning: Evidence for a common executive attention construct. Neuropsychology, 24(2), 222.

McCabe, S. E., Teter, C. J., \& Boyd, C. J. (2006). Medical use, illicit use and diversion of prescription stimulant medication. Journal of Psychoactive Drugs, 38(1), 43-56.

McCabe, S. E., West, B. T., Morales, M., Cranford, J. A., \& Boyd, C. J. (2007). Does early onset of nonmedical use of prescription drugs predict subsequent prescription drug abuse and dependence? Results from a national study. Addiction, 102(12), 1920-1930.

McCann, U. D., Kuwabara, H., Kumar, A., Palermo, M., Abbey, R., Brasic, J., ... \& Ricaurte, G. A. (2008). Persistent cognitive and dopamine transporter deficits in abstinent methamphetamine users. Synapse, 62(2), 91-100.

McGovern, R., \& McGovern, W. (2011). Voluntary risk-taking and heavy-end crack cocaine use: An edgework perspective. Health, Risk \& Society, 13(5), 487-500.

MacGregor, S. N., Keith, L. G., Chasnoff, I. J., Rosner, M. A., Chisum, G. M., Shaw, P., \& Minogue, J. P. (1987). Cocaine use during pregnancy: Adverse perinatal outcome. American Journal of Obstetrics and Gynecology, 157(3), 686-690. 
MacInnes, J. (2016). An introduction to secondary data analysis with IBM SPSS statistics. SAGE.

McKetin, R., Sutherland, R., Bright, D. A., \& Norberg, M. M. (2011). A systematic review of methamphetamine precursor regulations. Addiction, 106(11), 1911-1924.

MacKillop, J. (2013). Integrating behavioral economics and behavioral genetics: Delayed reward discounting as an endophenotype for addictive disorders. Journal of the Experimental Analysis of Behavior, 99(1), 14-31.

MacKillop, J. (2016). The behavioral economics and neuroeconomics of alcohol use disorders. Alcoholism: Clinical and Experimental Research, 40(4), 672-685.

MacKillop, J., Amlung, M. T., Acker, J., Gray, J. C., Brown, C. L., Murphy, J. G., ... \& Sweet, L. H. (2014). The neuroeconomics of alcohol demand: An initial investigation of the neural correlates of alcohol cost-benefit decision making in heavy drinking men. Neuropsychopharmacology, 39(8), 1988.

MacKillop, J., Amlung, M. T., Few, L. R., Ray, L. A., Sweet, L. H., \& Munafò, M. R. (2011). Delayed reward discounting and addictive behavior: A meta-analysis. Psychopharmacology, 216(3), 305321.

MacKillop, J., Murphy, J. G., Tidey, J. W., Kahler, C. W., Ray, L. A., \& Bickel, W. K. (2009). Latent structure of facets of alcohol reinforcement from a behavioral economic demand curve. Psychopharmacology, 203(1), 33-40.

MacKillop, J., O'hagen, S., Lisman, S. A., Murphy, J. G., Ray, L. A., Tidey, J. W., ... \& Monti, P. M. (2010). Behavioral economic analysis of cue-elicited craving for alcohol. Addiction, 105(9), 15991607.

MacLeod, C., \& Mathews, A. (1988). Anxiety and the allocation of attention to threat. The Quarterly journal of experimental psychology, 40(4), 653-670.

MacPherson, L., Magidson, J. F., Reynolds, E. K., Kahler, C. W., \& Lejuez, C. W. (2010). Changes in sensation seeking and risk-taking propensity predict increases in alcohol use among early adolescents. Alcoholism: Clinical and Experimental Research, 34(8), 1400-1408.

Macintyre, S., Ellaway, A., \& Cummins, S. (2002). Place effects on health: How can we conceptualise, operationalise and measure them?. Social Science \& Medicine, 55(1), 125-139.

Mack, K. A., Jones, C. M., \& Ballesteros, M. F. (2017). Illicit drug use, illicit drug use disorders, and drug overdose deaths in metropolitan and nonmetropolitan areas-United States. American Journal of Transplantation, 17(12), 3241-3252.

Mack, J. L. IndyStar. (2016, November 28). Convicted heroin dealer sentenced to life without parole. Retrieved from https://www.indystar.com/story/news/crime/2016/11/28/convicted-heroin-dealersentenced-life-without-parole/94558372/

Mackesy-Amiti, M. E., Fendrich, M., \& Goldstein, P. J. (1997). Sequence of drug use among serious drug users: Typical vs atypical progression. Drug and Alcohol Dependence, 45(3), 185-196.

Madden, G. J., \& Bickel, W. K. (Eds.). (2010). Impulsivity: The behavioral and neurological science of discounting. Washington, DC, US: American Psychological Association.

Madden, G. J., Bickel, W. K., \& Jacobs, E. A. (1999). Discounting of delayed rewards in opioid-dependent outpatients: Exponential or hyperbolic discounting functions?. Experimental and Clinical Psychopharmacology, 7(3), 284. 
Madden, G. J., Petry, N. M., Badger, G. J., \& Bickel, W. K. (1997). Impulsive and self-control choices in opioid-dependent patients and non-drug-using control patients: Drug and monetary rewards. Experimental and Clinical Psychopharmacology, 5(3), 256.

Madras, B. K. (2017). The surge of opioid use, addiction, and overdoses: Responsibility and response of the US health care system. JAMA Psychiatry, 74(5), 441-442.

Major, B., Dovidio, J. F., \& Link, B. G. (Eds.). (2017). The Oxford handbook of stigma, discrimination, and health. Oxford University Press.

Mäkelä, K. (2004). Studies of the reliability and validity of the Addiction Severity Index. Addiction, 99(4), $398-410$.

Mallatt, J. (2018). The effect of Prescription Drug Monitoring Programs on opioid prescriptions and heroin crime rates. Available at SSRN 3050692.

Mallik-Kane, K., \& Visher, C. A. (2008). Health and prisoner reentry: How physical, mental, and substance abuse conditions shape the process of reintegration (p. 82). Washington, DC: Urban Institute Justice Policy Center.

Maner, J. K., Richey, J. A., Cromer, K., Mallott, M., Lejuez, C. W., Joiner, T. E., \& Schmidt, N. B. (2007). Dispositional anxiety and risk-avoidant decision-making. Personality and Individual Differences, 42(4), 665-675.

Mann, W. E. (2004) Ethics. In J.E. Brower \& K. Guilfoy, (Eds.) The Cambridge Companion to Abelard. Cambridge, MA: Cambridge University Press. 279-304.

Mantsch, J. R., Baker, D. A., Funk, D., Lê, A. D., \& Shaham, Y. (2016). Stress-induced reinstatement of drug seeking: 20 years of progress. Neuropsychopharmacology, 4l(1), 335.

Marissen, M. A., Franken, I. H., Waters, A. J., Blanken, P., Van Den Brink, W., \& Hendriks, V. M. (2006). Attentional bias predicts heroin relapse following treatment. Addiction, 101(9), 1306-1312.

Marissen, M. A., Franken, I. H., Waters, A. J., Blanken, P., van den Brink, W., \& Hendriks, V. M. (2005). Attentional bias predicts heroin dependence following treatment. Cue Exposure Therapy for the treatment of heroin addiction, 82 .

Mark, T. L., Woody, G. E., Juday, T., \& Kleber, H. D. (2001). The economic costs of heroin addiction in the United States. Drug and Alcohol Dependence, 61(2), 195-206.

Marlatt, G. A., Baer, J. S., Donovan, D. M., \& Kivlahan, D. R. (1988). Addictive behaviors: Etiology and treatment. Annual Review of Psychology, 39(1), 223-252.

Marlatt, G. A. (1996). Models of relapse and relapse prevention: A commentary. Experimental and Clinical Psychopharmacology, 4(1), 55-60.

Marlatt, G. A., Baer, J. S., \& Quigley, L. A. (1997). 10. Self-efficacy and addictive behavior. Selfefficacy in Changing Societies, 289-315.

Marlowe, D. B., \& Wong, C. J. (2008). Contingency management in adult criminal drug courts. Contingency management in substance abuse treatment, 334-354.

Marmot, M. (2004). Status syndrome. Significance, 1(4), 150-154. 
Marazziti, D., Consoli, G., Picchetti, M., Carlini, M., \& Faravelli, L. (2010). Cognitive impairment in major depression. European Journal of Pharmacology, 626(1), 83-86.

Marrink, S. J., Risselada, H. J., Yefimov, S., Tieleman, D. P., \& De Vries, A. H. (2007). The MARTINI force field: Coarse grained model for biomolecular simulations. The Journal of Physical Chemistry B, 111(27), 7812-7824.

Marriott, M. (1989, February 20). After 3 Years, crack plague in New York only gets worse. New York Times. Retrieved from https://www.nytimes.com/1989/02/20/nyregion/after-3-years-crack-plaguein-new-york-only-gets-worse.html

Mars, S. G., Bourgois, P., Karandinos, G., Montero, F., \& Ciccarone, D. (2014). “Every 'never' I ever said came true": Transitions from opioid pills to heroin injecting. International Journal of Drug Policy, 25(2), 257-266.

Mars, S. G., Rosenblum, D., \& Ciccarone, D. (2018). Illicit fentanyls in the opioid street market: Desired or imposed?. Addiction.

Marsh, W., Copes, H., \& Linnemann, T. (2017). Creating visual differences: Methamphetamine users perceptions of anti-meth campaigns. International Journal of Drug Policy, 39, 52-61.

Marshall, B. D., Krieger, M. S., Yedinak, J. L., Ogera, P., Banerjee, P., Alexander-Scott, N. E., ... \& Green, T. C. (2017). Epidemiology of fentanyl-involved drug overdose deaths: A geospatial retrospective study in Rhode Island, USA. International Journal of Drug Policy, 46, 130-135.

Martel, M. O., Dolman, A. J., Edwards, R. R., Jamison, R. N., \& Wasan, A. D. (2014). The association between negative affect and prescription opioid misuse in patients with chronic pain: The mediating role of opioid craving. The Journal of Pain, 15(1), 90-100.

Martel, M. O., Finan, P. H., McHugh, R. K., Issa, M., Edwards, R. R., Jamison, R. N., \& Wasan, A. D. (2016). Day-to-day pain symptoms are only weakly associated with opioid craving among patients with chronic pain prescribed opioid therapy. Drug and Alcohol Dependence, 162, 130-136.

Maruna, S., \& King, A. (2009). Once a criminal, always a criminal?:‘ Redeemability’ and the psychology of punitive public attitudes. European Journal on Criminal Policy and Research, 15(1-2), 7-24.

Mather, M. (2004). Households and families in Appalachia. Washington, DC: Appalachian Regional Commission.

Matheson, C., Jaffray, M., Ryan, M., Bond, C. M., Fraser, K., Kirk, M., \& Liddell, D. (2014). Public opinion of drug treatment policy: Exploring the public's attitudes, knowledge, experience and willingness to pay for drug treatment strategies. International Journal of Drug Policy, 25(3), 407415.

Matthews, K. A., Croft, J. B., Liu, Y., Lu, H., Kanny, D., Wheaton, A. G., ... \& Eke, P. I. (2017). Healthrelated behaviors by urban-rural county classification-United States, 2013. Morbidity and Mortality Weekly Report. Surveillance Summaries (Washington, DC: 2002), 66(5), 1-8.

Mathews, E. M., Jeffries, E., Hsieh, C., Jones, G., \& Buckner, J. D. (2019). Synthetic cannabinoid use among college students. Addictive Behaviors.

Mattison, R. E., Hooper, S. R., \& Carlson, G. A. (2006). Neuropsychological characteristics of special education students with serious emotional/behavioral disorders. Behavioral Disorders, 31(2), 176188. 
Mauer, M. (2004). Race, class, and the development of criminal justice policy 1. Review of Policy Research, 21(1), 79-92.

Maxwell, J. C. \& Rutkowski, B. A. (2008). The prevalence of methamphetamine and amphetamine abuse in North America: A review of the indicators, 1992-2007. Drug and Alcohol Review, 27(3), 229235 .

Mayer, A. R., Mannell, M. V., Ling, J., Gasparovic, C., \& Yeo, R. A. (2011). Functional connectivity in mild traumatic brain injury. Human Brain Mapping, 32(11), 1825-1835.

Mayes, S. D., Calhoun, S. L., \& Crowell, E. W. (2000). Learning disabilities and ADHD: Overlapping spectrum disorders. Journal of Learning Disabilities, 33(5), 417-424.

Mayock, P., Cronly, J., \& Clatts, M. C. (2015). The risk environment of heroin use initiation: Young women, intimate partners, and "drug relationships". Substance Use \& Misuse, 50(6), 771-782.

Mcallister, T. W., Flashman, L. A., Sparling, M. B., \& Saykin, A. J. (2004). Working memory deficits after traumatic brain injury: Catecholaminergic mechanisms and prospects for treatment-A review. Brain Injury, 18(4), 331-350.

McCabe, S. E., Teter, C. J., Boyd, C. J., Wilens, T. E., \& Schepis, T. S. (2018). Sources of prescription medication misuse among young adults in the United States: The role of educational status. The Journal of Clinical Psychiatry, 79(2).

McCabe, S. E., West, B. T., Morales, M., Cranford, J. A., \& Boyd, C. J. (2007). Does early onset of nonmedical use of prescription drugs predict subsequent prescription drug abuse and dependence?

Results from a national study. Addiction, 102(12), 1920-1930.

McCance-Katz, E. F., Price, L. H., McDougle, C. J., Kosten, T. R., Black, J. E., \& Jatlow, P. I. (1993). Concurrent cocaine-ethanol ingestion in humans: Pharmacology, physiology, behavior, and the role of cocaethylene. Psychopharmacology, 111(1), 39-46.

McCann, K. S., Barker, S., Cousins, R., Franks, A., McDaniel, C., Petrany, S., \& Riley, E. (2018). Structured management of chronic nonmalignant pain with opioids in a rural primary care office. The Journal of the American Board of Family Medicine, 31(1), 57-63.

McClure, S. M., Ericson, K. M., Laibson, D. I., Loewenstein, G., \& Cohen, J. D. (2007). Time discounting for primary rewards. Journal of Neuroscience, 27(21), 5796-5804.

McClure, S. M., Laibson, D. I., Loewenstein, G., \& Cohen, J. D. (2004). Separate neural systems value immediate and delayed monetary rewards. Science, 306(5695), 503-507.

McCrady, B. S. (2004). To have but one true friend: Implications for practice of research on alcohol use disorders and social network. Psychology of Addictive Behaviors, 18(2), 113.

McCorkle, R. C. (1993). Research note: Punish and rehabilitate? Public attitudes toward six common crimes. Crime \& Delinquency, 39(2), 240-252.

McCrory, P., Meeuwisse, W., Johnston, K., Dvorak, J., Aubry, M., Molloy, M., \& Cantu, R. (2009). Consensus statement on concussion in sport-the 3rd international conference on concussion in sport held in Zurich, November 2008. South African Journal of Sports Medicine, 21(2).

McEwen, B. S. (1998). Stress, adaptation, and disease: Allostasis and allostatic load. Annals of the New York academy of sciences, 840(1), 33-44. 
McEwen, B. S. (2005). Stressed or stressed out: What is the difference?. Journal of Psychiatry and Neuroscience, 30(5), 315.

McEwen, B. S. (2008). Central effects of stress hormones in health and disease: Understanding the protective and damaging effects of stress and stress mediators. European Journal of Pharmacology, 583(2), 174-185.

McEwen, B. S., \& Gianaros, P. J. (2010). Central role of the brain in stress and adaptation: Links to socioeconomic status, health, and disease. Annals of the New York Academy of Sciences, 1186(1), 190-222.

McEwen, B. S. (2006). Protective and damaging effects of stress mediators: Central role of the brain. Dialogues in Clinical Neuroscience, 8(4), 367.

McEwen, B. S. (2012). Brain on stress: How the social environment gets under the skin. Proceedings of the National Academy of Sciences, 109 (Supplement 2), 17180-17185.

McEwen, B. S., \& Sapolsky, R. M. (1995). Stress and cognitive function. Current Opinion in Neurobiology, 5(2), 205-216.

McGue, M., Iacono, W. G., Legrand, L. N., Malone, S., \& Elkins, I. (2001). Origins and consequences of age at first drink. I. Associations with substance-use disorders, disinhibitory behavior and psychopathology, and P3 amplitude. Alcoholism: Clinical and Experimental Research, 25(8), $1156-1165$.

Mcgregor, C., Darke, S., Ali, R., \& Christie, P. (1998). Experience of non-fatal overdose among heroin users in Adelaide, Australia: Circumstances and risk perceptions. Addiction, 93(5), 701-711.

McHugh R. K., Park S., \& Weiss R., 2014. Cue-induced craving in prescription opioid and heroin dependence. Drug and Alcohol Dependence 140: e141.

McKenzie-Quirk, S. D., \& Miczek, K. A. (2008). Social rank and social separation as determinants of alcohol drinking in squirrel monkeys. Psychopharmacology, 201(1), 137.

McKnight, P. E., McKnight, K. M., Sidani, S., \& Figueredo, A. J. (2007). Missing data: A gentle introduction. Guilford Press.

McKnight, P. E., \& McKnight, K. M. (2011). Missing data in secondary data analysis in (eds. Trzesniewski, K. H., Donnellan, M., \& Lucas, R. E.) Secondary data analysis: An introduction for psychologists, 83-101.

McLellan, A., Cacciola, J., Kushner, H., Peters, R., Smith, I., \& Pettinati, H. (1992). The fifth edition of the Addiction Severity Index: Historical critique and normative data. Journal of Substance Abuse Treatment, 9, 199-213.

McLellan, A., Luborsky, L., Cacciola, J., Griffith, B., Evans, R., Barr, H., \& O’Brien, C. (1985). New data from the Addiction Severity Index: Reliability and validity in three centers. Journal of Nervous and Mental Disease, 173, 412-423.

McLellan, A. T., Lewis, D. C., O'brien, C. P., \& Kleber, H. D. (2000). Drug dependence, a chronic medical illness: Implications for treatment, insurance, and outcomes evaluation. Jama, 284(13), 16891695.

McKay, J. R. (2017). Making the hard work of recovery more attractive for those with substance use disorders. Addiction, 112(5), 751-757. 
McKee, A. C., Cantu, R. C., Nowinski, C. J., Hedley-Whyte, E. T., Gavett, B. E., Budson, A. E., ... \& Stern, R. A. (2009). Chronic traumatic encephalopathy in athletes: Progressive tauopathy after repetitive head injury. Journal of Neuropathology \& Experimental Neurology, 68(7), 709-735.

McLaughlin, D. K., \& Stokes, C. S. (2002). Income inequality and mortality in US counties: does minority racial concentration matter?. American Journal of Public Health, 92(1), 99-104.

McLean, K. (2016). “There's nothing here": Deindustrialization as risk environment for overdose. International Journal of Drug Policy, 29, 19-26.

McNamara, R., Dalley, J. W., Robbins, T. W., Everitt, B. J., \& Belin, D. (2010). Trait-like impulsivity does not predict escalation of heroin self-administration in the rat. Psychopharmacology, 212(4), 453464.

Meier, S., \& Sprenger, C. (2010). Present-biased preferences and credit card borrowing. American Economic Journal: Applied Economics, 2(1), 193-210.

Meehan, W. P., 3rd, \& Mannix, R. (2010). Pediatric concussions in United States emergency departments 889-893 in the years 2002-2006. Journal of Pediatrics, 157 (6),

Mehling, W. E., Daubenmier, J., Price, C. J., Acree, M., Bartmess, E., \& Stewart, A. L. (2013). Selfreported interoceptive awareness in primary care patients with past or current low back pain. Journal of Pain Research, 6, 403.

Meier, S., \& Sprenger, C. (2010). Present-biased preferences and credit card borrowing. American Economic Journal: Applied Economics, 2(1), 193-210.

Meit, M., Heffernan, M., Tanenbaum, E., \& Hoffmann, T. (2017). Appalachian diseases of despair. Report for the Appalachian Regional Commission. The Walsh Center for Rural Health Analysis National Opinion Research Center (NORC) at the University of Chicago, editor.

Mejía-Cruz, D., Green, L., Myerson, J., Morales-Chainé, S., \& Nieto, J. (2016). Delay and probability discounting by drug-dependent cocaine and marijuana users. Psychopharmacology, 233(14), 2705-2714.

Melrose, A. J., Hsu, E., \& Monterosso, J. (2015). the Potent but Inconsistent Motivations Characteristic of Addiction. The Wiley Handbook on the Cognitive Neuroscience of Addiction, 440.

Menard, S. (2002). Applied logistic regression analysis (Vol. 106). Sage.

Merikangas, K. R., Mehta, R. L., Molnar, B. E., Walters, E. E., Swendsen, J. D., Aguilar-Gaziola, S., ... \& Kolody, B. (1998). Comorbidity of substance use disorders with mood and anxiety disorders: Results of the International Consortium in Psychiatric Epidemiology. Addictive Behaviors, 23(6), 893-907.

Mericle, A. A., Karriker-Jaffe, K. J., Gupta, S., Sheridan, D. M., \& Polcin, D. L. (2016). Distribution and neighborhood correlates of sober living house locations in Los Angeles. American Journal of Community Psychology, 58(1-2), 89-99.

Merrall, E. L., Kariminia, A., Binswanger, I. A., Hobbs, M. S., Farrell, M., Marsden, J., ... \& Bird, S. M. (2010). Meta-analysis of drug-related deaths soon after release from prison. Addiction, 105(9), $1545-1554$

Messick, S. (1975). The standard problem: Meaning and values in measurement and evaluation. American Psychologist, 30(10), 955. 
Meurk, C., Carter, A., Partridge, B., Lucke, J., \& Hall, W. (2014a). How is acceptance of the brain disease model of addiction related to Australians' attitudes towards addicted individuals and treatments for addiction?. BMC Psychiatry, 14(1), 373.

Meurk, C., Partridge, B., Carter, A., Hall, W., Morphett, K., \& Lucke, J. (2014b). Public attitudes in Australia towards the claim that addiction is a (brain) disease. Drug and Alcohol Review, 33(3), 272-279.

Meyers, M. (2004). Crack mothers in the news: A narrative of paternalistic racism. Journal of Communication Inquiry, 28(3), 194-216.

Meyers, L. S., Gamst, G., \& Guarino, A. J. (2016). Applied multivariate research: Design and interpretation. Thousand Oaks, CA. Sage publications.

Midanik, L. T., Tam, T. W., \& Weisner, C. (2007). Concurrent and simultaneous drug and alcohol use: Results of the 2000 National Alcohol Survey. Drug and Alcohol Dependence, 90(1), 72-80.

Midi, H., Sarkar, S. K., \& Rana, S. (2010). Collinearity diagnostics of binary logistic regression model. Journal of Interdisciplinary Mathematics, 13(3), 253-267.

Miller, W. R. (2004). The phenomenon of quantum change. Journal of Clinical Psychology, 60(5), 453460.

Miller, L. (1992). Neuropsychology, personality, and substance abuse in the head injury case: Clinical and forensic issues. International Journal of Law and Psychiatry, 15(3), 303-316.

Miller, J. W., Naimi, T. S., Brewer, R. D., \& Jones, S. E. (2007). Binge drinking and associated health risk behaviors among high school students. Pediatrics, 119(1), 76-85.

Miller, M. L., Ren, Y., Szutorisz, H., Warren, N. A., Tessereau, C., Egervári, G., ... \& Schumann, G. (2018). Ventral striatal regulation of CREM mediates impulsive action and drug addiction vulnerability. Molecular Psychiatry, 23(5), 1328.

Misailidi, N., Papoutsis, I., Nikolaou, P., Dona, A., Spiliopoulou, C., \& Athanaselis, S. (2018). Fentanyls continue to replace heroin in the drug arena: the cases of ocfentanil and carfentanil. Forensic Toxicology, 36(1), 12-32.

Mischel, W. (1973). Toward a cognitive social learning reconceptualization of personality. Psychological Review, 80(4), 252.

Mischel, W., Ebbesen, E. B., \& Raskoff Zeiss, A. (1972). Cognitive and attentional mechanisms in delay of gratification. Journal of Personality and Social Psychology, 21(2), 204.

Mischel, W., Ayduk, O., Berman, M. G., Casey, B. J., Gotlib, I. H., Jonides, J., ... \& Shoda, Y. (2010). 'Willpower'over the life span: Decomposing self-regulation. Social Cognitive and Affective Neuroscience, 6(2), 252-256.

Mishna, F. (2003). Learning disabilities and bullying: Double jeopardy. Journal of Learning Disabilities, 36(4), 336-347.

Mishra, S., \& Lalumière, M. L. (2010). You can't always get what you want: The motivational effect of need on risk-sensitive decision-making. Journal of Experimental Social Psychology, 46(4), 605611. 
Mistry, C. J., Bawor, M., Desai, D., C Marsh, D., \& Samaan, Z. (2014). Genetics of opioid dependence: A review of the genetic contribution to opioid dependence. Current Psychiatry Reviews, 10(2), 156167.

Mital, S., Windle, M., Cooper, H. L., \& Crawford, N. D. (2018). Trends in non-medical prescription opioids and heroin co-use among adults, 2003-2014. Addictive Behaviors, 86, 17-23.

Mitchell, S. H. (2004). Effects of short-term nicotine deprivation on decision-making: Delay, uncertainty and effort discounting. Nicotine \& Tobacco Research, 6(5), 819-828.

Mitchell, S. G., Gryczynski, J., \& Schwartz, R. P. (2018). Commentary on "the more things change: Buprenorphine/naloxone diversion continues while treatment is inaccessible". Journal of Addiction Medicine, 12(6), 424-425.

Mobbs, D., Marchant, J. L., Hassabis, D., Seymour, B., Tan, G., Gray, M., ... \& Frith, C. D. (2009). From threat to fear: The neural organization of defensive fear systems in humans. Journal of Neuroscience, 29(39), 12236-12243.

Moeller, F. G., Barratt, E. S., Dougherty, D. M., Schmitz, J. M., \& Swann, A. C. (2001). Psychiatric aspects of impulsivity. American Journal of Psychiatry, 158(11), 1783-1793.

Moeller, K. E., Kissack, J. C., Atayee, R. S., \& Lee, K. C. (2017, May). Clinical interpretation of urine drug tests: what clinicians need to know about urine drug screens. In Mayo Clinic Proceedings (Vol. 92, No. 5, pp. 774-796). Elsevier.

Mohler-Kuo, M., Lee, J. E., \& Wechsler, H. (2003). Trends in marijuana and other illicit drug use among college students: Results from 4 Harvard School of Public Health College Alcohol Study surveys: 1993-2001. Journal of American College Health, 52(1), 17-24.

Mohr, C. D., Arpin, S., \& McCabe, C. T. (2015). Daily affect variability and context-specific alcohol consumption. Drug and Alcohol Review, 34(6), 581-587.

Molfenter, T., Fitzgerald, M., Jacobson, N., McCarty, D., Quanbeck, A., \& Zehner, M. (2019). Barriers to buprenorphine expansion in Ohio: A time-elapsed qualitative study. Journal of Psychoactive Drugs, 1-8.

Monico, L. B., \& Mitchell, S. G. (2018). Patient perspectives of transitioning from prescription opioids to heroin and the role of route of administration. Substance Abuse Treatment, Prevention, and Policy, 13(1), 4.

Monnat, S. M., \& Rigg, K. K. (2016). Examining rural/urban differences in prescription opioid misuse among US adolescents. The Journal of Rural Health, 32(2), 204-218.

Monnat, S. M. (2018). The contributions of socioeconomic and opioid supply factors to US drug mortality rates: Urban-rural and within-rural differences. Journal of Rural Studies.

Montague, P. R., \& Berns, G. S. (2002). Neural economics and the biological substrates of valuation. Neuron 36.

Monterosso, J., \& Ainslie, G. (1999). Beyond discounting: Possible experimental models of impulse control. Psychopharmacology, 146(4), 339-347.

Monterosso, J. R., \& Luo, S. (2010). An argument against dual valuation system competition: Cognitive capacities supporting future orientation mediate rather than compete with visceral motivations. Journal of Neuroscience, Psychology, and Economics, 3(1), 1. 
Monte, A. A., Mandell, T., Wilford, B. B., Tennyson, J., \& Boyer, E. W. (2009). Diversion of buprenorphine/naloxone coformulated tablets in a region with high prescribing prevalence. Journal of Addictive Diseases, 28(3), 226-231.

Monterosso, J., \& Ainslie, G. (2007). The behavioral economics of will in recovery from addiction. Drug and Alcohol Dependence, 90, S100-S111.

Monti, K. (2019). The Experience of Transitioning from Opioid Analgesics to Heroin: A Life Course Perspective (Doctoral dissertation, Adelphi University).

Monti, P. M., Rohsenow, D. J., \& Hutchison, K. E. (2000). Toward bridging the gap between biological, psychobiological and psychosocial models of alcohol craving. Addiction, 95(8s2), 229-236.

Moody, L. N., Satterwhite, E., \& Bickel, W. K. (2017). Substance use in rural Central Appalachia: Current status and treatment considerations. Journal of Rural Mental Health, 41(2), 123.

Moore, D. (2008). Erasing pleasure from public discourse on illicit drugs: On the creation and reproduction of an absence. International Journal of Drug Policy, 19(5), 353-358.

Moore, T. M., Seavey, A., Ritter, K., McNulty, J. K., Gordon, K. C., \& Stuart, G. L. (2014). Ecological momentary assessment of the effects of craving and affect on risk for relapse during substance abuse treatment. Psychology of Addictive Behaviors, 28(2), 619.

Moore, K., Stuewig, J., \& Tangney, J. (2013). Jail inmates' perceived and anticipated stigma: Implications for post-release functioning. Self and Identity, 12(5), 527-547.

Moore, K. E., Stuewig, J. B., \& Tangney, J. P. (2016). The effect of stigma on criminal offenders' functioning: A longitudinal mediational model. Deviant Behavior, 37(2), 196-218.

Moore, K. E., Tangney, J. P., \& Stuewig, J. B. (2016). The self-stigma process in criminal offenders. Stigma and Health, 1(3), 206.

Morgado, P., Sousa, N., \& Cerqueira, J. J. (2015). The impact of stress in decision making in the context of uncertainty. Journal of Neuroscience Research, 93(6), 839-847.

Morgan, D., Grant, K. A., Gage, H. D., Mach, R. H., Kaplan, J. R., Prioleau, O., ... \& Nader, M. A. (2002). Social dominance in monkeys: Dopamine D 2 receptors and cocaine self-administration. Nature Neuroscience, 5(2), 169.

Mowen, T. J., \& Visher, C. A. (2015). Drug use and crime after incarceration: The role of family support and family conflict. Justice Quarterly, 32(2), 337-359.

Mulia, N., Schmidt, L., Bond, J., Jacobs, L., \& Korcha, R. (2008). Stress, social support and problem drinking among women in poverty. Addiction, 103(8), 1283-1293.

Mullainathan, S., \& Shafir, E. (2013). Scarcity: Why having too little means so much. Macmillan.

Mullainathan, S., \& Shafir, E. (2009). Savings policy and decision-making in low-income households. Insufficient funds: Savings, assets, credit, and banking among low-income households, 121, 140-142.

Müller, C. P., \& Schumann, G. (2011). Drugs as instruments: A new framework for non-addictive psychoactive drug use. Behavioral and Brain Sciences, 34(6), 293-310. 
Mumola, C. J., \& Karberg, J. C. (2006). Drug use and dependence, state and federal prisoners, 2004 (pp. 1-12). Washington, DC: US Department of Justice, Office of Justice Programs, Bureau of Justice Statistics.

Muramatsu, N. (2003). County-level income inequality and depression among older Americans. Health Services Research, 38(6p2), 1863-1884.

Muriach, M., López-Pedrajas, R., Barcia, J. M., Sanchez-Villarejo, M. V., Almansa, I., \& Romero, F. J. (2010). Cocaine causes memory and learning impairments in rats: Involvement of nuclear factor kappa B and oxidative stress, and prevention by topiramate. Journal of Neurochemistry, 114(3), 675-684.

Murphy, J. G., Correia, C. J., Colby, S. M., \& Vuchinich, R. E. (2005). Using behavioral theories of choice to predict drinking outcomes following a brief intervention. Experimental and Clinical Psychopharmacology, 13(2), 93.

Murphy, J. G., \& Dennhardt, A. A. (2016). The behavioral economics of young adult substance abuse. Preventive Medicine, 92, 24-30.

Murphy, F. C., Rubinsztein, J. S., Michael, A., Rogers, R. D., Robbins, T. W., Paykel, E. S., \& Sahakian, B. J. (2001). Decision-making cognition in mania and depression. Psychological Medicine, 31(4), 679-693.

Myerson, J., Green, L., van den Berk-Clark, C., \& Grucza, R. A. (2015). Male, but not female, alcoholdependent African Americans discount delayed gains more steeply than propensity-score matched controls. Psychopharmacology, 232(24), 4493-4503.

Nader, M. A., \& Banks, M. L. (2014). Environmental modulation of drug taking: Nonhuman primate models of cocaine abuse and PET neuroimaging. Neuropharmacology, 76, 510-517.

Nader, M. A., Morgan, D., Gage, H. D., Nader, S. H., Calhoun, T. L., Buchheimer, N., ... \& Mach, R. H. (2006). PET imaging of dopamine D2 receptors during chronic cocaine self-administration in monkeys. Nature Neuroscience, 9(8), 1050.

Nader, M. A., Nader, S. H., Czoty, P. W., Riddick, N. V., Gage, H. D., Gould, R. W., ... \& Morton, D. (2012). Social dominance in female monkeys: Dopamine receptor function and cocaine reinforcement. Biological Psychiatry, 72(5), 414-421.

Nagy, M. S. (2002). Using a single-item approach to measure facet job satisfaction. Journal of Occupational and Organizational Psychology, 75(1), 77-86.

Nargiso, J. E., Ballard, E. L., \& Skeer, M. R. (2015). A systematic review of risk and protective factors associated with nonmedical use of prescription drugs among youth in the United States: A social ecological perspective. Journal of Studies on Alcohol and Drugs, 76(1), 5-20.

Nation, M., \& Heflinger, C. A. (2006). Risk factors for serious alcohol and drug use: The role of psychosocial variables in predicting the frequency of substance use among adolescents. The American Journal of Drug and Alcohol Abuse, 32(3), 415-433.

National Institute on Drug Abuse. (2018). Drugs, Brains, and Behavior: The Science of Addiction. Retrieved from National Institutes of Health; U.S. Department of Health and Human Services. website: https://d14rmgtrwzf5a.cloudfront.net/sites/default/files/soa.pdf

Naqvi, N. H., \& Bechara, A. (2010). The insula and drug addiction: An interoceptive view of pleasure, urges, and decision-making. Brain Structure and Function, 214(5-6), 435-450. 
Naqvi, N. H., Rudrauf, D., Damasio, H., and Bechara, A. (2007). Damage to the insula disrupts addiction to cigarette smoking. Science 315, 531-534. doi: 10.1126/science.1135926

Naqvi, N., Shiv, B., \& Bechara, A. (2006). The role of emotion in decision making: A cognitive neuroscience perspective. Current Directions in Psychological Science, 15(5), 260-264.

Narita, M., Kishimoto, Y., Ise, Y., Yajima, Y., Misawa, K., \& Suzuki, T. (2005). Direct evidence for the involvement of the mesolimbic $\kappa$-opioid system in the morphine-induced rewarding effect under an inflammatory pain-like state. Neuropsychopharmacology, 30(1), 111.

Neaigus, A., Miller, M., Friedman, S. R., Hagen, D. L., Sifaneck, S. J., Ildefonso, G., \& Des Jarlais, D. C. (2001). Potential risk factors for the transition to injecting among non-injecting heroin users: A comparison of former injectors and never injectors. Addiction, 96(6), 847-860.

Nebes, R. D., Butters, M. A., Mulsant, B. H., Pollock, B. G., Zmuda, M. D., Houck, P. R., \& Reynolds, C. F. (2000). Decreased working memory and processing speed mediate cognitive impairment in geriatric depression. Psychological Medicine, 30(3), 679-691.

Neisewander, J. L., Peartree, N. A., \& Pentkowski, N. S. (2012). Emotional valence and context of social influences on drug abuse-related behavior in animal models of social stress and prosocial interaction. Psychopharmacology, 224(1), 33-56.

Nelson, A., \& Killcross, S. (2006). Amphetamine exposure enhances habit formation. Journal of Neuroscience, 26(14), 3805-3812.

Nesse, R. M. (1994). Fear and fitness: An evolutionary analysis of anxiety disorders. Ethology and Sociobiology, 15(5-6), 247-261.

Netherland, J., \& Hansen, H. B. (2016). The war on drugs that wasn't: Wasted whiteness, "dirty doctors," and race in media coverage of prescription opioid misuse. Culture, Medicine, and Psychiatry, 40(4), 664-686.

Newschaffer, C. (1998). Validation of Behavioral Risk Factor Surveillance System (BRFSS) HRQOL measures in a statewide sample. Atlanta: U.S. Department of health and Human Services, Public Health Service, Centers for Disease Control and Prevention, National Center for Chronic Disease Prevention and Health Promotion.

Newcombe, V. F., Outtrim, J. G., Chatfield, D. A., Manktelow, A., Hutchinson, P. J., Coles, J. P., ... \& Menon, D. K. (2011). Parcellating the neuroanatomical basis of impaired decision-making in traumatic brain injury. Brain, 134(3), 759-768.

Nichols, T. T., \& Wilson, S. J. (2015). Working memory functioning and addictive behavior. The Wiley Handbook On the Cognitive Neuroscience of Addiction, 55-75. Hoboken, NJ: John Wiley and Sons

Newman, J. P. (1987). Reaction to punishment in extraverts and psychopaths: Implications for the impulsive behavior of disinhibited individuals. Journal of Research in Personality, 21(4), 464480 .

Nielsen, D. A., \& Kreek, M. J. (2012). Common and specific liability to addiction: Approaches to association studies of opioid addiction. Drug and Alcohol Dependence, 123, S33-S41.

Nixon, S. J., Paul, R., \& Phillips, M. (1998). Cognitive efficiency in alcoholics and polysubstance abusers. Alcoholism: Clinical and Experimental Research, 22(7), 1414-1420. 
Noble, K. G., McCandliss, B. D., \& Farah, M. J. (2007). Socioeconomic gradients predict individual differences in neurocognitive abilities. Developmental Science, 10(4), 464-480.

Noël, X., Brevers, D., \& Bechara, A. (2013). A neurocognitive approach to understanding the neurobiology of addiction. Current Opinion in Neurobiology, 23(4), 632-638.

Noid, W. G., Chu, J. W., Ayton, G. S., Krishna, V., Izvekov, S., Voth, G. A., ... \& Andersen, H. C. (2008). The multiscale coarse-graining method. I. A rigorous bridge between atomistic and coarse-grained models. The Journal of Chemical Physics, 128(24), 244114.

Nolan, M. L., Shamasunder, S., Colon-Berezin, C., Kunins, H. V., \& Paone, D. (2019). Increased presence of fentanyl in cocaine-involved fatal overdoses: Implications for prevention. Journal of Urban Health, 96(1), 49-54.

Noonan, M. P., Sallet, J., Mars, R. B., Neubert, F. X., O'Reilly, J. X., Andersson, J. L., ... \& Rushworth, M. F. (2014). A neural circuit covarying with social hierarchy in macaques. PLoS Biology, 12(9), e1001940.

Nordahl, T. E., Salo, R., \& Leamon, M. (2003). Neuropsychological effects of chronic methamphetamine use on neurotransmitters and cognition: A review. The Journal of Neuropsychiatry and Clinical Neurosciences, 15(3), 317-325.

Norden, L., Saxon, L., Kåberg, M., Käll, K., Franck, J., \& Lidman, C. (2009). Knowledge of status and assessment of personal health consequences with hepatitis $\mathrm{C}$ are not enough to change risk behaviour among injecting drug users in Stockholm County, Sweden. Scandinavian Journal of Infectious Diseases, 41(10), 727-734.

Novak, S. P., \& Kral, A. H. (2011). Comparing injection and non-injection routes of administration for heroin, methamphetamine, and cocaine users in the United States. Journal of Addictive Diseases, 30(3), 248-257.

Nummenmaa, L., \& Tuominen, L. (2018). Opioid system and human emotions. British Journal of Pharmacology, 175(14), 2737-2749.

O'Connor, L., \& Berry, J. W. (1990). The drug-of-choice phenomenon: Why addicts start using their preferred drug. Journal of Psychoactive Drugs, 22(3), 305-311.

Oddy, M., Moir, J. F., Fortescue, D., \& Chadwick, S. (2012). The prevalence of traumatic brain injury in the homeless community in a UK city. Brain injury, 26(9), 1058-1064.

O’Donnell, J. K., Halpin, J., Mattson, C. L., Goldberger, B. A., \& Gladden, R. M. (2017). Deaths involving fentanyl, fentanyl analogs, and U-47700—10 states, July-December 2016. MMWR. Morbidity and Mortality Weekly Report, 66(43), 1197.

O'Curry, S., \& Strahilevitz, M. (2001). Probability and mode of acquisition effects on choices between hedonic and utilitarian options. Marketing Letters, 12(1), 37-49.

O'Donoghue, T., \& Rabin, M. (1999). Doing it now or later. American Economic Review, 89(1), 103-124.

O’Donoghue, T., \& Rabin, M. (2002). Addiction and present-biased preferences.

Ostafin, B. D., \& Feyel, N. (2019). The effects of a brief meaning in life intervention on the incentive salience of alcohol. Addictive behaviors, 90, 107-111. 
Offices of the United States Attorneys. (2017, July 28). Top of the Chain Heroin Trafficker Sentenced to Life in Federal Prison. Retrieved from https://www.justice.gov/usao-sc/pr/top-chain-herointrafficker-sentenced-life-federal-prison

O’Donnell, J. K., Gladden, R. M., \& Seth, P. (2017). Trends in deaths involving heroin and synthetic opioids excluding methadone, and law enforcement drug product reports, by census regionUnited States, 2006-2015. MMWR. Morbidity and Mortality Weekly Report, 66(34), 897.

O'Connor, T. (2009). Degrees of freedom. Philosophical Explorations, 12(2), 119-125.

Ogborne, A. C., Smart, R. G., Weber, T., \& Birchmore-Timney, C. (2000). Who is using cannabis as a medicine and why: An exploratory study. Journal of Psychoactive Drugs, 32(4), 435-443.

Okrent, D. (2010). Last call: The rise and fall of prohibition. Simon and Schuster.

Oleson, E. B., \& Roberts, D. C. (2009). Behavioral economic assessment of price and cocaine consumption following self-administration histories that produce escalation of either final ratios or intake. Neuropsychopharmacology, 34(3), 796.

Olmstead, T. A., Alessi, S. M., Kline, B., Pacula, R. L., \& Petry, N. M. 2015. The price elasticity of demand for heroin: Matched longitudinal and experimental evidence. Journal of Health Economics 41: 59-71.

Onnela, J. P., \& Rauch, S. L. (2016). Harnessing smartphone-based digital phenotyping to enhance behavioral and mental health. Neuropsychopharmacology, 41(7), 1691.

Oquendo, M. A., \& Volkow, N. D. (2018). Suicide: A silent contributor to opioid-overdose deaths. New England Journal of Medicine, 378(17), 1567-1569.

Ornstein, T. J., Iddon, J. L., Baldacchino, A. M., Sahakian, B. J., London, M., Everitt, B. J., \& Robbins, T. W. (2000). Profiles of cognitive dysfunction in chronic amphetamine and heroin abusers. Neuropsychopharmacology, 23(2), 113.

Ornstein, S. I., \& Levy, D. (1983). Price and income elasticities of demand for alcoholic beverages. In Genetics Behavioral Treatment Social Mediators and Prevention Current Concepts in Diagnosis (pp. 303-345). Boston, MA: Springer.

Orrick, E. A., Worrall, J. L., Morris, R. G., Piquero, A. R., Bales, W. D., \& Wang, X. (2011). Testing social support theory: A multilevel analysis of recidivism. Journal of Criminal Justice, 39(6), 499-508.

Orsi, R., Yuma-Guerrero, P., Sergi, K., Pena, A. A., \& Shillington, A. M. (2018). Drug overdose and child maltreatment across the United States' rural-urban continuum. Child Abuse \& Neglect, 86, 358367.

Oser, C. B., Harp, K. L., O'Connell, D. J., Martin, S. S., \& Leukefeld, C. G. (2012). Correlates of participation in peer recovery support groups as well as voluntary and mandated substance abuse treatment among rural and urban probationers. Journal of Substance Abuse Treatment, 42(1), 95101.

Oser, C. B., Knudsen, H. K., Staton-Tindall, M., Taxman, F., \& Leukefeld, C. (2009). Organizational-level correlates of the provision of detoxification services and medication-based treatments for substance abuse in correctional institutions. Drug and Alcohol Dependence, 103, S73-S81.

Oser, C., Leukefeld, C., Staton-Tindall, M., Duvall, J., Garrity, T., Stoops, W., ... \& Wright, P. (2011). Criminality among rural stimulant users in the United States. Crime \& Delinquency, 57(4), 600621. 
Ouellette, J. A., \& Wood, W. (1998). Habit and intention in everyday life: The multiple processes by which past behavior predicts future behavior. Psychological Bulletin, 124(1), 54.

Overbeck, J. R., \& Park, B. (2006). Powerful perceivers, powerless objects: Flexibility of powerholders' social attention. Organizational Behavior and Human Decision Processes, 99(2), 227-243.

Ozaki, S., Narita, M., Iino, M., Miyoshi, K., \& Suzuki, T. (2003). Suppression of the morphine-induced rewarding effect and G-protein activation in the lower midbrain following nerve injury in the mouse: Involvement of G-protein-coupled receptor kinase 2. Neuroscience, 116(1), 89-97.

Ozaki, S., Narita, M., Narita, M., Iino, M., Sugita, J., Matsumura, Y., \& Suzuki, T. (2002). Suppression of the morphine-induced rewarding effect in the rat with neuropathic pain: Implication of the reduction in $\mu$-opioid receptor functions in the ventral tegmental area. Journal of Neurochemistry, 82(5), 1192-1198.

Paasche, C., Weibel, S., Wittmann, M., \& Lalanne, L. (2018). Time perception and impulsivity: A proposed relationship in addictive disorders. Neuroscience \& Biobehavioral Reviews.

Pachankis, J. E. (2007). The psychological implications of concealing a stigma: A cognitive-affectivebehavioral model. Psychological Bulletin, 133(2), 328.

Pachankis, J. E., Hatzenbuehler, M. L., Wang, K., Burton, C. L., Crawford, F. W., Phelan, J. C., \& Link, B. G. (2017). The burden of stigma on health and well-being: A taxonomy of concealment, course, disruptiveness, esthetics, origin, and peril across 93 stigmas. Personality and Social Psychology Bulletin, 0146167217741313.

Pacula R. L. \& Powell D., 2018. A supply side perspective on the opioid crisis. Journal of Policy Analysis and Management, 37(2), 438-446.

Pager, D. (2003). The mark of a criminal record. American Journal of Sociology, 108(5), 937-975.

Palamar, J. J., Rutherford, C., \& Keyes, K. M. (2019). "Flakka" use among high school seniors in the United States. Drug and Alcohol Dependence.

Paliwal, P., Hyman, S. M., \& Sinha, R. (2008). Craving predicts time to cocaine relapse: Further validation of the Now and Brief versions of the cocaine craving questionnaire. Drug and Alcohol Dependence, 93(3), 252-259.

Pampel, F. C. (2000). Logistic regression: A primer (Vol. 132). Sage.

Pandria, N., Kovatsi, L., Vivas, A. B., \& Bamidis, P. D. (2018). Resting-state abnormalities in heroindependent individuals. Neuroscience, 378, 113-145.

Panebianco, D., Gallupe, O., Carrington, P. J., \& Colozzi, I. (2016). Personal support networks, social capital, and risk of relapse among individuals treated for substance use issues. International Journal of Drug Policy, 27, 146-153.

Parker, A. M., Strunk, D., \& Fiellin, D. A. (2018). State Responses to the Opioid Crisis. The Journal of Law, Medicine \& Ethics, 46(2), 367-381.

Parker, H., Williams, L., \& Aldridge, J. (2002). The normalization of 'sensible'recreational drug use: Further evidence from the north west England longitudinal study. Sociology, 36(4), 941-964.

Parker, H. J., Parker, H., Aldridge, J., \& Measham, F. (1998). Illegal leisure: The normalization of adolescent recreational drug use. London, UK. Psychology Press. 
Passetti, F., Clark, L., Mehta, M. A., Joyce, E., \& King, M. (2008). Neuropsychological predictors of clinical outcome in opiate addiction. Drug and Alcohol Dependence, 94(1-3), 82-91.

Paterline, B. (2013). A market in transition: Drug trafficking in southern Appalachia. Journal of Law and Criminal Justice, 1(1), 1.

Patorno, E., Glynn, R. J., Levin, R., Lee, M. P., \& Huybrechts, K. F. (2017). Benzodiazepines and risk of all cause mortality in adults: Cohort study. BMJ,358, j2941.

Patrick, S. W., Faherty, L. J., Dick, A. W., Scott, T. A., Dudley, J., \& Stein, B. D. (2019). Association among county-level economic factors, clinician supply, metropolitan or rural location, and neonatal abstinence syndrome. JAMA, 321(4), 385-393.

Patrick, S. W., Buntin, M. B., Martin, P. R., Scott, T. A., Dupont, W., Richards, M., \& Cooper, W. O. (2018). Barriers to accessing treatment for pregnant women with opioid use disorder in Appalachian states. Substance Abuse, 1-7.

Patrick, S. W., Fry, C. E., Jones, T. F., \& Buntin, M. B. (2016). Implementation of prescription drug monitoring programs associated with reductions in opioid-related death rates. Health Affairs, 35(7), 1324-1332.

Patrick, S. W., Buntin, M. B., Martin, P. R., Scott, T. A., Dupont, W., Richards, M., \& Cooper, W. O. (2018). Barriers to accessing treatment for pregnant women with opioid use disorder in Appalachian states. Substance Abuse, 1-7.

Patterson, E. B. (1991). Poverty, income inequality, and community crime rates. Criminology, 29(4), 755776.

Patton, R., Deluca, P., Kaner, E., Newbury-Birch, D., Phillips, T., \& Drummond, C. (2013). Alcohol screening and brief intervention for adolescents: The how, what and where of reducing alcohol consumption and related harm among young people. Alcohol and Alcoholism, 49(2), 207-212.

Paulozzi, L. J. (2012). Prescription drug overdoses: A review. Journal of Safety Research, 43(4), 283-289.

Paulus, M. P., \& Angela, J. Y. (2012). Emotion and decision-making: Affect-driven belief systems in anxiety and depression. Trends in Cognitive Sciences, 16(9), 476-483.

Paulus, M. P., Hozack, N. E., Zauscher, B. E., Frank, L., Brown, G. G., Braff, D. L., \& Schuckit, M. A. (2002). Behavioral and functional neuroimaging evidence for prefrontal dysfunction in methamphetamine-dependent subjects. Neuropsychopharmacology, 26(1), 53.

Paulus, M. P., Hozack, N., Zauscher, B., McDowell, J. E., Frank, L., Brown, G. G., \& Braff, D. L. (2001). Prefrontal, parietal, and temporal cortex networks underlie decision-making in the presence of uncertainty. Neuroimage, 13(1), 91-100.

Paulus, M. P., Lovero, K. L., Wittmann, M., \& Leland, D. S. (2008). Reduced behavioral and neural activation in stimulant users to different error rates during decision making. Biological Psychiatry, 63(11), 1054-1060.

Paulus, M. P., \& Stewart, J. L. (2014). Interoception and drug addiction. Neuropharmacology, 76, 342-350.

Pearlin, L. I., Menaghan, E. G., Lieberman, M. A., \& Mullan, J. T. (1981). The stress process. Journal of Health and Social behavior, 337-356. 
Peavy, K. M., Banta-Green, C. J., Kingston, S., Hanrahan, M., Merrill, J. O., \& Coffin, P. O. (2012). "Hooked on" prescription-type opiates prior to using heroin: Results from a survey of syringe exchange clients. Journal of Psychoactive Drugs, 44(3), 259-265.

Pedhazur, E. J., \& Pedhazur-Schmelkin, L. P. (2013). Measurement, design, and analysis: An integrated approach. Psychology Press.

Peiper, N. C., Clarke, S. D., Vincent, L. B., Ciccarone, D., Kral, A. H., \& Zibbell, J. E. (2019). Fentanyl test strips as an opioid overdose prevention strategy: Findings from a syringe services program in the Southeastern United States. International Journal of Drug Policy, 63, 122-128.

Peirce, R. S., Frone, M. R., Russell, M., Cooper, M. L., \& Mudar, P. (2000). A longitudinal model of social contact, social support, depression, and alcohol use. Health Psychology, 19(1), 28.

Pepper, G. V., \& Nettle, D. (2013). Death and the time of your life: Experiences of close bereavement are associated with steeper financial future discounting and earlier reproduction. Evolution and Human Behavior, 34(6), 433-439.

Pepper, G. V., \& Nettle, D. (2014). Socioeconomic disparities in health behaviour: An evolutionary perspective. In Applied Evolutionary Anthropology (pp. 225-243). Springer, New York, NY.

Pergolizzi, J. V., LeQuang, J. A., Berger, G. K., \& Raffa, R. B. (2017). The basic pharmacology of opioids informs the opioid discourse about misuse and abuse: A review. Pain and Therapy, 6(1), 1-16.

Perry, B. D., Pollard, R. A., Blakley, T. L., Baker, W. L., \& Vigilante, D. (1995). Childhood trauma, the neurobiology of adaptation, and "use-dependent" development of the brain: How "states" become "traits". Infant Mental Health Journal, 16(4), 271-291.

Peters, A., McEwen, B. S., \& Friston, K. (2017). Uncertainty and stress: Why it causes diseases and how it is mastered by the brain. Progress in Neurobiology, 156, 164-188.

Peterson, A. B. (2016). Increases in fentanyl-related overdose deaths-Florida and Ohio, 20132015. MMWR. Morbidity and Mortality Weekly Report, 65.

Peterson, J. C., Gubrium, A., \& Fiddian-Green, A. (2018). Meth mouth, white trash, and the pseudoracialization of methamphetamine use in the US. Health Communication, 1-10.

Petry, N. M. (2000). Effects of increasing income on polydrug use: A comparison of heroin, cocaine and alcohol abusers. Addiction, 95(5), 705-717.

Petry, N. M. (2001). Pathological gamblers, with and without substance abuse disorders, discount delayed rewards at high rates. Journal of Abnormal Psychology, 110(3), 482.

Petry, N. M. (2001). Delay discounting of money and alcohol in actively using alcoholics, currently abstinent alcoholics, and controls. Psychopharmacology, 154(3), 243-250.

Petry, N. M., Alessi, S. M., Carroll, K. M., Hanson, T., MacKinnon, S., Rounsaville, B., \& Sierra, S. (2006). Contingency management treatments: Reinforcing abstinence versus adherence with goalrelated activities. Journal of Consulting and Clinical Psychology, 74(3), 592.

Petry, N. M., \& Bickel, W. K. (1999). A behavioral economic analysis of polydrug abuse in heroin addicts. In The Economic Analysis of Substance Use and Abuse: An Integration of Econometrics and Behavioral Economic Research (pp. 213-250). University of Chicago Press.

Petry, N. M., Bickel, W. K., \& Arnett, M. (1998). Shortened time horizons and insensitivity to future consequences in heroin addicts. Addiction, 93(5), 729-738. 
Petry, N. M., Tedford, J., \& Martin, B. (2001). Reinforcing compliance with non-drug-related activities. Journal of Substance Abuse Treatment, 20(1), 33-44.

Pew Research Center, Nov. 20, 2018, "Where Americans find meaning in life".

Phelan, J. C., Link, B. G., \& Tehranifar, P. (2010). Social conditions as fundamental causes of health inequalities: Theory, evidence, and policy implications. Journal of Health and Social Behavior, 51(1_suppl), S28-S40.

Phelan, J., Link, B. G., Moore, R. E., \& Stueve, A. (1997). The stigma of homelessness: The impact of the label" homeless" on attitudes toward poor persons. Social Psychology Quarterly, 323-337.

Phillips, J. K., Ford, M. A., Bonnie, R. J., \& National Academies of Sciences, Engineering, and Medicine. (2017). Trends in opioid use, harms, and treatment. In Pain Management and the Opioid Epidemic: Balancing Societal and Individual Benefits and Risks of Prescription Opioid Use. National Academies Press (US).

Phillips, L. A., \& Lindsay, M. (2011). Prison to society: A mixed methods analysis of coping with reentry. International Journal of Offender Therapy and Comparative Criminology, 55(1), 136154.

Phillips, L. A., \& Shaw, A. (2013). Substance use more stigmatized than smoking and obesity. Journal of Substance Use, 18(4), 247-253.

Piaget, J. (2005). The psychology of intelligence. Abingdon-on-Thames, UK. Routledge.

Pickett, K. E., \& Pearl, M. (2001). Multilevel analyses of neighbourhood socioeconomic context and health outcomes: A critical review. Journal of Epidemiology \& Community Health, 55(2), 111-122.

Pickard, H. (2011). Responsibility without blame: Empathy and the effective treatment of personality disorder. Philosophy, Psychiatry, \& Psychology: PPP, 18(3), 209.

Pickard, H. (2014). Responsibility without blame: Therapy, philosophy, law. Prison Service Journal, 213, 10.

Pickard, H. (2017). Responsibility without blame for addiction. Neuroethics, 10(1), 169-180.

Pickard, H., \& Ahmed, S. H. (2016). How do you know you have a drug problem? The role of knowledge of negative consequences in explaining drug choice in humans and rats. Addiction and choice: Rethinking the relationship, 29-48.

Pickens, C. L., Airavaara, M., Theberge, F., Fanous, S., Hope, B. T., \& Shaham, Y. (2011). Neurobiology of the incubation of drug craving. Trends in Neurosciences, 34(8), 411-420.

Pickering, H., \& Stimson, G. V. (1994). Prevalence and demographic factors of stimulant use. Addiction, 89(11), 1385-1389.

Pitel, A. L., Rivier, J., Beaunieux, H., Vabret, F., Desgranges, B., \& Eustache, F. (2009). Changes in the episodic memory and executive functions of abstinent and relapsed alcoholics over a 6-month period. Alcoholism: Clinical and Experimental Research, 33(3), 490-498.

Pizzagalli, D. A., Bogdan, R., Ratner, K. G., \& Jahn, A. L. (2007). Increased perceived stress is associated with blunted hedonic capacity: Potential implications for depression research. Behaviour Research and Therapy, 45(11), 2742-2753. 
Plunk, A. D., Grucza, R. A., \& Peglow, S. L. (2018). It is past time to think more inclusively about "deaths of despair". The American Journal of Bioethics, 18(10), 29-31.

Polites, G. L., \& Karahanna, E. (2012). Shackled to the status quo: The inhibiting effects of incumbent system habit, switching costs, and inertia on new system acceptance. MIS Quarterly, 36(1).

Pollatos, O., Herbert, B. M., Wankner, S., Dietel, A., Wachsmuth, C., Henningsen, P., \& Sack, M. (2011). Autonomic imbalance is associated with reduced facial recognition in somatoform disorders. Journal of Psychosomatic Research, 71(4), 232-239.

Pollatos, O., Laubrock, J., \& Wittmann, M. (2014). Interoceptive focus shapes the experience of time. PloS One, 9(1), e86934.

Ponton, R., \& Scott, J. (2004). Injection preparation processes used by heroin and crack cocaine injectors. Journal of Substance Use, 9(1), 7-19.

Pope, S. K., Falck, R. S., Carlson, R. G., Leukefeld, C., \& Booth, B. M. (2011). Characteristics of rural crack and powder cocaine use: Gender and other correlates. The American Journal of Drug and Alcohol Abuse, 37(6), 491-496.

Porcelli, A. J., \& Delgado, M. R. (2009). Acute stress modulates risk taking in financial decision making. Psychol Sci.

Post, R. M. (2016). Epigenetic basis of sensitization to stress, affective episodes, and stimulants: implications for illness progression and prevention. Bipolar Disorders, 18(4), 315-324.

Potvin, S., Stavro, K., Rizkallah, É., \& Pelletier, J. (2014). Cocaine and cognition: A systematic quantitative review. Journal of addiction medicine, 8(5), 368-376.

Potvin, S., Pelletier, J., Grot, S., Hebert, C., Barr, A. M., \& Lecomte, T. (2018). Cognitive deficits in individuals with methamphetamine use disorder: A meta-analysis. Addictive Behaviors, 80, 154160.

Prater, C. D., Zylstra, R. G., \& Miller, K. E. (2002). Successful pain management for the recovering addicted patient. Primary Care Companion to the Journal of Clinical Psychiatry, 4(4), 125.

Premack, D. (2017). Mechanisms of self-control. In Learning mechanisms in smoking (pp. 107-123). Abingdon-on-Thames, UK. Routledge.

Powell D. \& Pacula R. L., 2017. Prescription opiates and opioid Abuse: Regulatory efforts to limit diversion from medical markets to black markets in the United States. In Dual Markets (pp. 3754). Springer, Cham.

Preston, K. L., \& Epstein, D. H. (2011). Stress in the daily lives of cocaine and heroin users: relationship to mood, craving, relapse triggers, and cocaine use. Psychopharmacology, 218(1), 29-37

Preston, K. L., Kowalczyk, W. J., Phillips, K. A., Jobes, M. L., Vahabzadeh, M., Lin, J. L., ... \& Epstein, D. H. (2017). Context and craving during stressful events in the daily lives of drug-dependent patients. Psychopharmacology, 234(17), 2631-2642.

Preston, K. L., Kowalczyk, W. J., Phillips, K. A., Jobes, M. L., Vahabzadeh, M., Lin, J. L., ... \& Epstein, D. H. (2018). Before and after: Craving, mood, and background stress in the hours surrounding drug use and stressful events in patients with opioid-use disorder. Psychopharmacology, 235(9), 27132723. 
Prison Policy Initiative. (2018). States of incarceration: The global context $\mid$ Prison Policy Initiative. Retrieved November 6, 2018, from https://www.prisonpolicy.org/global/

Prunuske, J. P., Hill, C. A. S., Hager, K. D., Lemieux, A. M., Swanoski, M. T., Anderson, G. W., \& Lutfiyya, M. N. (2014). Opioid prescribing patterns for non-malignant chronic pain for rural versus non-rural US adults: A population-based study using 2010 NAMCS data. BMC Health Services Research, 14(1), 563.

Pulcu, E., Trotter, P. D., Thomas, E. J., McFarquhar, M., Juhász, G., Sahakian, B. J., ... \& Elliott, R. (2014). Temporal discounting in major depressive disorder. Psychological Medicine, 44(9), 18251834.

Purser, G. (2012). "STILL DOIN'TIME:” CLAMORING FOR WORK IN THE DAY LABOR INDUSTRY. WorkingUSA, 15(3), 397-415.

Quick, S. L., Pyszczynski, A. D., Colston, K. A., \& Shahan, T. A. (2011). Loss of alternative non-drug reinforcement induces relapse of cocaine-seeking in rats: Role of dopamine D 1 receptors. Neuropsychopharmacology, 36(5), 1015.

Quinn, D. M., \& Chaudoir, S. R. (2009). Living with a concealable stigmatized identity: The impact of anticipated stigma, centrality, salience, and cultural stigma on psychological distress and health. Journal of Personality and Social Psychology, 97(4), 634.

Quintana Mathé P., Ventura M., Grifell Guardia M., Palma Conesa A., Galindo Guarín L., Fornís Espinosa I., ... \& Torrens M., 2017. The hidden web and the fentanyl problem: Detection of ocfentanil as an adulterant in heroin. International Journal of Drug Policy 40: 78-83.

Rachlin, H. (1995). Self-control: Beyond commitment. Behavioral and Brain Sciences, 18(1), 109-121.

Rachlin, H. (1997). Four teleological theories of addiction. Psychonomic bulletin \& review, 4(4), 462-473.

Rachlin, H. (2006). Notes on discounting. Journal of the Experimental Analysis of Behavior, 85(3), 425435.

Rachlin, H., Raineri, A., \& Cross, D. (1991). Subjective probability and delay. Journal of the Experimental Analysis of Behavior, 55(2), 233-244.

Raghunathan, R., \& Pham, M. T. (1999). All negative moods are not equal: Motivational influences of anxiety and sadness on decision making. Organizational Behavior and Human Decision Processes, 79(1), 56-77.

Raine, A., Moffitt, T. E., Caspi, A., Loeber, R., Stouthamer-Loeber, M., \& Lynam, D. (2005). Neurocognitive impairments in boys on the life-course persistent antisocial path. Journal of Abnormal Psychology, 114(1), 38.

Ramaekers, J. G., Kauert, G., van Ruitenbeek, P., Theunissen, E. L., Schneider, E., \& Moeller, M. R. (2006). High-potency marijuana impairs executive function and inhibitory motor control. Neuropsychopharmacology, 31(10), 2296.

Ranaldi, R., \& Munn, E. (1998). Polydrug self-administration in rats: Cocaine-heroin is more rewarding than cocaine-alone. Neuroreport, 9(11), 2463-2466.

Randesi, M., van den Brink, W., Levran, O., Blanken, P., Butelman, E. R., Yuferov, V., ... \& Kreek, M. J. (2016). Variants of opioid system genes are associated with non-dependent opioid use and heroin dependence. Drug and Alcohol Dependence, 168, 164-169. 
Randesi, M., van den Brink, W., Levran, O., Yuferov, V., Blanken, P., van Ree, J. M., ... \& Kreek, M. J. (2018). Dopamine gene variants in opioid addiction: Comparison of dependent patients, nondependent users and healthy controls. Pharmacogenomics, 19(2), 95-104.

Ratcliff, R., \& McKoon, G. (2008). The diffusion decision model: Theory and data for two-choice decision tasks. Neural Computation, 20(4), 873-922.

Razali, N. M., \& Wah, Y. B. (2011). Power comparisons of shapiro-wilk, kolmogorov-smirnov, lilliefors and anderson-darling tests. Journal of Statistical Modeling and Analytics, 2(1), 21-33.

Real, L., \& Caraco, T. (1986). Risk and foraging in stochastic environments. Annual Review of Ecology and Systematics, 17(1), 371-390.

Reiman, A. (2009). Cannabis as a substitute for alcohol and other drugs. Harm Reduction Journal, 6(1), 35.

Reinarman, C., \& Levine, H. G. (1997). Crack in context. Crack in America: Demon drugs and Social Justice, 1. Oakland, CA. University of California Press.

Reinarman, C., \& Levine, H. G. (2017). The crack attack: America's latest drug scare, 1986-1992. In Images of Issues (pp. 147-186).Abingdon-on-Thames, UK. Routledge.

Reutter, L. I., Stewart, M. J., Veenstra, G., Love, R., Raphael, D., \& Makwarimba, E. (2009). "Who do they think we are, anyway?": Perceptions of and responses to poverty stigma. Qualitative Health Research, 19(3), 297-311.

Reynolds, B. (2006). A review of delay-discounting research with humans: Relations to drug use and gambling. Behavioural Pharmacology, 17(8), 651-667.

Rich, J. D., Boutwell, A. E., Shield, D. C., Key, R. G., McKenzie, M., Clarke, J. G., \& Friedmann, P. D. (2005). Attitudes and practices regarding the use of methadone in US state and federal prisons. Journal of Urban Health, 82(3), 411-419.

Richman, W. L., Kiesler, S., Weisband, S., \& Drasgow, F. (1999). A meta-analytic study of social desirability distortion in computer-administered questionnaires, traditional questionnaires, and interviews. Journal of Applied Psychology, 84(5), 754.

Rigg, K. K., \& Nicholson, H. L. (2019). Prescription opioid misuse among African-American adults: A rural-urban comparison of prevalence and risk. Drug and Alcohol Dependence.

Rigg, K. K., Monnat, S. M., \& Chavez, M. N. (2018). Opioid-related mortality in rural America: Geographic heterogeneity and intervention strategies. International Journal of Drug Policy, 57, 119-129.

Rigg, K. K., Kurtz, S. P., \& Surratt, H. L. (2012). Patterns of prescription medication diversion among drug dealers. Drugs: Education, Prevention and Policy, 19(2), 145-155.

Rigg, K. K., \& Monnat, S. M. (2015). Urban vs. rural differences in prescription opioid misuse among adults in the United States: Informing region specific drug policies and interventions. International Journal of Drug Policy, 26(5), 484-491.

Ritsher, J. B., Otilingam, P. G., \& Grajales, M. (2003). Internalized stigma of mental illness: Psychometric properties of a new measure. Psychiatry Research, 121(1), 31-49.

Ritter, A. (2014). Where is the pleasure?. Addiction, 109(10), 1587-1588.

Robbins, T. W., \& Costa, R. M. (2017). Habits. Current Biology, 27(22), R1200-R1206. 
Robbins, T. W., Ersche, K. D., \& Everitt, B. J. (2008). Drug addiction and the memory systems of the brain. Annals of the New York Academy of Sciences, 1141(1), 1-21.

Robbins, T. W., \& Everitt, B. J. (1999). Drug addiction: Bad habits add up. Nature, 398(6728), 567.

Robbins, T. W., \& Everitt, B. J. (2002). Limbic-striatal memory systems and drug addiction. Neurobiology of Learning and Memory, 78(3), 625-636.

Robinson, T. E., \& Berridge, K. C. (1993). The neural basis of drug craving: an incentive-sensitization theory of addiction. Brain Research Reviews, 18(3), 247-291.

Robinson, T. E., \& Berridge, K. C. (2000). The psychology and neurobiology of addiction: an incentivesensitization view. Addiction, 95(8s2), 91-117.

Roberti, J. W. (2004). A review of behavioral and biological correlates of sensation seeking. Journal of Research in Personality, 38(3), 256-279.

Roberto, C. A., \& Kawachi, I. (Eds.). (2015). Behavioral economics and public health. Oxford University Press.

Roddy, J., \& Greenwald, M. (2009). An economic analysis of income and expenditures by heroin-using research volunteers. Substance Use \& Misuse, 44(11), 1503-1518.

Roddy, J., Steinmiller, C. L., \& Greenwald, M. K. (2011). Heroin purchasing is income and price sensitive. Psychology of Addictive Behaviors, 25(2), 358.

Rodriguez, S. D., Vanderford, N. L., Huang, B., \& Vanderpool, R. C. (2018). A social-ecological review of cancer disparities in Kentucky. Southern Medical journal, 111(4), 213-219.

Rogers, R. D., Everitt, B. J., Baldacchino, A., Blackshaw, A. J., Swainson, R., Wynne, K., ... \& London, M. (1999). Dissociable deficits in the decision-making cognition of chronic amphetamine abusers, opiate abusers, patients with focal damage to prefrontal cortex, and tryptophan-depleted normal volunteers: Evidence for monoaminergic mechanisms. Neuropsychopharmacology, 20(4), 322339.

Rogers, R. D., \& Robbins, T. W. (2001). Investigating the neurocognitive deficits associated with chronic drug misuse. Current Opinion in Neurobiology, 11(2), 250-257.

Rogers, R. E., Higgins, S. T., Silverman, K., Thomas, C. S., Badger, G. J., Bigelow, G., \& Stitzer, M. (2008). Abstinence-contingent reinforcement and engagement in non-drug-related activities among illicit drug abusers. Psychology of Addictive Behaviors, 22(4), 544.

Ronis, D. L., Yates, J. F., \& Kirscht, J. P. (1989). Attitudes, decisions, and habits as determinants of repeated behavior. Attitude Structure and Function, 213, 39.

Rokach, A. (1997). Loneliness in jail: Coping strategies. International Journal of Offender Therapy and Comparative Criminology, 41(3), 260-271.

Rönkä, S., \& Katainen, A. (2017). Non-medical use of prescription drugs among illicit drug users: A case study on an online drug forum. International Journal of Drug Policy, 39, 62-68.

Roschelle, A. R., \& Kaufman, P. (2004). Fitting in and fighting back: Stigma management strategies among homeless kids. Symbolic Interaction, 27(1), 23-46. 
Ross, C. E., \& Mirowsky, J. (2001). Neighborhood disadvantage, disorder, and health. Journal of Health and Social Behavior, 258-276.

Ross, M. W., Timpson, S. C., Williams, M. L., Amos, C., \& Bowen, A. (2007). Stigma consciousness concerns related to drug use and sexuality in a sample of street-based male sex workers. International Journal of Sexual Health, 19(2), 57-67.

Roth, A. M., Felsher, M., Reed, M., Goldshear, J. L., Truong, Q., Garfein, R. S., \& Simmons, J. (2017). Potential benefits of using ecological momentary assessment to study high-risk polydrug use. mHealth, 3 .

Roussell, A., Holmes, M. D., \& Anderson-Sprecher, R. (2013). Community characteristics and methamphetamine use in a rural state: An analysis of preincarceration usage by prison inmates. Crime \& Delinquency, 59(7), 1036-1063.

Roxburgh, A., Hall, W. D., Dobbins, T., Gisev, N., Burns, L., Pearson, S., \& Degenhardt, L. (2017). Trends in heroin and pharmaceutical opioid overdose deaths in Australia. Drug and Alcohol Dependence, 179, 291-298.

Rubin, D. B. (2004). Multiple imputation for nonresponse in surveys (Vol. 81). John Wiley \& Sons.

Rubinov, M., Sporns, O., Thivierge, J. P., \& Breakspear, M. (2011). Neurobiologically realistic determinants of self-organized criticality in networks of spiking neurons. PLoS Computational Biology, 7(6), e1002038.

Rubinsten, O. (2009). Co-occurrence of developmental disorders: The case of developmental dyscalculia. Cognitive Development, 24(4), 362-370.

Rubinsztein, J. S., Michael, A., Underwood, B. R., Tempest, M., \& Sahakian, B. J. (2006). Impaired cognition and decision-making in bipolar depression but no 'affective bias' evident. Psychological Medicine, 36(5), 629-639.

Ruekert, R. W., \& Churchill Jr, G. A. (1984). Reliability and validity of alternative measures of channel member satisfaction. Journal of Marketing Research, 226-233.

Ruhm, C. J. (2018). Corrected US opioid-involved drug poisoning deaths and mortality rates, 19992015. Addiction, 113(7), 1339-1344.

Ruhm, C. J. (2019). Drivers of the fatal drug epidemic. Journal of Health Economics, 64, 25-42.

Rush, C. R., \& Baker, R. W. (2001). Behavioral pharmacological similarities between methylphenidate and cocaine in cocaine abusers. Experimental and Clinical Psychopharmacology, 9(1), 59.

Rush, C. R., Stoops, W. W., Sevak, R. J., \& Hays, L. R. (2010). Cocaine choice in humans during Damphetamine maintenance. Journal of Clinical Psychopharmacology, 30(2), 152-159.

Rutkow, L., Chang, H. Y., Daubresse, M., Webster, D. W., Stuart, E. A., \& Alexander, G. C. (2015). Effect of Florida's prescription drug monitoring program and pill mill laws on opioid prescribing and use. JAMA Internal Medicine, 175(10), 1642-1649.

Sackett, D. L. (1997, February). Evidence-based medicine. In Seminars in Perinatology (Vol. 21, No. 1, pp. 3-5). WB Saunders.

Saffer, H., \& Chaloupka, F. (1999). The demand for illicit drugs. Economic Inquiry, 37(3), 401-411. 
Salo, R., Nordahl, T. E., Galloway, G. P., Moore, C. D., Waters, C., \& Leamon, M. H. (2009). Drug abstinence and cognitive control in methamphetamine-dependent individuals. Journal of Substance Abuse Treatment, 37(3), 292-297.

Sampson, R. J., Laub, J. H., \& Wimer, C. (2006). Does marriage reduce crime? A counterfactual approach to within-individual causal effects. Criminology, 44(3), 465-508.

Sanders, B., Lankenau, S. E., Jackson-Bloom, J., \& Hathazi, D. (2008). Multiple drug use and polydrug use amongst homeless traveling youth. Journal of Ethnicity in Substance Abuse, 7(1), 23-40.

Santiago, C. D., Wadsworth, M. E., \& Stump, J. (2011). Socioeconomic status, neighborhood disadvantage, and poverty-related stress: Prospective effects on psychological syndromes among diverse lowincome families. Journal of Economic Psychology, 32(2), 218-230.

Sallet, J., Mars, R. B., Noonan, M. P., Andersson, J. L., O'reilly, J. X., Jbabdi, S., ... \& Rushworth, M. F. (2011). Social network size affects neural circuits in macaques. Science, 334(6056), 697-700.

Sapolsky, R. (2003). Taming stress. Scientific American, 289(3), 86-95.

Sapolsky, R. M. (2005). The influence of social hierarchy on primate health. Science, 308(5722), 648-652.

Sapolsky, R. M. (2005a). The influence of social hierarchy on primate health. Science, 308(5722), 648-652.

Sapolsky, R. (2005b). Sick of poverty. Scientific American, 293(6), 92-99.

Sapolsky, R. M. (2017). Behave: The biology of humans at our best and worst. London, England, UK: Penguin.

Sapolsky, R. M., Alberts, S. C., \& Altmann, J. (1997). Hypercortisolism associated with social subordinance or social isolation among wild baboons. Archives of General Psychiatry, 54(12), 1137-1143.

Sanfey, A. G., \& Chang, L. J. (2008). Multiple systems in decision making. Annals of the New York Academy of Sciences, 1128(1), 53-62.

Santoiemma, P. P., Dinwiddie, S. H., \& Angarone, M. P. (2017). Infectious disease complications in people who inject drugs. Psychiatric Annals, 47(1), 33-37.

Sanvicente-Vieira, B., Kommers-Molina, J., De Nardi, T., Francke, I., \& Grassi-Oliveira, R. (2016). Crackcocaine dependence and aging: Effects on working memory. Revista Brasileira de Psiquiatria, 38(1), 58-60.

Sarstedt, M., \& Wilczynski, P. (2009). More for less? A comparison of single-item and multi-item measures. Die Betriebswirtschaft, 69(2), 211.

Sayette, M. A. (2016). The role of craving in substance use disorders: Theoretical and methodological issues. Annual Review of Clinical Psychology, 12, 407-433.

Sayette, M.A. et al. (2005) Effects of smoking urge on temporal cognition. Psychol. Addictive Behaviors, $19,88-93$

Sayette, M. A.. \& Creswell, K. G. (2016). Self-Regulatory Failure and Addiction. In (Eds. Vohs, K. E., \& Baumeister, R. R.) Handbook of Self-Regulation, $3^{\text {rd }}$ Edition. The Guildford Press. New York, NY. FIX 
Schacter, D. L., Benoit, R. G., De Brigard, F., \& Szpunar, K. K. (2015). Episodic future thinking and episodic counterfactual thinking: Intersections between memory and decisions. Neurobiology of Learning and Memory, 117, 14-21.

Schaefer, H. S., Larson, C. L., Davidson, R. J., \& Coan, J. A. (2014). Brain, body, and cognition: Neural, physiological and self-report correlates of phobic and normative fear. Biological psychology, 98, 59-69.

Schafer, J. L. (1999). Multiple imputation: A primer. Statistical methods in medical research, 8(1), 3-15.

Schafer, J. L., \& Graham, J. W. (2002). Missing data: Our view of the state of the art. Psychological Methods, 7(2), 147.

Schilbach, F., Schofield, H., \& Mullainathan, S. (2016). The psychological lives of the poor. American Economic Review, 106(5), 435-40.

Schoenbaum, G., Roesch, M. R., \& Stalnaker, T. A. (2006). Orbitofrontal cortex, decision-making and drug addiction. Trends in Neurosciences, 29(2), 116-124.

Schoenberg, N. E., Huang, B., Seshadri, S., \& Tucker, T. C. (2015). Trends in cigarette smoking and obesity in Appalachian Kentucky. Southern Medical Journal, 108(3), 170-177.

Schomerus, G., Schwahn, C., Holzinger, A., Corrigan, P. W., Grabe, H. J., Carta, M. G., \& Angermeyer, M. C. (2012). Evolution of public attitudes about mental illness: a systematic review and metaanalysis. Acta Psychiatrica Scandinavica, 125(6), 440-452.

Schroeder, J. R., Latkin, C. A., Hoover, D. R., Curry, A. D., Knowlton, A. R., \& Celentano, D. D. (2001). Illicit drug use in one's social network and in one's neighborhood predicts individual heroin and cocaine use. Annals of Epidemiology, 11(6), 389-394.

Schulkin, J., Morgan, M. A., \& Rosen, J. B. (2005). A neuroendocrine mechanism for sustaining fear. Trends in Neurosciences, 28(12), 629-635.

Schulenberg, J. E., \& Maggs, J. L. (2002). A developmental perspective on alcohol use and heavy drinking during adolescence and the transition to young adulthood. Journal of Studies on Alcohol, Supplement, (14), 54-70.

Schulte, M. H., Cousijn, J., den Uyl, T. E., Goudriaan, A. E., van den Brink, W., Veltman, D. J., ... \& Wiers, R. W. (2014). Recovery of neurocognitive functions following sustained abstinence after substance dependence and implications for treatment. Clinical Psychology Review, 34(7), 531550 .

Schulz, A. J., Mentz, G., Lachance, L., Johnson, J., Gaines, C., \& Israel, B. A. (2012). Associations between socioeconomic status and allostatic load: Effects of neighborhood poverty and tests of mediating pathways. American Journal of Public Health, 102(9), 1706-1714.

Schulz, A., \& Vögele, C. (2015). Interoception and stress. Frontiers in Psychology, 6, 993.

Schuman-Olivier, Z., Albanese, M., Nelson, S. E., Roland, L., Puopolo, F., Klinker, L., \& Shaffer, H. J. (2010). Self-treatment: Illicit buprenorphine use by opioid-dependent treatment seekers. Journal of Substance Abuse Treatment, 39(1), 41-50.

Schwabe, L., \& Wolf, O. T. (2013). Stress and multiple memory systems: From 'thinking'to 'doing'. Trends in Cognitive Sciences, 17(2), 60-68. 
Schwabe, L., \& Wolf, O. T. (2011). Stress-induced modulation of instrumental behavior: From goaldirected to habitual control of action. Behavioural Brain Research, 219(2), 321-328.

Schwabe, L., \& Wolf, O. T. (2009). Stress prompts habit behavior in humans. Journal of Neuroscience, 29(22), 7191-7198.

Schwartz, B., Ward, A., Monterosso, J., Lyubomirsky, S., White, K., \& Lehman, D. R. (2002). Maximizing versus satisficing: Happiness is a matter of choice. Journal of Personality and Social Psychology, 83(5), 1178.

Schwartz, R. P., Kelly, S. M., O'Grady, K. E., Peterson, J. A., Reisinger, H. S., Mitchell, S. G., ... \& Brown, B. S. (2008). In-treatment vs. out-of-treatment opioid dependent adults: Drug use and criminal history. The American Journal of Drug and Alcohol Abuse, 34(1), 17-28.

Scott, S. B., Graham-Engeland, J. E., Engeland, C. G., Smyth, J. M., Almeida, D. M., Katz, M. J., ... \& Sliwinski, M. J. (2015). The effects of stress on cognitive aging, physiology and emotion (ESCAPE) project. BMC Psychiatry, 15(1), 146.

Searle, J. R. (2008). Mind, language and society: Philosophy in the real world. Basic books.

Sebold, M., Nebe, S., Garbusow, M., Guggenmos, M., Schad, D. J., Beck, A., ... \& Zimmermann, U. S. (2017). When habits are dangerous: Alcohol expectancies and habitual decision making predict relapse in alcohol dependence. Biological Psychiatry, 82(11), 847-856.

Segal, L. M., De Biasi, A., Mueller, J. L., May, K., Warren, M., Miller, B. F., ... \& Olson, G. (2017). Pain in the Nation: The Drug, Alcohol and Suicide Crises and the Need for a National Resilience Strategy.

Semrud-Clikeman, M., Biederman, J., Sprich-Buckminster, S., Lehman, B. K., Faraone, S. V., \& Norman, D. (1992). Comorbidity between ADDH and learning disability: A review and report in a clinically referred sample. Journal of the American Academy of Child \& Adolescent Psychiatry, 31(3), 439-448.

Seth, P., Scholl, L., Rudd, R. A., \& Bacon, S. (2018). Overdose deaths involving opioids, cocaine, and psychostimulants-United States, 2015-2016. Morbidity and Mortality Weekly Report, 67(12), 349.

Sevak, R. J., Stoops, W. W., Hays, L. R., \& Rush, C. R. (2009). Discriminative stimulus and subject-rated effects of methamphetamine, d-amphetamine, methylphenidate, and triazolam in methamphetamine-trained humans. Journal of Pharmacology and Experimental Therapeutics, 328(3), 1007-1018.

Sexton, R. L., Carlson, R. G., Leukefeld, C. G., \& Booth, B. M. (2006). Patterns of illicit methamphetamine production (" cooking") and associated risks in the rural south: An ethnographic exploration. Journal of Drug Issues, 36(4), 853-876.

Sexton, R. L., Carlson, R. G., Leukefeld, C. G., \& Booth, B. M. (2008). Barriers to formal drug abuse treatment in the rural south: A preliminary ethnographic assessment. Journal of Psychoactive Drugs, 40(2), 121-129.

Shadish, W. R., Cook, T. D., \& Campbell, D. T. (2002). Experimental and quasi-experimental designs for generalized causal inference/William R. Shedish, Thomas D. Cook, Donald T. Campbell. Boston: Houghton Mifflin. 
Shaffer, H. J., LaPlante, D. A., LaBrie, R. A., Kidman, R. C., Donato, A. N., \& Stanton, M. V. (2004). Toward a syndrome model of addiction: Multiple expressions, common etiology. Harvard Review of Psychiatry, 12(6), 367-374.

Shah, A. K., Shafir, E., \& Mullainathan, S. (2015). Scarcity frames value. Psychological Science, 26(4), $402-412$.

Shackman, A. J., Sarinopoulos, I., Maxwell, J. S., Pizzagalli, D. A., Lavric, A., \& Davidson, R. J. (2006). Anxiety selectively disrupts visuospatial working memory. Emotion, 6(1), 40.

Shannon, L. M., Havens, J. R., Mateyoke-Scrivner, A., \& Walker, R. (2009). Contextual differences in substance use for rural Appalachian treatment-seeking women. The American Journal of Drug and Alcohol Abuse, 35(2), 59-62.

Shaw, S. Y., Shah, L., Jolly, A. M., \& Wylie, J. L. (2008). Identifying heterogeneity among injection drug users: A cluster analysis approach. American Journal of Public Health, 98(8), 1430-1437.

Sheehy-Skeffington, J. (2017). Decision-making up against the wall: A framework for understanding the behavioural dimension of low socioeconomic status. New York, NY: Oxford University Press

Sheffer, C., MacKillop, J., McGeary, J., Landes, R., Carter, L., Yi, R., ... \& Bickel, W. (2012). Delay discounting, locus of control, and cognitive impulsiveness independently predict tobacco dependence treatment outcomes in a highly dependent, lower socioeconomic group of smokers. The American Journal on Addictions, 21(3), 221-232.

Schacter, D. L., Benoit, R. G., De Brigard, F., \& Szpunar, K. K. (2015). Episodic future thinking and episodic counterfactual thinking: Intersections between memory and decisions. Neurobiology of Learning and Memory, 117, 14-21.

Scheltema, J. F. (1910). The opium question. American Journal of Sociology, 16(2), 213-235.

Semple, S. J., Zians, J., Grant, I., \& Patterson, T. L. (2005). Impulsivity and methamphetamine use. Journal of Substance Abuse Treatment, 29(2), 85-93.

Shah, A. K., Mullainathan, S., \& Shafir, E. (2012). Some consequences of having too little. Science, 338(6107), 682-685.

Shah, A. K., Shafir, E., \& Mullainathan, S. (2015). Scarcity frames value. Psychological Science, 26(4), 402-412.

Shaham, Y., \& Stewart, J. (1995). Stress reinstates heroin-seeking in drug-free animals: An effect mimicking heroin, not withdrawal. Psychopharmacology, 119(3), 334-341.

Shane, F., Loewenstein, G., \& O'Donoghue, T. (2002). Time discounting and time preference: A critical review. Journal of Economic Literature, 40(2), 351.

Shaw, P., Kabani, N. J., Lerch, J. P., Eckstrand, K., Lenroot, R., Gogtay, N., ... \& Giedd, J. N. (2008). Neurodevelopmental trajectories of the human cerebral cortex. Journal of Neuroscience, 28(14), 3586-3594.

Sheehy-Skeffington, J. (2018). Behavior as a Factor in Rising Inequalities. Socio-Economic Environment and Human Psychology: Social, Ecological, and Cultural Perspectives.

Shiels, M. S., Freedman, N. D., Thomas, D., \& de Gonzalez, A. B. (2018). Trends in US drug overdose deaths in non-Hispanic black, Hispanic, and non-Hispanic white persons, 2000-2015. Annals of Internal Medicine, 168(6), 453-455. 
Shiffman, S., Stone, A. A., \& Hufford, M. R. (2008). Ecological momentary assessment. Annual Review of Clinical Psychology., 4, 1-32.

Shifrer, D. (2013). Stigma of a label: Educational expectations for high school students labeled with learning disabilities. Journal of Health and Social Behavior, 54(4), 462-480.

Shivy, V. A., Wu, J. J., Moon, A. E., Mann, S. C., Holland, J. G., \& Eacho, C. (2007). Ex-offenders reentering the workforce. Journal of Counseling Psychology, 54(4), 466.

Shortt, N. K., Rhynas, S. J., \& Holloway, A. (2017). Place and recovery from alcohol dependence: A journey through photovoice. Health \& Place, 47, 147-155.

Silber, M., Almkvist, O., Larsson, B., \& Uvnäs-Moberg, K. (1990). Temporary peripartal impairment in memory and attention and its possible relation to oxytocin concentration. Life Sciences, 47(1), 5765.

Siegel, S., \& Castellan, N. J. (1988). Nonparametric statistics for the Behavioral Sciences $2^{\text {nd }}$ Edition. New York: McGraw-Hill.

Sigmon, S. T., Pells, J. J., Boulard, N. E., Whitcomb-Smith, S., Edenfield, T. M., Hermann, B. A., ... \& Kubik, E. (2005). Gender differences in self-reports of depression: The response bias hypothesis revisited. Sex Roles, 53(5-6), 401-411.

Sigurdsson, T., Doyère, V., Cain, C. K., \& LeDoux, J. E. (2007). Long-term potentiation in the amygdala: A cellular mechanism of fear learning and memory. Neuropharmacology, 52(1), 215-227.

Silver, C. H., Ruff, R. M., Iverson, G. L., Barth, J. T., Broshek, D. K., ... \& Planning Committee. (2008). Learning disabilities: The need for neuropsychological evaluation. Archives of Clinical Neuropsychology, 23(2), 217-219.

Simon, S. L., Richardson, K., Dacey, J., Glynn, S., Domier, C. P., Rawson, R. A., \& Ling, W. (2001). A comparison of patterns of methamphetamine and cocaine use. Journal of Addictive Diseases, 2l(1), 35-44.

Sindelar, J. L. (2008). Paying for performance: The power of incentives over habits. Health Economics, 17(4), 449-451.

Singh-Manoux, A., Marmot, M. G., \& Adler, N. E. (2005). Does subjective social status predict health and change in health status better than objective status?. Psychosomatic Medicine, 67(6), 855-861.

Sinha, R., Fuse, T., Aubin, L. R., \& O'Malley, S. S. (2000). Psychological stress, drug-related cues and cocaine craving. Psychopharmacology, 152(2), 140-148

Sinha, R. (2001). How does stress increase risk of drug abuse and relapse?. Psychopharmacology, 158(4), 343-359.

Sinha, R. (2008). Chronic stress, drug use, and vulnerability to addiction. Annals of the New York Academy of Sciences, 1141(1), 105-130.

Sinha, R. (2011). New findings on biological factors predicting addiction relapse vulnerability. Current Psychiatry Reports, 13(5), 398.

Sinha, R. (2012). How does stress lead to risk of alcohol relapse?. Alcohol Research: Current Reviews, 34(4), 432. 
Sinha, R., Catapano, D., \& O’Malley, S. (1999). Stress-induced craving and stress response in cocaine dependent individuals. Psychopharmacology, 142(4), 343-351.

Sinha, R., \& Jastreboff, A. M. (2013). Stress as a common risk factor for obesity and addiction. Biological Psychiatry, 73(9), 827-835.

Sinha, R., Lacadie, C., Skudlarski, P., Fulbright, R. K., Rounsaville, B. J., Kosten, T. R., \& Wexler, B. E. (2005). Neural activity associated with stress-induced cocaine craving: A functional magnetic resonance imaging study. Psychopharmacology, 183(2), 171-180.

Sinha, R., \& Li, C. S. R., (2007). Imaging stress-and cue-induced drug and alcohol craving: Association with relapse and clinical implications. Drug and Alcohol Review, 26(1), 25-31.

Sinha, R. (2013). The clinical neurobiology of drug craving. Current Opinion in Neurobiology, 23(4), 649654.

Sinha, R., Talih, M., Malison, R., Cooney, N., Anderson, G. M., \& Kreek, M. J. (2003). Hypothalamicpituitary-adrenal axis and sympatho-adreno-medullary responses during stress-induced and drug cue-induced cocaine craving states. Psychopharmacology, 170(1), 62-72.

Simon, S. L., Domier, C. P., Sim, T., Richardson, K., Rawson, R. A., \& Ling, W. (2001). Cognitive performance of current methamphetamine and cocaine abusers. Journal of Addictive Diseases, 21(1), 61-74.

Skiba, R. J., Simmons, A. B., Ritter, S., Gibb, A. C., Rausch, M. K., Cuadrado, J., \& Chung, C. G. (2008). Achieving equity in special education: History, status, and current challenges. Exceptional Children, 74(3), 264-288.

Skinner, N., Feather, N. T., Freeman, T., \& Roche, A. (2007). Stigma and discrimination in health-care provision to drug users: The role of values, affect, and deservingness judgments. Journal of Applied Social Psychology, 37(1), 163-186.

Skosireva, A., O’Campo, P., Zerger, S., Chambers, C., Gapka, S., \& Stergiopoulos, V. (2014). Different faces of discrimination: Perceived discrimination among homeless adults with mental illness in healthcare settings. BMC Health Services Research, 14(1), 376.

Slavova, S., Costich, J. F., Bunn, T. L., Luu, H., Singleton, M., Hargrove, S. L., ... \& Ingram, V. (2017). Heroin and fentanyl overdoses in Kentucky: Epidemiology and surveillance. International Journal of Drug Policy, 46, 120-129.

Slovic, P., Finucane, M., Peters, E., \& MacGregor, D. G. (2002). Rational actors or rational fools: Implications of the affect heuristic for behavioral economics. The Journal of SocioEconomics, 31(4), 329-342.

Small, W., Rhodes, T., Wood, E., \& Kerr, T. (2007). Public injection settings in Vancouver: physical environment, social context and risk. International Journal of Drug Policy, 18(1), 27-36.

Smallwood, R. F., Laird, A. R., Ramage, A. E., Parkinson, A. L., Lewis, J., Clauw, D. J., ... \& Robin, D. A. (2013). Structural brain anomalies and chronic pain: A quantitative meta-analysis of gray matter volume. The Journal of Pain, 14(7), 663-675.

Smith, M. A. (2012). Peer influences on drug self-administration: Social facilitation and social inhibition of cocaine intake in male rats. Psychopharmacology, 224(1), 81-90. 
Smith, M. A., Chisholm, K. A., Bryant, P. A., Greene, J. L., McClean, J. M., Stoops, W. W., \& Yancey, D. L. (2005). Social and environmental influences on opioid sensitivity in rats: Importance of an opioid's relative efficacy at the mu-receptor. Psychopharmacology, 181(1), 27-37.

Smith, P. K., Dijksterhuis, A., \& Wigboldus, D. H. (2008). Powerful people make good decisions even when they consciously think. Psychological Science.

Smith, K. E., Bunting, A. M., Golder, S., Hall, M. T., Higgins, G. E., \& Logan, T. K. (2019). Prevalence and correlates of disability among a sample of victimized women on probation and parole. Journal of Correctional Health Care, 1078345819833387.

Smith, K. E., \& Staton, M. (2018). Synthetic cannabinoid use among a sample of individuals enrolled in community-based recovery programs: Are synthetic cannabinoids actually preferred to other drugs?. Substance Abuse, 1-10.

Smith, K. E., \& Tickamyer, A. R. (2011). Economic restructuring and family well-being in rural America. University Park, PA: Penn State Press.

Smith, P. K., \& Trope, Y. (2006). You focus on the forest when you're in charge of the trees: Power priming and abstract information processing. Journal of Personality and Social Psychology, 90(4), 578.

Smoski, M. J., Lynch, T. R., Rosenthal, M. Z., Cheavens, J. S., Chapman, A. L., \& Krishnan, R. R. (2008). Decision-making and risk aversion among depressive adults. Journal of Behavior Therapy and Experimental Psychiatry, 39(4), 567-576.

Snell-Rood, C., \& Carpenter-Song, E. (2018). Depression in a depressed area: Deservingness, mental illness, and treatment in the contemporary rural US. Social Science \& Medicine, 219, 78-86.

Snider, S. E., LaConte, S. M., \& Bickel, W. K. (2016). Episodic future thinking: Expansion of the temporal window in individuals with alcohol dependence. Alcoholism: Clinical and Experimental Research, 40(7), 1558-1566.

Snijders, T. A. (2005). Power and sample size in multilevel modeling. Encyclopedia of Statistics in Behavioral Science, 3, 1570-1573.

Soares, J. M., Sampaio, A., Ferreira, L. M., Santos, N. C., Marques, F., Palha, J. A., ... \& Sousa, N. (2012). Stress-induced changes in human decision-making are reversible. Translational Psychiatry, 2(7), e131.

Social Security Administration. (2019). SSI Recipients by State and County, 2017. Retrieved January 2, 2019, from https://www.ssa.gov/policy/docs/statcomps/ssi_sc/

Social Security Administration. (2016). Annual statistical report on the Social Security Disability Insurance program, 2015 (13-11826). Retrieved from https://www.ssa.gov/policy/docs/statcomps/di_asr/

Solinas, M., Chauvet, C., Thiriet, N., El Rawas, R., \& Jaber, M. (2008). Reversal of cocaine addiction by environmental enrichment. Proceedings of the National Academy of Sciences, 105(44), 1714517150.

Solomon, A. (2014). The noonday demon: An atlas of depression. New York: Simon and Schuster.

Song, M. K., Lin, F. C., Ward, S. E., \& Fine, J. P. (2013). Composite variables: When and how. Nursing Research, 62(1), 45. 
Sönmez, M. B., Kahyacı Kılıç, E., Ateş Çöl, I., Görgülü, Y., \& Köse Çınar, R. (2017). Decreased interoceptive awareness in patients with substance use disorders. Journal of Substance Use, 22(1), 60-65.

Soobader, M., Cubbin, C., Gee, G. C., Rosenbaum, A., \& Laurenson, J. (2006). Levels of analysis for the study of environmental health disparities. Environmental Research, 102(2), 172-180.

Sorrells, S. F., Paredes, M. F., Cebrian-Silla, A., Sandoval, K., Qi, D., Kelley, K. W., ... \& Chang, E. F. (2018). Human hippocampal neurogenesis drops sharply in children to undetectable levels in adults. Nature, 555(7696), 377.

Sparks, P. J., McLaughlin, D. K., \& Stokes, C. S. (2009). Differential neonatal and postneonatal infant mortality rates across US counties: the role of socioeconomic conditions and rurality. The Journal of Rural Health, 25(4), 332-341.

Spear, L. P. (2007). The developing brain and adolescent-typical behavior patterns: An evolutionary approach. Adolescent psychopathology and the developing brain: Integrating brain and Prevention Science, 9-30.

Spears, D. (2011). Economic decision-making in poverty depletes behavioral control. The BE Journal of Economic Analysis \& Policy, 11(1).

Spencer, N., Devereux, E., Wallace, A., Sundrum, R., Shenoy, M., Bacchus, C., \& Logan, S. (2005). Disabling conditions and registration for child abuse and neglect: A population-based study. Pediatrics, 116(3), 609-613.

Spitzer, R. L., Kroenke, K., Williams, J. B., \& Löwe, B. (2006). A brief measure for assessing generalized anxiety disorder: The GAD-7. Archives of Internal Medicine, 166(10), 1092-1097.

Spreng, R. N., Mar, R. A., \& Kim, A. S. (2009). The common neural basis of autobiographical memory, prospection, navigation, theory of mind, and the default mode: A quantitative metaanalysis. Journal of Cognitive Neuroscience, 21(3), 489-510.

Sproull, N. L. (2002). Handbook of research methods: A guide for practitioners and students in the social sciences. Scarecrow Press.

Snyder, H. R., \& Hankin, B. L. (2016). Spiraling out of control: Stress generation and subsequent rumination mediate the link between poorer cognitive control and internalizing psychopathology. Clinical Psychological Science, 4(6), 1047-1064.

Stacy, A. W., Ames, S. L., Wiers, R. W., \& Krank, M. D. (2010). Associative memory in appetitive behavior: Framework and relevance to epidemiology and prevention. Handbook of Drug Use Etiology: Theory, Methods, and Empirical Findings, 165-182.

Stafford, D., LeSage, M. G., \& Glowa, J. R. (1998). Progressive-ratio schedules of drug delivery in the analysis of drug self-administration: a review. Psychopharmacology, 139(3), 169-184.

Stahl, S. T., \& Schulz, R. (2014). Changes in routine health behaviors following late-life bereavement: A systematic review. Journal of Behavioral Medicine, 37(4), 736-755.

Stanovich, K. E., \& West, R. F. (2000). Individual differences in reasoning: Implications for the rationality debate?. Behavioral and Brain Sciences, 23(5), 645-665.

Stanwood, G. D., \& Levitt, P. (2004). Drug exposure early in life: Functional repercussions of changing neuropharmacology during sensitive periods of brain development. Current Opinion in Pharmacology, 4(1), 65-71. 
StataCorp. 2017. Stata Statistical Software: Release 15. College Station, TX: StataCorp LLC

Staton M., Ciciurkaite G., Havens J., Tillson M., Leukefeld C., Webster M., ... \& Peteet B., 2018. Correlates of injection drug use among rural Appalachian women. Journal of Rural Health, 34(1), $31-41$.

Staton, M., Leukefeld, C., \& Logan, T. K. (2001). Health service utilization and victimization among incarcerated female substance users. Substance Use \& Misuse, 36(6-7), 701-715.

Staton, M., Leukefeld, C., \& Webster, J. M. (2003). Substance use, health, and mental health: Problems and service utilization among incarcerated women. International Journal of Offender Therapy and Comparative Criminology, 47(2), 224-239.

Staton, M., Strickland, J. C., Webster, J. M., Leukefeld, C., Oser, C., \& Pike, E. (2018). HIV Prevention in Rural Appalachian Jails: Implications for Re-entry Risk Reduction Among Women Who Use Drugs. AIDS and Behavior, 22(12), 4009-4018.

Staton-Tindall, M., Harp, K. L., Minieri, A., Oser, C., Webster, J. M., Havens, J., \& Leukefeld, C. (2015). An exploratory study of mental health and HIV risk behavior among drug-using rural women in jail. Psychiatric Rehabilitation Journal, 38(1), 45.

Staton-Tindall, M., Royse, D., \& Leukfeld, C. (2007). Substance use criminality, and social support: An exploratory analysis with incarcerated women. The American Journal of Drug and Alcohol Abuse, 33(2), 237-243.

Staton-Tindall M., Webster J. M., Oser C. B., Havens J. R. \& Leukefeld C. G., 2015. Drug use, hepatitis C, and service availability: Perspectives of incarcerated rural women. Social Work in Public Health, 30(4), 385-396.

Stein, D. J., van Honk, J., Ipser, J., Solms, M., \& Panksepp, J. (2007). Opioids: From physical pain to the pain of social isolation. CNS Spectrums, 12(9), 669-674.

Stein, J. S., Wilson, A. G., Koffarnus, M. N., Daniel, T. O., Epstein, L. H., \& Bickel, W. K. (2016). Unstuck in time: Episodic future thinking reduces delay discounting and cigarette smoking. Psychopharmacology, 233(21-22), 3771-3778.

Steinberg, L. (2004). Risk taking in adolescence: What changes, and why?. Annals of the New York Academy of Sciences, 1021(1), 51-58.

Steinberg, L., Albert, D., Cauffman, E., Banich, M., Graham, S., \& Woolard, J. (2008). Age differences in sensation seeking and impulsivity as indexed by behavior and self-report: Evidence for a dual systems model. Developmental Psychology, 44(6), 1764.

Steinberg, L., Graham, S., O’Brien, L., Woolard, J., Cauffman, E., \& Banich, M. (2009). Age differences in future orientation and delay discounting. Child Development, 80(1), 28-44.

Stephens, N. M., Fryberg, S. A., \& Markus, H. R. (2011). When choice does not equal freedom: A sociocultural analysis of agency in working-class American contexts. Social Psychological and Personality Science, 2(1), 33-41.

Stewart, J. L., Connolly, C. G., May, A. C., Tapert, S. F., Wittmann, M., \& Paulus, M. P. (2014). Striatum and insula dysfunction during reinforcement learning differentiates abstinent and relapsed methamphetamine-dependent individuals. Addiction, 109(3), 460-471. 
Stewart, J., De Wit, H., \& Eikelboom, R. (1984). Role of unconditioned and conditioned drug effects in the self-administration of opiates and stimulants. Psychological Review, 91(2), 251.

Stewart, K., Cao, Y., Hsu, M. H., Artigiani, E., \& Wish, E. (2017). Geospatial analysis of drug poisoning deaths involving heroin in the USA, 2000-2014. Journal of Urban Health, 94(4), 572-586.

Stockdale, S. E., Wells, K. B., Tang, L., Belin, T. R., Zhang, L., \& Sherbourne, C. D. (2007). The importance of social context: Neighborhood stressors, stress-buffering mechanisms, and alcohol, drug, and mental health disorders. Social Science \& Medicine, 65(9), 1867-1881

Stone, D. M., Simon, T. R., Fowler, K. A., Kegler, S. R., Yuan, K., Holland, K. M., ... \& Crosby, A. E. (2018). Vital signs: Trends in state suicide rates-United States, 1999-2016 and circumstances contributing to suicide—27 states, 2015. Morbidity and Mortality Weekly Report, 67(22), 617.

Stoops, W. W., Tindall, M. S., Havens, J. R., Oser, C. B., Webster, J. M., Mateyoke-Scrivner, A., ... \& Leukefeld, C. G. (2007). Kentucky rural stimulant use: A comparison of methamphetamine and other stimulant users. Journal of Psychoactive Drugs, 39(sup4), 407-417.

Stoops, W. W., Staton-Tindall, M. S., Mateyoke-Scrivner, A., \& Leukefeld, C. (2005). Methamphetamine use in nonurban and urban drug court clients. International Journal of Offender Therapy and Comparative Criminology, 49(3), 260-276.

Stover, A. N., Winstanley, E. L., Zhang, Y., \& Feinberg, J. (2018). The impact of rural classification systems on a comparison of risky drug-related behaviors in Kentucky and Ohio counties. Journal of Human Behavior in the Social Environment, 1-7.

Strait, C. E., Sleezer, B. J., \& Hayden, B. Y. (2015). Signatures of value comparison in ventral striatum neurons. PLoS biology, 13(6), e1002173.

Strickland, J. C., Wagner, F. P., Stoops, W. W., \& Rush, C. R. (2015). Profile of internet access in active cocaine users. The American Journal on Addictions, 24(7), 582-585.

Strickland, J. C., Reynolds, A. R., \& Stoops, W. W. (2016). Regulation of cocaine craving by cognitive strategies in an online sample of cocaine users. Psychology of Addictive Behaviors, 30(5), 607.

Strickland, J. C., \& Smith, M. A. (2014). The effects of social contact on drug use: Behavioral mechanisms controlling drug intake. Experimental and Clinical Psychopharmacology, 22(1), 23.

Strickland, J. C., \& Smith, M. A. (2015). Animal models of social contact and drug selfadministration. Pharmacology Biochemistry and Behavior, 136, 47-54.

Stringer, E. C. (2009). 'Keeping the faith': How incarcerated African American mothers use religion and spirituality to cope with imprisonment. Journal of African American Studies, 13(3), 325-347.

Stueve, A., \& O'donnell, L. N. (2005). Early alcohol initiation and subsequent sexual and alcohol risk behaviors among urban youths. American Journal of Public Health, 95(5), 887-893.

Stull, S. W., Panlilio, L. V., Moran, L. M., Schroeder, J. R., Bertz, J. W., Epstein, D. H., ... \& Phillips, K. A. (2019). The chippers, the quitters, and the highly symptomatic: A 12-month longitudinal study of DSM-5 opioid-and cocaine-use problems in a community sample. Addictive Behaviors, 96, 183-191.

Styron, W. (2010). Darkness visible: A memoir of madness. New York, NY: Open Road Media. 
Su, Z. I., Wenzel, J., Baird, R., \& Ettenberg, A. 2011. Comparison of self-administration behavior and responsiveness to drug-paired cues in rats running an alley for intravenous heroin and cocaine. Psychopharmacology 214: 769-778.

Sumnall, H. R., Tyler, E., Wagstaff, G. F., \& Cole, J. C. (2004). A behavioural economic analysis of alcohol, amphetamine, cocaine and ecstasy purchases by polysubstance misusers. Drug and Alcohol Dependence, 76(1), 93-99.

Surratt, H. L., Staton, M., Leukefeld, C. G., Oser, C. B., \& Webster, J. M. (2018). Patterns of buprenorphine use and risk for re-arrest among highly vulnerable opioid-involved women released from jails in rural Appalachia. Journal of Addictive Diseases, 1-4.

Sutton, R. S., \& Barto, A. G. (2018). Reinforcement learning: An introduction.Cambridge, MA: MIT press.

Subramanian, S. V., Kawachi, I., \& Kennedy, B. P. (2001). Does the state you live in make a difference? Multilevel analysis of self-rated health in the US. Social Science \& Medicine, 53(1), 9-19.

Sullivan, M. (2018). Depression Effects on Long-term Prescription Opioid Use, Abuse, and Addiction. The Clinical Journal of Pain.

Sun Y., Liu L., Feng J., Yue W., Lu L., Fan Y., \& Shi J., 2017. MAOA rs1137070 and heroin addiction interactively alter gray matter volume of the salience network. Scientific Reports 7: 45321.

Sundquist, J., Sjöstedt, C., Winkleby, M., Li, X., Kendler, K. S., \& Sundquist, K. (2016). Neighborhood linking social capital as a predictor of drug abuse: A Swedish national cohort study. Addictive Behaviors, 63, 37-44

Sutherland, R., Peacock, A., Whittaker, E., Roxburgh, A., Lenton, S., Matthews, A., ... \& Bruno, R. (2016). New psychoactive substance use among regular psychostimulant users in Australia, 2010 2015. Drug and Alcohol Dependence, 161, 110-118.

Szalavitz, M. (2017). Squaring the circle: Addiction, disease and learning. Neuroethics, 10(1), 83-86.

Szott, K. (2018). 'Heroin is the devil': Addiction, religion, and needle exchange in the rural United States. Critical Public Health, 1-11.

Tabachnick, B. G., \& Fidell, L. S. (2007). Using multivariate statistics. Boston, MA: Allyn \& Bacon/Pearson Education.

Tabassam, W., \& Grainger, J. (2002). Self-concept, attributional style and self-efficacy beliefs of students with learning disabilities with and without attention deficit hyperactivity disorder. Learning Disability Quarterly, 25(2), 141-151.

Taber, D. F., Neubert, T. D., \& Rheingold, A. L. (2002). Synthesis of (-)-morphine. Journal of the American Chemical Society, 124(42), 12416-12417.

Tai-Seale, T., \& Chandler, C. (2010). Nutrition and overweight concerns in rural areas: A literature review. Rural Healthy People, 2, 115-130.

Takahashi, T. (2004). Cortisol levels and time-discounting of monetary gain in humans. Neuroreport, 15(13), 2145-2147.

Takeuchi, K. (2011). Non-parametric test of time consistency: Present bias and future bias. Games and Economic Behavior, 71(2), 456-478. 
Tan, G., Jensen, M. P., Thornby, J. I., \& Shanti, B. F. (2004). Validation of the Brief Pain Inventory for chronic nonmalignant pain. The Journal of Pain, 5(2), 133-137.

Tapert, S. F., \& Brown, S. A. (1999). Neuropsychological correlates of adolescent substance abuse: Fouryear outcomes. Journal of the International Neuropsychological Society, 5(6), 481-493.

Tarou, L. R., \& Bashaw, M. J. (2007). Maximizing the effectiveness of environmental enrichment: Suggestions from the experimental analysis of behavior. Applied Animal Behaviour Science, 102(3-4), 189-204.

Tarter, R. E., Kirisci, L., Mezzich, A., Cornelius, J. R., Pajer, K., Vanyukov, M., ... \& Clark, D. (2003). Neurobehavioral disinhibition in childhood predicts early age at onset of substance use disorder. American Journal of Psychiatry, 160(6), 1078-1085.

Tarter, R. E., Kirisci, L., Habeych, M., Reynolds, M., \& Vanyukov, M. (2004). Neurobehavior disinhibition in childhood predisposes boys to substance use disorder by young adulthood: Direct and mediated etiologic pathways. Drug and Alcohol Dependence, 73(2), 121-132.

Tasca, M., Mulvey, P., \& Rodriguez, N. (2016). Families coming together in prison: An examination of visitation encounters. Punishment \& Society, 18(4), 459-478.

Taylor, B. G., Brownstein, H. H., Mulcahy, T. M., Fernandes-Huessy, J., Woods, D. J., \& Hafford, C. (2011). The characteristics of methamphetamine markets and their impact on communities. Criminal Justice Review, 36(3), 312-331.

Taylor, S. E., \& Lobel, M. (1989). Social comparison activity under threat: Downward evaluation and upward contacts. Psychological Review, 96(4), 569.

Teasdale, B., \& Silver, E. (2009). Neighborhoods and self-control: Toward an expanded view of socialization. Social Problems, 56(1), 205-222.

Terracciano, A., Löckenhoff, C. E., Crum, R. M., Bienvenu, O. J., \& Costa, P. T. (2008). Five-Factor Model personality profiles of drug users. BMC Psychiatry, 8(1), 22.

Tetrault, J. M., Desai, R. A., Becker, W. C., Fiellin, D. A., Concato, J., \& Sullivan, L. E. (2008). Gender and non-medical use of prescription opioids: Results from a national US survey. Addiction, 103(2), 258-268.

Thaler, R. H., \& Ganser, L. J. (2015). Misbehaving: The making of behavioral economics (p. 358). New York, NY: WW Norton.

Thaler, R. H., Sunstein, C. R., \& Balz, J. P. (2014). Choice architecture. Princeton, NJ: Princeton University Press.

Theirman Buck Law Firm. (2019, May 28). Labor Ready. Retrieved June 6, 2019, from https://thiermanbuck.com/labor-ready/

Thorne, D., Tickamyer, A., \& Thorne, M. (2004). Poverty and income in Appalachia. Journal of Appalachian Studies, 10(3), 341-357.

Thorndike, E. L. (1998). Animal intelligence: An experimental study of the associate processes in animals. American Psychologist, 53(10), 1125.

Tice, D. M., Baumeister, R. F., Shmueli, D., \& Muraven, M. (2007). Restoring the self: Positive affect helps improve self-regulation following ego depletion. Journal of Experimental Social Psychology, 43(3), 379-384. 
Tice, D. M., Bratslavsky, E., \& Baumeister, R. F. (2001). Emotional distress regulation takes precedence over impulse control: If you feel bad, do it!. Journal of Personality and Social Psychology, 80(1), 53.

Tiffany, S. T. (1999). Cognitive concepts of craving. Alcohol Research and Health, 23(3), 215-224.

Tiffany, S. T., Warthen, M. W., \& Goedeker, K. C. (2008). The functional significance of craving in nicotine dependence. In The motivational impact of nicotine and its role in tobacco use (pp. 171197). Springer, New York, NY.

Tiesman, H. M., Konda, S., Cimineri, L., \& Castillo, D. N. (2019). Drug overdose deaths at work, 20112016. Injury Prevention, injuryprev-2018.

Tobin, K. E., Davey, M. A., \& Latkin, C. A. (2005). Calling emergency medical services during drug overdose: An examination of individual, social and setting correlates. Addiction, 100(3), 397-404.

Todd, P. M., \& Gigerenzer, G. (2000). Précis of simple heuristics that make us smart. Behavioral and Brain Sciences, 23(5), 727-741.

Tomassoni, A. J., Hawk, K. F., Jubanyik, K., Nogee, D. P., Durant, T., Lynch, K. L., ... \& D’Onofrio, G. (2017). Multiple fentanyl overdoses-New Haven, Connecticut, June 23, 2016. MMWR. Morbidity and Mortality Weekly Report, 66(4), 107.

Tomer, J. F. (2001). Addictions are not rational: A socio-economic model of addictive behavior. The Journal of Socio-Economics, 30(3), 243-243.

Tomer, J. F. (2007). What is behavioral economics?. The Journal of Socio-Economics, 36(3), 463-479.

Tonkiss, F. (2005). Space, the city and social theory: Social relations and urban forms. Cambridge, UK: Polity.

Torregrossa, M. M., \& Taylor, J. R. (2016). Neuroscience of learning and memory for addiction medicine: From habit formation to memory reconsolidation. In Progress in Brain Research (Vol. 223, pp. 91-113). London, UK: Elsevier.

Tourangeau, R., Couper, M. P., \& Conrad, F. (2004). Spacing, position, and order: Interpretive heuristics for visual features of survey questions. Public Opinion Quarterly, 68(3), 368-393.

Tramullas, M., Martínez-Cué, C., \& Hurlé, M. A. (2008). Chronic administration of heroin to mice produces up-regulation of brain apoptosis-related proteins and impairs spatial learning and memory. Neuropharmacology, 54(4), 640-652.

Travis III, L. F., \& Stacey, J. (2010). A half century of parole rules: Conditions of parole in the United States, 2008. Journal of Criminal Justice, 38(4), 604-608.

Treede, R. D., Rief, W., Barke, A., Aziz, Q., Bennett, M. I., Benoliel, R., ... \& Giamberardino, M. A. (2015). A classification of chronic pain for ICD-11. Pain, 156(6), 1003.

Treloar, C., \& Abelson, J. (2005). Information exchange among injecting drug users: A role for an expanded peer education workforce. International Journal of Drug Policy, 16(1), 46-53.

Trenz, R. C., Scherer, M., Harrell, P., Zur, J., Sinha, A., \& Latimer, W. (2012). Early onset of drug and polysubstance use as predictors of injection drug use among adult drug users. Addictive Behaviors, 37(4), 367-372. 
Trescot, A. M., Datta, S., Lee, M., \& Hansen, H. (2008). Opioid pharmacology. Pain Physician, 11(2 Suppl), S133-53.

Trzesniewski, K. H., Donnellan, M., \& Lucas, R. E. (2011). Secondary data analysis: An introduction for psychologists. American Psychological Association.

Tsuang, M. T., Lyons, M. J., Meyer, J. M., Doyle, T., Eisen, S. A., Goldberg, J., ... \& Eaves, L. (1998). Cooccurrence of abuse of different drugs in men: The role of drug-specific and shared vulnerabilities. Archives of General Psychiatry, 55(11), 967-972.

Tsui, J. I., Anderson, B. J., Strong, D. R., \& Stein, M. D. (2014). Craving predicts opioid use in opioiddependent patients initiating buprenorphine treatment: A longitudinal study. The American Journal of Drug and Alcohol Abuse, 40(2), 163-169.

Tsui, J. I., Lira, M. C., Cheng, D. M., Winter, M. R., Alford, D. P., Liebschutz, J. M., ... \& Samet, J. H. (2016). Chronic pain, craving, and illicit opioid use among patients receiving opioid agonist therapy. Drug and Alcohol Dependence, 166, 26-31.

Tucker, J. A., Vuchinich, R. E., Black, B. C., \& Rippens, P. D. (2006). Significance of a behavioral economic index of reward value in predicting drinking problem resolution. Journal of Consulting and Clinical Psychology, 74(2), 317.

Turnbull, A. P. (1995). Exceptional lives: Special education in today's schools. Merrill/Prentice Hall: Old Tappan, NJ.

Turner, R. J., Wheaton, B., \& Lloyd, D. A. (1995). The epidemiology of social stress. American Sociological Review, 104-125.

Turel, O., \& Bechara, A. (2016). A triadic reflective-impulsive-interoceptive awareness model of general and impulsive information system use: Behavioral tests of neuro-cognitive theory. Frontiers in Psychology, 7, 601.

Turner, C., McClure, R., \& Pirozzo, S. (2004). Injury and risk-taking behavior-a systematic review. Accident Analysis \& Prevention, 36(1), 93-101.

Tymms, P. (2004). Effect sizes in multilevel models. The Mere, UK: National Foundation for Educational Research.

Uhl, G. R., Hall, F. S., \& Sora, I. (2002). Cocaine, reward, movement and monoamine transporters. Molecular Psychiatry, 7(1), 21.

Unick, G. J., \& Ciccarone, D. (2017). US regional and demographic differences in prescription opioid and heroin-related overdose hospitalizations. International Journal of Drug Policy, 46, 112-119.

Unick, G., Rosenblum, D., Mars, S., \& Ciccarone, D. (2014). The relationship between US heroin market dynamics and heroin-related overdose, 1992-2008. Addiction, 109(11), 1889-1898.

Urminsky, O., \& Zauberman, G. (2017). The health consequences of intertemporal preferences. Routledge International Handbook of Self-Control in Health and Well-Being, 61.

Ursache, A., Noble, K. G., \& Blair, C. (2015). Socioeconomic status, subjective social status, and perceived stress: Associations with stress physiology and executive functioning. Behavioral Medicine, 41(3), 145-154.

U.S. Census Bureau. (2019a). Income Inequality. Retrieved January 6, 2019, from https://www.census.gov/topics/income-poverty/income-inequality/data/data-tables.2016.html 
U.S. Census Bureau. (2019b). American FactFinder. Retrieved February 5, 2019, from https://factfinder.census.gov/faces/nav/jsf/pages/index.xhtml

U.S. Census Bureau. (2018, October 31). FAQs. Retrieved from https://www.census.gov/programssurveys/saipe/about/faq.html

U.S. Department of Agriculture. (2013). Measuring rurality: Rural-urban continuum codes. Retrieved from United States Department of Agriculture Economic Research Service website: https://www.ers.usda.gov/data-products/rural-urban-continuum-codes/documentation/.

U.S. Department of Health and Human Services. (2019, January 11). Poverty Guidelines. Retrieved from https://aspe.hhs.gov/poverty-guidelines

U.S. Department of Justce. (2015). Arrest Table. Retrieved February 20, 2019, from

https://ucr.fbi.gov/crime-in-the-u.s/2015/crime-in-the-u.s.-

2015/tables/arrest_table_arrests_for_drug_abuse_violations_percent_distribution_by_regions_201 5.xls

U.S. Department of Labor (2010). 2010 Standard Occupational Classification Major Groups. Retrieved from http://www.bls.gov/soc/major_groups.htm

Vadhan, N. P., Hart, C. L., Haney, M., van Gorp, W. G., \& Foltin, R. W. (2009). Decision-making in longterm cocaine users: Effects of a cash monetary contingency on Gambling task performance. Drug and Alcohol Dependence, 102(1-3), 95-101.

van Beek, Robyn Dwyer, Annie Malcolm, I. (2001). Cocaine injecting: The sharp end of drug-related harm!. Drug and Alcohol Review, 20(3), 333-342.

van Boekel, L. C., Brouwers, E. P., van Weeghel, J., \& Garretsen, H. F. (2013). Stigma among health professionals towards patients with substance use disorders and its consequences for healthcare delivery: systematic review. Drug and Alcohol Dependence, 131(1), 23-35.

van Boekel, L. C., Brouwers, E. P., van Weeghel, J., \& Garretsen, H. F. (2014). Healthcare professionals' regard towards working with patients with substance use disorders: comparison of primary care, general psychiatry and specialist addiction services. Drug \& Alcohol Dependence, 134, 92-98.

Van der Meere, J., Marzocchi, G. M., \& De Meo, T. (2005). Response inhibition and attention deficit hyperactivity disorder with and without oppositional defiant disorder screened from a community sample. Developmental Neuropsychology, 28(1), 459-472.

van Honk, J., Hermans, E. J., Putman, P., Montagne, B., \& Schutter, D. J. (2002). Defective somatic markers in sub-clinical psychopathy. Neuroreport, 13(8), 1025-1027.

Van den Bergh, O., Zacharioudakis, N., \& Petersen, S. (2018). Interoception, categorization, and symptom perception. The Interoceptive Mind: From Homeostasis to Awareness, 212.

van den Berg, R. A., Hoefsloot, H. C., Westerhuis, J. A., Smilde, A. K., \& van der Werf, M. J. (2006). Centering, scaling, and transformations: improving the biological information content of metabolomics data. BMC Genomics, 7(1), 142.

van Randenborgh, A., de Jong-Meyer, R., \& Hüffmeier, J. (2010). Decision making in depression: Differences in decisional conflict between healthy and depressed individuals. Clinical psychology \& psychotherapy, 17(4), 285-298. 
van Ree, J. M., Gerrits, M. A., \& Vanderschuren, L. J. (1999). Opioids, reward and addiction: An encounter of biology, psychology, and medicine. Pharmacological Reviews, 51(2), 341-396.

Vandaele, Y., \& Janak, P. H. (2018). Defining the place of habit in substance use disorders. Progress in Neuro-Psychopharmacology and Biological Psychiatry, 87, 22-32.

Vanderschuren, L. J., Achterberg, E. M., \& Trezza, V. (2016). The neurobiology of social play and its rewarding value in rats. Neuroscience \& Biobehavioral Reviews, 70, 86-105.

Vanderschuren, L. J., Spruijt, B. M., Hol, T., Niesink, R. J., \& Van Ree, J. M. (1995). Sequential analysis of social play behavior in juvenile rats: effects of morphine. Behavioural Brain Research, 72(1-2), 89-95.

Vansickel, A. R., Stoops, W. W., \& Rush, C. R. (2010). Human sex differences in d-amphetamine selfadministration. Addiction, 105(4), 727-731.

Vaughn, M. G., Beaver, K. M., DeLisi, M., Perron, B. E., \& Schelbe, L. (2009). Gene-environment interplay and the importance of self-control in predicting polydrug use and substance-related problems. Addictive Behaviors, 34(1), 112-116.

Venniro, M., Caprioli, D., \& Shaham, Y. (2016). Animal models of drug relapse and craving: from drug priming-induced reinstatement to incubation of craving after voluntary abstinence. Progress in Brain Research, 224, 25-52.

Venniro, M., Zhang, M., Shaham, Y., \& Caprioli, D. (2017). Incubation of methamphetamine but not heroin craving after voluntary abstinence in male and female rats. Neuropsychopharmacology, 42(5), 1126.

Venniro, M., Zhang, M., Caprioli, D., Hoots, J. K., Golden, S. A., Heins, C., ... \& Shaham, Y. (2018). Volitional social interaction prevents drug addiction in rat models. Nature Neuroscience, 21(11), 1520 .

Verdejo-García, A. (2011). Novel therapies for cognitive dysfunction secondary to substance abuse: Brief screening, referral, and cognitive rehabilitation. Psychiatric Times, 28(6), 45-45.

Verdejo-Garcia, A. (2016). Cognitive training for substance use disorders: Neuroscientific mechanisms. Neuroscience \& Biobehavioral Reviews, 68, 270-281.

Verdejo-García, A., Bechara, A., Recknor, E. C., \& Perez-Garcia, M. (2006). Executive dysfunction in substance dependent individuals during drug use and abstinence: An examination of the behavioral, cognitive and emotional correlates of addiction. Journal of the International Neuropsychological Society, 12(3), 405-415.

Verdejo-Garcia, A., Benbrook, A., Funderburk, F., David, P., Cadet, J. L., \& Bolla, K. I. (2007). The differential relationship between cocaine use and marijuana use on decision-making performance over repeat testing with the Iowa Gambling Task. Drug and Alcohol Dependence, 90(1), 2-11.

Verdejo-Garcia, A., Chong, T. T. J., Stout, J. C., Yücel, M., \& London, E. D. (2018). Stages of dysfunctional decision-making in addiction. Pharmacology Biochemistry and Behavior, 164, 99105.

Verdejo-García, A., Lawrence, A. J., \& Clark, L. (2008). Impulsivity as a vulnerability marker for substance-use disorders: Review of findings from high-risk research, problem gamblers and genetic association studies. Neuroscience \& Biobehavioral Reviews, 32(4), 777-810. 
Verdejo-García, A. J., Perales, J. C., \& Pérez-García, M. (2007). Cognitive impulsivity in cocaine and heroin polysubstance abusers. Addictive Behaviors, 32(5), 950-966.

Verdejo-García, A., Rivas-Pérez, C., Vilar-López, R., \& Pérez-García, M. (2007). Strategic self-regulation, decision-making and emotion processing in poly-substance abusers in their first year of abstinence. Drug and Alcohol Dependence, 86(2-3), 139-146.

Visher, C. A., Knight, C. R., Chalfin, A., \& Roman, J. K. (2009). The impact of marital and relationship status on social outcomes for returning prisoners. Washington, DC: Urban Institute.

Vivolo-Kantor, A. M., Seth, P., Gladden, R. M., Mattson, C. L., Baldwin, G. T., Kite-Powell, A., \& Coletta, M. A. (2018). Vital signs: Trends in emergency department visits for suspected opioid overdoses-United States, July 2016-September 2017. Morbidity and Mortality Weekly Report, 67(9), 279.

Vogt Yuan, A. S. (2010). Black-White differences in aging out of substance use and abuse. Sociological Spectrum, 31(1), 3-31.

Vohs, K. D., \& Baumeister, R. F. (Eds.). (2016). Handbook of self-regulation: Research, theory, and applications. New York, NY: Guilford Publications.

Volkow, N. D. (2014). America's addiction to opioids: Heroin and prescription drug abuse. Senate Caucus on International Narcotics Control, 14.

Volkow, N. D., Baler, R. D., \& Goldstein, R. Z. (2011). Addiction: Pulling at the neural threads of social behaviors. Neuron, 69(4), 599-602.

Volkow, N. D., Chang, L., Wang, G. J., Fowler, J. S., Franceschi, D., Sedler, M., ... \& Logan, J. (2001). Loss of dopamine transporters in methamphetamine abusers recovers with protracted abstinence. Journal of Neuroscience, 21(23), 9414-9418.

Volkow, N. D., \& Collins, F. S. (2017). The role of science in addressing the opioid crisis. New England Journal of Medicine, 377(4), 391-394.

Volkow, N. D., Fowler, J. S., Wolf, A. P., Hitzemann, R., Dewey, S., Bendriem, B., ... \& Hoff, A. (1991). Changes in brain glucose metabolism in cocaine dependence and withdrawal. American Journal of Psychiatry, 148(5), 621-626.

Volkow, N. D., Frieden, T. R., Hyde, P. S., \& Cha, S. S. (2014). Medication-assisted therapies-Tackling the opioid-overdose epidemic. New England Journal of Medicine, 370(22), 2063-2066.

Volkow, N. D., \& Koob, G. (2015). Brain disease model of addiction: Why is it so controversial?. The Lancet Psychiatry, 2(8), 677-679.

Volkow, N. D., \& Li, T. K. (2004). Drug addiction: The neurobiology of behaviour gone awry. Nature Reviews Neuroscience, 5(12), 963.

Volkow, N. D., \& McLellan, A. T. (2016). Opioid abuse in chronic pain-misconceptions and mitigation strategies. New England Journal of Medicine, 374(13), 1253-1263.

Vosburg, S. K., Haynes, C., Besharat, A., \& Green, J. L. (2017). Changes in drug use patterns reported on the web after the introduction of ADF OxyContin: Findings from the Researched Abuse, Diversion, and Addiction-Related Surveillance (RADARS) System web monitoring program. Pharmacoepidemiology and Drug Safety, 26(9), 1044-1052. 
Vosburg, S. K., Jones, J. D., Manubay, J. M., Ashworth, J. B., Shapiro, D. Y., \& Comer, S. D. (2013). A comparison among tapentadol tamper-resistant formulations (TRF) and $\mathrm{O}$ xy $\mathrm{C}$ ontin ${ }^{(n)}$ (non-TRF) in prescription opioid abusers. Addiction, 108(6), 1095-1106.

Votaw, V. R., Wittenauer, J., Connery, H. S., Weiss, R. D., \& McHugh, R. K. (2017). Perceived risk of heroin use among nonmedical prescription opioid users. Addictive Behaviors, 65, 218-223.

Vowles, K. E., McEntee, M. L., Julnes, P. S., Frohe, T., Ney, J. P., \& van der Goes, D. N. (2015). Rates of opioid misuse, abuse, and addiction in chronic pain: A systematic review and data synthesis. Pain, 156(4), 569-576.

Vuchinich, R. E., \& Heather, N. (Eds.). (2003). Choice, behavioral economics, and addiction. London, UK: Elsevier.

Vuchinich, R. E., \& Simpson, C. A. (1998). Hyperbolic temporal discounting in social drinkers and problem drinkers. Experimental and Clinical Psychopharmacology, 6(3), 292.

Wade-Galuska, T., Winger, G., \& Woods, J. H. (2007). A behavioral economic analysis of cocaine and remifentanil self-administration in rhesus monkeys. Psychopharmacology, 194(4), 563-572.

Wagenmakers, E. J., van der Maas, H. L., \& Farrell, S. (2012). Abstract concepts require concrete models: Why cognitive scientists have not yet embraced nonlinearly coupled, dynamical, self-organized critical, synergistic, scale-free, exquisitely context-sensitive, interaction-dominant, multifractal, interdependent brain-body-niche systems. Topics in Cognitive Science, 4(1), 87-93.

Wagner, K. D., Armenta, R. F., Roth, A. M., Maxwell, J. C., Cuevas-Mota, J., \& Garfein, R. S. (2014). Use of synthetic cathinones and cannabimimetics among injection drug users in San Diego, California. Drug and Alcohol Dependence, 141, 99-106.

Wahler, E. A. (2015). Social disadvantage and economic hardship as predictors of follow-Up addiction severity after substance abuse treatment: Does referral to treatment by the criminal justice system matter?. Alcoholism Treatment Quarterly, 33(1), 6-27.

Waldorf, D., Reinarman, C., \& Murphy, S. (1992). Cocaine changes: The experience of using and quitting (Vol. 49). Philadelphia, PA: Temple University Press.

Walker, R., Godlaski, T. M., \& Staton-Tindall, M. (2013). Spirituality, drugs, and alcohol: A philosophical analysis. Substance Use \& Misuse, 48(12), 1233-1245.

Walker, E. R., Pratt, L. A., Schoenborn, C. A., \& Druss, B. G. (2017). Excess mortality among people who report lifetime use of illegal drugs in the United States: A 20-year follow-up of a nationally representative survey. Drug and Alcohol Dependence, 171, 31-38.

Walker, R., Hiller, M., Staton, M., \& Leukefeld, C. G. (2003). Head injury among drug abusers: An indicator of co-occurring problems. Journal of Psychoactive Drugs, 35(3), 343-353.

Walker, R., Logan, T. K., Clark, J. J., \& Leukefeld, C. (2005). Informed consent to undergo treatment for substance abuse: A recommended approach. Journal of Substance Abuse Treatment, 29(4), 241251.

Walker, R., Staton, M., \& Leukefeld, C. G. (2001). History of head injury among substance users: Preliminary findings. Substance Use \& Misuse, 36(6-7), 757-768.

Walker, S. P., Wachs, T. D., Grantham-McGregor, S., Black, M. M., Nelson, C. A., Huffman, S. L., ... \& Gardner, J. M. M. (2011). Inequality in early childhood: Risk and protective factors for early child development. The Lancet, 378(9799), 1325-1338. 
Wallace, D., Fahmy, C., Cotton, L., Jimmons, C., McKay, R., Stoffer, S., \& Syed, S. (2016). Examining the role of familial support during prison and after release on post-incarceration mental health. International Journal of Offender Therapy and Comparative Criminology, 60(1), 3-20.

Walsh, S. L., Stoops, W. W., Moody, D. E., Lin, S. N., \& Bigelow, G. E. (2009). Repeated dosing with oral cocaine in humans: Assessment of direct effects, withdrawal, and pharmacokinetics. Experimental and Clinical Psychopharmacology, 17(4), 205.

Walter, M., Gerber, H., Kuhl, H. C., Schmid, O., Joechle, W., Lanz, C., ... \& Borgwardt, S. J. (2013). Acute effects of intravenous heroin on the hypothalamic-pituitary-adrenal axis response: A controlled trial. Journal of Clinical Psychopharmacology, 33(2), 193-198.

Walter, M., Denier, N., Gerber, H., Schmid, O., Lanz, C., Brenneisen, R., ... \& McGuire, P. (2015). Orbitofrontal response to drug-related stimuli after heroin administration. Addiction Biology, 20(3), 570-579.

Walter, M., Wiesbeck, G. A., Degen, B., Albrich, J., Oppel, M., Schulz, A., ... \& Dürsteler-MacFarland, K. M. (2011). Heroin reduces startle and cortisol response in opioid-maintained heroin-dependent patients. Addiction Biology, 16(1), 145-151.

Walter, C., Knothe, C., \& Lötsch, J. (2016). Abuse-deterrent opioid formulations: Pharmacokinetic and pharmacodynamic considerations. Clinical Pharmacokinetics, 55(7), 751-767.

Walters, G. D., \& Urban, H. (2014). Age of substance use onset as a predictor of early adult substance dependence and offending in male and female delinquents: Simple and mediated moderation. Journal of Drug Issues, 44(4), 442-456.

Walteros, C., Sánchez-Navarro, J. P., Muñoz, M. A., Martínez-Selva, J. M., Chialvo, D., \& Montoya, P. (2011). Altered associative learning and emotional decision making in fibromyalgia. Journal of Psychosomatic Research, 70(3), 294-301.

Walton, M. A., Blow, F. C., Bingham, C. R., \& Chermack, S. T. (2003). Individual and social/environmental predictors of alcohol and drug use 2 years following substance abuse treatment. Addictive Behaviors, 28(4), 627-642.

Wang, B., Shaham, Y., Zitzman, D., Azari, S., Wise, R. A., \& You, Z. B. (2005). Cocaine experience establishes control of midbrain glutamate and dopamine by corticotropin-releasing factor: A role in stress-induced relapse to drug seeking. Journal of Neuroscience, 25(22), 5389-5396.

Wang, G., Shi, J., Chen, N., Xu, L., Li, J., Li, P., ... \& Lu, L. (2013). Effects of length of abstinence on decision-making and craving in methamphetamine abusers. PloS One, 8(7), e68791.

Waninger, K. N., Gotsch, P. B., Watts, D., \& Thuahnai, S. T. (2008). Use of lemon juice to increase crack cocaine solubility for intravenous use. Journal of Emergency Medicine, 34(2), 207-209.

Wanous, J. P., \& Hudy, M. J. (2001). Single-item reliability: A replication and extension. Organizational Research Methods, 4(4), 361-375.

Wanous, J. P., \& Reichers, A. E. (1996). Estimating the reliability of a single-item measure. Psychological Reports, 78(2), 631-634.

Wanous, J. P., Reichers, A. E., \& Hudy, M. J. (1997). Overall job satisfaction: How good are single-item measures?. Journal of Applied Psychology, 82(2), 247. 
Ward, L., Mathias, J. L., \& Hitchings, S. E. (2007). Relationships between bereavement and cognitive functioning in older adults. Gerontology, 53(6), 362-372.

Ward, A., \& Mann, T. (2000). Don't mind if I do: Disinhibited eating under cognitive load. Journal of Personality and Social Psychology, 78(4), 753.

Warner-Smith, M., Darke, S., \& Day, C. (2002). Morbidity associated with non-fatal heroin overdose. Addiction, 97(8), 963-967.

Warner, M., Trinidad, J. P., Bastian, B. A., Miniño, A. M., \& Hedegaard, H. (2016). Drugs most frequently involved in drug overdose deaths: United States, 2010-2014.

Warner-Smith, M., Darke, S., Lynskey, M., \& Hall, W. (2001). Heroin overdose: Causes and consequences. Addiction, 96(8), 1113-1125.

Wasan, A. D., Ross, E. L., Michna, E., Chibnik, L., Greenfield, S. F., Weiss, R. D., \& Jamison, R. N. (2012). Craving of prescription opioids in patients with chronic pain: A longitudinal outcomes trial. The Journal of Pain, 13(2), 146-154.

Watson, J., Crawley, J., \& Kane, D. (2016). Social exclusion, health and hidden homelessness. Public Health, 139, 96-102.

Webster, B. S., Cifuentes, M., Verma, S., \& Pransky, G. (2009). Geographic variation in opioid prescribing for acute, work-related, low back pain and associated factors: A multilevel analysis. American Journal of Industrial Medicine, 52(2), 162-171.

Webster, J. M., Dickson, M. F., Mannan, F., \& Staton, M. (2018). Characteristics of prescription-opioidimpaired and other substance-impaired drivers in rural Appalachian Kentucky. Journal of Psychoactive Drugs, 50(5), 373-381.

Webster, J. M., Pimentel, J. H., \& Clark, D. B. (2008). Characteristics of DUI offenders convicted in wet, dry, and moist counties. Accident Analysis \& Prevention, 40(3), 976-982.

Webster, L. R., Johnson, F. K., Stauffer, J., Setnik, B., \& Ciric, S. (2011). Impact of intravenous naltrexone on intravenous morphine-induced high, drug liking, and euphoric effects in experienced, nondependent male opioid users. Drugs in $R \& D, 11(3), 259-275$.

Wei, Y. J. J., Zhu, Y., Liu, W., Bussing, R., \& Winterstein, A. G. (2018). Prevalence of and factors associated with long-term concurrent use of stimulants and opioids among adults with attentiondeficit/hyperactivity disorder. JAMA Network Open, 1(4), e181152-e181152.

Wen, H., Hockenberry, J. M., Borders, T. F., \& Druss, B. G. (2017). Impact of Medicaid expansion on Medicaid-covered utilization of buprenorphine for opioid use disorder treatment. Medical Care, 55(4), 336-341.

Weich, S., \& Lewis, G. (1998). Poverty, unemployment, and common mental disorders: Population based cohort study. BMJ, 317(7151), 115-119.

Weidner, R. R. (2009). Methamphetamine in three small Midwestern cities: Evidence of a moral panic. Journal of Psychoactive Drugs, 41(3), 227-239.

Weisheit, R. A., \& Brownstein, H. (2016). Drug production in the rural context. Routledge International Handbook of Rural Criminology, 235-244.

Weiss, F. (2005). Neurobiology of craving, conditioned reward and relapse. Current Opinion in Pharmacology, 5(1), 9-19. 
Werts, C. E., \& Linn, R. L. (1970). Cautions in applying various procedures for determining the reliability and validity of multiple-item scales. American Sociological Review, 35(4), 757-759.

Wertz, J. S., Cleaveland, B. L., \& Stephens, R. S. (1995). Problems in the application of the Addiction Severity Index (ASI) in rural substance abuse services. Journal of Substance Abuse, 7(2), 175-188.

West, S. L., \& O'Neal, K. K. (2004). Project DARE outcome effectiveness revisited. American Journal of Public Health, 94(6), 1027-1029.

Western, B., Braga, A. A., Davis, J., \& Sirois, C. (2015). Stress and hardship after prison. American Journal of Sociology, 120(5), 1512-1547.

Wheeler, E., Davidson, P. J., Jones, T. S., \& Irwin, K. S. (2012). Community-based opioid overdose prevention programs providing naloxone-United States, 2010. MMWR. Morbidity and Mortality Weekly Report, 61(6), 101.

White, J. M. (2004). Pleasure into pain: the consequences of long-term opioid use. Addictive Behaviors, 29(7), 1311-1324.

Wiens, T. K., \& Walker, L. J. (2015). The chronic disease concept of addiction: Helpful or harmful?. Addiction Research \& Theory, 23(4), 309-321.

Wiers, R. W., Field, M., \& Stacy, A. W. (2016). Passion's slave?: Conscious and unconscious cognitive processes in alcohol and drug abuse. The Oxford Handbook of Substance Use and Substance Use Disorders, 1, 311-350.

Wildberger, J. I., John, C. N., \& Hallock, R. M. (2017). Perceptions of the medicinal value of hallucinogenic drugs among college students. Journal of Psychedelic Studies, 1(2), 50-54.

Wildt, A. R., Ahtola, O. T., H. (1978). Analysis of covariance (Vol. 12). Sage. Newbury Park, CA.

Wilens, T. E., Adler, L. A., Adams, J., Sgambati, S., Rotrosen, J., Sawtelle, R., ... \& Fusillo, S. (2008). Misuse and diversion of stimulants prescribed for ADHD: A systematic review of the literature. Journal of the American Academy of Child \& Adolescent Psychiatry, 47(1), 21-31.

Wilkinson, R. G., \& Marmot, M. (Eds.). (2003). Social determinants of health: The solid facts. World Health Organization.

Wilkinson, R. G., \& Pickett, K. E. (2009). Income inequality and social dysfunction. Annual Review of Sociology, 35, 493-511.

Williams, L., \& Parker, H. (2001). Alcohol, cannabis, ecstasy and cocaine: Drugs of reasoned choice amongst young adult recreational drug users in England. International Journal of Drug Policy, 12(5-6), 397-413.

Williams, M. V., Palar, K., \& Derose, K. P. (2011). Congregation-based programs to address HIV/AIDS: Elements of successful implementation. Journal of Urban Health, 88(3), 517-532.

Wilson, S. J. (Ed.). (2015). The Wiley handbook on the cognitive neuroscience of addiction. Hoboken, NJ: John Wiley \& Sons.

Wilson, A. M., Deri Armstrong, C., Furrie, A., \& Walcot, E. (2009). The mental health of Canadians with self-reported learning disabilities. Journal of Learning Disabilities, 42(1), 24-40. 
Wilson, R. J., Ryerson, A. B., Singh, S. D., \& King, J. B. (2016). Cancer incidence in Appalachia, 20042011. Cancer Epidemiology and Prevention Biomarkers, 25(2), 250-258.

Wingo, T., Nesil, T., Choi, J. S., \& Li, M. D. (2016). Novelty seeking and drug addiction in humans and animals: From behavior to molecules. Journal of Neuroimmune Pharmacology, 11(3), 456-470.

Wittmann, M. (2016). Felt time: The psychology of how we perceive time. Mit Press.

Wittmann, M. (2018). Altered States of Consciousness: Experiences out of time and self. Cambridge, MA: MIT Press.

Wittmann, M., \& Paulus, M. P. (2008). Decision making, impulsivity and time perception. Trends in Cognitive Sciences, 12(1), 7-12.

Wolffgramm, J., \& Heyne, A. (1995). From controlled drug intake to loss of control: The irreversible development of drug addiction in the rat. Behavioural Brain Research, 70(1), 77-94.

Wollman, S. C., Alhassoon, O. M., Hall, M. G., Stern, M. J., Connors, E. J., Kimmel, C. L., ... \& Radua, J. (2017). Gray matter abnormalities in opioid-dependent patients: A neuroimaging metaanalysis. The American Journal of Drug and Alcohol Abuse, 43(5), 505-517.

Wood, S. C., \& Anagnostaras, S. G. (2009). Memory and psychostimulants: Modulation of Pavlovian fear conditioning by amphetamine in C57BL/6 mice. Psychopharmacology, 202(1-3), 197-206.

Wood, W., \& Neal, D. T. (2007). A new look at habits and the habit-goal interface. Psychological review, 114(4), 843.

Wood, W., \& Rünger, D. (2016). Psychology of habit. Annual Review of Psychology, 67, 289-314.

Wood, T. O. (1904). The Repetition of Prescriptions containing Dangerous Drugs. British Medical Journal, 1(2247), 215.

Woolf, S. H., Schoomaker, H., Hill, L., \& Orndahl, C. M. (2019). The social determinants of health and the decline in US life expectancy: Implications for Appalachia. Journal of Appalachian Health, l(1), 2 .

Woolverton, W. L., \& Johnson, K. M. (1992). Neurobiology of cocaine abuse. Psychopharmacology, 86, 280.

Worley, J. (2012). Prescription drug monitoring programs, a response to doctor shopping: Purpose, effectiveness, and directions for future research. Issues in Mental Health Nursing, 33(5), 319-328.

Worley, M. J., Shoptaw, S. J., Bickel, W. K., \& Ling, W. (2015). Using behavioral economics to predict opioid use during prescription opioid dependence treatment. Drug and Alcohol Dependence, 148, 62-68.

Wright, C. J. (2012). Becoming to remain: Community college students and post-secondary pursuits in central Appalachia. Journal of Research in Rural Education, 27(6).

Wu, L. T., Blazer, D. G., Patkar, A. A., Stitzer, M. L., Wakim, P. G., \& Brooner, R. K. (2009). Heterogeneity of stimulant dependence: A national drug abuse treatment clinical trials network study. American Journal on Addictions, 18(3), 206-218.

Wu, L. T., \& Blazer, D. G. (2011). Illicit and nonmedical drug use among older adults: A review. Journal of Aging and Health, 23(3), 481-504. 
Yamamoto, T., Dargan, P. I., Dines, A., Yates, C., Heyerdahl, F., Hovda, K. E., ... \& Euro-DEN Research Group. (2019). Concurrent use of benzodiazepine by heroin users-What are the prevalence and the risks associated with this pattern of use?. Journal of Medical Toxicology, 15(1), 4-11.

Yan, W. S., Li, Y. H., Xiao, L., Zhu, N., Bechara, A., \& Sui, N. (2014). Working memory and affective decision-making in addiction: A neurocognitive comparison between heroin addicts, pathological gamblers and healthy controls. Drug and Alcohol Dependence, 134, 194-200.

Yarnell, S. C. (2015). Cocaine abuse in later life: A case series and review of the literature. The Primary Care Companion for CNS Disorders, 17(2).

Yates, J. R., Bardo, M. T., \& Beckmann, J. S. (2017). Environmental enrichment and drug value: A behavioral economic analysis in male rats. Addiction Biology.

Yen, I. H., \& Kaplan, G. A. (1999). Poverty area residence and changes in depression and perceived health status: Evidence from the Alameda County Study. International Journal of Epidemiology, 28(1), 90-94.

Yeung, N., \& Summerfield, C. (2012). Metacognition in human decision-making: Confidence and error monitoring. Philosophical Transactions of the Royal Society B: Biological Sciences, 367(1594), $1310-1321$

Yokell, M., D Zaller, N., C Green, T., \& D Rich, J. (2011). Buprenorphine and buprenorphine/naloxone diversion, misuse, and illicit use: An international review. Current Drug Abuse Reviews, 4(1), 2841.

Vivolo-Kantor, A. M., Seth, P., Gladden, R. M., Mattson, C. L., Baldwin, G. T., Kite-Powell, A., \& Coletta, M. A. (2018). Vital signs: Trends in emergency department visits for suspected opioid overdoses-United States, July 2016-September 2017. Morbidity and Mortality Weekly Report, 67(9), 279.

Young, A. M., Havens, J. R., \& Leukefeld, C. G. (2010). Route of administration for illicit prescription opioids: A comparison of rural and urban drug users. Harm Reduction Journal, 7(1), 24.

Young, A. M., Havens, J. R., \& Leukefeld, C. G. (2012). A comparison of rural and urban nonmedical prescription opioid users' lifetime and recent drug use. The American Journal of Drug and Alcohol Abuse, 38(3), 220-227.

Young, S., \& Thome, J. (2011). ADHD and offenders. The World Journal of Biological Psychiatry, 12(sup1), 124-128.

Youngblut, J. M., \& Casper, G. R. (1993). Focus on psychometrics single-item indicators in nursing research. Research in Nursing \& Health, 16(6), 459-465.

Yurasek, A. M., Aston, E. R., \& Metrik, J. (2017). Co-use of alcohol and cannabis: A review. Current Addiction Reports, 4(2), 184-193.

Zanis, D. A., McLellan, A. T., \& Corse, S. (1997). Is the Addiction Severity Index a reliable and valid assessment instrument among clients with severe and persistent mental illness and substance abuse disorders?. Community Mental Health Journal, 33(3), 213-227.

Zanis, D. A., McLellan, A. T., \& Randall, M. (1994). Can you trust patient self-reports of drug use during treatment?. Drug and Alcohol Dependence, 35(2), 127-132.

Zapata, A., Minney, V. L., \& Shippenberg, T. S. (2010). Shift from goal-directed to habitual cocaine seeking after prolonged experience in rats. Journal of Neuroscience, 30(46), 15457-15463. 
Zapolski, T. C., Baldwin, P., Banks, D. E., \& Stump, T. E. (2017). Does a crossover age effect exist for African American and Hispanic binge drinkers? Findings from the 2010 to 2013 National Study on Drug Use and Health. Alcoholism: Clinical and Experimental Research, 41(6), 1129-1136.

Zauberman, G., Ratner, R. K., \& Kim, B. K. (2008). Memories as assets: Strategic memory protection in choice over time. Journal of Consumer Research, 35(5), 715-728.

Zauberman, G., Kim, B. K., Malkoc, S. A., \& Bettman, J. R. (2009). Discounting time and time discounting: Subjective time perception and intertemporal preferences. Journal of Marketing Research, 46(4), 543-556.

Zeng, H., Lee, T. M., Waters, J. H., So, K. F., Sham, P. C., Schottenfeld, R. S., ... \& Chawarski, M. C. (2013). Impulsivity, cognitive function, and their relationship in heroin-dependent individuals. Journal of Clinical and Experimental Neuropsychology, 35(9), 897-905.

Zernike, K. (2006). Potent Mexican meth floods in as states curb domestic variety. New York Times, 1(23), 06.

Zernig, G., Kummer, K. K., \& Prast, J. M. (2013). Dyadic social interaction as an alternative reward to cocaine. Frontiers in Psychiatry, 4, 100.

Zernig, G., Giacomuzzi, S., Riemer, Y., Wakonigg, G., Sturm, K., \& Saria, A. (2003). Intravenous drug injection habits: Drug users' self-reports versus researchers' perception. Pharmacology, 68(1), 4956.

Zhu, M., \& Ratner, R. K. (2015). Scarcity polarizes preferences: The impact on choice among multiple items in a product class. Journal of Marketing Research, 52(1), 13-26.

Zijlstra, F., Veltman, D. J., Booij, J., van den Brink, W., \& Franken, I. H. (2009). Neurobiological substrates of cue-elicited craving and anhedonia in recently abstinent opioid-dependent males. Drug and Alcohol Dependence, 99(1-3), 183-192.

Zibbell J. E., Iqbal K., Patel R. C., Suryaprasad A., Sanders K. J., Moore-Moravian L., ... \& Holtzman D., 2015. Increases in hepatitis $C$ virus infection related to injection drug use among persons aged $\leq 30$ years-Kentucky, Tennessee, Virginia, and West Virginia, 2006-2012. MMWR. Morbidity Mortality Weekly Report 64(17), 453-458.

Zibbell, J. E., Asher, A. K., Patel, R. C., Kupronis, B., Iqbal, K., Ward, J. W., \& Holtzman, D. (2018). Increases in acute hepatitis $C$ virus infection related to a growing opioid epidemic and associated injection drug use, United States, 2004 to 2014. American Journal of Public Health, 108(2), 175181.

Zinberg, N. E. (1984). Drug, set, and setting: The basis for controlled intoxicant use. New Haven, CT: Yale University Press.

Zinberg, N. E., Harding, W. M., \& Winkeller, M. (1977). A study of social regulatory mechanisms in controlled illicit drug users. Journal of Drug Issues, 7(2), 117-133.

Zinberg, N. E., Jacobson, R. C., \& Harding, W. M. (1975). Social sanctions and rituals as a basis for drug abuse prevention. The American journal of drug and alcohol abuse, 2(2), 165-182.

Zlebnik, N. E., \& Carroll, M. E. (2015). Prevention of the incubation of cocaine seeking by aerobic exercise in female rats. Psychopharmacology, 232(19), 3507-3513. 
Zlotnick, C., Najavits, L. M., Rohsenow, D. J., \& Johnson, D. M. (2003). A cognitive-behavioral treatment for incarcerated women with substance abuse disorder and posttraumatic stress disorder: Findings from a pilot study. Journal of Substance Abuse Treatment, 25(2), 99-105.

Zoorob, M. J. (2018). Polydrug epidemiology: Benzodiazepine prescribing and the drug overdose epidemic in the United States. Pharmacoepidemiology and Drug Safety, 27(5), 541-549.

Zoorob, M. J., \& Salemi, J. L. (2017). Bowling alone, dying together: the role of social capital in mitigating the drug overdose epidemic in the United States. Drug and Alcohol Dependence, 173, 1-9.

Zuckerman, S. L., Lee, Y. M., Odom, M. J., Solomon, G. S., \& Sills, A. K. (2013). Baseline neurocognitive scores in athletes with attention deficit-spectrum disorders and/or learning disability. Journal of Neurosurgery: Pediatrics, 12(2), 103-109 
APPENDIX A

Institutional Authorization Agreement Study Renewal Letter 


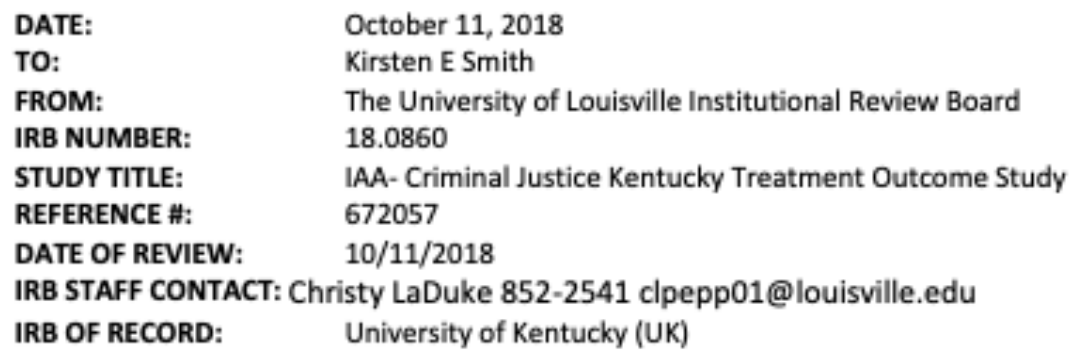

The continuation request was received by the Human Subjects Protection Program Office, reviewed by the HSPPO staff and was found to be complete.

UK IRB is the IRB of Record for this study. Thank you for submitting the updated IRB Continuation Approval letter with the new expiration listed as $9 / 17 / 2019$. Please submit the continuing review application in iRIS prior to the study expiration date.

This letter will be kept on file with the Human Subjects Protection Program office. All direct correspondence with the other institution involved should be kept with the study records by the Principal Investigator.

As a reminder, the investigator is responsible for ensuring the reporting requirements to the external IRB are met.

In addition, local University of Louisville IRB reporting is required for:

- Local personnel/Investigator changes

- Submitting the Continuation Approval letter upon renewal of the study by the external IRB

- Serious Issues of Non-Compliance

- Unanticipated Problems (UPIRSTOs)

- Data Safety Monitoring Reports that indicate safety concerns (e.g. suspending treatment, placing enrollment on hold)

- Study Closure

If you have any questions, please contact the IRB analyst listed above or the Human Subjects Protection Program office at hsppofc@louisulle.edu.

$$
\text { Chusty laphe }
$$

Christy LaDuke, MA, CCRP

Associate Director, HSPPO 
APPENDIX B

Participant Counties of Residence Frequencies 
Figure 3.0. Participant counties of residence prior to incarceration with $\leq 10$ observations per county

Green, Oldham, Robertson, Washington, Woodford

Bracken, Edmonson, Henry, Jackson, Leslie, Trimble

Anderson, Bourbon, Caldwell, Carlisle, Clinton, Crittenden, Cumberland, Knott, Lyon, McLean, Trigg, Wolfe

Allen, Estill, Hickman, Johnson, Larue, Livingston, Martin, Metcalfe, Morgan, Powell

Ballard, Garrard, Lee, Lewis, McCreary, Magoffin, Meade, Monroe, Owen, Owsley, Todd, Webster

Breathitt, Butler, Elliott, Fleming, Pendleton, Simpson, Spencer, Taylor, Union

Adair, Bath, Boyle, Clay, Fulton, Lawrence, Letcher, Menifee, Shelby

Breckinridge, Casey, Franklin, Grayson, Harrison, Nicholas, Pike

Gallatin, Harlan, Rockcastle, Scott

Barren, Floyd, Grant, Graves, Lincoln, Marion, Russell

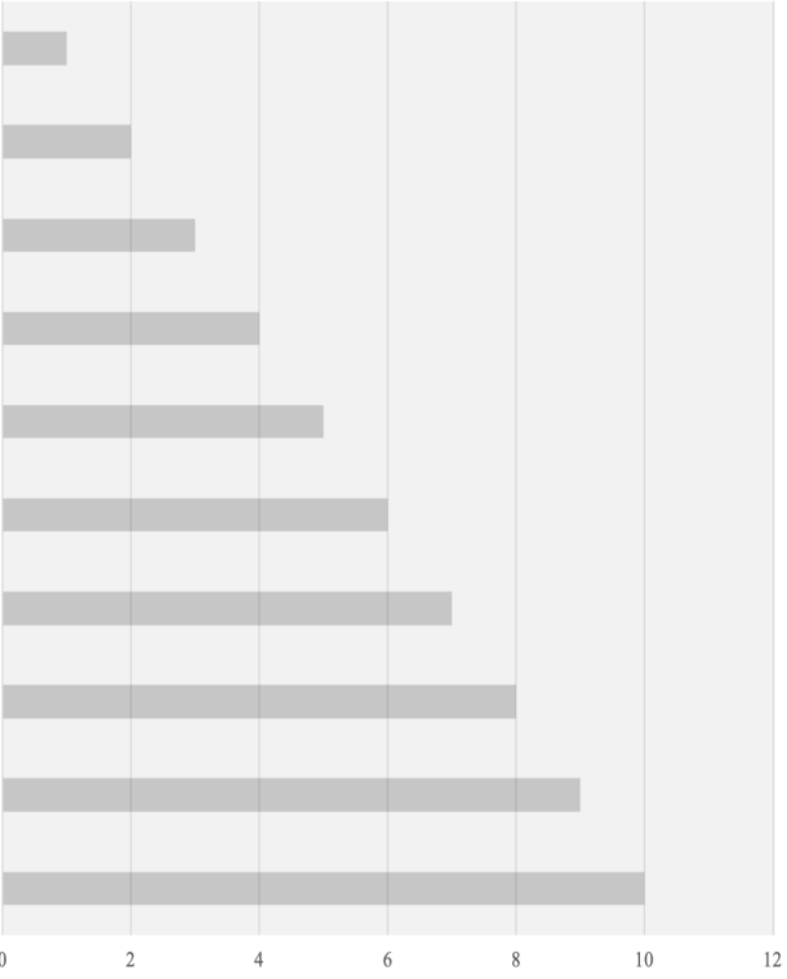


Figure 3.1. Participant counties of residence subsequent to incarceration with $\leq 10$ observations per county

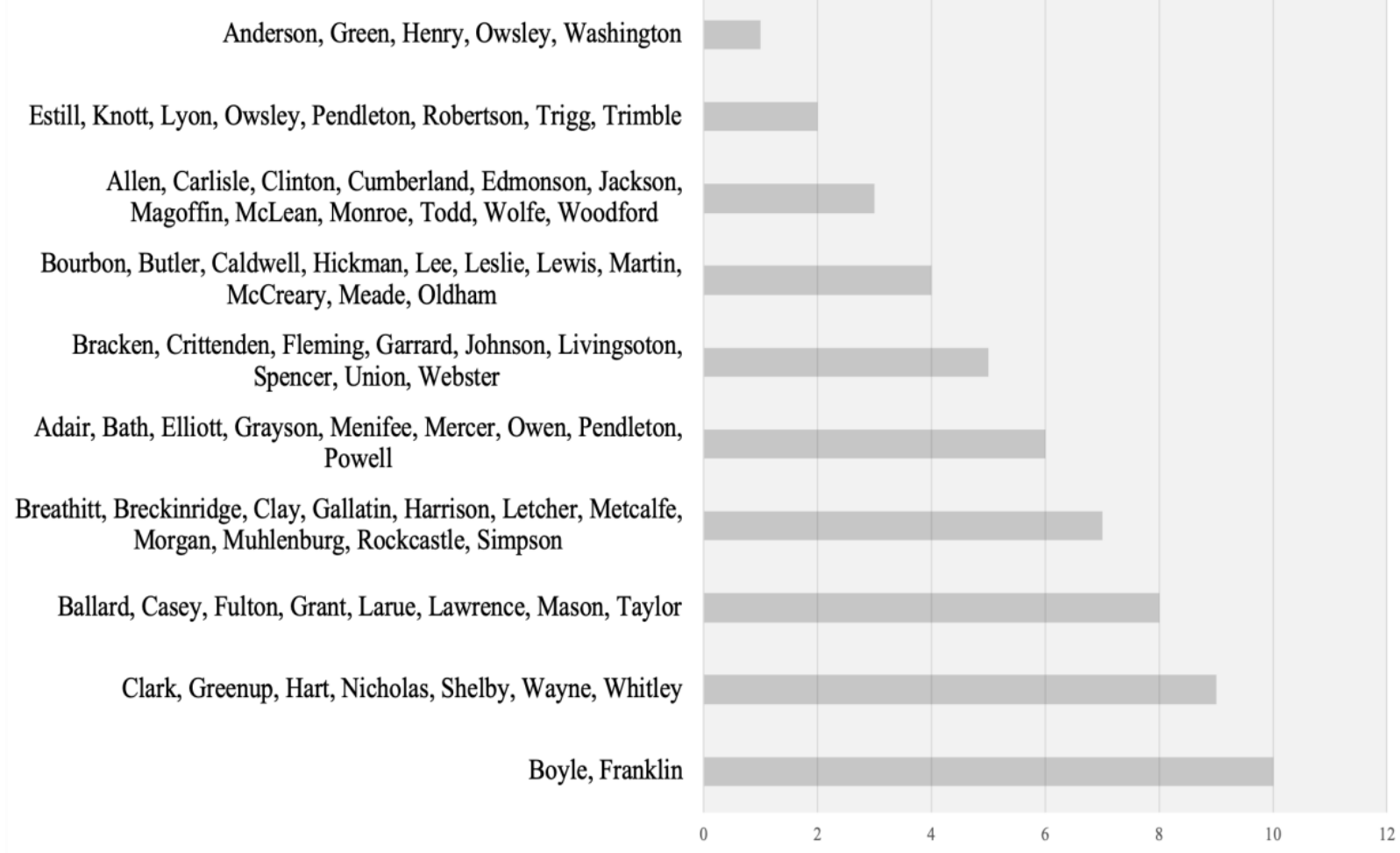


Figure 4.0

Participant counties of residence prior to incarceration with $>10$ observations per county

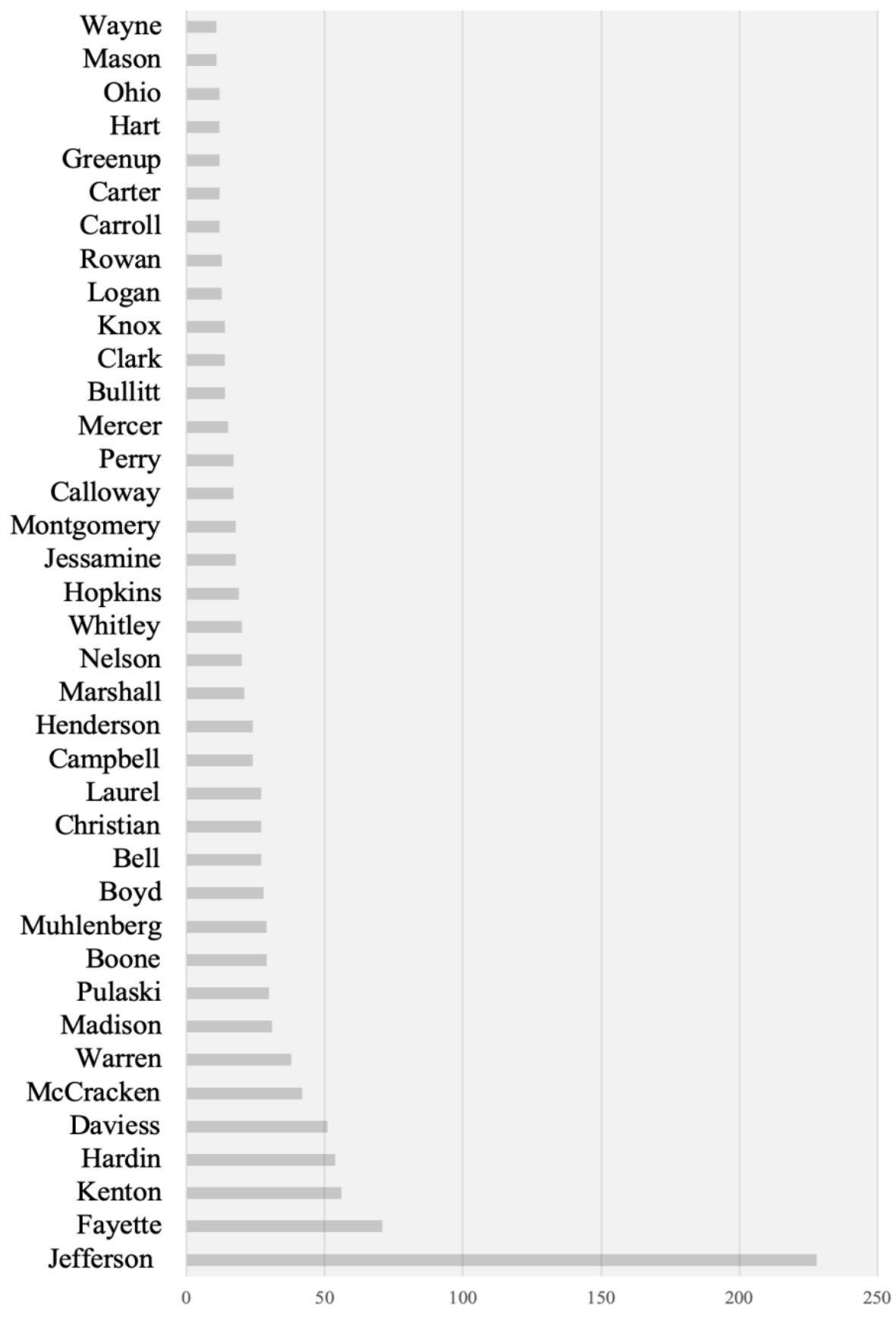


Figure 4.1. Participant counties of residence subsequent to incarceration with $>10$ observations per county.

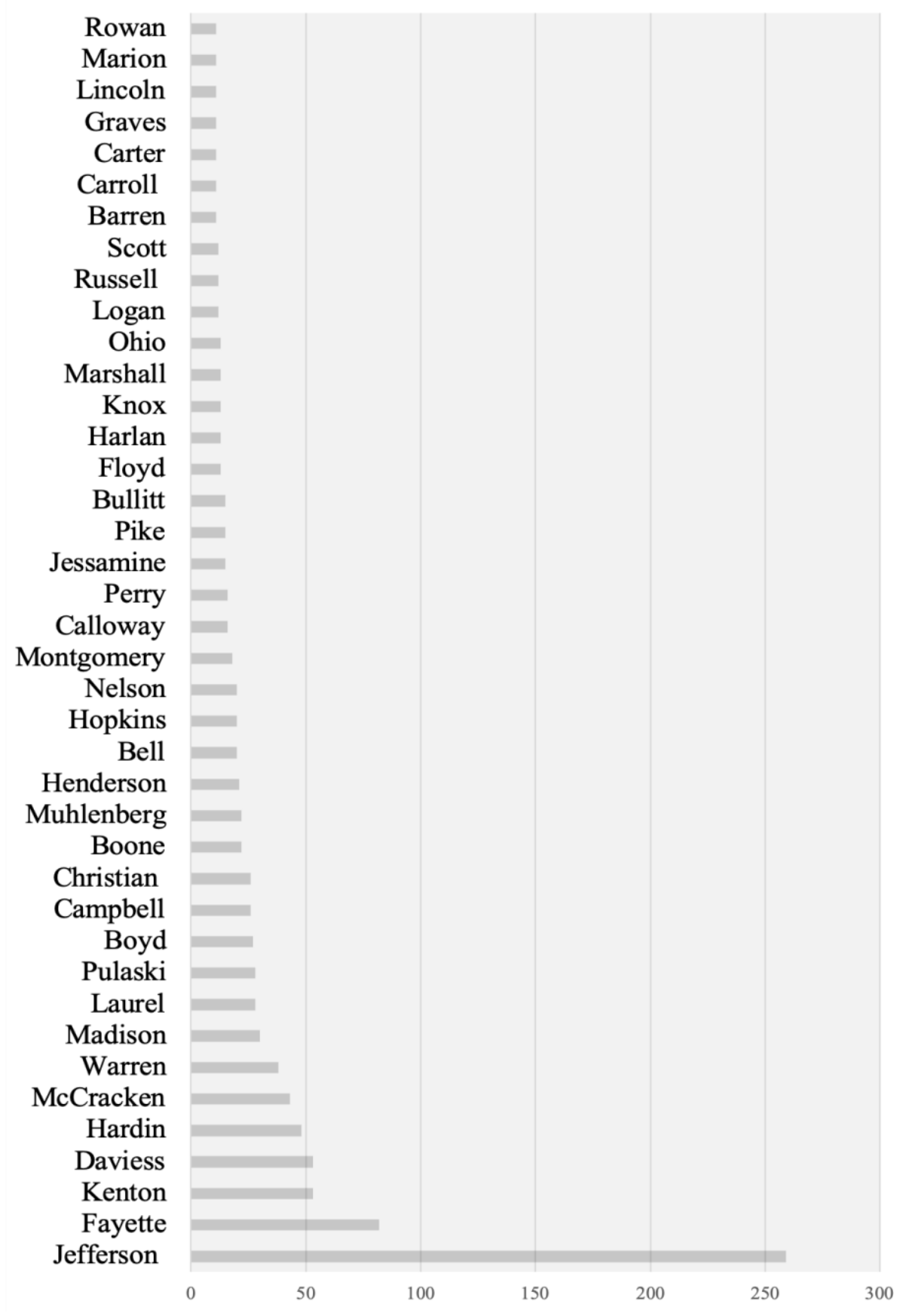




\section{CURRICULUM VITA}

Kirsten Elin Smith, M.S.W.

kirstenelin.smith@louisville.edu

859-475-2021

\section{EDUCATION}

Doctoral Candidate, Kent School of Social Work, University of Louisville

2016-2019

Post-Baccalaureate Independent Study, University of Kentucky

Department of Behavioral Science, Lexington, Kentucky

Master's Social Work, University of Kentucky

Lexington, Kentucky

Summa Cum Laude

Bachelor's Social Work, Minor in History, University of Kentucky

2014

Lexington, Kentucky

Summa Cum Laude

\section{$\underline{\text { RESEARCH }}$}

(PI) Lawson, T, Smith, K. E. Alternative psychoactive substances: Prevalence and emerging trends in a sample of drug-using individuals enrolled in community-based treatment (2017-2018). This study sought to examine lifetime and recent use of kratom, novel psychoactive substances, and traditional drugs of abuse (e.g., alcohol, nicotine, cocaine).

\section{PUBLICATIONS}

\section{PUBLISHED:}

Smith, K. E., Bunting, A., Walker, R., Hall, M. T., Grundmann, O., Castillo, O. (2019). Non-prescribed buprenorphine use mediates the relationship between recent heroin and kratom use among a sample of polysubstance users. Journal of Psychoactive Drugs. 
Smith, K. E., Stoops, W. W. (2019). Synthetic cathinone use among a sample of polysubstance users: Indirect indicator of indiscriminate drug taking or preferred drug of abuse? Journal of Drug Issues, 49(2), 369-386.

Smith, K. E., Bunting, A. M., Golder, S., Hall, M. T., Higgins, G. E., Logan, TK. (2019). Prevalence and correlates of disability among a sample of victimized women on probation and parole. Journal of Correctional Healthcare, 33(3), 250-256.

Smith, K. E., Staton, M. (2018). Synthetic cannabinoid use among a sample of individuals enrolled in community-based recovery programs: Are synthetic cannabinoids actually preferred to other drugs? Substance Abuse, 1-10.

Smith, K. E. (2018). Prevalence and correlates of electronic cigarette use among a clinical sample of polysubstance users in Kentucky: Long live the cigarette? Substance Use and Misuse, 54(2), 225-235.

Smith, K. E., \& Lawson, T. (2017). Prevalence and motivations for kratom use in a sample of substance users enrolled in a residential treatment program. Drug and Alcohol Dependence, 180, 340-348.

Dickson, M. F., Staton-Tindall, M., Smith, K. E., Leukefeld, C., Webster, J. M., \& Oser, C. B. (2017). A Facebook follow-up strategy for rural drug-using women. The Journal of Rural Health, 33(3), 250-256.

Smith, K. E., Bunting, A., Staton, M., Walker, J. R., Shalash, S., McNees, E., \& Pangburn, K. (2017). Examination of synthetic cannabinoid and cathinone use in drug-using offender sample 2013-2015. Journal of Psychoactive Drugs, 49(5), 436-445.

\section{SUBMITTED FOR REVIEW:}

Garcia-Romeu, A., Dunn, K. E., Smith, K. E., Griffiths, R. R. Kratom (Mitragyna speciosa): User demographics, use patterns, and implications for the opioid epidemic.

Under review: Drug and Alcohol Dependence.

Bath, R., Buchholz, T., Buros, A. F., Singh, D., Smith, K. E., Veltri, C. A., Grundmann, O. Self-reported health diagnoses and demographic correlates with Kratom use: Results from an online survey. Journal of Addiction Medicine.

Pope, N. D., Baldwin, P. K., Gibson, A., Smith, K E. Exploring How Young adult caregivers adopt family caregiving roles. Journal of Intergenerational Relationships.

Victor, G., Smith, K. E., Walker, J. R., Logan, T. K, Cole, J. Residential peer-led recovery program: An exploratory examination of psychosocial outcomes.

Under review: American Journal of Drug and Alcohol Abuse

\section{IN PREPARATION:}

Garcia-Romeu, A., Dunn, K. E., Smith, K. E., \& Griffiths, R. R. An online survey of kratom (Mitragyna speciosa) users: Motivations for use and perceived therapeutic potentials

Smith, K. E., Tillson, M., Staton, M., \& Winston, E. Diverted buprenorphine use among adults entering corrections-based treatment.

Smith, K. E. Ethical analysis: Potential harms associated with peer-led, 12-step recovery approaches for addressing opioid use disorder in the United States 


\section{PRESENTATIONS}

Center for Clinical \& Translational Science Spring Conference (April 2016). Lexington, KY. Poster Presentation: Profile of synthetic drug users in corrections-based substance abuse treatment program. Smith, K. E., Shalash, S., Staton-Tindall, M., McNees, E., \& Pangburn, K.

Society for Social Work Research Conference (January 2018). Washington, DC. Poster Presentation: Prevalence and features of Social Security Disability Insurance (SSDI) utilization in a sample of women on community corrections. Smith, K. E., Bunting, A., Golder, S., Hall, M., Higgins, G. E., \& Logan, T. K.

Graduate Student Regional Research Conference (March 2018). Louisville, KY. Oral Presentation: Profile of kratom use among a sample of polysubstance users enrolled in residential drug treatment. Smith, K. E.

Academic and Health Policy Conference on Correctional Health (March 2018). Houston, TX. Poster Presentation: Prevalence and correlates of disability among a sample of women on probation and parole. Smith, K. E., Bunting, A., Golder, S., Hall, M., Higgins, G. E., \& Logan, T. K.

College on Problems of Drug Dependence 2018 Annual Meeting (June 2018). San Diego, CA. Oral Presentation: Kratom use patterns among opioid users in the United States: A novel form of harmreduction? Smith, K. E., Bunting, A. M., Walker, R., Hall, M., Grundmann, O, Castillo, O.

College on Problems of Drug Dependence 2018 Annual Meeting (June 2018). San Diego, CA. Media Forum Oral Presentation: Kratom use patterns among opioid users in the United States: A novel form of harm-reduction? Smith, K. E., Bunting, A. M., Walker, R., Hall, M., Grundmann, O, Castillo, O.

Appalachian Translational Research Network Summit. (September 2018). Lexington, KY. Poster Presentation: A profile of 'diverted' buprenorphine use among treatment-seeking offenders: Implications for Appalachian Kentucky. Tillson, M., Smith, K. E., Staton, M., Winston, E.

Substance Use Research Day. (March 2019). Lexington, KY. Poster Presentation: Chemical bedfellows: Illicit use of prescription opioids and buprenorphine associated with methamphetamine use among a sample of polydrug users in Kentucky. Smith, K. E.

Academic and Health Policy Conference on Correctional Health (March 2019). Las Vegas, NV. Oral Presentation: Prevalence and correlates of past-year non-prescribed buprenorphine use among a sample of adults entering Kentucky Department of Corrections drug treatment programs. Smith, K. E., Tillson, M., Staton, M., Winston, E.

\section{Chaired/co-chaired sessions and panels:}

Chilcoat H., \& Smith, K. E. Oral Communications: Is there a downside? Harm reduction. College on Problems of Drug Dependence 2018 annual meeting (June 2018). San Diego, CA. 
PUBLICATIONS REVIEWED \& CONSULTING

Reviewer, Addiction [2]

2018-2019

Reviewer, Drug and Alcohol Dependence [1]

2018

Reviewer, Journal of Psychoactive Drugs [5]

2017-2019

Reviewer, Journal of Ethnopharmacology [2]

2017-2018

Reviewer, Neuropsychiatric Disease and Treatment [1]

2018

Reviewer, Substance Abuse Treatment, Prevention, and Policy [1]

2017-2018

Reviewer, Journal of Pain Research [2]

2018

The Commonwealth of Kentucky's Department of Public Advocacy:

2016-2018

Death penalty mitigation case reviews

\section{PROFESSIONAL EXPERIENCE}

INTRAMURAL RESEARCH TRAINING AWARD,

2019-

POST-DOCTORAL FELLOW

National Institutes of Health, National Institute on Drug Abuse

Real-world Assessment, Prediction, and Treatment Unit,

Clinical Pharmacology and Therapeutics Research Branch

Baltimore, Maryland

DATA COORDINATOR/ANALYST

2018-2019

University of Kentucky, Center on Drug and Alcohol Research

Lexington, Kentucky

Major Responsibilities: Conducted baseline interviews and consolidated tracking and locating efforts for post-intervention follow-up data collection among a clinical sample of substance users residing in rural Appalachia. Track and locate difficult-to-reach participants for follow-up data collection as part of the Criminal Justice Kentucky Treatment Outcome Study (CJKTOS), an ongoing study examining effectiveness of the KY Department of Correction's (DOC) Substance Abuse Treatment Program (SAP) and KY DOC-referred medication-assisted treatment pilot programs. Assisted in data management and analyses for five ongoing outcome evaluations. Prepared annual technical reports and manuscripts for academic publication.

RESEARCH ASSISTANT

2016-2018

Kent School of Social Work, University of Louisville

Louisville, Kentucky

Major Responsibilities: To fulfill objectives for a SAMHSA-funded grant, conducted follow-up interviews pertaining to substance use, mental health, victimization, and perinatal/postnatal health behaviors among a sample of drug-using women exiting residential treatment. Oversaw data collection tasks of 1 Masters-level student. Assisted with statistical analyses and manuscript preparation using longitudinal Women's Health Study (NIDA R01DA027981) data which captures health behaviors of women on probation/parole with a history of victimization.

SENIOR DATA COORDINATOR

2015-2016

University of Kentucky, Center on Drug and Alcohol Research

Lexington, Kentucky

Major Responsibilities: Tracked and located difficult-to-reach study participants for post-intervention data collection as part of the Criminal Justice Kentucky Treatment Outcome Study (CJKTOS), an ongoing study 
examining effectiveness of the KY DOC SAP program. Conducted follow-up interviews with paroled and incarcerated individuals. Drafted annual reports for state legislators. Trained DOC clinicians to conduct baseline assessments of incarcerated SAP participants. Created a pilot assessment instrument for DOC clinical training workgroups on substance use intervention practices. Assisted in data management, analyses, and manuscript preparation.

RESEARCH ASSISTANT

2016

University of Kentucky College of Social Work, Lexington, Kentucky

Major Responsibilities: Helped prepare academic presentations and publications on issues pertaining to substance use, incarceration, health care policy, and use of social media in conducting research with hardto-reach populations. Assisted with transcribing, coding, and rating data obtained under an ongoing project concerned with harm-reduction interventions for rural, female substance users involved with the criminal justice system (NIDA R01DA033866 \& K02DA035116).

MASTER'S PRACTICUM

2014-2015

Hope Center Recovery Program for Men, Lexington, Kentucky

Major Responsibilities: Conducted strengths-based, trauma-informed biopsychosocial assessments for incoming clients. Founded and facilitated weekly process group and led classes for Phase I clients. Created curriculum for and taught a monthly psychoeducational class pertaining to the neurobiology of addiction. Established therapeutic alliances with clients and collaboratively explored issues related to reentry, relapse prevention, and strategies for improving self-efficacy.

UNDERGRADUATE PRACTICUM

United Way of the Bluegrass, Lexington, Kentucky

Major Responsibilities: Worked as part of an interdisciplinary financial stability team to assist economically marginalized clients with establishing individualized development accounts (IDAs). Revised UWBG participant follow-up survey. Collected and analyzed UWBG participant data and prepared for quarterly and annual reports. Conducted client orientations, helped participants complete individualized savings plans and establish financial goals. Coordinated financial literacy and education opportunities with community partners. Participated in community outreach events and grant reviews.

UNDERGRADUATE PRACTICUM

2013

Chrysalis House, Inc. Lexington, Kentucky

Major Responsibilities: Assisted in client admission screening, intake. Conducted psychosocial and drug history assessment. Assisted with weekly home visits at off-site housing and administered urine analysis tests. Facilitated weekly cognitive behavioral therapy group. Updated electronic client files with progress notes and assessed for relapse risk. Helped prepare clients for GED testing, job-seeking, and independent living.

TEACHING

Teaching Practicum

University of Louisville, Kent School of Social Work

Louisville, Kentucky

SW 662-75 Substance Use and Substance Use Disorders 
ACADEMIC AND PROFESSIONAL AWARDS

Academic and Health Policy Conference on Correctional Health

Travel award

College on Problems of Drug Dependence

Early Career Investigator travel award

Society for Social Work \& Research

2018

Travel award

Academic and Health Policy Conference on Correctional Health

Travel award

University of Louisville

2016-2018

Doctoral scholarship \& Research assistantship

Idea Festival Scholarship award

University of Kentucky

Virginia Lane Award, full undergraduate scholarship

University of Kentucky

Virginia Lane Award, full undergraduate scholarship

University of Kentucky

Two-time recipient of the Academic Excellence Scholarship

\section{FUNDING}

University of Louisville Graduate Student Research

Fund Grant Recipient

\section{MEMBERSHIPS \& AFFILIATIONS}

College on Problems of Drug Dependence, member-in training Academic Consortium on Criminal Justice Health

American Society of Criminology

Society for Social Work and Research

Sociologists for Women in Society

Students for Sensible Drug Policy, University of Kentucky

National Association of Social Workers

Center for Inquiry

American Humanist Association

2018-Present 2017-Present 2017-Present 2017-2019

2016-Present 2015-2016 2014-2016 2016-Present 2015-Present

\section{SERVICE WORK}

Volunteer instructor, Hope Center Women's Recovery Program

2018-2019

Bluegrass Reentry Council

2016-2018

Disability Resource Center

2016-2017

Guest lecturer, University of Kentucky College of Social Work

2014-2016

Volunteer, United Way of the Bluegrass

2014-2015

Volunteer, AIDS Volunteers of America Inc.

2013

Volunteer instructor, Hope Center Men's Recovery Program

2012-2019

Volunteer, Lexington Rescue Mission, Lexington, KY

2012 